\title{
Justyna Jaroszewska \\ Das Instrument der Direktzahlungen in der Agrarpolitik der USA und der EU
}

Eine Konvergenzanalyse 

Justyna Jaroszewska

Das Instrument der Direktzahlungen in der Agrarpolitik der USA und der EU

Dieses Werk ist lizenziert unter einer

Creative Commons

Namensnennung - Weitergabe unter gleichen Bedingungen

4.0 International Lizenz.

(c) (i) (2) 
erschienen im Universitätsverlag Göttingen 2018 
Justyna Jaroszewska

Das Instrument der Direktzahlungen in der Agrarpolitik der USA und der EU

Eine Konvergenzanalyse

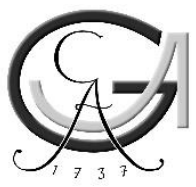

Universitätsverlag Göttingen 2018 


\section{Bibliographische Information der Deutschen Nationalbibliothek}

Die Deutsche Nationalbibliothek verzeichnet diese Publikation in der Deutschen Nationalbibliographie; detaillierte bibliographische Daten sind im Internet über $<$ http://dnb.dnb.de $>$ abrufbar.

Dieses Buch ist auch als freie Onlineversion über die Homepage des Verlags sowie über den Göttinger Universitätskatalog (GUK) bei der Niedersächsischen Staats- und Universitätsbibliothek Göttingen (http://www.sub.uni-goettingen.de) erreichbar. Es gelten die Lizenzbestimmungen der Onlineversion.

Satz und Layout: Annett Eichstaedt Umschlaggestaltung: Margo Bargheer

(C) 2018 Universitätsverlag Göttingen https:/ / univerlag.uni-goettingen.de ISBN: 978-3-86395-391-1

DOI: https://doi.org/10.17875/gup2018-1117 


\section{Danksagung}

Für die Chance, extern promovieren zu können, und für seine Anleitung während der drei Jahre der Erstellung dieser Arbeit danke ich meinem Doktorvater und ersten Gutachter, Prof. Dr. Stephan von Cramon-Taubadel. Prof. Dr. Stefan Tangermann gebührt mein Dank für das Überlassen dieses hochinteressanten Themas, für wertvolle Anregungen und die Zeit, die er meiner Arbeit gewidmet hat, einschließlich der Erstellung der Korreferate und des Gutachtens. Der Austausch mit ihm hat mich fachlich und auch persönlich sehr bereichert.

Ganz besonders möchte ich mich bei meinem Vorgesetzten, Dr. Thomas Kirchberg, bedanken. Ohne seine großzügige Unterstützung wäre diese Arbeit schlicht nicht möglich gewesen. Joachim Kellermann von Schele danke ich herzlich für die Durchsicht dieser Abhandlung und die vielfältigen Aufmunterungen, die vor allem in schwierigeren Phasen für einen willkommenen Ausgleich gesorgt haben. Ein ganz großes Dankeschön geht auch an Gabriele Retterath für die freundliche Fürsorge und dass sie nie müde war, diese Arbeit Korrektur zu lesen. Anette Radowski danke ich herzlich für ihre verständnisvolle Unterstützung bei Südzucker. 
Über den gesamten Arbeitszeitraum hinweg bekam ich sehr viel Zuspruch, der mich immer wieder darin bestärkte weiterzumachen. Ich danke allen, die mit mir mitgefiebert haben.

Meinen Eltern danke ich dafür, dass sie stets - und auch bei diesem Projekt an mich geglaubt und mich unterstützt haben. Ihnen widme ich diese Arbeit. 


\section{Inhaltsverzeichnis}

Danksagung................................................................................................. I

Inhaltsverzeichnis......................................................................................... III

Tabellenverzeichnis ............................................................................ VII

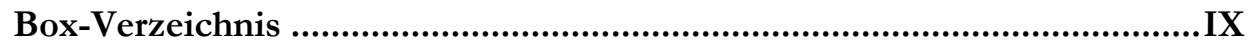

Abkürzungsverzeichnis .................................................................................XI

1 Forschungsfrage: Analyse der Konvergenz der Agrarpolitiken der USA und der EU hinsichtlich der Direktzahlungen.......................................... 1

2 Theoretischer Analyserahmen .................................................................. 7

2.1 Klärung der Begriffe der staatlichen Zahlungen und der Direktzahlungen ........................................................................................... 8

2.2 Konvergenztheorie als Analyserahmen.......................................................... 14

2.3 Literaturauswertung.................................................................................. 17

2.4 Forschungskonzept und Aufbau der Arbeit.................................................. 21 
3 Das Instrument der Direktzahlungen in der Wissenschaft ...................... 25

3.1 Ursprung des Instruments der staatlichen Zahlungen ................................... 26

3.2 Wissenschaftliche Konzepte in den USA …………….................................... 30

3.3 Wissenschaftliche Konzepte in den USA: Zusammenfassung..................... 42

3.4 Wissenschaftliche Konzepte in der EU.......................................................... 45

3.5 Wissenschaftliche Konzepte in der EU: Zusammenfassung .........................58

3.6 Direktzahlungen in der Wissenschaft der USA und der EU: Fazit ............. 59

4 Geschichte der staatlichen Zahlungen in den USA.................................. 63

4.1 Anfänge der staatlichen Zahlungen ................................................................. 63

4.2 Abkehr von der Preisstützung: Ausgleichszahlungen ..................................... 85

4.3 Einführung der Direktzahlungen .................................................................. 96

4.4 Teilweise Abkehr vom Entkopplungskurs ………………………………... 98

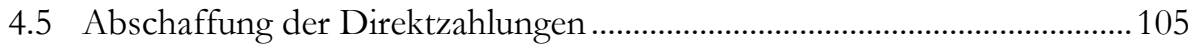

5 Geschichte der staatlichen Zahlungen in der EU .................................. 113

5.1 Großbritannien als Vorreiter...........................................................................113

5.2 Anfänge der staatlichen Zahlungen ..............................................................118

5.3 Direktzahlungen in der EU: Der Weg dorthin ...........................................127

5.4 Abkehr von der Preisstützung: Ausgleichszahlungen ...................................136

5.5 Einführung der Direktzahlungen ............................................................... 147

5.6 Beibehaltung der Direktzahlungen................................................................153

6 Vergleich der Bedeutung der Direktzahlungen für die Landwirtschaft

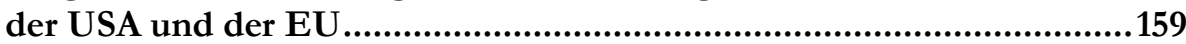

7 Auswahl der Milestones in der Entwicklung der Direktzahlungen beidseits des Atlantiks ..........................................................................177

7.1 Ausgewählte Milestones in den USA.......................................................178

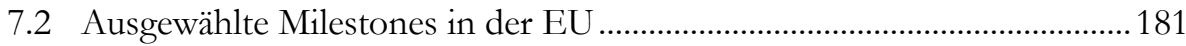

7.3 Verlauf des Konvergenzprozesses zwischen den USA und der EU in Bezug auf die Direktzahlungen 
8 Zusammenstellung der für die Milestones relevanten Einflussfaktoren 189

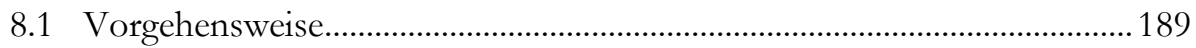

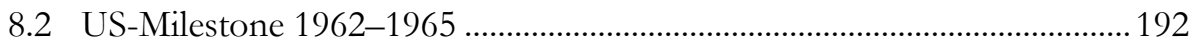

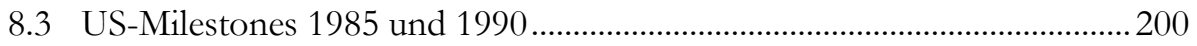

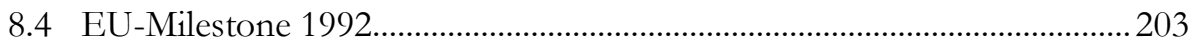

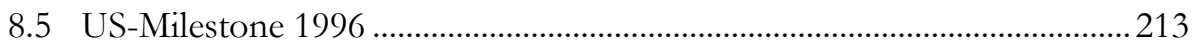

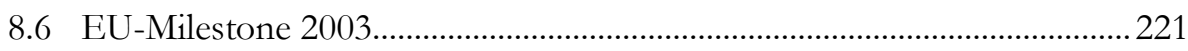

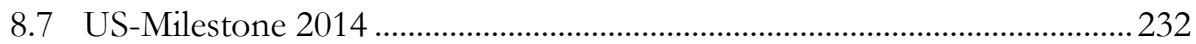

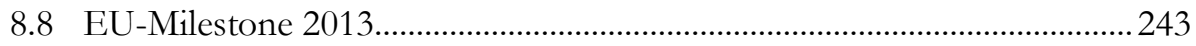

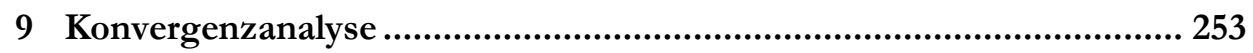

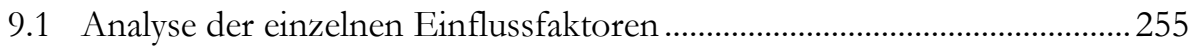

9.2 Paarvergleich der Milestones: Gemeinsamkeiten und Unterschiede ........ 263

9.3 Analyse der Konvergenzmuster ............................................................... 270

9.3.1 Untersuchung der Kausalmechanismen der Politikkonvergenz.... 270

9.3.2 Untersuchung der intervenierenden Faktoren ............................... 274

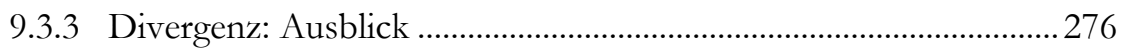

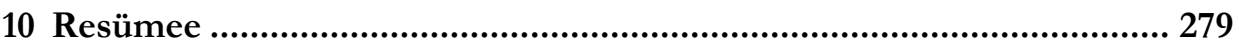

Literaturverzeichnis ................................................................... 285

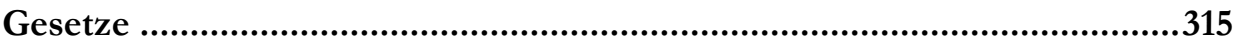

Anhang: Übersicht über die Höhe der Agrarzahlungen in den USA im

Zeitablauf.....................................................................................321

Tabelle A1: Gesamtbetrag der staatlichen Zahlungen an Landwirte in den

USA seit 1932 (ausgedrückt in jeweiligen Preisen) ..................................... 321

Tabelle A2: Gesamtbetrag der primären Zahlungstypen in den USA seit 1933 (ausgedrückt in jeweiligen Preisen) ....................................................... 325

Tabelle A3: Gesamtbetrag der primären Zahlungstypen in den USA seit 1933 (ausgedrückt in Preisen von 2009) . 328 



\section{Tabellenverzeichnis}

Tabelle 1: Höhe der Stützung für Weizen und Mais in den USA 1954-1966 .........88

Tabelle 2: „Deficiency payments“-System für Weizen in den USA 1978-1994 ....94

Tabelle 3: „PFC payments“-System für Weizen in den USA 1996-2002 ................98

Tabelle 4: „Direct payments“-System für Weizen in den USA 2003-2008 ...........100

Tabelle 5: Gesamtbetrag der Ausgleichszahlungen in den betreffenden EULändern 1968-1970

Tabelle 6: Gesamtausgaben für Marktmaßnahmen und Direktbeihilfen bei pflanzlichen Erzeugnissen vs. Ausgaben für Direktbeihilfen in der EU in ausgewählten Jahren

Tabelle 7: Anteil der gekoppelten Direktzahlungen an den gesamten

EU-Direktzahlungen im Zeitablauf.

Tabelle 8: Gekoppelte Direktzahlungen und ihre Budgets laut dem EU-

Haushaltsplan 2016

Tabelle 9: Budget der Farm Bill von 2014 zum Zeitpunkt der Verabschiedung .161

Tabelle 10: Tatsächliche Ausgaben für „commodity payments“ und die Ernteversicherung in den USA 2000-2015.

Tabelle 11: Ausgaben für die einzelnen Arten von „commodity payments“ in den USA 1996-2016.

Tabelle 12: Durch ,government payments“ begünstigte Betriebe in den USA im Zeitablauf 
Tabelle 13: Durch „conservation payments“ begünstigte Betriebe in den USA im Zeitablauf

Tabelle 14: Verteilung von „commodity payments“ in den USA je nach

Betriebsgröße 2012

Tabelle 15: Von EU-Direktzahlungen begünstigte Betriebe 2014

Tabelle 16: Zahlungen für Agrarumweltmaßnahmen in ausgewählten EU-Mitgliedstaaten 2007-2012

Tabelle 17: Durchschnitts- und Medianeinkommen landwirtschaftlicher

Haushalte im Vergleich zum Einkommen aller Haushalte in den USA im

Zeitablauf (zu laufenden Preisen)

Tabelle 18: Entwicklung des PSE in der EU und den USA in Prozent der

Bruttoeinnahmen der Landwirtschaft seit dem Jahr 1986

Tabelle 19: Entwicklung der Zahlungen auf der Grundlage historischer Werte

(Produktion nicht erforderlich) in der EU und den USA seit dem Jahr 1986

Tabelle 20: Die ausgewählten Milestones in der Entwicklung der

Direktzahlungen in den USA und in der EU

Tabelle 21: Der US-Reformen 1962-1965 als Ganzes und dem Milestone zugrunde liegende Faktoren.

Tabelle 22: Der US-Reformen 1985 und 1990 als Ganzes und den Milestones zugrunde liegende Faktoren.

Tabelle 23: Der EU-Reform von 1992 als Ganzes und dem Milestone zugrunde liegende Faktoren.

Tabelle 24: Der US-Reform von 1996 als Ganzes und dem Milestone zugrunde liegende Faktoren.....

Tabelle 25: Der EU-Reform von 2003 als Ganzes und dem Milestone zugrunde liegende Faktoren.

Tabelle 26: Der US-Reform von 2014 als Ganzes und dem Milestone zugrunde liegende Faktoren....

Tabelle 27: Der EU-Reform von 2013 als Ganzes und dem Milestone zugrunde liegende Faktoren.

Tabelle 28: Zusammenstellung der für die ausgewählten Milestones relevanten Einflussfaktoren.

Tabelle A1: Gesamtbetrag der staatlichen Zahlungen an Landwirte in den USA seit 1932 (ausgedrückt in jeweiligen Preisen).

Tabelle A2: Gesamtbetrag der primären Zahlungstypen in den USA seit 1933 (ausgedrückt in jeweiligen Preisen)

Tabelle A3: Gesamtbetrag der primären Zahlungstypen in den USA seit 1933 (ausgedrückt in Preisen von 2009). 


\section{Box-Verzeichnis}

Box 1: Konzept der „direct income payments“ der OECD .......................................12

Box 2: Agrarzahlungen in frühen Konzepten des Wissenschaftlichen Beirats beim Bundeslandwirtschaftsministerium .....................................................49

Box 3: Konzepte der 1920er-Jahre zur Ausgestaltung der US-Agrarpolitik ............64

Box 4: Ausgestaltung der Agrarzahlungen unter dem „Agricultural Adjustment

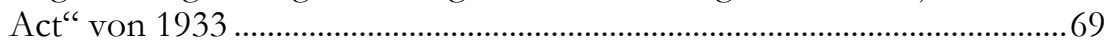

Box 5: Agrarzahlungen im US-Zuckersektor im Zeitraum 1939 bis 1945 ................78

Box 6: Bericht der „Food and Fiber Commission“ zur US-Agrarpolitik von

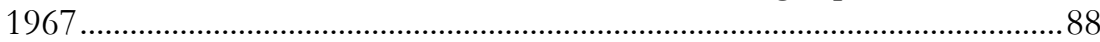

Box 7: Bericht der „Commission on 21 ${ }^{\text {st }}$ Century Production Agriculture“ zur US-Agrarpolitik von 2001 ...........................................................................99

Box 8: WTO-Panel zu US-Agrarsubventionen im Baumwollsektor.........................108

Box 9: Reform der Agrarpolitik in Schweden von 1990 ..........................................135

Box 10: Kommissionspapier zur EU-Agrarpolitik für das 21. Jahrhundert .........142

Box 11: EU-Modell einer multifunktionalen Landwirtschaft....................................146

Box 12: „Disaster payments“ in der US-Agrarpolitik.................................................186

Box 13: Weg zur Einführung von „price support payments“ in den USA im

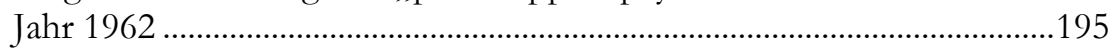

Box 14: Bedeutung der WTO bei der Vorbereitung der Farm Bill von 2008 ......241 



\title{
Abkürzungsverzeichnis
}

\author{
AAA Agricultural Adjustment Act \\ ACRE Average Crop Revenue Election \\ AMS Aggregate Measure of Support \\ AMTA Agricultural Market Transition Act \\ ARA Agricultural Reconciliation Act \\ ARC Agriculture Risk Coverage \\ ARP Acreage Reserve Program \\ $\mathrm{CBO} \quad$ Congressional Budget Office \\ CCC Commodity Credit Corporation \\ CED Committee for Economic Development \\ CEPR Centre for Economic Policy Research \\ CRP Conservation Reserve Program \\ CRS Congressional Research Service \\ DCP Direct Compensation Payments \\ DSB Dispute Settlement Body \\ EAGFL Europäischer Ausrichtungs- und Garantiefonds für die Landwirtschaft \\ ERS Economic Research Service \\ EU Europäische Union \\ EUR Euro \\ FAIR Federal Agriculture Improvement and Reform Act \\ FAPRI Food and Agricultural Policy Research Institute
}


GAO Government Accountability Office

GAP Gemeinsame Agrarpolitik

GATT General Agreement on Tariffs and Trade

MFR Mehrjähriger Finanzrahmen

MLA Market Loss Assistance

MTR Mid-Term Review

NAFTA North American Free Trade Agreement

NASS National Agricultural Statistics Service

OECD Organisation for Economic Co-operation and Development

OMB Office of Management and Budget

PFC Production Flexibility Contracts

PLC Price Loss Coverage

PSE Producer Support Estimate

SNAP Supplemental Nutrition Assistance Program

STAX Stacked Income Protection Plan

TTIP Transatlantische Handels- und Investitionspartnerschaft

URAA Uruguay Round Agricultural Agreement

USA United States of America

USD US-Dollar

USDA United States Department of Agriculture

WTO World Trade Organization 


\section{Forschungsfrage: Analyse der Konvergenz der Agrarpolitiken der USA und der EU hinsichtlich der Direktzahlungen}

Im Juli 2013 haben die Europäische Union ${ }^{1}$ (EU) und die Vereinigten Staaten von Amerika (USA), ,the two elephants of the developed world“ (Haniotis 2006: 66), Verhandlungen über die Transatlantische Handels- und Investitionspartnerschaft (TTIP) aufgenommen. Diese befinden sich zwar seit der Präsidentschaftswahl in den USA im Herbst 2016 im Stillstand, aber bereits die Absicht, ein solches Abkommen abzuschließen, genügte, um die Aufmerksamkeit auf die Agrar- und Lebensmittelpolitik beider Parteien zu lenken und eine zum Teil sehr kontrovers geführte öffentliche Diskussion in diesem Bereich zu entfachen.

Die Kritiker befürchten insbesondere eine Absenkung von Umwelt- und Verbraucherschutzstandards in der EU. Die Befürworter hingegen weisen auf neue Chancen hin, die die Beseitigung von Handelshemmnissen in einer solchen Freihandelszone mit sich bringen würde. Die USA und die EU sind zwar immer noch die größten Wirtschaftsakteure weltweit: Auf sie zusammen entfallen $12 \%$ der Weltbevölkerung, 45\% des Welt-Bruttoinlandsprodukts, 30\% des Weltgüterhandels, $60 \%$ des Bestands der weltweiten Direktinvestitionen und $40 \%$ der weltwei-

\footnotetext{
${ }^{1}$ In der vorliegenden Arbeit wird durchgehend der Begriff „Europäische Union“ („Union“) verwendet - auch für die Zeit vor 1992.
} 
Analyse der Konvergenz der Agrarpolitiken der USA und der EU

ten Patente (Deutsch 2013: 5). Allerdings erodiert ihre Welthandelsposition: Zwischen 2001 und 2011 sind die Anteile der EU im globalen Güter- und Dienstleistungsaustausch um jeweils 3 Prozentpunkte, die der USA um 6 bzw. 2 Prozentpunkte gesunken. Gleichzeitig verzeichnete beispielsweise China ein Plus von 10 Prozentpunkten im Güterhandel und 2 Prozentpunkten im Dienstleistungsaustausch (ibid.: 3).

Das TTIP-Abkommen sollte helfen, die Schwächung der Welthandelsposition beider Parteien abzubremsen und ihre Wettbewerbsfähigkeit zu erhöhen. Laut der Folgenabschätzung des „Centre for Economic Policy Research“ (CEPR) in London kann die EU durch den Abschluss des TTIP bis zum Jahr 2027 mit einem zusätzlichen Wirtschaftswachstum von $0,5 \%$ des Bruttoinlandsprodukts und die USA von $0,4 \%$ des Bruttoinlandsprodukts gegenüber dem Szenario ohne TTIP rechnen. Die bilateralen Exporte der EU würden dabei um $28 \%$ und die der USA um rund $37 \%$ ansteigen, ohne dass dieser Anstieg negative Auswirkungen auf andere Handelsströme hätte (CEPR 2013). Auch nach Einschätzung des USLandwirtschaftsministeriums (USDA, United States Department of Agriculture) soll das Bruttoinlandsprodukt sowohl in der EU (je nach Szenario um bis zu $0,29 \%$ gegenüber 2011) als auch in den USA (um bis zu 0,1\%) dank TTIP wachsen. Während die USA insbesondere aus der Steigerung der Agrarexporte Nutzen ziehen könnten, würden die EU bzw. die europäischen Verbraucher von niedrigeren Importpreisen profitieren sowie von den Steigerungen der Exporte im nichtlandwirtschaftlichen Bereich (Beckman et al. 2015).

Seit Jahren schon sind die USA der wichtigste Handelspartner der EU im Agrarbereich. Auf sie entfielen im Jahr 2015 mit 19,4 Mrd. Euro (EUR) 15\% der gesamten EU-Agrarexporte, Tendenz seit 2010 steigend. Wertmäßig machen Getränke (Spirituosen, Wein, Bier sowie alkoholfreie Getränke) etwa die Hälfte dieser Exporte aus. Die USA sind der wichtigste Absatzmarkt der EU für re-exportierten Kaffee und Tee (53\% der EU-Exporte von Kaffee und Tee), Bier (44\%), Spirituosen (37\%) und Olivenöl (35\%). Gleichzeitig sind sie die zweitgrößte Quelle nach Brasilien - für die EU-Agrarimporte. Der Wert solcher Einfuhren aus den USA belief sich 2015 auf insgesamt 12 Mrd. EUR. Zu den wichtigsten Importgütern zählen tropische Früchte und Nüsse sowie Soja, gefolgt von Spirituosen und Lebensmittelzubereitungen. Die USA sind der wichtigste EU-Lieferant von Spirituosen (54\% der EU-Importe von Spirituosen), lebenden Tieren (53\%), Soja (35\%), Haustiernahrung $(30 \%)$ und tropischen Früchten und Nüssen $(21 \%)$ (Kommission 2016a: 9ff). Im bilateralen Handel mit Erzeugnissen der Land- und Ernährungswirtschaft ist die EU also Netto-Exporteur mit einem Handelsbilanzüberschuss von 7,4 Mrd. EUR.

Die Zölle im transatlantischen Handel liegen im Durchschnitt in allen Bereichen auf einem niedrigen Niveau von etwa 3,5\% (Felbermayr et al. 2013: 9). Im Agrar- und Nahrungsmittelhandel aber liegt das Zollniveau oftmals darüber. Es beträgt 14,6\% auf EU-Seite und 3,3\% auf US-Seite für verarbeitete Lebensmittel- 
produkte sowie jeweils 3,7 \% für Erzeugnisse der Land- und Forstwirtschaft sowie Fischerei (CEPR 2013: 14). Neben der Senkung bzw. Abschaffung der Zölle wird in diesem Bereich besonders der Abbau der nichttarifären Hemmnisse (insbesondere durch die Harmonisierung der Vorschriften und die Angleichung der auf beiden Seiten des Atlantiks geltenden Normen) als Treiber für die Gewinngenerierung identifiziert (CEPR 2013).

Die öffentlichen Debatten zum T'TIP-Abkommen sind stark von gesundheitspolizeilichen und pflanzenschutzrechtlichen Maßnahmen bestimmt (Stichwort: "Chlorhühnchen"). Aber nicht nur ausgewählte Standards und Anforderungen, sondern auch die Agrarförderung gehören laut einer Studie im Auftrag der Europäischen Kommission zu den wichtigsten regulierungsbezogenen Divergenzen bzw. nichttarifären Hemmnissen, die den Handel im Agrar- und Lebensmittelbereich zwischen den USA und der EU beeinflussen (Ecorys 2009: 81ff). Die EUAgrarförderung belegt Platz 5, die US-Agrarförderung sogar Platz 1 unter den jeweils aus der Perspektive der anderen Seite bewerteten und anhand von Umfragen und Experteninterviews identifizierten - wichtigsten Divergenzen (ibid.: 81f und 85). Die Agrarförderung wird hier als ,direct and indirect government support by means of subsidies, protective legislation and tax policies to farmers" definiert.

Ein wichtiges Element der Agrarförderung sowohl in der EU als auch in den USA stellen bzw. stellten die Direktzahlungen für landwirtschaftliche Erzeuger dar. Als solches standen sie im Fokus der Agrarreformen der letzten Jahrzehnte. Im Einklang mit den Empfehlungen der Organisation für wirtschaftliche Zusammenarbeit und Entwicklung (OECD, Organisation for Economic Co-operation and Development) für eine stärker marktorientierte Agrarpolitik waren sie das Instrument der Wahl für den Übergang von der Preisstützung zur Einkommensstützung. In der EU sind sie sogar zum Eckpfeiler der Gemeinsamen Agrarpolitik (GAP) mit entsprechend hohem Anteil am EU-Agrarhaushalt geworden. Auch in den neuesten Reformen - 2013 in der EU und 2014 in den USA - spielten die Überlegungen zur Zukunft der Direktzahlungen eine prominente Rolle.

Zum Zeitpunkt der Abfassung dieser Untersuchung kann nicht verlässlich abgeschätzt werden, ob und wann das TTIP-Abkommen beschlossen und wie umfassend es sein wird. Es ist nicht ausgeschlossen, dass es - angesichts der protektionistischen Strömungen in den USA und des erheblichen Widerstands in Teilen der Gesellschaft in Europa - nicht zustande kommt. Zurzeit deutet auch nichts darauf hin, dass die Agrarförderung Teil des T'TIP-Abkommens sein wird. Eine Vereinbarung über inländische Agrarsubventionen zwischen den USA und der EU könnte zwar als Vorbild und Impulsgeber für spätere Abkommen dienen wie Josling und Tangermann (2015: 228) bemerken -, sie wenden aber gleichzeitig ein: „[B]ilateral agreement on domestic subsidy disciplines in TTIP would give away negotiating chips that the US and the EU might need in the multilateral negotiations in the Doha Round, in particular vis-à-vis developing countries, which 
are trying to change the rules of that game in their favor in the current negotiations“ (ibid.: 228). Deshalb sei es unwahrscheinlich, dass ,the TTIP negotiations will deviate from the tradition of not including disciplines on domestic subsidies in agriculture in FTAs" (ibid.). Nichtsdestotrotz lohnt aus vorgenannten Gründen ein genauerer Blick auf die Agrarförderung auf beiden Seiten des Atlantiks, insbesondere auf das wichtige Instrument der Direktzahlungen.

Darauf, dass ein Vergleich der agrarpolitischen Entwicklung in den USA und der EU interessante Erkenntnisse liefern kann, weisen Josling und Tangermann (2015: 162) in ihrem Buch über die Geschichte der Agrarhandelsbeziehungen zwischen den beiden Wirtschaftsakteuren hin: „A particularly interesting aspect of farm policy developments on both sides of the Atlantic in the recent past is their comparative nature. "Die Autoren machen darauf aufmerksam, dass sich die Agrarpolitiken der USA und der EU eine Zeit lang einander annäherten: ,The development over a 50-year period of farm policies on the two sides of the Atlantic can certainly be described as one of convergence“ (ibid.: 264). In diesem Zusammenhang nennen sie das sinkende Stützungsniveau, die stärkere Marktorientierung, die Entkopplung der Stützung von der Produktion und schließlich das konvergierende agrarpolitische Instrumentarium. Allerdings wurde dieser Trend zur Konvergenz in jüngerer Zeit gebrochen. Dieses stellen die Autoren mit dem Hinweis auf die unterschiedliche Entwicklung des Instruments der entkoppelten Direktzahlungen im Zuge der letzten Reformen 2013 bzw. 2014 fest. Somit eröffnete sich eine interessante Forschungslücke, die einer Bearbeitung bedarf. Sie besteht darin zu untersuchen, welche Ursachen der neuerlichen Divergenz der Agrarpolitiken der USA und der EU zugrunde liegen: „The political economy mechanisms behind this new divergence of policies between the US and the EU await more research, and the outcome will be of great interest" (ibid.: 162). Dabei rückt das Instrument der Direktzahlungen in den Fokus.

Josling und Tangermann (2015) streifen diese Frage nur kurz. Sie stellen die Hypothese auf, dass die Auseinanderentwicklung der Agrarpolitiken der USA und der EU dem Entstehen eines unterschiedlichen innenpolitischen Umfelds, in dem die jüngsten Agrarpolitikreformen beschlossen wurden, geschuldet ist (ibid.: 265). Deshalb wurde auf den Verlust der Legitimation der Direktzahlungen - ihre ursprüngliche Begründung als Kompensation für den Abbau der Preisstützung griff nicht mehr - unterschiedlich reagiert. Die EU versuchte, den entkoppelten Direktzahlungen über das sogenannte „Greening“ eine neue Legitimation zu verschaffen. Josling und Tangermann (2015: 265) formulieren es zugespitzt so: „In Europe today the importance of shaping policy toward environmental goals cannot be overstated: it appears that nearly any rural policy can be sold on the political market if it can be argued that it benefits the environment." Die USA schafften die entkoppelten Direktzahlungen ab und setzten auf antizyklische Instrumente, da diese von der Öffentlichkeit eher akzeptiert werden als die auch in Zeiten ho- 
her Marktpreise für Agrarerzeugnisse zu gewährenden Direktzahlungen. Josling und Tangermann (2015: 265) fassen die Lage in den USA wie folgt zusammen:

In the US the politics of the direct payments was different: they were never linked to environmental goods. They proved however to be a political liability, as the public was unsupportive of payments to (large) farmers unrelated to their income status: farm incomes were in any case at record levels. But there appears to be general public sympathy for farmers as entrepreneurs running businesses susceptible to multiple risks.

Für Josling und Tangermann (2015) sind die letzten Entscheidungen beidseits des Atlantiks auf die Erosion der gesellschaftlich-politischen Basis für die Direktzahlungen zurückzuführen. Sie konstatieren: „If this hypothesis is correct the recent emergence of diverging trends of farm policies on both sides of the Atlantic has relatively little to do with new and fundamentally diverging conditions in agriculture, but with different political calculations as to how benefits derived from farm support policies can be best maintained“" (ibid.: 163).

Die vorliegende Arbeit setzt hier an und greift die Aufgabenstellung aus dem Buch von Josling und Tangermann auf. Sie beschränkt sich dabei nicht auf die Analyse der neuerlichen Divergenz der Agrarpolitiken von USA und EU, sondern schaut noch tiefer in die Vergangenheit, um die von Josling und Tangermann (2015) festgestellte Konvergenz am Beispiel der Direktzahlungen zu untersuchen. In theoretischer Hinsicht stehen dabei zwei Fragen im Fokus. Erstens wird untersucht, inwiefern eine Angleichung zwischen den Agrarpolitiken der USA und der EU hinsichtlich des Instruments der Direktzahlungen tatsächlich erfolgte. Es wird geprüft, welches Konvergenzmuster zu erkennen ist. Zweitens wird der Frage nachgegangen, welche Faktoren den Konvergenzprozess beeinflussten. Das übergeordnete Ziel ist es, die Ursachen hinter dem Prozess der Konvergenz und der anschließenden Divergenz der Agrarpolitiken der USA und der EU am Beispiel der Direktzahlungen herauszuarbeiten. Auf dieser Grundlage wird es abschließend auch möglich sein, die oben angeführte Hypothese von Josling und Tangermann (2015) zur Divergenz zu überprüfen. In empirischer Hinsicht liegt der Analyse dieser Fragen ein Vergleich der Entwicklungen des Instruments der Direktzahlungen auf beiden Seiten des Atlantiks zugrunde.

Der Ertrag der vorliegenden Untersuchung besteht in den zu erzielenden Einsichten über die Konvergenz der Agrarpolitiken der beiden stärksten Wirtschaftsakteure der Welt im Laufe der letzten Jahrzehnte einschließlich der jeweiligen Gründe dafür. Auch soll sie zu einem besseren Verständnis des Instruments der Direktzahlungen beitragen. Die Gemeinsame Agrarpolitik der EU und die USAgrarpolitik waren zwar bereits Gegenstand einiger vergleichender Studien (z. B. Ingersent und Rayner 1999, Moyer und Josling 2002, Josling und Tangermann 2015). Allerdings fehlt bislang ein expliziter Vergleich des Instruments der Direkt- 
zahlungen auf beiden Seiten. Dabei geben die empirischen Untersuchungen Hinweise darauf, dass „the choice of instruments is non random“ (Swinnen et al. 2016: 108). Die politische Ökonomie der Politikinstrumentenwahl gilt aber grundsätzlich als eher unterentwickelt. Wie Swinnen et al. (2016: 107) bemängeln, konzentrieren sich die polit-ökonomischen Studien zur Agrarpolitik eher auf die Erklärungen über den Umfang der politischen Interventionen, als dass sie die Gründe für die Wahl der Instrumente untersuchen. Dieses Bias sei „an important shortcoming of the literature" (ibid.).

Außerdem beschränken sich die bisherigen Arbeiten oft auf die Analyse eines bestimmten, zeitlich beschränkten Reformabschnittes bzw. es werden die neuesten Reformen nicht erfasst, weil die meisten der relevanten Arbeiten weiter in der Vergangenheit liegen. Des Weiteren - auch wenn sich die vergleichenden Arbeiten der Mechanismen der Politikkonvergenz bedienen - beschäftigen sie sich laut Feindt (2007: 496) mit den Entwicklungen in der Agrarpolitik weniger unter diesem Gesichtspunkt als unter politikspezifischen Aspekten. Die Konvergenzforschung baut zwar auf einer vergleichenden Politikanalyse auf. Es wird aber darüber hinaus gezielt untersucht, ob die Ähnlichkeiten in der Politik zweier oder mehrerer Staaten im Zeitverlauf zu- oder abgenommen haben und welche Ursachen dieser Entwicklung zugrunde liegen. In der Forschung zur Politikkonvergenz wird der Agrarpolitik bisher insgesamt nur sehr geringe Aufmerksamkeit geschenkt. Die vorliegende Untersuchung hingegen bedient sich explizit der Konvergenztheorie. 


\section{Theoretischer Analyserahmen}

Im Mittelpunkt der folgenden Untersuchung steht das Instrument der Direktzahlungen an landwirtschaftliche Erzeuger. Der Begriff der Direktzahlungen wird in der Literatur sowie der politischen Diskussion nicht einheitlich verwendet. In den USA galt die Bezeichnung „direct payments“ zuletzt für eine Kategorie der Zahlungen, sogenannte „,fixed direct payments“, die von der Erzeugung entkoppelt waren. In der EU wird sie ebenfalls für eine Kategorie der Zahlungen verwendet, wobei nicht alle EU-Direktzahlungen entkoppelt sind. Der Begriff „direct payments" wird allerdings auch als Sammelbezeichnung für alle staatlichen Zahlungen an landwirtschaftliche Erzeuger verwendet. Beispielsweise nutzt Benedict (1953: 315) den Begriff „direct payments“ bereits für die ersten systematischen Zahlungen an Landwirte in der Geschichte der föderalen US-Agrarpolitik im Rahmen des „Agricultural Adjustment Act" von 1933. Zudem werden Bezeichnungen wie Ausgleichszahlungen oder „deficiency payments“, „income payments“ oder „income aids“, direkte Einkommensübertragungen, „commodity payments“ usw. in diesem Zusammenhang häufig synonym verwendet und tragen zum Begriffschaos bei. Vor diesem Hintergrund ist eine Definition der Direktzahlungen für die Zwecke der vorliegenden Arbeit unerlässlich. Anschließend wird das Analysekonzept dargestellt. 


\subsection{Klärung der Begriffe der staatlichen Zahlungen und der Direktzahlungen}

In der vorliegenden Arbeit werden unter Direktzahlungen Stützungsmaßnahmen für die Landwirtschaft verstanden, die unter den Kriterien der Green Box der Welthandelsorganisation (WTO, World Trade Organization) als „decoupled income support" notifiziert waren/sind bzw. ihnen entsprechen. Im Rahmen des Übereinkommens der Uruguay-Runde über die Landwirtschaft aus dem Jahr 1994 verständigten sich die Unterzeichnerstaaten darauf, die Inlandsstützung im Agrarbereich zu verringern (WTO-GAT'T 1994). Ausgenommen von dieser Verpflichtung wurden Stützungsmaßnahmen, die den Kriterien des Anhangs 2 zum besagten Übereinkommen entsprechen (sogenannte Green Box). Alle Maßnahmen nach Absatz 6 bis 13 dieses Anhangs nennt die WTO „direct payments to producers“, u. a. „payments for relief from natural disasters“ und „payments under environmental programmes“. Der hier interessierende „decoupled income support" ist in Absatz 6 definiert (s. unten). Ein weiterer Baustein seiner Definition findet sich in Absatz 1, in dem die allgemeinen Kriterien für die Stützungsmaßnahmen, für die eine Ausnahme von der Senkungsverpflichtung beansprucht wird, festgehalten werden. Zudem verdeutlicht Absatz 5, dass „decoupled income support“ zur Kategorie der „direct payments to producers“ gehört (konkret werden die Maßnahmen nach Absatz 6 bis 13 ,individual types of direct payment“ genannt).

Die Grundbedingung nach Absatz 1 ist, dass die Stützung keine oder höchstens geringe Handelsverzerrungen oder Auswirkungen auf die Erzeugung hervorruft. Folglich muss „decoupled income support“" von den Steuerzahlern - und nicht über Transfers von den Verbrauchern - finanziert werden und darf sich nicht wie eine Preisstützung für die Erzeuger auswirken. Um sich als „decoupled income support" zu qualifizieren, müssen die Maßnahmen folgende spezifische Kriterien nach Absatz 6 erfüllen:

- Die Berechtigung zum Erhalt solcher Zahlungen wird anhand von eindeutigen Kriterien wie Einkommen, Status als Erzeuger oder Landbesitzer, Einsatz von Produktionsfaktoren oder Produktionsleistung in einem gegebenen begrenzten Bezugszeitraum festgelegt.

- Die Höhe solcher Zahlungen in einem gegebenen Jahr ist nicht abhängig von oder bezogen auf Art oder Menge der Erzeugung (einschließlich Großvieheinheiten) eines Erzeugers in einem beliebigen Jahr nach dem Bezugszeitraum.

- Die Höhe solcher Zahlungen in einem gegebenen Jahr ist nicht abhängig von den Preisen oder bezogen auf die Preise, die national oder international in einem beliebigen Jahr nach dem Bezugszeitraum für eine Erzeugung gelten. 
- Die Höhe solcher Zahlungen in einem gegebenen Jahr ist nicht abhängig von oder bezogen auf Produktionsfaktoren, die in einem beliebigen Jahr nach dem Bezugszeitraum eingesetzt werden.

- Der Erhalt solcher Zahlungen ist nicht von einer Erzeugung abhängig.

Der erste Teil der Definition bezieht sich auf die Produktions- und Handelsauswirkungen der Maßnahmen. Der zweite Teil basiert auf Kriterien, die nach der Aufstellung des Stützungsprogramms grundsätzlich nicht verändert werden sollen. Insbesondere ist „decoupled income support“ nicht an die aktuelle und zukünftige Erzeugung sowie an Marktpreise gebunden.

Wie von Lopez (2001: 9) berichtet, wird der Begriff „Entkopplung“ sehr häufig sowohl in der Wissenschaft als auch in der Politik verwendet, allerdings oft unterschiedlich definiert. Zum Beispiel verwenden Burfisher und Hopkins (2003) eine Definition der Entkopplung, die auf Kriterien basiert, die die Zahlungen erfüllen müssen, um als entkoppelte Zahlungen zu gelten. Nach dieser Definition sind entkoppelte Zahlungen pauschale Einkommenszahlungen, die nicht von der aktuellen Erzeugung, dem Einsatz von Produktionsfaktoren oder von den Preisen abhängig sind und für die der Anspruch auf festen, historischen Kriterien basiert. Die OECD verwendet hingegen eine Ex-post-Definition der Entkopplung, wonach die Klassifizierung der Maßnahmen nicht auf den ex ante definierten Kriterien basiert, sondern auf der empirischen Ex-post-Bewertung ihrer Produktionsund Handelsauswirkungen (OECD 2005: 5).

Die Definition der WTO wurde aus verschiedenen Gründen für die Zwecke der vorliegenden Arbeit gewählt. Zum einen ist sie vielseitig: Sie basiert - wie Swinbank et al. (2004: 22) bemerken - sowohl auf Kriterien als auch auf möglichen Auswirkungen der Maßnahmen. Zum anderen sind die betreffenden Direktzahlungen klar durch die Notifikation als „decoupled income support“ an die WTO abzugrenzen. Die OECD (2005: 13) weist darauf hin, dass alle landwirtschaftsspezifischen Stützungsmaßnahmen gewisse - wenn auch unterschiedlich ausgeprägte - Auswirkungen auf die Produktion haben. Grundsätzlich genüge die Auflage, dass der Begünstigte in der Landwirtschaft tätig bleiben muss, damit die Zahlungen Produktionsauswirkungen haben. So auch im Falle anderer auf die aktuelle Situation bezogener Bedingungen, wie der Ausschluss bestimmter Erzeugnisse oder die Bindung an Cross-Compliance-Bedingungen, selbst wenn die Zahlungen auf historischen Ansprüchen basieren und keine Produktion erforderlich ist (ibid.: 15). Die Wahl der an die WTO notifizierten Maßnahmen bringt Klarheit hinsichtlich dieser komplexen Frage.

Die so definierten Direktzahlungen interessieren in Hinsicht auf die Fragestellung der vorliegenden Arbeit, weil sie sowohl in den USA als auch in der EU eine zentrale Rolle im Reformprozess weg von der produktionsabhängigen Preisstützung spielten. Vor dem Hintergrund der teilweise sehr ausgeprägten Unterschiede zwischen den USA und der EU in Bezug auf die agrarpolitischen Ziele und In- 
strumente (s. Bureau 2012) stellten sie eine Zeitlang eine der wenigen Gemeinsamkeiten dar, weil sie beidseits des Atlantiks im Einsatz waren. Entsprechend setzte eine divergierende Entwicklung ein, als die USA sie abschafften. Auch können die Direktzahlungen Indikatoren für eine „moderne“ Agrarpolitik sein. Laut OECD-Einschätzung sei „decoupling support from production decisions [...] an essential step in limiting unwanted impacts of many traditional [Herv. durch die Verf.] policy approaches“ (OECD 2005: 16).

Die Direktzahlungen sind das Instrument der Wahl für den Übergang vom Paradigma einer schutzbedürftigen Landwirtschaft (,,protected development paradigm") zu einem marktliberalen Paradigma und können auch nach dem erfolgten Übergang eine gewisse Rolle spielen (Coleman 2001). Dabei eignen sie sich für unterschiedliche Zielsetzungen. Swinbank (2004: 2) nennt in diesem Zusammenhang neben der Kompensation für die Abschaffung von Preis- oder Einkommensstützung auch permanente Einkommensstützung sowie Vergütung für die Bereitstellung von öffentlichen Gütern im Kontext einer multifunktionalen Landwirtschaft. Auch die OECD unterstreicht die vielseitigen Einsatzmöglichkeiten der entkoppelten Zahlungen (OECD 1994). Sie nennt diese „direct income payments“ (die Kriterien für „direct income payments“ ähneln den spezifischen Kriterien aus der WTO-Definition von „decoupled income support“, vgl. Box 1). Die Organisation identifiziert vier Bereiche, in denen „direct income payments" verwendet werden können: Agrarstrukturanpassung, Einkommensstabilisierung bzw. Ausgleich für Preis- und Einkommensschwankungen, Sicherung des Mindesteinkommens sowie Verbesserung der Umweltleistung. Je nach Ausgestaltung können sich die Zahlungen als „direct income payments“ qualifizieren. Im Allgemeinen sieht die OECD den Vorteil solcher Zahlungen darin, eine bessere Ausrichtung der Stützung auf bestimmte Gruppen der Landwirte bzw. eine Kopplung an angestrebte Ergebnisse zu ermöglichen (ibid.). Die Erklärung der Minister der OECD-Länder aus dem Jahr 1987 erwähnt explizit „low-income farmers, those in particularly disadvantaged regions, or those affected by structural adjustment in agriculture" als die möglichen Zielgruppen (OECD 1987: o.S.).

Bei den Direktzahlungen ${ }^{2}$ im Sinne dieser Arbeit handelt es sich somit um entkoppelte Direktzahlungen nach Absatz 6 des Anhangs 2 zum Übereinkommen über die Landwirtschaft von 1994. Die Direktzahlungen der WTO-Ära sind durch ihre Notifikation als „decoupled income support“ klar erkennbar. Die vorliegende Analyse beschränkt sich aber nicht auf diesen Zeitraum. Bereits vor der Gründung der WTO im Jahr 1995 waren sowohl in den USA als auch in der EU unterschied-

\footnotetext{
2 Das jetzige Hauptinstrument der EU-Agrarpolitik wird Direktzahlungen genannt. Um Missverständnisse zu vermeiden, wird für die gesamten - entkoppelten und gekoppelten - Direktzahlungen in der EU grundsätzlich der Begriff „EU-Direktzahlungen“ verwendet, außer in klaren Fällen, wie beispielsweise bei der Beschreibung der Entwicklungen in der EU (s. Kapitel 5). Für die Direktzahlungen im Sinne dieser Arbeit wird des Öfteren der Ausdruck „(entkoppelte) Direktzahlungen“ verwendet, um für Klarheit zu sorgen.
} 
liche staatliche Zahlungen an landwirtschaftliche Erzeuger in Kraft. Es ist von Interesse herauszufinden, ob sie möglicherweise die Kriterien für die Direktzahlungen im Sinne dieser Arbeit erfüllen.

Für sämtliche staatliche Zahlungen an landwirtschaftliche Erzeuger - also nicht nur die Direktzahlungen wie oben definiert - wird in der vorliegenden Untersuchung die Definition (aber nicht der Begriff) der "direct payments" der OECD aus dem Jahr 1987 verwendet. Sie wurde für Zwecke der Berechnung des „Producer Subsidy Equivalent" (Vorgänger des „Producer Support Estimate“, PSE), der der Quantifizierung der Agrarstützung in den OECD-Ländern dienen sollte, etabliert. Dabei beschränkte sich die OECD nicht auf die Messung des Gesamtstützungsniveaus, sondern zeigte auf, aus welchen Kategorien der Politikmaßnahmen sich diese Stützung zusammensetzt. Eine davon waren „direct payments“, die als ,all measures which transfer money directly to producers (Direct Payments) without raising prices to consumers" definiert wurden (Cahill und Legg 1989-1990: 16). Daraus wird ersichtlich, dass der Begriff „direct payments“ (Direktzahlungen) damals anders verwendet wurde als er für die Zwecke der vorliegenden Arbeit genutzt wird. Zwischen den „direct payments“ der OECD-Definition von 1987 und den späteren „direct income payments“ besteht ein erheblicher Unterschied.

Die Kategorien der Politikmaßnahmen, die der „Producer Subsidy Equivalent“ von 1987 umfasste, unterscheiden sich zunächst dadurch, ob die Unterstützung landwirtschaftlicher Erzeuger durch die Einkommenstransfers von Verbrauchern oder von Steuerzahlern erfolgt. Die Marktpreisstützung umfasst Transfers von Verbrauchern, die zu einer Differenz zwischen dem inländischen Marktpreis und dem Weltmarktpreis für ein bestimmtes Erzeugnis führen. Im Gegensatz dazu handelt es sich bei den sonstigen Maßnahmen um Mitteltransfers aus dem Staatshaushalt, die eine solche Differenz nicht verursachen. Neben den hier interessierenden „direct payments“ gehören dazu Zahlungen, welche die Vorleistungskosten reduzieren (,input subsidies") sowie allgemeine Maßnahmen (,general services") und sonstige, indirekte Zahlungen und Steuervergünstigungen. Dabei induzieren „direct payments“ im Unterschied zu den letzteren drei Maßnahmenkategorien die Zahlungen an Landwirte direkt bzw. führen direkt zur Einkommensverbesserung (Cahill und Legg 1989-1990).

Die „direct payments“ im Sinne des „Producer Subsidy Equivalent“ von 1987 umfassen eine Vielzahl unterschiedlicher Zahlungstypen, die unterschiedliche Wirkungen auf den Sektor haben können. Dazu gehören u. a. ,deficiency payments, area and headage payments [Herv. im Original], insurance and disaster payments, diversion payments for temporary retirement of resources and compensatory payments as part of the reform process“ (OECD 1998: 153). Bei den so definierten „direct payments" handelt es sich somit um eine Sammelbezeichnung ohne weitere Differenzierung der Zahlungen. Deshalb eignet sie sich ungeachtet des Namens als Bezeichnung für sämtliche staatliche Zahlungen an Landwirte in dieser Arbeit. Als 
Synonym werden der Begriff „Agrarzahlungen“ und - für die USA - der in der USDA-Statistik genutzte Begriff „government payments“ verwendet. Darüber hinaus wird der Begriff „commodity payments“ aus den USDA-Dokumenten als Bezeichnung für einkommensstützende Zahlungen im Rahmen des Titels I „Commodities“ einer Farm Bill verwendet. Das Gegenstück der „commodity payments" in der EU sind die EU-Direktzahlungen.

Zudem bleibt zu erwähnen, dass es sich bei den hier interessierenden staatlichen Zahlungen um Transfers an die Primärlandwirtschaft und nicht an die Verarbeiter- oder Vertriebssektoren handelt. Des Weiteren werden nur die landwirtschaftsspezifischen Politiken berücksichtigt. Somit werden beispielsweise die klassischen Sozialzahlungen, die den landwirtschaftlichen Betrieben zufließen, oder die Transportzahlungen, die auch anderen Sektoren zur Verfügung gestellt werden, nicht berücksichtigt.

Box 1: Konzept der „direct income payments“ der OECD

Das Konzept der „direct income payments“ der OECD kann als ein früher Versuch gewertet werden, entkoppelte Zahlungen zu definieren und zu beschreiben, auch wenn keine der relevanten OECD-Studien (von 1990 und 1994) das Wort „Entkopplung“ erwähnten. Dieses Konzept geht auf die Erklärung der Minister der OECD-Länder aus dem Jahr 1987 zurück, in der die OECD-Mitgliedstaaten ihr Bekenntnis zur Reform der Agrarpolitik und zur Uruguay-Runde bekräftigten und eine Reihe von Grundsätzen für die Reform aufstellten. Unter anderem legten sie fest, dass „, $[\mathrm{r}]$ ather than being provided through price guarantees or other measures linked to production or to factors of production, farm income support should, as appropriate, be sought through direct income support" (OECD 1987: o.S.). Das langfristige Ziel war ,to allow market signals to influence by way of a progressive and concerted reduction of agricultural support, as well as by all other appropriate means, the orientation of agricultural production“" (ibid.). Dieses würde zu einer besseren Ressourcenallokation führen, wovon sowohl die Verbraucher als auch die Wirtschaft insgesamt profitieren würden, so die Ministererklärung von 1987.

„Direct income payments“ würden erlauben, die vorgegebenen politischen Ziele zu erreichen ,with lower levels of assistance and the least economic distortions" (OECD 1990: 47). Ihre Rolle wird zum einen in der Einkommensumverteilung zwischen Einzelpersonen, Gruppen oder Regionen gesehen (z. B. Ausgleichszahlungen beim Abbau der Marktpreisstützung oder Zahlungen für benachteiligte Regionen). Zum anderen können „direct income payments“ auf die Korrektur von Marktversagen abzielen (z. B. Zahlungen für die Bereitstellung öffentlicher Güter). Möglich wäre es auch, diese zwei Rollen in einer Zahlung zu 
kombinieren (OECD 1994). Die OECD definiert „direct income payments“ als ,all those payments made directly from public authorities ' budgets to individual farmers that have the effect of increasing farmers' incomes" (OECD 1994: 7).

Die Organisation formulierte zunächst allgemeine Kriterien, die die Zahlungen erfüllen müssen, um sich als „direct income payments“ zu qualifizieren. Zum einen müssen sie von der öffentlichen Hand finanziert werden. Solche Finanzierung schließt eine Verzerrung der Marktpreise aus und ermöglicht Transparenz bezüglich des Umfangs der Unterstützung an die Landwirtschaft, so die OECD. Zum anderen sollte die Höhe der „direct income payments“ entweder fest oder außerhalb der Kontrolle der Begünstigten sein. Dieses bedeutet, dass es für den Landwirt nicht möglich sein sollte, durch die Produktionsentscheidungen einen Einfluss auf die Höhe der Zahlungen zu nehmen. Eine Ausnahme bilden hier die freiwilligen Zahlungen für die Bereitstellung der Umweltgüter: Die Entscheidung, Umweltgüter zu erzeugen, liegt durchaus im Ermessen des Landwirts. Da aber für diese Güter kein Markt existiert, können auch keine Marktsignale verzerrt werden, argumentiert die OECD. Insbesondere sollte die Höhe der „direct income payments“ nicht durch den Umfang der aktuellen oder künftigen Produktion eines Erzeugnisses bestimmt sein (OECD 1994).

Zudem erarbeitete die OECD Best Practices für die Ausgestaltung der Zahlungen in jedem der identifizierten Einsatzbereiche: Agrarstrukturanpassung, Einkommensstabilisierung bzw. Ausgleich für Preis- und Einkommensschwankungen, Sicherung des Mindesteinkommens sowie Verbesserung der Umweltleistung. Die Empfehlungen betreffen insbesondere die Fragen der Berechtigung, Höhe und Laufzeit der Zahlungen sowie deren Finanzierung. Unter anderem betont die OECD, dass bei den Zahlungen zwecks der Sicherung des Mindesteinkommens die Höhe des Gesamteinkommens eines Landwirts, inklusive des Einkommens aus nichtlandwirtschaftlichen Tätigkeiten (Haushaltseinkommen), für seine Berechtigung entscheidend sein sollte (ibid.). Auch sollten die „direct income payments“ unabhängig von den aktuellen Preisen für Agrarerzeugnisse sein (OECD 1990). Gleichzeitig warnt die OECD (1994: 39) davor, in den „direct income payments“ ein Allheilmittel zu sehen: „It should be stressed that, among the less distortionary reform-oriented alternatives available to policy makers, direct income payments may not always be the only or the most appropriate type of instrument for pursuing a given policy objective, nor may they always be suitable in the conditions prevailing in particular Member countries." 


\subsection{Konvergenztheorie als Analyserahmen}

Da sich die vorliegende Arbeit mit der Frage der Angleichung von Politiken zweier ausgewählter Länder bzw. Wirtschaftsblöcke befasst, bietet sich als Analyserahmen die Konvergenztheorie an. Es handelt sich hierbei um einen Ansatz aus den Politikwissenschaften, der im Bereich der Agrarpolitik bisher relativ selten verwendet wurde. Im vorliegenden Abschnitt wird eine Bestandsaufnahme zum aktuellen Stand der Konvergenzforschung dargestellt. Es wird auf ihre Entstehung und die Begriffsdefinition eingegangen. Anschließend werden die Typen der Politikkonvergenz sowie die Erklärungsansätze für die Entwicklung von Konvergenz geschildert.

Die Konvergenzforschung befasst sich mit der Frage, ob und inwieweit unterschiedliche Länder ihre Politik im Zeitablauf angleichen und welche Faktoren für dieses Phänomen der Konvergenz verantwortlich sind (Knill 2005). Die Politikkonvergenz ist kein neues Forschungsthema. An akademischer Popularität gewann sie aber erst in den 1990er-Jahren vor dem Hintergrund der zunehmenden internationalen Interdependenzen von Nationalstaaten im Zuge von Europäisierung und Globalisierung (Holzinger und Knill 2005). In den letzten Jahren erschienen verschiedene Analysen, die der Frage der zunehmenden Ähnlichkeit von nationalen Politiken und ihrer jeweiligen - transnationalen und/oder innenpolitischen - Gründe nachgingen. Dieses ist insbesondere in den Bereichen der Wirtschafts-, Sozial-, Umwelt- und Hochschulpolitik der Fall (Heichel und Sommerer 2007: 112f).

Definiert wird der Begriff der Politikkonvergenz als „tendency of policies to grow more alike, in the form of increasing similarity in structures, processes, and performances“ (Drezner 2001: 53). Eine detailliertere Definition von Politikkonvergenz liefert Knill (2005: 768): „[P]olicy convergence can be defined as any increase in the similarity between one or more characteristics of a certain policy (e.g. policy objectives, policy instruments, policy settings) across a given set of political jurisdictions (supranational institutions, states, regions, local authorities) over a given period of time." Den Gegenpol zur Konvergenz stellt die Divergenz dar, die als eine Abnahme der Ähnlichkeiten zwischen den betrachteten Zeitpunkten definiert wird (Holzinger et al. 2007b: 23). Die Konvergenz kann in Bezug auf verschiedene Politikdimensionen untersucht werden. Dazu gehören nach Heichel und Sommerer (2007: 114) Ziele, Inhalte, Auswirkungen sowie Politikstile, aber auch hier interessierende Instrumente. Des Weiteren wird zwischen Output-Konvergenz und Outcome-Konvergenz unterschieden. Als Output werden die von der Regierung ergriffenen Maßnahmen und als Outcome die tatsächliche Wirkung dieser Maßnahmen in Bezug auf die Zielerreichung verstanden (ibid.: 114f).

Die Konvergenzforschung unterscheidet zwischen vier Konvergenztypen (Knill 2005). Die Sigma-Konvergenz beschreibt die Abnahme der Variation nationaler Politiken, also das Zusammenwachsen von Politiken, Politikinstrumenten oder Standards, ohne das die Bewegungsrichtung betrachtet wird. Die Delta-Kon- 
vergenz bezeichnet die Annäherung der Politik an ein bestimmtes Modell (Politikideal), während die Beta-Konvergenz die Annäherung der Politik der NachfolgerStaaten an einen Vorreiter (Pionier) erfasst. Auf diese Weise lässt sich die Bewegungsrichtung erfassen. Schließlich beleuchtet die Gamma-Konvergenz die Veränderung von Rangplätzen der Länder einer Gruppe in Bezug auf eine bestimmte Politik.

Hinsichtlich der Erklärung von Politikkonvergenz werden mögliche Faktoren in Kausalmechanismen und intervenierende Faktoren unterteilt (Holzinger et al. 2007b). Erstere beschreiben, „auf welchem Weg die politischen Entscheidungen eines politischen Systems die Entscheidungen anderer beeinflussen können“ (ibid.: 30). Wie Feindt (2007: 498) bemerkt, können sie „sowohl auf der nationalen wie der transnationalen Ebene wirken“, allerdings müssen sie „länderübergreifend wirksam sein“, um Konvergenz erklären zu können. Die große Zahl von Kausalmechanismen, die in der Literatur genannt werden, ordnet Knill (2005) fünf Gruppen zu. Zum einen kann Politikkonvergenz Ergebnis eines ähnlichen Problemdrucks sein, der unterschiedliche Länder zu ähnlichen politischen Maßnahmen veranlasst, die aber unabhängig voneinander erfolgen. Dabei ist die Frage entscheidend, ob das objektiv bestehende Problem auch jeweils gleich wahrgenommen und interpretiert wird (Feindt 2007). Zum zweiten kann Politikkonvergenz aus der Ausübung des Zwangs resultieren: Staaten oder internationale Organisationen nutzen Machtasymmetrien aus, um andere Staaten zur Übernahme von bestimmten Politiken zu zwingen, z. B. nach einem gewonnenen Krieg oder als Bedingung für die Kreditvergabe. Die dritte Gruppe von Kausalmechanismen umfasst die Harmonisierung nationaler Politiken über internationales oder supranationales Recht. Die Staaten verpflichten sich, gemeinsam ausgehandelte Regeln zu beachten bzw. Programme zu implementieren (z. B. im Rahmen der WTO). Viertens kann auch der Regulierungswettbewerb zu Politikkonvergenz führen: Staaten, die einem Wettbewerbsdruck ausgesetzt sind, passen ihre Politik an, um eine Belastung der heimischen Industrie gegenüber der Konkurrenz aus dem Ausland zu vermeiden (Knill 2005).

Schließlich kann Politikkonvergenz Ergebnis der sogenannten transnationalen Kommunikation sein. Darunter werden Mechanismen subsumiert, die ausschließlich auf Kommunikation zwischen den Staaten basieren (Holzinger und Knill 2005). Sie umfassen bilaterales Politiklernen, in dem eine Regierung von einer anderen Regierung in Bezug auf die Lösung eines gemeinsamen Problems lernt (sowohl was zu tun als auch was zu unterlassen ist), sowie die durch den Wunsch nach Konformität mit anderen Staaten getriebene Nachahmung. Im Falle der gemeinsamen Suche nach Problemlösungen in transnationalen Netzwerken ist die Konvergenz nicht das Ergebnis eines bilateralen Transfers, sondern es wird multilateral eine Lösung entwickelt. Bei der Verbreitung von Politikmodellen durch internationale Organisationen hingegen sind nicht Staaten, sondern Organisationen 
wie die EU, die OECD, die Weltbank, aber auch Nichtregierungsorganisationen federführend:

In constantly searching for new policy ideas, disseminating best practice and evaluating domestic policy performance, they function as mediators of cross-national policy transfer, urging national governments to adopt successful policy models [...]. Countries that deviate from recommended policy models or rank low in international league tables face pressure to legitimate their policy approaches in light of ,international scrutiny'. (ibid.: 785)

Nach Drezner (2001) können die Kausalmechanismen der Politikkonvergenz in solche wirtschaftlicher und ideeller Natur unterteilt werden. Die erste Gruppe hat ihren Ursprung in Sorgen vor dem Verlust der Wettbewerbsfähigkeit: „[T] he pressure to modify regulatory policies comes from the threat of mobile capital to exit, causing nonconverging states to lose their competitiveness in the global economy“. Im Falle der zweiten Gruppe hingegen „,states alter institutions and regulations because a set of beliefs has developed sufficient normative power that leaders fear looking like laggards if they do not adopt similar policies“" (ibid.: 57). Holzinger und Knill (2005: 777) weisen darauf hin, dass die Kausalmechanismen einzeln, aber auch in Kombinationen auftreten können und eine Interaktion mehrerer Mechanismen möglich ist.

Neben den Kausalmechanismen identifiziert die Konvergenzforschung auch sogenannte intervenierende Faktoren. Das sind diejenigen nationalen Faktoren, die die Wirksamkeit von Kausalmechanismen beeinflussen und somit die Geschwindigkeit und den Umfang der Konvergenz bestimmen (Holzinger et al. 2007b: 25). Sie werden in drei Gruppen unterteilt: kulturelle, institutionelle und sozio-ökonomische Faktoren. Es wird angenommen, dass ähnliche Voraussetzungen in verschiedenen Ländern als Katalysatoren in internationalen Konvergenzprozessen wirken, z. B. institutionelle Übereinstimmungen sowie kulturelle und sozio-ökonomische Gemeinsamkeiten (ibid.: 30). Auch die spezifischen Eigenschaften und Merkmale von Politiken spielen für ihre Übernahme in anderen Ländern eine Rolle. Beispielsweise konvergieren stark umverteilend wirkende Politiken in geringerem Maße als Politiken mit weniger weitreichenden redistributiven Folgen (ibid.: 30f).

Des Weiteren kommt es bei Politikkonvergenz auf die Reichweite von Politikwandel an. Nach Hall (1993) wird zwischen drei Dimensionen einer Politik unterschieden: Paradigmen, Instrumente und die spezifischen Ausprägungen dieser Instrumente („policy settings“, z. B. Höhe einer Sozialleistung). Paradigmen sind grundsätzlich nur schwer zu verändern. Bei Instrumenten und „policy settings“ hingegen ist Wandel - und damit auch Konvergenz - wahrscheinlicher (Knill 2005). Diesem widersprechen allerdings einige Untersuchungen, die zeigen, dass die Politikinstrumente und Einzelbestimmungen betreffende Konvergenz weniger 
ausgeprägt ist als die Konvergenz auf der Ebene allgemeiner Ideen (Holzinger et al. 2007b: 31). Lenschow et al. (2005) verknüpfen diese Unterscheidung von Politikdimensionen mit den Hauptgruppen der intervenierenden Faktoren und zeigen auf, welche Faktoren die Konvergenz bei den einzelnen Dimensionen am stärksten beeinflussen. Bei Paradigmen spielen laut ihnen kulturelle Faktoren eine besondere Rolle. Auf Ebene der Instrumente sind institutionelle, bei „,policy settings“" sozio-ökonomische Aspekte die dominierenden, wenn auch nicht die einzigen Faktoren.

\subsection{Literaturauswertung}

Nachstehend werden die Arbeiten zum Thema der Politikkonvergenz im Bereich der Agrarpolitik vorgestellt. Daneben existieren einige vergleichende Arbeiten in Bezug auf die Agrarpolitik der USA und der EU (Koester et al. 1988, Moyer und Josling 1990, Ingersent und Rayner 1999, Moyer und Josling 2002, Josling und Tangermann 2015). Die aus der Sicht der vorliegenden Untersuchung wichtigsten Arbeiten finden sich in Kapitel 8 bei der Untersuchung der Einflussfaktoren für die ausgewählten Milestones wieder. Auf die Analyse von Josling und Tangermann (2015), die den Impuls für die vorliegende Arbeit setzte, wurde bereits in der Einleitung eingegangen.

Nur ganz wenige Arbeiten befassen sich mit den Entwicklungen in der Agrarpolitik unter dem Gesichtspunkt der Politikkonvergenz. Aus Sicht der vorliegenden Arbeit ist insbesondere die Untersuchung von Feindt (2007) relevant. Zum einen, weil sie neben anderen Ländern auch die hier interessierenden USA und EU umfasst. Zum anderen identifiziert Feindt (2007) die relevanten Kausalmechanismen für die Politikkonvergenz im Agrarbereich. Allerdings unterscheidet sich sein quantitativer Ansatz grundlegend vom qualitativen Ansatz der vorliegenden Arbeit. Zudem liegt bei Feindt (2007) das Interesse in der Agrarpolitik als Ganzes, ohne dass einzelne Instrumente berücksichtigt werden. Auch ist der behandelte Zeitraum - von 1986 bis 2004 - nicht mehr aktuell. Feindt selbst sieht Bedarf für weitere Untersuchungen von Konvergenzprozessen in der Agrarpolitik. Er merkt an, dass, wenn Konvergenz auf der Ebene des „Producer Support Esti-

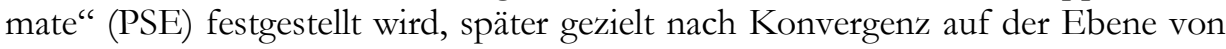
Politikideen, Maßnahmen und „policy settings“ gesucht werden kann (ibid.: 506).

Für seine Untersuchung verwendet Feindt (2007) das PSE in Prozent der Einkommen der Erzeuger. Mithilfe dieses Indikators stellt er die Delta-Konvergenz der Agrarpolitiken der OECD-Länder zwischen den Jahren 1986 und 2004 fest. Zum einen wurde die Entwicklung in Richtung einer Verringerung des Stützungsniveaus erkennbar (der Mittelwert des relativen PSE sank im Zeitverlauf, mit PSE nahe Null als Zielwert). Auch konnte eine Entwicklung in Richtung der Senkung des Anteils des Wertes produktionsbezogener Maßnahmen in Form von Marktpreisstützung sowie produktions- und vorleistungsbezogener Zahlungen am PSE 
konstatiert werden (der Mittelwert des Anteils solcher Maßnahmen am PSE sank im Zeitverlauf, mit dem Anteil von null Prozent als Zielwert). Die festgestellte Delta-Konvergenz bezieht sich zum einen auf das Stützungsniveau, zum anderen auf die Struktur der Agrarpolitik (produktionsbezogene vs. produktionsunabhängige Maßnahmen). Feindt (2007) zeigt, dass diese Konvergenz auf Effekte der Harmonisierungsprozesse im Rahmen der WTO sowie des ähnlichen Problemdrucks in Form von Haushaltsproblemen und Produktionsüberschüssen in den 1980er-Jahren (insbesondere in den USA und der EU) zurückzuführen ist. Dafür variiert er die Gruppe der untersuchten Länder (OECD-Länder mit vergleichbaren Problemen und WTO-Länder, die zugleich OECD-Mitglieder sind). Des Weiteren nennt er die transnationale Kommunikation im Forum der OECD als wichtigen Kausalmechanismus für die Konvergenz der nationalen Agrarpolitiken.

Gleichzeitig konnte Feindt (2007) keine Sigma-Konvergenz der Agrarpolitik der OECD-Länder zwischen 1986 und 2004 in Bezug auf das Stützungsniveau und die Struktur feststellen. Sie wäre zu erwarten, wenn in den Ländern mit dem höchsten Stützungsniveau bzw. mit dem höchsten Anteil produktionsbezogener Maßnahmen am PSE die Reduktion des Stützungsniveaus bzw. des Anteils produktionsbezogener Maßnahmen stärker wäre als in den restlichen Ländern. Dieses konnte aber nicht festgestellt werden: Die Unterschiede in der Struktur der Agrarpolitik sowie in der Höhe der Stützung in der Gruppe der untersuchten Länder wurden nicht kleiner (Standardabweichung des relativen PSE und Standardabweichung des Anteils produktionsbezogener Maßnahmen am PSE sanken nicht). Dieses sei laut Feindt (2007: 518f) u. a. dem WTO-Übereinkommen über die Landwirtschaft geschuldet, das eine Delta-Konvergenz in Richtung einer stärkeren Marktorientierung erforderte, aber den Mitgliedern große Spielräume in der Ausgestaltung der Stützung gewährte. Eine Aufschlüsselung der Datenreihen je Land lässt allerdings Konvergenztendenzen innerhalb der verschiedenen Ländergruppen feststellen. Feindt (2007: 512) weist u. a. kurz auf die Konvergenz zwischen den USA und der EU in den Jahren 1995 bis 2001 in Bezug auf das (steigende) Stützungsniveau hin, die durch wachsende produktionsabhängige Zahlungen in den USA Ende der 1990er-Jahre bedingt war.

Als Fazit konstatiert Feindt (2007: 518), dass die Mechanismen der Politikkonvergenz zwischen den Agrarpolitiken der OECD-Länder von 1986 bis 2004 „nicht in einer größeren Ähnlichkeit der Politiken“ resultierten, sondern ,in einer gleichsinnigen, das landwirtschaftliche Schutzniveau langsam senkenden und von der Produktion entkoppelnden Entwicklungsrichtung“. Er erwartet für die Zukunft „tendenziell eine weitere Verminderung des landwirtschaftlichen Schutzniveaus in den OECD-Ländern und ein[en] Rückgang des Anteils produktionsbezogener Maßnahmen“, glaubt aber, dass „[a]ufgrund politikspezifischer Faktoren [...] die Liberalisierung voraussichtlich langsamer und weniger umfassend verlaufen [wird] als in den meisten anderen Wirtschaftssektoren" (ibid.: 519). Im Hinblick auf Faktoren, die eine Konvergenz im Bereich der Agrarpolitik bremsen, 
weist Feindt (2007) u. a. auf den stark redistributiven Charakter der Agrarpolitik hin. Er nennt in diesem Zusammenhang auch die niedrige Priorität der Agrarpolitik in den Augen der Öffentlichkeit sowie potenziell hohe Kosten einer weitgehenden Agrarreform in einigen Ländern, sollte es zu einer Aufgabe der landwirtschaftlichen Produktion aufgrund der Senkung des Stützungsniveaus kommen. Neben den politikspezifischen Faktoren nennt Feindt (2007) auch weitere Aspekte, z. B. länderspezifische Faktoren wie klimatische und geografische Besonderheiten, aufgrund derer die Landwirtschaft im jeweiligen Land einen hohen gesellschaftlichen Wert genießt (Japan, Norwegen). Auch Kombinationen aus länderund politikspezifischen Faktoren können die anhaltenden Unterschiede in der Agrarpolitik der OECD-Länder erklären. Als Beispiel gibt er die besondere Stellung der Agrarpolitik in den politischen Systemen der USA und der EU an, wo Entscheidungsträger agrarischen Interessen nahestehen.

Um die begrenzte Konvergenz in der Agrarpolitik zu erklären, bedient sich Feindt (2007) außerdem des Konzeptes des Paradigmenwechsels. Er merkt an, dass ähnliche Problemlagen wie Haushaltsprobleme und Produktionsüberschüsse zur Konvergenz der nationalen Agrarpolitiken führen können. Dabei sei allerdings die Frage entscheidend, ob das objektiv bestehende Problem auch jeweils gleich wahrgenommen und interpretiert wird. Dieses war in der Agrarpolitik der 1990erund frühen 2000er-Jahre nicht der Fall, argumentiert Feindt (2007: 501) mit Blick auf die USA und die EU. Denn in den USA herrschte das marktliberale Paradigma, in Europa das Paradigma einer multifunktionalen Landwirtschaft. Mit der unterschiedlichen Interpretation ähnlicher Probleme erklärt er den Mangel an SigmaKonvergenz in Bezug auf die Agrarpolitik der OECD-Länder im untersuchten Zeitraum.

Coleman und Grant (1998) erklären eine begrenzte Konvergenz der Agrarkreditpolitiken mit dem Konzept der institutionellen Pfadabhängigkeit. Ihre qualitative Analyse richtet den Fokus auf die Merkmale der Politiken. Untersucht wird, inwiefern sich die Agrarkreditpolitiken von Australien, Großbritannien, Irland, Kanada und den USA im Zeitraum von 1970 bis 1995 an das marktliberale Modell als Politikideal annäherten (Delta-Konvergenz). Das marktliberale Modell bedeutet, dass die Landwirtschaft wie jeder andere Sektor behandelt wird: „,L]ending takes place at market-determined interest rates [...]. If state-owned corporations remain active under this model, they have the legal status of banks and operate as commercial entities. Like other banks, their mandate will not be restricted to financing agricultural producers only, but will extend to all commercial opportunities“" (ibid.: 229). Es wird zwischen Output (Politikinhalte) und Outcome (Zielerreichung gemessen als Anteil der privaten Unternehmen auf dem Agrarkreditmarkt) unterschieden, deren Konvergenz jeweils separat untersucht wurde.

Coleman und Grant (1998) stellen fest, dass, auch wenn sich jedes der untersuchten Länder dem marktliberalen Modell mehr oder weniger annäherte, wichtige Unterschiede zwischen den nationalen Agrarkreditpolitiken erhalten blieben. 
Diese erklären sie durch die historische Wahl der Politikinstrumente und die Pfadabhängigkeit nach Pierson (Politiken können Anreize bieten, die Entwicklung durch positive Feedback-Effekte auf dem einst eingeschlagenen Pfad zu halten): „Policy convergence [...] may be shaped by national institutional repertoires and by the properties of policy instruments and the approaches to policy implementation“ (ibid.: 238). Die Autoren betonen, dass die Analyse der Institutionen auf makropolitischer Ebene nicht vernachlässigt werden sollte, allerdings ,an analysis of these institutions must be coupled to a micro-level analysis of the institutional characteristics of policy instruments themselves if we are to explain fully variations among nation-states in policy convergence“ (ibid.: 244). In ihrer Fallstudie identifizieren sie vier solche Merkmale: Vertragsbeziehung zwischen Staat und Landwirten (direkt vs. indirekt), Dauer des Bestehens des staatlichen Kreditinstitutes, Grad der Ähnlichkeit zwischen den staatlichen und privaten Kreditinstituten in Bezug auf Struktur und Tätigkeit sowie Gruppen der Landwirte, die mit Krediten bedient werden (nichtwettbewerbsfähig vs. wettbewerbsfähig). Je länger die Staatsagentur aktiv ist, je direkter ihre Beziehungen zu Landwirten, je unterschiedlicher ihre Struktur gegenüber den Privatbanken und je unattraktiver ihre Klientel für private Kreditinstitute, desto höher die Wahrscheinlichkeit eines „lock-in“ (Beharren auf dem Entwicklungspfad). Dieses erschwert die Politikkonvergenz zwischen den Ländern. Darüber hinaus merken Coleman und Grant (1998: 236) an, dass es Hinweise darauf gibt, dass Konvergenz der Outcomes der Konvergenz der Politikinhalte und Instrumente folgt.

Coleman (2001) untersucht den Politikwandel in den Milch- und Getreidesektoren in Australien, Kanada und den USA weg vom Paradigma einer schutzbedürftigen Landwirtschaft („protected development policy paradigm“) in Richtung des marktliberalen Paradigmas, in dem landwirtschaftliche Einkommen nur in genau abgegrenzten, limitierten Fällen unterstützt werden können. Im Fokus seiner Analyse steht somit die Delta-Konvergenz, die, wenn die Länder das Modell vollständig übernehmen würden, eine Sigma-Konvergenz nach sich ziehen würde. Coleman (2001: 221) stellt fest, dass Politikkonvergenz stärker ausgeprägt ist, wenn der Sektor dem internationalen Wettbewerb ausgesetzt ist: „Whether an economic sector is open or closed to the global economy appears to be a crucial background condition for the degree of convergence across nation-states in policies governing the sector.“ Insgesamt konstatiert er, dass „pressures for policy convergence have increased even in a sector as singular as agriculture“ (ibid.: 239). Folglich sind sich die nationalen Agrarpolitiken im Jahr 2001 ähnlicher als in der Vergangenheit. Deshalb: „If the internationalization of agricultural policy continues, we can expect that policies will converge even more over the next two decades" (ibid.).

Auch wenn sich die Arbeiten von Coleman und Grant (1998) und Coleman (2001) lediglich mit einem Konvergenzmuster auseinandersetzen, geben sie wichtige Hinweise für die Analyse der Politikkonvergenz im Bereich der Agrarpolitik 
im Allgemeinen. Alle drei Veröffentlichungen zeigen eine begrenzte, aber wachsende Konvergenz in diesem Bereich auf.

\subsection{Forschungskonzept und Aufbau der Arbeit}

Der Gegenstand der vorliegenden Untersuchung ist das Instrument der Direktzahlungen für landwirtschaftliche Erzeuger in den USA und der EU. Die Arbeit ist ein auf dieses einzelne Instrument bezogener Zwei-Länder-Vergleich, der sich mit Output-Daten beschäftigt. Auf der Grundlage der Ausführungen von Josling und Tangermann (2015) wird von einem Konvergenzprozess zwischen den USA und der EU in Bezug auf die Agrarpolitik, der durch die neuesten Reformen (2014 in den USA und 2013 in der EU) unterbrochen wurde, ausgegangen.

In der vorliegenden Arbeit wird zunächst genauer untersucht, wie dieser Prozess beim Instrument der Direktzahlungen verlief. Die Frage lautet, inwiefern sich die USA und die EU bei der Anwendung des Instruments der Direktzahlungen im Zeitverlauf einander annäherten. Es wird allerdings keine Sigma-Konvergenz im eigentlichen Sinne erfasst. Denn der Fokus der Arbeit besteht nicht darin zu überprüfen, wie das Instrument der Direktzahlungen in den USA und in der EU zu bestimmten, gleichen Zeitpunkten ausgestaltet war. Vielmehr wird mit ausgewählten Milestones in der Geschichte der Direktzahlungen, die zeitlich versetzt sind, operiert, weil die jeweilige Politik relevante Entscheidungen zu unterschiedlichen Zeitpunkten traf.

Im Fokus dieser Untersuchung stehen vielmehr die Beta-Konvergenz, die die Annäherung an einen Vorreiter beschreibt, und die Delta-Konvergenz, die die Annäherung an ein Modell bzw. Politikideal beschreibt. Die Gegenüberstellung der Milestones der US-amerikanischen und der europäischen Seite ist dazu prädestiniert, die Beta-Konvergenz zu untersuchen. Die Frage, die sich dabei stellt, ist, welcher der beiden Akteure hinsichtlich der Direktzahlungen die Vorreiterrolle innehatte und wer der „Nachzügler“ war. Auch die Delta-Konvergenz kann durch die Milestones abgebildet werden. Das anstrebenswerte Modell bilden hier die Direktzahlungen im Sinne der vorliegenden Arbeit („decoupled income support"). Es stellt somit kein idealtypisches Benchmark dar, sondern es ist in der Realität vorzufinden. Es wird geprüft, wie schnell sich die USA und die EU dem Modell annäherten. Anschließend wird untersucht, welches der beiden Konvergenzmuster besser geeignet ist, die Entwicklungen zu beschreiben.

Der Kern der Arbeit besteht darin, Erklärungen für die Konvergenz bzw. die Divergenz zwischen den Agrarpolitiken der USA und der EU hinsichtlich des Instruments der Direktzahlungen herauszuarbeiten. Von besonderem Interesse ist dabei die Frage, ob die jüngste Divergenz eher auf die Veränderung der Kausalmechanismen oder mehr auf länderspezifische Faktoren zurückzuführen ist. Oder aber es steckt politisches Kalkül dahinter, wie von Josling und Tangermann (2015) 
vermutet. Außerdem stellt sich die Frage, ob die festgestellte Divergenz ein kurzfristiges Phänomen ist oder auf Dauer bestehen bleibt.

Um diese Fragen zu beantworten, wird für die ausgewählten Milestones in der Entwicklung der Direktzahlungen beidseits des Atlantiks ein Vergleich der Faktoren, die der jeweiligen Entscheidung zugrunde liegen, vorgenommen. Die Faktoren werden anhand der Auswertung der Literatur zu den einzelnen Reformen zusammengestellt und anschließend den Kausalmechanismen der Politikkonvergenz zugeordnet. Auf dieser Basis werden Gemeinsamkeiten und Unterschiede zwischen den USA und der EU herausgearbeitet und bewertet. Es wird ergebnisoffen mit vier Kausalmechanismen operiert: der Harmonisierung im Rahmen der WTO, der transnationalen Kommunikation, dem regulativen Wettbewerb und dem vergleichbaren Problemdruck. Zusätzlich wird untersucht, welche intervenierenden Faktoren eine Rolle im hier untersuchten Konvergenzprozess spielten.

Die vorliegende Arbeit ist als qualitative Untersuchung konzipiert. Solche Untersuchungen haben gegenüber quantitativen Studien zur Politikkonvergenz den Vorteil, dass sie es eher ermöglichen, unterschiedliche Politikdimensionen in die Analyse einzubeziehen (Heichel und Sommerer 2007). Des Weiteren machen sie eine detailliertere Beschreibung von Politiken und Politikentwicklungen möglich: Die einzelnen Elemente einer Politik können besser erfasst, Veränderungen sowie ihre Ursachen erläutert und konvergierende bzw. nicht-konvergierende Entwicklungen aufgezeigt werden (ibid.). Diese Vorzüge sind für die vorliegende Arbeit entscheidend. Gleichzeitig geben Heichel und Sommerer (2007: 114) zu bedenken: „Qualitative Untersuchungen dürften, aufgrund der Berücksichtigung mehrerer Politikdimensionen, Konvergenz insgesamt eher unterschätzen, da dies mit zunehmender Dimensionsanzahl unwahrscheinlicher wird." Für quantitative Arbeiten stellen sie hingegen fest, dass diese die Konvergenz aufgrund der eindimensionalen Datengrundlage tendenziell überschätzen (ibid.).

Der Aufbau dieser Arbeit ergibt sich wie folgt: Die Einleitung beschreibt den Hintergrund der vorliegenden Studie, die Forschungslücke und die Zielsetzung einschließlich der Vorgehensweise. Im Anschluss daran wird der theoretische Rahmen dargestellt und die terminologische Klärung der Begriffe der staatlichen Zahlungen und der Direktzahlungen vorgenommen. Als nächstes wird die relevante Literatur ausgewertet und die Konzeption für die vorliegende Arbeit vorgestellt. Nachfolgend wird die Entwicklung der staatlichen Zahlungen an Landwirte in den USA und in der EU in separaten Kapiteln geschildert. Den Ausgangspunkt bildet ihre Ersteinführung auf breiterer Basis beidseits des Atlantiks, um die Erfahrungen mit dem Instrument der Agrarzahlungen in seiner Gesamtheit erfassen zu können, was nicht zuletzt für die Schlussfolgerungen von Bedeutung ist. Darauf aufbauend wird die Bestimmung der Milestones in der Entwicklung der Direktzahlungen auf beiden Seiten des Atlantiks vorgenommen. Unter Milestones werden in der Untersuchung substanzielle Reformen der Agrarpolitik in Bezug auf die Direktzahlungen verstanden. Ihre Bestimmung ist aus zwei Gründen ein ent- 
scheidender Schritt: Zum einen ermöglicht es einen direkten Vergleich zwischen den beiden Agrarpolitiken hinsichtlich der Direktzahlungen, u. a. des Tempos ihrer Einführung. Durch die Gegenüberstellung der jeweiligen Milestones wird der Verlauf des Prozesses der Konvergenz bzw. der Divergenz der Agrarpolitiken der USA und der EU gezeigt. Zum anderen wird für die ausgewählten Milestones ein Vergleich der Faktoren, die der jeweiligen Entscheidung zugrunde liegen, vorgenommen. Welche Antriebskräfte dabei in Betracht gezogen werden, wird in der Einführung zum Kapitel 8 detailliert erläutert. Sobald klar wird, woher der größte Reformdruck rührte, werden Gemeinsamkeiten und Unterschiede zwischen den USA und der EU identifiziert und bewertet. Auch die Konvergenzmuster werden dort untersucht.

Die Arbeit befasst sich schwerpunktmäßig mit konkreten agrarpolitischen Entwicklungen in zwei ausgewählten Ländern bzw. Wirtschaftsräumen. Nichtsdestotrotz wird in einem separaten Kapitel die Diskussion von staatlichen Zahlungen und insbesondere von Direktzahlungen in der wissenschaftlichen Literatur dargestellt. Der Bezug zu den übrigen Kapiteln besteht darin, dass herausgearbeitet wird, inwiefern die Ideen und Vorschläge von Wissenschaftlern in der praktischen Agrarpolitik umgesetzt wurden. Dabei wird auch der Frage nach dem Ursprung des Instruments der staatlichen Zahlungen sowie der Direktzahlungen nachgegangen. Das Resümee gibt einen systematischen Überblick über die Ergebnisse der Analyse und bewertet diese abschließend. 



\section{Das Instrument der Direktzahlungen in der Wissenschaft}

Im vorliegenden Kapitel wird die Behandlung von staatlichen Zahlungen und insbesondere von Direktzahlungen in der wissenschaftlichen Literatur dargestellt. ${ }^{3}$ Es wird dabei zwischen den US-amerikanischen und den europäischen, darunter auch deutschen, Konzepten unterschieden. Der Bezug zu den übrigen Kapiteln besteht darin, dass untersucht wird, ob und wann die in der Wissenschaft entwickelten Ideen und Konzepte in der praktischen Agrarpolitik umgesetzt wurden. Dieses muss allerdings nicht zwingend bedeuten, dass sie tatsächlich einen direkten Einfluss auf die agrarpolitische Entscheidungsfindung hatten und somit ein kausaler Zusammenhang besteht. Die Wirkung der wissenschaftlichen Konzepte zu beurteilen ist nur eingeschränkt möglich, weil nicht abzuschätzen ist, wie die jeweilige Agrarpolitik aussehen würde, hätte es sie nicht gegeben. Schließlich wird den Fragen nach dem Ursprung der staatlichen Zahlungen und der Direktzahlungen gemäß der Definition in dieser Arbeit - sowie den Ideenflüssen zwischen den

\footnotetext{
3 Trotz der umfassenden Recherche nach relevanten Veröffentlichungen kann diese Arbeit keinen Anspruch auf Vollständigkeit erheben. Die in den zitierten Publikationen verwendeten Begriffe für staatliche Zahlungen und Direktzahlungen werden in Anführungszeichen wiedergegeben.
} 
USA und der EU nachgegangen. Die Literatur scheint an diesen Fragen bisher erstaunlich wenig Interesse zu zeigen. ${ }^{4}$

\subsection{Ursprung des Instruments der staatlichen Zahlungen}

Das Instrument der staatlichen Zahlungen an die Landwirtschaft ist keine Erfindung des 20. Jahrhunderts. Bereits im 18. Jahrhundert unterstützte Großbritannien die Erzeugung ausgewählter Agrarrohstoffe in seinen damaligen Kolonien mithilfe von sogenannten „direct bounties“, die für die nach Großbritannien exportierten Mengen gewährt wurden (Beer 1907). Das Ziel war, die Eigenversorgung des Britischen Empire zu erhöhen. Beispielsweise sollte damit Faserhanf aus Nordamerika mittels der Anfang des 18. Jahrhunderts eingeführten Beihilfe den günstigeren russischen Faserhanf vom britischen Markt verdrängen. Diese Unterstützung der Koloniallandwirtschaft war allerdings nicht unstrittig: „,[T] here was some objection to the colonial planter receiving bounties, while the British farmer was not entitled to them though he paid the taxes that they necessitated" (ibid.: 202).

Dasselbe Ziel der Erhöhung der Selbstversorgung hatten die „bounties“, die die US-Bundesstaaten gewährten, nachdem die USA im Jahr 1776 unabhängig von der britischen Kolonialmacht wurden. Die Vielzahl dieser Maßnahmen lässt Powell (1913: 192) konkludieren, dass „the scope of state activity in this direction has been limited only to the extent that the imagination of legislators and of lobbyists has been limited". Insbesondere wurde auf diese Weise versucht, die Seidengewinnung in größerem Maßstab zu etablieren. Bereits im Jahr 1623 förderte Virginia die Haspelseide mit einem „bounty“ und schrieb die Anpflanzung von Maulbeeren vor. Im Jahr 1783 führte Connecticut ein „bounty“ sowohl für Maulbeeren (gezahlt pro Baum) als auch für Rohseide (gezahlt je Unze) ein. Mehrere Bundesstaaten folgten im Laufe des 19. Jahrhunderts, nachdem die Initiative, Unterstützung auf Bundesebene zu gewinnen, im US-Kongress scheiterte. Wie Powell (1913: 197) feststellt, war Seide ,the favorite subject for encouragement by means of bounties". Allerdings waren viele der Maßnahmen von kurzer Dauer.

Mit den pro Tonne des Erzeugnisses gezahlten „bounties“ wurde punktuell auch der Anbau von Hanf, Flachs, Ramie und Jute für den Einsatz als Faser gefördert. Der Bundesstaat Maine führte 1837 ein „bounty“ für Weizen ein und ein Jahr später eines für indischen Mais, die pro Bushel gezahlt wurden. Beide Beihilfen wurden bereits 1839 abgeschafft. In Massachusetts war von 1838 bis 1841 ein „bounty“ für Weizen in Kraft (Powell 1913).

\footnotetext{
${ }^{4}$ Baffes und de Gorter (2005: 38) erwähnen unter „,early decoupling proposals“ in den USA und der EU die Konzepte von Cochrane, Swerling, Uri et al. und Nash. Bei Little et al. (2004: 40f) werden insgesamt vier europäische „early proposals for decoupled payments“ der 1960er- und 1970er-Jahre aufgelistet: die Konzepte von Nash, Uri et al., Koester und Tangermann sowie ein Vorschlag der britischen „Agricultural Study Group of the Federal Trust for Education“ von 1970.
} 
Auch der Anbau von Zuckerrüben wurde mit „,bounties“ gefördert. Das „bounty“ in Massachusetts von 1837 wurde noch je Pfund Zucker gezahlt und richtete sich somit an die Zuckererzeuger. Im Jahr 1883 führte dieser Bundesstaat ein „bounty“ für die heimischen Zuckerrüben ein. Maine, Michigan, New Jersey, Kansas und Nebraska folgten mit eigenen „bounties“ für Zucker oder Zuckerrüben bzw. -rohr. Letzterer führte 1895 auch ein „bounty“ für Chicorée ein. Laut Cherington (1912: 381) wurden insbesondere von 1895 bis 1898 auf Ebene der US-Bundesstaaten viele „bounties“ im Zuckersektor beschlossen, um die Rübenzuckerproduktion zu fördern. In der Regel wurden sie pro Pfund Zucker gezahlt, allerdings unter der Bedingung, dass den Rübenanbauern ein bestimmter Rübenmindestpreis gezahlt wird. Die Höhe der Beihilfen und der Mindestpreise war so festgelegt, dass die Landwirte am meisten von diesen ,sugar bounties“ profitierten (ibid.: 382).

Neben den Maßnahmen der Bundesstaaten waren im 19. Jahrhundert auch zwei „bounties“ auf Bundesebene in Kraft (Powell 1913). Mit dem ersten von 1813 wurde die Fischerei und damit nicht die Landwirtschaft gefördert (ibid.). Das zweite „bounty“ wurde von 1891 bis 1894 im Zuckersektor angewandt. Es belief sich auf 2 Cent/Pfund Zucker und sollte dem heimischen Zuckersektor die Aussetzung der Importzölle für Rohzucker erträglicher machen (Ballinger 1975: 11). Die Begünstigten waren mit der Beihilfe allerdings nicht zufrieden aus Gründen, die auch Jahrzehnte später gegen die Agrarzahlungen erhoben werden: „The subsidy was visible to everyone, whereas the benefit obtained from the tariff was less so“ (ibid.). Das „sugar bounty“ hatte Charakter einer Ausgleichszahlung. Da es aber nicht den Landwirten, sondern den Zuckererzeugern zugutekam, erfüllt es nicht die Kriterien für die staatlichen Zahlungen, die die vorliegende Arbeit verwendet. 5

Anders das „sugar bounty“, das im Zuckersektor in Australien im Jahr 1903 durch den „Sugar Bounty Act“ eingeführt wurde. Gewährt wurde es den Anbauern von Zuckerrohr und Zuckerrüben, die auf ihren Plantagen ausschließlich „white labour" beschäftigten (und somit auf Zwangsarbeiter von Pazifik-Inseln verzichteten). Mit dem nachfolgenden „Sugar Bounty Act“ von 1905 konnte das „bounty“ gekürzt oder vollständig zurückgehalten werden, wenn die Löhne der Landarbeiter unter dem regionalen Standardsatz für weiße Arbeitskräfte lagen. Dieses „bounty“ - gezahlt für die gelieferte Menge von Rohr und Rüben und damit gekoppelt - hatte also eine klare soziale Komponente.

Ein „bounty“ - für Weizen - wurde in Großbritannien am Anfang des 20. Jahrhunderts diskutiert. Der damalige Kolonialminister J. Chamberlain stieß

\footnotetext{
5 Auch in einigen europäischen Ländern wurden „sugar bounties“ für die Zuckererzeuger relativ früh eingeführt, allerdings hatten sie einen indirekten Charakter. Die Subvention ergab sich aus der günstigen Relation einer Steuer, die nach dem Gewicht der Rüben berechnet wurde, zu einer vom Staat je Mengeneinheit Zucker gewährten Exportvergütung, die nach dem Steuerbetrag für die zu seiner Herstellung durchschnittlich erforderlichen rohen Rübenmenge berechnet wurde. Gewinn machte, wer überdurchschnittlich hohe Zuckerausbeuten erzielte (Ambrosius 2005: 83f).
} 
eine Zolltarifreform an, die von der 1904 gegründeten „Tariff Commission“ wissenschaftlich begleitet werden sollte. Chamberlain wollte die CommonwealthStaaten durch Präferenzzölle enger an das Empire binden, was eine Schutzzollpolitik voraussetzte, darunter auch Besteuerung der Importe von wichtigen Agrarprodukten wie Weizen und Fleisch aus den Ländern außerhalb des Commonwealth. Vor dem Hintergrund, dass die Einführung des Weizenzolls für politisch nicht durchsetzbar gehalten wurde, wurde als Alternative ein je Hektar Anbaufläche gezahltes „bounty“ für heimische Landwirte diskutiert (Marrison 1986). Allerdings war auch dieses Instrument nicht unproblematisch. „[B]ounties were, in the age of the Brussels Sugar Convention, regarded as ,the worst form of protection ... and ... very unpopular “", zitiert Marrison (1986: 176) einen Zeitzeugen. ${ }^{6}$ Am Ende empfahl die „Tariff Commission“ das „bounty“ für Weizen nicht. Seine Befürworter akzeptierten, dass es „,for the time being ... beyond the scope of practical politics“ war (ibid.: 178). Wie Collins (2000: 63) bemerkt: „Pressure for a bounty on wheat was resisted on the grounds that it would be difficult to administer and would lead to demands for it to be extended to other products."

Die Idee eines „bounty“ an Landwirte wurde in Großbritannien im Jahr 1917 wieder aufgegriffen als „deficiency payments“ für Weizen und Hafer eingeführt wurden mit dem Ziel, die heimische Erzeugung angesichts der Kriegssituation zu erhöhen (s. Kapitel 5). Dieser Schritt ging auf die Empfehlungen des MilnerAusschusses von 1915 zurück, über die Tracy (1989: 147) schreibt, dass „this seems to be the first mention of a system of ,deficiency payments "“. Allerdings scheint die Idee der „deficiency payments“ noch älter zu sein. Sie stammt offenbar von H. R. Haggard, einem Agrarexperten und Schriftsteller (u. a. „König Salomos Schatzkammer"), der 1895 erfolglos für die Konservativen für das Parlament kandidierte. Wie es aussieht, regte er dieses Instrument im Jahr 1898 an. Wie Collins (2000) bemerkt, gewannen die von Haggard ins Spiel gebrachten Zahlungen im Laufe der Zeit an Unterstützung. Ihr Vorteil wurde darin gesehen, dass sie den Verbraucher nicht in Form höherer Lebensmittelpreise belasteten: „H. Rider Haggard [...] declared himself in favour of a bounty to wheat growers by a system of deficiency payments, a form of subsidy then gaining favour due to the cost of it falling not on consumers but on the Exchequer" (ibid.: 61). Bis dahin konzentrierte sich die Debatte über mögliche Maßnahmen zur Unterstützung der Landwirtschaft angesichts der seit den 1870er-Jahren herrschenden Niedrigpreise auf den Aufbau eines Außenschutzes, der aber, so die Befürchtung und einer der Ablehnungsgründe, zur Verteuerung der Lebensmittel führen würde und deshalb äuBerst unpopulär war (Collins 2000). Konkret schrieb Haggard (1899: 413f) in seinem Tagebuch für das Jahr 1898:

\footnotetext{
${ }^{6}$ Die wichtigsten Rübenzucker produzierenden Länder subventionierten ihre Zuckerexporte durch direkte und indirekte Exportprämien. Darunter litten Großbritannien bzw. das zuckerexportierende britische Indien. Die Brüsseler Zuckerkonvention von 1902 schaffte die Exportprämien ab (Ambrosius 2005).
} 
I do $[\ldots]$ think it desirable that whenever wheat falls below a certain unprofitable price - say 30s. a quarter - a moderate bounty should be paid from the Imperial Exchequer to those who continue to grow it. Probably it is futile to expect that such a measure will be adopted except under the stern compulsion of conditions which we cannot foresee, and perhaps this also may be held to savour of Protection. Still, it will be admitted that in view of national and other contingencies, it is not to the interest of the country that wheat should go out of cultivation, or indeed that the present area under that crop should be further contracted. Nor is it to the interest of the country that the classes $[\ldots]$ who for generations have been the backbone of England, should, for the lack of a reasonable wage, which under present circumstances it is impossible to pay them, be driven from the land that bore them and herded together in the towns.

Diese Worte stammen ursprünglich aus seinem Brief an eine Zeitung, die Haggard vorwarf, für die Einführung von protektionistischen Maßnahmen zu sein und somit gegen den damaligen Freihandel-Mainstream. Haggard glaubte nicht, dass „bounties“ unter normalen Umständen eingeführt werden. Vielmehr schrieb er vorausschauend: „A bounty on corn might help, but will there ever be a bounty unless some great war has first taught the people how necessary it is that a certain proportion of our acreage should be kept under wheat? I doubt it" (ibid.: 455).

Dass Großbritannien in der Zwischenkriegszeit auf „deficiency payments“ und nicht - wie die meisten kontinentaleuropäischen Länder - auf die Schutzzollpolitik setzte, war der Tatsache geschuldet, dass es die Importe aus den Kolonien nicht beschränken wollte (Tracy 1989: 125). Aber auch in einigen anderen europäischen Ländern wurden in der Zeit punktuell staatliche Zahlungen zur Unterstützung der Landwirte beschlossen. Hervorzuheben sind die Zahlungen an Getreideanbauer in Belgien, die je Hektar gewährt wurden (Swinnen 2010b: 151) sowie direkte Beihilfen für Zuckerrüben- und Kartoffelanbauer in den Niederlanden (Ingersent und Rayner 1999: 92). In Frankreich wiederum wurde 1931 den Weinerzeugern eine Beihilfe zur Rodung von Rebstöcken gewährt mit dem Ziel, der Überproduktion von Wein entgegenzuwirken (Meloni und Swinnen 2013).

Die aufgeführten Beispiele machen deutlich, dass die Politik staatliche Zahlungen an die Landwirtschaft bereits vor dem 2. Weltkrieg relativ stark einsetzte. So, wie die Zahlungen ausgestaltet wurden, waren sie an die aktuelle Erzeugung und/ oder an die Marktpreise gekoppelt. Ihr vorrangiges Ziel war, die Erhöhung der heimischen Erzeugung zu bewirken. Es lohnt ein Blick auf die wissenschaftlichen Konzepte zu diesem Thema. 


\subsection{Wissenschaftliche Konzepte in den USA}

Johnson (1947: 145) bemerkt, dass - auch wenn das Instrument der staatlichen Zahlungen an landwirtschaftliche Erzeuger schon in der Zeit zwischen dem 1. und dem 2. Weltkrieg in Großbritannien und in den USA in Form von preisabhängigen „supplementary payments“ im Einsatz war - eine erste systematische Analyse erst im Jahr 1944 durch T. Schultz - Professor an der Chicago University und späterer Nobelpreisträger - erfolgte.

Für Schultz (1944: 301) resultierten die niedrigen Preise für Agrarerzeugnisse aus einem ,excess supply of resources in agriculture [...], primarily labour“. Daraus folge, dass die nationale Agrarpolitik als Hauptziel ,the attainment of a better equilibrium in the distribution of the labour force, with special emphasis upon reducing to a minimum the excess labour supply in agriculture" haben sollte (ibid.: 303). Dieses könne die bisherige US-Agrarpolitik, die auf Angebotskontrolle und Preisstützung setzte, nicht bewirken. Des Weiteren bemerkte Schultz (1944: 307), dass die Abwanderung der Arbeitskräfte aus der Landwirtschaft eher bei höheren, und nicht niedrigeren, Agrarpreisen stattfindet, denn die treibende Kraft für diese Migration sei die Verfügbarkeit von nichtlandwirtschaftlichen Arbeitsplätzen.

Die Politik sollte sich darauf konzentrieren, „to maintain farm incomes and, thus, when necessary, offset the adverse effects of cyclical developments on farm income“ (ibid.: 308). In diesem Zusammenhang schlug Schultz „compensatory price payments" vor, die in Zeiten von Wirtschaftskrisen und somit geringer Nachfrage nach Agrarprodukten greifen sollten, um ,the drop in prices received by farmers (all or as large a part as public policy may find adequate) caused by a business depression and unemployment" (ibid.: 310) auszugleichen. Die Zahlungen sollten der Differenz zwischen dem aktuellen Marktpreis für ein Erzeugnis und seinem Preis vor der Krise (oder einem Teil davon) entsprechen. Sie sollten so lange in Kraft bleiben, bis die Marktpreise das Vorkrisenniveau erreichten oder überschritten bzw. bis sich die Arbeitslosigkeit verringert (und somit die Nachfrage nach Lebensmitteln steigt). Eine Angebotskontrolle war nicht vorgesehen. Sein Konzept hatte laut Schultz (1944: 311) folgende Vorteile: ${ }^{7}$

(1) It would leave market prices free to clear whatever supplies are marketed and therefore it would not interfere either with internal or external trade. (2) It would permit the prices of farm products and accordingly the price of food to drop during a depression. (3) It would entail a claim for governmental expenditures during a depression, and the amount would increase as the depression deep-

\footnotetext{
${ }^{7}$ Schultz setzte sich 1974 mit seinem Vorschlag aus jungen Jahren auseinander und stellte kritisch fest: „Economic policy is a tricky business. The demand for it is ever so fickle; what is in fashion today is old hat tomorrow. [...] Out of my analysis of economic fluctuations, there emerged the idea of compensatory prices for agriculture. It was a new idea. [...] Although the underlying economic analysis is solid, it has not been a feasible policy proposal“" (zitiert nach Pasour 2013: 599f).
} 
ened, namely as farm prices fell. (4) Compensatory price payments would go primarily to families in the lower income brackets because most farm families receive low incomes. (5) It would not curtail agricultural production when a business depression gets under way and reduces the demand for farm products. [...] (6) Nor would this approach permit major distortions in the relative prices of agricultural products to occur as a consequence of a recession in business. (7) It would maintain the demand of farm people for industrial and other products.

Schultz entwickelte zudem das Konzept von „forward prices“ mit dem Ziel, die Preisunsicherheit zu reduzieren und damit die Effizienz in der Landwirtschaft zu steigern (Schultz 1943). Dabei ging es nicht darum, Agrarpreise auf möglichst hohem Niveau festzulegen, sondern darum, den Landwirten einen Preis für ihre Erzeugung zu sichern, ,that will induce as large a production as is consistent with the best use of agricultural resources" (ibid.: 58). In den meisten Fällen würde ein solcher Preis „be less than that which consumers with their present purchasing power are prepared to pay for farm products" (ibid.). Diese Idee wurde von D. G. Johnson weiterentwickelt (s. unten).

Zu jener Zeit schlug Schultz auch ein Konzept für „supplementing farm incomes“ vor (Schultz 1943). Er kritisierte die „benefit payments“ unter dem „Agricultural Adjustment Act“ von 1933, die auf „,criteria associated with the production side of a farm“ (ibid.: 65) bezogen waren (heute würden sie ,an die Produktion gekoppelt" genannt), denn ,the farm families needing additional income most urgently have been granted the least, while those needing it least have received the largest payments" (ibid.: 66). Sein Vorschlag war, allen Familien von der Betriebsgröße und Erzeugung unabhängige, personenbezogene Zahlungen zur Verfügung zu stellen. Er bevorzugte allerdings Sachleistungen (Lebensmittel, Bildung, medizinische Leistungen) gegenüber Barzahlungen.

Im Jahr 1944 veröffentlichte der Hochschulverband „Association of LandGrant Colleges and Universities“ den Bericht „Postwar Agricultural Policy“ im Rahmen der Debatte über die Ausgestaltung der US-Agrarpolitik nach der Beendigung der kriegsbedingten Notstandsmaßnahmen. Dieses war „[o]ne of the most serious efforts to come to grips with the nonemergency aspects of the farm policy problem“ (Benedict 1953: 469). Der Verband schlug vor, die Preisstützung und die Produktionskontrolle über mehrere Jahre schrittweise abzubauen. Den Landwirten sollten - aber nur während langen und schweren Krisen - „income payments“ gewährt werden. In wirtschaftlich guten Zeiten hingegen besteht hohe Nachfrage nach Agrarprodukten, deshalb kann die Landwirtschaft dann dem freien Markt überlassen werden. Benedict (1953: 469) stellte zusammenfassend fest, dass der ,report [...] was widely read and discussed but did not, apparently, have much effect on the course of agricultural legislation in the Congress". 
Zu jener Zeit waren preisabhängige und produktgebundene „deficiency payments“ ein unter US-Wissenschaftlern beliebtes Instrument. Eine Umfrage der „American Farm Economic Association" unter ihren Mitgliedern von 1945 zeigte, dass 30\% der Befragten „payments direct to farmers to make up the difference between the announced support level and the market price" als agrarpolitisches Instrument bevorzugten. 37\% der Befragten wählten Ernährungsprogramme, 9\% andere, nicht näher spezifizierte Instrumente und 8\% staatliche Lagerhaltung (16\% der Befragten - vornehmlich die, die freie Märkte bevorzugten - gaben dazu keine Antwort). Entsprechend sprachen sich $40 \%$ der Befragten für das Konzept der produktgebundenen „forward prices“ zur Verhinderung plötzlicher Veränderungen der landwirtschaftlichen Einkommen und $37 \%$ für Einkommensstützung ausschließlich in Krisenzeiten aus, ohne dass die Preise einzelner Produkte gestützt werden. Lediglich $4 \%$ der Befragten befürworteten das damals geltende System der hohen Preisstützung und 19\% die völlige Marktliberalisierung (Black 1946a: $605 \mathrm{f}$ ).

Im Jahr 1945 zeichnete die „American Farm Economic Association“ die besten Arbeiten über ,a price policy for agriculture, consistent with economic progress, that will promote adequate and more stable income from farming" aus (Nicholls und Johnson 1946). Insgesamt 18 Autoren wurden prämiert. Lediglich zwei Preisträger sahen für die Nachkriegszeit freie Marktpreise bei allen Agrarerzeugnissen vor. 16 Preisträger hielten mehr oder weniger ausgeprägte Preisgarantien für einige oder alle Erzeugnisse für notwendig, davon befürworteten 14 eine Form von „forward prices“ „to call forth no more than the quantities of those foods and fibers for which there is an effective demand at full employment" (ibid.: 272). 16 Autoren - also die große Mehrheit - schlugen eine Form staatlicher Zahlungen an landwirtschaftliche Erzeuger vor. Zum einen waren das, bei zehn Preisträgern, „direct payments“. Diese sollten greifen, wenn Preise und/oder Einkommen unter eine festgesetzte Schwelle (z. B. „forward price") sinken, um die Lücke zwischen dieser Schwelle und dem tatsächlichen Preis bzw. Einkommen auszugleichen. Damit sollte die Regierung die eingegangenen Preisgarantien einhalten.

Zum anderen wurden auch Zahlungen - „income payments“ - für andere Zwecke als die Umsetzung der Preispolitik vorgeschlagen, nämlich „for lessening the effects of fluctuating income, price levels and employments on farm incomes" (ibid.: 275). Wie Nicholls und Johnson (1946: 276) feststellten, herrschte „a unanimous agreement among the award winners discussing the problem that any minimum income commitments should be made good by direct price or income payments to producers rather than through market price supports". Es wurde dabei zwischen der Zeit des Übergangs vom Kriegs- in den Friedensmodus und der Zeit danach - und hier zwischen Krisen- und wirtschaftlich guten Zeiten - unterschieden (Krisenzeiten - „depressions“ - waren als Zeiten mit hoher Arbeitslosigkeit definiert). Drei Preisträger wollten die Zahlungen an die Einhaltung bestimmter 
Umweltauflagen binden. Nur einer sprach sich für Angebotskontrollen, fünf für eine Ernteversicherung aus.

Darunter regte Cochrane (1945) entkoppelte, zeitlich begrenzte „income payments" bei freien Marktpreisen an (s. unten). Eggert (1945) sprach sich für die Übernahme des britischen Systems der „deficiency payments“ aus. Waugh (1945: 776) schlug den Einsatz von „deficiency payments“ zwar für die Übergangszeit nach Kriegsende vor, hob jedoch hervor, dass sie „no panacea for the problems of food and agriculture" darstellen, denn sie ,like market price supports [...] tend to maintain a high rate of production even if demand falls off". Für die Zeit danach empfahl er für Krisenzeiten nicht näher spezifizierte ,income payments“, am besten „conditioned upon compliance with a program of sound readjustments in the agriculture“ (ibid.: 780). Einer der Preisträger schlug für Krisenzeiten „direct income payments“ vor, deren Höhe „at least slightly in excess of the variable costs of the farmers whose production is needed" festgelegt sein sollte (Nicholls und Johnson 1946: 275). Froker (1945: 848) wiederum brachte an die aktuelle Erzeugung gekoppelte „parity income payments“ ins Spiel: „If total net farm income from normal operations is below the prescribed minimum then it should be supplemented with parity income payments direct to individual farmers in proportion to the market value of the products which they produce and sell." Zudem sahen vier Preisträger für die Übergangszeit von drei bis zehn Jahren degressiv ausgestaltete „income payments“ für die Überschusserzeugnisse „to supplement free market prices" vor (Nicholls und Johnson 1946: 281). Diese sollten den Unterschied zwischen dem aktuellen Durchschnittsmarktpreis für ein Erzeugnis und einem Paritätsniveau (in der Regel dem für zwei Jahre nach Kriegsende garantierten Niveau von $90 \%$ der Parität) ausgleichen.

Johnson (1945) schlug sowohl „supplementary payments“ als auch „income payments“ (die er "compensatory payments“ nannte) vor. Beide Arten der Zahlungen sollten die Differenz zwischen dem durchschnittlichen Marktpreis für ein einzelnes Erzeugnis in einer Region und dem staatlich festgelegten „forward price“ ausgleichen. Der Unterschied bestand darin, dass bei „supplementary payments" die „forward prices“ leicht unter den Gleichgewichtspreisen liegen sollten. Bei „compensatory payments“, die in Wirtschaftskrisen mit hoher Arbeitslosigkeit greifen sollten, sollten die „forward prices" so festgelegt werden, dass die landwirtschaftlichen Einkommen ein bestimmtes Niveau (75\% des Durchschnittseinkommens in den zwei der Krise vorausgegangenen Jahren) erreichen. Beide Arten von Zahlungen sollten produktgebunden für die verkaufte Menge gewährt werden. Eine Mindestzahlung von 20 US-Dollar (USD) pro Jahr und Familienangehörigen wurde vorgesehen, um die einkommensschwächeren Betriebe mit niedrigen Verkaufsmengen (und somit niedrigen Zahlungen) zu unterstützen.

In seinem Buch „Forward Prices for Agriculture“ von 1947 entwickelte Johnson das Konzept von „forward prices“ weiter. Er sprach sich darin für die Abschaffung der Angebotskontrolle aus „because it is ineffective as a method and 
because the effects upon resources allocation are undesirable" (Johnson 1947: 142). Sein Anliegen war, Preisschwankungen (bzw. Unsicherheiten) auf den Agrarmärkten zu bekämpfen, weil sie das Investitionsverhalten der Landwirte negativ beeinflussen und somit die Effizienzsteigerungen im Sektor bremsen können. Die Aufteilung in „supplementary payments“ und „,compensatory payments“ behielt er bei.

Sein Konzept sah vor, dass die Regierung - rechtzeitig bevor die Landwirte ihre Produktionsentscheidungen treffen - die erwarteten Gleichgewichtspreise („forward prices") verkündet und später sicherstellt, dass die Landwirte diese Preise auch erzielen. ${ }^{8}$ In den Jahren, in denen die „forward prices“ über den tatsächlichen Marktpreisen liegen, sollten Maßnahmen wie staatliche Lagerhaltung und „supplementary payments“ vorgenommen werden, um die „forward prices“-Garantie einzuhalten. Die Zahlungen sollten die Differenz zwischen den beiden Preisen ausgleichen. „Compensatory payments“ wiederum sollten in Wirtschaftskrisen greifen, um den Landwirten ein Mindesteinkommen (75\% des Nettoeinkommens in der Vorkrisenzeit) zu gewährleisten. Den Begriff lieh Johnson (1947: 216) dabei von Schultz. Er gab zu, dass , [t] $[$ he integration of the two programs may present certain problems" (ibid.: 218). Zum Einwand, die Einführung der Zahlungen wäre mit enormen Ausgaben verbunden, wies er auf ihren volkswirtschaftlichen Nutzen hin: „The contribution of the payments during a depression to regaining full employment and to the longer-run effective use of resources must be considered. In no sense do such payments represent a real cost to society and if they lead to fuller use of resources, the payments will result in a net social gain to society" (ibid.: 147).

Das Konzept von „forward prices“ kritisierten u. a. Tsou und Black (1944), die empfahlen, dass ,additions to income should take the form of direct supplements, not price enhancements" (ibid.: 550). Sie sprachen sich für den Einsatz von staatlichen Zahlungen zum Zwecke der „production adjustments“ aus, um chronische Überschüsse zu vermeiden und Landwirte zur Produktion anderer Erzeugnisse zu bewegen: „If there are to be subsidies, they should be in the form of income payments, rather than of price supports; and they best take the form of specific aid in shifting production to other lines" (ibid.: 546).

Vor diesem Hintergrund sticht das Entkopplungskonzept von W. W. Cochrane hervor. ${ }^{9}$ Seine Arbeit für den oben erwähnten Wettbewerb der „American Farm Economic Association“ im Jahr 1945 beginnt mit der Kritik der damaligen Preispolitik. Cochrane (1945: 813) stellte fest:

\footnotetext{
${ }^{8}$ Gardner (1996) stellt dar, wie sich die Auffassungen von Johnson mit der Zeit weg von Preisstützungsprogrammen veränderten.

${ }^{9}$ Später sprach sich Cochrane gegen den freien Markt und für einen umfassenden „supply management plan" in der Landwirtschaft aus (Cochrane und Ryan 1976: 37).
} 
Within the framework of this policy prices have come to be regarded primarily as implemental tools for the maintenance of income [Herv. im Original]; the function of the price system as a mechanism for directing continuously and efficiently the use of productive resources to meet changes in consumer demand and changes in production techniques has been reduced to a subsidiary role.

In seinem Konzept sollten die Preise von der Einkommensfunktion entlastet und dem freien Markt überlassen werden. Der Übergang vom gestützten in den freien Markt sollte den Landwirten durch im Laufe der Zeit sinkende „income payments" erleichtert werden. Cochrane (1945) schätzte diesen Zeitraum auf etwa fünf Jahre. Die neuen Zahlungen sollten auf Basis der individuellen Erzeugung der Jahre 1939 bis 1941 gewährt werden, sodass die Landwirte nicht auf die Produktion der Überschusserzeugnisse angewiesen wären, um die Stützung zu erhalten. Sie könnten zu anderen Kulturen wechseln, ohne den Zahlungsanspruch zu verlieren (die Möglichkeit der Aufgabe der landwirtschaftlichen Produktion wird an der Stelle nicht erwähnt). Es handelte sich somit um von der aktuellen und zukünftigen Produktion entkoppelte Zahlungen und höchstwahrscheinlich ein erstes solches Konzept in der Wissenschaft. Allerdings waren die „income payments“ von Cochrane nicht von den Preisen entkoppelt, weil - wie so oft zu der Zeit die Paritätspreise (und damit auch die aktuellen Produktionskosten) zur Berechnung der vorgeschlagenen Zahlungen herangezogen wurden.

Dass zu jener Zeit staatliche Zahlungen an die Landwirtschaft vielfach diskutiert werden mussten, zeigt der Beitrag von Jesness (1946), der sich mit der damaligen Kritik an diesem Instrument auseinandersetzte. Er schrieb:

The most potent argument leveled against income payments is that they are a form of subsidy. Supporters of price maintenance through the use of government funds do not seem to realize that it likewise constitutes subsidy. The difference on these grounds is more apparent than real. Another objection raised against income payments is that their continuance depends upon the willingness of Congress to appropriate the necessary funds. However, this limitation may in fact be an advantage in that it may give some promise that a program of income payments will be reserved for conditions of extreme depression or for other unusually abnormal situations instead of becoming a permanent source of farm income. (ibid.: 7)

Wie Schultz und viele andere Wissenschaftler zu der Zeit wollte Jesness staatliche Zahlungen nur als Instrument für Krisenzeiten verstanden wissen. Denn wenn sie dauerhaft etabliert würden, würden sie tendenziell ,be capitalized in land values; $[\ldots]$ interfere with production shifts, particularly if they are based on past production; [...] slow up movement of farm people into other fields" (ibid.). Als Dauerinstrument wurden die staatlichen Zahlungen hier kritisch gesehen. 
Auch wenn der sogenannte Brannan-Plan von 1949, der ,income payments“ für nichtlagerfähige Erzeugnisse vorsah, nicht umgesetzt wurde (s. Kapitel 4), verfolgten und entwickelten Wissenschaftler in den 1950er-Jahren die Idee der staatlichen Zahlungen weiter. Beispielsweise schlug Brandow (1955) im Rahmen des „modified compensatory payment plan“ „,ompensatory payments“ an landwirtschaftliche Erzeuger als Ersatz für das zu der Zeit geltende System der Preisstützung vor. Die Zahlungen sollten gewährt werden, wenn die Marktpreise unter eine bestimmte Schwelle (90\% der Parität) sinken. Um Anreize für Produktionssteigerungen zu minimieren, sollten die Zahlungen nur für einen Teil der aktuellen Erzeugung eines Landwirts geleistet werden, und zwar für den Teil, der der ihm zugewiesenen „marketing allotment“ entsprach. Die über die „allotment“ hinaus gehenden Mengen sollten von den Zahlungen nicht profitieren können, sie könnten aber auf dem Markt verkauft werden. Die „marketing allotment“ für ein Erzeugnis auf nationaler Ebene sollte $75 \%$ der gesamten Verkaufsmenge eines Erzeugnisses im ausgewählten historischen Basiszeitraum entsprechen; sie sollte nicht von Jahr zu Jahr angepasst werden. Diese Gesamtmenge sollte dann auf die Landwirte in Abhängigkeit von ihren individuellen Verkaufsmengen im gleitenden Basiszeitraum der letzten drei Jahre verteilt werden.

Wiederum Galbraith (1955) regte zur selben Zeit ein System ohne Angebotskontrollen an, in dem an die aktuelle Erzeugung gebundene „direct payments“ für pflanzliche Erzeugnisse den Unterschied zwischen dem gewünschten Paritätsniveau und dem Marktpreis ausgleichen sollten. Statt Angebotskontrollen empfahl er „supplementary payments“ für tierische Erzeugnisse, um die Nachfrage nach Futtermitteln zu steigern und für ,a movement of resources from the sector where they are an embarrassment to the sector where they can be accommodated with comparative ease“ zu sorgen (ibid.: 303). Auch sollten „direct resource transfer payments" für die Umwandlung der Ackerfläche in Dauergrünland eingeführt werden. Er argumentierte dabei wie folgt: „A small subsidy of livestock products would, in effect, be used to eliminate the need for a much larger and much less useful one for cereals. With the surplus would go the [production] controls" (ibid.).

Auch der Vorschlag von Clawson (1958) sah - mit Ausnahmen für Milch sowie Obst und Gemüse - keine Angebotskontrolle vor. Die Preisstützung sollte aufgegeben werden; die Agrarpreise sollte gänzlich der freie Markt regeln. Gleichzeitig empfahl sie, den Landwirten in drei unterschiedlichen Situationen „,supplemental payments" zu gewähren: Wenn vorübergehend kein Gleichgewicht zwischen Angebot und Nachfrage besteht (z. B. bei beschränkter Nachfrage oder besonders großer Ernte, in Wirtschaftskrisen) sowie „even on a long-term basis as a means of raising agricultural incomes in general to a new level" (ibid.: 276). Clawson (1958: 275) begründete diese Stützung damit, dass die Landwirte, neben „a flow of farm commodities“, für die sie am Markt bezahlt werden, auch ,a productive capacity“ zur Verfügung stellen „to take care of our needs now and in the 
future, including reserve capacity to meet emergency needs“. Das neue System sollte, wenn nicht alle, dann zumindest die wichtigsten 20 Agrarerzeugnisse umfassen. Die Zahlungen sollten für $80 \%$ der gleitenden betrieblichen Basismenge des betreffenden Produktes gewährt werden. Diese „output base“ entsprach der durchschnittlichen Produktmenge, die ein Betrieb in den jeweils letzten fünf Jahren erzeugte. Der Betrag pro Einheit sollte sich aus der Differenz zwischen dem durchschnittlichen Marktpreis für ein Erzeugnis und einem auf der Parität basierten Einkommensstandard ergeben.

Im Konzept von Clawson (1958) sind Elemente erkennbar, die auf eine zumindest teilweise Entkopplung der Stützung von der aktuellen Erzeugung deuten. Die „output base“ sollte „freely transferable, either to accompany lands transferred or separately, from farm to farm" sein (ibid.: 275) und ,[t] farming might be allowed to take his base with him" (ibid.: 276). Zudem betont Clawson, dass sie „would not require any production to qualify for such payments in a particular year" (ibid.: 275f). Allerdings würde dann eine Kürzung und - nach fünf Jahren - der Verlust der Zahlungen erfolgen; damit hätten die Zahlungen einen Übergangscharakter. Die gleitende „output base“ bot zudem einen Anreiz zur Flächenausweitung, auch wenn Clawson (1958) bemerkt, dass die Begrenzung der Zahlungen auf „output base“ bei niedrigen Marktpreisen dämpfend wirken würde. Wäre die „output base“ im Konzept von Clawson (1958) nach ihrer Festlegung unveränderbar, wären die Zahlungen von der Erzeugung entkoppelt (Die Unabhängigkeit von Preisen wäre allerdings nicht gegeben, da mit den Paritätspreisen operiert wurde.).

Einen neuen Reformansatz mit Entkopplungselementen bot Swerling (1959) an. Wie er selbst bemerkte, war zu der Zeit ,[t]he need for forging and testing new tools of agricultural policy" breit anerkannt (ibid.: 173). Er betonte gleichzeitig, dass die künftige Agrarpolitik „,will have to stand more on its merits in the broad public interest" und dass „legislation that blatantly confers special privileges will have hard going in Congress" (ibid.: 174). Sein Konzept zielte auf die Ablösung der Preisstützung durch eine obligatorische Einkommensversicherung für Landwirte ab. Konkret bestand es darin, ,income protection for individual farm operators by the application of standard principles of social insurance" anzubieten (ibid.). Dieses würde heißen, dass in der Landwirtschaft ein ähnlicher Mechanismus als Sicherheitsnetz zum Tragen kommen würde, wie in anderen Wirtschaftssektoren (z. B. Arbeitslosenversicherung). Das Ziel war, starke Einbrüche landwirtschaftlicher Einkommen zu verhindern und somit Einkommensschwankungen, unabhängig von ihrer Ursache, vorzubeugen. Wie von Swerling (1959: 181) festgehalten: „Farm operators would enjoy substantial relief as needed during recessions of the length and severity experienced in the postwar period. [...] protection would be particularly effective against fortuitous variations in crop yields and prices or against natural disaster." 
Bei Swerling (1959) sollten die Zahlungen nicht von den Preisen, sondern von dem in der Vergangenheit in der Einkommenssteuererklärung deklarierten individuellen Einkommen eines jeden Landwirts abhängen. Konkret schlug er vor, das versicherte Einkommen auf $75 \%$ des Basiseinkommens des Landwirts festzulegen. Dabei sollte das Basiseinkommen dem gleitenden Durchschnitt des individuellen Nettogewinns aus der selbstständigen landwirtschaftlichen Tätigkeit in den letzten fünf Jahren entsprechen. Der Landwirt sollte Zahlungen dann erhalten, wenn sein Nettogewinn in dem betreffenden Jahr unter das versicherte Einkommen fiel; die Differenz zwischen diesen beiden Größen würde er ausgeglichen bekommen. Um in den Genuss der Zahlungen zu kommen, müsste der Landwirt keine bestimmten Erzeugnisse produzieren oder gar in der Landwirtschaft weiter tätig sein. Der Anspruch auf die Zahlungen würde nicht an die Fläche oder den Betrieb, sondern an die Person gebunden und nicht übertragbar oder vererbbar sein. Darüber hinaus empfahl Swerling (1959) - neben Höchstgrenzen pro Person - auch Kürzung der Zahlungen, wenn das Einkommen des Landwirts aus nichtlandwirtschaftlichen Tätigkeiten eine Schwelle überschritt. Einen Teil des Versicherungsbeitrags müsste der Landwirt selbst finanzieren, der restliche - größere Teil würde durch Finanzmittel aus dem Staatshaushalt gedeckt.

Anfang der 1960er-Jahre wurden in den USA (entkoppelte) Direktzahlungen vermehrt vorgeschlagen, um die Auswirkungen des empfohlenen Abbaus der Preisstützung aufzufangen. Aufmerksamkeit verdienen insbesondere drei wohl erste Konzepte: das des Wirtschaftswissenschaftlers der Harvard University H. Houthakker, das des „Committee for Economic Development" (CED) unter der Mitarbeit von T. Schultz und das des Agrarökonoms E. O. Heady. Diese Arbeiten enthalten konkrete Handlungsempfehlungen für die damalige praktische Agrarpolitik der USA. Die Zeitzeugen Hadwiger und Talbot (1965: 6) bemerken mit Blick auf den Anfang der 1960er-Jahre: „Although most prominent economists (even in 1962) passed up [...] opportunities as existed to be active in the process of making government policy, a breed of economists did emerge which wanted not only to understand the problems, but to find acceptable solutions and sell them."

Houthakker (1961: 63) stellte zunächst die Frage: „How is it that [the agricultural price support programs] have survived so much criticism?" Er beantwortete sie mit dem Hinweis auf das Fehlen praktikabler Alternativen. Ein abruptes Ende der Preisstützungsprogramme sah er kritisch, weil dieses die Wirtschaft stark belasten würde. Er lobte das Versicherungskonzept von Swerling (1959), merkte aber an, dass gerade der Übergang in ein solches System geregelt werden sollte. Mit seinem Vorschlag zu „transitional acreage payments“ wollte er diese Lücke schließen. Darunter sind Zahlungen pro Acre Anbaufläche zu verstehen, die den Unterschied zwischen dem aktuellen (hohen) Stützpreis und dem Preis im freien Markt, der vermutlich niedriger ausfallen würde, ausgleichen. Nur die Erzeugnisse, die vor der Reform der Preisstützung unterlagen, wären förderfähig. Die Begüns- 
tigten sollten Landwirte sein, die diese Erzeugnisse zu einem noch zu bestimmenden Stichtag in der jüngeren Vergangenheit anbauten.

Die Höhe der Zahlung je Acre könnte sich, so der Vorschlag, am Anfang aus der Differenz zwischen dem alten und neuen Preis multipliziert mit dem Landesdurchschnittsertrag ergeben und würde somit je Kultur variieren. Sie sollte für alle Anbauer des jeweiligen Erzeugnisses gleich ausfallen. Allerdings würden effizientere (größere) Betriebe im Vergleich zum System der an die aktuelle Erzeugung gekoppelten Maßnahmen schlechter gestellt, da sie höhere Erträge als Durchschnitt vorweisen können. Folglich ,[a] much larger part of the subsidies to agriculture $[\ldots]$ would reach those for whom they are presumably intended" (ibid.: 66). Houthakker meinte damit die weniger produktiven, kleineren Betriebe.

Die Innovation seines Konzeptes bestand insbesondere darin, dass „[t]he amount received would be independent of past, present, or future output on the land concerned“" (ibid.: 64). Das bedeute konkret, erklärte Houthakker (1961: 65), dass eine Erhöhung der Anbaufläche für das betreffende Erzeugnis nach dem Stichtag keinen Einfluss auf die Höhe der Zahlungen haben würde, ebenso wie der Anbau anderer Kulturen. Auch Begünstigte, die nach dem Stichtag die Landwirtschaft ganz aufgeben würden, würden die „transitional acreage payments“ nicht verlieren. Somit wäre die Zahlung nicht von der aktuellen und zukünftigen Erzeugung abhängig und damit entkoppelt (auch wenn Houthakker diesen Begriff nicht verwendete). Nach dem Inkrafttreten des neuen Stützungsprogramms sollten seine Kriterien grundsätzlich unverändert bleiben. Eine einzige Ausnahme ließ Houthakker zu: Falls sich der prognostizierte Marktpreis - der Einfluss auf die Höhe der Zahlung hat - als unrealistisch erweist, darf er korrigiert werden. Er regte zudem an, die Zahlungen zeitlich zu begrenzen (z. B. auf fünf Jahre) und sie degressiv auszugestalten. Nach der Übergangsperiode sollten keine staatlichen Eingriffe in die Agrarmärkte mehr zulässig sein, das heißt, dass die Landwirtschaft dem freien Markt überlassen wäre.

Houthakker (1961: 65) hob folgende Vorteile des „transitional acreage payments"-Systems hervor:

The great merit of [,the transitional acreage payment"] is that it does not provide an incentive for excessive production, as high price supports do. The amount received by the individual farmer on account of [transitional acreage payments] is independent of his production. The market price alone is his guide in deciding what to produce. If he is efficient, he will find it worth his while to produce more; if he is inefficient he may change to other crops or even leave farming altogether.

Auch seien die mit den „transitional acreage payments“ verbundenen Ausgaben niedriger als die des aktuellen Systems. Zudem würde im freien Markt die Gefahr der stetig steigenden Überschüsse verschwinden. Das Instrument der Angebots- 
kontrolle, das Houthakker (1961) sehr kritisch bewertete, wäre - zusammen mit Exportsubventionen und staatlichen Ankäufen - nicht mehr nötig. Ein weiterer Vorteil der „transitional acreage payments" sei, dass sie mit einer Absenkung der Preise für Verbraucher und Verarbeiter einhergehen würden (ibid.: 65). Houthakker (1961) mahnte an, die Zahlungen tatsächlich zeitlich strikt zu begrenzen. Eine permanente Zahlung würde nach seinen Worten ,a caste of rural pensioners“ kreieren und ,even the most sentimental devotees of the family farm would hardly advocate this“ (ibid.: 66). Er schloss mit folgenden Worten:

The appeal of this proposal is not to those who want to preserve the present structure of agriculture regardless of the burden on consumers and taxpayers; it is to those who recognize that in a progressive economy agriculture must change along with all other sectors, but who also recognize the wisdom of tempering the wind to the shorn lamb. (ibid.)

Diese Arbeit von Houthakker wurde explizit in der Studie des „Committee for Economic Development" von 1962 erwähnt, die ebenfalls ein Entkopplungskonzept vorschlug: „While the Committee was at work on this Statement a plan very similar to the one suggested here was independently proposed by Hendrik S. Houthakker" (CED 1962: 49). Neben dem Reformkonzept bot CED (1962) eine detaillierte Analyse der Wurzeln des herrschenden „farm problems“, der bis dato ergriffenen Maßnahmen sowie deren Auswirkungen auf die Landwirtschaft an.

Für das Umgehen mit dem „farm problem“ sah CED (1962) grundsätzlich drei mögliche Handlungsansätze: der ,laissez-faire approach“, in dem sich der Staat zurückhält und die Einkommensentwicklung in der Landwirtschaft dem freien Markt überlässt, der „protectionist approach“, in dem der Staat zwecks der Einkommensstützung stark in die Märkte eingreift und schließlich der ,adaptive approach“, der „calls for action by government working with the free market, not against it" (ibid.: 12). Schon zu Beginn der Studie sprachen sich die Autoren für letzteren aus und rechtfertigten ihn wie folgt: „Small steps will not do. We are dealing with a big and difficult problem. [...] We are recommending many governmental activities here that we would usually regard as inappropriate. The circumstances, however, are unusual“" (ibid.: 26). Diesen Ansatz zu verfolgen wäre kostengünstiger als die Beibehaltung des geltenden Systems, aber „,it will not be cheap and easy; if it were, it would not be effective“ (ibid.). Das oberste Ziel war, der Agrarpolitik innerhalb einer Übergangsperiode von fünf Jahren zu ermöglichen, „to work its way out of a larger number of otherwise permanent governmental operations in the economy" (ibid.).

Das Reformkonzept des CED ist breiter aufgestellt als das von Houthakker (1961) und enthält zwei Kategorien von Maßnahmen. Zum einen sind das Maßnahmen mit dem Ziel, die Landwirte zur Aufgabe der landwirtschaftlichen Produktion zu bewegen. Dazu zählt - neben den Weiterbildungs- und Umschulungs- 
maßnahmen für Landwirte - der Abbau der Preisstützung. Die Stützpreise für Weizen, Futtergetreide, Baumwolle und Reis sollten reduziert werden und zwar nicht sukzessiv, sondern in einem einzelnen Schritt. CED (1962) empfahl, die Preise auf das Niveau der ,adjustment prices“ abzusenken - also der Preise, die auf dem Markt nach den avisierten Anpassungsmaßnahmen herrschen würden. Die zweite Kategorie von Maßnahmen umfasst solche Maßnahmen, die die negativen Auswirkungen des Anpassungsprozesses abfedern sollten. Ihre Ziele sind sowohl eine starke Einkommensverringerung als auch weitere Überschüsse zu verhindern (ibid.: 31). Das CED schlug das vorübergehende „Cropland Adjustment Program" vor, in dessen Rahmen Anreize geschaffen werden sollten, in weniger wettbewerbsfähigen Regionen 20 Mio. Acre Ackerfläche in Grünland umzuwandeln, sowie das ebenfalls vorübergehende „Soil Bank Program“ zur Stilllegung ganzer Betriebe.

Darüber hinaus sollte der Abbau der Preisstützung fünf Jahre lang durch ,income protection payments" als Ausgleich für den Einkommensrückgang in den Sektoren Weizen, Reis und Baumwolle, in denen der stärkste Preisrückgang zu erwarten war, begleitet werden (ibid.: 47f). Ihre Höhe errechnet sich auf Basis der dem Anbauer zugewiesenen ,acreage allotment“ (also unabhängig davon, wie viel er tatsächlich anbaut) und seines individuellen Ertrags in den zwei Jahren vor dem Programmstart, multipliziert mit dem Unterschied zwischen dem Stützpreis im Jahr 1960 und dem neuen Preis. Die betreffenden Erzeugnisse müssten nicht mehr angebaut werden. Die Unabhängigkeit von der Erzeugung nannte CED (1962: 49) „essential“ (der Begriff der Entkopplung wird dabei nicht verwendet). Diese Entkopplungsidee unterstützten allerdings nicht alle Autoren. In einer Sondernote zur CED-Publikation steht: „Payment of public funds to anyone for something not produced is economically wrong. The idea of the recommended Temporary Income Protection Payment being independent of further production of the crops in question is a serious mistake" (ibid.: 49). Die vom CED vorgeschlagenen Zahlungen sollten mit jedem Jahr sinken, um im sechsten Jahr bei null zu liegen.

Auch Heady (1962) schlug zu dieser Zeit eine Reform der US-Agrarpolitik Richtung „direct payments“ vor. Seiner Meinung nach wäre dieses „, [t] he least-cost method for society to place a given amount of compensation from treasury outlay in the hands of farmers" (ibid.: 408). Solche Zahlungen bedeuten nicht nur geringe Verwaltungskosten, sondern auch, dass ,nearly all of the money appropriated for compensation purposes can be put in the hands of farmers" (ibid.). Zu ihren Vorteilen gehöre auch Folgendes:

Unlike price supports above market levels with required storage, none of the funds from direct payments need be siphoned off into commodity storage and similar sectors. Neither does investment need to be made in a large staff to administer and police the program, as in the case of input or output quotas. Finally, under certain 
conditions $[\ldots]$, direct payments can give more complete freedom to the market in allocating resources of agriculture among commodities in line with consumer preference. (ibid.)

„Direct payments leave greater power to the market“, schlussfolgerte Heady (1962: 411). Dafür aber müssen sie ,a lump-sum quantity, devoid of relation to units of output or inputs used in future periods“ sein (ibid.: 409). Denn „[o]nce direct payments become scheduled to inputs or outputs, they promise to draw or hold an ,overage" of resources in agriculture and of products onto the market", erklärte Heady (1962: 409). Allerdings: „[L]ump-sum payment can still be based on past or historic base without affecting future output“ (ibid.). Darüber hinaus könnten sie mit Elementen ausgestattet werden, ,which would not retard but would catalyze migration of labor and capital" (ibid.: 412). Er schlug in diesem Zusammenhang vor, dass der Anspruch auf die neuen Zahlungen auch nach Aufgabe der landwirtschaftlichen Produktion nicht verloren geht. Im Konzept von Heady (1962) sollten „direct payments“ 20 Jahre lang die Einkommensverluste ausgleichen, die die Landwirte aufgrund der Reform erfahren würden. Der Differenzbetrag zwischen dem alten und dem neuen Niveau der Einnahmen (,revenu-

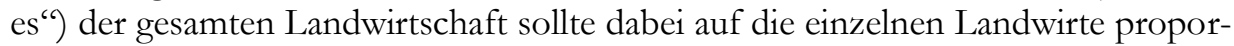
tional zu ihrem Anteil an der gesamten Erzeugung vor der Reform heruntergebrochen werden.

\subsection{Wissenschaftliche Konzepte in den USA: Zusammenfassung}

Zusammenfassend lässt sich feststellen, dass in den USA in den Jahren nach dem 2. Weltkrieg zahlreiche Vorschläge zum Einsatz der staatlichen Zahlungen unterbreitet wurden. Der Schwerpunkt dieser Ausarbeitungen lag zunächst auf „deficiency payments"-artigen, antizyklischen Zahlungen zur Umsetzung der Preispolitik. Die Preise sollten nicht durch staatliche Ankäufe und Lagerhaltung sowie Angebotskontrolle, sondern mittels „deficiency payments“ gestützt werden. Auch wenn das nach Kriegsende geltende, sehr hohe Niveau der Preisstützung von $90 \%$ der Parität von der Mehrheit der Ökonomen abgelehnt wurde, scheinen die von ihnen empfohlenen Preisgarantien immer noch großzügig gewesen zu sein. Selbst ansonsten eher liberal ausgerichtete Ökonomen sahen eine starke Rolle des Staates auf den Agrarmärkten vor. Dieses erklärt Gardner (1996: 231) mit den zu der Zeit noch lebhaften Erinnerungen an die „Great Depression“: „[F]ear of the agricultural consequences of depression led the dominant market-oriented wing of economists in 1945-50 to recommend producer price guarantees more generous than even the prairie populists in Congress were recommending in 1990." Die vorgeschlagenen Zahlungen bezweckten die Stabilisierung von Preisen und Einkommen. Sie sollten nicht dauerhaft greifen, sondern lediglich in Krisenzeiten (Vorkri- 
sen-Preisniveau als Benchmark) bzw. um das gewünschte Preisniveau („forward prices") zu erreichen, greifen. Die Ökonomen schienen bei „deficiency payments“ von der Konjunktur- und Krisentheorie von Keynes inspiriert worden zu sein. Schultz schrieb 1974 zurückblickend auf diese Zeit: , [] was, no doubt, influenced all too much by Keynesian thinking" (zitiert nach Pasour 2013: 599). Auch die Zeitzeugen Tsou und Black (1944) stellten solche Einflüsse bei Schultz fest.

Im Gegensatz zu Zahlungen zur Umsetzung der Preisgarantien waren die Reformkonzepte, die Gardner (1996: 226) ,attempts to find creative ways to accomplish the desirable consequences of price supports without (as much of) the undesirable effects" nennt, in den ersten Jahren nach dem 2. Weltkrieg relativ selten. Zu nennen sind die im Jahr 1945 vorgeschlagenen Ausgleichszahlungen, um negative Auswirkungen einer Senkung der sehr hohen Preisstützung abzufedern, wobei dieses damals noch nicht bedeutete, dass nach der Übergangszeit gänzlich auf sie verzichtet würde (es war vielmehr ein Übergang vom sehr hohen und starren zu niedrigerem und flexiblem Preisstützungsniveau). Eine Ausnahme bildete Cochrane (1945), dessen ,income payments“ einen Übergang vom gestützten in den freien Markt begleiten sollten. Auch wurden Zahlungen vorgeschlagen, die nicht an Preise für einzelne Erzeugnisse, sondern an das Agrareinkommen gebunden waren. Unter den vorgenannten Arbeiten der US-Wissenschaftler stechen insbesondere die ausgereiften Konzepte Houthakker (1961), CED (1962) und Heady (1962) hervor, weil sie von der aktuellen und zukünftigen Erzeugung entkoppelte Zahlungen vorsahen. Aber auch frühere Konzepte - von Cochrane (1945), Clawson (1958) und Swerling (1959) - enthielten bereits Entkopplungselemente. Eine diesbezügliche, konkretere Bewertung fällt allerdings schwer, weil oft wichtige Details zu den vorgeschlagenen Zahlungen fehlen, wie z. B. bei den 1945 vorgeschlagenen „income payments“.

Es stellt sich die Frage, wann die hier vorgestellten wissenschaftlichen Konzepte in der praktischen Agrarpolitik umgesetzt wurden. Die erste Kategorie der Zahlungen - die preisabhängigen, auf Produktbasis gewährten „deficiency payments" - wurde nach dem 2. Weltkrieg in den USA vielfach eingesetzt. Bereits im Jahr 1954 wurden solche Zahlungen für Wolle beschlossen, nachdem 1953 der sogenannte „Bell Report“ ausdrücklich empfahl, in diesem Sektor die Zölle zu reduzieren und „direct payments“ einzuführen. Der Bericht „stated that increased imports of wool would meet the greater demand for the commodities at the lower price while domestic production would be unaffected“" (Rau 1957: 131). Wie Pasour (2013: 595) bemerkt, wurden die „,complementary payments“ von Schultz durch die US-Politik in der Farm Bill von 1973 in Form von „deficiency payments“ aufgegriffen. Gardner (1996: 231) hingegen sieht mehr Ähnlichkeiten mit den „marketing loan“-Zahlungen, da hier - im Gegensatz zum „deficiency payment"-Programm - keine Angebotskontrollen vorgesehen waren. Die Festlegung der „forward prices“ vor Aussaat wurde spätestens ab 1974 in den USA zur gängi- 
gen Praxis, jedoch waren die Stützpreise keine Gleichgewichtspreise. Dafür waren sie zu hoch festgelegt, was in Überschüssen resultierte.

Ungeachtet dieser Entwicklungen gibt es Hinweise darauf, dass nicht die vorgenannten Konzepte zu preisabhängigen bzw. nicht-entkoppelten Zahlungen von der Politik aufgegriffen wurden, sondern dass - umgekehrt - sich die Wissenschaftler von der praktischen Politik inspirieren ließen. ${ }^{10}$ Schultz (1943: 57f) selbst erwähnt die Festlegung eines ,specific price“ für Soja durch die US-Regierung vor Aussaat 1942, um zu illustrieren, ,how an assured forward price works“. Benedict (1953: 403) nennt die im Jahr 1941 verkündeten garantierten Preise für Schweine und andere Erzeugnisse ,, a good illustration of the device later to be referred to as ,forward pricing““. Wie Black (1946b: 683) bemerkt, erinnern die von Schultz vorgeschlagenen „compensatory payments“ an „parity payments“ der späteren 1930er-Jahre. Sie unterschieden sich darin, dass für letztere eine weiter in der Vergangenheit liegende Periode den Benchmark darstellte, wohingegen es bei Schultz der unmittelbare Vorkrisenzeitraum war. Nicht zuletzt kamen die vom britischen Schriftsteller Haggard angeregten „deficiency payments“ in der Zwischenkriegszeit in Großbritannien zur Anwendung.

Der Einsatz der zweiten Kategorie der Zahlungen - der „income payments“, die nicht zur Umsetzung der Preisgarantien gedacht waren - war in der praktischen Politik zunächst verhaltener als der der „deficiency payments“. Die Ausgleichszahlungen der 1960er-Jahre zur Entschädigung der Erzeuger für den Rückgang der „loan rates“ weisen gewisse Ähnlichkeiten mit den im Jahr 1945 für eine Übergangszeit vorgeschlagenen „,income payments“ für die Überschusserzeugnisse auf. Allerdings waren sie - entgegen dem Vorschlag - zeitlich nicht begrenzt und nicht degressiv. Sie hatten zudem eine weitere wichtige Funktion: den Anreiz zur Flächenstilllegung zu verstärken.

Besonders lange dauerte es, bis die (entkoppelten) Direktzahlungen in der praktischen Politik eingesetzt wurden. Der Entkopplungsprozess bei den „deficiency payments“ erfolgte in den USA schrittweise ab 1985 und mündete in ,production flexibility contract payments“ von 1996 (s. Kapitel 4). Somit wurde das Konzept der Direktzahlungen viel später in der praktischen Politik umgesetzt als staatliche Zahlungen an sich. Es lässt sich zudem feststellen, dass die Politik die staatlichen Zahlungen, wie die „deficiency payments“, einsetzte, bevor sie in der Wissenschaft vorkamen, die Idee der (entkoppelten) Direktzahlungen aber ihren

\footnotetext{
${ }^{10}$ Eine Ausnahme bildeten die 1933 in den USA eingesetzten „rental payments“, die offenbar zunächst in der Wissenschaft vorgeschlagen wurden, bevor die Politik sie nutzte. Die Idee von staatlichen Zahlungen als Entgelt für Produktionsbeschränkungen scheint ursprünglich von J. S. Davis (Chefökonom des „Federal Farm Board“ von 1929 bis 1931 und langjähriger Direktor des „Food Research Institute" in Stanford) und F. Graham (Ökonom in Princeton) zu stammen. Im Jahr 1931 schlugen sie vor, Landwirten eine staatlich finanzierte Zahlung in Höhe von 2 USD pro Acre zu gewähren, damit sie Flächen aus der Weizenproduktion nehmen. Den Vorteil dieser „direct payments“ sahen sie in der Schaffung eines wirksamen Anreizes zur Produktionsbeschränkung (Hamilton 1991).
} 
Ursprung in der Wissenschaft zu haben scheint. Es konnten vor dem Jahr 1945, als Cochrane sein Konzept vorstellte, keine (entkoppelten) Direktzahlungen in den USA (aber auch in Europa) identifiziert werden.

\subsection{Wissenschaftliche Konzepte in der EU}

Es scheint, dass die Idee einer von der Produktion entkoppelten Zahlung an die Landwirte in Europa zuallererst dort entwickelt wurde, wo produktionsbezogene „deficiency payments“ breit eingesetzt worden waren: in Großbritannien. Bereits Anfang der 1960er-Jahre plädierte Nash ${ }^{11}$ (1961: 187f) dafür, das geltende System der „deficiency payments“ abzuschaffen und durch die von der aktuellen Erzeugung entkoppelten, personenbezogenen und zeitlich befristeten Ausgleichszahlungen zu ersetzen:

But in removing the system of price guarantees and re-establishing free market prices the state is by no means debarred from action to assist agriculture in the process of reorganization or to alleviate the hardships likely to be suffered by individuals in consequence of it. Instead of obstructing the withdrawal of farmers from an industry which cannot adequately reward them, the state should use its financial power to give active encouragement to it [...]. [T] he simplest method of compensating agriculture for the withdrawal of the protective system would be an unconditional payment [Herv. durch die Verf.] to all those at present engaged in farming, or to those of them deemed to be in need of compensation, calculated by the reference to the difference between the incomes now earned under the protective system and those capable of being earned under a system of free market prices. An annuity calculated in this way and payable for life to all engaged in farming, but not transferable to their successors, would, in theory at least, make it possible to bring the protective system to an end while fully making good the loss of income to its present beneficiaries.

Im Unterschied zu den vom deutschen Wissenschaftlichen Beirat im Jahr 1973 in Betracht gezogenen Einkommenszahlungen, die ausdrücklich zur Erhaltung der Landbewirtschaftung gedacht waren (s. Box 2), sollten die Zahlungen im Konzept von Nash an die von der Reform betroffenen Landwirte gezahlt werden, unabhängig davon, ob sie in der Landwirtschaft bleiben (Nash und Attwood 1961). Auffällig ist die zeitliche Überlappung mit der Welle von Entkopplungskonzepten in den USA. Gegenseitige Einflüsse, womöglich durch die gemeinsame Sprache befördert, sind nicht auszuschließen.

\footnotetext{
${ }^{11}$ E. F. Nash war Professor für Agrarökonomie am University College of Wales.
} 
In Kontinentaleuropa war die im Zusammenhang mit den Vorschlägen zur Harmonisierung der Getreidepreise in den EU-Gründerstaaten erhobene Forderung nach einer Kompensation für die von Preissenkungen betroffenen Landwirte Anlass zur Diskussion der sogenannten „direkten Einkommensübertragungen“ (Wille 1976: 38). Dieses bestätigt Schlotter (1963: 451) mit der Bemerkung, dass ein solches Instrument - neben den USA - „seit kurzem auch in den EWG-Ländern“ diskutiert wird. Sein Einsatz wurde im Juni 1962 in dem berühmten „Professorengutachten" vorgeschlagen. Das Gutachtergremium setzte sich aus vier Mitgliedern des Wissenschaftlichen Beirats des Bundeslandwirtschaftsministeriums und vier von der EU-Kommission nominierten Beratern zusammen. Vor dem Hintergrund der Senkung des Agrarpreisniveaus in Deutschland nach der Schaffung eines gemeinsamen Marktes für Agrarerzeugnisse empfahl das Gutachten, neben gezielten wirtschaftlichen und sozialen Anpassungshilfen auch zeitlich begrenzte „direkte Einkommensübertragungen“ in Erwägung zu ziehen (Plate 1968). Eine ähnlich ausgestaltete direkte Einkommensstützung schlugen Plate und Woermann (1962) in Deutschland im Mai 1962 vor. Die Ähnlichkeiten mit dem Konzept aus dem „Professorengutachten“ sind kein Zufall, denn beide Autoren waren Mitglieder des Gutachtergremiums; ihre Untersuchung vom Mai floss laut Plate (1968) in das Gutachten mit ein.

In Bezug auf die Verbesserung der landwirtschaftlichen Einkommen bietet die „Preispolitik [...] keine Lösungsmöglichkeit mehr“, so Plate und Woermann (1962: 74). Sie schrieben weiter, dass das Einkommensproblem in der Landwirtschaft „,nur durch Anpassungsprozesse gelöst werden [kann], die auf einen grundlegenden Wandel der Agrarstruktur und der Arbeitswirtschaft in den landwirtschaftlichen Betrieben hinauslaufen“" (ibid.: 75). Vor diesem Hintergrund seien gezielte Maßnahmen erforderlich, um „die notwendigen Anpassungsprozesse zu fördern und dabei auftretende Härten zu mildern“ (ibid.). Neben Investitionshilfen und Agrarstrukturmaßnahmen zogen sie auch „eine zeitlich begrenzte direkte Einkommensstützung" in Betracht (ibid.: 76). Sie sei insbesondere für den Fall „einer fühlbaren und schnellen Agrarpreissenkung“ erwägenswert (ibid.: 77). Zwar wurden die vorgeschlagenen Zahlungen nicht (direkt) als entkoppelte Stützung dargestellt, allerdings besaßen sie eine Reihe von Merkmalen, die in den späteren Vorschlägen für Direktzahlungen zu finden sind. Zuallererst sollten sie ,in einer Form gewährt werden, bei der die Anpassung der Produktion an die Nachfrage sowie die notwendigen Änderungen der Betriebsorganisation und der Agrarstruktur möglichst wenig behindert werden“" (ibid.: 76). Sie sollten nicht nur zeitlich begrenzt, sondern auch degressiv ausgestaltet sein, also im Laufe der Zeit sinken, sowie unter bestimmten Voraussetzungen kapitalisiert werden können (z. B. bei Aufgabe nicht lebensfähiger Betriebe oder zur Finanzierung von Investitionen). Die Bemessungsgrundlage könnte die landwirtschaftliche Nutzfläche, ggf. verknüpft mit anderen - nicht näher spezifizierten - Merkmalen sein. Diese Form der Stützung sei sowohl für Deutschland als auch für die gesamte EU möglich. 
Dem britischen Modell mit an einzelne Erzeugnisse gebundenen „deficiency payments“ erteilten Plate und Woermann (1962) eine klare Absage. In den späteren Gutachten des Wissenschaftlichen Beirats beim Bundeslandwirtschaftsministerium ist bis in die 1980er-Jahre ein Vorschlag für eine vollständige Entkopplung der Zahlungen von der aktuellen und zukünftigen Erzeugung nicht zu erkennen (vgl. Box 2).

Weniger bekannt als das „Professorengutachten“ dürfte die Arbeit des schweizerischen Wirtschaftswissenschaftlers H. Bachmann ${ }^{12}$ aus dem Jahr 1958 sein, die er mit Blick auf die Integrationsbestrebungen in Europa im Bereich der Landwirtschaft verfasste. Bachmann (1958) plädierte für eine Neuorientierung der westeuropäischen Agrarpolitik, bei der Marktordnungen und staatliche finanzielle Unterstützung der Landwirtschaft voneinander getrennt und direkte Subventionen von dauerhaftem Charakter eingeführt werden. Dadurch sollte das Integrationsproblem, das sich aus dem Nebeneinander der verschiedenen nationalen Agrarpolitiken und Marktordnungen für Agrarprodukte sowie der unterschiedlichen Interessen der Agrarüberschussstaaten und der Länder mit Importüberschüssen ergab, gelöst werden. Insbesondere schlug er drei Arten von Zahlungen vor. Sie könnten zum einen nach dem Rohertrag der einzelnen Betriebe (globale Produktionssubvention), zum anderen nach der Zahl der Arbeitskräfte (Arbeitssubvention) oder auch nach der Betriebsfläche (Hektarsubvention) bemessen werden, wobei Bachmann letzterem den Vorzug gab. Diese sollten nicht homogen pro Flächeneinheit eingesetzt werden, sondern je nach Bodenfruchtbarkeit oder Anbauintensität (mit Bevorzugung der Bergregionen) variieren. Ihre Höhe sollte sich aus der jeweiligen Relation von Produktionskosten zu Weltmarktpreisen ergeben.

Das neue System wäre ,endlich [...] in die Form globaler Subventionen gekleidet, welche sich bezüglich der Produktionsausrichtung der Landwirtschaft neutral verhalten, so daß sich der einzelne Landwirt ausschließlich nach Produktpreisen, welche weitgehend oder vollständig den Weltmarktpreisen entsprechen, und nach seinen Gestehungskosten zu richten hat“ (ibid.: 48). Dieses würde „dem staatlichen Agrardirigismus ein Ende“ setzen, „die Landwirtschaft und die mit ihr verbundenen Wirtschaftszweige wieder in den Rahmen der freien Marktwirtschaft“ zurückführen und „Klarheit über die Kosten des Agrarschutzes“ schaffen (ibid.: 38). Bachmann (1958: 36) bemerkte jedoch gleichzeitig: „Die Realisierung eines ,neutralen' Subventionssystems dürfte sich als eine schwierige Aufgabe erweisen, in der Theorie sowohl wie in der praktischen Verwirklichung. "

Auch Weinschenck (1964) beschäftigte sich zu der Zeit mit den Auswirkungen der Schaffung des gemeinsamen Agrarmarktes in Westeuropa und der anstehenden Preisharmonisierung auf die deutsche Landwirtschaft. Er bemerkte: „Auf der Suche nach einem Ausweg aus dieser Situation wird in letzter Zeit viel über direk-

\footnotetext{
${ }^{12}$ Die Arbeit des Schweizers Bachmann wird hier unter EU-Konzepten geführt, weil sie sich mit den damaligen Integrationsbemühungen innerhalb Europas beschäftigt.
} 
te Einkommensübertragungen diskutiert“ (ibid.: 129). Diese definiert er als „Subventionen, die unter Umgehung des Marktes den landwirtschaftlichen Betrieben direkt gewährt werden“ (ibid.). Er nimmt insbesondere „produktneutrale deficiency payments“ und „Ausgleichszahlungen“ unter die Lupe. Erstere fungieren als Garantie eines Mindest-Betriebseinkommens je Hektar mit dem Ziel, eine bestimmte Agrarstruktur zu erhalten. Letztere sollten Einkommensausfälle bei sinkenden Agrarpreisen ausgleichen und nach der (wohl aktuellen) Größe der landwirtschaftlichen Nutzfläche bemessen werden. Diese beiden Formen „direkter Einkommensübertragungen" lehnte Weinschenck entschieden ab. Zeitlich unbefristete „produktneutrale deficiency payments“ müssten in der gesamten EU eingeführt werden, was er für politisch kaum realistisch und darüber hinaus schwer mit einem marktwirtschaftlichen System vereinbar hielt. Ausgleichszahlungen wiederum kritisierte er, weil sie insbesondere bei ungünstiger Betriebsgrößenstruktur und unter ungünstigen natürlichen Verhältnissen nicht genügen würden, um der Landwirtschaft die notwendigen Anpassungen an die Marktverhältnisse zu ermöglichen (ibid.: 148). Vielmehr plädierte Weinschenck dafür, die direkten Einkommensübertragungen in Form von Investitionsbeihilfen zu gewähren. Offenbar interpretierte er letztere als direkte Einkommensstützung.

Dass die zu der Zeit diskutierten produkt- bzw. produktionsneutralen Zahlungen nicht unbedingt dem Begriff der Direktzahlungen im Sinne der vorliegenden Untersuchung entsprechen, zeigt die Arbeit von Schlotter über „produktionsneutrale Einkommenshilfen" an die Landwirtschaft aus dem Jahr 1963. Danach sei die „Produktionsneutralität“ dieser Zahlungen nur in Verbindung mit einer restriktiven Agrarpreispolitik gegeben: Jede Einführung oder Erhöhung der Einkommenshilfen sollte mit einer Preissenkung verbunden werden, um ihnen die Erweiterungsinvestitionen stimulierende Wirkung zu nehmen. Schlotter (1963: 463) zweifelt aber daran, „ob die Frage der Produktionsneutralität einkommenspolitischer Agrarinterventionen in der praktischen Agrarpolitik relevant wird“. Damit das geschieht, bedarf es aus seiner Sicht zwei schwer zu erfüllenden Voraussetzungen. Zum einen muss ein Selbstversorgungsgrad von mehr als $100 \%$ erreicht werden. Zum anderen muss es ausgeschlossen sein, dass Agrarüberschüsse exportiert werden können. Ansonsten sei die Erörterung produktionsneutraler Einkommenshilfen angesichts des massiven Hungerproblems auf der Welt „grotesk“ (ibid.: 451). Wie Wille (1976: 31) bemerkt:

Der Begriff „direkte Einkommensübertragungen“ ist, obwohl er in der agrarpolitischen Diskussion in jüngster Zeit immer wieder verwendet wird, bislang ohne klare Definition und Abgrenzung geblieben. Bezeichnungen wie „produktneutrale“ oder „produktionsneutrale“ Einkommenshilfen, Ausgleichszahlungen oder „deficiency payments“, Einkommenszuschüsse etc. werden in diesem Zusammenhang häufig synonym verwendet und haben zu einer allgemeinen Begriffsverwirrung geführt. 
Box 2: Agrarzahlungen in frühen Konzepten des Wissenschaftlichen Beirats beim Bundeslandwirtschaftsministerium

Nach dem „Professorengutachten“ von 1962 beschäftigte sich der Wissenschaftliche Beirat beim Bundeslandwirtschaftsministerium mit dem Instrument der staatlichen Zahlungen an Landwirte in regelmäßigen Abständen. Beispielsweise empfahl er 1968 in seinen Vorschlägen zur Milchpolitik, den Interventionspreis für Butter zu senken und den Grünlandbetrieben die Differenz zwischen dem durchschnittlichen EU-Erzeugerpreis und einem garantierten Preis wenigstens teilweise durch „Subventionen“ auszugleichen (Wissenschaftlicher Beirat 1968). Der garantierte Preis sollte im ersten Reformjahr leicht unter dem Richtpreis festgelegt und danach schrittweise in Richtung des vermutlichen Gleichgewichtspreises abgesenkt werden. Dafür, dass es sich hier jedoch um keinen „forward price“ handelt, spricht die Tatsache, dass die vorgeschlagenen „Subventionen“ als Übergangsmaßnahme gedacht waren: Sie sollten greifen „,[s]olange der garantierte Erzeugerpreis noch nicht aus dem Markt erwirtschaftet wird“" (ibid.: 10). Da ihre Zuteilung nach der Milchanlieferung an die Molkereien erfolgen sollte, waren sie an die aktuelle Erzeugung gekoppelt. Plate war zu jener Zeit Vorsitzender des Beirats.

In seinen Empfehlungen von 1973 zur Reform der GAP analysierte der Beirat mehrere Alternativen der Einkommenspolitik (Wissenschaftlicher Beirat 1973). Darunter zog er auch den Einsatz von „direkten Einkommensübertragungen“ zum Teilausgleich für den Einkommensausfall infolge des Abbaus des Außenhandelsschutzes bzw. der Senkung der Preise für einzelne Erzeugnisse in Betracht. Dabei wurde auf die positiven deutschen Erfahrungen mit den als Anpassungshilfe konzipierten Ausgleichszahlungen im Rahmen der Getreidepreisharmonisierung von 1967 (s. Kapitel 5) hingewiesen.

Der Beirat diskutierte zu jener Zeit detailliert verschiedene Ausgestaltungen der Ausgleichszahlungen. Produktgebundenen Zahlungen nach Muster der englischen „deficiency payments“ erteilte er von vornherein eine Absage, weil sie keine Anpassung des Angebots bewirken würden. In Betracht gezogen wurden hingegen betriebsbezogene „Einkommenszahlungen“ zur „Erhaltung eines bestimmten Mindesteinkommens aus landwirtschaftlicher Tätigkeit in allen Regionen, in denen die Landbewirtschaftung nach dem Willen der Träger der politischen Entscheidungsgewalt aufrecht erhalten werden soll“", deren Höhe vom regionalen Durchschnittseinkommen abgeleitet ist (ibid.: 41). Da solche Zahlungen in der Regel „mit sinkender Betriebsgröße und abnehmender Gunst der natürlichen Verhältnisse“ ansteigen würden, hätten sie eine Verlangsamung des nötigen Anpassungsprozesses an das neue Preisniveau zur Folge (ibid.). An dieser 
Stelle kommentierte der Beirat den Vorschlag des Atlantischen Instituts, solche Ausgleichszahlungen maximal auf 15 Jahre zu begrenzen (s. unten). Er äußert sich dazu skeptisch, denn „[s]oziale Gründe würden auch nach Ablauf der Übergangszeit eine Fortsetzung der Einkommenszahlungen erforderlich machen“ (ibid.: 42). Diskutiert, aber nicht weiter erwogen, wurden „direkte Einkommensübertragungen“, die sich nach dem in einer bestimmten Basisperiode hergestellten Produktionsvolumen, nach der in einer Basisperiode genutzten landwirtschaftlichen Fläche oder nach dem in einer Basisperiode vorhandenen Arbeitskräfteaufwand richten und somit zumindest zum Teil entkoppelt sein sollten (ibid.: $21 \mathrm{ff}$ ).

Am Ende sprach sich der Beirat dagegen aus, Ausgleichszahlungen EU-weit einzusetzen, denn diese seien ,wenig geeignet, die agrarpolitischen Probleme der EWG einer Lösung näherzubringen“ (ibid.: 56). Befürchtet wurde die damit verbundene hohe Belastung der öffentlichen Haushalte sowie, dass die Landwirtschaft „,im überwiegenden Teil zu einem permanenten Empfänger von Subventionen“ wird (ibid.). Der Beirat empfahl stattdessen den „,vorrangige[n] Einsatz preispolitischer Instrumente unter Abstimmung des Außenhandelsschutzes auf die Einkommenspolitik“ zusammen mit der „Differenzierung der Agrarpreisniveaus zwischen den Mitgliedsstaaten“ (ibid.: 29 und 30). Diese Preispolitik sollte durch strukturpolitische Maßnahmen flankiert werden. Auch ließ der Beirat zeitlich befristete „direkte Einkommensübertragungen“ in sehr begrenzten Ausmaß zu, um die negativen Folgen der empfohlenen Maßnahmen für Landwirte in bestimmten benachteiligten Regionen abzumildern und ihnen ggf. das Ausscheiden aus der landwirtschaftlichen Produktion zu erleichtern (ibid.: 57). Explizit empfohlen wurde, „Einkommensübertragungen nicht produktionsneutral einzusetzen, sondern so, daß Anreize zur Umstellung auf die als wünschenswert betrachteten Betriebsorganisationen oder zur Herstellung bestimmter Produkte entstehen“ (ibid.: 14 der Anlage 313).

In einem Gutachten von 1987 untersuchte der Beirat Handlungsalternativen für die europäische Agrarpolitik (Wissenschaftlicher Beirat 1987). Er stellte fest, dass eine „Umorientierung der Gemeinsamen Agrarpolitik [...] unumgänglich“ sei und empfahl für die zukünftige Gestaltung der GAP eine „,mittlere“ Preispolitik (ibid.: 49). Mit einer solchen Preispolitik war die reale Preissenkung von jährlich 3 bis $4 \%$ über einen mittelfristigen Zeitraum verbunden (ein Minder-

\footnotetext{
${ }^{13}$ Die Anlage 3 des Gutachtens „Direkte Einkommensübertragungen“ wurde von G. Weinschenck verfasst.
} 
heitsvotum hielt dieses für zu stark), flankiert durch eine Reihe von Strukturmaßnahmen. ${ }^{14}$ Eine „marktorientierte“ Preispolitik mit der jährlichen Absenkungsrate von $5 \%$, begleitet durch „direkte Einkommensübertragungen“ zum Ausgleich bzw. Abmilderung der mit der Preissenkung einhergehenden Einkommensverluste, wurde vom Beirat als nicht geeignet bewertet. Er wies auf erhebliche Probleme hin, die mit einem solchem Reformschritt verbunden wären, z. B. sehr hohe Transferzahlungen, die die Sonderstellung der Landwirtschaft noch deutlicher hervortreten lassen würden (ibid.: 41). Zudem wies er auf die Entscheidungsprobleme hinsichtlich der Verteilung und mangelnde Erfahrungswerte in Bezug auf längerfristig angelegte, umfangreiche Zahlungen hin. Vor diesem Hintergrund „fällt es schwer, konkrete Vorschläge zur spezifischen Ausgestaltung direkter Einkommenstransfers hinsichtlich Bemessungsgrundlage, Höhe, Dauer und Form zu machen, und die jeweiligen Auswirkungen auf das Agrargüterangebot und den landwirtschaftlichen Strukturwandel abzuschätzen“, schlussfolgerte der Beirat (ibid.: 42). Ergänzend zum Gutachten von 1987 empfahl der Beirat zwei Jahre später zeitlich begrenzte „Einkommenshilfen“ zur Kompensation der Einkommensverluste im Falle einer stärkeren Senkung der Preise als die durch eine „mittlere“ Preispolitik vorgegebene Kürzung (Wissenschaftlicher Beirat 1989).

Aus den vorgenannten Arbeiten geht hervor, dass staatliche Zahlungen an die Landwirte - auch solche, die zumindest teilweise entkoppelt sind - in den 1960erJahren in Kontinentaleuropa diskutiert wurden. Allerdings wurden sie als Alternative zur Preisstützung generell noch nicht in Erwägung gezogen und es ist auch kein klares Entkopplungskonzept zu erkennen. Wie es scheint, wurde die europäische Wissenschaft Anfang der 1970er-Jahre verstärkt auf die Entkopplungsidee aufmerksam. Zu jener Zeit erschien eine Reihe von konkreten, detaillierten Vorschlägen zu (entkoppelten) Direktzahlungen, sodass es schwer ist, den wahren Vorreiter zu benennen.

Es scheint jedoch, dass Ausgleichszahlungen als Kompensation für den Abbau der Preisstützung in Kontinentaleuropa zum ersten Mal von J. F. van Riemsdijk von der Universität Wageningen vorgeschlagen wurden. Sein bekanntester Aufsatz zu diesem Thema stammt aus dem Jahr 1973, basiert aber nach eigenen Angaben auf seinen früheren in niederländischer Sprache verfassten Arbeiten aus den Jahren 1960, 1968 und 1972 (van Riemsdijk 1973: 162). Van Riemsdijk regte eine Reform der EU-Agrarpolitik hin zu „direct compensation payments“ für Landwirte an und stellte detailliert dar, wie ein solches System der direkten Einkommensstüt-

\footnotetext{
${ }^{14}$ Dazu gehörten neben einer Vorruhestandsregelung und der Aufstockung der Ausgleichszulage für benachteiligte Gebiete auch eine Flächenstilllegung bzw. -umwidmung für Umweltzwecke (Wissenschaftlicher Beirat 1987).
} 
zung ausgestaltet werden sollte. Sein Anspruch sei gewesen, die Interessen der bereits aktiven Landwirte und der Neueinsteiger sowie des Rests der Gesellschaft in Einklang zu bringen. Er schlug vor, die Stützpreise in der EU in einem Schritt um etwa $20 \%$ in Richtung der „,normalized prices“ abzusenken. Der daraus resultierende Einkommensverlust sollte den Landwirten durch „direct compensation payments" ausgeglichen werden. Zusätzlich zu dieser Aufgabe sollten die Zahlungen auch „reasonable claims for higher prices of the agricultural sector which have not been met by the present system of market and price policy" decken (ibid.: 169). Für diese Zwecke sollte der ursprüngliche Zahlungsbetrag ggf. um $5 \%$ erhöht werden. Für tierische Erzeugnisse waren trotz Preissenkungen keine Zahlungen vorgesehen.

Der Beihilfesatz pro Hektar sollte sich durch Multiplizieren des regionalen Durchschnittsertrags der betreffenden Kultur mit der Höhe der Preissenkung pro Einheit (ggf. erhöht um 5\%) ergeben. Die Anbaufläche eines Landwirts für diese Kultur in den drei der Reform vorausgehenden Jahren war die Bemessungsgrundlage. Somit waren die „direct compensation payments“ von der aktuellen Erzeugung entkoppelt, auch wenn dieser Begriff in der Veröffentlichung nicht fällt. Das britische System der „deficiency payments“ lehnte van Riemsdijk aufgrund des Einflusses dieser Zahlungen auf die Produktionsentscheidungen der Landwirte ab. In seinem Konzept waren die Zahlungen personenbezogen, zeitlich auf die Übergangsphase ins neue System befristet (max. 20 Jahre bzw. bis zum 65. Lebensjahr des Landwirts) und nur an Ehegatten vererbbar, falls der Betrieb fortgeführt würde.

Vor dem Hintergrund der Verbesserung der Agrarstruktur sah van Riemsdijk eine Reihe von weiteren Regeln für die Ausgleichszahlungen vor. Erstens sollten lediglich Landwirte unter 65 Jahren von ihnen profitieren. Zweitens sollten sie auch Neueinsteigern, die sich während der Übergangsphase in der Landwirtschaft niederlassen, gewährt werden (in den ersten drei Jahren ihrer landwirtschaftlichen Tätigkeit sollten sie darüber hinaus von einem Zuschlag in Höhe von $20 \%$ der regionalen Zahlung profitieren). Drittens waren zusätzliche Zahlungen auch für Landwirte, die ihre Betriebe während der Übergangszeit vergrößerten, vorgesehen. Schließlich sollten den Landwirten, die in dieser Zeit die Betriebsfläche reduzierten, die Zahlungen gekürzt werden, wobei die Zahlungen proportional schwächer als die Betriebsfläche zurückgehen sollten, denn das Ziel des neuen Systems ,is not simply to get agriculture adequately restructured, but also to provide for an adaptation which is socially acceptable $[\ldots]$ preventing too rapid a reduction of the agricultural labour force on the one hand and avoiding, on the other hand, its opposite“ (ibid.: 170). Überschritt der individuelle Betrag eines Betriebes eine Obergrenze, würden nach den ersten fünf Jahren die darüber hinaus gehenden Zahlungen schrittweise sinken und gleichzeitig zusätzliche Auflagen greifen. Die Obergrenze sollte der Hälfte des Arbeitslohns (inklusive Sozialleistungen) eines qualifizierten landwirtschaftlichen Arbeiters entsprechen (damals etwa 10 Hektar auf Gunststandorten) und würde damit jährlich und regional variieren. Ein Landwirt, 
der seinen Arbeitern ohne Genehmigung kündigen würde, würde die über die Obergrenze hinausgehenden Zahlungen gänzlich verlieren (ibid.: 171). Damit waren die Ausgleichszahlungen von van Riemsdijk nicht bedingungslos.

Zur Akzeptanz der neuen Maßnahme bei den Landwirten bemerkte van Riemsdijk (1973: 182): „Farmers might prefer, at first sight, increased producer prices or additional deficiency payments because such instruments, unlike the DCP [direct compensation payments] system, would not make them feel responsible to the parish.“ Allerdings: ,[i]f they have to choose either DCPs or no income improvement at all, the remaining minority, although perhaps disliking such payments, will also come to their senses“ (ibid.: 182). Wichtig war für ihn, dass ,agricultural population will be informed clearly, fully and in good time about the new system " und dass ,adequate employment will be available outside of the agricultural sector“ (ibid.: 176). Für die Finanzierung der Zahlungen schlug van Riemsdijk (1973) eine Erhöhung der Mehrwertsteuer bzw. Einführung einer speziellen Verbrauchersteuer auf Lebensmittel vor.

Van Riemsdijk war - neben weiteren 21 Wissenschaftlern aus allen neun Mitgliedstaaten der EU - Unterzeichner des sogenannten Memorandums von Wageningen von 1973, das Vorschläge für eine Reform der GAP enthielt (Corbet und van Riemsdijk 1973). Unter anderem regten die Agrarökonomen auf 20 Jahre begrenzte „direct compensatory payments“ zum Ausgleich der Einkommensverluste infolge des gleichzeitig empfohlenen Abbaus der Preisstützung an. Aus Deutschland unterzeichneten T. Heidhues, W. Scheper, G. Schmitt und W. von Urff das Memorandum.

Ein weiterer Entkopplungsvorschlag vom Anfang der 1970 er-Jahre ${ }^{15}$ stammt von den Ökonomen aus dem Umfeld des Pariser Atlantischen Instituts, die „[e]in radikales Umdenken in der Agrarpolitik“ für notwendig hielten und die Einführung von „Ausgleichszahlungen“ für pflanzliche und tierische Erzeugnisse empfahlen (Uri et al. 1971: 53). Die im Mansholt-Plan von 1968 enthaltenen Reformvorschläge gingen ihnen nicht weit genug, denn diese würden - wenn nicht durch einen Abbau der Preisstützung begleitet - zum weiteren Anstieg der Überschüsse führen. Sie empfahlen deshalb, die Preisstützung durch eine Einkommensstützung zu ersetzen, aber „,[e]s ist eine Sache, auf die direkte Einkommensstützung zu verweisen und eine andere, sie in die Tat umzusetzen, d.h. konkrete Formeln für ihre praktische Anwendung zu finden“ (ibid.: 54). Deshalb setzten sie sich zum Ziel, „präzise Lösungsmöglichkeiten anzubieten und zur Diskussion zu stellen“ (ibid.). Parallel zu den Maßnahmen aus ihrem Konzept sollten unterstützend die Maßnahmen aus dem Mansholt-Plan greifen.

Es wurde vorgeschlagen, die Einkommensstützung in Form von zeitlich begrenzten (max. 15 Jahre) Ausgleichszahlungen an Landwirte, die nicht älter als 55 Jahre waren, zu gestalten. Um einen Strukturwandel zu unterstützen, sollten sie

${ }^{15}$ Die Fassung auf Englisch erschien 1970. 
nur an die damals aktiven Erzeuger fließen und nicht übertragbar bzw. vererbbar sein. Zudem sollten sie mit der Größe des Betriebs abnehmen. Die Höhe der Zahlung sollte sich aus der Multiplikation der Differenz zwischen dem alten und neuen Preis mit dem EU-Durchschnittsertrag ergeben (es wurde nicht präzisiert, ob historisch oder aktuell) und wäre somit per Hektar EU-weit einheitlich. Als Bemessungsgrundlage sollte die individuelle, der durchschnittlichen Hektarzahl der letzten drei Jahre vor der Reform entsprechende Fläche dienen. Somit würde eine Flächenausweitung nach der Preissenkung nicht mit höheren Zahlungen belohnt. Uri et al. (1971) empfahlen zudem, eine Flächenreduzierung nicht mit einer Kürzung der Zahlungen zu bestrafen. Die Entkopplung von der Erzeugung war allerdings nicht vollständig. Denn im Falle einer sukzessiven Preissenkung ließen die Autoren zu, dass als Bemessungsgrundlage für die zusätzlich erforderlichen Ausgleichszahlungen die vor der jeweils letzten Preissenkung genutzte Fläche gilt. Somit würden die Landwirte zumindest die Höhe der mit den kommenden Preissenkungsschritten verbundenen Ausgleichszahlungen durch die Flächenausweitungen beeinflussen können. Wie van Riemsdijk lehnten auch Uri et al. (1971) das Instrument der gekoppelten „deficiency payments“ ab, da ihre Produktionsanreize kaum geringer als die einer hohen Preisstützung seien.

Ebenfalls um diese Zeit herum erschien in Deutschland ein weithin beachtetes und intensiv diskutiertes Gutachten zweier Göttinger Ökonomen U. Koester und S. Tangermann, das sie im Auftrag des Bundeslandwirtschaftsministeriums erstellt hatten. ${ }^{16}$ Sie präsentierten darin einen detaillierten Reformvorschlag zur Einführung der Direktzahlungen (Koester und Tangermann 1976 17 ). Die Autoren übten Kritik an dem zu dieser Zeit in der EU geltenden System der Preisstützung: „Das System ist vorwiegend durch ein Kurieren an Symptomen in der gegenwärtigen Ausprägung entstanden. [...] Erwartete Änderungen in den Rahmenbedingungen sprechen dafür, daß das gegenwärtige System zukünftig eher schlechter als besser die angegebenen Funktionen erfüllen wird" (ibid.: 5). Vor diesem Hintergrund empfahlen Koester und Tangermann (1976) eine neue Ausrichtung der Agrarpolitik, welche der Fehlallokation der Produktionsressourcen entgegenwirken und somit die gesamtwirtschaftliche Wohlfahrt steigern würde. Zwar wurde die Kosten-Nutzen-Analyse der alternativen agrarpolitischen Systeme (das damalige Preisstützungssystem und ein System direkter Einkommensübertragungen mit partieller Kontingentierung) nur für Deutschland durchgeführt, die Autoren regten aber eine Übertragung des vorgeschlagenen Systems auf die Ebene der EU an. Dabei wurden Kosten nicht nur als Staatsausgaben für den Agrarsektor, sondern vornehmlich als Verzicht am Sozialprodukt definiert.

\footnotetext{
16 Tangermann schlug bereits in seiner Dissertation die Einführung „direkter Einkommensübertragungen“" vor, die von Land zu Land unterschiedlich ausfallen und über die Erhöhung der Mehrwertsteuer finanziert werden könnten (Tangermann 1974).

${ }_{17}$ Die englische Fassung erschien 1977.
} 
In diesem System waren „direkte Einkommensübertragungen“ als Kompensation für die mit den angeregten jährlichen realen Preissenkungen in Höhe von 2 bis 2,5\% einhergehende Einkommensminderung der Landwirte vorgesehen. Diese Zahlungen waren personenbezogen und sollten - in Form von Zertifikaten - nur an damalige Landwirte fließen; Neueinsteiger sollten keinen Anspruch darauf haben. Sie sollten zudem zeitlich begrenzt sein (höchstens die gesamte Erwerbstätigkeit der betroffenen Generation - möglichst aber nicht länger als 15 bis 20 Jahre). Eine Kernempfehlung war zudem, „direkte Einkommensübertragungen“ unabhängig von der betrieblichen Produktionsmenge, der bearbeiteten Fläche und der Zahl der Arbeitskräfte zu gewähren. Die Möglichkeit der Kapitalisierung dieser Zahlungen wurde ausdrücklich vorgesehen, beispielsweise im Fall eines Verkaufs oder langfristiger Verpachtung der landwirtschaftlichen Nutzfläche bzw. wenn der Betriebsinhaber einen Arbeitsplatz außerhalb der Landwirtschaft fand. Somit waren die „direkten Einkommensübertragungen“ von Koester und Tangermann (1976) von der aktuellen und zukünftigen Erzeugung entkoppelt (dieser Begriff wurde aber nicht genutzt).

Bei Übertragung des vorgeschlagenen Systems auf die Gemeinschaft sollten auf EU-Ebene nur die Agrarpreise verbindlich und einheitlich für alle Mitgliedstaaten festgesetzt werden. Die Zuständigkeit für die „direkten Einkommensübertragungen" sollte hingegen in nationaler Hand liegen. Insbesondere sollten die Mitgliedstaaten für die Festlegung der Höhe der Zahlungen zuständig sein. Somit würde sie von Land zu Land unterschiedlich ausfallen, „,[u]m den unterschiedlichen ökonomischen und sozialen Umweltbedingungen in den einzelnen Ländern Rechnung zu tragen“ (ibid.: 259). Als Finanzierungsgrundlage wurde entweder der allgemeine Haushalt (was aber als unrealistisch bewertet wurde) oder - in ausdrücklicher Anlehnung an van Riemsdijk (1973) - eine Strukturabgabe zu Lasten der Verbraucher, deren Erhebung wie bei der Mehrwertsteuer durch den Einzelhandel erfolgt, vorgeschlagen. Die Höhe der Abgabe sollte die Differenz zwischen dem Niveau der Erzeugerpreise im damals geltenden System und dem im neuen System nicht überschreiten, um eine zusätzliche Belastung der Verbraucher zu vermeiden.

Koester und Tangermann (1976) präsentierten nicht nur konkrete Vorschläge zur Gestaltung der „direkten Einkommensübertragungen“, sondern setzten sich ausführlich mit der Kritik an diesem Instrument auseinander und legten seine zahlreichen Vorteile dar. Erstens würde die finanzielle Belastung der Verbraucher im Falle des neuen Systems die Belastung bei der Fortsetzung der damaligen Preispolitik nicht überschreiten. Zweitens minimiere die Ausgestaltung der Einkommensübertragungen in Form von personengebundenen, an der gesamtsektoralen Einkommenslage orientierten Zertifikaten den Verwaltungsaufwand. Hier helfe auch, dass die vorgeschlagenen Zahlungen zeitlich befristet und von den betrieblichen Entscheidungen unabhängig sind. Drittens werde der Strukturwandel in der Landwirtschaft nicht gehindert, denn die Zahlungen können kapitalisiert 
werden. Viertens werde das Produktionswachstum (und damit die Überschusskumulierung) gebremst, weil die preislichen Anreize abnehmen würden. Zusammenfassend schreiben die Autoren, dass die Reform der Agrarpolitik hin zu den vorgeschlagenen „direkten Einkommensübertragungen“ „das Sozialprodukt erhöhen, den Staatshaushalt entlasten, die internationalen Handelsbeziehungen verbessern, die soziale Lage der Landwirte nicht verschlechtern und die Flexibilität der Agrarpolitik erhöhen" würde (ibid.: 253).

Auch T. Josling regte zu dieser Zeit die Einführung von Zahlungen an Landwirte im Rahmen einer GAP-Reform an (Josling 1973). Die von ihm vorgeschlagenen ,income supplements“ sollten personenbezogen und situationsspezifisch gewährt und zugleich mit den Zielen von Strukturreformen verknüpft werden. Daneben sollten den Landwirten - insbesondere im Getreide und Milchsektor auch „deficiency payments“-ähnliche Zahlungen zur Verfügung stehen. Der schwerwiegendste politische Einwand gegen eine solche Reform könnte seiner Ansicht nach ,the expressed desire of farm groups that all their income should come ,through the market“" sein (ibid.: 95). Nichtsdestotrotz äußerte er die Hoffnung: ,The conjunction of high world prices and concern about inflation with the challenge of the next round of multilateral trade talks makes the next year or two a particularly appropriate time for re-evaluation of the aims and instruments of the CAP“ (ibid.: 98).

Die Notwendigkeit einer Reform der GAP und neuer agrarpolitischer Instrumente wurde zu jener Zeit nicht nur unter Agrarökonomen diskutiert. Für „eine neue Agrarordnung in Europa“ sprach sich z. B. der liberal ausgerichtete Kronberger Kreis (der wissenschaftliche Beirat der Stiftung Marktwirtschaft), der mit diesem Titel seine Veröffentlichung zur EU-Agrarpolitik versah, aus (Engels et al. 1985). Er empfahl einen Abbau der Preisstützung über einige Jahre, damit die Landwirte ihre Erzeugung der Nachfrage anpassen können. Die Abnahmegarantien in Form von Interventionen sollten auf das Sicherheitsnetz begrenzt und Kontingente ganz abgeschafft werden. „[D]ie Umstellung auf die neue Agrarordnung erträglich machen“ sollte die Aufgabe der vorgeschlagenen „direkten Zahlungen“" sein (ibid.: 124). Diese „Überbrückungshilfe als Auffangnetz“ sollte nicht allen Erzeugern zur Verfügung gestellt werden, sondern nur denjenigen, die aufgrund ihrer schwachen Einkommens- und Vermögenslage die Umstellung aus eigener Kraft nicht bewerkstelligen können (ibid.). Entscheidend hier sollte die Höhe des Gesamteinkommens eines Landwirts sein, inklusive seines Einkommens aus nichtlandwirtschaftlichen Tätigkeiten. Bei der Betriebsaufgabe sollten die Zahlungen dem (ehemaligen) Landwirt weiter gewährt werden. Um sie möglichst einfach zu verwalten, sollten sie über die Finanzämter, z. B. mithilfe von Steuergutscheinen, abgewickelt werden.

Auf keinen Fall sollten die „direkten Zahlungen“ ein Dauerinstrument werden, denn es sei nicht möglich, sie völlig produktionsneutral zu gestalten. Darüber hinaus „belohnen sie Tüchtige wie Untüchtige gleichermaßen und lindern den Druck 
und die Bereitschaft, sich anzupassen“, sie würden daher den nötigen Strukturwandel hemmen (ibid.). Engels et al. (1985: 125) bemerkten auch, dass „die moralische Berechtigung“ für einen Dauerausgleich nicht gegeben ist: „Die gegenwärtige Agrarpolitik der Preisstützung ist von der Landwirtschaft durchgesetzt worden. Folglich hat die Landwirtschaft diese Politik entscheidend mit zu verantworten. Es geht aber nicht an, diese Politik, vor der von Beginn an dringend gewarnt worden ist, erst zu erzwingen und sich dann den Verzicht darauf ewig bezahlen zu lassen.“ Eine Ausnahme empfahlen die Autoren lediglich für Betriebe in benachteiligten Gebieten zum „Erhalten der landwirtschaftlichen Bevölkerung und des Landschaftsbildes“, denn dieses sei ein knappes Gut, dessen Bereitstellung über ständige direkte Beihilfen vergütet werden sollte (ibid.: 126).

Ein neueres Entkopplungskonzept stellt der sogenannte „Tangermann-Bond“ dar. Vorgeschlagen wurde er von S. Tangermann im Jahr 1990 in einem Bericht für die „Land Use and Food Policy Intergroup“ des Europäischen Parlaments. Um den Landwirten den durch Preissenkung erlittenen Einkommensverlust auszugleichen, sollte der Staat zeitlich begrenzte, handelbare Zertifikate ausgeben. Später empfahlen Swinbank und Tangermann (2004: 55) die Einführung solcher Bonds als „,the next stage in CAP reform“. In diese sollten die gegenwärtigen Direktzahlungen verpflichtend umgewandelt werden. Die handelbaren Bonds würden ihren Besitzern einen mit der Zeit abnehmenden Zahlungsfluss garantieren. Sie sollten zeitlich befristet sein, wobei, wie die Autoren betonen, ,it is not the precise duration of future payments that is so important (to us), but rather that a fixed deadline is firmly agreed and publicised“ (ibid.: 63). Als Basiszeitraum für die Zuteilung der Bonds an die Betriebe empfahlen Swinbank und Tangermann (2004) eine ausreichend weit in der Vergangenheit liegende Periode, um sicherzustellen, dass nach der Ankündigung der Reform die Landwirte die Höhe ihrer Zahlungen nicht mehr beeinflussen können. Auch könnten die Bonds so ausgestaltet werden, dass die Zahlungen für größere Betriebe moduliert sind. Ihr alleiniges Ziel sollte sein, den Erzeugern die aus dem Politikwechsel resultierenden Einkommenseinbußen zu kompensieren, deshalb sollten sie nicht an zusätzliche Anforderungen, beispielsweise im Umweltbereich, gebunden werden.

Laut Swinbank und Tangermann (2004: 58) ist das erste und maßgebliche Ziel der vorgeschlagenen Reform, Sicherheit bezüglich der Zukunft der Direktzahlungen zu schaffen:

[1]n exchange for the limitation of payment duration (which is what most farmers expect in any case), there would be an explicit legal commitment that the payments would be made with absolute certainty over a given future period and at a predetermined level. Investments decisions, and decisions regarding the professional future of farmers and their children, could then be taken on firmer ground. Agricultural policy makers could plan future budget allocations and the scope for other measures under the CAP. 
Ein weiterer Vorteil der Bonds sei, dass sie nicht nur - wie die Betriebsprämie von der aktuellen und zukünftigen Erzeugung, sondern auch vom Faktor Boden vollkommen entkoppelt sind und somit die Produktionsentscheidungen der Landwirte nicht beeinflussen. Zudem sahen Swinbank und Tangermann (2004) in den Bonds „logical conclusion“ des Reformprozesses der GAP an, der 1992 mit einer Teilentkopplung begann (ibid.: 58). Weiter würden die vollkommen entkoppelten Bonds die Stellung der EU in der WTO verbessern sowie EU-Finanzmittel für „more constructive uses in rural areas“ freisetzen (ibid.: 59). Schließlich wiesen Swinbank und Tangermann (2004: 66) darauf hin, dass „[f]or the future policy process, a significant advantage is that the policy change made in this way is locked in“ und dass ,as the bonds can be held by anybody, future payments would in effect no longer be a matter of agricultural policy, but rather a predetermined matter of a relatively simple financial nature". Damit wäre die Einführung der zusätzlichen Zahlungen bei einer verschlechterten Marktlage, wie sie in den USA zwei Jahre nach der Entkopplung von 1996 erfolgte, ausgeschlossen.

\subsection{Wissenschaftliche Konzepte in der EU: Zusammenfassung}

Ähnlich wie in den USA, wurden auch in der EU nach dem 2. Weltkrieg zahlreiche Vorschläge zum Einsatz der staatlichen Zahlungen bzw. der Direktzahlungen in der Agrarpolitik unterbreitet. Auch wenn sie auf 30 Jahre verteilt sind, haben sie durchaus Gemeinsamkeiten. Sie fokussieren auf die Erarbeitung von Konzepten zur Neuausrichtung der Agrarpolitik, um die negativen Auswirkungen des für notwendig gehaltenen Abbaus der Preisstützung abzufedern. Den „deficiency payments" wurde aufgrund der Tatsache, dass sie Produktionsanreize boten, vielfach explizit eine Absage erteilt. Auch mehrmals - aber aus einem anderen Grund - wurden die dreijährigen ,gemeinschaftlichen Ausgleichsmaßnahmen“ von 1967 erwähnt, die im Rahmen der Getreidepreisharmonisierung u. a. in Deutschland zum Einsatz kamen. Sie wurden als Beispiel dafür angeführt, dass die Idee der Ausgleichszahlungen praktikabel ist. Darin scheint das Konzept von Plate und Woermann aus dem Jahr 1962 relativ schnell verwirklicht zu sein. Als Beispiel für solche Zahlungen können auch die mit der Aufwertung der Deutschen Mark verbundenen Zahlungen in Deutschland von 1969 und die Norwegen zugestandenen Beihilfen von 1972 dienen (s. Kapitel 5).

Hinsichtlich der vollständigen Entkopplung der Zahlungen von der Erzeugung war die Wissenschaft in der EU der praktischen Politik weit voraus. In diesem Fall dauerte es aber sehr lange, bis die theoretischen Konzepte umgesetzt wurden. Der erste Anlauf mit der landwirtschaftlichen Einkommensbeihilfe von 1989, die weder an Mengen noch an Preise gekoppelt und dazu vorübergehend, degressiv ausgestaltet und durch Mitgliedstaaten ko-finanziert war - und damit ei- 
nige Kernelemente der vorgenannten Konzepte umfasste -, schlug bekanntlich fehl. Die Entkopplung der Zahlungen von der aktuellen und zukünftigen Erzeugung erfolgte dann schrittweise über mehrere Jahre ab 1992 (s. Kapitel 5). Damit näherte sich die praktische Politik den wissenschaftlichen Positionen an. In der Betriebsprämie von 2003 finden sich einige der zentralen Empfehlungen der 1970er-Jahre wieder. Zu den Elementen, die bis heute in der EU-Agrarpolitik keine Beachtung fanden, gehören insbesondere die Tatsache, dass die Zahlungen personen- statt flächenbezogen ausgestaltet sind sowie ihre zeitliche Befristung. Beides bilden wichtige Merkmale der Tangermann-Bonds. Bereits während der MacSharry-Reform diskutiert - Daugbjerg (2004) beschreibt den politischen Widerstand dagegen - wurde das Bond-Konzept bis heute in der EU nicht umgesetzt. In den USA scheinen die zeitlich befristeten und im Voraus festgelegten Ausgleichszahlungen als Kompensation für die Abschaffung der Erdnussquote von 2002 Ähnlichkeiten mit dem Bond zu haben, wäre nicht die gleichzeitige Ausweitung des Systems der zur Zeit des „,buyout“ gültigen Agrarzahlungen auf Erdnüsse gewesen; die ähnlich ausgestalteten Ausgleichszahlungen für Tabak von 2004, der in dieses Zahlungssystem nicht integriert wurde, waren nicht aus dem Staatshaushalt finanziert (s. Kapitel 4).

\subsection{Direktzahlungen in der Wissenschaft der USA und der EU: Fazit}

Mit Blick auf beide Seiten des Atlantiks lässt sich basierend auf den vorgenannten Publikationen zusammenfassend feststellen, dass während in den USA - zumindest zunächst - eher antizyklische Zahlungen zur Umsetzung der Preispolitik den Schwerpunkt der Reformkonzepte darstellten, in der EU eher befristete Ausgleichszahlungen, die die Senkung der Preisstützung flankieren sollten, interessierten. Nichtsdestotrotz wurden die ersten Entkopplungskonzepte in den USA erarbeitet. Anfang der 1960er-Jahre waren dort detaillierte Reformvorschläge zur Einführung von Direktzahlungen vorhanden. In der EU erfolgte eine ähnliche Entwicklung erst zehn Jahre später. Zu der Zeit wurden auch in den Dokumenten der Europäischen Kommission solche Zahlungen erwähnt (s. Kapitel 5.3). Bereits davor waren auf beiden Seiten des Atlantiks Entkopplungselemente punktuell in wissenschaftlichen Arbeiten zu finden.

Anders als bei preisabhängigen Zahlungen, die in der praktischen Politik zum Einsatz kamen bevor die entsprechenden Konzepte in der Wissenschaft erarbeitet wurden (Haggard der Wissenschaft zuzuordnen wäre weit hergeholt), scheint die Idee der Entkopplung der Zahlungen von der aktuellen und zukünftigen Erzeugung und von den Marktpreisen ihren Ursprung in der Wissenschaft zu haben.

Allerdings hatte sowohl in den USA als auch in der EU keines der vorgenannten Entkopplungskonzepte zur Zeit seiner Vorstellung einen unmittelbaren Ein- 
fluss auf die praktische Politik, im Gegenteil - diese lag noch lange Zeit nicht auf der Linie der Wissenschaft. Auf beiden Seiten des Atlantiks dauerte es mehrere Jahre, bis die Idee der Entkopplung umgesetzt wurde. Auch andere Länder waren diesbezüglich nicht schneller. Wie van Tongeren (2008: 10) feststellt, wurden entsprechende Reformen - weg von der Preisstützung und hin zur (flächengebundenen) Einkommensstützung - außer von den USA und der EU auch von Mexiko, der Schweiz und Norwegen umgesetzt. Nicht nur die Reformen in den USA und der EU fanden erst in den 1990er-Jahren statt (und später). In Mexiko wurden „procampo payments“ 1996, in der Schweiz „income support payments“ 1993 und in Norwegen „payments based on non-current area“ Anfang der 2000er-Jahre eingeführt (OECD 1998, OECD 2016c). Ahner (2010: 34) bemerkt mit dem Hinweis auf das Konzept von Koester und Tangermann von 1976 wie folgt: „Wie so oft mit neuen radikalen Ideen, die Realität brauchte lange, bis sie bereit war, diese Gedanken zumindest teilweise aufzunehmen.“

Es bleibt anzumerken, dass die hier diskutierten Ideen zur Zeit ihrer Veröffentlichung auch in wissenschaftlichen Kreisen selbst nicht unumstritten waren. Als Beispiel dafür kann der zwischen Weinschenck und Plate im Jahr 1970 ausgetragene Meinungsstreit über die künftige Ausrichtung der Agrarpolitik dienen (Scholz 1972). Während Weinschenck die Preissenkungen für unzumutbar hielt und stattdessen eine Kontingentierung der landwirtschaftlichen Erzeugung auf Teilmärkten bevorzugte, lehnte Plate letztere ab und plädierte für direkte Einkommensübertragungen als Einkommensausgleich für die betroffenen Betriebe. Anfang der 1970er-Jahre wurden von Agrarökonomen neben „direct compensation payments" auch „deficiency payments“, „quantitative controls“ sowie „Community production grants to lower farmers' costs" als Alternativen zur Preisstützung in der EU ins Spiel gebracht (Corbet und van Riemsdijk 1973: 159). Eine Mitte der 1980er-Jahre unter europäischen Agrarökonomen durchgeführte Befragung ergab, dass diese die damalige EU-Agrarpolitik zwar beklagten, sich aber über die Lösungen nicht einig waren (Herrmann und Schäfer 1985). In diesem Zusammenhang bemerkt Schmitt (1988: 15): „Diese Ambivalenz der Agrarökonomen gilt indes nicht nur für die Gegenwart, sondern begleitet die Geschichte der Agrarpolitik gleichsam von Anfang an.“

Es stellt sich die Frage, inwiefern die in der Wissenschaft erarbeiteten Konzepte tatsächlich Einfluss auf die politischen Entscheidungen hatten. Hierzu besteht Grund zur Skepsis, was anhand der folgenden Beispiele illustriert werden kann. Gardner (1996: 240) stellt mit Blick auf die Entwicklung der US-Agrarpolitik fest:

Perhaps the most sobering set of observations involves the policies that agricultural economists earlier recommended not be adopted. Instead of a governmental tune-up of price supports so that their economic advantages to society can be realized without the distortions that unwise policies can cause, the political tendency has been to continue the distortions and to ignore possible improvements 
from an economy-wide viewpoint. Only when new political interests came into the picture, notably environmental interests and the pressures associated with a general necessity to cut government spending, has there been a significant policy change.

In Bezug auf die Einführung der (entkoppelten) Direktzahlungen in den USA stellt Conley (1996: 43) zwar fest, dass im Gesetzgebungsverfahren, das in der Farm Bill von 1996 mündete, „[p]ast economic analysis of farm policy certainly provided guidance for policymakers". Gleichzeitig aber nannte er das Reformergebnis ,an economist's solution without economists“, denn „[e]conomists have not played their usual role of raising questions and issues and explicitly identifying tradeoffs for the political process to resolve"; den entscheidenden Reformfaktor stellte die „baseline capture“ dar (ibid.: 41).

In der EU bemerkte der Mitautor des „Professorengutachtens“, Hanau (1971: 332), zwar rückblickend: „Wohl konnte sich die Gewährung von Kompensationen für die Landwirtschaft [bei der Getreidepreisangleichung 1967] auf das Gutachten stützen“. Allerdings bewertet er den Beitrag des Gutachtens zur agrarpolitischen Willensbildung insgesamt als gering. Er gibt anderen Faktoren klar den Vorzug: „Für das Zustandekommen des Brüsseler Beschlusses über die Getreidepreisangleichung auf mittlerem Niveau der nominalen Getreidepreise in den EWGLändern war nämlich letztlich entscheidend das Interesse aller EWG-Länder an der Verwirklichung der Gemeinschaft" (ibid.).

Einen Rückschluss auf den spürbaren Einfluss der wissenschaftlichen Konzepte auf die agrarpolitische Entscheidungsfindung daraus zu ziehen, dass die durch Wissenschaftler ausgesprochenen Empfehlungen von der Politik - teilweise und verspätet - befolgt worden sind, ist nicht möglich. Aber auch wenn es offensichtlich anderer - mächtigerer - Faktoren bedarf, um Politikwechsel zu vollziehen, kann die Politik auf die in der Wissenschaft erarbeiteten Ideen, Konzepte und Reformvorschläge im Sinne des „policy stream“ nach Kingdon (1995) zurückgreifen, wenn Reformdruck aufkommt. Gleichzeitig mit seiner Geringschätzung des Einflusses des „Professorengutachtens“ auf die agrarpolitische Willensbildung weist Hanau (1971: 332) auf den Beitrag des Gutachtens zur öffentlichen Meinungsbildung hin. Diese Unterscheidung zeigt, dass die Wirkungsmöglichkeiten der wissenschaftlichen Konzepte nicht auf den direkten Einfluss auf die Entscheidungsfindung zu beschränken sind. Sie können somit durchaus eine wichtige Rolle im politischen Prozess spielen - auch wenn nicht unbedingt eine direkte -, indem die Öffentlichkeit informiert und Reformbefürworter mit wissenschaftsbasierten Argumenten ausgestattet werden.

Es bleibt noch die Frage nach eventuellen Ideenflüssen zwischen den wissenschaftlichen Kreisen in den USA und der EU in Bezug auf die Idee der Direktzahlungen. Für diesen Zweck wurde geprüft, ob die hier angeführten Autoren einander zitierten. Es stellte sich heraus, dass die europäischen Agrarökonomen, einschließlich der Vordenker Bachmann, Plate und Nash, ihre US-amerikanischen 
Kollegen wie z. B. Cochrane, Clawson und Houthakker, die offensichtlich früher über Direktzahlungen veröffentlicht hatten, nicht zitieren bzw. keine Verweise auf deren Arbeiten tätigen. Auch umgekehrt ist das nicht der Fall. ${ }^{18}$ Vor diesem Hintergrund konnten - basierend auf den untersuchten Veröffentlichungen - keine direkten gegenseitigen Einflüsse festgestellt werden. Dieses bedeutet verständlicherweise nicht, dass es gar keine Einflüsse gab. Voraussetzungen dafür waren gegeben. Denn wie es scheint, war die einschlägige US-amerikanische Literatur zumindest in Deutschland - in den 1960er- und 1970er-Jahren bekannt. Schlotter (1963: 451) bemerkte, dass der Einsatz von Einkommenshilfen an die Landwirtschaft zu der Zeit „vorwiegend in den USA“ erörtert wurde, was darauf hindeutet, dass diese Diskussion in Europa Anfang der 1960er-Jahre durchaus verfolgt wurde. Wille (1976) stellte verschiedene Modi direkter Einkommensübertragungen, sowohl als Ausgleichszahlungen als auch dauerhafte Einkommensstützung, zusammenfassend dar. Er untersuchte nicht nur ihre Formen und Ausgestaltungsmöglichkeiten, sondern auch Wirkungen. Dabei beschrieb er die Erfahrungen Großbritanniens und der USA mit den produktgebundenen Einkommensübertragungen. Und nicht zuletzt nahm er auf die damaligen Vorschläge der US-amerikanischen Wissenschaftler zu Direktzahlungen Bezug, indem er u. a. Brandow, Houthakker und Swerling erwähnte. Diese Verweise enthielten bereits seine Beiträge in der „Agrarwirtschaft“ von 1971 und auf der GEWISOLA-Tagung im Jahr 1973.

\footnotetext{
18 Innerhalb Europas wurde mehr zitiert. Beispielsweise erwähnen Koester und Tangermann (1976) das Konzept von van Riemsdijk. Der Holländer nahm auch an der Jahrestagung der deutschen Gesellschaft für Wirtschafts- und Sozialwissenschaften des Landbaues im Jahr 1969 teil (GEWISOLA 1969). Dort machte er in einer Wortmeldung deutlich, dass er eine „einmalige Einkommensübertragung“, die „bei einem langfristigen Preisniveau, das ein angemessenes Einkommen ermöglicht und gleichzeitig auf den Weltmarkt abgestimmt ist", für erforderlich hielt (ibid.: 81).
} 


\section{Geschichte der staatlichen Zahlungen in den USA}

\subsection{Anfänge der staatlichen Zahlungen}

Der Ursprung einer gezielten staatlichen Unterstützung der Landwirtschaft in den USA - einschließlich der Zahlungen an landwirtschaftliche Erzeuger - liegt in der schwierigen wirtschaftlichen Lage des Sektors nach dem 1. Weltkrieg. Agrarpreise und -einkommen sanken bedingt durch den Zusammenbruch der Nachfrage nach US-Agrarprodukten in Europa. Der technische Fortschritt, gekennzeichnet durch eine zunehmende Mechanisierung, trug zur Überschusssituation bei. Wegen der allgemeinen Wirtschaftskrise, die in den Jahren 1929 bis 1932 ihren Höhenpunkt erreichte, fiel auch die inländische Nachfrage nach Lebensmitteln. Dieses ließ die Agrarüberschüsse weiter steigen (Benedict 1953). Auch wenn die Krise alle Wirtschaftsbereiche traf, gaben die Preise für landwirtschaftliche Erzeugnisse stärker nach als die Preise für Industrieprodukte (ibid.: 277). Ihr Verhältnis zueinander bezogen auf den Zeitraum von 1910 bis 1914 (100) - fiel von 92 im Jahr 1929 auf 58 im Jahr 1932 (USDA 1951: Tabelle 667). Mit 1,9 Mrd. USD lag das Nettoeinkommen landwirtschaftlicher Betriebsleiter im Jahr 1932 nur noch bei $31 \%$ des Niveaus von 1929 (ibid.: Tabelle 676). Die Hypothekenschuld der landwirtschaftlichen Betriebe stieg von 3,3 Mrd. USD im Jahr 1910 auf 9,2 Mrd. USD im Jahr 1930 (USDA 1939: Tabelle 649). Im Durchschnitt der 1920er-Jahre gingen 13\% 
aller Agrarbetriebe bankrott (ibid.: Tabelle 648). Dabei machte zu jener Zeit die landwirtschaftliche Bevölkerung mit knapp 30 Mio. ein Viertel der Gesamtbevölkerung aus (ibid.: Tabelle 622).

In dieser Situation sah sich die US-Regierung in der Pflicht, in das Marktgeschehen einzugreifen, um das bestehende ,agricultural problem“ (Davis 1939: 63) zu lösen. Die Proteste auf dem Land erhöhten den Druck auf die Politik, aktiv zu werden (Hamilton 1991: 212). Bis dahin fand die föderale Hilfe für die Landwirtschaft fast ausschließlich in Form von Landgrants zur Finanzierung der landwirtschaftlichen Hochschulen sowie Dienstleistungen, z. B. im Bereich der Agrarforschung und -beratung, statt (Benedict 1953: 393). Ingersent und Rayner (1999: 111) formulieren es folgendermaßen: ,There was little direct government support for farm products, except sugar, in contrast to a policy of high protectionism for manufacturers.“19 Zwar wurde der Präsident bereits mit dem „Food and Fuel Control Act" vom August 1917 bevollmächtigt, die Preise ausgewählter landwirtschaftlicher Erzeugnisse über öffentliche Aufkäufe und im Fall von Weizen über einen garantierten Abnahmepreis zu stützen (Benedict 1953). Laut Rucker und Pasour (2007: 458) stellte die US-Regierung damit ihre Fähigkeit, Agrarpreise zu beeinflussen, unter Beweis. Allerdings war dieses Experiment von kurzer Dauer, denn die neue Befugnis des Präsidenten lief mit Kriegsende aus. Vor diesem Hintergrund stellte das umfangreiche Eingreifen des Staates in Agrarpreise und -einkommen Anfang der 1930er-Jahre agrarpolitisch eine ganz neue Entwicklung dar. Die ersten Überlegungen für entsprechende Maßnahmen wurden schon in den 1920er-Jahren angestellt (s. Box 3). Es herrschte dabei die Auffassung, dass eine Stärkung der Landwirtschaft die Wirtschaft im Allgemeinen stärken würde (Benedict 1953).

Box 3: Konzepte der 1920er-Jahre zur Ausgestaltung der US-Agrarpolitik

Insbesondere drei Initiativen verdienen Aufmerksamkeit. Der sogenannte McNary-Haugen-Plan basierte auf der Broschüre „Equality for Agriculture“ von G. N. Peek und H. S. Johnson vom Unternehmen Moline Plow (Benedict 1953). In dieser forderten sie 1922 eine Gleichstellung der Landwirtschaft gegenüber der Industrie. Ziel war es, für Überschusserzeugnisse ihre „fair exchange value“ zu erreichen. Dieses Konzept wurde später im McNary-Haugen-Plan weiterentwickelt. Er sah die Gründung eines staatlichen Unternehmens vor, das Agrarerzeugnisse in solchem Umfang ankaufen sollte, dass deren Inlandspreis auf das Niveau von „ratio-price“ steigen würde. Diese angekauften Produkte sollten dann im Ausland zum Weltmarktpreis verkauft werden. Um Verluste, die dadurch entstehen würden, aufzufangen, sollte auf die erste Verarbeitungsstufe der Erzeugnisse eine Abgabe („,equalization fee“) erhoben werden. Der „,ratio-

${ }^{19}$ Von 1891 bis 1894 wurde den Zuckererzeugern ein „sugar bounty“ gewährt (s. Kapitel 3.1). 
price“ wurde definiert als der Preis eines Erzeugnisses, der im selben Verhältnis zum allgemeinen Preisniveau steht, wie es der Preis dieses Erzeugnisses vor dem 1. Weltkrieg tat. Dieses wurde für eine den Landwirten zustehende gerechte Entlohnung gehalten. Trotz mehrerer Versuche im Zeitraum von 1924 bis 1928 wurde der McNary-Haugen-Plan nicht verabschiedet. Laut Benedict (1953: 238) trug er aber zur Veränderung der Haltung der Politik und Öffentlichkeit gegenüber der Landwirtschaft bei.

Die zweite bedeutende Initiative - der sogenannte „domestic allotment plan“ - wurde im Jahr 1926 von H. N. Owen erstmals vorgestellt und in den nächsten Jahren - insbesondere von Professor J. D. Black von der Harvard University - weiterentwickelt. Im Gegensatz zum McNary-Haugen-Plan befasste sich der „domestic allotment plan“ unmittelbar mit dem individuellen Landwirt. In seiner ursprünglichen Version sah er die Einführung von ,allotment rights“ für Landwirte vor, die ihrem Anteil an der Ernte entsprechen sollten, der im Inland verbraucht wird. „Allotments rights“ würden dann von Verarbeitern erworben, die für die Menge, die auf dem heimischen Markt verkauft wird, solche Rechte nachweisen müssten. Als Ergebnis würden die Erzeugerpreise in den USA über dem Niveau der Weltmarktpreise liegen (Benedict 1953). Darüber hinaus wurde auch der sogenannte „Export Debenture Plan“ diskutiert, der größtenteils von Professor C. L. Stewart, Illinois erarbeitet wurde. Er sah die Zahlung einer Prämie für den Export von Agrarerzeugnissen in Form von handelbaren „debentures“, die von Importeuren für die Zahlung der Importzölle genutzt werden könnten, vor. Infolge dessen sollten Erzeugerpreise im Umfang der Prämie steigen (ibid.: 226).

Den ersten großen Versuch, die wirtschaftliche Lage der Landwirtschaft zu verbessern, startete die US-Regierung mit dem „Agricultural Marketing Act“ von 1929.20 Die beschlossenen Maßnahmen zielten darauf ab, die Vermarktungsorganisation und die Verhandlungsposition der Landwirte sowie die Finanzierung in dem Sektor zu verbessern. Insbesondere wurden der Aufbau und die Organisation von landwirtschaftlichen Kooperativen unterstützt, u. a. durch zinsverbilligte Darlehen für den Absatz von Erzeugnissen oder für den Bau bzw. Erwerb von Lagerhäusern. Darüber hinaus sah der Rechtsakt die Gründung des „Federal Farm Board" mit einem revolvierenden Fonds von 500 Mio. USD vor. Das Board war ermächtigt, Agrarerzeugnisse aufzukaufen und zu lagern, um damit die Preise zu stabilisieren. Nur an der Vermarktungsseite anzusetzen erwies sich allerdings als unzureichend, um Agrarpreise und -einkommen zu stabilisieren. Immer wieder

${ }^{20}$ Neben dem „Hawley-Smoot Tariff Act“ von 1930, mit dem die USA Importzölle für Industrieund Agrarprodukte anhob (Benedict 1953). 
warb das „Federal Farm Board“ für freiwillige Produktionsbeschränkungen als Ergänzung zur Lagerhaltung, konnte sich aber nicht durchsetzen (Hamilton 1991).

Mit dem Scheitern des „Agricultural Marketing Act“ stieg angesichts der unverändert schwierigen Lage der Landwirtschaft die Akzeptanz - sowohl in der Politik als auch unter Landwirten - für radikalere Maßnahmen zur Bekämpfung der Krise, „even things that would have been anathema to them only a few years earlier“ (Benedict 1953: 276). Allerdings „, [e]ven in 1933, when the severity and duration of the depression had created receptiveness to more drastic measures, the idea of direct arrangements with individual farmers and a great army of federal administrative employees was accepted reluctantly and only as the lesser of two evils“ (ibid.: 269). Weiter schreibt Benedict (1953: 348):

In an economy plagued by unmarketable surpluses, especially one so recently composed of aggressive, individualistic pioneers as the United States, such ideas [staatliche Eingriffe in den Markt, Anm. der Verf.] are not warmly received. Only the desperate conditions faced by farmers in the early 1930's could have induced them to accept as much "regimentation" as they did accept in those years. They still looked back nostalgically to the days when they could produce and sell what they wanted to, and as much as they wanted to. Nevertheless, a major change in public attitudes had occurred as a result of the experiences of these years. Plans and controls were thereafter to be more readily accepted and lived with. This attitude was to be further developed as a result of the intensive general mobilization of resources that characterized World War II.

Beginnend im Jahr 1933 wurde unter Präsident Roosevelt der „New Deal““ - eine Serie von Wirtschafts- und Sozialreformen - beschlossen. Der „Agricultural Adjustment Act“ (AAA) vom Mai 1933 sollte für die Linderung der Not in der Landwirtschaft sorgen. Die Bemühungen der Regierung, höhere Preise wiederherzustellen, Einkommen zu erhöhen und die Produktion zu regulieren, waren aber nicht auf die Landwirtschaft begrenzt, sondern betrafen - über den „National Industrial Recovery Act" vom Juni 1933 - auch andere Wirtschaftssektoren (Benedict 1953). Der AAA von 1933 steht für einen Übergang von der Laissez-faireHaltung der US-Regierung gegenüber den Agrarmärkten zu einer gezielten Agrarstützung und markierte somit agrarpolitisch eine Zäsur: „The whole AAA program [...] marked a considerable shift in attitude on the part of American agriculture from that which had characterized it through most of its history" (ibid.: 347). In seiner Rede vor dem Kongress am 16. März 1933 äußerte sich Präsident Roosevelt über den geplanten Rechtsakt: „I tell you frankly that it is a new and untrod path [Herv. durch die Verf.], but I tell you with equal frankness that an unprecedented condition calls for the trial of new means to rescue agriculture" (zitiert nach Davis 1939: 240). Als Notmaßnahme beschlossen (Benedict 1953: 346), 
werden die 1933 eingeführten Instrumente die Agrarpolitik der USA für den Rest des Jahrhunderts prägen. Von Anfang an waren staatliche Zahlungen an die Landwirte Teil des agrarpolitischen Instrumentariums.

Das Hauptziel des „Agricultural Adjustment Act“ war die Herstellung und Aufrechterhaltung eines Gleichgewichts zwischen Erzeugung und Verbrauch landwirtschaftlicher Güter - mit den dazugehörenden Marktbedingungen -, um Preise für Landwirte auf solchem Niveau wiederherzustellen, das ihren Gütern eine Kaufkraft in Bezug auf die von Landwirten gekauften Waren zusicherte, die der Kaufkraft landwirtschaftlicher Güter im Referenzzeitraum entsprach. Als Referenzzeitraum wurde der Zeitraum zwischen August 1909 und Juli 1914 gewählt (AAA 1933). Laut Ingersent und Rayner (1999: 96) standen US-Landwirte in dieser Zeit besser da denn je seit dem Sezessionskrieg in den Jahren 1861 bis 1865. Ausdruck fand dieses Konzept in den sogenannten Paritätspreisen, die sich durch Multiplikation des Durchschnittspreises für das Erzeugnis im Referenzzeitraum mit einem Index der Preise für die Aufwendungen einer Farmerfamilie, bezogen auf den Referenzzeitraum (Paritätsindex) ergaben (Bowers et al. 1984). ${ }^{21}$ Der Paritätsindex setzte sich nicht nur aus Preisen für Produkte und Dienstleistungen für landwirtschaftliche Produktion (Futtermittel, Maschinen, Saatgut, Dünger, Baumaterialien für andere Verwendungszwecke als Hausbau) und aus Lohnsätzen für Fremdarbeiter, sondern auch aus Preisen für Produkte und Dienstleistungen für das Leben (Lebensmittel, Bekleidung, Kosten der Haushaltsführung, Möbel und Einrichtungsgegenstände, Materialien für Hausbau) zusammen (USDA 1936: Tabelle 450). Im Jahr 1938 wurde er um die Zinsen pro Acre auf grundpfandrechtlich gesicherte Landwirtschaftsschulden, Steuerzahlungen pro Acre auf landwirtschaftliche Grundstücke sowie Frachtsätze erweitert (Benedict 1953: 376). Seit seiner Einführung im Jahr 1933 war das Paritätskonzept Gegenstand von Kritik (z. B. Committee on Parity Concepts 1946). Insbesondere wurde darauf hingewiesen, dass das Konzept die Effizienzsteigerungen ignorierte, die seit seiner Einführung und noch mehr seit dem Referenzzeitraum im Agrarsektor erfolgten.

Staatliche Zahlungen an Landwirte waren als Teil des Produktionsanpassungsprogramms namens „Agricultural Adjustment Program“, das das zentrale Element des AAA 1933 darstellte, vorgesehen. In dessen Rahmen wurde der Landwirtschaftsminister ermächtigt, Kürzungen der Anbaufläche bzw. Erzeugung von Grunderzeugnissen durch Verträge mit Landwirten oder andere freiwillige Instrumente zu sichern. Vom dadurch bewirkten Produktionsrückgang erhoffte man sich den Anstieg der Agrarpreise auf das Paritätsniveau. Als Gegenleistung für die Reduzierung der Erzeugung sollten die kooperierenden Landwirte („,cooperators“)

\footnotetext{
${ }^{21}$ Bei dem Index der Preise der von Landwirten benötigten Güter und Dienstleistungen von $152 \mathrm{im}$ Jahr 1942 müssten die Erzeugerpreise in diesem Jahr bei 152\% der im Referenzzeitraum vorherrschenden Preise liegen, um dieselbe Kaufkraft pro Einheit zu haben. Lag der Durchschnittspreis für Weizen im Referenzzeitraum bei 88,4 Cent/Bushel, müsste er sich 1942 auf 134,4 Cent/Bushel belaufen, um $100 \%$ der Parität zu erreichen (Beispiel nach Bowers et al. 1984: 3f).
} 
„benefit payments“ oder „rental payments“ erhalten, die zusammen mit den gestiegenen Preisen ihr Einkommen verbessern würden. Als eine befristete Maßnahme konzipiert, sollte dieses Programm seine Gültigkeit mit dem Ende der Notsituation in der Landwirtschaft verlieren (AAA 1933). Als Grunderzeugnisse wurden von Anfang an Weizen, Baumwolle, Mais, Reis, Tabak, Milch und Milchprodukte sowie Schweine definiert. Später kamen auch Roggen, Flachs, Gerste, Sorghum, Milch- und Rindvieh, Zuckerrüben und -rohr, Kartoffeln sowie Erdnüsse dazu. Je nach Erzeugnis variierte das Programm in seinen Einzelheiten. Der Mechanismus war aber für alle Erzeugnisse gleich. Für jeden Landwirt sollte die Basisanbaufläche für beihilfefähige Grundkulturen ermittelt werden. Schloss er einen Vertrag mit dem Landwirtschaftsminister ab, verpflichtete er sich, diese Kultur auf nicht mehr als dem vereinbarten Teil der Basisanbaufläche anzubauen. ${ }^{22}$ Somit sah das Programm eine horizontale, prozentuale Kürzung der Anbaufläche einer bestimmten Kultur auf allen kooperierenden Betrieben vor, unabhängig davon, wie wirtschaftlich sie produzierten und wie ertragsreich die betreffende Fläche war..$^{23}$ Darüber hinaus war der Landwirtschaftsminister ermächtigt, für alle Erzeugnisse zwecks Preiserhöhung Vermarktungsvereinbarungen mit Verarbeitern zu schlieBen und Lizenzen an Verarbeiter zu vergeben, was insbesondere bei Gemüse und Milch Anwendung fand (Benedict 1953).

Weil die Maßnahmen zur Produktionsanpassung mindestens eine Saison brauchten, um ihre Wirkung zu entfalten, wurden sogenannte „, nonrecourse loans" konzipiert, um die bereits erzeugten Mengen vom Markt nehmen zu können (Orden et al. 1999). Dieses waren unwiderrufliche Darlehen, bei denen die eingelagerte Ernte als Sicherheit diente. Konnte der Erzeuger seine Ernte zu einem Preis verkaufen, der den Wert der staatlich festgelegten „loan rate“ überstieg, zahlte er das Darlehen zurück. Lag der Verkaufspreis unter diesem Wert, verwirkte er die Ernte, musste jedoch das Darlehen nicht zurückzahlen. Bei dieser Regelung handelte es sich somit um eine Art Preisgarantie, wo „loan rates“ - pro Einheit festgesetzt - einen garantierten Mindestpreis darstellten (ibid.). Nur die im Rahmen des Anpassungsprogramms kooperierenden Landwirte konnten von „nonrecourse loans" profitieren. Diese wurden 1933 für Mais und Baumwolle eingeführt und über die neu eingerichtete „Commodity Credit Corporation“ (CCC) abgewickelt. Ergänzend dazu fanden direkte Aufkäufe von Erzeugnissen, wie beispielsweise Rindern und Mastschweinen, statt. Eine solche staatliche Lagerhaltung zur Stabilisierung der Preise wurde als wichtige Ergänzung zum Anpassungspro-

\footnotetext{
22 Die Nutzung der aus der Produktion eines Grunderzeugnisses genommenen Fläche war eingeschränkt. Möglich waren die Flächenstilllegung, der Anbau von bodenverbessernden und erosionsverhindernden Kulturen sowie die Nutzung für den eigenen Verbrauch. Allerdings wurden diese Restriktionen aufgrund der schweren Dürre im Jahr 1934 gelockert (Davis 1939).

23 Im Jahr 1933 wurden 10,5 Mio. Acres Baumwolle, im Jahr 1934 insgesamt fast 36 Mio. und 1935 30 Mio. Acres an Baumwolle, Mais, Weizen und Tabak aus der Produktion genommen bei einer Gesamtackerfläche von etwa 416 Mio. Acres (USDA 1937: Tabelle 513; USDA 1956: Tabelle 609).
} 
gramm gesehen. Während der Markt durch die erste Maßnahme von bereits erzeugten Agrarprodukten entlastet werden sollte, sollten sie durch die zweite Maßnahme gar nicht auf den Markt gelangen. Teile der gelagerten Produktion wurden über die 1933 gegründete „Federal Surplus Relief Corporation“ an Bedürftige verteilt. Gemäß den Vorschriften des sogenannten Abschnitts 32 des „Agricultural Adjustment Act“, der im Jahr 1935 hinzugefügt wurde, konnte der Landwirtschaftsminister $30 \%$ der Zolleinnahmen für eine breite Palette von preisstützenden und Überschüsse beseitigenden Maßnahmen verwenden, darunter auch für Exportsubventionen (Benedict 1953).

Box 4: Ausgestaltung der Agrarzahlungen unter dem „Agricultural Adjustment Act" von 1933

Für Baumwollerzeuger waren im Rahmen von AAA 1933 verschiedene Zahlungen vorgesehen. Weil die Baumwolle zum Programmstart im Juni 1933 bereits gepflanzt worden war, mussten Landwirte einen Teil ihrer Anbaufläche umpflügen, um sich für „,rental payments“ zu qualifizieren. Die Zahlungen standen den Erzeugern zur Verfügung, die ihre Baumwollanbaufläche für 1933 um mindestens $25 \%$ - aber nicht mehr als um $50 \%$ - verringerten. Die Baumwollanbaufläche für 1934 sollten die teilnehmenden Landwirte um 35 bis $45 \%$ und für 1935 um 25\% gegenüber ihrer durchschnittlichen Anbaufläche für Baumwolle im Basiszeitraum 1928 bis 1932 reduzieren. Als Gegenleistung erhielten sie „rental payments“ auf der Grundlage der aus der Baumwollproduktion genommenen Fläche sowie des Durchschnittsertrags der Jahre 1928 bis 1932; eine Obergrenze pro Acre wurde eingeführt. Zusätzlich wurden ihnen „benefit payments" für $40 \%$ ihrer individuellen Erzeugung im Basiszeitraum gewährt (diese Menge entsprach der Baumwollmenge für den Inlandsmarkt; die entsprechende Fläche hieß „allotment“) (Davis 1935). Die Teilnehmer an den Programmen für 1935 und 1936 erhielten außerdem die den Unterschied zwischen dem aktuellen Marktpreis und dem festgelegten Stützpreis ausgleichenden „,price adjustment payments“ als Ergänzung zu „nonrecourse loans“ (Bowers et al. 1984).

Weizenerzeuger wurden aufgefordert, die Weizenanbaufläche für 1934 und 1935 um 15\% bzw. 10\% gegenüber dem Durchschnitt der Jahre 1930 bis 1932 zu reduzieren. Als Gegenleistung bekamen sie 1933, 1934 und 1935 pro Bushel gezahlte ,adjustment payments“ für $54 \%$ der durchschnittlichen Weizenmenge, die sie im Basiszeitraum 1928 bis 1932 erzeugt hatten (die entsprechende Fläche hieß ,allotment"). Diese Menge stellte den Teil der Ernte eines Landwirts dar, der seinem proportionalen Anteil an der im Inland verbrauchten nationalen Weizenerzeugung entsprach (Davis 1935). Maisanbauer sollten die Maisanbaufläche für 1934 um mindestens 20\% gegenüber dem Durchschnitt der Jahre 
1932 und 1933 (Basiszeitraum) reduzieren. Im Gegenzug erhielten sie „rental payments" auf der Grundlage der aus der Maisproduktion genommenen Fläche sowie des Durchschnittsertrags der letzten zehn Jahre. Damit kombiniert waren die entsprechenden Maßnahmen für Mastschweine. Die Zahl der für den Markt produzierten Mastschweine sollte um mindestens 25\% gegenüber dem Basiszeitraum reduziert werden. Dafür erhielten die Landwirte separate „adjustment payments" pro Tier für $75 \%$ der in diesem Zeitraum gehaltenen Mastschweine (Davis 1935).

„Benefit payments“ wurden Erdnussbauern, deren Anbaufläche für das Jahr 1935 nicht mehr als 90\% der Anbaufläche 1933 oder 1935 oder nicht mehr als $90 \%$ des Durchschnitts der Jahre 1933 und 1935 betrug, gewährt. Darüber hinaus erhielten die Erzeuger, die Erdnüsse für Öl sowie Futtermittelzwecke - und nicht für die Nahrungsmittelproduktion - verwendeten, sogenannte „diversion payments“ (McArthur et al. 1982). Reisanbauer bekamen für eine Produktionsanpassung Zahlungen, die am Anfang durch die von Erzeugern gezahlte Umlage und erst ab 1935 durch eine Verarbeitungsabgabe finanziert wurde (Bowers et al. 1984). Für Tabakerzeuger wurden für 1933, 1934 und 1935 „benefit payments“ für die Reduzierung der Ernte- bzw. Anbaufläche eingeführt (Davis 1935).

Eine Besonderheit stellten die Zahlungen im Zuckersektor im Rahmen des "Jones-Costigen Act" von 1934 dar: „Rental payments" und „benefit payments“ für Zuckerrüben- und Zuckerrohranbauer waren an die Einhaltung des Verbots von Kinderarbeit und verbesserter Standards im Bereich der Vergütung der Feldarbeiter gebunden. Dieses war in keinem anderen Programm zu finden (Benedict 1953).

Unter AAA 1933 gab es eine Vielzahl verschiedener Zahlungen an landwirtschaftliche Erzeuger (s. Box 4). Deren Ziel war aber gleich: Die Landwirte für die Reduzierung der Erzeugung zu entschädigen und ihre Einkommenssituation zu verbessern. Die gewährten Zahlungen waren nicht flächendeckend, sondern standen nur für ausgewählte Grunderzeugnisse zur Verfügung. Ihre Höhe basierte auf der historischen Produktion des teilnehmenden Betriebes (Anbaufläche und Erträge in der Vergangenheit); allerdings musste das betreffende Erzeugnis weiter produziert werden. Dazu kommt, dass der Anspruch auf die Zahlungen grundsätzlich an die Fläche und nicht an die Person gebunden war. Insgesamt bot das Anpassungsprogramm den flächenstärkeren Landwirten einen größeren Anreiz teilzunehmen, da sie insgesamt höhere Zahlungen erhielten und auf einen Teil der Erzeugung leichter verzichten konnten (Benedict 1953).

Hervorzuheben ist der Grundsatz der Freiwilligkeit. Beispielsweise nahm an dem ersten Programm für Weizen für 1934 weniger als die Hälfte der beihilfefähigen Weizenerzeuger teil, deren Fläche etwa $75 \%$ der Weizenanbaufläche im Ba- 
siszeitraum ausmachte (Davis 1935: 47). Allerdings wurden in einigen Sektoren die freiwilligen Maßnahmen mit der Zeit durch obligatorische ersetzt. Damit sollte vermieden werden, dass die nicht kooperierenden Landwirte von den durch die Produktionsanpassung gestiegenen Preisen für ihre unbeschränkte Erzeugung profitierten. Für Baumwolle ${ }^{24}$ wurde dieses Instrument im Jahr 1934 eingeführt, Tabak folgte noch in demselben Jahr (Benedict 1953). Aufgrund dessen war die Produktionsanpassung für diese zwei Erzeugnisse effektiver als bei anderen Kulturen, stellt Benedict (1953: 313) fest. Auch für Zucker wurden Quoten eingeführt (Davis 1935).

Neben Freiwilligkeit war Haushaltsneutralität ein wichtiges Merkmal des Anpassungsprogramms aus dem AAA 1933. Die Zahlungen sollten grundsätzlich nicht aus dem Staatshaushalt, sondern durch eine Abgabe finanziert werden, die speziell für diesen Zweck auf die primäre Verarbeitung der betroffenen Kultur erhoben wurde. Der Steuersatz sollte dem Unterschied zwischen dem aktuellen Durchschnittspreis eines Erzeugnisses und seinem „fair exchange value“ (dem errechneten Paritätspreis) entsprechen, es sei denn, ein solches Niveau würde den Verbrauch übermäßig reduzieren. Das Letztere festzustellen - und somit auch die Höhe der Zahlungen - lag im Ermessen des Landwirtschaftsministers (AAA 1933). Dieses bedeutete, dass mit den durch das Programm bewirkten Preissteigerungen die Verarbeitungssteuer automatisch sinken würde. Laut Benedict (1953: 315) wurden die Abgaben zum Großteil an Verbraucher weitergegeben. Auch waren im AAA 1933 selbst 100 Mio. USD für „benefit payments“ sowie „rental payments" und den damit verbundenen Verwaltungsaufwand vorgesehen. Mit den Finanzmitteln aus dem Staatshaushalt wurden insbesondere Teile des Programms für Baumwolle, Mais und Mastschweine finanziert (Davis 1939).

$\mathrm{Zu}$ Beginn der gezielten Agrarstützung in den USA stellten die Zahlungen neben den weiteren Maßnahmen zur Produktionsanpassung - das primäre Instrument der Agrarpolitik dar, während die Preisstützung zweitrangig war. Johnson (1954: 8) bemerkt dazu:

In the first four or five years of the development of farm programs under the New Deal, the role given to price supports was secondary to other aspects of the programs. It was generally believed that the adjustment features of the programs - restrictions on acreages, transfer of land from one product to another, payments directly to farmers [Herv. durch die Verf.], and creation of orderly markets - were more important than the non-recourse loans.

\footnotetext{
${ }^{24}$ Auf Baumwolle wurde eine Abgabe von $50 \%$ des Durchschnittspreises erhoben. Ausgenommen wurden Kleinerzeuger mit bis 5 Acres Anbaufläche und 2 Baumwollballen im Jahr 1935. Andere Erzeuger erhielten Freistellungsbescheinigungen, allerdings nur für die Menge, die sie im Rahmen des Vertrages mit dem Landwirtschaftsminister erzeugen durften. Die genehmigte Gesamtmenge durfte 10 Mio. Baumwollballen nicht überschreiten (Benedict 1953).
} 
„Nonrecourse loans“ wurden konzipiert, weil die bevorzugte Produktionsanpassung nicht sofort Wirkung entfalten konnte (Orden et al. 1999: 21). Bowers et al. (1984: 6) weisen zudem auf den ursprünglich vorübergehenden Charakter der „nonrecourse loans“ hin: „USDA officials justified loans as an emergency measure enabling growers to hold their cotton until the price could advantage as a result of the production control program“. Später allerdings gewann die Preisstützung die Oberhand, sodass Johnson im Jahr 1954 feststellen konnte: „Today major reliance is placed upon purchases and loans, with acreage restrictions being imposed when the former result in stocks that become difficult to manage" (Johnson 1954: 7).

Orden et al. (1999: 21) stellen fest, dass, auch wenn das Produktionsanpassungsprogramm von 1933 am Ende einen eher marginalen Effekt auf die Kürzung der Agrarproduktion ${ }^{25}$ hatte, es die Einkommenssituation der Landwirte mittels Zahlungen verbesserte. In den drei Jahren zwischen 1933 und 1935 flossen rund 1,4 Mrd. USD an die Landwirte, darunter 452 Mio. USD für Baumwolle und 315 Mio. USD für Weizen (USDA 1938: Tabelle 555); ausgedrückt in Preisen von 2009 waren das insgesamt etwa $15 \mathrm{Mrd}$. USD (vgl. Tabelle A3). Das landwirtschaftliche Nettoeinkommen, das mit 2 Mrd. USD im Jahr 1932 einen Tiefpunkt erreichte, erholte sich vom Jahr zu Jahr (vgl. Tabelle A1). Allerdings muss bedacht werden, dass die Verteilung der Agrarzahlungen unter den Erzeugern nicht einheitlich war. Außerdem bemerkt Benedict (1953: 313f), dass es nicht möglich war, die Wirkung der Zahlungen von der Wirkung der anderen Krisenmaßnahmen, sowohl in der Landwirtschaft als auch in der allgemeinen Wirtschaft, zu trennen.

Im Januar 1936 wurden die wesentlichen Teile des AAA von 1933, einschließlich der Befugnis der Regierung, eine Verarbeitungsabgabe zu erheben und anbaureduzierende Verträge mit einzelnen Landwirten zu schließen, vom Obersten Gerichtshof für verfassungswidrig erklärt (Hoosac-Mills-Entscheidung). Da die Landwirtschaft eine lokale Angelegenheit sei, habe die föderale Regierung kein Recht, sie zu regulieren, entschied das Gericht. Darüber hinaus kritisierte es, dass von der Verarbeitungsabgabe lediglich eine bestimmte Gruppe von Landwirten profitierte (Bowers et al. 1984). Die Hoosac-Mills-Entscheidung bedeutete ein abruptes Ende des bisherigen Produktionsanpassungsprogramms, aber kein Ende der staatlichen Agrarstützung in den USA. Ihre Kontinuität gewährleistete der kurzfristig aufgestellte „Soil Conservation and Domestic Allotment Act“ vom Februar 1936, der ein neues Konzept der Agrarstützung lieferte, nicht zuletzt um

\footnotetext{
25 Ab 1934 verschleiert die schwere Dürre die Ergebnisse des Anpassungsprogramms. Es ist unklar, inwieweit die schlechten Wetterbedingungen oder die Maßnahmen unter dem „Agricultural Adjustment Act" für den stattgefundenen Produktionsrückgang verantwortlich waren. Die Effektivität des Programms wurde durch gleichzeitig geschaffene Anreize zur Erhöhung der Erzeugung, wie „,nonrecourse loans" oder günstige Kredite, untergraben. Außerdem konnten Landwirte weniger produktive Flächen aus der Produktion nehmen, die Anbaueinschränkungen trotz Vertrag umgehen oder aber die Zahlungen dafür nutzen, um die Anbauintensität auf der verbleibenden Fläche zu erhöhen (Benedict 1953).
} 
ihre Verfassungskonformität zu sichern. Er legte - zumindest nominell - andere Ziele als der „Agricultural Adjustment Act“ von 1933 fest. Die Preisparität wurde durch die Einkommensparität ersetzt, definiert als das Verhältnis der Kaufkraft des Nettoeinkommens einer Person in der Landwirtschaft ${ }^{26}$ zur Kaufkraft des Nettoeinkommens einer Person im nichtlandwirtschaftlichen Sektor, das zwischen August 1909 und Juli 1914 herrschte. Dieses bedeutete eine Umstellung vom Preisziel auf das Einkommensziel; Agrarpreise durften schwanken, ohne mit dem neuen Ziel in Konflikt zu geraten. Mit der Einkommensstützung wurde die Förderung des Bodenschutzes kombiniert. Sie diente der Erhöhung der Akzeptanz für die Agrarförderung seitens der Gesellschaft, die spätestens seit den schweren Staubstürmen „Dust Bowl“, von denen in den 1930er-Jahren weite Teile der USA betroffen waren, für das Thema sensibilisiert war. In Wirklichkeit aber war der Bodenschutz nur ein zweitrangiges Ziel (Benedict 1953).

Den Landwirten, die sich bereit erklärten, anstelle von Boden gefährdenden Kulturen bodenschonende Kulturen - wie Gräser, Leguminosen und andere Futterpflanzen - anzubauen, wurden „,soil-conserving payments“ gewährt. Als Boden gefährdende Kulturen wurden Weizen, Baumwolle, Mais, Tabak und Zuckerrüben definiert - also grundsätzlich die Kulturen, deren Erzeugung reduziert werden sollte. Somit diente diese neue Zahlung in erster Linie eher der Stilllegung von Ressourcen als dem Umweltschutz. Ihre Höhe lag durchschnittlich bei 10 USD/ Acre. Die Teilnahme am Bodenschutzprogramm war für Landwirte freiwillig. Ähnlich wie die Agrarzahlungen unter AAA 1933 standen „soil-conserving payments“ nicht allen Landwirten zur Verfügung, sondern nur jenen, die in der Vergangenheit die ausgewählten, Boden gefährdenden Kulturen angebaut hatten. Im Gegensatz zu den Zahlungen unter AAA 1933 wurden sie aber direkt aus dem Staatshaushalt finanziert. Darüber hinaus wurden Zahlungen für Umweltleistungen, wie Einsatz von Düngemitteln, Gründüngerpflanzen oder Aufforstung, von max. einem USD pro Acre angeboten - sogenannte ,soil-building payments“ (Benedict 1953).

Aufgrund der schwierigen Preissituation im Baumwollsektor wurden 1937 bereits die aus dem Staatshaushalt finanzierten Zahlungen vorgezogen, die sich noch in der Planung des neuen Agrargesetzes befanden. Diese „price adjustment payments" wurden für die Baumwollerzeuger eingeführt, die sich bereit erklärten, an dem für 1938 avisierten Produktionsanpassungsprogramm teilzunehmen. Sie erhielten den Unterschied zwischen 0,12 USD je Pfund Baumwolle und dem Durchschnittspreis am Verkaufstag, höchstens aber 0,03 USD je Pfund Baumwolle. Aus Kostengründen wurden die Zahlungen nur für $65 \%$ der individuellen Baumwollmenge im Referenzjahr 1937 getätigt (Bowers et al. 1984: 11).

\footnotetext{
${ }^{26} \mathrm{Im}$ „Agricultural Adjustment Act“ von 1938 wurde präzisiert, dass es sich hier um das Einkommen aus landwirtschaftlichen Tätigkeiten handelt.
} 
Im Jahr 1937 wurden auch die Zahlungen im Zuckersektor modifiziert, um sie verfassungskonform zu gestalten. Als Finanzierungsgrundlage ersetzte eine in den Staatshaushalt fließende Verbrauchssteuer die Verarbeitungsabgabe. Die Zuckererzeuger und -raffineure, die die Verbrauchssteuer entrichten mussten, leiteten diese Belastung allerdings tendenziell nicht an die Verbraucher, sondern an ihre Lieferanten - die Anbauer - weiter (Ballinger 1975: 36). Die Erzeuger von Zuckerrüben und -rohr konnten weiter von den „benefit payments“ profitieren, vorausgesetzt, dass sie ihren Anteil an der Quote nicht überschritten. Diese Zahlungen waren an die Vorgaben im Bereich Arbeitsrecht (Verbot der Kinderarbeit mit Ausnahme der Kinder aus der Familie, angemessene Löhne für die Feldarbeiter) und Umwelt (Erhaltung der Bodenfruchtbarkeit) gebunden. Darüber hinaus mussten die Erzeuger, die gleichzeitig Verarbeiter waren, die von anderen Erzeugern eingekauften Zuckerrüben bzw. Zuckerrohr angemessen bezahlen. Aufgrund der Bindung an diese zahlreichen Auflagen wurden „benefit payments“ im Zuckersektor auch „conditional payments“ genannt. Zusätzlich dazu waren sogenannte „abandonment payments“ und „deficiency payments“ bei Aufgabe der Anbaufläche bzw. gesunkenen Erträgen infolge natürlicher Katastrophen vorgesehen (Ballinger 1946: 8).

Die nächste Farm Bill - „Agricultural Adjustment Act“ (AAA) von 1938 führte die „soil-conserving payments“ mit einigen Modifikationen weiter. Mit dem sogenannten „Agricultural Conservation Program“ bezuschusste der Staat ausgewählte Umweltleistungen der Landwirte auf Basis vertraglicher Vereinbarungen. Bis zu 75\% der Gesamtkosten der Umsetzung von etwa 100 Verfahren überwiegend zum Erosions- und Wasserschutz konnten vom Staat übernommen werden (,cost-sharing payments"). ${ }^{27}$ Auch wurde eine Kappung der Zahlungen bei 10.000 USD pro Erzeuger und Jahr eingeführt, um der Kritik zu begegnen, die Programme wären so konzipiert, dass sie ausschließlich den Großbetrieben zugutekommen (Benedict 1953). Auf die Verarbeitungsabgabe wurde endgültig verzichtet, aber ähnlich wie AAA von 1933 sah die neue Farm Bill eine Reihe von Maßnahmen zur Angebotssteuerung vor. Ihre Verfassungskonformität wurde letzten Endes im Jahr 1942 vom Obersten Gericht bestätigt, das urteilte, dass der Kongress unter der „Commerce Clause“ der US-Verfassung befugt ist, die landwirtschaftliche Erzeugung zu regulieren, einschließlich des Anbaus von Erzeugnissen für den eigenen Verbrauch. ${ }^{28}$ Als Generallinie für die Festlegung der Agrar-

\footnotetext{
27 Die Ausgaben für dieses Programm lagen bei etwa 200 Mio. USD im Jahr. Im Jahr 1995 nutzten es rund 85.000 Landwirte (Anderson und Magleby 1997: 258). Im Jahr 1996 wurde es durch das „Environmental Quality Incentive Program“ ersetzt. Die Kürzung der Anbaufläche für Überschusserzeugnisse wurde allerdings schon im Jahr 1947 beendet (ibid.: Abbildung 1.1.4).

${ }^{28}$ Im Jahr 1941 überschritt Landwirt Filburn die ihm zugeteilte Vermarktungsquote für Weizen. Seine Erzeugung innerhalb der Quote war für den Markt, die darüber hinausgehende für den eigenen Verbrauch vorgesehen. Trotzdem musste er für diese Übererzeugung eine Strafabgabe zahlen. Er klagte gegen Landwirtschaftsminister Wickard mit der Begründung, die Erzeugung von Weizen für den Eigenverbrauch hätte keine unmittelbare Wirkung auf den zwischenstaatlichen Handel und fiele
} 
stützung galt wieder die Preisparität. Die Zahl der Grunderzeugnisse wurde auf fünf reduziert: Weizen, Mais, Baumwolle, Tabak und Reis. Im Jahr 1941 kamen noch Erdnüsse dazu (Bowers et al. 1984).

Der AAA von 1938 führte eine neue Kategorie der Agrarzahlungen - sogenannte „parity payments“ - ein. Sie waren Teil eines komplexen Programms zur Angebotssteuerung mit dem Ziel, die Überschussproduktion der Grunderzeugnisse zu bewältigen. Sowohl Anbau- als auch Absatzbeschränkungen waren möglich. In der Regel sollte der Landwirtschaftsminister jedes Jahr sogenannte ,acreage allotments“ für die Grunderzeugnisse festsetzen. Die Definition einer ,acreage allotment" variierte dabei je nach Produkt. Für Weizen war das beispielsweise eine Anbaufläche, auf der - auf Basis der Durchschnittserträge der letzten zehn Jahre einschließlich Übertragsbestände $130 \%$ des Inlandsverbrauchs und des Exports eines normalen Jahres erzeugt werden konnte. Nachdem diese Zielproduktionsmenge festgelegt wurde, wurde sie auf Basis der historischen Anbaufläche auf die Bundesstaaten, Landkreise und schließlich Betriebe heruntergebrochen. Nur ein ganz geringer Anteil der ,acreage allotments“ auf Landkreis-Ebene (z. B. 3\% bei Weizen) konnte den Betrieben zugeteilt werden, die in den letzten drei Jahren das betreffende Erzeugnis nicht angebaut hatten. Die Einhaltung der individuellen „allotments“ war für Landwirte Voraussetzung für den Erhalt von sowohl „,nonrecourse loans“ als auch „parity payments“. „Allotment“ galt allerdings nur dann als nicht eingehalten, wenn der teilnehmende Landwirt es wissentlich überschritten hatte. Für die „noncooperators“ - Landwirte, die ihre individuelle ,allotments“ nicht einhielten - waren keine Strafabgaben vorgesehen (Benedict 1953).

Wenn das Angebot eines Erzeugnisses das im AAA von 1938 festgelegte Niveau überschritten hatte, war der Landwirtschaftsminister verpflichtet, eine sogenannte Vermarktungsquote in bestimmter Höhe für das nächste Vermarktungsjahr festzusetzen. Diese Auslöseschwelle variierte je nach Erzeugnis und betrug beispielsweise bei Weizen 135\% des Inlandsverbrauchs und des Exports eines normalen Jahres, lag also leicht über der Erzeugung auf der ,allotment“ (AAA 1938). Wenn die Vermarktungsquote in Kraft war, wurde die Einhaltung der individuellen ,allotments“ für alle Landwirte verpflichtend. „Noncooperators“ mussten eine Strafabgabe zahlen. Im Gegensatz zu „allotments“ mussten die Vermarktungsquoten von den Erzeugern gebilligt werden. Sie traten erst in Kraft, wenn sie im Referendum von mehr als zwei Dritteln der Erzeuger genehmigt wurden. Im Falle einer Ablehnung standen den Landwirten allerdings „,nonrecourse loans" bis zum Anfang des übernächsten Vermarktungsjahres nicht mehr zur Verfügung (Benedict 1953). Ergänzt wurden die Produktions- und Vermark-

somit nicht in den Rahmen rechtlicher Befugnisse des Kongresses nach der „Commerce Clause“ der Verfassung. Das Oberste Gericht entschied allerdings, dass eine Aktivität keine unmittelbare Wirkung auf den Handel haben muss, um unter die „Commerce Clause“ zu fallen, solange ihre Wirkung wesentlich und wirtschaftlich ist. Somit sei der Kongress befugt, die Erzeugung von Weizen für den eigenen Verbrauch zu regulieren (Supreme Court 1942). 
tungsbeschränkungen durch „,nonrecourse loans“, die mit AAA 1938 fest etabliert und für ausgewählte Erzeugnisse (Weizen, Mais und Baumwolle) verpflichtend gemacht wurden. Die „loans“ standen nur den „cooperators“ zur Verfügung, das heißt nur den Landwirten, die ihre „allotment" nicht überschritten bzw. die die vom Landwirtschaftsminister ggf. vorgeschlagene Vermarktungsquote nicht ablehnten. Auch hier variierten die Bedingungen je nach Erzeugnis. Beispielsweise musste für Weizen eine „loan rate“ auf dem Niveau von 52 bis $75 \%$ der Parität zur Verfügung gestellt werden, wenn der Preis zum Ende des Vermarktungsjahres unter $52 \%$ der Parität lag oder wenn die voraussichtliche Erzeugung für das aktuelle Jahr den Inlandsverbrauch und Exporte eines normalen Jahres überstieg. Darüber hinaus wurde für Weizen das erste Versicherungsprogramm als Pilotprogramm eingerichtet, um die Erzeuger vor Ernteausfällen zu schützen (AAA 1938).

Ferner wurde der Landwirtschaftsminister ermächtigt, „parity payments“ für die kooperierenden Erzeuger von Baumwolle, Mais, Weizen, Reis und Tabak zu tätigen. Ihre Höhe sollte so festgelegt werden, dass sie die Differenz zwischen den tatsächlichen Erzeugerpreisen und Paritätspreisen möglichst ausglichen. Als Basis für die Berechnung von „parity payments“ galt die „,normale Erzeugung“ eines Landwirts, die sich durch Multiplikation seiner Durchschnittserträge der letzten zehn bzw. fünf Jahre mit der Zahl der Acres seiner Anbaufläche, die auf die ,allotment" beschränkt war, ergab (AAA 1938). Wieder einmal waren die Zahlungen nur an Erzeuger ausgewählter Erzeugnisse gerichtet, um sie zur Teilnahme an Produktionsbeschränkungsmaßnahmen zu bewegen. Allerdings wurden sie nicht automatisch gezahlt, sondern nur dann, wenn Finanzmittel aus dem Staatshaushalt zugewiesen wurden (Bowers et al. 1984). Beispielsweise wurden 1944 und 1945 keine „parity payments“ genehmigt. Außerdem wurden 1938-1940 keine „parity payments “ für Tabak, 1938 keine für Weizen und 1942-1943 keine für Baumwolle gewährt. Im ersten Anwendungsjahr 1938 beliefen sich „parity payments“ auf 123 Mio. USD. Sie entfielen ausschließlich auf Baumwolle. Ein Jahr später stiegen sie auf insgesamt fast 212 Mio. USD, wovon 96 Mio. USD für Baumwolle gewährt wurden (USDA 1946: Tabelle 705).

Mit dem Beginn des 2. Weltkriegs musste die Agrarpolitik der USA eine neue Richtung einschlagen (Benedict 1953: 402). War der Fokus der 1930er-Jahre darauf gerichtet, die Agrarproduktion einzudämmen, galt es nun, sie zu erhöhen, um den steigenden Bedarf sowohl für militärische Zwecke als auch für „lend-lease“ zu decken. Am Anfang dienten die unter AAA 1938 bereits verfügbaren Instrumente, insbesondere „nonrecourse loans“, als Produktionsanreiz. Das Niveau von „loan rates" wurde erst für Grunderzeugnisse und dann für alle anderen Erzeugnisse, für die eine Produktionssteigerung vom Landwirtschaftsminister gefordert war (sogenannte Steagall-Erzeugnisse) erhöht. Im Jahr 1942 wurden für diese Erzeugnisse die „loan rates“ auf dem Niveau von 90\% der Parität für zwei Jahre nach der Erklärung über die Einstellung der Feindseligkeiten garantiert (Benedict 1953). Laut Cochrane und Ryan (1976: 72) umfasste das Preisstützungsprogramm im 
Jahr 1945 etwa 166 landwirtschaftliche Erzeugnisse. Gleichzeitig wurden für Agrarprodukte - anders als bei nichtlandwirtschaftlichen Produkten - im Rahmen des zur Inflationsbekämpfung eingeführten Höchstpreissystems großzügige Preisobergrenzen festgelegt (110\%, später $100 \%$ der Parität) (Benedict 1953).

Die genannten Maßnahmen boten Anreize, die Gesamtagrarproduktion zu steigern. Allerdings erwiesen sie sich als wenig geeignet, alte Produktionsmuster zu ändern und den Fokus der Landwirte von Grunderzeugnissen, insbesondere Baumwolle, auf andere Erzeugnisse, wie Ölsaaten oder Fleisch- und Milchprodukte, zu legen. Zwar wurde die Preisstützung für die Steagall-Erzeugnisse erhöht, dieses galt aber auch für die Grunderzeugnisse. In dieser Situation griff die Regierung zum Instrument der Zahlungen an Landwirte. Der „Emergency Price Control Act" vom Januar 1942 sah die Möglichkeit der direkten Subventionierung der heimischen Erzeuger vor, um die Agrarproduktion aufrechtzuerhalten bzw. zu erhöhen. Konkret konnte die Preisbehörde („Office of Price Administration“) „subsidy payments" in dem Umfang, in einer solchen Weise und zu solchen Bedingungen, die sie zum Erhalt der erforderlichen Produktion für notwendig erachtete, tätigen. Außerdem konnte sie landwirtschaftliche Erzeugnisse ankaufen, um sie dann zu einem niedrigeren Preis an Verbraucher oder Verarbeiter zu verkaufen. Allerdings konnten diese neuen Befugnisse vom „Office of Price Administration“ nur dann ausgeübt werden, wenn die Produktionsziele im laufenden Jahr oder im darauffolgenden Jahr nicht erreicht werden würden (ibid.).

Angesichts der steigenden Nachfrage nach Agrarprodukten wurden zudem die produktionsbeschränkenden Maßnahmen gelockert und Vermarktungsquoten ausgesetzt. Ab Dezember 1942 wurde die Höhe der bestehenden Zahlungen an Landwirte davon abhängig gemacht, inwiefern die jährlich festgelegten Zielvorgaben für die Produktion kriegsrelevanter Erzeugnisse durch einzelne Betriebe erfüllt wurden. Für jeden Acre, der unter $90 \%$ des Ziels lag, waren Abzüge vorgesehen. Einen Monat später wurde auch ein spezielles, 100 Mio. USD schweres Programm erlassen, in dessen Rahmen den Landwirten, die die Zielvorgaben mindestens zu $90 \%$ erfüllten, „incentive payments“ gewährt wurden. Darüber hinaus wurden ebenfalls im Januar 1943 ,production payments“ zur Erhöhung der Produktion von Kartoffeln, Bohnen und gartenbaulichen Kulturen eingeführt. Die geltenden Regelungen zum Bodenschutz wurden dahingehend gelockert, dass auf der aus der Produktion genommenen Fläche außer bodenschonenden Kulturen auch kriegsrelevante Erzeugnisse angebaut werden konnten (ibid.). Somit brachte der Krieg Agrarzahlungen ins Spiel, deren Ziel es nicht mehr war, Landwirte für die Produktionsbeschränkung zu entschädigen, sondern sie für Produktionssteigerungen zu belohnen.

Mit dem „Hold-the-Line Order“ von Präsident Roosevelt vom April 1943 wurden die Preis- und Lebensmittelbehörden ermächtigt, strikte Preisobergrenzen für alle die Lebenshaltungskosten beeinflussenden Produkte einzuführen, darunter auch für landwirtschaftliche Erzeugnisse. So konnten sie die Agrarpreise, die „ex- 
cessively high, unfair, or inequitable“ waren, reduzieren (Benedict 1953: 421). Auf der anderen Seite wurden die Behörden ermächtigt, Preisanpassungen, Stützpreise, Subventionen oder andere Anreize, die als notwendig für die Beibehaltung oder Erhöhung der Agrarerzeugung erachtet wurden, zu erlassen, vorausgesetzt, dass diese Maßnahmen nicht zu einer Erhöhung der Lebenshaltungskosten führen würden. Im Mai 1943 wurden Preisobergrenzen für Fleisch und Trocken-Lebensmittelsortimente im Einzelhandel festgelegt. Darüber hinaus wurden die Preise für Fleisch, Butter und Kaffee sowohl im Einzel- als auch im Großhandel um etwa $10 \%$ gekürzt. Der Unterschied zwischen dem neuen und dem alten Preis wurde den Produzenten durch eine gleichwertige Subventionszahlung ausgeglichen. Die Landwirte erhielten zudem direkte Subventionszahlungen beispielsweise für Milch, Zuckerrüben, Zuckerrohr, Gemüse und Bohnen. Außerdem konnten sie durch den Verkauf der Erzeugnisse an staatliche Behörden einen höheren Preis erzielen als am freien Markt. Dieses betraf Produkte wie Sojabohnen, Erdnüsse und Milch. Von Verarbeitern kaufte die CCC Cheddar-Käse, Rosinen und Zucker an, um sie verbilligt an Händler oder Verbraucher zu verkaufen. Da einige der Subventionen für Verarbeiter an die Bedingung geknüpft waren, dass den Landwirten „support prices“ gezahlt wurden, profitierten auch Primärerzeuger davon. Diese Form der Subventionen wurde - mit einigen Ausnahmen - zum 30. Juni 1945 bzw. 1946 beendet (ibid.).

Das vorrangige Ziel der genannten Subventionszahlungen war, Verbraucherpreise für Nahrungsmittel stabil zu halten bzw. Preisobergrenzen aufrechtzuerhalten, indem den Erzeugern bzw. Verarbeitern einen Teil der gestiegenen Kosten ausgeglichen wurde. Sie dienten auch als Anreiz für Produktionssteigerungen der ausgewählten Erzeugnisse, da die Preise diese Funktion nicht wahrnehmen konnten, weil sie nicht steigen durften. Das Ziel, den Erzeugern höhere Einkommen zu ermöglichen, stand erst an nachrangiger Stelle, denn das landwirtschaftliche Nettoeinkommen befand sich auf hohem Niveau und erreichte 1945 12,3 Mrd. USD (vgl. Tabelle A1). Der Fokus der Kriegsprogramme lag auf - in den 1930er-Jahren von der Politik mit kleineren Ausnahmen vernachlässigten - tierischen Erzeugnissen. Die Ausgaben für die Zahlungen und die gezielten Ankäufe betrugen im Jahr 1945 knapp 1,6 Mrd. USD; mehr als zwei Drittel davon im Fleisch- und Milchbereich. An dritter Stelle folgte der einem System der Rationierung unterworfene Zucker mit 103,6 Mio. USD (vgl. Box 5) (Benedict 1953: 428f).

Box 5: Agrarzahlungen im US-Zuckersektor im Zeitraum 1939 bis 1945

Die Entwicklung der US-Agrarpolitik während des 2. Weltkriegs lässt sich gut am Beispiel von Zucker verfolgen. Es herrschte eine Zuckerknappheit, die durch den Ausfall der Lieferanten auf den Philippinen und Java, den Rückgang der Erzeugung in Europa, die schlechte Zuckerrübenernte in den USA in den Jahren 1943-1945 sowie die schlechte Zuckerrohrernte in Kuba im Jahr 1945 
bedingt war. Obendrein wurde Zucker in größerem Maße für industrielle Zwecke genutzt: Den daraus gewonnenen Alkohol verwendete man für die Kautschukerzeugung, nachdem der Zugang zu asiatischen Naturkautschukplantagen kriegsbedingt abgeschnitten wurde. Zucker war das erste Lebensmittel, das der Rationierung unterworfen wurde sowie das einzige, das 1946 noch rationiert war (Ballinger 1946).

Um dem Preisanstieg entgegenzuwirken, wurde zuerst die Quotenregelung vom 11. September 1939 bis zum 26. Dezember 1939 ausgesetzt. Im August 1941 wurde eine Preisobergrenze für verzollten Rohrzucker und im Dezember 1941 für Zucker im Großhandel sowie für durch Erstvertreiber verkauften Zucker zum unmittelbaren Verbrauch erlassen. Ab Mai 1942 wurde auch der Zuckerpreis im Einzelhandel gedeckelt und die Quoten wurden nach oben angepasst. Darüber hinaus setzte das USDA das neue Instrument der Zielvorgaben ein. Die Zielvorgabe für die Erzeugung von Zuckerrüben und Zuckerrohr für das Erntejahr 1943 belief sich auf 1,050 Mio. Acres Zuckerrüben und 0,34 Mio. Acres Zuckerrohr. In den Jahren 1944 und 1945 sollten 0,951 Mio. Acres Zuckerrüben und 0,337 Mio. Acres Zuckerrohr angebaut werden. Allerdings wurden diese Zielvorgaben in keinem der Jahre erfüllt (Ballinger 1946: 6). Zum Vergleich: 1938 lag die geerntete Anbaufläche für Rüben bei 0,93 Mio. Acres und für Rohr bei 0,313 Mio. Acres (USDA 1940: Tabelle 182 und 195).

Ab 1942 mussten die Landwirte ihre Zuckerrüben- bzw. Zuckerrohranbaufläche nicht mehr beschränken, um in den Genuss der durch die Verbrauchssteuer finanzierten ,benefit payments“ zu kommen. Der Basissatz für die Zahlungen betrug in den Jahren 1939 bis 1941 0,6 USD je 100 Pfund von wirtschaftlich gewinnbarem Rohrzucker und lag damit auf dem Vorkriegsniveau. Erzeuger, die 500 Tonnen und mehr Zucker produzierten, erhielten niedrigere Zahlungen. In den nachfolgenden Kriegsjahren wurde der Basissatz auf 0,8 USD pro 100 Pfund von gewinnbarem Zucker erhöht. Außerdem wurden ab dem Jahr 1942 die Zahlungen auf Erzeuger auf den Amerikanischen Jungferninseln ausgeweitet (bis dahin waren sie nur für den kontinentalen Teil der USA sowie Hawaii und Puerto Rico vorgesehen). Die Regelung zu „abandonment payments“ und „deficiency payments“ war weiterhin in Kraft (Ballinger 1946: 8).

Darüber hinaus gab es Subventionszahlungen aus dem Staatshaushalt in Form eines Ausgleichs für die gestiegenen Transport- und Lagerungskosten von Zucker sowie Transportkosten von Zuckerrüben. Außerdem erhielten die Erzeuger von Zuckerrüben und -rohr ,,incentive payments“, die den Anreiz für die Produktionserhöhung bieten sollten. Denn der Preis von Rüben bzw. Rohr konnte diese Funktion nicht mehr erfüllen, da er vom Zuckerpreis abhing, der 
wiederum gedeckelt war. „Incentive payments“ im Zuckersektor wurden zuerst in Louisiana im Jahr 1943 gezahlt. Der Zahlungssatz von 0,33 USD je Tonne Zuckerrohr wurde für 1944 auf 0,85 USD und für 1945 auf 1,6 USD je Tonne erhöht. Den Erzeugern in Florida wurden ,incentive payments“ in Höhe von 1,6 USD pro Tonne Rohr im Jahr 1945 gewährt. Die Zuckerrübenanbauer erhielten ,incentive payments“ im Zeitraum von 1943 bis 1945: im ersten Jahr 1,5 USD, dann 3 USD pro Tonne Zuckerrüben (Ballinger 1946: 9). Da sich die Preisobergrenzen auch auf die Margen der Zuckererzeuger negativ auswirkten, hatte die Regierung auch ihnen „subsidy payments“ gewährt. Darüber hinaus kaufte die staatliche CCC sowohl Zucker als auch Melasse auf (ibid.: 18f).

Insgesamt wurden im Zuckerbereich in den Jahren 1942 bis 1945 knapp 201 Mio. USD an „,benefit payments“, ,abandonment payments“ und „,deficiency payments" gezahlt. Ein ähnlicher Betrag wurde für die übrigen Maßnahmen ausgegeben (ibid.: 32).

Die Agrarzahlungen der Kriegsjahre trafen auf heftigen Widerstand von Seiten der Landwirte, ihrer Verbände und Kongressvertreter - auch wenn eine gezielte Unterstützung der Landwirtschaft spätestens seit 1933 kein neues Phänomen war. Sie betrachteten die Zahlungen als Beihilfen für Verbraucher und nicht für die Erzeuger. Diese seien unnötig, inflationär und würden die Staatsausgaben vergrößern, lautete die Kritik. Nicht unwichtig war auch, dass höhere Verbraucherpreise ein höheres Niveau der Preisstützung nach dem Krieg ermöglichen würden. Das Ringen zwischen Präsident Roosevelt, einem Anhänger der Subventionen, und dem Kongress, der dagegen war, dauerte bis Kriegsende. Der Kongress versuchte, die neuen Programme abzuschaffen; jedoch legte der Präsident sowohl 1943 als auch 1944 sein Veto ein. Da die neuen Zahlungen mit der Verlängerung der Laufzeit von CCC verknüpft waren, war der Präsident in diesem Machtkampf besser aufgestellt. Denn: Würde die Laufzeit von CCC nicht verlängert werden, würden auch die von den Landwirten gewünschten Programme wie „nonrecourse loans“ abgeschafft (Benedict 1953).

In den Kriegsjahren verloren die staatlichen Zahlungen als Beitrag zum landwirtschaftlichen Einkommen an Bedeutung, auch wenn ihr Niveau höher als in den Vorjahren war. Im Jahr 1939 machten sie noch 17\% des landwirtschaftlichen Nettoeinkommens aus. Aufgrund von gestiegenen Preisen und Einkommen waren es im Jahr 1941 nur noch 8,4\% und im Jahr 1945 6\% (vgl. Tabelle A1). In demselben Zeitraum halbierte sich die Gesamthöhe von „conservation payments“. Ihr Anteil an den Gesamtzahlungen sank von $73 \%$ im Jahr 1939 auf $35 \%$ im Jahr 1945, was den Fokus auf die Erhöhung der Erzeugung widerspiegelt (vgl. Tabelle A2).

Die Auseinandersetzung über die Ausgestaltung der Agrarpolitik setzte sich nach Kriegsende fort. Sie war u. a. durch die jüngsten Erfahrungen mit der Stüt- 
zung der Kartoffelpreise geprägt. Alleine im Jahr 1948 wurden mehr als 4 Mio. Tonnen Kartoffeln (ein Drittel der Erzeugung) vom Staat aufgekauft und zum größten Teil vernichtet, da Lagerung und Export nur begrenzt möglich waren. Dieses belastete den Staatshaushalt mit knapp 222 Mio. USD und stieß in der Öffentlichkeit auf scharfe Kritik. Die Schwächen bzw. Grenzen des aktuellen Systems wurden sichtbar. Vor diesem Hintergrund empfahl der sogenannte ColmerAusschuss des Repräsentantenhauses in seinen Berichten von 1944 bis 1946, die Preisstützung flexibler zu machen und sie zusammen mit den Produktionsbeschränkungsmaßnahmen zurückzufahren (aber nicht abzuschaffen). In Zeiten niedriger Preise sollten die landwirtschaftlichen Einkommen durch „compensatory payments“ oder ,supplemental income payments“ gestützt werden (Benedict 1953).

Im April 1949 wurde der sogenannte Brannan-Plan vorgestellt, benannt nach dem damaligen Landwirtschaftsminister C. F. Brannan. Auch er schlug „income payments" vor. Mit diesen Zahlungen sollten die Erzeuger nichtlagerfähiger Produkte unterstützt werden. Für die Erzeuger lagerfähiger Produkte, wie Baumwolle oder Getreide, war weiter eine Preisstützung vorgesehen. Als Grundlage für die Berechnung der Agrarstützung sollte statt der Preisparität ein neuer Einkommensstandard als gleitender Durchschnitt der letzten zehn Jahre dienen (zunächst von 1939 bis 1948). Aus der Relation des landwirtschaftlichen Einkommens in diesem neuen Referenzzeitraum zum Einkommen im Berechnungsjahr sollte die Stützungshöhe ermittelt werden. Konkret sollten die Zahlungen den Unterschied zwischen dem Marktpreis und dem vom Landwirtschaftsminister festgelegten Niveau ausgleichen. Das Konzept der Preisparität sollte endgültig abgeschafft werden (ibid.). Die Förderung des Landwirtschaftssektors über die „income payments“ sollte dazu führen, dass die Erzeugnisse zu niedrigeren Preisen auf den Markt gelangten. Somit erinnerten sie an die Subventionszahlungen im 2. Weltkrieg. Auch Hersteller tierischer Erzeugnisse sollten in den Genuss von Zahlungen kommen. Die staatliche Unterstützung für Baumwolle, Weizen, Mais, Tabak, Eier, Milch, Geflügel, Schweine, Lamm und Vieh sollte verpflichtend sein (situationsabhängig sollte der Landwirtschaftsminister dafür bestimmte Instrumente anwenden). Die Unterstützung für alle anderen Erzeugnisse lag nach dem Brannan-Plan im Ermessen des Landwirtschaftsministers. Er sollte dabei die Angebotssituation im Verhältnis zur Nachfrage, die Verfügbarkeit von Finanzmitteln, die Verderblichkeit des Erzeugnisses und seine Bedeutung für die inländische Wirtschaft, die Fähigkeit, Vorräte zu veräußern, die Notwendigkeit vorübergehende Exportmarktverluste wettzumachen sowie die Fähigkeit und Bereitschaft der Erzeuger, ihre Produktion an die Nachfrage anzupassen, berücksichtigen (ibid.).

Der Brannan-Plan sah die Beschränkung der Preis- und Einkommensstützung auf die Größe eines typischen Familienbetriebes vor, definiert als Produktionsmenge bis 1.800 Einheiten. Mengen, die darüber hinausgingen, sollte keine Stützung mehr gewährt werden. Eine Einheit entsprach 10 Bushel Mais, etwa 8 Bushel Weizen oder etwa 50 Pfund Baumwolle. Somit konnte ein Betrieb, der nur Mais 
produzierte, für maximal 18.000 Bushel Unterstützung bekommen (ibid.: 487). Beim durchschnittlichen Maisertrag von 38,8 Bushel/Acre im Jahr 1949 entsprach dieses 464 Acres oder 187 Hektar. Im Fall von Weizen entsprachen 14.400 Bushel beim Durchschnittsertrag von 14,9 Bushel/Acre - 966 Acres oder 390 Hektar, im Fall der Baumwolle entsprachen 90.000 Pfund beim Durchschnittsertrag von 284 Pfund/Acre - 316 Acres oder 127 Hektar (eigene Berechnungen; Erträge nach USDA 1951: Tabelle 1, 38 und 82). Zum Vergleich: Die durchschnittliche Betriebsgröße lag 1950 bei 215 Acres oder 87 Hektar (errechnet nach USDA 1960: Tabelle 631).

Dieser erste breite Versuch eines Übergangs weg von Preisstützung hin zur Einkommensstützung, die über Geldzuwendungen erfolgen sollte, blieb letztendlich erfolglos. Laut Cochrane und Ryan (1976: 88f) scheiterte der Brannan-Plan insbesondere aus drei Gründen. Wie schon in den Kriegsjahren sprachen sich Landwirte gegen die Einführung der staatlichen Zahlungen aus. Der Berufsstand kritisierte, dass der Brannan-Plan zu einer „cheap food philosophy“ (Benedict 1953: 488) führen würde. Auch die Kappung der Agrarstützung stieß bei ihnen auf heftigen Widerstand. Gefürchtet wurden außerdem die mit den Zahlungen einhergehende Transparenz sowie die Notwendigkeit, jedes Mal, wenn ein Agrargesetz neu beschlossen wird (bis 1965 wurden Farm Bills auf jährlicher Basis verabschiedet), die Zahlungen vor Kongress und Öffentlichkeit rechtfertigen zu müssen. Die Subventionen sollten lieber versteckt bleiben, wie es bei der Preisstützung der Fall war. Zum Zweiten war der Einkommensstandard so hoch angesetzt, dass die hohen Umsetzungskosten des Brannan-Plans die Politik abschreckten. Im gewählten Referenzzeitraum 1939 bis 1948 erreichten die landwirtschaftlichen Einkommen in den USA ihren historischen Höchststand. Daraus würden sich ein hohes Beihilfeniveau und somit auch hohe Budgetausgaben ergeben. Die Kostenschätzungen reichten von $3 \mathrm{Mrd}$. bis zu $8 \mathrm{Mrd}$. USD pro Jahr. Schließlich fürchteten die oppositionellen Republikaner die Attraktivität der Idee einer hohen Agrarstützung bei gleichzeitig niedrigen Nahrungsmittelpreisen, weshalb sie den Plan mit ganzer Kraft bekämpften. Im Jahr 1954 führte der „National Wool Act““ „direct income payments " für Wollproduzenten ${ }^{29}$ ein, die dem Unterschied zwischen dem Erzeugerpreis und dem von der Regierung festgesetzten Niveau entsprachen - ein Instrument, das an die Zahlungen aus dem Brannan-Plan erinnert (Bowers et al. 1984). Flächendeckend wurden die Ideen aus diesem Plan jedoch erst 1973 mit den „deficiency payments“ umgesetzt.

Das Ergebnis war, dass Ende der 1940er-Jahre „[p]rice support coupled with some cursory attempts at supply control remained as the politically acceptable

\footnotetext{
${ }^{29}$ Die „direct income payments“ für Wolle wurden von 1955 bis 1972 jedes Jahr gewährt. Erst hohe Wollpreise im Jahr 1973 ermöglichten eine Aussetzung der Zahlungen bis 1977 (Cochrane und Ryan 1976). Das Programm wurde aufgrund hoher Haushaltskosten 1993 beendet, die letzten Zahlungen flossen an die Erzeuger 1995 (Orden et al. 1999).
} 
farm policy" (Cochrane und Ryan 1976: 29).30 Entsprechend wurden die Farm Bills in den Jahren 1948 und 1949 gestaltet: Der Fokus lag auf der Preisstützung, nicht auf den Agrarzahlungen. Die Überschusserzeugnisse konnten an Bedürftige oder in Schulen verteilt oder aber exportiert werden. Das prominente Beispiel ist das „Public Law 480“ von 1954, das die gesetzliche Grundlage für den Einsatz von Agrarüberschüssen im Rahmen der amerikanischen Entwicklungshilfe bildete („Food for Peace Program“). Flankiert wurden diese Maßnahmen von Zahlungen zur Honorierung von Umweltleistungen der Landwirte (Cochrane und Ryan 1976). Die Regierungspolitik zielte darauf ab, einen Absturz der Agrarpreise und -einkommen, wie es nach dem 1. Weltkrieg der Fall war, zu verhindern (Benedict 1953: 456).

Die Preisstützung wurde erst mit dem „Agricultural Act“ von 1954 flexibel gestaltet. Innerhalb einer festgelegten Bandbreite konnte das Preisstützungsniveau bei wachsenden Überschüssen auf 82,5\% der Parität (ab 1956 75\%) gesenkt werden. Allerdings bekam man damit die Überschüsse nicht in den Griff. Durch Innovationen und bessere Produktionsverfahren konnten Landwirte kräftige Produktivitätszuwächse erzielen. Da die Nutzung des technischen Fortschritts die Produktionskosten fallen ließ, war auch das neue Niveau der Preisstützung attraktiv, insbesondere für größere Betriebe (Cochrane und Ryan 1976). Beispielsweise wurde im Zeitraum von 1955 bis 1959 gegenüber 1950-1954 weiter durchschnittlich etwa ein Drittel der US-Weizenerzeugung mit „loans“ unterstützt. Bei Mais stieg dieser Anteil von durchschnittlich knapp 9\% auf 13,5\% der Erzeugung, bei Baumwolle von $16 \%$ auf $48 \%$ (eigene Berechnungen nach USDA 1963: Tabelle 16, 51 und 105). Im Juli 1960 betrugen die außerhalb der Agrarbetriebe gelagerten Bestände von Weizen 1,3 Mrd. Bushel, von Mais 1,2 Mrd. Bushel und von Baumwolle 1,7 Mio. Ballen (USDA 1963: Tabelle 10, 43 und 105). Zum Vergleich: 1960 betrug die Erzeugung von Weizen 1,4 Mrd. Bushel, Mais 4,3 Mrd. Bushel und Baumwolle 14,3 Mio. Ballen (ibid.: Tabelle 1, 38 und 86). Nicht zuletzt führten die Zahlungen für ausgewählte Umweltleistungen im Rahmen des „Agricultural Conservation Program“ zur Verbesserung der Bodenqualität und somit auch zu Ertragssteigerungen. Für den bezuschussten Einsatz von Mineraldüngern und den Bau von Drainage- und Bewässerungssystemen wurden erhebliche Teile des Programmbudgets ausgegeben. Beispielsweise wurden im Jahr 1951 42\% bzw. 7\% des 257 Mio. USD schweren Budgets für diese Zwecke verwendet (PMA-USDA 1952: Tabelle 1).

Belief sich der Wert der aus der Preisstützung resultierenden Bestände von „Commodity Credit Corporation“ zum Ende des Jahres 1948 auf knapp 322 Mio.

\footnotetext{
30 Zum einen wurden die Instrumente zur Produktionsbeschränkung nicht rigoros verwendet (z. B. waren „allotments“ für Weizen von 1944 bis 1949 gar nicht verkündet). Zum anderen waren sie wenig effektiv, weil ihre Basis die Anbaufläche war. Dazu kommt, dass sie freiwillig waren. Ob sie eingehalten wurden, hing insbesondere von der Höhe der angebotenen „loan rates“ in Relation zu Marktpreisen ab (Cochrane und Ryan 1976).
} 
USD, lag er acht Jahre später schon bei 5,9 Mrd. USD (USDA 1949: Tabelle 751 und USDA 1958: Tabelle 739). Die großen Mengenüberschüsse führten Mitte der 1950er-Jahre dazu, dass zu einer Regelung ähnlich dem Bodenschutzprogramm der 1930er-Jahre gegriffen wurde. Wieder einmal diente die Förderung des Umweltschutzes, die über staatliche Zahlungen an Landwirte erfolgte, der Produktionsbeschränkung sowie Einkommensstützung. Die sogenannte „Soil Bank“ wurde durch den „Agricultural Act“ aus dem Jahr 1956 eingeführt. Dieses Programm hatte zwei Komponenten: ,acreage reserve“ und „conservation reserve“ (Cochrane und Ryan 1976: 257ff). Im Rahmen der ersten wurde den Landwirten die jährliche Reduzierung der Anbaufläche der Grunderzeugnisse - Weizen, Baumwolle, Mais, Tabak, Erdnüsse und Reis - vergütet. Die aus der Produktion genommene Fläche wurde stillgelegt. Als Entschädigung erhielten die teilnehmenden Landwirte „rental payments“, die mindestens den Nettoeinkommensverlust ausgleichen sollten, der dem Landwirt aufgrund der Stilllegung entstand. Die Höhe der Zahlungen variierte je nach Erzeugnis und Region. Im Jahr 1957 befanden sich etwa 21 Mio. Acres in ,acreage reserve“. Ein Jahr später wurde eine Kappung der Zahlungen von 3.000 USD pro Betrieb eingeführt. Das Programm wurde nach 1958 aufgrund der Unwirksamkeit, die Gesamterzeugung zu reduzieren, der hohen Budgetausgaben (614 Mio. USD 1957 und 696 Mio. USD 1958) und des Widerstands seitens der Öffentlichkeit, Landwirte für „doing nothing“ zu bezahlen, beendet (ibid.: 78).

Im Rahmen von „conservation reserve“ wiederum wurden Landwirte für eine langfristige Stilllegung der Ackerfläche und ihre Zuweisung für Umweltzwecke entschädigt. Dieses Programm war für alle Landwirte offen. Die Ackerfläche musste mindestens für drei Jahre und maximal für zehn bzw. 15 Jahre aus der Produktion genommen werden. Die Zahlungen umfassten ein jährliches „,rental payment" und ggf. eine Zahlung, die einen Teil der Kosten der Durchführung von umweltfreundlichen Maßnahmen auf der stillgelegten Fläche, wie die Aufforstung oder Anlage von Gründecken, deckte. Eine Kappung der Zahlungen auf 5.000 USD pro Betrieb wurde 1958 eingeführt. Aufgrund der niedrigen Höhe der Zahlungen war die Teilnahme am Programm schwach und die vorgesehenen Finanzmittel von 450 Mio. USD jährlich wurden nicht ausgeschöpft. Die Teilnahme verbesserte sich etwas ab 1959, als die „acreage reserve“ abgeschafft wurde. Den Höchststand erreichte „conservation reserve“ 1960 mit 28,7 Mio. Acres. Im Jahr 1961 wurde dieses Programm eingestellt (Cochrane und Ryan 1976).

Ähnlich wie in den 1940er-Jahren spielten auch in den 1950er-Jahren die staatlichen Zahlungen an Landwirte agrarpolitisch keine große Rolle. Ihr Anteil am landwirtschaftlichen Nettoeinkommen lag im Zeitraum von 1950 bis 1960 unter $10 \%$. Den höchsten Anteil erreichten sie mit über 9\% bzw. 8\% in den Jahren 1957 und 1958, bedingt durch die hohen Zahlungen im Rahmen von ,acreage reserve“ der „Soil Bank“ (vgl. Tabelle A1). 
Trotz der ergriffenen Maßnahmen blieben die aus der Preisstützung resultierenden Bestände von CCC auf hohem Niveau: Zwischen 1956 und 1965 überstieg ihr Wert in jedem der Jahre zum Jahresende 4 Mrd. USD, mit dem Spitzenwert von 7,5 Mrd. USD im Jahr 1959 (USDA 1958 bis 1966). Gleichzeitig lag das landwirtschaftliche Nettoeinkommen Anfang der 1960er-Jahre niedriger als eine Dekade früher (vgl. Tabelle A1). Wie Rucker und Pasour (2007: 471) bemerken, wurde deutlich, dass die angestrebte Lösung des Einkommensproblems in der Landwirtschaft nicht durch die alten Instrumente der Preisstützung und der Produktionsbeschränkung herbeigeführt werden konnte. Angesichts dessen wurde im März 1961 mit dem „Feed Grain Program“ ein Notprogramm für Futtergetreide verabschiedet. Maiserzeuger konnten nur dann von der Preisstützung profitieren („loan rate" wurde von 1,06 auf 1,2 USD/Bushel erhöht), wenn sie mindestens $20 \%$ und maximal 40\% ihrer durchschnittlichen Maisanbaufläche von 1959-1960 für Umweltzwecke - hauptsächlich Stilllegung - auswiesen (diese Kopplung mit der Preisstützung fehlte bei der „Soil Bank“). Als zusätzlicher Anreiz für die Teilnahme an dem Programm wurden sogenannte ,acreage diversion payments“ eingeführt. Bei einer Flächenkürzung von $20 \%$ wurden Zahlungen für $50 \%$, bei höheren Kürzungen für $60 \%$ der normalen Produktion auf der stillgelegten Fläche gewährt (für die Berechnung der normalen Produktion wurden individuelle Durchschnittserträge der Jahre 1959 und 1960 herangezogen). Die Teilnehmer konnten zwischen Barzahlungen und „payments-in-kind“ in Form von sich in staatlicher Lagerhaltung befindendem Mais wählen (Cochrane und Ryan 1976).

Ein ähnliches Programm wurde für Sorghum und Gerste aufgestellt. Im Jahr 1962 wurde der Anbau von Früchten, die nicht zu Überschusserzeugnissen gehörten, auf der stillgelegten Fläche erlaubt, allerdings wurden in diesem Fall „acreage diversion payments“ dem Landwirt gekürzt. Ein Stilllegungsprogramm wurde mit dem „Agricultural Act“ vom August 1961 auch für Weizen aufgelegt. Die Weizenerzeuger mussten 1962 ihre ,allotments“ um einen festgesetzten Prozentsatz kürzen, um von der Preisstützung (2 USD/Bushel gegenüber 1,8 USD/Bushel im Vorjahr) und ,acreage diversion payments “ für $45 \%$ der normalen Produktion auf der stillgelegten Fläche zu profitieren. Für größere - freiwillige - Kürzungen der Weizenanbaufläche waren höhere „acreage diversion payments“ (60\% der normalen Produktion) vorgesehen. Für die Berechnung der normalen Produktion wurden individuelle historische Durchschnittserträge herangezogen (ibid.).

\subsection{Abkehr von der Preisstützung: Ausgleichszahlungen}

Eine große Neuerung kam mit dem „Food and Agriculture Act“ vom September 1962. Er senkte das Preisstützungsniveau für Futtergetreide ab 1963 auf etwa Weltmarktpreisniveau. Der Unterschied zwischen der alten (bei Mais 1,2 USD/ Bushel) und der neuen „loan rate“ (1,07 USD/Bushel - etwa die Höhe von 1960) sollte durch eine neue Art von Zahlungen, sogenannte „price support payments“, 
ausgeglichen werden. Für Mais lag ihre Höhe im Jahr 1963 bei 0,18 USD/Bushel, gewährt für die normale Produktion eines Landwirts ${ }^{31}$ (jeweilige Maisanbaufläche multipliziert mit dem Durchschnitt der Erträge von 1959 und 1960). Die neuen Zahlungen hatten eine zweifache Funktion: In erster Linie entschädigten sie die Erzeuger für den Rückgang der Stützpreise (,loan rates“). Darüber hinaus verstärkten sie den Anreiz, Fläche stillzulegen. Denn nur Landwirte, die einen Teil ihrer Maisanbaufläche stilllegten (mindestens $20 \%$ und höchstens $40 \%$ der Maisanbaufläche im Basiszeitraum 1959-1960, „base acreage“32 genannt), konnten von der Preisstützung in Form von „,nonrecourse loans“ und den neuen Zahlungen profitieren. „Acreage diversion payments“ wurden parallel weiter gezahlt. Je mehr Fläche der Landwirt über die Mindestvorgabe von 20\% hinaus stilllegte, desto mehr „diversion payments“, aber weniger „price support payments“ erhielt er (und umgekehrt). Dieses entfiel im Jahr 1966, als die „price support payments“ auf die Erzeugung auf $50 \%$ der „,base acreage“ beschränkt wurden. Das Programm wurde später mehrmals modifiziert. Insbesondere sollten ab 1971 „price support payments" die Differenz zwischen dem durchschnittlichen Marktpreis und dem Stützpreis (für Mais 1,35 USD/Bushel oder $70 \%$ der Parität) ausgleichen. Außerdem wurden die für die Berechnung der Zahlungen relevanten Erträge regelmäßig aktualisiert (Cochrane und Ryan 1976). Nach ähnlichem Muster wurde im „Food and Agriculture Act" von 1962 auch das Programm für Weizen konzipiert. Die Höhe der „loan rate“ wurde für 1963 von 2 USD/Bushel auf 1,82 USD/Bushel (Höhe von 1961) gesenkt. Von den „,nonrecourse loans“ konnten nur Landwirte, die ihre ,allotment" einhielten, profitieren. Für Landwirte, die einen Teil der „allotment" stilllegten, waren zwei Arten von Zahlungen vorgesehen. Zum einen waren das „price support payments“, die bei 0,18 USD/Bushel für die normale Produktion (aktuelle Anbaufläche multipliziert mit dem Durchschnitt der Erträge von 1959 und 1960) festgesetzt wurden; zum anderen „diversion payments“ für einen Teil der normalen Produktion auf der stillgelegten Fläche (ibid.).

Als der Versuch scheiterte, für Weizen ein verpflichtendes Programm zur Angebotssteuerung zu etablieren (es wurde im Mai 1963 in einem Referendum durch die Landwirte abgelehnt ${ }^{33}$ ) wurden mit dem „Agricultural Act“ vom April 1964 wichtige Änderungen im Weizenprogramm vorgenommen. Die „loan rate“ wurde ab 1964 weiter gesenkt (auf 1,3 USD/Bushel, was etwa dem Niveau des Welt-

\footnotetext{
31 „Nonrecourse loans“ wurden dem Landwirt für seine gesamte Produktion eines Erzeugnisses gewährt (Cochrane und Ryan 1976).

32 „Allotments“ wurden für Mais mit der Wirkung ab 1959 aufgehoben (Cochrane und Ryan 1976).

33 Die von der Kennedy-Regierung konzipierte langfristige Angebotssteuerung wäre für die Branche verpflichtend gewesen. Danach hätten die Erzeugerorganisationen das Angebot für einzelne Erzeugnisse selbst managen müssen, um Preissteigerungen zu erzielen. Geplant für alle wichtigsten Erzeugnisse, wurde das Programm in einer abgeänderten Form am Ende nur für Weizen angeboten und im Referendum 1963 von den Landwirten abgelehnt (Cochrane und Ryan 1976).
} 
marktpreises entsprach). Die „price support payments“ wurden durch sogenannte Vermarktungszertifikate ersetzt, dessen Wert davon abhing, ob Weizen für den Verbrauch im Inland oder im Ausland bestimmt war. Ihr Ziel war es auch, die Absenkung des Stützpreises auszugleichen. „Domestic certificate payments“ wurden für den Teil der Weizenerzeugung eines Landwirts, der für die Deckung des Inlandsverbrauchs vorgesehen war, gezahlt (im Jahr 1964 0,7 USD/Bushel für $45 \%$ der Erzeugung auf der „allotment“; es wurde der Durchschnittsertrag von 1958-1962 herangezogen). Entsprechend wurden „export certificate payments“ für den Teil der individuellen Weizenerzeugung gewährt, der exportiert werden sollte (im Jahr 1964 0,25 USD/Bushel für 45\% der Erzeugung auf der ,allotment"). Ihre Finanzierungsquelle war unterschiedlich: „Domestic certificate payments“ waren durch eine Verarbeitungssteuer, „export certificate payments“ aus dem Staatshaushalt finanziert. Um von den Zahlungen profitieren zu können, mussten Landwirte ihre vorab um $10 \%$ gekürzten „allotments“ einhalten. Darüber hinaus wurden weiter ,acreage diversion payments“ gewährt (ibid.).

In den folgenden Jahren wurde das Weizenprogramm immer wieder modifiziert. Ab 1965 war die Stilllegung erforderlich, auch um sich für „nonrecourse loans“ zu qualifizieren. Ab 1966 wurden „export certificates payments“ abgeschafft bei gleichzeitiger Erhöhung des Wertes der „domestic certificate payments“, die für $45 \%$ der Erzeugung auf der ,allotment“ zu zahlen waren. Der neue Wert sollte den Unterschied zwischen dem Stützpreis („loan rate“) und $100 \%$ der Parität am 1. Juli eines jeweiligen Jahres ausgleichen. Ab 1971 wurde er als Unterschied zwischen dem durchschnittlichen Marktpreis und 100\% der Parität berechnet. Die Ausgaben für „domestic certificate payments“ wurden zum Teil aus dem Staatshaushalt, zum Teil über eine Verarbeitungsabgabe finanziert. Ein ähnliches Programm wurde ab 1966 auch für Baumwolle aufgestellt (ibid.). Darüber hinaus wurde 1964 das „Dairy Indemnity Payment Program“ für Milch aufgestellt, im dessen Rahmen Zahlungen für die Milcherzeuger vorgesehen wurden, die ihre Milch aufgrund des Vorliegens von chemischen Rückständen vom Markt nehmen mussten (Bowers et al. 1984). Ab 1965 konnten Erzeuger, die sowohl eine „base acreage“ für Futtergetreide als auch eine ,allotment" für Weizen besaBen, Weizen durch Futtergetreide und umgekehrt substituieren, ohne die Zahlungen zu verlieren. Ab 1971 war dieses auch für Erzeuger möglich, die entweder nur eine „base acreage“ für Futtergetreide oder eine „allotment“ für Weizen besaßen. Ab 1972 konnten sie auf dieser Fläche auch Soja anbauen. Grundsätzlich musste der Landwirt das für die Zahlungen relevante Erzeugnis aber weiter anbauen, ansonsten wäre seine „base acreage“ (Mais) bzw. „allotment“ (Weizen und Baumwolle) um $20 \%$ gekürzt worden. Sie ging komplett verloren, wenn dort in drei aufeinander folgenden Jahren das Erzeugnis oder seine zugelassenen Alternativen nicht angebaut wurden (Cochrane und Ryan 1976). 
Tabelle 1: Höhe der Stützung für Weizen und Mais in den USA 1954-1966

\begin{tabular}{|c|c|c|c|c|c|c|c|c|}
\hline & 1954 & 1960 & 1961 & 1962 & 1963 & 1964 & 1965 & 1966 \\
\hline $\begin{array}{l}\text { Weizen } \\
\text { USD/bu. }\end{array}$ & 2,24 & 1,78 & 1,79 & 2,0 & 2,0 & 2,00 & 2,00 & 2,57 \\
\hline $\begin{array}{l}\text { davon „loan } \\
\text { rate“ }\end{array}$ & 2,24 & 1,78 & 1,79 & 2,0 & 1,82 & 1,30 & 1,25 & 1,25 \\
\hline $\begin{array}{l}\text { Mais } \\
\text { USD/bu. }\end{array}$ & 1,62 & 1,06 & 1,20 & 1,20 & 1,25 & 1,25 & 1,25 & 1,30 \\
\hline $\begin{array}{l}\text { davon „loan } \\
\text { rate“ }\end{array}$ & 1,62 & 1,06 & 1,20 & 1,20 & 1,07 & 1,10 & 1,05 & 1,00 \\
\hline
\end{tabular}

Quelle: USDA (1972: Tabelle 675).

Durch die schrittweise Absenkung der Stützpreise in Form von „loan rates“ (vgl. Tabelle 1) und die Einführung von „price support payments“ sollten die Nahrungsmittelpreise für die Verbraucher sinken, ohne dass dieses einen negativen Einfluss auf das Einkommensniveau der Landwirte hatte. Entsprechend wuchs die Bedeutung der staatlichen Zahlungen für das landwirtschaftliche Nettoeinkommen. Ihr Anteil daran betrug in den Jahren von 1961 bis 1965 jeweils über 10\%, in den Jahren von 1966 bis 1970 sogar über 20\% (vgl. Tabelle A1). Dabei blieb die Gesamthöhe der „conservation payments“ mit knapp über 200 Mio. USD konstant (vgl. Tabelle A2). Laut Orden et al. (1999: 66) war dieser partielle „Cash out“34 bei Exporterzeugnissen - weg von den Preisstützungsinstrumenten der „New Deal“Zeit und hin zu einer durch produktionsbeschränkende Maßnahmen flankierten Einkommensstützung - die einzige Reformstrategie, die zu jener Zeit politisch möglich und haltbar war. Eine Bestätigung für die neue Richtung in der USAgrarpolitik lieferte die „Food and Fiber Commission“ (vgl. Box 6).

Box 6: Bericht der „Food and Fiber Commission“ zur US-Agrarpolitik von 1967

Die „Food and Fiber Commission“ wurde einberufen, um die aktuelle Agrarund Handelspolitik in Bezug auf nationale Interessen sowie das Wohl von ländlicher Bevölkerung, Landwirten, Arbeitern und Verbrauchern zu bewerten. In ihrem Bericht „Food and Fiber for the Future“ von 1967 kam die Kommission zu dem Ergebnis, dass es Zeit für einen wesentlichen Wechsel in der US-Agrar-

\footnotetext{
34 Orden et al. (1999) analysieren die US-Agrarpolitik anhand von vier Reformstrategien. Staatliche Eingriffe können entweder schnell oder langsam und entweder mit oder ohne Entschädigung abgebaut werden. Eine langsame Transformation, begleitet durch Entschädigungen, nennen sie „cash out“. Eine schnellere Transformation mit Entschädigungen ist „buyout“. Ein langsamer, unentschädigter Abbau ist „squeeze out“, ein schneller, unentschädigter Abbau ist „cut out“"
} 
politik in Richtung Marktorientierung sei. Das Ziel sollte sein, die Einkommen der Landwirte langfristig zu verbessern. Dabei sollte die staatliche Unterstützung für die Landwirtschaft so konzipiert werden, dass sich eine marktorientierte Landwirtschaft entwickelt. Dazu empfahl die Kommission die Höhe der Preisstützung unter dem Weltmarktpreisniveau festzulegen. Als besonders geeignet, die Agrareinkommen zu verbessern wurde das Instrument der Agrarzahlungen („deficiency payments“) identifiziert. Es wurde den anderen Instrumenten (hohe Preisstützung, Exportsubventionen und Importquoten) vorgezogen. Die Zahlungen sollten dann angewandt werden, wenn die Einnahmen aus Verkäufen am Markt den Landwirten keine Paritätseinkommen sichern würden (Cochrane und Ryan 1976). Eine Minderheit der Kommissionsmitglieder war hingegen der Meinung, dass „,only market forces should determine prices“ und ,incomes could be protected by supplemental government payments similar to unemployment compensation, if farmers experienced a temporary income deficiency“(ibid.: 53).

Im Jahr 1970 wurde eine Reihe von Neuerungen der bestehenden Programme eingeführt. Ab 1971 war die Einhaltung von „,allotments“ keine Voraussetzung mehr für den Erhalt der Stützung. „Allotments“ dienten aber weiterhin als Basis für die Berechnung der Zahlungen und der Fläche, die stillgelegt werden sollte. „Acreage diversion“ wurde für die Anbauer der relevanten Erzeugnisse Weizen, Futtergetreide und Baumwolle durch ein neues Instrument der Produktionsbeschränkung - den sogenannten „set-aside“ - ergänzt. Der Unterschied bestand darin, dass unter „set-aside“ keine Reduzierung der Anbaufläche eines bestimmten Erzeugnisses verpflichtend war. Beispielsweise konnte der Landwirt - sobald er einen Teil seiner Gesamtanbaufläche, der dem für das bestimmte Jahr vorgeschriebenen Prozentsatz seiner ,allotment“ für Weizen entsprach (z. B. 130\%), stilllegte - auf der Restfläche alle Kulturen anbauen, inklusive Weizen. Somit erhielt der Landwirt mehr Flexibilität in seinen Anbauentscheidungen. Das ,setaside“-Programm war mit der Teilnahme an den Preis- und Einkommensstützungsprogrammen verknüpft. Als Gegenleistung erhielten die Landwirte „setaside payments“; eine Zusatzzahlung war vorgesehen, wenn die stillgelegte Fläche der Öffentlichkeit für Freizeitzwecke zugänglich war (Cochrane und Ryan 1976).

Eine weitere Neuerung aus dem Jahr 1970 war die Kappung der Zahlungen an landwirtschaftliche Erzeuger. Bereits der „Agricultural Act“ von 1961 hatte die Förderung und Stärkung des Familienbetriebes zum Ziel. Übermäßige Zahlungen pro Betrieb wurden von Kritikern als eine nicht vertretbare Einkommensstützung angesehen. Darüber hinaus würde ihre Kürzung zur angestrebten Kostenreduzierung beitragen. Es wurde allerdings auch argumentiert, dass für eine wirksame Produktionsbeschränkung die Teilnahme größerer Betriebe unabdingbar war. Eine Kappung würde den Anreiz für sie mindern, an den Anpassungsprogrammen zu partizipieren. Laut der Kompromisslösung von 1970 konnte ab 1971 jeder Er- 
zeuger von Weizen, Baumwolle oder Futtergetreide nicht mehr als 55.000 USD unter jedem einzelnen kulturspezifischen Programm erhalten. Dieses bezog sich auf „price-support payments“, „, set-aside payments“, „,diversion payments“ sowie „certificate payments“. Das Volumen der im Rahmen der „loan rates“-Regelung gewährten Subventionen war weiterhin nicht begrenzt (ibid.).

Institutionalisiert wurde die neue Art der Agrarzahlungen im Jahr 1973 - genau 40 Jahre nach der Einführung der preisstützenden „loan rates"-Regelung. „Deficiency payments“ genannt, sollten die Zahlungen immer dann gewährt werden, wenn der Weltmarktpreis unter ein bestimmtes Niveau - den Richtpreis fiel. ${ }^{35}$ Dieses Konzept war eine Weiterentwicklung der Idee, die Landwirte für die Kürzung der Stützpreise zu entschädigen und galt ab 1974 für Weizen, Baumwolle und Futtergetreide, ab 1976 auch für Reis. Der Beihilfesatz entsprach etwa der Differenz zwischen dem Richtpreis und der „loan rate“ oder dem Marktpreis, je nachdem, was höher war. Darüber hinaus sah die Farm Bill von 1973 die Einführung von „disaster payments“ im Falle natürlicher Katastrophen vor (Bowers et al. 1984). Somit wurden den Landwirten nicht nur die aufgrund der Preis-, sondern auch die aufgrund der Mengenschwankungen erlittenen Einkommenseinbußen ausgeglichen.

Am Anfang waren „allotments“, die auf der historischen Erzeugung basierten, die Grundlage für die Berechnung der „deficiency payments“. Sie wurden also unabhängig davon gezahlt, wie groß die aktuelle Anbaufläche für das bestimmte, der „deficiency payments“-Regelung unterliegende Erzeugnis eines Landwirts war. Bei Überschreitung der ,allotment“ erhielt der Landwirt denselben Betrag an „deficiency payments“, den er bei ihrer Einhaltung bekommen hätte. Wenn er die „allotment" unterschritt, erhielt er auch für den Teil der ,allotment", auf dem das bestimmten Erzeugnis nicht angebaut wurde, „deficiency payments“, allerdings unter Kürzung des Zahlungssatzes. Die Grundlage für die Berechnung von „deficiency payments“ war ab 1978 die aktuelle produktbezogene Anbaufläche eines Landwirts, die mittels eines Zuweisungskoeffizienten ${ }^{36}$ angepasst wurde. Diese Zahlungen mussten aber für mindestens $80 \%$ der betreffenden, aktuellen Anbaufläche eines Landwirts gezahlt werden. Allerdings durfte in den Jahren, in denen „set-aside“ galt, die darunter stillgelegte Fläche und die aktuelle Anbaufläche für die relevanten Erzeugnisse die sogenannte „normal crop acreage“ eines Betriebes nicht überschreiten. Für 1978 war das die vorjährige Anbaufläche für die betref-

\footnotetext{
35 Wenn der nationale Durchschnittspreis für ein Erzeugnis während der ersten fünf Monate des Vermarktungsjahres niedriger lag als der für dieses Jahr festgesetzte Richtpreis (Glaser 1986).

${ }^{36}$ Für Weizen, Futtergetreide (Mais) und Baumwolle legte der Landwirtschaftsminister ab 1978 statt „allotments“ die sogenannte „,national program acreage“ fest als eine Anbaufläche, die nötig war, um im nächsten Jahr den Bedarf an einem Erzeugnis für Inlandsverbrauch, Nettoexporte und den gewünschten Aufbau bzw. Abbau der Lagerbestände zu decken. Der Zuweisungskoeffizient ergab sich aus der Teilung der „,national program acreage“ durch die prognostizierte Anbaufläche in dem jeweiligen Jahr (Johnson und Ericksen 1977).
} 
fenden Erzeugnisse (Johnson und Ericksen 1977). Mit der Farm Bill von 1977 ersetzte die aktuelle Anbaufläche die historische ,allotment" auch als Grundlage für die Festlegung von „set-aside“. Bei „set-aside“ konnte der Landwirt frei entscheiden, was er auf der Restfläche anbaute. Insbesondere konnte er die Anbaufläche für das bestimmte Erzeugnis im Vergleich zum Vorjahr erhöhen, ohne die Zahlungen zu verlieren, solange er die Vorgaben zu „set-aside“ und „normal crop acreage“ erfüllte (ibid.: 15). Das einzelbetriebliche Ziel, „deficiency payments“ zu maximieren, konnte in dieser Konstellation viel flexibler als früher erreicht werden. So regten die neuen Regeln die Landwirte an, die Intensität zu erhöhen bzw. neue Flächen in die Produktion zu nehmen. Insbesondere konnten Landwirte ihre beihilfefähige Fläche erhöhen, indem sie für ein Jahr aus dem Stützungsprogramm ausschieden und das beihilfefähige Erzeugnis auf zusätzlicher Fläche anbauten.

Da sich somit der Bedarf für „deficiency payments“ erhöhte, wurde $1981 \mathrm{zu}$ einem neuen Flächenstilllegungsprogramm „Acreage Reserve Program“ (ARP) gegriffen, um die Produktionsüberschüsse und Haushaltsausgaben zu reduzieren. Die Teilnahme am ARP-Programm war freiwillig und unentgeltlich in dem Sinne, dass dafür - im Gegensatz zu dem somit abgeschafften „set-aside“-Programm keine spezifischen Zahlungen gewährt wurden. Dabei ging den Landwirten ihre erst 1970 gewonnene Entscheidungsfreiheit bezüglich der Restfläche verloren, weil die im Rahmen von ARP stillzulegende Fläche je Erzeugnis bestimmt wurde (als Prozentsatz der auf der Vorjahres-Anbaufläche eines Erzeugnisses beruhenden Basisanbaufläche, genannt „crop acreage base“). Dank dieses Instruments konnte die Anbaufläche eines Betriebes für „deficiency payments“-fähige Erzeugnisse relativ zum Vorjahr gekürzt werden. Sofern es in einem Jahr beschlossen wurde, war die Teilnahme der Landwirte am ARP-Programm Voraussetzung für den Erhalt der Preisstützung und der „deficiency payments“ (Orden et al. 1999). Zusätzlich war „paid land diversion“ - ebenfalls produktbezogen - möglich (Harwood und Young 1989). Die Obergrenze für die Zahlungen an Getreide- und Baumwollerzeuger wurde 1973 von 55.000 USD auf 20.000 USD pro Person gesenkt, bezogen allerdings nur auf „deficiency payments“. Mit der Farm Bill von 1985 wurde die Kappung bei „deficiency payments“ und „diversion payments“ auf 50.000 USD pro Person festgelegt (Ingersent und Rayner 1999).

Während im Zeitraum von 1966 bis 1972 der Anteil der staatlichen Zahlungen am landwirtschaftlichen Nettoeinkommen bei über $20 \%$ lag, betrug er im Jahr 1974 nur noch knapp 2\%. Die Zahlungen fielen auf 530 Mio. USD (36\% davon entfielen auf „,conservation payments") - den niedrigsten Stand seit 1956. Denn in den Jahren 1974 bis 1976 mussten aufgrund von hohen Marktpreisen weder „deficiency payments“ noch „set-aside payments“ greifen. Gleichzeitig erreichten die „conservation payments“ (ausgedrückt in Preisen von 2009) ihr bisheriges Tief seit der Einführung im Jahr 1936 (vgl. Tabelle A3). Ab 1977 lagen die staatlichen 
Zahlungen aber wieder deutlich bei über 1 Milliarde USD (s. Tabelle A1 und Tabelle A2). ${ }^{37}$

Das „deficiency payments“-System bedeutete die Abkehr von der reinen Preisstützungspolitik der letzten 40 Jahre, die die Verbraucher mit höheren Lebensmittelpreisen belastete und zur Verteuerung der US-Agrarerzeugung auf den Auslandsmärkten führte. Entworfen in Zeiten hoher Marktpreise erwies sich das neue System mit der Zeit als sehr kostenintensiv. Anfang der 1980er-Jahre stürzte der US-Agrarsektor in die schlimmste Krise seit der „Great Depression“ der 1930erJahre. Ein starker Dollar führte zu stark rückläufigen Exporten; die Marktpreise fielen. Als Ergebnis schossen die Ausgaben für die staatlichen Zahlungen in die Höhe (vgl. Tabelle A1), wodurch wiederum Druck entstand, Änderungen in der Stützungsregelung vorzunehmen. Allerdings erfüllte die neue Farm Bill von 1985, der „Food Security Act“, die in sie gesetzten Erwartungen nicht (Orden et al. 1999).

Unter anderem führte sie eine neue Zahlung ein, indem sie die „,nonrecourse loan“-Regelung um Vorschriften zu „marketing assistance loans“ ergänzte (am Anfang verpflichtend nur für Hochland-Baumwolle und Reis). Wenn der Marktpreis unter der „loan rate“ lag, konnte der Landwirt das Darlehen innerhalb von neun Monaten zum (niedrigeren) Marktpreis statt zur ursprünglichen „loan rate“ zurückzahlen. Die Differenz zwischen dem Marktpreis und der „loan rate“ entsprach einer Beihilfe (,marketing loan gain“). Die Ernte durfte auf dem Markt auch zu einem späteren Zeitpunkt als die Rückzahlung des Darlehens - verkauft werden. Um die Ausgaben für die öffentliche Lagerhaltung zu reduzieren, wurde das Ersetzen der Darlehen bzw. „marketing loan gains" durch Zahlungen vorgesehen: Bei Marktpreisen niedriger als die „loan rates“ konnten die Landwirte, die auf ihren Anspruch, Darlehen aufzunehmen, verzichtet hatten, sogenannte „loan deficiency payments" beantragen. Sie glichen die Differenz zwischen dem Marktpreis und der „loan rate“ aus (und entsprachen somit der „marketing loan gain“). Diese Differenz multipliziert mit der Erzeugung, für die das Darlehen beantragt werden konnte, ergab den Betrag von „loan deficiency payments“ eines Landwirts (Glaser 1986). Im Gegensatz zu den klassischen „,nonrecourse loans“, wo Erzeugnisse - um ihre Preise zu stützen - durch die öffentliche Hand vom Markt genommen wurden, war das Ziel der „marketing loan“-Regelung nicht die Preise,

\footnotetext{
${ }^{37}$ Im Juli und August 1972 exportierten die USA rund 440 Mio. Bushel Weizen an die von einer Dürre geplagte Sowjetunion. Die verkaufte Menge entsprach 30\% der jährlichen durchschnittlichen Weizenerzeugung der USA der letzten fünf Jahre. Deshalb - und zusätzlich gestützt durch das aufgrund ungünstigerer Witterungsverhältnisse knappere Angebot weltweit - schossen die Getreidepreise und anschließend auch die Preise für Soja und Vieh in die Höhe, was zu höheren Lebensmittelpreisen führte. In dieser Situation animierte der damalige Landwirtschaftsminister Earl Butz die Landwirte, die Anbaufläche zu vergrößern (,plant fence row to fence row“, zitiert nach Wyant 2008: 1), was zu Lasten der umweltschonend bewirtschafteten Fläche ging. Mit diesem als „Great Grain Robbery" bekannten Ereignis rückte die Agrarpolitik, insbesondere das Exportsubventionsprogramm, ins Blickfeld der breiten Öffentlichkeit (Luttrell 1973).
} 
sondern das Einkommen der Landwirte bei niedrigen Marktpreisen zu stützen. Wie Westcott und Price (2001: 1) bemerken: „[L]oan programs have effectively moved from providing price support to providing income support without supporting market prices.“ Darüber hinaus wurde der Landwirtschaftsminister 1985 ermächtigt, „loan rates“ für Weizen und Futtergetreide um maximal 20\% gegenüber dem Basissatz zu senken. In diesem Fall sollte den Erzeugern eine Zahlung in Höhe der Differenz zwischen dem Basissatz der „loan rate“ und der gekürzten „loan rate“ oder dem Marktpreis, je nachdem, was höher war, gewährt werden (sogenannte „Findley payments") (Glaser 1986).

Die Farm Bill von 1985 brachte eine Neuausrichtung der „conservation payments" mit sich. Es war die allererste Farm Bill, die einen gesonderten Teil zum Umweltschutz enthielt. Sie initiierte den Prozess, bei „conservation payments“ die Umweltziele in den Vordergrund zu stellen (Cain und Lovejoy 2004: 39). Diese gestiegene Relevanz der Umweltbelange spiegelte sich in der Steigerung der Gesamthöhe der „conservation payments“ seit dem Jahr 1987 wider (vgl. Tabelle A2). Im Jahr 1985 wurde auch ein neues - längerfristiges - Flächenstilllegungsprogramm namens „Conservation Reserve Program“ (CRP) eingeführt. Es sah Zahlungen an Landwirte vor, die sich bereit erklärten, Teile ihrer Fläche für zehn Jahre aus der Produktion zu nehmen und für Umweltzwecke auszuweisen. Von Umweltorganisationen begrüßt, war das CRP-Programm auch im Interesse der Landwirte, die sich dadurch eine Verringerung des Bedarfs für die unbezahlte Flächenstilllegung (ARP-Programm) versprachen. Gleichzeitig sollten Landwirte, die bestimmte Verpflichtungen in Bezug auf die Umwelt nicht einhielten, von der Agrarstützung ausgeschlossen werden („,conservation compliance“). Dazu zählte u. a., dass Landwirte bereits extensiv genutztes Land („sodbuster") bzw. Feuchtgebiete (,swampbuster") nicht in intensive Nutzung überführen konnten. Wie Orden et al. (1999: 77) feststellen, trug dieses Bündnis der Landwirte mit Umweltorganisationen dazu bei, dass die Agrarstützung auf hohem Niveau bleiben konnte.

Trotz der produktionsbeschränkenden Maßnahmen erreichten die staatlichen Zahlungen an Landwirte und ihr Anteil am landwirtschaftlichen Nettoeinkommen (er betrug im Jahr 1983 sogar über 65\%) Mitte der 1980er-Jahre Rekordhöhen (vgl. Tabelle A1). Das gilt auch für die Teilnahmerate am „deficiency payment“System (vgl. Tabelle 2). Dieses ist auf die durch die Krise bedingten hohen „deficiency payments“ und „set-aside payments“38 zurückzuführen. Der Gesamtbetrag von „,commodity payments“ erhöhte sich zwischen 1980 und 1983 um das Vierfache; zwischen 1985 und 1987 verdoppelte er sich und erreichte im Jahr 1987 mit 13,2 Mrd. USD das bisherige Rekordniveau. Auch in Preisen von 2009 ist dieses das höchste Niveau, das die „commodity payments“ in der Geschichte der USAgrarpolitik je erreichten. Die „conservation payments“ erhöhten sich ebenfalls,

\footnotetext{
38 Seinen höchsten Stand erreichte „set-aside“ im Jahr 1983, in dem 77,9 Mio. Acres aus der Produktion genommen wurden; im Jahr 1987 waren es 53,5 Mio. Acres. Zum Vergleich: Die Ackerfläche lag in der Zeit bei etwa 445 Mio. Acres (Orden et al. 1999: 59).
} 
bedingt durch die Einführung von „Conservation Reserve Program“, erheblich. Im Jahr 1986 nicht einmal bei 300 Mio. USD, erreichten sie ein Jahr später 1,5 Mrd. USD. Die höheren Marktpreise Ende des Jahrzehntes spiegelten sich in niedrigeren „commodity payments“ wider. „Conservation payments“ aber blieben weiter auf dem Niveau von etwa 2 Mrd. USD (vgl. Tabelle A2 und Tabelle A3).

Tabelle 2: „Deficiency payments“-System für Weizen in den USA 1978-1994

\begin{tabular}{lccccc} 
& $\begin{array}{c}\text { Richtpreis } \\
\text { USD/bu. }\end{array}$ & $\begin{array}{c}\text { "Loan rate“ } \\
\text { USD/bu. }\end{array}$ & $\begin{array}{c}\text { „Def. payment“ } \\
\text { USD/bu. }\end{array}$ & $\begin{array}{c}\text { „Acreage base“ } \\
\text { Mio. Acres }\end{array}$ & $\begin{array}{c}\text { Teilnahmerate } \\
\text { \% }\end{array}$ \\
\hline 1978 & 3,40 & 2,35 & 0,52 & 58,8 & 63 \\
1982 & 4,05 & 3,55 & 0,50 & 90,7 & 48 \\
\hline 1986 & 4,38 & 2,40 & 1,98 & 91,6 & 85 \\
1990 & 4,00 & 1,95 & 1,28 & 80,5 & 83 \\
1994 & 4,00 & 2,58 & 0,61 & 78,2 & 87 \\
\hline
\end{tabular}

Quelle: FSA-USDA (2010).

Anmerkung: Die Teilnahmerate entspricht der Anbaufläche der teilnehmenden Betriebe als Prozentsatz der Gesamtanbaufläche.

Mit der Farm Bill von 1985 wurden erste Schritte unternommen, die Zahlungen von der aktuellen Erzeugung zu entkoppeln. Erträge, die als Grundlage für die Berechnung der Erzeugung dienten, für die „deficiency payments“ zu gewähren waren, wurden „eingefroren“ (im Zeitraum von 1986 bis 1987 entsprachen sie dem individuellen Durchschnitt der Erträge der Jahre 1981 bis 1985 unter Ausschluss des höchsten und des niedrigsten Wertes). Produktionszuwächse konnten also nicht mehr zur Erhöhung des individuellen Betrags an „deficiency payments“ führen. Die Farm Bill von 1985 änderte auch die Methode, nach der die Basisanbaufläche (,crop acreage base“) festgelegt wurde. Ab nun entsprach sie dem gleitenden Durchschnitt der individuellen produktspezifischen Anbaufläche der letzten fünf Jahre sowie der Fläche, die während der letzten fünf Jahre als bepflanzt betrachtet wurde (u. a. die unter den staatlichen Programmen stillgelegte Fläche). „Deficiency payments“ wurden grundsätzlich für die aktuelle Anbaufläche gewährt, die die genehmigte Anbaufläche eines Landwirts (,permitted acreage") nicht überschreiten durfte. Die genehmigte Anbaufläche wiederum entsprach der Basisanbaufläche für ein Erzeugnis abzüglich der unter staatlichen Programmen stillgelegten Fläche im gegebenen Jahr. Damit wurde die Ausweitung der Basisanbaufläche erschwert: Stieg der Landwirt aus dem Stützungsprogramm für ein Jahr aus, konnte er seine Basisanbaufläche lediglich um $20 \%$ der zusätzlich in diesem Jahr angebauten Fläche erweitern. Außerdem wurde eine begrenzte Produktionsflexibilität eingeführt. Die Getreideerzeuger erhielten die Möglichkeit, 92\% der „deficiency payments“ für ihre genehmigte Anbaufläche zu bekommen, auch 
wenn sie nur auf 50 bis $92 \%$ dieser Fläche das beihilfefähige Erzeugnis anbauten, vorausgesetzt, dass sie die Restfläche für Umweltzwecke auswiesen (Harwood und Young 1989).

Mit der Farm Bill von 1990 wurde ab 1991 die sogenannte „triple base“-Regel eingeführt, die die Basisanbaufläche („,crop acreage base“) eines Landwirts aufteilte in:

- Fläche, die unter ARP für Umweltzwecke ausgewiesen werden musste, wie vom Landwirtschaftsminister jährlich festgelegt. Diese Fläche wurde von „deficiency payments“ und anderer Stützung ausgeschlossen.

- Fläche, auf der beliebige Erzeugnisse, außer Obst und Gemüse, angebaut werden konnten („flexible acreage") und die von vornherein von „deficiency payments“ ausgeschlossen wurde. Sie wurde auf $15 \%$ der Basisanbaufläche festgesetzt. Zusätzlich konnten Landwirte weitere $10 \%$ der Basisanbaufläche als ,optional flexible acreage“ ausweisen. Dieses hatte keine negativen Auswirkungen auf die Größe seiner Basisanbaufläche. Eine Unterstützung im Rahmen der „nonrecourse loan“- und „,marketing loan“-Regelungen war für die Erzeugung auf „flexible acreage“ möglich, falls die beihilfefähigen Erzeugnisse angebaut wurden.

- Restanbaufläche („permitted acreage"), die der Landwirt nur mit der Kultur, für die „deficiency payments“ vorgesehen waren, bepflanzen konnte. Die Erzeugung auf dieser Fläche profitierte sowohl von „deficiency payments“ als auch von der Preisstützung („nonrecourse loans“ und „marketing loans“) (Ingersent und Rayner 1999).

Mithilfe der „triple base“-Regel wurde die Fläche, für die „deficiency payments“ $\mathrm{zu}$ zahlen waren, um mindestens $15 \%$ reduziert. Auch wenn die stillzulegende Fläche bei null lag, wurden „deficiency payments“ für maximal 85\% der Basisanbaufläche gewährt. Gleichzeitig erhielten die Landwirte mehr Flexibilität bei den Produktionsentscheidungen. Sie konnten die Erzeugung auf „flexible acreage“ am Markt - und nicht mehr an erzielbaren Zahlungen - ausrichten (Pollack und Lynch 1991). In der ersten Hälfte der 1990er-Jahre herrschten hohe Agrarpreise, die 1995 und 1996 einen Rekordstand erreichten. Entsprechend sanken die Zahlungen und ihr Anteil am landwirtschaftlichen Nettoeinkommen (vgl. Tabelle A1).

Orden et al. (1999: 166) beklagen einen „conflicting mix of incentives“, der die US-Agrarpolitik dieser Zeit auszeichnete und zu Marktverzerrungen führte. „Deficiency payments" schufen Anreize zur Überschussproduktion und verfälschten den Wert landwirtschaftlicher Grundstücke, insbesondere in den Regionen, wo die Zahlungen einen großen Anteil am landwirtschaftlichen Nettoeinkommen ausmachten. Gleichzeitig war das Angebot durch Flächenstilllegungsprogramme und durch geringe Flexibilität bei den Produktionsentscheidungen beschränkt. In die- 
sem System musste der Staat sich selbst permanent überbieten (ibid.: 78f). Die Beihilfen für die auf der genehmigten Anbaufläche angebauten Erzeugnisse mussten hoch genug sein, um die Erzeuger zur Teilnahme am jährlichen unentgeltlichen ARP zu bewegen. Hohe „deficiency payments“ führten aber zur erhöhten Anbauintensität, was wiederum zu Forderungen nach einem bezahlten Flächenstilllegungsprogramm für Umweltzwecke führte. Da das CRP-Programm auch freiwillig war, musste die Zahlung pro Acre höher als Alternativeinnahmen liegen, „deficiency payments" eingeschlossen.

\subsection{Einführung der Direktzahlungen}

Weitere substanzielle Fortschritte bei der Entkopplung der Zahlungen von der Erzeugung brachte der weithin beachtete „Federal Agriculture Improvement and Reform Act" von 1996 („FAIR Act") mit sich. Die größte Neuerung betraf die sogenannten Vertragserzeugnisse Weizen, Mais, Sorghum, Gerste, Hafer, Baumwolle und Reis. Erzeuger dieser Produkte, die an den Stützungsprogrammen im Zeitraum von 1991 bis 1995 teilgenommen hatten, konnten sogenannte „production flexibility contracts“ (PFC) für sieben Jahre abschließen. Mit diesen erhielten sie die Berechtigung zu fixierten, jährlichen Pauschalzahlungen ,production flexibility contract (PFC) payments", die von den zukünftig tatsächlich erzeugten Mengen und Preisen entkoppelt wurden (auch „AMTA payments“ genannt nach „Agricultural Market Transition Act“- dem Titel I des „FAIR Act"). Sie ersetzten die von Marktpreisen abhängigen „deficiency payments“, die zusammen mit den Richtpreisen abgeschafft wurden (Ingersent und Rayner 1999).

Die Grundlage für die Berechnung der neuen Zahlungen bildeten $85 \%$ der Vertragsanbaufläche eines Landwirts ${ }^{39}$ multipliziert mit dem individuellen, auf historischen Werten basierenden, „eingefrorenen“ Ertrag. Die „PFC payments“ eines Betriebes für ein Erzeugnis ergaben sich aus der Multiplikation dieser „payment quantity“ mit dem vom Gesetzgeber fixierten einheitlichen Zahlungssatz per Bushel (Young und Shields 1996). Die Gesamthöhe der neuen Zahlungen wurde bis 2002 auf 35,6 Mrd. USD festgesetzt, davon 5,6 Mrd. USD im ersten Anwendungsjahr 1996 und 4 Mrd. USD im letzten Jahr 2002 (ibid.: 1). Entsprechend sollte auch der Zahlungssatz pro Bushel mit der Zeit sinken (vgl. Tabelle 3). Die Höchstgrenze für die „PFC payments“ belief sich auf 40.000 USD pro Person. Allerdings konnte eine Person unter der sogenannten „three entity“-Regel „PFC payments“ bis zu 80.000 USD für drei verschiedene Betriebseinheiten erhalten. Analog konnten die auf 75.000 USD limitierten Zahlungen aus „marketing loans“ unter der „three entity“-Regel 150.000 USD pro Person erreichen (Young und

\footnotetext{
39 Die Vertragsanbaufläche umfasste die Basisanbaufläche für ein Erzeugnis von 1996, errechnet nach den Regeln der Farm Bill von 1990, zuzüglich rückkehrender CRP-Fläche und abzüglich neuer, unter CRP für Umweltzwecke ausgewiesenen Fläche (Young und Shields 1996).
} 
Shields 1996). Mit dem Agrargesetz von 1996 erhielten die Landwirte weitgehende Freiheit bei den Produktionsentscheidungen. Insbesondere wurde das jährliche „Acreage Reduction Program“ abgeschafft (das CRP-Programm wurde beibehalten). Die Landwirte konnten auf der Vertragsanbaufläche und der Restfläche verschiedene Kulturen anbauen (mit Beschränkungen für Obst und Gemüse) oder diese Fläche stilllegen, ohne dabei die Zahlungen zu verlieren. Sie mussten sich allerdings verpflichten, die Umweltvorschriften in Bezug auf Feuchtgebiete und Bodenschutz einzuhalten (Cross-Compliance). Außerdem mussten sie das Land weiterhin landwirtschaftlich nutzen, eine Erzeugung war aber nicht notwendig (Young und Shields 1996).

Laut Orden et al. (1999: 166ff) gehört die Abschaffung von „deficiency payments“, die Beendigung der produktionsbeschränkenden Maßnahmen sowie die Entkopplung der Stützung von den Produktionsentscheidungen, Preisen und Erzeugung zu den wichtigsten Errungenschaften des „FAIR Act“. Letzteres würde die Position der USA in den künftigen internationalen Handelsverhandlungen verbessern. ${ }^{40}$ Ein weiterer positiver Punkt war die Kappung der nominalen „loan rates“. Darüber hinaus brachte die Farm Bill von 1996 mit „PFC payments“ eine klare und transparente Einkommensstützung mit sich und mehr Budgetsicherheit als schwer zu prognostizierende - weil von Marktpreisen abhängige - „deficiency payments“. Harvey (1998: 14) sieht die Zusage, die Rolle des Staates im Agrarbereich durch eine Kommission überprüfen und bewerten zu lassen, als weiteren Vorteil an (vgl. Box 7).

Trotz dieser wichtigen Erfolge hatte der „FAIR Act“ auch seine negativen Seiten (Orden et al. 1999: 168ff). Die Reform von 1996 beschränkte sich auf ausgewählte Exporterzeugnisse. Insbesondere wurde keine ähnliche Deregulierung für Zucker, Milch und Milchprodukte, Erdnüsse und Tabak beschlossen. Die „marketing loan“-Regelung wurde beibehalten und die Exportpolitik nur moderat geändert. Die Voraussetzungen für den Erhalt von „PFC payments“ waren im Vergleich zu diesen von „deficiency payments“ leicht zu erfüllen. Als Ergebnis erreichte die Teilnahmerate bei dem neuen Einkommensstützungsprogramm fast $100 \%$ (vgl. Tabelle 3). Da die Zahlungen von der historischen Anbaufläche abhingen, konzentrierten sie sich auf Großbetriebe. Schließlich war die neue Regelung aufgrund der in der zweiten Hälfte der 1990er-Jahre herrschenden hohen Marktpreise am Ende in der Umsetzung teurer als es eine Fortführung der Farm Bill von 1990 mit „deficiency payments“ gewesen wäre. Für Ingersent und Rayner (1999: 401) spiegelt diese gemischte Bewertung des „FAIR Act“ den politischen Kompromiss wider. Die Farm Bill von 1996 ,,appears to be a compromise measure giving something to both the advocates of radical agricultural policy reform and the defenders of status quo" (ibid.: 403).

\footnotetext{
40 Die USA benötigten dank der Entkopplung von der aktuellen und zukünftigen Erzeugung keine Blue Box mehr, die in der Uruguay-Runde des GATT entstanden war und die „direct payments“ im Rahmen von produktionsbeschränkenden Maßnahmen umfasst.
} 
Tabelle 3: „PFC payments“-System für Weizen in den USA 1996-2002

\begin{tabular}{|c|c|c|c|c|c|}
\hline & $\begin{array}{r}\text { Richtpreis } \\
\text { USD/bu. }\end{array}$ & $\begin{array}{c}\text { „Loan rate“ } \\
\text { USD/bu. }\end{array}$ & $\begin{array}{r}\text { „PFC payment } \\
\text { USD/bu. }\end{array}$ & $\begin{array}{r}\text { „Acreage base“ } \\
\text { Mio. Acres }\end{array}$ & $\begin{array}{r}\text { Teilnahmerate } \\
\%\end{array}$ \\
\hline 1996 & - & 2,58 & 0,87 & 76,7 & 99 \\
\hline 1997 & - & 2,58 & 0,63 & 76,7 & 99 \\
\hline 1998 & - & 2,58 & 0,66 & 78,9 & 99 \\
\hline 1999 & - & 2,58 & 0,64 & 79,0 & 99 \\
\hline 2000 & - & 2,58 & 0,59 & 78,9 & 99 \\
\hline 2001 & - & 2,58 & 0,59 & 78,5 & 99 \\
\hline 2002 & - & 2,80 & 0,59 & 76,2 & 98 \\
\hline
\end{tabular}

Quelle: FSA-USDA (2010).

Anmerkung: Die Teilnahmerate entspricht der Anbaufläche der teilnehmenden Betriebe als Prozentsatz der Gesamtanbaufläche.

\subsection{Teilweise Abkehr vom Entkopplungskurs}

Nach der Verabschiedung des „FAIR Act“ stellte sich die Frage nach der Nachhaltigkeit der in der Agrarpolitik vorgenommenen Änderungen (Harvey 1998). Die Farm Bill von 1996 ließ die Zukunft von „PFC payments“ nach 2002 offen. Auch hob sie die unbefristete Gesetzgebung von 1938 und 1949 nicht auf und somit war die Rückkehr zu den alten Stützungsprogrammen weiter möglich.

Bereits für das Vermarktungsjahr 1998 wurden sogenannte „market loss assistance (MLA) payments“ beschlossen, um den Landwirten die Verluste aufgrund sinkender Marktpreise auszugleichen. Die neuen Zahlungen sollten bis zum Vermarktungsjahr 2001 für Weizen, Futtergetreide, Baumwolle und Reis proportional zu „PFC payments“ gewährt werden. Ihre Gesamthöhe wurde vom Gesetzgeber festgelegt. ${ }^{41}$ Zusätzlich wurden sie für Ölsaaten gewährt. Von „MLA payments“ profitierten 1999 bis 2001 auch Milchproduzenten; die Preisstützung für Milch, deren Abschaffung Ende 1999 durch den „FAIR Act“ vorgesehen war, wurde verlängert (Young 2002). Im Jahr 2000 erreichten die staatlichen Zahlungen an landwirtschaftliche Erzeuger mit insgesamt 23,2 Mrd. USD (28,3 Mrd. USD in Preisen von 2009) einen neuen Rekord, der später nur noch im Jahr 2005 überboten wurde (s. Tabelle A2 und Tabelle A3).

${ }^{41}$ Der Gesamtbetrag belief sich auf 2,9 Mrd. USD im Jahr 1998, 5,5 Mrd. USD 1999, 5,5 Mrd. USD 2000 und 4,6 Mrd. USD 2001 (jeweils Vermarktungsjahr) (Young 2002: o.S.). 
Box 7: Bericht der „Commission on $21^{\text {st }}$ Century Production Agriculture“ zur US-Agrarpolitik von 2001

Im Januar 2001 - im Vorfeld der Debatte über eine neue Farm Bill - veröffentlichte die unter dem „FAIR Act“ zum Skizzieren der zukünftigen Agrarpolitik einberufene Kommission (die sogenannte „Commission on 21 ${ }^{\text {st }}$ Century Production Agriculture“) ihren Endbericht „Directions for Future Farm Policy: The Role of Government in Support of Production Agriculture“. Darin sprach sie sich für die Schaffung eines effektiven und adäquaten Sicherheitsnetzes für landwirtschaftliche Einkommen aus. Die Kommission empfahl ein duales System der Zahlungen: „PFC payments“ aus dem „FAIR Act“ mit dem Budget von etwa 4 Mrd. USD pro Jahr und ein zusätzliches „Supplemental Income Support“-Programm. Letzteres sollte immer dann aktiviert werden, wenn die Bruttoeinnahmen aus den Programmerzeugnissen (Weizen, Mais, Soja, Sorghum, Reis, Baumwolle, Hafer und Gerste) auf nationaler oder regionaler Ebene unter ein bestimmtes Niveau fallen. Wie „PFC payments“ sollten die neuen Zahlungen von aktuellen Marktpreisen und Erzeugung entkoppelt sein. Zusätzlich sollte die „marketing loan“-Regelung, inklusive der „loan deficiency payments“ beibehalten werden (Young und Effland 2001).

Die Befürworter einer stärkeren Marktorientierung in der Agrarpolitik waren in der Kommission in der Minderheit. Sie sprachen sich gegen die Einführung von „counter-cyclical income payments“ in jeglicher Form (weil sie in landwirtschaftlichen Boden- und Pachtpreisen kapitalisiert würden) und Flächenstilllegungen aus. Gleichzeitig forderten sie die Abschaffung von „PFC payments“, insbesondere wenn „,counter-cyclical payments“ beschlossen würden. Die damit frei gewordenen Finanzmittel sollten statt für einkommensstützende Zahlungen für zwei Arten der Programme bereitgestellt werden. Mit dem ersten Programm sollten größere, für den Markt produzierende Betriebe insbesondere im Risikomanagement (z. B. über Versicherungsprogramme) unterstützt werden. Die Förderung kleiner Betriebe wiederum sollte ihren Schwerpunkt auf die ländliche Entwicklung setzen sowie auf die Beratung bei der Einführung neuer Technologien mit dem Ziel größerer Skaleneffekte. Die Stützung sollte außerdem stärker an Umweltziele gebunden werden (Effland und Young 2001).

Die wesentlichen Grundregeln der US-Agrarpolitik Anfang der 2000er-Jahre definierte der „Farm Security and Rural Investment Act“ von 2002. Dieser sah die Einkommensstützung in Form verschiedener Zahlungen an Landwirte vor. Dazu zählten „direct payments“, die die „PFC payments“ ersetzten, sowie „counter- 
cyclical payments“ und die Zahlungen aus der „,marketing loans“-Regelung. 42 Des Weiteren führte er „,incentive payments“ für den Anbau von Hartweizen ein. Diese Zahlungen sollten von 2003 bis 2005 in der Gesamthöhe von 20 Mio. USD für maximal 2 Mio. Acres gewährt werden (Young 2002). „Direct payments“ (auch „fixed direct payments“ genannt) wurden - wie „PFC payments“ - für Erzeuger von Weizen, Mais, Gerste, Sorghum, Hafer, Baumwolle, Reis und - neu - Ölsaaten sowie Erdnüssen (sogenannte „,covered commodities“) auf Basis jährlicher Verträge und unabhängig von den Marktentwicklungen geleistet. Der Zahlungssatz wurde produktbezogen in der Farm Bill festgelegt und betrug beispielswiese für Weizen 0,52 USD/Bushel (vgl. Tabelle 4), für Mais 0,28 USD/Bushel und für Baumwolle 0,0667 USD/Pfund (die Zahlungssätze für 2002 - das letzte Jahr der „PFC payments“ - sollten entsprechend angepasst werden) (Young 2002).

Tabelle 4: „Direct payments“-System für Weizen in den USA 2003-2008

\begin{tabular}{rrrrrr} 
& $\begin{array}{r}\text { Richtpreis } \\
\text { USD } \text { bu. }\end{array}$ & $\begin{array}{r}\text { „Loan rate“ } \\
\text { USD/bu. }\end{array}$ & $\begin{array}{r}\text { \#irect payment“ } \\
\text { USD/bu. }\end{array}$ & $\begin{array}{r}\text { „Acreage base“ } \\
\text { Mio. Acres }\end{array}$ & $\begin{array}{r}\text { Teilnahmerate } \\
\text { \% }\end{array}$ \\
\hline 2003 & 3,86 & 2,80 & 0,52 & 76,2 & 98 \\
\hline 2004 & 3,92 & 2,75 & 0,52 & 76,0 & 98 \\
\hline 2005 & 3,92 & 2,75 & 0,52 & 75,4 & 98 \\
2006 & 3,92 & 2,75 & 0,52 & 74,8 & 97 \\
2007 & 3,92 & 2,75 & 0,52 & 74,8 & 97 \\
2008 & 3,92 & 2,75 & 0,52 & 74,8 & 96 \\
\hline
\end{tabular}

Quelle: FSA-USDA (2010).

Anmerkung: Die Teilnahmerate entspricht der Anbaufläche der teilnehmenden Betriebe als Prozentsatz der Gesamtanbaufläche.

Mit den neuen „,counter-cyclical payments“ wurden „MLA payments“ institutionalisiert. Sie galten für dieselben Erzeugnisse wie „direct payments“ und sollten erfolgen, sobald der effektive Preis unter ein bestimmtes Niveau, den Richtpreis, fällt. Ihr Ziel war es also, Landwirte vor niedrigen Marktpreisen zu schützen. Der effektive Preis ergab sich aus der Summe folgender Werte: der Durchschnittspreis des Erzeugnisses im Vermarktungsjahr oder seine „loan rate“, je nachdem, was höher lag, sowie der „direct payment“-Zahlungssatz für dieses bestimmte Erzeugnis. Der Richtpreis wurde direkt in der Farm Bill festgelegt und belief sich für 2003 beispielsweise für Weizen auf 3,86 USD/Bushel, Mais auf 2,60 USD/Bushel

\footnotetext{
42 Ab dem Jahr 2000 wurde die Rückzahlung von „,marketing loans“ in Form von „,commodity certificates", die die Erzeuger beim USDA kaufen konnten, möglich gemacht. Der Vorteil dieser Lösung für die Landwirte lag darin, dass im Falle der Nutzung von „commodity certificates“ die Höchstgrenzen für die Zahlungen aus „marketing loans“ nicht griffen (Monke 2004).
} 
und Baumwolle auf 0,724 USD/Pfund (ibid.). Von der Wirkungsweise waren diese teilweise entkoppelten Zahlungen weitgehend vergleichbar mit „deficiency payments" (die Bindung an die Teilnahme an Flächenstilllegungen wurde allerdings aufgehoben). Entsprechend wurden sie der WTO als nicht-produktspezifische Amber-Box-Beihilfen notifiziert (Schnepf 2014c: 14).

Für die Berechnung der beiden Zahlungstypen „direct payments“ und „counter-cyclical payments" wurden die historische Basisanbaufläche (85\% davon) und Erträge herangezogen. Das betroffene Produkt musste auf dieser Fläche jedoch nicht mehr angebaut werden. Landwirte hatten die Wahl, entweder ihre Basisanbaufläche aus dem „FAIR Act“ zu aktualisieren (die durchschnittliche Anbaufläche 1981 bis 1985 würde durch die durchschnittliche Anbaufläche 1998 bis 2001 ersetzt) oder sie zu belassen, ggf. ergänzt um die entsprechende Basisanbaufläche für Ölsaaten. Allerdings konnte die Basisanbaufläche die aktuelle Anbaufläche nicht überschreiten. Die Erträge, die bereits auf dem Niveau von 1995 eingefroren waren, wurden für „direct payments“ nicht verändert. Nur für „counter-cyclical payments" konnten sie aktualisiert werden. Beide Zahlungstypen waren an die Einhaltung von Umweltschutz-Anforderungen (,sodbuster" und „swampbuster") gebunden. Die „marketing loan“-Regelung, inklusive der „loan deficiency payments", wurde auf Erdnüsse, Wolle, Mohair, Honig, Kichererbsen, Linsen und trockene Erbsen ausgedehnt. Die Höchstdauer des Darlehens betrug neun Monate (Young 2002).

Die Höchstgrenze für „direct payments“ pro Person wurde bei 40.000 USD beibehalten. Die Höchstgrenze für „,counter-cyclical payments“ wurde auf 65.000 USD und für die Zahlungen unter der „marketing loan“-Regelung auf 75.000 USD pro Person festgesetzt. Erzeuger mit Bruttoeinnahmen (,adjusted gross income") von mehr als 2,5 Mio. USD als dreijährigem Durchschnitt wurden von den Zahlungen ausgeschlossen, es sei denn, dass mehr als $75 \%$ dieser Einnahmen aus landwirtschaftlichen Tätigkeiten stammte (ibid.).

Auch „MLA payments“ für Milch von 1999 bis 2001 wurden durch die Farm Bill von 2002 institutionalisiert. Im Rahmen des neu aufgestellten „Dairy Market Loss Payments Program“ wurden Milcherzeugern monatliche Zahlungen gewährt, wenn der Marktpreis unter ein in der Farm Bill festgesetztes Niveau (16,94 USD/ cw $\left.{ }^{43}\right)$ fiel. Die Zahlung entsprach $45 \%$ der Differenz zwischen dieser Schwelle und dem Marktpreis und wurde für die vermarktete Milcherzeugung eines Landwirts gewährt (bis zur Obergrenze von 2,4 Mio. Pfund Milch im Jahr). Auch rückwirkende Zahlungen für die Verluste, die Milcherzeuger aufgrund niedriger Preise seit dem 1. Dezember 2001 erlitten, wurden vorgesehen (ibid.). Das Instrument der staatlichen Zahlungen wurde im Milchbereich zwar bereits in den 1980erJahren genutzt, allerdings als Entschädigung für Produktionskürzung bzw. -einstellung („Milk Diversion Program“ und „Milk Production Termination Pro-

${ }^{43}$ Die US-Maßeinheit „short hundredweight“ (100 Pfund) entspricht 45,35 Kilo. 
gram“). Das Ziel dieser freiwilligen Programme war - ähnlich wie bei den Flächenstilllegungsprogrammen für die Feldkulturen - die Vermeidung von Überschüssen und die Reduzierung der Ausgaben für Preisstützungsprogramme (Glaser 1986).

Von den sechs Grunderzeugnissen des "Agricultural Adjustment Act" von 1938 war damit für Weizen, Mais, Baumwolle und Reis die Abkehr von den ursprünglichen Regelungen hin zu größerer Marktorientierung über den oben beschriebenen Weg, begonnen mit der Einführung von „price support payments“ in den 1960er-Jahren, erfolgt. Im Jahr 2002 wurde das System der zu der Zeit geltenden Agrarzahlungen auch auf Erdnüsse ausgedehnt. „Direct payments“ und „counter-cyclical payments" sollten den Landwirten, die in der Vergangenheit Erdnüsse angebaut hatten, auf Grundlage der individuellen Durchschnittserträge der Jahre 1998 bis 2001 und 85\% der Durchschnittsanbaufläche in denselben Jahren (Basisanbaufläche) gewährt werden; „marketing loans“ sollten allen Erdnüsse anbauenden Landwirten zur Verfügung stehen. Gleichzeitig wurde die Quote für Erdnüsse, die ihre Wurzeln in den 1930er-Jahren hatte, abgeschafft. Ihre Inhaber erhielten für den Wertverlust ihrer Quote einen Ausgleich in Form einer separaten Zahlung, die in den Jahren 2002 bis 2006 in fünf Tranchen erfolgte und sich auf 0,11 USD/Pfund der im Jahr 2001 zugewiesenen Quote belief (,peanuts buyout"). Auf eigenen Wunsch konnten die betroffenen Erzeuger den Ausgleich in Form einer einmaligen Zahlung erhalten (Young 2002). Der Gesamtbetrag der Zahlungen an Erdnussquoteninhaber betrug insgesamt 1,3 Mrd. USD und wurde aus dem Staatshaushalt finanziert (Dohlman et al. 2009: 6).

Die Stützungsregelung für das sechste Grunderzeugnis Tabak wurde 2004 separat mit dem „Fair and Equitable Tobacco Reform Act“ reformiert („tobacco buyout $^{\prime \prime}$ ). Nicht nur den Quoteninhabern, wie bei Erdnüssen, sondern auch den aktiven Erzeugern von Quotentabak wurden sogenannte "transition payments“ für zehn Jahre (2005 bis 2014) gewährt. Sie wurden ausdrücklich als „full and fair consideration for the termination of such tobacco marketing quotas and related price support" konzipiert (Fair and Equitable Tobacco Reform Act 2004: Sec. 622). Dass der Ausgleich als vollständig beschrieben wurde, implizierte, dass nach 2014 keine weiteren Zahlungen gewährt werden würden. Dieses war auch der Fall. Die „transition payments“ wurden durch eine bei Herstellern und Importeuren von Tabakprodukten erhobene Abgabe finanziert. Die Zahlung für Quoteninhaber belief sich auf 0,7 USD pro Pfund der individuellen sogenannten Basisquote im Jahr. Für Erzeuger von Quotentabak war eine zusätzliche Zahlung von 0,3 USD je Pfund und Jahr vorgesehen. Die Zahlung konnte in zehn jährlichen Tranchen oder einmalig entrichtet werden. Insgesamt erhielten Tabakquoteninhaber und Tabakerzeuger Zahlungen im Wert von 9,6 Mrd. USD. Im Gegensatz zu den Erdnussanbauern wurden die Tabakerzeuger nicht in das System der zur Zeit des „,buyout“ gültigen Agrarzahlungen (,direct payments“, „, counter-cyclical payments" und „marketing loans") einbezogen (Dohlman et al. 2009: 18). 
Die nächste Farm Bill - der „Food, Conservation and Energy Act“ von 2008 behielt die einkommensstützenden Zahlungen „direct payments“, „,counter-cyclical payments“ und „marketing loans" der vorausgegangenen Farm Bill mit einigen Modifikationen bei. Unter anderem wurden die Richtpreise und „loan rates“ geändert. Ab dem Jahr 2009 wurden „,counter-cyclical payments“ auf trockene Erbsen, Linsen und Kichererbsen sowie „marketing loans“ auf Kichererbsen ausgedehnt. Betriebe mit weniger als 10 Acres (4 Hektar) Basisanbaufläche für alle relevanten Kulturen wurden mit einigen Ausnahmen von "commodity payments“ ausgeschlossen. Darüber hinaus wurde ein Pilotprojekt initiiert, im Rahmen dessen auf der Basisanbaufläche der Anbau von ausgewählten Gemüsesorten zur Verarbeitung erlaubt wurde (allerdings nur auf insgesamt 75.000 Acres). 83,3\%und nicht mehr $85 \%$ - der Basisanbaufläche waren Grundlage für die Berechnung von „direct payments“. Auch die einkommensstützenden, im Falle niedriger Marktpreise zu gewährenden Zahlungen für Milcherzeuger wurden mit einigen Änderungen beibehalten („milk income loss contract payments“) (Johnson 2008).

Nicht nur, dass die bisherigen Agrarzahlungen weitergeführt wurden: Die Farm Bill von 2008 sah für die Erzeuger von Weizen, Futtergetreide, Baumwolle, Soja und Erdnüsse ab 2009 einen weiteren Zahlungstyp vor - sogenannte ,average crop revenue election payments" im Rahmen eines neuen Programms zur Einkommensstützung „Average Crop Revenue Election“ (ACRE). Das Programm sorgte dafür, dass die Begünstigten einen garantierten Erlös gegenüber einer Referenzperiode erzielen konnten (Erlös wurde als das Produkt aus Preis und Flächenertrag definiert). Im Gegenzug mussten sie aber Abschläge bei den anderen Subventionen hinnehmen (Kürzung der „direct payments“ um 20\% sowie der „loan rates“ um $30 \%$ und Verzicht auf die „counter-cyclical payments") und durften bis zum Ablauf der Farm Bill von 2008 das Programm nicht verlassen. Einmal gewählt, umfasste das ACRE-Programm alle relevanten Erzeugnisse eines Landwirts, die Zahlungen wurden aber produktspezifisch gewährt (ibid.). Insbesondere der nationale Verband der Maisanbauer („National Corn Growers Association“) machte sich im Laufe der Debatte über die Ausgestaltung der neuen Farm Bill für ein neues Instrument stark, das auf Erlösänderungen basieren sollte (Woolverton und Young 2009: 4).

Die teilnehmenden Betriebe sollten Anspruch auf die Zahlungen bekommen, wenn zwei Auslöseschwellen erreicht wurden: Wenn der tatsächliche Erlös pro Acre auf Bundesstaatsebene für ein beihilfefähiges Erzeugnis geringer ausfällt als die sogenannte Erlösgarantie auf gleicher Ebene und wenn der tatsächliche Erlös eines Landwirts unter seinen Benchmarkerlös fällt. Die Erlösgarantie auf Bundesstaatsebene belief sich auf $90 \%$ des Durchschnitts der nationalen produktspezifischen Marktpreise der letzten zwei Jahre multipliziert mit dem „olympischen“ Durchschnitt der Erträge in dem betreffenden Bundestaat in den letzten fünf Jahren. Sie konnte sich von Jahr zu Jahr um maximal $10 \%$ ändern. Der tatsächliche Erlös auf Bundesstaatsebene wiederum entsprach dem Ertrag in dem betreffen- 
den Bundesstaat und Erntejahr multipliziert mit dem Durchschnitt der nationalen Marktpreise im Vermarktungsjahr oder mit $70 \%$ der „loan rate“, je nachdem, was höher lag. „ACRE payment“ pro Acre entsprach der Differenz zwischen der Erlösgarantie und dem tatsächlichen Erlös auf Bundesstaatsebene, durfte $25 \%$ der Erlösgarantie allerdings nicht überschreiten. Auch musste der Erzeuger Verluste vorweisen, das heißt, sein tatsächlicher Erlös musste unter seinem Benchmarkerlös (der Durchschnitt der nationalen Marktpreise der letzten zwei Jahre multipliziert mit dem „olympischen“ Durchschnitt der individuellen Erträge der letzten fünf Jahre zuzüglich der Ernteversicherungsprämie je Acre) liegen. Der Zahlungsbetrag eines Erzeugers errechnete sich aus der Multiplikation des „ACRE payment" mit dem Verhältnis zwischen dem „olympischen“ Durchschnitt seiner Erträge in den letzten fünf Jahren und dem „olympischen“ Durchschnitt der Erträge in dem betreffenden Bundestaat in demselben Zeitraum. Dieses wurde anschlieBend mit 83,3\% der Anbaufläche multipliziert ( $85 \%$ ab 2012); allerdings konnte die Fläche, für die „ACRE payments“ gezahlt wurden, die Basisanbaufläche eines Landwirts nicht übertreffen (Johnson 2008).

Angeboten als Alternative zum Bezug der „counter-cyclical payments“ wurden „ACRE payments“ nicht durch sinkende Marktpreise für erzeugte Produkte, sondern durch sinkende Erlöse der Erzeuger ausgelöst. Damit sollte eine Übersubventionierung verhindert werden, wenn die Preise zwar niedrig ausfallen, aber durch die hohen Erträge überkompensiert werden (Zulauf und Orden 2009: 1). Eine solche Situation mit niedrigen Marktpreisen und hohen Erträgen entstand z. B. 2004 und 2005. Die in diesen zwei Jahren getätigten „,counter-cyclical payments" sowie die Zahlungen aus „marketing loans" betrugen für Mais insgesamt 12,8 Mrd. USD. Zulauf und Orden (2009: 17f) schätzten die „ACRE payments“ 2004-2005 aufgrund von hohen Erträgen auf nur 0,5 Mrd. USD, wären sie zu dieser Zeit bereits in Kraft gewesen. Beachtenswert ist, dass bei „ACRE payments“im Gegensatz zu den traditionellen antizyklischen Agrarzahlungen „countercyclical payments“ und „marketing loans“ - keine feste Untergrenze vorgesehen war: Die Erlösgarantie schwankte je nach historischen Markterlösen. Wie Zulauf und Orden (2009: 1) weiter feststellen, würden somit die Zahlungen aus dem ACRE-Programm in Zeiten der über mehrere Jahre herrschenden niedrigen Marktpreise weniger Unterstützung als die traditionellen Agrarzahlungen sichern. Auf der anderen Seite bedeuteten hohe Preise und daraus resultierende höhere Markterlöse eine höhere ACRE-Erlösgarantie. „ACRE payments“ müssten bei Unterschreitung dieser hoch angesetzten Schwelle gewährt werden. Für das Auslösen der traditionellen antizyklischen Agrarzahlungen hingegen müssten die Marktpreise tiefer sinken. „ACRE payments“ wurden unter der Amber Box der WTO als produktspezifische Beihilfen notifiziert (Schnepf 2014c: 14).

Die „three entity“-Regel wurde durch die Farm Bill von 2008 abgeschafft. Die Obergrenze für die Zahlungen aus „marketing loans“ in Höhe von 75.000 USD pro Person wurde ebenfalls beendet. Die Höchstbeträge pro Person sowie die 
Obergrenzen der anrechenbaren Bruttoeinnahmen für den Anspruch auf „direct payments“ und „counter-cyclical payments“ wurden hingegen beibehalten. Für die am ACRE-Programm teilnehmenden Landwirte wurde der Höchstbetrag für „,direct payments“ um $20 \%$ gekürzt. Für „ACRE payments“ selbst sollte die Obergrenze für „counter-cyclical payments“ gelten (65.000 USD pro Person), zuzüglich des Betrags, um den der Höchstbetrag für „direct payments“ reduziert wurde (8.000 USD) (Johnson 2008).

\subsection{Abschaffung der Direktzahlungen}

Die Farm Bill von 2014 gestaltete das System der Agrarzahlungen um. Sie schaffte „fixed direct payments“, „counter-cyclical payments" und „ACRE payments“ ab (Shields 2014a). Allerdings waren die Veränderungen nur auf den ersten Blick gravierend. Denn in Wirklichkeit wurden lediglich die „fixed direct payments“ endgültig beendet, während die Landwirte immer noch die Wahl zwischen einem preis- und einem erlösbasierten Absicherungsprogramm haben. Im Rahmen des ersten Programms werden „price loss coverage (PLC) payments“ gewährt, die „counter-cyclical payments“ ersetzten. Die Alternative - das Programm mit ,agriculture risk coverage (ARC) payments“ - ersetzte „ACRE payments“. Die neuen Zahlungen sind an die laufende Produktion nicht gebunden (weder an die aktuelle Anbaufläche noch an die aktuellen Erträge) und können somit als teilweise entkoppelt angesehen werden. ${ }^{44}$ Es herrscht noch Unklarheit diesbezüglich, wie sie der WTO notifiziert werden. Schnepf (2015: 31) geht davon aus, dass diese Zahlungen als Amber-Box-Stützungen gemeldet werden (analog zu „counter-cyclical payments"). Eine Alternative stelle die Blue Box dar (im Rahmen der DohaRunde wurde die Umschichtung der „counter-cyclical payments“ von der Amber Box in die Blue Box diskutiert). Im Vergleich zu ihren Vorgängern gewähren die neuen Programme laut Shields (2014a: 6) einen höheren Schutz vor niedrigen Marktpreisen bzw. Umsatzverlusten durch höhere Referenzpreise und Erlösgarantien. Darüber hinaus behielt die neueste Farm Bill die Zahlungen unter der „marketing loan“-Regelung bei. Im Gegensatz zu „price loss coverage payments“ und „agriculture risk coverage payments“ stehen sie allen Erzeugern beihilfefähiger Produkte zur Verfügung und nicht nur denen, die das Produkt in der Vergangenheit anbauten und somit über die entsprechende Basisanbaufläche verfügen. Sie werden unter der Amber Box als produktspezifische Beihilfen notifiziert (Schnepf 2014c: 14).

\footnotetext{
${ }^{44}$ Der Entwurf des Repräsentantenhauses wollte als Grundlage für „PLC payments“ die aktuelle Anbaufläche etablieren. Dieser Punkt entwickelte sich zu einem der größten Streitpunkte in der Debatte zur Ausgestaltung der Farm Bill 2014. Im Fall von „ARC payments“ wollten ursprünglich sowohl der Senat als auch das Repräsentantenhaus die aktuelle Anbaufläche als die Basis für ihre Berechnung etablieren (Chite 2014: 7).
} 
Zu den beihilfefähigen Erzeugnissen unter den PLC- und ARC-Programmen gehören Weizen, Hafer, Gerste, Mais, Sorghum, mittel- und langkörniger Reis, trockene Erbsen, Linsen, Kichererbsen, Soja und andere Ölsaaten sowie Erdnüsse (Hochland-Baumwolle ist in Reaktion auf das verlorene WTO-Panel kein beihilfefähiges Erzeugnis mehr ${ }^{45}$ ). Die historische Basisanbaufläche konnte gegenüber der Basisanbaufläche unter der Farm Bill von 2008 unverändert bleiben; sie konnte aber auch einmalig vom Landwirt aktualisiert werden, indem seine Durchschnittsanbaufläche der Jahre 2009 bis 2012 für die Berechnung der Zahlungen herangezogen wird. Der Landwirt kann auf der Basisanbaufläche jedes Erzeugnis anbauen. Allerdings werden seine Zahlungen in dem Erntejahr gekürzt, in dem er Obst, Gemüse (außer Mung-Bohnen und Hülsenfrüchte) oder Wildreis auf mehr als $15 \%$ der Basisanbaufläche (oder 35\% im Falle des auf der individuellen Erlösgarantie basierten ARC-Programms) anbaut. Die Landwirte mit Basisanbauflächen sollten sich einmalig und unwiderruflich - produktbezogen - zwischen dem PLCund dem auf der Landkreis-Erlösgarantie basierenden ARC-Programm entscheiden. Als Alternative konnte - nicht mehr produktbezogen, sondern für alle relevanten Erzeugnisse - die Teilnahme an dem auf der individuellen Erlösgarantie basierenden ARC-Programm beantragt werden. Wurde keine Auswahl getroffen, erhielt der Landwirt im Jahr 2014 keine dieser Zahlungen; 2015 bis 2018 stand ihm nur das PLC-Programm zur Verfügung (Shields 2014a).

„PLC payments“ werden dann gewährt, wenn der nationale Durchschnittspreis für das beihilfefähige Erzeugnis unter den vom Gesetzgeber festgesetzten Referenzpreis fällt. In solchem Fall erhalten die Landwirte den Differenzbetrag zwischen dem Referenzpreis und dem nationalen Durchschnittspreis bzw. der „loan rate“, je nachdem was höher liegt. Dieses bedeutet, dass der Zahlungssatz je Bushel mit den sinkenden Preisen steigt, aber nur, bis der Marktpreis das „loan rate“-Niveau erreicht. Danach wächst der Zahlungssatz zwar nicht mehr, aber dafür werden die Zahlungen aus „marketing loans“ aktiviert. Die Auslöseschwelle für „PLC payment“ (der Referenzpreis) wurde gegenüber der früheren Auslöseschwelle für „counter-cyclical payments“ (der Richtpreis abzüglich des „direct payments"-Zahlungssatzes) beispielsweise für Weizen um 51\% und für Mais um $57 \%$ erhöht. Die Zahlungen werden für $85 \%$ der Basisanbaufläche gewährt. Die Erträge für die Berechnung der individuellen „PLC payments“ konnten bei den aus der vorausgegangenen Farm Bill belassen oder aber aktualisiert werden ( $90 \%$ der individuellen Durchschnittserträge 2008 bis 2012) (ibid.).

Unter dem Alternativprogramm ARC werden die Zahlungen dann gewährt, wenn der Erlös, definiert als das Produkt aus Preis und Flächenertrag pro Acre Anbaufläche, unter die Erlösgarantie fällt. Bei dem auf der Landkreis-Erlösgaran-

\footnotetext{
45 Im Baumwollsektor spielten „commodity payments“ in der Vergangenheit eine besonders wichtige Rolle: Von 1996 bis 2013 erhielten Baumwollerzeuger bzw. Inhaber der Basisanbauflächen für Baumwolle über 10 Mrd. USD an „direct payments“ und von 2003 bis 2013 7,6 Mrd. USD an „counter-cyclical payments“ (Schnepf 2014a: 8).
} 
tie basierenden ARC-Programm werden die Zahlungen dann ausgelöst, wenn der tatsächliche Erlös auf Landkreisebene unter die Landkreis-Erlösgarantie fällt. Letztere entspricht $86 \%$ des historischen Erlöses, der wiederum als Produkt aus „olympischem“ Durchschnitt der Erträge im Landkreis in den letzten fünf Jahren und „olympischem“ Durchschnitt der nationalen Marktpreise in demselben Zeitraum definiert ist. Der Zahlungssatz pro Acre darf 10\% des historischen Erlöses pro Acre nicht überschreiten. In diesem Konzept fängt der Erzeuger die ersten $14 \%$ der Erlösausfälle und der Staat - durch „ARC payments“ - die nächsten $10 \%$ davon auf. Der Zahlungsbetrag des Landwirts errechnet sich aus der Multiplikation der Differenz zwischen der Erlösgarantie und dem tatsächlichen Landkreis-Erlös mit 85\% der Basisanbaufläche. Bei dem auf der individuellen Erlösgarantie basierten ARC-Programm lösen Erlösverluste auf Betriebsebene „ARC payments“ aus. Für die Berechnung der Erlöse werden die individuellen Erträge des betreffenden Landwirts herangezogen. Sein Zahlungsbetrag errechnet sich hier aus der Multiplikation der Differenz zwischen der Erlösgarantie und dem tatsächlichen Erlös mit $65 \%$ der Basisanbaufläche (ibid.).

Die „marketing loan“-Regelung wurde durch die Farm Bill von 2014 beibehalten. Die beihilfefähigen Erzeugnisse sind alle Erzeugnisse, für die „PLC payments“ bzw. „ARC payments“ gelten, zuzüglich Hochland-Baumwolle, ExtraLangstapel-Baumwolle, Wolle, Mohair und Honig. Die für den Zeitraum von 2014 bis 2018 festgesetzten „loan rates“ blieben im Vergleich zu den „loan rates“ unter der Farm Bill von 2008 mit Ausnahme von Hochland-Baumwolle unverändert. Die Höchstgrenze für die aus „PLC payments“, „ARC payments“ und, neu, auch aus den Zahlungen unter der „marketing loan“-Regelung kombinierten Leistungen wurde auf 125.000 USD pro Person festgelegt. Dieses betrifft alle beihilfefähigen Erzeugnisse - außer Erdnüsse, für die eine separate Obergrenze in Höhe von ebenfalls 125.000 USD pro Person gilt. Die Obergrenze der anrechenbaren Bruttoeinnahmen für den Anspruch auf die Agrarzahlungen beträgt nun 900.000 USD (ibid.).

Hochland-Baumwolle wurde infolge des verlorenen WTO-Panels (vgl. Box 8) von den neu eingeführten Stützungsprogrammen „Price Loss Coverage“ und "Agricultural Risk Coverage“ ausgeschlossen. Dafür wurde für sie ein separates Stützungsprogramm in Form einer Erlösversicherung vorgesehen, der sogenannte „Stacked Income Protection Plan“ (STAX), der entweder alleine oder zusätzlich zu den klassischen Ernteversicherungen abgeschlossen werden kann. Der STAX deckt Verluste von 10 bis 30\% der erwarteten Erlöse auf Landkreisebene ab und greift, wenn die tatsächlichen Erlöse auf Landkreisebene um mindestens $10 \%$ gegenüber dieser Schwelle sinken. Im Gegensatz zu den früheren Stützungsprogrammen für Baumwolle ist die Teilnahme am STAX-Programm für Baumwollerzeuger über einen Versicherungsbeitrag kostenpflichtig. 80\% des Versicherungsbeitrags sollen dabei aus dem Staatshaushalt finanziert werden - eine Subvention, 
die höher liegt als bei anderen Programmen. ${ }^{46}$ Weil als Versicherungsprogramm gestaltet, unterliegen die Zahlungen unter dem STAX-Programm nicht der Obergrenze für individuelle Agrarzahlungen. Für das Jahr 2014 und ggf. auch für 2015 wurden für Baumwollproduzenten „transition payments“ vorgesehen, die die ihnen aus der Reform entstandenen Verluste kompensieren sollen (Schnepf 2014a). Wie Orden und Zulauf (2015: 12) bemerken: „A success of the 2014 farm bill is the termination of the WTO Brazil-U.S. upland cotton case.“

Box 8: WTO-Panel zu US-Agrarsubventionen im Baumwollsektor

Welche Einflüsse die US-Agrarstützung auf den Weltmarktpreis und den internationalen Handel hatte, wurde Untersuchungsgegenstand der auf Antrag Brasiliens initiierten WTO-Streitschlichtung zur Überprüfung der Stützungsmaßnahmen der USA im Baumwollsektor in den Jahren 1999 bis 2002 (DS267). Es handelte sich dabei sowohl um inländische Stützungsmaßnahmen als auch um Exportsubventionen. Laut Brasilien senkten sie die Baumwollpreise auf dem Weltmarkt und reduzierten somit die Mengen und den Wert der brasilianischen Baumwollexporte, was zu Nachteilen für den brasilianischen Baumwollsektor führte (Schnepf 2014a).

In seiner Analyse zur Auswirkung der einzelnen US-Subventionen auf den Weltmarktpreis berücksichtigte das WTO-Streitbeilegungsgremium folgende Kriterien: die Größenordnung der US-Baumwollerzeugung und -exporte, die allgemeine Preisentwicklung sowie die Art der betreffenden Subventionen. Das Gremium stellte 2004 fest, dass die USA in den Jahren 1999 bis 2002 der zweitgrößte Erzeuger und der größte Exporteur von Baumwolle weltweit waren, dass in diesem Zeitraum Baumwollpreise rückläufig waren und dass die „marketing loan"-Regelung, inklusive „marketing loan gains“ und „loan deficiency payments“, sowie „counter-cyclical payments“, „Step 2 payments“47 und „MLA payments" von Preisen abhingen. In Anbetracht dessen befand es, dass sich die oben genannten (antizyklischen) Agrarzahlungen auf den Weltmarkt in Form eines erheblichen Preisdrucks auswirkten. Dadurch wurden die Interessen Brasili-

\footnotetext{
46 Bei dem ähnlichen, für andere Erzeugnisse als Hochland-Baumwolle aufgestellten „Supplemental Coverage Option"-Programm beläuft sich die Subvention auf 65\% des Versicherungsbeitrags (Chite 2014: 17).

47 Das „Upland Cotton User Marketing Certificate Program“ („Step 2“) wurde 1991 für einheimische Baumwollverarbeiter und -exporteure aufgestellt. Die im Rahmen dieses Programms gewährten Zahlungen („Step 2 payments") sollten Preisnachteile beim Kauf einheimischer Hochland-Baumwolle kompensieren, weil ihr Preis tendenziell höher als der Weltmarktpreis lag. Die USA schafften „Step 2 payments“ zum 1. August 2006 ab (Schnepf 2014a: 2). In den Jahren 1991 bis 2006 erhielten die Begünstigten insgesamt fast 3,9 Mrd. USD an diesen Zahlungen (ibid.: 5).
} 
ens im Sinne des Art. 6 Absatz 3 Buchstabe c des Übereinkommens über Subventionen und Ausgleichsmaßnahmen ernsthaft geschädigt. ${ }^{48}$ Diese Feststellungen wurden 2005 vom WTO-Berufungsgremium bestätigt. Die USA wurden aufgefordert, die schädigenden Auswirkungen der oben genannten Stützungsmaßnahmen zu beseitigen oder diese zu streichen (WTO 2004 und 2005).

Die anderen von Brasilien beanstandeten Agrarzahlungen - „direct payments“ und ihre Vorgänger „production flexibility contract payments“ - wurden hingegen als die Interessen Brasiliens nicht schädigende Maßnahmen eingestuft. Dieses galt auch für die Ernteversicherungsprogramme (Schnepf 2014a: 2). Hervorzuheben ist die Feststellung des Streitbeilegungsgremiums, dass , direct payments“ und „production flexibility contract“ kein „decoupled income support“ im Sinne des Anhangs 2 des Übereinkommens über die Landwirtschaft und damit auch keine Green-Box-Maßnahmen, wie von den USA behauptet, sind (WTO 2004: 118). Zwar wurde für die Zeit nach dem Referenzzeitraum kein Anbau eines bestimmten Erzeugnisses als Voraussetzung für die Gewährung dieser Zahlungen verlangt. Allerdings war praktisch ein Anbauverbot von Obst und Gemüse in Kraft. Auch in diesem Fall, so befand das Gremium, entsteht eine Produktionsabhängigkeit der Zahlungen. Deshalb können sie nicht als vollständig entkoppelt betrachtet werden. Dieses wurde vom Berufungsgremium aufrechterhalten (WTO 2005: 128).

Darüber hinaus stellte Brasilien die Entkopplung der „fixed direct payments“ vor dem Panel infrage, weil die Farm Bill von 2002 eine Aktualisierung der Basisanbaufläche im Vergleich zur Basisanbaufläche für „production flexibility contract payments“ zuließ. In diesem Zusammenhang argumentierte die EU, die vor dem Panel als „dritte Partei“ auftrat, dass kontinuierliche Aktualisierungen von Referenzperioden für eine Stützungsmaßnahme Erwartungen entstehen lassen, dass die Erzeugung von bestimmten Produkten in Zukunft mit erhöhten Zahlungsansprüchen belohnt werden könnte. Damit untergraben sie tendenziell die entkoppelte Natur solcher Zahlungen (WTO 2004: 112). Das Panel sah allerdings keine Notwendigkeit, sich mit dieser Frage zu beschäftigen, nachdem es die Unvereinbarkeit der Zahlungen mit den Green-Box-Kriterien aufgrund des Anbauverbots für Obst und Gemüse festgestellt hatte.

\footnotetext{
48 Dieser Artikel besagt, dass ernsthafte Beeinträchtigungen auf jeden Fall dann entstehen können, wenn die Wirkung einer Subvention eine erhebliche Preisunterbietung bei dem subventionierten Produkt - verglichen mit dem Preis einer gleichartigen Ware eines anderen WTO-Mitglieds auf demselben Markt - oder eine Verhinderung von Preiserhöhungen oder Preisrückgänge oder Umsatzverluste auf demselben Markt ist.
} 
Da die durch die USA nach dem verlorenen Panel vorgenommenen Anpassungen der Agrarpolitik (Abschaffung der „Step 2“-payments und Abschaffung bzw. Modifikation der „Export Credit Guarantee“-Programme) als unzulänglich befunden wurden, wurde Brasilien im Jahr 2009 ermächtigt, Gegenmaßnahmen einzuführen. Dieses erhöhte den Druck auf die USA und ein Jahr später schlossen die beiden Parteien das „Framework for a Mutually Agreed Solution to the Cotton Dispute in the WTO“ ab. Es wurde vereinbart, dass Brasilien die Gegenmaßnahmen aussetzt, während sich die USA verpflichteten, das neu gegründete „Brazil’s Cotton Institute“ mit jährlich 147,3 Mio. USD zu finanzieren und Änderungen der Stützungsregelung für den Baumwollsektor in der nächsten, für 2012 geplanten Farm Bill vorzunehmen (Schnepf 2014a).

Bei Milch wurde das Stützungsprogramm mit „milk income loss contract payments“ abgeschafft. An seiner Stelle wurde das „Margin Protection Program“ aufgelegt, das die Milcherzeuger vor niedrigen Margen schützen soll und wie ein Versicherungsprogramm ${ }^{49}$ funktioniert. Alle Milcherzeuger sind beihilfefähig. Die Marge ist definiert als die Differenz zwischen dem Erzeugerpreis pro Mengeneinheit Milch, ausgedrückt in cwt (kalkuliert als nationaler Durchschnitt) und den durchschnittlichen, auf Grundlage der Preise für Mais, Sojabohnenmehl und Luzerneheu ermittelten Futterkosten für die Erzeugung von 1 cwt Milch. „Margin protection payments" werden für die historische Milcherzeugung eines Landwirts bezahlt, die seiner höchsten Jahreserzeugung zwischen 2011 und 2013 entspricht. Diese individuelle Milcherzeugung wird ab 2014 jährlich angepasst, um die Steigerungen in der nationalen Milchproduktion widerzuspiegeln. Wächst die individuelle Erzeugung aber stärker als der Landesdurchschnitt, ist diese zusätzliche Milchmenge nicht beihilfefähig. Die Zahlungen werden gewährt, wenn die Marge unter die Schwelle von 4 USD/cwt fällt (dafür ist kein Versicherungsbeitrag zu zahlen). Des Weiteren kann der Landwirt wählen, ab wann weitere Zahlungen aktiviert (Schwellenwert zwischen 4 und $8 \mathrm{USD} / \mathrm{cwt}$ ) und für welchen Anteil seiner historischen Erzeugung (zwischen 25 und $90 \%$ ) sie gewährt werden sollen; dieses beeinflusst jedoch die Höhe seines Versicherungsbeitrags. „Margin protection payments" greifen, sobald für einen Zeitraum von zwei aufeinanderfolgenden Monaten die ermittelte nationale Referenzmarge unter die vom Landwirt gewählte Schwelle fällt. Der Zahlungsbetrag des Landwirts entspricht der Differenz zwischen diesen beiden Werten. Diese wird anschließend mit dem gewählten Anteil seiner Erzeugung multipliziert und durch 6 dividiert (das heißt anteilig auf zwei Monate aufgeteilt). „Margin protection payments“ werden eingestellt, sobald die Referenzmarge für einen Zeitraum von zwei aufeinanderfolgenden Monaten den

\footnotetext{
49 Im Sinne der Budgetberichterstattung gehören „margin protection payments“ zu „commodity payments".
} 
vom Landwirt gewählten Schwellenwert erreicht bzw. übersteigt. „Indemnity payments" im Falle von unverschuldeten Milchkontaminationen wurden von der Farm Bill von 2014 beibehalten (Schnepf 2014b: 7ff).

Das Stützungsprogramm für Zucker wurde unverändert beibehalten. Es sind keine Zahlungen für Anbauer oder Verarbeiter vorgesehen (Shields 2014a: 3).

Die Gesamthöhe der Agrarzahlungen stieg 2005 aufgrund der antizyklischen Natur der „,counter-cyclical payments“ und „marketing loans“ mit dem Rückgang der Marktpreise. Von 2006 bis 2012 sank die Gesamthöhe der Zahlungen bedingt durch den Preisanstieg. Entsprechend sank auch der Anteil der Agrarzahlungen am landwirtschaftlichen Nettoeinkommen (s. Tabelle A1). Zu beachten ist der Anstieg von „conservation payments“ seit dem Jahr 2003. Beachtenswert ist auch der Rückgang von „commodity payments“ im Jahr 2014, der auf die Abschaffung von „fixed direct payments“ zurückzuführen ist. Die verbliebenen „commodity payments" in Höhe von fast 1,5 Mrd. USD sind zum größten Teil „transition payments" für Hochland-Baumwolle sowie für Tabak. Nominal befanden sich die Agrarzahlungen im Jahr 2014 auf dem niedrigsten Niveau seit 1997, real sogar seit 1982 (s. Tabelle A2 und Tabelle A3).

Mit der Entscheidung von 2014, „, fixed direct payments“ abzuschaffen, wurde der 1996 gewagte Entkopplungskurs beendet..$^{50}$ Im Kapitel 8 wird versucht, die Frage nach den Gründen für diese Abschaffung zu beantworten. An der Stelle gilt anzumerken, dass mit diesem Schritt das vom WTO-Panel im Jahr 2004 festgestellte Problem der Konformität der „fixed direct payments“ mit den Green-BoxKriterien beseitigt wurde. Beachtenswert ist, dass gerade die Zahlungen, die am weitesten entkoppelt waren und sich somit am wenigsten markt- und handelsverzerrend auswirkten, aufgehoben wurden..$^{51}$ Laut Zulauf und Orden (2014: 28) verdeutlichen die Änderungen der Stützungsprogramme für Baumwolle und Milch in Richtung eines Versicherungsprogramms „the evolution of US agricultural policy toward insurance as a key pillar of support“. Es sei nicht auszuschließen, dass in Zukunft vermehrt „margin insurance“ angeboten werden (ibid.: 29).

\footnotetext{
${ }^{50}$ Eine weitere Ausnahme von der Entkopplung bilden die sogenannten ,generic base acres“, die frühere Basisanbaufläche für Hochland-Baumwolle. Wenn auf dieser Fläche die für „price loss coverage payments“ und ,agriculture risk coverage payments“ berechtigten Erzeugnisse angebaut werden, werden den Anbauern diese Zahlungen gewährt. Somit sind sie an die aktuelle Erzeugung gekoppelt (Zulauf und Orden 2014).

${ }^{51}$ Wie Bureau (2012: 42) es formulierte: „[] $\mathrm{n}$ all forms of commodity support, the direct payments are clearly those [in the US] that generate the fewest market distortions.“
} 



\section{Geschichte der staatlichen Zahlungen in der EU}

\subsection{Großbritannien als Vorreiter}

Wie Ingersent und Rayner (1999: 26) bemerken, waren in Westeuropa vor dem 1. Weltkrieg staatliche Interventionen in die Agrarmärkte unterschiedlich ausgeprägt. Während Großbritannien, Dänemark und die Niederlande auf den freien Markt setzten, waren in Frankreich und Deutschland landwirtschaftliche Erzeuger u. a. durch hohe Zölle geschützt. Staatliche Zahlungen an Landwirte zählten in dieser Zeit nicht zum agrarpolitischen Instrumentarium. Interessanterweise wurden die ersten breit aufgestellten Agrarzahlungen an landwirtschaftliche Erzeuger in Europa im liberalen Großbritannien beschlossen. Die Einführung von „deficiency payments“ im Jahr 1917 stellte dort den ersten größeren staatlichen Eingriff in die Agrarmärkte seit der Abschaffung der die Getreideimporte beschränkenden „Corn Laws“ im Jahr 1846 dar. Noch im Jahr 1960 bezeichnete sie Mollett (1960: 20) als Vorreiter der modernen Stützungsprogramme für die Landwirtschaft. Whetham (1974: 36) fasste die britische Agrarpolitik im Zeitraum von 1846 bis zur Einführung der Agrarzahlungen wie folgt zusammen:

During the seventy years which followed the repeal of the corn laws, the national policy for British agriculture was limited to paying farmers, for the food and raw materials they produced, prices determined by competition in markets increasingly dominated by 
overseas produce. This policy was considered so fundamental to the welfare of the country that when grain and meat prices fell sharply in the last quarter of the nineteenth century, and successive governments showed real concern over the agricultural depression, protection by way of tariffs was never contemplated for any of the basic foods. At any time up to 1916 there was strong opposition to any policy which provided support for British farmers by raising the prices of imported grain, meat, or dairy produce.

Das Jahr 1917 bildete eine Zäsur. Im diesem Jahr wurde der „Corn Production Act" verabschiedet, der die Einführung von Mindestpreisen für Weizen und Hafer vorsah. Sein Ziel war, die heimische Erzeugung zu steigern, denn der Krieg auf See schnitt Großbritannien von Lebensmittelimporten zunehmend ab. Gleichzeitig fiel die Ernte 1916 mager aus, was den Anstieg der Lebensmittelpreise zusätzlich befeuerte. Der im „Corn Production Act“ verankerte Mechanismus war folgender: Wenn die Marktpreise für Weizen und Hafer unter das Niveau der Mindestpreise fielen, sollte ihren Erzeugern der Unterschied durch die aus dem Staatshaushalt finanzierten „deficiency payments“ ausgeglichen werden. Diese sollten nicht - wie ursprünglich vorgeschlagen - für die verkaufte, sondern für die angebaute Menge mit Durchschnittserträgen als Berechnungsgrundlage gezahlt werden. Die Mindestpreise wurden unter dem Niveau der damalig vom KriegsErnährungsministerium gezahlten Kaufpreise festgesetzt und sollten von Jahr zu Jahr sinken. Somit waren sie eher als Signal an Landwirte gedacht, dass sich eine höhere Erzeugung lohnen würde. Gleichzeitig führte der „Corn Production Act“ einen Mindestlohn für landwirtschaftliche Arbeiter ein und fror praktisch die Pachtpreise ein (Whetham 1978).

Ursprünglich sollten diese Regelungen ab der Ernte 1917 bis einschließlich der Ernte 1922 in Kraft bleiben (ibid.). Der anschließende „Agriculture Act“ vom Dezember 1920 setzte das Instrument der „deficiency payments“ für Weizen und Hafer aber fort. Sie sollten für die angenommenen Durchschnittserträge von einer Tonne Weizen und 1,25 Tonnen Hafer pro Acre getätigt werden. Die parallel festgelegten Mindestpreise für Weizen und Hafer orientierten sich an den Produktionskosten und waren zeitlich unbegrenzt. Deren Abschaffung musste vier Jahre im Voraus angekündigt werden, um den Landwirten Planungssicherheit zu geben (Whetham 1974). Diese frühen „deficiency payments“ kamen nie zum Einsatz. Als die Weizenpreise kurz nach der Verabschiedung des „Agriculture Act“ abstürzten, schaffte die Regierung - angesichts der auszuufern drohenden Ausgaben für die Zahlungen - die Regelung im August 1921 ab. Als Entschädigung erhielten die betroffenen Landwirte eine einmalige Zahlung pro Acre Weizen und Hafer der Ernte 1921. Zudem wurde der Mindestlohn für landwirtschaftliche Arbeiter aufgehoben. Die Nichteinhaltung der „deficiency payments“-Regelung ging in die britische Geschichte als „der große Betrug“ („Great Betrayal“) ein (ibid.: 48). 
Die Einführung der „deficiency payments“ im Jahr 1917 ging auf die Empfehlungen des „Committee on Food Production“ unter Vorsitz von Lord Milner zurück. Dieser sogenannte Milner-Ausschuss erhielt 1915 von der Regierung den Auftrag zu untersuchen, ,what measures, if any, were desirable to increase the output of agriculture in England and Wales, assuming that the war would continue beyond the harvest of 1916“ (Whetham 1978: 75). In seinem Zwischenbericht „HomeGrown Food Supplies“ vom Juli 1915 empfahl der Ausschuss, einen Mindestpreis für Weizen für die nächsten vier Jahre einzuführen, um angesichts der Kriegssituation die heimische Produktion zu erhöhen. Fiel der Weizenpreis unter den Mindestpreis, sollten Landwirte für ihre angebaute Weizenmenge „deficiency payments" erhalten. Die Begünstigten müssten nachweisen, dass sie im Oktober 1913 Weizen auf mindestens 20\% ihrer Fläche anbauten oder dass sie ihre Weizenanbaufläche seit dieser Zeit um $20 \%$ erhöhten (Whetham 1978). Whetham (1978: 76f) stellt fest, dass ,[t]he Milner Committee reflected the opinion of many people who felt that the uncertainties of war required a food production policy akin to that already adopted in Germany, with an emphasis on high output of crops for human consumption". Nichtsdestotrotz stießen der Zwischenbericht sowie der finale Bericht vom Oktober 1915 bei der Regierung zunächst auf Ablehnung. Diese begründete sie mit der Reduzierung der Aktivitäten der deutschen U-Boote, Erhöhung der weltweiten Weizenanbaufläche, zunehmenden Einberufung der landwirtschaftlichen Arbeiter in die Armee sowie hohen Ausgaben für „deficiency payments“. Die vorgeschlagenen Zahlungen wurden erst zwei Jahre später eingeführt (Whetham 1978).

Nach der plötzlichen Abschaffung der „deficiency payments“ im Jahr 1921 kehrte Großbritannien zur liberalen Agrarpolitik zurück, ließ aber einige Ausnahmen von diesem Kurs zu, beispielsweise die staatlichen Zahlungen im Zuckersektor, die allerdings nicht den Rübenanbauern, sondern den Zuckerherstellern zugutekamen und als Hilfe für den Aufbau einer neuen Industrie begründet wurden (Ingersent und Rayner 1999: 54). Ihre Laissez-faire-Haltung in der Agrarpolitik gab die britische Regierung im Jahr 1932 im Zuge der schweren Wirtschaftskrise wieder auf. Die veränderte politische Situation in Europa und die in anderen Ländern breit angenommenen protektionistischen Maßnahmen begünstigten den Politikwechsel (Mollett 1960). Insbesondere wurden mit den Stimmen der „Conservative Party“ die „deficiency payments“ - diesmal nur für Weizen - mit dem „Wheat Act" von 1932 wieder ins Leben gerufen, um die landwirtschaftlichen Einkommen zu stützen. Die Zahlungen sollten den Unterschied zwischen dem festgesetzten Mindestpreis (,standard price“) und dem durchschnittlichen Marktpreis im jeweiligen Vermarktungsjahr ausgleichen. Dabei machte der Mindestpreis fast das Doppelte der Erzeugerpreise für Weizen im Zeitraum 1930 bis 1931 aus. Die Zahlungen wurden für den verkauften Brotweizen getätigt. Die beihilfeberechtigte Weizenmenge war dabei auf eine vom Landwirtschaftsministerium jährlich festzu- 
setzende Menge beschränkt, durfte aber über 27 Mio. cwt ${ }^{52}$ nicht hinausgehen. Diese Obergrenze war großzügig, denn in den Jahren 1923 bis 1932 fiel die britische Weizenerzeugung nur ein Mal höher aus. Falls die tatsächliche Erzeugung die festgesetzte Obergrenze überschritt, sollten die Zahlungen proportional gekürzt werden (ibid.). Finanziert wurden die neuen „deficiency payments“ nicht aus dem Staatshaushalt, sondern durch eine von den Mühlen zu zahlende Abgabe auf Mehl, unabhängig davon, ob es aus einheimischem oder aus importiertem Weizen hergestellt wurde. Dabei stammten über $90 \%$ des für die menschliche Ernährung in Großbritannien eingesetzten Weizens aus Importen. Ungeachtet dessen zählte Weizen zu der Zeit zu den wichtigsten für den Markt produzierten Erzeugnissen im Land (ibid.: 21) und als solches geriet er in den Fokus der Politik.

Mollett (1960: 27) berichtet, dass durch die „deficiency payments“ die Zahl der Weizenanbauer rasch stieg (von 77.000 in 1932/33 auf 95.000 in 1934/35). Die Mehrmenge von heimischem Weizen konnte aber vom Markt absorbiert werden und es kam nicht zu Überschüssen: „Demand for wheat to feed livestock proved to be relatively elastic. This, and the fact that the Wheat Act did not attempt to reduce the feed outlet for millable wheat, were undoubtedly, safety valves" of the scheme“ (ibid.: 31). Von den „deficiency payments" profitierten insbesondere die Großbetriebe. Die Belastung für Verbraucher hielt sich in Grenzen, weil der beihilfeberechtigte heimische Brotweizen nur einen kleinen Teil der benötigten Brotweizenmenge ausmachte und somit die Mehlabgabe entsprechend niedrig gehalten werden konnte. Vor dem 2. Weltkrieg variierte die jährliche Gesamthöhe der „deficiency payments" zwischen 1,3 Mio. Britischen Pfund in 1936/37 und 9,2 Mio. Britischen Pfund in 1938/39 (ibid.: 27).

Laut Mollett (1960: 34) weckte das britische System der "deficiency payments“ das Interesse der US-Agrarpolitik ,as offering a better means of relieving the distress prevailing among United States growers than the domestic allotment plan“. Vor diesem Hintergrund wurde die Möglichkeit der Einführung solcher Zahlungen in den USA in den 1930er-Jahren analysiert. Aufgrund der großen Unterschiede in Bezug auf die Landwirtschaft zwischen den beiden Ländern kam man jedoch zu dem Schluss, dass es kaum Chancen geben würde, das britische System für die USA zu übernehmen. Dort deckte die heimische Erzeugung den Marktbedarf an Brotweizen vollständig. Entsprechend hoch wären die beihilfeberechtigte Weizenmenge und die benötigte Mehlabgabe. Letztere würde sich negativ auf die Nachfrage auswirken. Schließlich gäbe es verwaltungstechnische Schwierigkeiten.

Im Jahr 1934 wurde in Großbritannien das Instrument der „deficiency payments “ für Rinder und im Jahr 1937 für Gerste und Hafer eingeführt. Im Gegensatz zu Weizen war die Berechnungsgrundlage der Zahlungen für Gerste und Hafer nicht die verkaufte Menge, sondern die Anbaufläche. Darüber hinaus wurden sie aus dem Staatshaushalt finanziert (Whetham 1978). Ab Mai 1940 wurde der

\footnotetext{
52 Die britische Maßeinheit „long hundredweight“ (112 Pfund) entspricht 50,8 Kilo.
} 
Staatshaushalt auch Finanzierungsgrundlage für die „deficiency payments“ für Weizen. Bis zum Jahr 1953 wurden allerdings keine Zahlungen getätigt (Mollett 1960). In diesem Zeitraum war für Weizen, wie auch für die meisten anderen landwirtschaftlichen Erzeugnisse, ein System der garantierten Preise in Kraft, in dem der Staat als einziger Erstkäufer dieser landwirtschaftlichen Erzeugnisse fungierte. Erst ab 1953 wurden die landwirtschaftlichen Märkte wieder für private Akteure freigegeben und die garantierten Preise abgeschafft; das Instrument der „deficiency payments" wurde wieder eingeführt (Ingersent und Rayner 1999). Das ab der Ernte 1954 eingeführte neue Konzept für „deficiency payments“ sah Zahlungen für Weizen, Hafer, Gerste, Roggen und Menggetreide zwecks Einkommensstützung für ihre Erzeuger vor. Wie unter dem Vorkriegssystem sollten Landwirte ihre Ernte zu herrschenden Marktpreisen verkaufen können. Die Differenz zwischen den Durchschnittsmarktpreisen und den festgesetzten Mindestpreisen sollte ihnen durch die aus dem Staatshaushalt finanzierten „deficiency payments s" ausgeglichen werden. Im Unterschied zum Vorkriegssystem wurde keine Obergrenze für die beihilfeberechtigte Erzeugnismenge eingeführt (Mollett 1960). Die Mindestpreise sollten auf jährlicher Basis im Verhandlungsprozess mit den Bauernverbänden überprüft werden (Bowers 1985).

Mitte der 1960er-Jahre wurde eine Reihe protektionistischer Maßnahmen im Hinblick auf Agrarimporte ergriffen. Dieses bedeutete laut Bowers (1985: 71) eine klare Abkehr von der bisherigen Agrarpolitik. Hervorzuheben ist das Instrument der „minimum import prices“: Auf Agrareinfuhren, deren Preis einen bestimmten Schwellenwert unterschritt, wurden zusätzliche Abgaben erhoben. Auf Getreide fand dieses System bereits ab dem Jahr 1964 Anwendung. Im Gegensatz zur alten Politik, deren Kosten der Steuerzahler trug, war nun der Verbraucher über höhere Lebensmittelpreise belastet. Die Beschränkung der Einfuhren von in der Regel günstigeren Lebensmitteln sollte den Staatshaushalt im Hinblick auf die Ausgaben für „deficiency payments“ entlasten. Denn mit freien Importen schlugen die niedrigeren Weltmarktpreise auf den heimischen Markt durch, der Bedarf an Zahlungen stieg somit. Da Großbritannien ein Netto-Importeur von Nahrungsmitteln war, war dieses besonders herausfordernd (Ingersent und Rayner 1999).

Neben der Begrenzung der Ausgaben für „deficiency payments“ sollten mit den Importbeschränkungen die antizipierten Probleme der Harmonisierung der britischen Agrarpolitik mit der Politik der Europäischen Union angegangen werden (der erste Antrag des Landes auf die EU-Mitgliedschaft stammt aus dem Jahr 1961) (ibid.). Nicht weniger wichtig war der Versuch, über die Verdrängung von Importen durch die heimische Erzeugung den finanziellen Beitrag Großbritanniens zum EU-Haushalt im Falle des Beitritts zu reduzieren (Bowers 1985). Denn im Jahr 1970 - drei Jahre vor dem Beitritt Großbritanniens zur EU - wurde das Finanzierungssystem der Union reformiert. Es wurde zu einem Eigenmittelsystem, zu dem u. a. die Abgaben auf Importe von Agrarerzeugnissen zählten. Bowers (1985: 73) bemerkt deshalb: „Entry to the EEC can be seen as the logical 
culmination of UK agricultural policy in the 1960s." Mit dem Beitritt zur EU musste Großbritannien auf das Hauptinstrument seiner bisherigen Agrarpolitik die „deficiency payments“ - verzichten und das Preisstützungssystem der EU annehmen.

\subsection{Anfänge der staatlichen Zahlungen}

Die Gemeinsame Agrarpolitik (GAP) war ein Grundstein des europäischen Integrationsprozesses, und zwar aus mehreren Gründen (Hill 2012). Die Landwirtschaft war in den Gründerstaaten ein wichtiger Sektor, nicht zuletzt aufgrund des Erfordernisses der Ernährungssicherung in der Nachkriegszeit. Ohne eine gemeinsame Politik wäre die Schaffung eines gemeinsamen Marktes für landwirtschaftliche Erzeugnisse aufgrund der umfassenden einzelstaatlichen Interventionen in die Agrarmärkte sehr schwierig gewesen. Unterschiedliche Höhe der Stützung der Landwirtschaft in den Mitgliedstaaten hätte zudem zu unterschiedlichen Lebensmittelpreisen geführt, was die Wettbewerbsverhältnisse in anderen Sektoren sowie den Handel innerhalb der Union verzerren würde (ibid.). Bereits der auf der Konferenz in Messina 1955 in Auftrag gegebene Spaak-Bericht von 1956 stellte fest, dass der angestrebte gemeinsame Markt die Landwirtschaft einschließen sollte. Dort wurde die Idee einer gemeinsamen Agrarpolitik, deren Ziel es sein sollte, die ausreichende Versorgung der Bevölkerung mit Nahrungsmitteln sicherzustellen und Landwirten ein angemessenes Einkommen zu sichern, erstmals explizit erwähnt. Gleichzeitig mahnte der Bericht an, die Integration im Bereich Landwirtschaft behutsam voranzutreiben (Thiemeyer 1999). Die Entscheidung, gemeinsame Agrarmarktordnungen zu errichten, wurde im Januar 1962 getroffen. Sie sahen grundsätzlich gleiche Marktordnungspreise für die landwirtschaftlichen Erzeugnisse in allen EU-Mitgliedstaaten vor, die die tatsächlichen Marktpreise zum größten Teil bestimmten. Die Organisation der Märkte beruhte auf drei Grundprinzipien: Einheit des Marktes, Gemeinschaftspräferenz und finanzielle Solidarität. Der landwirtschaftlichen Bevölkerung durch die Steigerung der Produktivität eine angemessene Lebenshaltung zu sichern, stand zu Beginn der GAP an vorderster Stelle unter den in Art. 39 der Römischen Verträge proklamierten Zielen (Ingersent und Rayner 1999: 149). Die erste Marktordnung, für Getreide, trat im Jahr 1967 in Kraft. Mitte der 1970er-Jahre waren bereits 91\% der Agrarproduktion in Marktordnungen organisiert (Kommission 1975: 8).

Auf die negativen Folgen einer solchen Politikausrichtung wurde bereits parallel zur Entstehung der GAP in Wissenschaftskreisen gewarnt, z. B. im sogenannten „Professorengutachten“ aus dem Jahr 1962 (BML 1975). Die Kritik seitens der Wissenschaft an der GAP riss im Laufe der Zeit nicht ab. Es wurde u. a. beanstandet, dass sie Handelsspannungen provozieren (Koester und Bale 1984), Nettosozialprodukttransfers zwischen den Mitgliedsländern verursachen (Koester 2001) und die sektorale Einkommensdisparität nicht reduzieren würde (Koester und 
Tangermann 1976). Auch die Europäische Kommission wies relativ früh auf die mit der auf Preisstützung basierten Agrarpolitik verbundenen Probleme hin. Im als „Mansholt-Plan“ bekannten „Memorandum on the Reform of Agriculture“ von 1968 thematisierte sie u. a. die hohen volkswirtschaftlichen Kosten der bisherigen Politik, die weiterhin bestehende Einkommensdisparität zwischen der Landwirtschaft und anderen Wirtschaftssektoren sowie die Tatsache, dass sie für die nicht wettbewerbsfähigen Betriebe Anreize schafft, in der Landwirtschaft zu bleiben (Kommission 1968: 25). Im Jahr 1973 wies die Kommission darauf hin, dass die bisherige Politik das Problem der Einkommensdisparität innerhalb der Landwirtschaft nicht lösen konnte. Als problematisch sah sie auch die steigenden Ausgaben der Abteilung Garantie des Europäischen Ausrichtungs- und Garantiefonds für die Landwirtschaft (EAGFL), die mit den steigenden Überschüssen einhergingen sowie die nachteilige Wirkung der Währungskursschwankungen auf die Einheit des Binnenmarktes an (Kommission 1973: 7). Im Jahr 1980 hob die Kommission u. a. die Tatsache hervor, dass von der Preispolitik vor allem größere Betriebe profitierten, die diese Unterstützung am wenigstens nötig hätten. Damit verbunden war das Problem der regionalen Ungleichheit: Die wohlhabenden Regionen und Landwirte in der Union profitierten überproportional von der Stützung unter der GAP (Kommission 1980: 9).

Nichtsdestotrotz hielt die Kommission (1973: 31) an der durch die Strukturpolitik begleiteten Preispolitik fest: „The Commission believes that the achievement of the objectives set out in Article 39 of the Treaty should be based on the market and price policy and the agricultural structures policy, implemented jointly, supported by the regional policy and the social policy“. Dieses bedeutet nicht, dass das Instrument der staatlichen Zahlungen an Landwirte in der GAP nicht angewandt wurde. Bereits vor der Gründung der EU bediente sich die nationale Politik der Gründerstaaten dieses Instruments. Beispielsweise wurden in der Bundesrepublik Deutschland im Rahmen des Landwirtschaftsgesetzes von 1955 neben Subventionen für Produktionsmittel auch Zuschläge für einzelne Produkte eingeführt. Zu den bedeutendsten zählten Zahlungen im Eiersektor, um den Erzeugern die in Deutschland weit über dem Weltmarktpreis festgelegten Getreidepreise zu kompensieren, sowie im Milchsektor, um die Erzeugereinkommen und Milchqualität zu verbessern (Jákli 1990).

Die ersten Ideen zur Einführung der Agrarzahlungen auf EU-Ebene wurden bereits in den 1960er-Jahren im Zusammenhang mit den Verhandlungen über die Einführung einheitlicher Getreidepreise ins Spiel gebracht. Der Hintergrund war, angesichts des Beschlusses vom Dezember 1964 zur Festlegung eines gemeinsamen Getreidepreises ab dem 1. Juli 1967, den Landwirten in den Hochpreisländern die aus der Getreidepreissenkung resultierenden Einkommensverluste zu kompensieren. Zu diesem Zweck wurden 1967 ,gemeinschaftliche Ausgleichsmaßnahmen“ eingeführt (Verordnung Nr. 742/67/EWG). Bei den betroffenen Ländern handelte es sich um die Bundesrepublik Deutschland, Italien und Lu- 
xemburg. Beim EAGFL wurde vorübergehend eine Sonderabteilung errichtet, die die beschlossenen Ausgleichszahlungen abwickeln sollte. Die Zahlungen waren zeitlich befristet und degressiv ausgestaltet (das heißt, im Laufe der Zeit sanken sie). Die Budgets für die einzelnen Länder wurden in der Verordnung festgelegt (s. Tabelle 5). Es wurde aber den Ländern überlassen, über die Verwendung der Beträge zu beschließen. Deutschland beispielsweise entschied sich für die Getreideanbaufläche als Bemessungsgrundlage der neuen Zahlungen. Laut den Richtlinien des Bundesministeriums für Landwirtschaft vom 29. Februar 1968 waren die Landwirte beihilfefähig, die im Jahr 1967 Getreide zur Körnergewinnung ernteten. Die Höhe der Zahlung belief sich im ersten Anwendungsjahr auf 88 Deutsche Mark (DM) pro Hektar des im Jahr 1967 geernteten Getreides. Braugersteerzeuger erhielten eine zusätzliche Zahlung von 15 DM je Tonne der im Jahr 1967 vermarkteten Braugerste. Überstieg der Gesamtbetrag eines Landwirts 500 DM, war er verpflichtet, den übersteigenden Betrag für Betriebsinvestitionen oder zur Tilgung von Krediten zu verwenden. Diese gemeinschaftlichen, als Anpassungshilfe konzipierten Zahlungen im Rahmen der Getreidepreisharmonisierung von 1967 waren die ersten Ausgleichszahlungen in der EU.

Tabelle 5: Gesamtbetrag der Ausgleichszahlungen in den betreffenden EU-Ländern 1968-1970

\begin{tabular}{lrrr} 
& 1968 & 1969 & 1970 \\
\hline Deutschland & 140,00 Mio. RE & 93,50 Mio. RE & 46,75 Mio. RE \\
Italien & 65,00 Mio. RE & 44,00 Mio. RE & 22,00 Mio. RE \\
Luxemburg & 1,25 Mio. RE & 0,75 Mio. RE & 0,50 Mio. RE \\
\hline
\end{tabular}

Quelle: Verordnung Nr. 742/67/EWG.

Die Ausgleichszahlungen von 1967 waren nicht die einzigen staatlichen Agrarzahlungen der frühen Entwicklungsphase der GAP. Vom Preis abhängige, produktgebundene Zahlungen waren im Rahmen einiger Marktordnungen in Kraft, beispielsweise in der Marktordnung für Fette vom Jahr 1966. Ähnlich wie bei Getreide wurden für Ölsaaten auf Jahresbasis Richtpreise und Interventionspreise festgelegt. Der Gemeinschaftsmarkt für Ölsaaten war aber - im Gegensatz zum Getreidemarkt - nicht durch eine variable Abschöpfung geschützt. Um zu verhindern, dass Ölmühlen und Futtermittelhersteller günstigere Importware einsetzen, wurde ihnen eine Beihilfe gewährt. Sie entsprach dem Unterschied zwischen dem Marktpreis des betreffenden Erzeugnisses, der dem Weltmarktpreis glich, und seinem Richtpreis und diente der Kompensation für die höheren EU-Preise. Die Beihilfe funktionierte somit wie die „deficiency payments“ (Ingersent und Rayner 1999: 228).

Die anderen Marktordnungen, die zu ihrer Gründungszeit einen ähnlichen Mechanismus beinhalteten (Intervention, aber keine variable Abschöpfung), um- 
fassten Olivenöl (1966), Rohtabak (1970) und Schaf- und Ziegenfleisch (1980) (Henning 2000: 172). Sie hatten gemeinsam, dass die Beihilfe an Verarbeiter des primären Erzeugnisses gewährt wurde. Eine variable Beihilfe - allerdings für Erzeuger - wurde im Rahmen der Marktordnung für Getreide (1967) für Hartweizen eingeführt. Sie wurde getätigt, wenn der in der Region mit dem größten Überschuss gültige Interventionspreis unter dem garantierten Mindestpreis lag und entsprach der Differenz zwischen diesen beiden Preisen zu Beginn des Wirtschaftsjahres. Der Beihilfebetrag war für die gesamte Union gleich (Verordnung 120/67/EWG). Diese Art der Zahlungen nannte die Kommission „supplementary production aid“ (Kommission 1975: 8). Sie wurden grundsätzlich für Erzeugnisse mit niedrigem Selbstversorgungsgrad, deren Produktion begrenzt war und sich auf wenige Regionen beschränkte, gewährt. Die geringen Erzeugungsmengen bedeuteten, dass die Ausgaben für die Maßnahmen in Grenzen gehalten werden konnten. Insgesamt profitierten von diesen Zahlungen im Jahr 1975 etwa 2,5\% der Agrarproduktion der Union (ibid.).

Einkommensstützende Zahlungen spielten in der europäischen Agrarpolitik vor 1992 eine sehr geringe Rolle. Die Kommission spricht in diesem Fall über „aid at a fixed rate" (ibid.). Mitte der 1970er-Jahre umfassten diese Zahlungen lediglich $0,6 \%$ der EU-Agrarproduktion und wurden für Erzeugnisse gewählt, die nur in geringen Mengen bzw. in einigen wenigen Regionen der Union produziert wurden. Dieses galt für Sonderkulturen wie Flachs und Hanf (ab 1970) sowie Hopfen (1971), aber auch für Baumwollsaat (1971), Saatgut (1971), Seidenraupen (1972) und Trockenfutter (1974) (Kommission 1975; betreffende Verordnungen). Diese Marktordnungen (nur Baumwollsaat und Seidenraupen waren nicht in einer Marktordnung erfasst) hatten gemeinsam, dass sie über kein obligatorisches Interventionssystem und keinen effektiven Außenschutz in Form variabler Abschöpfungen verfügten (Henning 2000: 172). Ziel der Zahlungen war, den Erzeugern der betreffenden Produkte ein angemessenes Einkommen zu sichern. Dazu wurde ein fester Betrag pro Hektar Anbaufläche (bzw. bei Trockenfutter je Gewicht und bei Seidenraupen je in Betrieb genommene Samenschachtel), der in der gesamten EU gleich war, gezahlt. Um von den Zahlungen profitieren zu können, mussten die Landwirte das betreffende Erzeugnis weiter anbauen.

Eine erste solche Beihilfe wurde für Flachs und Hanf mit dem Ziel, die Erzeugereinkommen zu verbessern, eingeführt. Diese gemeinschaftliche Regelung sollte die bisherigen einzelstaatlichen Erzeugerbeihilfen ersetzen. Der Beihilfesatz war in der ganzen Union einheitlich festgelegt und wurde je Hektar Anbau- und Erntefläche gezahlt. Bei der Festlegung seiner Höhe sollte die voraussichtliche Entwicklung der Preise für Fasern und Saaten von Flachs und Hanf sowie der Preise ihrer natürlichen Konkurrenzprodukte berücksichtigt werden. Damit strebte der Gesetzgeber an, eine Balance zwischen dem Erzeugungsumfang und den Absatzmöglichkeiten zu gewährleisten, insbesondere bei Flachs, dessen Erzeugung in der EU über dem Inlandsverbrauch lag (Verordnung (EWG) Nr. 1308/70). Die Bei- 
hilfe wurde grundsätzlich den Erzeugern gewährt; bei Faserflachs war vorgesehen, dass sich Erzeuger und erster Käufer die Zahlung je zu Hälfte teilen, was der Gesetzgeber durch eine enge vertragliche Bindung zwischen ihnen begründete (Verordnung (EWG) Nr. 619/71).

Während das Instrument der staatlichen Zahlungen im Rahmen der Marktordnungen nur punktuell eingesetzt wurde, war die Kommission offensichtlich bereit, es im Rahmen der Agrarstrukturpolitik breiter anzuwenden. Mit dem sogenannten „Mansholt-Plan“ von 1968 schlug die Kommission die Einführung befristeter Beihilfen an bestimmte Gruppen landwirtschaftlicher Betriebsinhaber zur Ergänzung der bisherigen Preispolitik vor. Neben zahlreichen sozio-strukturellen Maßnahmen (z. B. Beihilfen für die Aufgabe der landwirtschaftlichen Produktion oder Investitionsbeihilfen) wurde „personal assistance not tied either to the volume of output or to the employment of factors of production " für Landwirte, die von den restlichen Maßnahmen nicht profitieren könnten, ins Spiel gebracht (Kommission 1968: 27). Auf dieses Konzept konnten sich die Gesetzgeber zu der Zeit allerdings nicht einigen (Ingersent und Rayner 1999: 157). „[T] he plan proved too radical to be politically acceptable. It was seen as destructive of too many family farms and too great a departure from the policy of market price support" (Kommission 1994b: 13). Im Jahr 1972 wurde im Rahmen des abgeschwächten „Mansholt-Plans“ zur Verbesserung der Agrarstruktur neben landwirtschaftlichen Renten lediglich eine Landaufgabeprämie eingeführt, deren Höhe sich nach der freigesetzten landwirtschaftlich genutzten Fläche richtete (Richtlinie 72/160/ EWG). Es wurde auch eine Investitionsbeihilfe, die grundsätzlich in Form von Zinsvergütungen gewährt werden sollte, beschlossen (Richtlinie 72/159/EWG). Diese Maßnahmen galten als sogenannte „gemeinsame Maßnahmen“ im Sinne des Art. 6 der Verordnung (EWG) Nr. 729/70 und wurden somit aus der Abteilung Ausrichtung des EAGFL mitfinanziert.

Darüber hinaus wurde im Jahr 1975 von der Abteilung Ausrichtung des EAGFL eine ko-finanzierte Zahlung an Betriebe in Berggebieten und in anderen von der Natur benachteiligten Gebieten zum Ausgleich der ungünstigeren Produktionsbedingungen eingeführt (die sogenannte „Zulage zum Ausgleich der ständigen natürlichen Nachteile“). Diese Beihilferegelung sollte die „Fortführung der Ausübung landwirtschaftlicher Erwerbstätigkeiten“ und somit die „Erhaltung eines Minimums an Bevölkerungsdichte“ bzw. die „Erhaltung der Landschaft" sichern (Art. 1 der Richtlinie 75/268/EWG). Später rückte angesichts der restriktiveren Preispolitik das Ziel, „die Anpassung und Neuorganisation dieser Betriebe zu unterstützen“ (Verordnung (EWG) Nr. 1760/87), in den Vordergrund.

Zudem wurden Zahlungen für die Stilllegung von Ressourcen angewandt, z. B. die gemeinschaftlich finanzierte Prämie von 1977 an Milchbauern, die auf die Vermarktung von Milch und Milcherzeugnissen verzichteten oder ihre Milchkuhbestände auf Fleischerzeugung umstellten (Verordnung (EWG) Nr. 1078/77). Im Jahr 1986 wurde eine aus dem EU-Haushalt finanzierte Vergütung für Milcher- 
zeuger, die die Milcherzeugung endgültig aufgaben, eingeführt (Verordnung (EWG) Nr. 1336/86). Im Weinsektor wurde bereits im Jahr 1976 eine Umstellungsprämie zur Verringerung der Weinernten, die als gemeinsame Maßnahme aus der Abteilung Ausrichtung des EAGFL ko-finanziert wurde, eingeführt (Verordnung (EWG) Nr. 1163/76). Eine vorübergehende oder endgültige Aufgabe von Rebflächen wurde mit einer im Jahr 1980 eingeführten Prämie gefördert. Gleichzeitig wurde auch eine Prämie für den Verzicht auf die Wiederbepflanzung beschlossen. An der Finanzierung beider Beihilfearten beteiligte sich die Abteilung Ausrichtung des EAGFL (Verordnung (EWG) Nr. 456/80).

Abgesehen von den genannten agrarstrukturpolitischen Maßnahmen wurden Agrarzahlungen punktuell im Milch- und Fleischsektor zur Förderung der Erzeugereinkommen angewandt. Im Rindfleischsektor wurden u. a. 1975 die Prämie bei der Geburt von Kälbern und die Prämie für Schlachtrinder (Verordnung (EWG) Nr. 464/75) sowie 1980 die Mutterkuhprämie (Verordnung (EWG) Nr. 1357/80) eingeführt. Ebenfalls vollständig aus dem EU-Haushalt finanziert war die Zusatzprämie für die Erhaltung des Mutterkuhbestandes in Irland und Nordirland von 1982 (Verordnung (EWG) Nr. 1199/82). Im Jahr 1968 wurde eine Beihilfe für Magermilch und Magermilchpulver, die für Futterzwecke verwendet oder zu Kasein verarbeitet wurden, eingeführt (Verordnung (EWG) Nr. 804/68).

Das Instrument der Zahlungen an Erzeuger wurde auch genutzt, um Landwirten in den neu beigetretenen Mitgliedstaaten die Umstellung der Agrarstützung zu erleichtern. Beispielsweise wurde Großbritannien 1974 ermächtigt, vorübergehende einzelstaatliche Beihilfen im Rindfleisch- (Verordnung (EWG) Nr. 920/74) und Schweinefleischsektor (Verordnung (EWG) Nr. 722/74) zu gewähren. Agrarzahlungen wurden auch von nationalen Regierungen unabhängig von den gemeinschaftlichen Agrarbeschlüssen angewandt. Beispielsweise gewährte Frankreich im Jahr 1981 - angesichts der restriktiveren Preispolitik der Union zwecks der Entlastung des EU-Haushalts - Landwirten direkte, umsatzbezogene Einkommensbeihilfen in Gesamthöhe von $1 \mathrm{Mrd}$. DM. Zwar wurden diese Beihilfen durch die Kommission für unvereinbar mit den Bestimmungen des EU-Vertrages befunden, deren Rücknahme konnte aber nicht erzwungen werden (von Urff 1982: 148f).

In einigen Fällen wurden Mitgliedstaaten von der Union ermächtigt, nationale Zahlungen - ggf. unter Ko-Finanzierung aus dem EU-Haushalt - an Erzeuger zu gewähren, z. B. eine ko-finanzierte Schlachtungsprämie zugunsten der Rindfleischerzeuger in Großbritannien von 1986 (Verordnung (EWG) Nr. 1347/86) oder eine rein einzelstaatliche Zusatzprämie für Kälber in Italien aus demselben Jahr (Verordnung (EWG) Nr. 1346/86). Für die deutschen Landwirte waren von 1970 bis 1973 durch die EU mitfinanzierte direkte Beihilfen als Ausgleich für Einkommensminderungen, die als Folge der Aufwertung der Deutschen Mark vom Oktober 1969 entstanden, vorgesehen. Denn die Aufwertung führte zu einer Senkung der in DM ausgedrückten Agrarpreise, die im Rahmen der GAP in Rechnungseinheiten festgesetzt waren. Diese Beihilfen durften nicht nach Maßgabe des Preises 
oder der Menge des Erzeugnisses bestimmt werden (Kommission 1970: 153f). Norwegen wurde im Beitrittsvertrag ein spezielles Stützungssystem vor dem Hintergrund zugestanden, dass die Angleichung der dortigen Agrarpreise an die EUPreise mit erheblichen Einkommensverlusten für die Landwirte in Norwegen verbunden gewesen wäre. Es wurde explizit festgehalten, dass diese nationalen Beihilfen nicht an das verkaufte Erzeugnis gebunden sein und auch keine Erzeugerpreissubventionen umfassen dürften (Europäische Gemeinschaften 1972).

Im Jahr 1985 wurden in der EU die nationalen Umweltzahlungen zugelassen. Die Mitgliedstaaten wurden ermächtigt, Landwirten in Gebieten mit gefährdeter Umwelt einzelstaatliche Beihilfen zu gewähren, wenn sie sich verpflichteten, Bewirtschaftungspraktiken durchzuführen, die der Erhaltung oder Verbesserung der Umwelt dienten (Verordnung (EWG) Nr. 797/85). Mit der Verordnung (EWG) Nr. 1760/87 wurden die „Beihilfen in Gebieten mit besonderer Notwendigkeit des Schutzes der Umwelt und der natürlichen Ressourcen sowie der Erhaltung des natürlichen Lebensraums und der Landschaft" zur gemeinsamen Maßnahme und als solche wurden sie von der Abteilung Ausrichtung ko-finanziert. Die Prämie wurde jährlich pro Hektar ausbezahlt, wenn der Landwirt in einem von dem Mitgliedstaat für diese Zwecke ausgewiesenen Gebiet wirtschaftete und sich verpflichtete, die ausgewählten umweltfreundlichen Produktionspraktiken für mindestens fünf Jahre auszuüben. Die Höhe des Beihilfesatzes sowie Art und Weise der Praktiken sollten von den Mitgliedstaaten festgelegt werden. Die Regelung war für sie fakultativ.

Im Jahr 1987 wurde in der EU eine sogenannte Extensivierungsbeihilfe in den Sektoren Getreide, Rindfleisch und Wein eingeführt. Sie hatte zum Ziel, die Landwirte zur Umstellung der Produktion auf nicht überschüssige Erzeugnisse oder zur Extensivierung der Produktion von Überschusserzeugnissen zu bewegen. Als Extensivierung im Sinne der Regelung galt die Verringerung der Produktion des betreffenden Erzeugnisses um mindestens $20 \%$, ohne dass die Produktionskapazitäten für andere Überschusserzeugnisse erhöht wurden. Die Union beteiligte sich an der Finanzierung der neuen Beihilfe über die Abteilung Ausrichtung des EAGFL (Verordnung (EWG) Nr. 1760/87). Bei der Extensivierungsbeihilfe handelte es sich um die erste Maßnahme aus der Gruppe der sogenannten ,structural stabilizers", also Maßnahmen, die "generally act upon agricultural markets by making an impact on supply whilst at the same time attenuating the negative effects of market reform on income via premiums paid in the framework of the scheme" (Kommission 1991c: 103).

Im Jahr 1988 wurde eine Beihilfe zur Förderung der freiwilligen Stilllegung von Anbauflächen eingeführt, um die Erzeugung dem Marktbedarf anzupassen. Sie sollte den Landwirten den aus der Flächenstilllegung resultierenden Einkommensverlust kompensieren. Bei Weidewirtschaft mit extensiver Viehhaltung, der Erzeugung von Linsen, Kichererbsen und Wicken sowie bei der nichtlandwirtschaftlichen Nutzung der stillgelegten Flächen sollte die Beihilfe angesichts der ge- 
ringeren Einkommensminderung gekürzt werden. Ansonsten war auf den stillgelegten Flächen Brache mit einem geeigneten Bewuchs sowie Aufforstung möglich. Die partizipierenden Landwirte waren verpflichtet, mindestens 20\% der Anbaufläche des Betriebes für fünf Jahre stillzulegen und auf den stillgelegten Flächen Maßnahmen zur Erhaltung zufriedenstellender agronomischer Bedingungen durchzuführen. Die EU beteiligte sich an der Finanzierung der neuen Beihilfe zu gleichen Teilen über die Abteilungen Garantie und Ausrichtung des EAGFL, da die Prämie sowohl eine gemeinsame Maßnahme als auch eine Intervention darstellte; ihre Höhe sollte aber von den einzelnen Mitgliedstaaten festgesetzt werden (Verordnung (EWG) Nr. 1094/88 und Nr. 1272/88).

Zwanzig Jahre nach der ersten Ausgleichszahlung als Anpassungshilfe vor dem Hintergrund des Abbaus der Preisstützung (Ausgleichszahlung im Rahmen der Getreidepreisharmonisierung von 1967) wurde eine Ausgleichszahlung im Rindfleischsektor eingeführt. In diesem Sektor wurde im Jahr 1987 eine restriktivere Interventionspolitik beschlossen, u. a. sollte der Interventionspreis dem Marktpreis wesentlich angenähert werden (Verordnung (EWG) Nr. 467/87). Um die Auswirkungen dieser Anpassungen aufzufangen, wurde eine Sonderprämie für Rindfleischerzeuger aus EU-Mitteln eingeführt. Diese Prämie sollte für eine begrenzte Anzahl der männlichen Rinder eines Bestandes einmal während ihres Lebens gezahlt werden. Die neue Regelung hatte eine begrenzte Dauer von 21 Monaten, wurde aber immer wieder verlängert (z. B. Verordnung (EWG) Nr. 571/89). Den Charakter einer Anpassungshilfe hatte auch die im Jahr 1989 beschlossene vorübergehende landwirtschaftliche Einkommensbeihilfe (Verordnung (EWG) Nr. 768/89), die zudem eine größere Reichweite hatte, weil sie nicht auf einen Sektor begrenzt war. Als unmittelbarer Vorgänger der Ausgleichszahlungen der MacSharry-Reform soll sie nachstehend detaillierter vorgestellt werden.

Die landwirtschaftliche Einkommensbeihilfe war für Betriebe vorgesehen, die durch „die im Zusammenhang mit der Reform der gemeinsamen Agrarpolitik entstandenen neuen Marktgegebenheiten in Schwierigkeiten geraten und wegen ihrer wirtschaftlichen und strukturellen Voraussetzungen nicht in der Lage sind, die Anpassung aus eigenen Kräften erfolgreich durchzuführen“" (Art. 1 der Verordnung (EWG) Nr. 768/89), und diente der Einkommensstützung während des Anpassungsprozesses. Konkret waren Betriebsinhaber, deren Familiengesamteinkommen je Arbeitseinheit eine bestimmte durch den jeweiligen Mitgliedstaat festgelegte Grenze nicht überschritt, beihilfefähig. Diese Grenze durfte nicht größer als $70 \%$ des nationalen oder $90 \%$ des regionalen Bruttoinlandsprodukts je Erwerbsperson ausfallen. Bei der Berechnung des Familieneinkommens waren Einkünfte aus nichtlandwirtschaftlichen Tätigkeiten mit berücksichtigt. Die Beihilfen konnten für höchstens fünf Jahre gewährt werden und waren degressiv gestaffelt. Die Höhe des Beihilfesatzes sollte auf Grundlage von aus den Änderungen der GAP resultierenden Verlusten erfolgen, wobei die Mitgliedstaaten die Wahl hat- 
ten, die Beihilfe auf pauschaler oder individueller Basis festzusetzen. Eine Höchstgrenze je Arbeitseinheit wurde vorgesehen.

Die EU beteiligte sich an der Finanzierung der Einkommensbeihilfen mit 70\% des Betrags bei Betrieben, die in den strukturschwachen Regionen (sogenannte Ziel-1-Fördergebiete) lagen und mit $25 \%$ in den restlichen Fällen. Dabei war die Ko-Finanzierung durch die EU auf hauptberuflich tätige Landwirte begrenzt und eine Obergrenze für die Unionsbeteiligung vorgesehen. Die Regelung war befristet: Nach dem 31. März 1993 konnten Beihilfen nicht mehr eingeführt und nach dem 31. März 1998 nicht mehr ausbezahlt werden. Auch war die Regelung für die Mitgliedstaaten - entgegen dem ursprünglichen Vorschlag der Kommission - fakultativ. Um die neuen Zahlungen anbieten zu können, mussten sie ein Programm für landwirtschaftliche Einkommensbeihilfen ausarbeiten, in dem u. a. Ziele, geografische Gebiete, ggf. ausgewählte Sektoren und Modalitäten für die Gewährung der Zahlung festgelegt werden sollten. Die allgemeine Bedingung war, dass die Einkommensbeihilfen nicht an die Preise oder die Menge der Erzeugung der Begünstigten gekoppelt sein durften. Darüber hinaus sollten sie nicht zu Wettbewerbsverzerrungen führen und keinen Anreiz für die Steigerung der landwirtschaftlichen Erzeugung bieten. Die Verordnung enthielt auch ein grundsätzliches Verbot aller einzelstaatlichen Einkommensbeihilfen, die nicht den Voraussetzungen und Modalitäten der Verordnung (EWG) Nr. 768/89 entsprachen. Insbesondere wurden Beihilfen untersagt, die an Preise, die Menge der landwirtschaftlichen Erzeugnisse oder die Produktionsfaktoren gekoppelt waren.

Die Einkommensbeihilfe gehörte zur Gruppe der sogenannten „structural stabilizers“. Neben der Extensivierungsprämie von 1987 beinhaltete sie auch die neue Vorruhestandsregelung von 1988 sowie die im selben Jahr eingeführte Beihilfe für die Förderung der Flächenstilllegung. Die Kommission (1991c: 103) beurteilte die Implementierung dieser Regelungen im Jahr 1990 als enttäuschend: „Measures have tended to be applied thinly and unevenly as between Member States or in some cases not at all." Beispielsweise wurden zwei Jahre nach dem Inkrafttreten der Regelung zur Flächenstilllegung lediglich 800.000 Hektar aus der Produktion genommen, was 2,4\% der Getreideanbaufläche der EU entsprach (Kommission 1991c: 103). Nur Deutschland entschied sich, den Landwirten Vorruhestandsprämien anzubieten (ibid.: 105). Schließlich wurde 1990 von der Einkommensbeihilferegelung nur in den Niederlanden (für Ackerbaubetriebe, die die ausgewählten Kulturen anbauten), Frankreich (alle Landwirte), Italien (Olivenölproduzenten im Süden) und Deutschland (Landwirte in Baden-Württemberg) Gebrauch gemacht (ibid.: 21). Insgesamt acht von damals zwölf Mitgliedstaaten führten am Ende die Einkommensbeihilfen ein (Belgien, Dänemark, Deutschland, Griechenland, Frankreich, Italien, Niederlande und Spanien) (Kommission 1994a: 22). Etwa 185.000 Landwirte profitierten davon; die Ausgaben beliefen sich bis zum Jahre 1993 auf nur 650 Mio. Europäische Währungseinheiten (ECU) (Kommission 1993: 22). Laut Hill (2012) ist der Misserfolg der Einkommensbeihilfen 
von 1989 u. a. darauf zurückzuführen, dass ihre Einführung für die Mitgliedstaaten fakultativ war.

\subsection{Direktzahlungen in der EU: Der Weg dorthin}

Nachstehend wird - basierend auf den offiziellen Texten der Europäischen Kommission - der Weg der EU zur ersten Anwendung des Instruments der Direktzahlungen dargestellt. Der Fokus liegt deshalb auf Dokumenten der Kommission, weil sie unter den EU-Institutionen mit dem exklusiven Initiativrecht ausgestattet ist. Für die Zahlungen werden die von der Kommission originär verwendeten Bezeichnungen übernommen.

Der Idee einkommensstützender Zahlungen an landwirtschaftliche Erzeuger wurde in der EU lange Zeit eine Absage erteilt. Laut Kommission (1994b: 11f) waren flächendeckende Agrarzahlungen zu Beginn der GAP aus finanziellen sowie verwaltungstechnischen Gründen nicht praktikabel:

In devising a policy to meet the objectives of the common agricultural policy, the six founding Member States had no hesitation in selecting market price support. [...] Alternative forms of intervention, such as deficiency payments $[\ldots]$, would have been much more costly to the initially modest Community budget. Neither did the Six then have the administrative infrastructures to make direct payments to farmers a realistic option for policy-makers.

Ihre Ablehnung der Zahlungen begründete die Kommission auch mit der Befürchtung, dass diese den Strukturwandel in der Landwirtschaft behindern können. Konkret schrieb sie:

The Commission has also reached the conclusion that additional measures in the form of more or less generalized direct aid to farm incomes might well form an obstacle to the structural changes needed in agriculture, engender major administrative difficulties in most of the Member States, and entail unduly heavy expenditure for official budgets. (Kommission 1973: 4)

Inwiefern die zu der Zeit hohen Weltmarktpreise für landwirtschaftliche Erzeugnisse, die die preisstützungsbedingte Haushaltsbelastung reduzierten, die Bereitschaft zu Reformen bremsten, kann nur spekuliert werden. Denn zwei Jahre später schloss die Kommission eine künftige Anwendung des Instruments der ,income subsidies“, zusätzlich zu den punktuell geltenden Fällen, nicht mehr aus. Dazu stellte sie im Bericht „Stocktaking of the common agricultural policy“, der eine Analyse der bisherigen Entwicklung der GAP sowie Evaluierung verschiedener agrarpolitischer Instrumente lieferte, zwar fest: 
Income subsidy implies that optimum use is not being made of the labour factor in the farms considered. It can therefore only be justified economically as a provisional solution pending the modernization of the farms or the retraining of farmers for other types of activity. (Kommission 1975: 26)

Nichtsdestotrotz führte die Kommission weiter aus:

It may be that the Commission will at some later date conclude that direct subsidies of a temporary nature, within the limits of reasonable budgetary expenditure, are necessary in other specific and clearly defined cases, especially where market and price policy measures together with the socio-structural and regional policies do not enable satisfactory results to be obtained within a reasonable time. (ibid.: 37f)

Eine Einkommensstützung über „direct subsidies“ wäre somit im Falle des Versagens der Preispolitik eine mögliche Option. Sie sollte nicht flächendeckend, sondern nur in klar definierten Fällen und in einem strikten zeitlichen und haushaltspolitischen Rahmen erfolgen. Bereits zu jener Zeit plädierte die Kommission (1975: 27) dafür, die Stützung so zu gestalten, dass sie nicht an die Produktion einzelner Erzeugnisse gebunden ist: „,T] he granting of aid must not be linked with specific types of production: so long as the beneficiary continues to practise farming it is preferable that he should concentrate on those products in relation to which - at market prices - his productivity is highest." Darüber hinaus könnte sie zu einem gewissen Grad umgekehrt proportional zu den vom Betrieb zugekauften Produktionsmitteln gewährt werden (ibid.). Fast zwanzig Jahre nach ihrem Bericht von 1975 bemerkte die Kommission (1994b: 14) zu ihren eigenen Vorschlägen selbstkritisch: „However, the proposals for policy changes were not as farreaching as the rigour of this analysis might have suggested“".

Im Jahr 1980 wiederholte die Kommission die allgemeine Aussage zu ihrer Bereitschaft, „direct income support“ in bestimmten Fällen anzuwenden, um ,problems of a special regional nature or concerning particular commodities“ (Kommission 1980: 7) zu lösen. „Price policy must reflect trends on the world market more than it has in the past" - diese Vorgabe für die künftige GAP stellte die Kommission in ihrem „Report on the mandate of 30 May 1980“ von 1981 (Kommission 1981a: 14) vor. Die vorgeschlagenen Anpassungen der Preispolitik (u. a. Begrenzung der Preisgarantie in Form von Garantieschwellen) sollten aber nicht ohne Kompensation für betroffene Erzeuger in Form von „direct income subsidies“ erfolgen. Diese sollten aus Kostengründen auf Kleinbetriebe beschränkt werden: „The Commission's guidelines on prices and production cannot be applied in a vacuum, ignoring the income problems of certain producers. This is why it is proposing the introduction, in certain specific cases, of direct income subsidies which, given the cost, would be confined to small producers" (ibid.: 16). 
Diese Beihilfen sollten vollständig oder teilweise aus dem EU-Haushalt finanziert werden. Konkret brachte die Kommission „aids to support the incomes“ im Rindfleischsektor vor dem Hintergrund der vorgeschlagenen restriktiveren Handhabung der Intervention für Rind- und Kalbfleisch ins Spiel. Somit sollten den Erzeugern die daraus resultierenden Einkommensverluste kompensiert werden (Ausgleichszahlungen). Grundsätzlich sollten alle rinderhaltenden Betriebe beihilfefähig sein; es wurde aber eine Obergrenze pro Betrieb vorgeschlagen (Kommission 1981b: 73). Gleichzeitig schloss die Kommission eine Erweiterung der Anwendung dieser Form der Beihilfe in der Zukunft nicht aus:

$[T]$ he Commission considers that any future extension of direct subsidies should be set in the context of the trend in prices and agricultural incomes, with account being taken of annual decisions on prices and other measures, and the budgetary situation. It reserves the right, if necessary, to make proposals to introduce or adjust direct subsidies in other cases. (ibid.: 73f)

Allerdings wurde die Idee der „aids to support the incomes“ für Rind- und Kalbfleischerzeuger zu der Zeit vom Rat ${ }^{53}$ nicht unterstützt (von Urff 1982). Ebenso im Jahr 1981 schlug die Kommission eine schrittweise Kürzung der realen Preise für Getreide vor. Sie sollten an das Getreidepreisniveau der USA, das etwa um $20 \%$ unter dem EU-Niveau lag, angepasst werden (Kommission 1981b: 76). Interessanterweise sah die Kommission zu der Zeit keinen Ausgleich für diese Preissenkung vor.

Im Grünbuch „Perspectives for the Common Agricultural Policy“ von 1985 befasste sich die Kommission umfassender mit neuen agrarpolitischen Instrumenten und zeigte in dieser Veröffentlichung Alternativen für die künftige Entwicklung der GAP auf (Kommission 1985a). Sie setzte sich zum Ziel, ein Gleichgewicht zwischen Angebot und Nachfrage auf dem Agrarmarkt herzustellen sowie neue Wege zur Reduzierung der Erzeugung in Problembereichen aufzuzeigen. Die dort dargelegte Option, „direct income aids“ auf breiterer Basis einzuführen, verstand die Kommission allerdings nicht als einen konkreten Reformvorschlag, sondern lediglich als Diskussionsgrundlage. Sie wies auf politische, administrative und finanzielle Fragen hin, die mit dieser Form der Agrarstützung und insbesondere mit ihrem selektiven Charakter einhergehen und antizipierte den Widerstand der Landwirte gegen Maßnahmen, die den Charakter einer direkten Hilfe haben (ibid.). Nichtsdestotrotz empfahl die Kommission, vor dem Hintergrund einer restriktiven Preispolitik die Möglichkeit von „direct income aids“ (im Grünbuch

\footnotetext{
${ }^{53}$ Das Europäische Parlament (1981) wiederum hielt zu der Zeit den Einsatz „direkter Ausgleichszahlungen" nur dann für notwendig, wenn sie als Entgelt für Leistungen gezahlt werden, die aus regional- und sozialpolitischen sowie aus ökologischen Gründen erforderlich sind und in der Regel über das am Markt zu erzielende Einkommen nicht abgegolten werden können (z. B. Aufrechterhaltung der Landbewirtschaftung in Berggebieten).
} 
auf Deutsch „direkte Einkommensbeihilfen“) insbesondere im Getreidesektor zu untersuchen. Sie wies auf die Schwierigkeiten hin, im Rat Beschlüsse zu Preissenkungen durchzusetzen, wenn sie nicht durch einkommenspolitische Maßnahmen begleitet werden. ${ }^{54}$ Diese sollten einen subsidiären Charakter gegenüber der Preispolitik haben und durch eine intensivere Regionalpolitik ergänzt werden, die den Bedarf an solcher Stützung mit der Zeit verringern würde. Als Alternative wurden verschiedene Maßnahmen zur Angebotssteuerung dargestellt, wobei sich die Kommission gegen Quotenregelungen aussprach (ibid.: 20). Diese Ablehnung von Quoten scheint dem Instrument der einkommensstützenden Zahlungen letztlich zum Erfolg verholfen zu haben.

Die neuen Zahlungen sollten selektiv gestaltet werden - die pro Produktionseinheit gezahlten „deficiency payments“, die grundsätzlich allen Erzeugern des betreffenden Produkts zur Verfügung stehen, erteilte die Kommission aus Kostengründen eine Absage. Aus demselben Grund regte sie eine Begrenzung der Zahlungen pro Hektar an. Außerdem betonte sie, dass "great care would have to be taken to keep, as far as possible, such schemes neutral with respect to production" (ibid.: 56). Auch aus diesem Grund wurden produktionsbezogene „deficiency payments" abgelehnt. Die Kommission (1985a: 56) identifizierte vier verschiedene Arten der möglichen Beihilferegelungen: eine Vorruhegehaltsregelung, eine Regelung mit einer strukturpolitischen Komponente, eine Regelung mit sozialer Ausrichtung sowie eine Umwidmungsregelung mit einer Umweltschutzkomponente. Alle diesen Optionen hatten gemeinsam, dass sie das landwirtschaftliche Einkommen direkt stützten. Sie schlossen sich gegenseitig nicht aus, sondern konnten auch gleichzeitig angewandt werden, so die Kommission.

Im Rahmen der Vorruhegehaltsregelung regte die Kommission eine Beihilfe an hauptberuflich tätige Landwirte ab 55 Jahre, die die landwirtschaftliche Tätigkeit aufgaben, an. Diese Zahlung sollte ihnen bis zum 65. Lebensjahr gewährt werden. Im Vergleich zur damals geltenden Vorruhestandsregelung nach Richtlinie 72/160/EWG sollten die Zahlungen attraktiver und die Bedingungen für die Teilnahme weniger restriktiv gestaltet werden (ibid.: $57 \mathrm{f}$ ).

Einen neuen, noch nicht erprobten Ansatz hingegen stellte die Einkommensbeihilfe mit einer strukturpolitischen Komponente dar. Darunter verstand die Kommission eine temporäre (z. B. auf fünf Jahre begrenzte) finanzielle Unterstützung für hauptberuflich tätige Landwirte, um ihnen mit der restriktiven Preispolitik einhergehende Einkommensverluste auszugleichen (ibid.: 58ff). Gleichzeitig wies die Kommission auf die Gefahr hin, dass die kleinen Betriebe nicht aus der Landwirtschaft ausscheiden würden, solange die Beihilfe ihre Verluste kompen-

\footnotetext{
54 Als Beispiel nannte die Kommission die schwierigen Verhandlungen über die Agrarpreise für 1985/86. Sie schlug für 1985/86 Preissenkungen für Getreide und Raps von 3,6\% vor. Nachdem Deutschland unter Berufung auf den Luxemburger Kompromiss Veto einlegte und damit kein Ratsbeschluss zustande kam, beschloss die Kommission eine Senkung der Preise für Getreide und Raps in Höhe von 1,8\% (von Urff 1986).
} 
siert. Die Zahlung sollte auf Landwirte begrenzt werden, deren landwirtschaftliches Einkommen unter einem gewissen Prozentsatz (z. B. $75 \%$ ) des vergleichbaren Einkommens (durchschnittlicher Bruttolohn außerlandwirtschaftlicher Arbeitnehmer in dem betreffenden Gebiet) liegt. Sie sollte mit der Zeit sinken und zur Vereinfachung der Verwaltung - als Pauschalsatz pro Produktionseinheit (Hektar oder Viehbestandseinheit) berechnet werden. Der Pauschalsatz sollte in Abhängigkeit vom Produktionstyp und regionalen Durchschnitt des Wirtschaftswertes je Produktionseinheit variieren. Schließlich sollte die Zahlung vorübergehend sein, ,giving the farmer a financial relief during some years in order to allow him to decide on his future and to make the necessary adjustments" (ibid.: 59). Diese Zahlung wurde somit als Anpassungshilfe konzipiert.

Im Rahmen der Regelung mit sozialer Ausrichtung brachte die Kommission eine Einkommensbeihilfe mit einem rein sozialen Charakter ins Spiel, mit der soziale Härten vermieden werden sollten, die womöglich aus den Strukturveränderungen resultierten. Gewährt würde sie nur den gegenwärtig wirtschaftenden Landwirten, deren Gesamteinkommen (Einkommen aus landwirtschaftlicher und außerlandwirtschaftlicher Tätigkeit) um einen bestimmten Prozentsatz unter dem vergleichbaren Einkommen - dem durchschnittlichen Bruttolohn außerlandwirtschaftlicher Arbeitnehmer in dem betreffenden Gebiet - oder noch niedriger liegt. Die Zahlung sollte dem Unterschied zwischen dem individuellen Gesamteinkommen und dem Prozentsatz des vergleichbaren Einkommens entsprechen. Dabei sollte von ihr ein Pauschalsatz abgezogen werden, der für die im selben Haushalt lebenden Familienmitglieder berechnet wird, die über außerlandwirtschaftliche Einkommen verfügen (ibid.: 60f).

Bei der Umwidmungsregelung handelte es sich um eine Zahlung für die Stilllegung landwirtschaftlicher Fläche mit dem Ziel, die Agrarproduktion zu vermindern. Die somit freigewordene Fläche sollte langfristig für nichtlandwirtschaftliche Zwecke, wie Aufforstung, Freizeitparks oder Schaffung ökologischer Schutzgebiete, genutzt werden. Grundsätzlich könnten alle Landwirte die Beihilfe beantragen. Ihre Höhe sollte von dem aufgegebenen Produktionsvolumen abhängen. Sie könnte aber auch dafür gezahlt werden, dass der Landwirt keine Überschusserzeugnisse mehr anbaut, sondern auf andere Erzeugnisse umstellt (ibid.: 61f).

Außer diesen Einkommensbeihilfen regte die Kommission (1985a: 52) im Grünbuch auch eine Zahlung für umweltfreundliche Praktiken an, die neben den positiven Effekten für die Umwelt auch zur Verminderung der landwirtschaftlichen Erzeugung beitragen würde: „Corresponding payments would at the same time support and diversify farmers' incomes and contribute to the control of production." Dieses waren einerseits Zahlungen für landwirtschaftliche Praktiken, die in die Landbewirtschaftung integriert werden könnten, wie Verringerung des Einsatzes von Dünge- und Pflanzenschutzmitteln. Diese Praktiken könnten gesetzlich vorgeschrieben werden (z. B. in Gebieten mit ausgeprägten Umweltproblemen) oder freiwillig sein. Andererseits könnte der Staat Flächen von Landwirten pach- 
ten bzw. kaufen, um sie für Umweltzwecke zu nutzen (insbesondere stillzulegen wie im Rahmen der Umwidmungsregelung).

Eine vollständige Finanzierung von „direct income aids“ aus dem EU-Haushalt wurde zu der Zeit nicht vorgesehen. Laut Kommission sollte sich aber die EU an ihrer Finanzierung beteiligen, weil die Last der Einkommensstützung der von ihr finanzierten Preispolitik zum Teil abnehmen würde. Darüber hinaus sah sie die gemeinschaftliche Finanzierung als komplementär zu den gemeinsamen Bestimmungen und Kriterien, um fairen Wettbewerb in der Landwirtschaft in der gesamten Union zu gewährleisten. Schließlich würden Umweltzahlungen sowohl im Interesse der Mitgliedstaaten als auch der Union liegen. Auch gab die Kommission die Solidarität mit den ärmeren EU-Regionen als Grund für die Partizipation der Union an der Finanzierung der Zahlungen an. Gleichzeitig sprach sie sich für eine nationale Beteiligung an der Finanzierung aus, um einen entsprechenden Grad der einzelstaatlichen Verantwortlichkeit für die Verwaltung und Kontrolle des Beihilfesystems zu sichern. Die Höhe der Unionsbeteiligung könnte in Abhängigkeit von der landwirtschaftlichen Situation in den einzelnen Mitgliedstaaten oder deren Finanzkraft variieren (ibid.: 56f).

In der dem Grünbuch folgenden Veröffentlichung „A future for Community agriculture", in der die Kommission die Ergebnisse der öffentlichen Konsultationen zur Ausgestaltung der GAP zusammenfasste, bemerkte sie, dass der Idee der „direct aids to incomes“ seitens der Landwirte mit Misstrauen begegnet wurde: „They believe that in agriculture, as in all other sectors, it is the market, and therefore prices, which should provide the yardstick of the profitability of the farm, and they cannot accept an arrangement which would leave many of them ,on welfare $_{\text {“، }}$ (Kommission 1985b: o.S.). In der Situation blieb sie im Hinblick auf einkommensstützende Zahlungen zunächst bei ihrer früheren Position, diese nur in bestimmten Fällen und ergänzend zur Preispolitik anzuwenden:

[S]erious difficulties stand in the way of generalized deployment of aid schemes of this kind, given the very diversified economic and structural situations in the Community at the present time, for administrative and budgetary reasons; on the other hand, direct aids of a more specific character could well form a valuable adjunct to market management or could help to temper certain structural problems. (ibid.)

Basierend auf dem Grünbuch schlug die Kommission im Jahr 1986 die Einführung einer Vorruhestandsregelung für Haupterwerbslandwirte verbunden mit der Aufgabe der landwirtschaftlichen Produktion vor. Dieser Vorschlag fand im Rat allerdings keine Zustimmung (von Urff 1987). In der Mittteilung vom Februar 1987 ,The Single Act: A new frontier for Europe“ kündigte die Kommission den Vorschlag von „a supplementary mechanism for supporting incomes“ auf EUEbene an, um eine bessere Balance ,between the imperatives of the market and 
the need for solidarity“ (Kommission 1987a: 12) zu erreichen. Sie übermittelte ihn dem Rat im April 1987; die Basis war das Grünbuch von 1985. Die vorgeschlagenen Einkommensbeihilfen waren als ein der Preispolitik untergeordnetes Instrument konzipiert. Zum einen regte die Kommission (1987b: 11) eine „gemeinschaftliche Einkommensbeihilfe" für potenziell leistungsfähige Betriebe an. Darunter waren solche Betriebe zu verstehen, die durch die Anpassungen der Preispolitik in Bedrängnis geraten waren, aber Überlebenschancen hatten. Die Beihilfe sollte aus dem EU-Haushalt (Garantieabteilung des EAGFL) ko-finanziert werden, auf fünf Jahre befristet und degressiv gestaffelt sein. Sie durfte nicht dazu führen, dass sich die Höhe des Einkommens der Begünstigten über 125\% des durchschnittlichen landwirtschaftlichen Einkommens der Region hinaus erhöht. Die Beihilfe erinnerte somit an die Regelung mit einer strukturpolitischen Komponente aus dem Grünbuch von 1985. Zum anderen schlug die Kommission (1987b: 11) eine Rahmenregelung für eine „einzelstaatliche Einkommensbeihilfe“ vor. Diese Beihilfe sollte sich auf den sozialen Bereich beschränken, das heißt nur an die in Schwierigkeiten geratenen Betriebe fließen. Das Einkommen der Begünstigten sollte sich dadurch nicht über das durchschnittliche landwirtschaftliche Einkommen der Region bzw. über $80 \%$ des landwirtschaftlichen Einkommens auf Landesebene hinaus erhöhen. Die Regelung mit sozialer Ausrichtung aus dem Grünbuch von 1985 sollte somit in die Kompetenz der Mitgliedstaaten fallen. Darüber hinaus griff die Kommission die Idee einer Vorruhestandsregelung für ältere Landwirte wieder auf, die durch die EU mitfinanziert werden sollte (Option Vorruhegehaltsregelung aus dem Grünbuch).

Die beiden vorgeschlagenen Einkommensbeihilfen würden zusammen mit den Maßnahmen der Preis- und Marktpolitik ein kohärentes und ausgewogenes Ganzes darstellen (Kommission 1987b: 12). Eine Regelung zu freiwilligen Flächenstilllegungen (Umwidmung aus dem Grünbuch) fand sich im Vorschlag der Bundesrepublik Deutschland wieder, der von der Kommission mit aufgenommen und dem Rat vorgelegt wurde. Auf dem Brüsseler Sondergipfel der Staats- und Regierungschefs im Februar 1988 wurde neben wichtigen Entscheidungen zu Agrarausgaben und Preispolitik (u. a. Agrarleitlinie) lediglich die Option Flächenstilllegungen (samt Kernpunkten) beschlossen sowie die Vorruhestandsregelung im Grundsatz angenommen (von Urff 1988). Im Hinblick auf die Vorschläge zu den übrigen Beihilfen konnte auf dem Brüsseler Sondergipfel keine Einigung erzielt werden. Der Europäische Rat forderte den Ministerrat lediglich auf, in Anlehnung an seine Schlussfolgerungen vom Juni des Vorjahres diesbezüglich eine Entscheidung bis zum 1. Juli 1988 zu treffen (Europäischer Rat 1988: 41). Im besagten Juni 1987 ließen die Staats- und Regierungschefs verlauten, dass aufgrund von negativen Auswirkungen der Maßnahmen der restriktiveren Preispolitik auf die landwirtschaftlichen Einkommen „die einzuführenden Reformen entsprechend den Vorschlägen der Kommission mit der Gewährung direkter und selektiver Einkommensbeihilfen einhergehen“ könnten, diese aber „subsidiären Charakter gegen- 
über der Preispolitik behalten müssten, keine Auswirkungen auf das Produktionsniveau haben dürften und im Gemeinschaftsrahmen zu erfolgen hätten “ (Europäischer Rat 1987: 10). Somit kann festgestellt werden, dass unter den Regierungsvertretern der Mitgliedstaaten grundsätzlich Konsens darüber bestand, dass die Einkommensbeihilfen notwendig waren. Die Deadline vom 1. Juli 1988 wurde allerdings vom Ministerrat nicht eingehalten (s. unten), was auf kontroverse Diskussionen unter den Mitgliedstaatsvertretern hindeutet.

Im Oktober 1987 bezog auch das Europäische Parlament Stellung zu den Kommissionsvorschlägen. Um die Auswirkungen der restriktiveren Preispolitik abzufedern und die landwirtschaftlichen Einkommen zu sichern, sprach sich das Parlament für die Einkommensbeihilfen aus. Es betonte die Wichtigkeit der Unionsbeteiligung an ihrer Finanzierung:

Schließlich ist es nach Auffassung des Parlaments von wesentlicher Bedeutung, daß die Gemeinschaft die weniger reichen Mitgliedstaaten bei der Finanzierung von Beihilfesystemen für landwirtschaftliche Einkommen unterstützt, um sicherzustellen, daß das bestehende Gefälle der regionalen Einkommen aus landwirtschaftlicher Tätigkeit nicht noch größer wird und den Landwirten in den reicheren Mitgliedstaaten nicht ein ungerechter Wettbewerbsvorteil zuteil wird. (Kommission 1987c: 49)

Im Mai 1988 legte die Kommission einen überarbeiteten Vorschlag zu direkten Einkommensbeihilfen vor. Sie fasste die Regelung für strukturpolitische EUBeihilfen und die Rahmenregelung für einzelstaatliche soziale Beihilfen zu einer einzigen Verordnung zusammen. Beihilfefähig sollten die strukturell und wirtschaftlich schwächsten Familienbetriebe sein. Die Beihilfe sollte für fünf Jahre gewährt werden; eine Höchstgrenze sowie die Möglichkeit, den Beihilfesatz auf pauschaler Grundlage zu berechnen, waren vorgesehen. Die Unionsbeteiligung an der Finanzierung der neuen Beihilfen war nur im Falle von Beihilfen für Haupterwerbsbetriebe geplant; die Beträge aus dem EU-Haushalt sollten aber mit der Zeit sinken (Kommission 1988a: 51). Am 13. Oktober 1987 billigte das Europäische Parlament den Vorschlag, wenn auch mit gewissen Änderungen. Es unterstrich, dass das Ziel der Beihilfe nicht darin bestehen soll, die Preispolitik zu ersetzen. Sie sollte für zunächst fünf Jahre gewährt werden und nicht, wie im Kommissionsvorschlag vorgesehen, für höchstens fünf Jahre (Kommission 1988b: 42). Trotz des überarbeiteten Vorschlags gestalteten sich die Verhandlungen im Rat der Landwirtschaftsminister langwierig. Der Zeitzeuge von Urff (1989: 90) schrieb:

Die Beratung ist aufgrund kontroverser Auffassungen im Ministerrat bislang sehr schleppend erfolgt. Während ein Teil der Mitgliedstaaten ein allgemeines Beihilfesystem einführen möchte, sind andere der Meinung, daß es nur darum gehen könne, die Einkommens- 
konsequenzen aufgrund der Anpassung der gemeinsamen Agrarpolitik während einer Übergangsperiode zu mildern.

Die Verordnung zur Einführung landwirtschaftlicher Einkommensbeihilfen wurde schließlich am 21. März 1989 vom Rat angenommen (s. Kapitel 5.2). Parallel dazu reformierte Schweden seine auf Preisstützung basierte Agrarpolitik, was zeigt, dass eine radikale Agrarreform zu jener Zeit durchaus möglich war (vgl. Box 9).

Zusammenfassend lässt sich feststellen, dass staatliche Zahlungen an landwirtschaftliche Erzeuger bereits seit den Anfängen der GAP Thema im agrarpolitischen Diskurs waren. Die wesentliche Frage war zunächst, ob sie überhaupt eingeführt werden sollten. Die Kommission hielt sie für kostenintensiv, bürokratisch und strukturkonservierend und somit höchstens als Ergänzung zur Preispolitik und in begrenzten, klar definierten Fällen denkbar. Das Hauptaugenmerk in der Debatte galt zwar nicht der Frage der Entkopplung der Zahlungen von der Produktion und den Marktpreisen, diese zog sich aber als Motiv durch die Texte der Kommission und wurde bereits 1975 von ihr angesprochen. Letztendlich waren die ersten breiter aufgestellten einkommensstützenden Zahlungen in der EU von Produktion und Preisen entkoppelt. Die landwirtschaftlichen Einkommensbeihilfen von 1989 erfüllten die Kriterien für ,decoupled income support" der Green Box und als solche wurden sie notifiziert (Swinbank et al. 2004: 23). Somit sind sie als Direktzahlungen im Sinne dieser Arbeit anzusehen.

Box 9: Reform der Agrarpolitik in Schweden von 1990

Mit der im Jahr 1990 beschlossenen Reform wurden in Schweden Exportsubventionen und Marktregulierungen inklusive der Preisgarantien (ggf. nach einer Übergangszeit) abgeschafft (der Außenschutz blieb bestehen). Um die Auswirkungen dieser Reform auf das landwirtschaftliche Einkommen abzumildern, gewährte man den Landwirten vier Jahre degressive (mit der Zeit sinkende) Ausgleichszahlungen („compensatory payments“). Die Zahlungen wiesen auch agrarstrukturelle Elemente auf: Bei Aufgabe der landwirtschaftlichen Produktion für mindestens fünf Jahre erhielt der Landwirt die gesamten Ausgleichsbeträge als einmalige Zahlung. Als Berechnungsgrundlage für die Zahlungen wurde die Fläche des Betriebes genommen. Rabinowicz (2003: 8) hebt hervor, dass trotz des radikalen Charakters der Reform die Zahlungen „relatively modest“ waren. Weiterhin bemerkt sie: „The underlying philosophy was that compensations/ adjustment measures should be paid not because past policy automatically creates an entitlement for future support but because the society has an obligation, in agriculture and elsewhere, to abolish past policies under socially acceptable conditions" (ibid.: 10). Die schwedische Reform war nicht durch den Haushaltsdruck bedingt. Im Fokus der Kritik standen nicht die Staatsausgaben für die bis- 
herige Agrarpolitik, sondern ihre indirekten Auswirkungen, wie Wohlfahrtsverluste und Inflation. Die treibende Kraft hinter der Reform war das schwedische Finanzministerium, das zu der Zeit auch in anderen Wirtschaftsbereichen des Landes Strukturreformen durchführte. Die politischen Rahmenbedingungen für eine Agrarreform waren laut Rabinowicz günstig: Die regierenden Sozialdemokraten waren nicht mehr auf die Unterstützung der Zentrumspartei angewiesen, die traditionell die Interessen der Landwirte vertrat. Fast alle Parteien unterstützten die Reform, denn die bisherige Agrarpolitik wurde nicht nur in Wirtschaftskreisen, sondern auch in der breiten Öffentlichkeit sehr kritisch betrachtet. Somit verlor sie ihre Legitimität, urteilt Rabinowicz (2003: 11). Sie weist auBerdem auf die frühere Agrarreform aus dem Jahr 1967 hin, aus deren Scheitern wichtige Lehren gezogen wurden.

\subsection{Abkehr von der Preisstützung: Ausgleichszahlungen}

Die negativen Folgen der bisherigen Agrarpreispolitik in Bezug auf Überproduktion und Haushaltsbelastung wurden bereits in den 1970er-Jahren sichtbar. Beispielsweise wies die Kommission im Jahr 1973 auf „heavy surpluses in the dairy sector" (Kommission 1973: 7) hin. Die vom Rat in den 1980er-Jahren beschlossenen Anpassungen der Politik, wie Produktionsquote für Milch, Mitverantwortungsabgaben oder Garantieschwellen, „did not solve the fundamental problems of the CAP“" (Kommission 1994b: 17). Zwischen 1989 und 1991 stiegen die Ausgaben für die GAP um $30 \%$ an. Zum Ende des Jahres 1990 betrugen die öffentlichen Lagerbestände bei Getreide 14,4 Mio. Tonnen, bei Milchprodukten 600.000 Tonnen und bei Rindfleisch 530.000 Tonnen (ibid.). Schmitt und Tangermann (1984: 788) kritisieren das Handeln der Politik Mitte der 1980er-Jahre mit folgenden Worten:

Die EG-Agrarpolitiker scheinen sich zur Zeit nicht auf den Rückzug aus einer überzogenen Preisstützung vorzubereiten, sondern sie suchen das gegenwärtige Stützungsniveau und seine weitere Anhebung durch Hilfskonstruktionen abzusichern, die die negativen Nebeneffekte unterdrücken oder verdecken sollen. Einzelbetriebliche Kontingente, weitere Importbeschränkungen und die Erschließung neuer Finanzquellen für die Bedienung steigender Haushaltslasten sind solche Hilfskonstruktionen.

Wie die Kommission (1994b: 11) feststellt: „Despite successive attempts at reform, market price support has remained the main policy instrument during the three decades of the common agricultural policy." Zu beachten ist jedoch, dass vor dem Jahr 1992 Zahlungstypen eingeführt wurden, die bis heute Teil des GAP- 
Instrumentariums darstellen, so die Agrarumweltzahlungen, die ersten Ausgleichszahlungen für die Einkommensverluste aufgrund der Senkung der Preisstützung oder die Zahlungen für benachteiligte Gebiete. Mit Blick auf solche soziostrukturellen Maßnahmen bemerkt von Urff (1988: 113), dass sich dort die Entwicklung etablierte, „diese mehr und mehr zu einem Instrument der Einkommenspolitik fortzuentwickeln, sie gleichzeitig aber auch in den Dienst der Produktionsbegrenzung zu stellen“. In Abwesenheit der breiter aufgestellten einkommensstützenden Zahlungen übernahmen also andere Zahlungen ihre Rolle. Beachtenswert ist darüber hinaus, dass die Anzahl der Agrarzahlungen im Laufe der Jahre stark anstieg. Trotz massiver Kritik, mehreren Reformvorschlägen und beschlossenen Anpassungen blieb es aber bis Anfang der 1990er-Jahre bei der uneingeschränkten Dominanz der Markt- und Preispolitik (Moyer und Josling 1990).

Erst die nach dem damaligen Agrarkommissar benannte MacSharry-Reform von 1992 brachte diesbezüglich eine Änderung. Die ersten Reformvorschläge gelangten Ende 1990 an die Öffentlichkeit (von Urff 1991: 104). Anfang Februar 1991 wurde mit der Kommissionsmitteilung „Die künftige Entwicklung der Gemeinsamen Agrarpolitik“ eine „schonungslose Analyse“ der bisherigen Agrarpolitik veröffentlicht, die als Grundlage für die Reform von 1992 diente (ibid.: 105). Als Ziel der Reform erklärte die Kommission, eine ausreichend große Zahl der Landwirte zum Bleiben auf dem Land bewegen zu wollen, denn es gäbe keinen anderen Weg, um ,the natural environment, traditional landscapes and a model of agriculture based on the family farm as favoured by the society generally" zu erhalten (Kommission 1991a: 9f). Es müsse anerkannt werden, dass Landwirte zwei wichtige Funktionen gleichzeitig erfüllen: Zum einen ist es die Produktion von Agrarrohstoffen für die menschliche Ernährung, aber auch für andere Verwendungen und zum anderen die Erbringung von Umweltschutzleistungen im ländlichen Raum (ibid.: 10). Hier knüpfte die Kommission an ihre Überlegungen aus dem Grünbuch 1985 an und baute diese aus.

Sie stellte fest, dass trotz massiv gestiegener GAP-Ausgaben die Einkommen in der Landwirtschaft stagnierten. Dieses zeige laut Kommission, dass „the mechanisms of the CAP as currently applied are no longer in a position to attain certain objectives prescribed for the agricultural policy under Article 39 of the Treaty of Rome" (ibid.: 3). Der Fehler der GAP läge darin, dass sie konzipiert wurde, als in der EU Produktionsdefizite herrschten; entsprechend wurden die agrarpolitischen Maßnahmen ausgerichtet. Die veränderte Situation Anfang der 1990er-Jahre erfordere eine Revision dieser Maßnahmen, wobei die Kommission die bisherigen Reformen als unzureichend erklärte. In diesem Zusammenhang wies sie u. a. darauf hin, dass $80 \%$ der Finanzmittel des EAGFL an nur $20 \%$ der Betriebe flieBen. Sie sprach auch von einer Vertrauenskrise innerhalb der EU sowie von einer Krise im Verhältnis mit Handelspartnern (ibid.: 9). Zur Wiederherstellung des Marktgleichgewichts müssten - neben der Förderung der Extensivierung der landwirtschaftlichen Produktion - die Stützpreise gesenkt werden. Dieses Vorhaben 
ließe sich allerdings nur dann durchsetzen, wenn es durch umfängliche, produktionsneutrale Maßnahmen zum Ausgleich der entstandenen Einkommensminderungen begleitet würde (,compensatory aid“). Die Einkommensbeihilfen von 1989 konnten diese Rolle nicht übernehmen, weil ,they have been tacked on to a system whose mechanisms have not changed“ (ibid.: 8). Daraus leitete die Kommission die Schlussfolgerung ab, dass „direct aids through premiums “, 55 um wirksam zu sein, nicht nur Teil der entsprechenden Marktorganisationen, sondern ihr Kernelement sein sollten.

Ein wichtiges Element des ursprünglichen Kommissionskonzeptes war die Modulation. So nannte die Kommission (1991a: 12) die Differenzierung der Höhe der geplanten Zahlungen entsprechend den ausgewählten Faktoren einschließlich der Betriebsgröße: „Direct aid measures, based generally on the livestock numbers or area of farms and modulated in function of factors such as size, income, regional situation or other relevant factors, should be integrated into the market organisations so as to guarantee the producers income." Grundsätzlich beabsichtigte die Kommission nur Betriebe mit einer Fläche unterhalb einer festgesetzten Grenze voll für die reformbedingten Einkommensverluste zu entschädigen. Am Beispiel von Getreide erläuterte sie ihr Konzept wie folgt:

Full compensation would apply up to a certain level of area and partial compensation thereafter on a degressive basis. Beyond a certain size the payment of the aid per hectare would be conditional on the withdrawal from production of part of the area devoted to arable crops, as defined each year in function of the state of the market. The land withdrawn from production could be used for non-food production. The level of aid per hectare would vary each year in function of developments on the market and of productivity. (ibid.: 13f)

Zugleich setzte sich die Kommission auch mit der antizipierten Kritik zur geplanten Reform auseinander. Dem Argument, dass die degressive Staffelung der Zahlungen mit zunehmender Betriebsgröße diskriminierend sei, setzte sie entgegen, dass vielmehr die Marktordnungsmechanismen in ihrer derzeitigen Form diskriminierend seien, weil sie größere und intensiv wirtschaftende Betriebe stärker förderten. Zum Argument, mit dem neuen Stützungsmechanismus würden sich die Haushaltsausgaben erhöhen, erklärte die Kommission, dass aus volkswirtschaftlicher Sicht dieser vorteilhafter sowie effizienter sei (ibid.: 16f). Vor diesem Hintergrund empfahl sie seine frühestmögliche Einführung. Er sollte jedoch schrittweise umgesetzt werden, um den Mitgliedstaaten und den Landwirten die Möglichkeit zu geben, sich der neuen Situation anzupassen (ibid.: 13).

\footnotetext{
55 In der Kommissionsmitteilung von 1991 ist der Begriff „Direktzahlungen“ („direct payments“) nicht zu finden. Im Kommissionsvorschlag von 1991 wird er ein einziges Mal verwendet.
} 
Das Ziel der Kommissionsmitteilung vom Februar 1991 war „to present the main outlines of the proposed reform and push the debate forward" (Cunha und Swinbank 2011: 75). Im Juli 1991 folgte der offizielle Vorschlag mit detaillierten Ausführungen und Kostenangaben, dessen Kernstück die Senkung des Preisstützungsniveaus begleitet durch „direct compensatory payments“ zum Ausgleich der daraus resultierenden Einkommensverluste darstellte (Kommission 1991b). Die Kommission schlug insbesondere vor:

- Bei Getreide sollte der Richtpreis innerhalb von drei Jahren schrittweise um $35 \%$ auf 100 ECU/Tonne gesenkt werden. Der Interventionspreis sollte nur noch bei 90 ECU/Tonne und der Schwellenpreis bei 110 ECU/Tonne liegen. Bei Rindfleisch war eine Kürzung des institutionellen Preises um 15\%, bei Butter und Magermilchpulver um durchschnittlich $10 \%$ vorgesehen.

- Bei Getreide, Rindfleisch und Milch sollten die Ausgleichszahlungen („,compensatory payments“ bzw. ,premiums“) die Preisstützung ergänzen. Bei Ölsaaten und Eiweißpflanzen sollte die Stützung vollständig über solche Zahlungen erfolgen. Die Zahlungen für Ackerbaukulturen waren als Flächenbeihilfe, die Zahlungen für Rindfleisch und Milch als je Tier gezahlte Prämie konzipiert. Ihnen zugrunde gelegt wurden die historischen, regionalen Durchschnittserträge (bzw. Herdengrößen). Beispielsweise sollte sich der Beihilfesatz pro Hektar bei Getreide aus der Multiplikation des „olympischen“ Durchschnitts der regionalen Erträge der letzten fünf Wirtschaftsjahre (1986/87 bis 1990/91) mit der Differenz zwischen dem alten und neuen Preis (55 ECU/Tonne in der Endstufe) errechnen. Die Ausgleichszahlungen sollten somit von Region zu Region variieren. Ihre durchschnittliche Höhe in der Union bei Getreide bezifferte die Kommission auf 253 ECU/Hektar. Für Erzeuger von Hartweizen wurde ein Zuschlag von 300 ECU/Hektar vorgesehen. Bei Rindfleisch und Schaffleisch handelte es sich um keine neuen Zahlungen, sondern um Aufstockung und ggf. Umgestaltung der bestehenden Zahlungen (Sonderprämie für männliche Rinder und Mutterkuh- bzw. Mutterschafprämie, s. Kapitel 5.2).

- Die Ausgleichszahlungen für Getreide, Ölsaaten und Eiweißpflanzen sollten auf die sogenannte regionale Grundfläche begrenzt werden. Diese umfasste die mit diesen Kulturen bestellten sowie die stillgelegten Flächen einer Region im Basiszeitraum 1989 bis 1991. Überschritt die Fläche, für die Beihilfen oder Stilllegungsprämien im bestimmten Jahr beantragt wurden, die Grundfläche, sollten die Ausgleichzahlungen gekürzt werden. Um die Zahlungen zu erhalten, sollten Erzeuger einen Teil ihrer mit den betreffenden Kulturen bebauten Fläche aus der Produktion nehmen (zunächst $15 \%$ ). Die 
Stilllegung sollte in der Regel rotierend erfolgen. Für die stillgelegte Fläche wurde eine Ausgleichszahlung in Höhe der für Getreide in der Endstufe geltenden Ausgleichszahlung vorgesehen. Allerdings sollten Betriebe mit über 50 Hektar relevanter Anbaufläche Ausgleichszahlungen für max. 7,5 Hektar stillgelegte Fläche bekommen. Bei Milch und Rindfleisch sollten neben regionalen Herdengrößen, die das Gegenstück der Grundfläche bei Ackerbaukulturen darstellten, auch einzelbetriebliche Obergrenzen gelten (40 bzw. 90 Tiere je Betrieb); außerdem sollten die Prämien nur dann gewährt werden, wenn eine gewisse Besatzdichte je Hektar nicht überschritten würde. Bei besonders niedriger Besatzdichte wurden Zusatzprämien vorgesehen.

- Die Milchquote sollte über das Jahr 1992 hinaus verlängert und um $3 \%$ gekürzt werden (von der Kürzung sollten kleinere Milcherzeuger allerdings ausgenommen werden). Als Ausgleich sollte den betroffenen Erzeugern für zehn Jahre eine aus dem EU-Haushalt finanzierte Beihilfe gewährt werden, wobei eine Aufstockung aus nationalen Mitteln möglich war. Diese Zahlungen sollten auf Basis von Schuldverschreibungen („,bonds“) erfolgen. Die Milcherzeuger sollten die Möglichkeit haben, die „,bonds“ entweder zu behalten und die entsprechenden Zahlungen selbst einzukassieren oder diese am Privatmarkt zu verkaufen.

- Für Landwirte, deren Anbaufläche einem jährlichen Ernteaufkommen von höchstens 92 Tonnen Getreide entsprach (sogenannte Kleinerzeuger) wurde eine vereinfachte Regelung vorgeschlagen, nach der sie auf Stilllegung verzichten und die Ausgleichszahlung in Höhe der für Getreide geltenden Ausgleichszahlung erhalten konnten. Diese Zahlung würde ihnen für die mit den betreffenden Kulturen bebaute Fläche gewährt, ungeachtet des tatsächlichen Anbaumixes.

- Es wurde eine Reihe von flankierenden Maßnahmen vorgesehen, die durch die Abteilung Garantie des EAGFL ko-finanziert werden sollten. Dieses waren Zahlungen für Landwirte, die sich bereit erklären würden, umweltfreundliche Produktionspraktiken durchzuführen (z. B. Verringerung des Einsatzes von Pflanzenschutzmitteln) oder auf umweltschädigende Methoden zu verzichten bzw. sie rückgängig zu machen (z. B. Umbruch von Grünland). An solchen Zahlungen sollte sich die EU mit bis zu 250 ECU/Hektar beteiligen. Die Agrarumweltprogramme, in deren Rahmen diese Zahlungen gewährt werden sollten, sollten mehrjährig aufgestellt sein. Auch die langfristige Stilllegung landwirtschaftlicher Flächen und ihre Nutzung für umweltspezifische Zwecke sollte prämiert werden. 
Höhere Zahlungen als bisher wurden für die Aufforstung und die Vorruhestandsregelung vorgesehen. Letztere sollte für die Mitgliedstaaten obligatorisch sein.

- Im Hinblick auf die Reformkosten ging die Kommission von einer jährlichen Mehrbelastung der Abteilung Garantie des EAGFL von 2,3 Mrd. ECU aus, womit diese Ausgaben unter der Agrarleitlinie bleiben würden. Die Ausgaben für die flankierenden Maßnahmen schätzte die Kommission auf etwa 4 Mrd. ECU für fünf Jahre.

Die MacSharry-Reform wurde im Jahr 1992 beschlossen und trat bereits 1993 in Kraft. Der Rat folgte grundsätzlich dem Kommissionsvorschlag. Zu den wichtigsten von ihm vorgenommenen Änderungen gehörte die Kürzung des Interventionspreises für Butter um nur 5\% statt 15\%. Damit entfiel die Notwendigkeit, als Ausgleich „premiums“ für Milchbauer einzuführen. Der Betrag für die Berechnung der Ausgleichszahlung für Getreide sollte bei 45 und nicht 55 ECU/Tonne liegen und ein hoher Außenschutz (Richtpreis bei 110 ECU/Tonne und Schwellenpreis bei $155 \mathrm{ECU} /$ Tonne) weiter gewährt werden (von Urff 1993). Das Konzept der „bonds“ im Milchsektor wurde nicht gebilligt (Little et al. 2004). Auch die vorgeschlagene Obergrenze bei der Gewährung der Ausgleichszahlung für die Flächenstilllegung wurde nicht übernommen, nicht zuletzt unter Druck von Deutschland, das sich für einen vollen Ausgleich der Einkommensverluste unabhängig von der Betriebsgröße aussprach (Cunha und Swinbank 2011).

Die im Zuge der MacSharry-Reform eingeführten Ausgleichszahlungen waren von der Produktion nicht vollständig entkoppelt. Zwar lagen dem Beihilfesatz historische regionale Erträge zugrunde. Außerdem wurden die neuen Zahlungen auf die sogenannte regionale Grundfläche begrenzt, die die stillgelegten sowie die mit Getreide, Ölsaaten und Eiweißpflanzen bestellten Flächen einer Region in der Vergangenheit umfasste. Allerdings wurden die Ausgleichszahlungen für die Fläche gewährt, die mit den relevanten Kulturen (Getreide, Ölsaaten und Eiweißpflanzen) bestellt war oder nach der Verordnung (EWG) Nr. 1765/92 stillgelegt wurde. Dieses bedeutete, dass der Landwirt, wenn er die Zahlungen nicht verlieren wollte, die betreffenden Erzeugnisse weiter produzieren musste (laut Art. 10 der Verordnung Nr. 1765/92 musste zumindest die Aussaat erfolgen). Baffes und de Gorter (2005: 24) weisen darauf hin, dass die Tatsache, dass die Begrenzung der Ausgleichszahlungen auf der regionalen bzw. nationalen Ebene und nicht einzelbetrieblich angesetzt war, einen Anreiz für Landwirte darstellte, ihre Flächen über die individuelle beihilfefähige Fläche hinaus mit den relevanten Kulturen zu bestellen. Denn eine mögliche Strafe erfolgte erst ex post und bezog sich auf die gesamte relevante Anbaufläche. Als teilweise entkoppelte Zahlungen qualifizierten sich die Ausgleichszahlungen der MacSharry-Reform als Blue-Box-Maßnahme.

Im Zuge der Reform von 1992 stieg die agrarpolitische Bedeutung der Direktbeihilfen an Landwirte, was sich in der Zusammenstellung der Agrarausgaben widerspiegelte (vgl. Tabelle 6). 
Tabelle 6: Gesamtausgaben für Marktmaßnahmen und Direktbeihilfen bei pflanzlichen Erzeugnissen vs. Ausgaben für Direktbeihilfen in der EU in ausgewählten Jahren

\begin{tabular}{lrrr} 
& 1990 & 1995 & 1998 \\
\hline Direktbeihilfen (ECU) & 592.000 .000 & 10.905 .000 .000 & 14.996 .000 .000 \\
$\begin{array}{l}\text { EAGFL, Abteilung Garantie } \\
\text { (ECU) }\end{array}$ & 16.802 .000 .000 & 23.466 .500 .000 & 26.316 .000 .000 \\
\hline
\end{tabular}

Quelle: Haushaltsplan 1990, (S. 292, 434, 506), Haushaltsplan 1995 (S. 359, 570, 572), Haushaltsplan 1998 (S. 359, 573, 574).

Anmerkungen: Direktbeihilfen umfassen die Einkommensbeihilfen 1989, Hektarbeihilfen der MacSharry-Reform und Beihilfen für Hartweizen. Außer der Einkommensbeihilfe sind die Direktbeihilfen Teil der Ausgaben der Abteilung Garantie des EAGFL.

Zum Zeitpunkt der Entscheidung über die Einführung von ,direct compensatory payments“ im Jahr 1992 wurde keine explizite Aussage zu ihrer Zukunft gemacht (Swinbank und Tangermann 2004: 57). Allerdings sprach die Kommission (1993: 11) bereits ein Jahr später von dauerhaften Ausgleichszahlungen („,permanent compensatory aid“). Im Jahr 1994 stellte sie ihre Vision für die zukünftige Entwicklung der GAP, inklusive der Einkommensstützung vor (vgl. Box 10). Wie Tangermann (1998: 35) schon im Jahr 1998 feststellte, markiert die MacSharryReform eher den Anfang als das Ende der fundamentalen Reform der EUAgrarpolitik.

Box 10: Kommissionspapier zur EU-Agrarpolitik für das 21. Jahrhundert

Die durch den Abbau der Preisstützung entstandenen Einkommensverluste sollten den Landwirten durch Ausgleichszahlungen kompensiert werden. Angesichts der Tatsache, dass ,[t]he need for compensation for decreases in prices will differ very much between Member States depending on the severity of the structural adjustment problems, and according to differences in pension schemes, unemployment benefits and other social provisions" sollte die Verantwortung für die direkte Einkommensstützung, u. a. in Bezug auf die Vergabekriterien, Höhe und Laufzeit der Zahlungen, in die Kompetenz der Mitgliedstaaten fallen (ibid.: 34). Die Zahlungen könnten somit z. B. im Hinblick auf ihre Höhe zwischen den Mitgliedstaaten variieren. Die Kommission berief sich dabei auf das Subsidiaritätsprinzip und stellte fest, dass „,[w]hen it comes to social and incomes policies, there is in general little, if any, a priori evidence that better results can be achieved with responsibility placed at the Community level“" (ibid.: 33). Die Zahlungen sollten von der aktuellen Erzeugung entkoppelt sein und den Wettbewerb zwischen den Mitgliedstaaten nicht verzerren. Insbesondere sollten „decoupled income payments“ möglich sein, die als solche Zahlungen definiert 
wurden, die die Kriterien der WTO-Green Box erfüllen (ibid.: 35). Als Ausnahme vom Entkopplungsprinzip sollten ,payments to achieve rural and environmental objectives" gestattet sein (ibid.).

Die Verantwortung der Mitgliedstaaten für die Einkommensstützung bedeutete für die Kommission nicht, dass die Zahlungen ausschließlich aus den nationalen Haushalten finanziert werden müssen. Allerdings sollten die Übertragungen aus dem EU-Haushalt über einen Zeitraum von höchstens zehn Jahren gewährt werden und mit der Zeit sinken. Außer für die direkte Einkommensstützung könnten EU-Gelder für Maßnahmen zur Förderung der ländlichen Entwicklung, des Umweltschutzes und der agrarstrukturellen Anpassungen verwendet werden (ibid.). Die Kommission vertrat die Überzeugung, dass „, [u]nder the new system, support may be more efficiently targeted to those most likely to be in need from a social or economic point of view" (ibid.: 37).

Im Jahr 1995 kündigte die Kommission die Notwendigkeit einer weiteren Reform der EU-Agrarpolitik vor dem Hintergrund der bevorstehenden Herausforderungen an:

$[\mathrm{N}]$ ew challenges are already visible and will determine the shape of the CAP for the first decades of the 21st century: increasing market imbalances in the EU after the turn of the century (and in some cases perhaps even before) [...]; the new financial perspectives after 1999; the next multilateral round of trade negotiations, starting in 1999; the internal debate on the stronger integration of environmental aspects into the CAP; the continuous challenge of rural development; and, last not least, the need for simplification. The time has come to reflect on these challenges and to prepare the choices for the next century. (Kommission 1995: 36)

In dieser Veröffentlichung sprach sich die Kommission für eine Weiterentwicklung des Reformansatzes von 1992 aus (ibid.: 23). Sie schlug vor, die Ausgleichszahlungen beizubehalten, sie aber stärker mit ökologischen und sozialen Belangen zu verzahnen. Darüber hinaus sollte die GAP vereinfacht werden, was die Kommission u. a. durch Einräumen größerer nationaler Gestaltungsspielräume erreichen wollte. Einer in Wissenschaftskreisen geforderten ,radikalen Agrarreform“ mit einer auf das Weltmarktniveau abgesenkten Preisstützung, degressiven, entkoppelten Ausgleichszahlungen sowie "direct income support payments“ und „payments for environmental services“ auf Ebene der Mitgliedstaaten mit eventueller Ko-Finanzierung durch die EU - erteilte die Kommission zu der Zeit eine Absage, weil sie „would imply a number of social and environmental risks which, at least in some regions, could lead to quite negative effects" (ibid.: 22). Entsprechend bestand die in der „Agenda 2000“ formulierte agrarpolitische Strategie in 
„deepening and extending the 1992 reform through further shifts from price support to direct payments, and developing a coherent rural policy to accompany this process" (Kommission 1997: 29). Gleichzeitig empfahl die Kommission, den Landwirten in den Beitrittsländern keine Ausgleichszahlungen im Sinne der MacSharry-Reform zu gewähren, denn sie mussten keinen Abbau der Preisstützung erleiden. Vielmehr würden dort nach dem Beitritt die Agrarpreise eher steigen. Als Fazit stellte die Kommission (1995: 26) fest: „[A] strong increase in incomes for farmers alone through direct (compensatory) payments (which would not correspond to any price cut and would in some cases even supplement price increases) risks to create income disparities that could rapidly lead to social unrest in the countries and regions concerned."

Den konkreten Vorschlag für die neue Reform legte die Kommission im März 1998 vor (Kommission 1998). Neben den schon 1995 aufgelisteten Herausforderungen für die GAP nannte die Kommission die Tatsache als problematisch, dass die Verteilung der Stützung ,somewhat unequally“ sei, weil sie sich auf Regionen und Erzeuger konzentrierte, „who are not among the most disadvantaged“ (ibid.: 3). Dieses zu korrigieren vermochte die Reform von 1992 nur sehr begrenzt, bemängelte die Kommission. Auch wies sie auf das schlechte Image der GAP in der Gesellschaft hin und machte sich zur Aufgabe, es zu verbessern: „Making the CAP more acceptable to the citizen in the street, to the consumer, is one of our primary tasks in the years ahead" (ibid.). Sie stellte zudem das EUModell der multifunktionalen Landwirtschaft dar (vgl. Box 11). Im Hinblick auf die Neugestaltung des agrarpolitischen Instrumentariums schlug die Kommission insbesondere vor (Kommission 1998):

- Bei Getreide sollte der Interventionspreis in einem Schritt um 20\% gesenkt werden. Die Rolle der Intervention sollte nicht mehr sein, Preise auf hohem Niveau zu garantieren, sondern als Sicherheitsnetz zu fungieren. Die Preissenkung sollte durch eine Erhöhung der Direktzahlungen (,direct payments") teilweise ausgeglichen werden. Die bis jetzt höhere Zahlung für Ölsaaten sollte an die für Getreide angeglichen werden. Für Eiweißpflanzen wurde eine zusätzliche Direktzahlung vorgesehen.

- Die Milchquote sollte im Jahr 2006 auslaufen. Die Interventionspreise für Butter und Magermilchpulver sollten ab 2000 in vier gleichen Schritten um insgesamt 15\% reduziert werden. Als Ausgleich wurden „direct payments“ für Milchkühe und die Erhöhung der Milchquote um $2 \%$ vorgeschlagen.

- Bei Rindfleisch sollte das Stützungsniveau um insgesamt 30\% gesenkt und die in der gesamten Union einheitlichen „,direct payments “ für Mastrinder und Mutterkühe erhöht werden. Ein Teil des Ausgleichs plante die Kommission, den Mitgliedstaaten in Form von aus der Garantieabteilung des EAGFL finanzierten „envelo- 
pes“ zuzuweisen, deren Umfang von der nationalen Produktionsmenge abhängt. Die Länder sollten selbst bestimmen, ob sie diese Finanzmittel zur Aufstockung der bestehenden Tierprämien oder zur Gewährung einer Grünlandprämie verwenden.

- Die verpflichtende Flächenstilllegung sollte als Instrument beibehalten, ihr Satz aber auf $0 \%$ festgesetzt werden. Freiwillige Flächenstilllegung sollte möglich sein.

- Die Kommission brachte eine Kürzung der Direktzahlungen für Großbetriebe ins Spiel, die alle EU-Mitgliedstaaten verpflichtend einführen sollten. Sie begründete diesen Schritt mit dem Streben, „,to avoid the reform of the CAP becoming a further reason for handing out over-generous public subsidies to agricultural holdings“" (Kommission 1998: 6). Für den Betrag zwischen 100.000 und 200.000 ECU pro Betrieb und Jahr sollte eine Kürzung um 20 \% und für darüber hinausgehende Beträge um $25 \%$ vorgenommen werden. Außerdem wollte die Kommission die Mitgliedstaaten ermächtigen, die Direktzahlungen um maximal 20\% zu kürzen, wenn im Betrieb der Arbeitskraftbesatz unterhalb der von ihnen festgesetzten Grenze liegt (sogenannte Modulation). Die somit gewonnenen Mittel sollten im jeweiligen Land für die Maßnahmen der 2. Säule verwendet werden. Ursprünglich hatte die Kommission vor, eine individuelle Obergrenze für alle Direktzahlungen einzuführen (Kommission 1997: 32).

- Die Mitgliedstaaten sollten ermächtigt werden, die Gewährung der Direktzahlungen von der Einhaltung bestimmter Umweltauflagen abhängig zu machen und, soweit diesen nicht entsprochen wird, Kürzungen vorzunehmen (Cross-Compliance).

- Die gestärkten Maßnahmen zur ländlichen Entwicklung und die Agrarumweltprogramme sollten in der 2. Säule der GAP gebündelt werden.

Die Reform wurde im Jahr 1999 beschlossen und trat ab 2000/01 in Kraft. Zu den wichtigsten vom Ministerrat vorgenommenen Änderungen des Kommissionsvorschlags gehörte die Senkung des Interventionspreises für Getreide um $20 \%$ in zwei Schritten sowie die Verschiebung der Reform bei Milch. Bei Rindfleisch wurde die „envelope“-Lösung stark begrenzt und eine neue Schlachtprämie beschlossen. Von der Kürzung der Direktzahlungen für Großbetriebe wurde abgesehen. Die freiwillige Modulation hingegen wurde eingeführt, wobei die Länder ermächtigt wurden, die Direktzahlungen eines Betriebes auch dann zu kürzen, wenn sein Deckungsbeitrag oder seine Zahlungen im Rahmen der Stützungsregelungen oberhalb einer bestimmten Grenze lagen. Der Basissatz für die obligatorische Flächenstilllegung wurde für die ersten zwei Jahre auf $10 \%$ festgesetzt, erst 
danach sollte er auf 0 \% sinken (von Urff 1999). Im März 1999 korrigierte der Europäische Rat die Reformbeschlüsse. Unter anderem wurde beschlossen, dass der Interventionspreis für Getreide in zwei gleichen Stufen um 15\% gesenkt und die Flächenzahlungen von 54 auf 63 EUR/Tonne angehoben werden. Der Basissatz für die obligatorische Flächenstilllegung wurde auf $10 \%$ für den gesamten Zeitraum 2000 bis 2006 festgesetzt. Die Reform des Milchsektors sollte erst ab 2005/06 in Kraft treten (Europäischer Rat 1999).

\section{Box 11: EU-Modell einer multifunktionalen Landwirtschaft}

In ihrem von 1998 datierten Reformvorschlag für die „Agenda 2000“ unterstrich die Kommission die Besonderheit des EU-Agrarsektors. Sie sprach vom „European model of agriculture“ (dem Modell einer multifunktionalen Landwirtschaft), das sie im Gegensatz zum Modell der Landwirtschaft der größten Wettbewerber definierte (Kommission 1998: 7). Mit Hinweis auf den US-amerikanischen „FAIR Act“ von 1996 schrieb sie: „This is not the same model as pursued by our major competitors elsewhere. There are many differences between ours and theirs. Seeking to be competitive should not be confused with blindly following the dictates of a market that is far from perfect" (ibid.). Das europäische Modell beabsichtige „to safeguard the earnings of farmers, above all keeping them stable, using the machinery of the market organisations and compensatory payments" (ibid.).

Der Unterschied zum Modell der Wettbewerber „lies in the multifunctional nature of Europe's agriculture and the part it plays in the economy and the environment, in society and in preserving the landscape" (ibid.: 8). Angesichts der wichtigen Funktionen, die die Landwirtschaft erfüllt, sei es notwendig, die Landbewirtschaftung in Europa aufrechtzuerhalten und die Einkommen der Landwirte zu sichern. Gleichzeitig schloss die Kommission (1998: 8) eine Rückkehr zum Protektionismus aus: „This model, if it can be called that, may offer shortterm comfort but means inevitable decline in the longer run." Interessanterweise wurde dieses Modell der europäischen Landwirtschaft sowohl von den Befürwortern der weiteren Reformen der GAP in Richtung mehr Marktorientierung als auch von ihren Gegnern bemüht (Moyer und Josling 2002: 186).

Erwähnenswert, weil unter der Green Box als „,decoupled income support“ notifiziert, sind die sogenannten Ausgleichsmaßnahmen infolge von Verringerungen bestimmter landwirtschaftlicher Umrechnungskurse (Verordnung (EG) Nr. 1527/ 95). In ausgewählten Mitgliedstaaten, inklusive Deutschland, sollten vor dem Hintergrund der Schaffung des Binnenmarktes den Landwirten EinkommenseinbuBen infolge von Währungsaufwertung durch von der EU ko-finanzierte Aus- 
gleichszahlungen kompensiert werden. Diese Regelung stützte sich auf Art. 9 der Verordnung (EWG) Nr. 3813/92.

\subsection{Einführung der Direktzahlungen}

Die „Agenda 2000“ zementierte die EU-Direktzahlungen „as an acceptable support model“ (Daugbjerg 2012: 98). Die Möglichkeit, die Bestimmungen dieser Reform zu überarbeiten, wurde in den Schlussfolgerungen des Europäischen Rates vom März 1999 explizit vorgesehen. Darin erteilte er der Kommission das Mandat, ihm im Jahr 2002 einen Bericht über die Entwicklung der Agrarausgaben, ggf. mit entsprechenden Änderungsvorschlägen, zu unterbreiten. Auch ein Bericht zur Lage auf dem Getreide- und Ölsaatenmarkt wurde für spätestens 2002 angefragt (Europäischer Rat 1999). Ihre ersten Reformvorschläge legte die Kommission im Juli 2002 vor (Kommission 2002); die detaillierten Vorschläge folgten Anfang 2003 (Kommission 2003). Zu den bisherigen Zielen der Agrarpolitik fügte die Kommission die Vereinfachung der Regelungen sowie die Rechtfertigung der Unterstützung durch das Erbringen von Leistungen, die die Gesellschaft von den Landwirten erwartet, hinzu (Kommission 2002: 2).

Den Direktzahlungen wurde agrarpolitisch eine zentrale Bedeutung beigemessen, denn „market revenues alone are not enough to ensure an acceptable standard of living for many farm households" (ibid.: 7). Im Hinblick auf die Verlagerung der Agrarstützung vom Erzeugnis auf den Erzeuger durch die Direktzahlungen zog die Kommission (2002: 18) ein positives Fazit: „Despite initial concerns, the positive impact of this process in promoting greater market orientation and competitiveness, stabilising agriculture incomes and reducing negative environmental incentives is evident." Gleichzeitig stellte sie fest, dass die 1992 beschlossenen Direktzahlungen ihre Ausgleichsfunktion bereits verloren und zu Einkommenszahlungen wurden, sodass sich die Frage stellt, „whether the distribution of direct support is optimal“ (ibid.: 8). Es ging der Kommission einerseits um das Ungleichgewicht zwischen den kleineren und größeren Betrieben, andererseits um das Ungleichgewicht zwischen den Direktzahlungen und den Maßnahmen zur Förderung der ländlichen Entwicklung: Zu der Zeit waren 16\% der Gesamtausgaben des EAGFL für die Maßnahmen der 2. Säule eingesetzt (ibid.: 9). In diesem Zusammenhang stellte sie fest, dass die Direktzahlungen von 1992 wenig geeignet für eine gezielte Unterstützung der landwirtschaftlichen Betriebe seien.

Die geltende freiwillige Modulation sollte durch die für die Mitgliedstaaten obligatorische „dynamische Modulation“ ersetzt werden. Hierfür sollten alle Direktzahlungen in arithmetischen Schritten um jeweils 3\% pro Jahr bis zu einem Satz von insgesamt $20 \%$ gekürzt werden. Den Betrieben sollte dabei ein Freibetrag von 5.000 EUR für die ersten beiden Vollzeit-Arbeitskräfte zugestanden werden. Nach Anwendung des Freibetrags und der Modulation sollte der Direktzahlungsbetrag je Betrieb und Jahr bei höchstens 300.000 EUR liegen. Die somit gewon- 
nenen Finanzmittel sollten auf der Grundlage der landwirtschaftlichen Fläche, der landwirtschaftlichen Beschäftigung sowie eines Wohlstandskriteriums auf die Mitgliedstaaten aufgeteilt und für die Förderung der 2. Säule der GAP verwendet werden (Kommission 2002). Der offizielle Kommissionsvorschlag vom Januar 2003 enthielt allerdings keine Obergrenze für die Direktzahlungen je Betrieb (Kommission 2003). Das Konzept der dynamischen Modulation ersetzte die Kommission mit der Regelung zur Degression und Modulation. Unter der Degression war eine Senkung der Direktzahlungen in Abhängigkeit vom dem Betrieb zufließenden Betrag zu verstehen. Konkret sollten die Direktzahlungen zwischen 5.001 und 50.000 EUR je Betrieb und Jahr einer im Zeitraum 2006 bis 2012 in ungleichen Schritten von 1\% auf 12,5\% steigenden Kürzung unterzogen werden. Die Direktzahlungen von über 50.000 EUR je Betrieb sollten 2006 um 1\%, 2012 um 19\% gekürzt werden. Die Direktzahlungen von 5.000 EUR und weniger pro Betrieb sollten von der Degression ausgenommen werden. Nur ein Teil der somit gewonnenen Mittel sollte als zusätzliche EU-Finanzierung für die Maßnahmen der 2. Säule eingesetzt werden (Modulation). Diese Mittel sollten nach den Kriterien der landwirtschaftlichen Fläche, der landwirtschaftlichen Beschäftigung und des Bruttoinlandsprodukts pro Kopf in Kaufkraftstandards unter den EU-Ländern aufgeteilt werden. Die restlichen etwa zwei Drittel der Mittel sollten für die Deckung des zusätzlichen Mittelbedarfs bei weiteren GAP-Reformen verwendet werden. In den neuen Mitgliedstaaten sollten die Degression und Modulation erst dann angewandt werden, wenn die Direktzahlungen dort das normale EU-Niveau erreicht haben (ibid.).

Das Kernelement des Reformvorschlags war die Entkopplung der Direktzahlungen von der Produktion (Cunha und Swinbank 2011: 130). Als Vorgabe galt: „Agricultural production must be more orientated to the products and services that the public wants and not to artificially created price incentives or productspecific aids. Direct income payments should not steer the production decisions of farmers" (Kommission 2002: 11). Die Vorteile der Entkopplung sah die Kommission in der Vereinfachung der GAP im Hinblick auf die Integration der Beitrittsländer und in der Konformität der neu gestalteten Direktzahlungen mit der WTO-Green Box. Sie betrachtete die Entkopplung als natürlichen Abschluss des bisherigen Reformprozesses und als "the final step in the shift of support from product to producer" (ibid.: 19). In diesem Zusammenhang schlug sie vor, ein System von „single income payments“ einzuführen. Diese Zahlungen sollten möglichst viele bisherige Direktzahlungen eines Landwirts ersetzen und auf der Grundlage historischer Referenzgrößen betriebsbezogen berechnet werden. Der Gesamtbetrag der Zahlungen, auf die der Landwirt im Referenzzeitraum Anspruch hatte, würde durch die Anzahl der Hektare, die im Referenzzeitraum zu diesem Betrag führten (einschließlich der Futterfläche) dividiert, um Zahlungsansprüche zu kreieren. Dieses Vorgehen sollte im Falle von Landverkauf bzw. Verpachtung eine Übertragung der Zahlungen erleichtern. Die Landwirte sollten auf 
ihrem Land alle Erzeugnisse, einschließlich derjenigen, für die weiterhin gekoppelte Zahlungen gewährt werden, anbauen können. Der Anbau von Obst und Gemüse sollte allerdings von der Förderung im Rahmen der neuen Regelung ausgeschlossen werden (Kommission 2002). Im Jahr 2003 präzisierte die Kommission, dass auf Basis des Referenzzeitraums 2000 bis 2002 Getreide, Ölsaaten, Eiweißpflanzen, Rindfleisch, Milch und Milchprodukte, Schaf- und Ziegenfleisch, Kartoffelstärke, Körnerleguminosen, Reis, Saatgut und Trockenfutter in die neue Regelung einbezogen werden sollten. Die neu vorgeschlagene Qualitätszahlung für Hartweizen, die Zahlung für Schalenfrüchte sowie ein Teil der Ausgleichszahlung für Reis sollten von vornherein von der Entkopplung ausgenommen werden. Jedem geltend gemachten Zahlungsanspruch sollte ein förderfähiger Hektar gegenüberstehen, wobei es sich hier grundsätzlich um jede landwirtschaftliche Fläche eines Betriebs handeln konnte. Für Tierhaltungsbetriebe ohne Bodenbindung wurden besondere Regeln vorgesehen. Zahlungsansprüche sollten mit oder ohne Land auf andere Landwirte im gleichen Mitgliedstaat übertragen werden können. Die Fläche könnten Landwirte für jede landwirtschaftliche Tätigkeit nutzen außer für Dauerkulturen (Kommission 2003). Darüber hinaus schlug die Kommission (2002) vor:

- Die Direktzahlungen sollten an die Einhaltung von bestimmten Auflagen in den Bereichen Umwelt, Lebensmittelsicherheit, Tiergesundheit und Tierschutz gebunden werden (obligatorisches Cross-Compliance).

- Eine verpflichtende Flächenstilllegung für zehn Jahre - ökologische Flächenstilllegung genannt - sollte die rotierende obligatorische Flächenstilllegung als Voraussetzung für den Erhalt der Direktzahlungen ersetzen. Ökobetriebe sollten von dieser Verpflichtung befreit werden.

- Eine Zahlung für Energiepflanzen in Höhe von 45 EUR/Hektar sollte eingeführt werden. Auf Stilllegungsflächen sollten Energiepflanzen nicht mehr angebaut werden können.

- Es wurde vorgesehen, die Zusatzprämie für Hartweizen zu kürzen. Gleichzeitig sollte aber eine Qualitätsprämie eingeführt werden (gewährt für die an Verarbeiter gelieferte Hartweizenmenge bestimmter Qualität).

- Die bisherige Produktionsbeihilfe für Trockenfutter sollte entkoppelt und in die Betriebsprämienregelung einbezogen werden.

- Es wurden vier alternative Optionen für die zukünftige Unterstützung des Milchsektors vorgelegt, inklusive der Abschaffung der Quoten im Jahr 2008.

Im Juni 2003 einigte sich der Rat über die Reform (sogenannte Luxemburger Beschlüsse). Das Prinzip der Entkopplung wurde angenommen, allerdings blieb die 
beschlossene Entkopplung deutlich hinter den Kommissionsvorschlägen zurück (Wissenschaftlicher Beirat 2003: 2). Zum einen wurde den Mitgliedstaaten die Möglichkeit der Teil-Entkopplung gewährt: 25\% der Direktzahlungen für Getreide, Ölsaaten und Eiweißpflanzen, 50 \% der Prämien für Schaf- und Ziegenfleisch sowie entweder bis zu 100\% der Mutterkuhprämien und gleichzeitig bis zu $40 \%$ der Schlachtprämien oder bis zu $100 \%$ der Schlachtprämien bzw. alternativ bis zu $75 \%$ der Sonderprämien für Mastbullen konnten weiterhin gekoppelt ausgezahlt werden. Zum anderen bekamen die Mitgliedstaaten die Möglichkeit, bis zu 10\% ihrer jährlichen nationalen Obergrenze einzubehalten, um „Ergänzungszahlungen " für besondere Formen der landwirtschaftlichen Tätigkeit, die für den Schutz oder die Verbesserung der Umwelt oder zur Verbesserung der Qualität und der Vermarktung landwirtschaftlicher Erzeugnisse wichtig sind, zu gewähren (Art. 69 der Verordnung (EG) Nr. 1782/2003). Wie Cunha und Swinbank (2011: 149) bemerken: „Partial decoupling was the price to pay for the support of the nonreformist countries. “ Die Umsetzung der Entkopplung sollte frühestens Anfang 2005 und spätestens Anfang 2007 beginnen. Im Jahr 2008 sollte mit der Entkopplung der Ausgleichszahlungen für Milch begonnen werden. Zur Wahl standen den Mitgliedstaaten grundsätzlich zwei Entkopplungsmodelle. Nach dem Betriebsmodell sollten individuelle Hektarprämien auf der Basis der jeweiligen Ansprüche im Referenzzeitraum ausgezahlt werden. Beim Regionalmodell sollte das gesamte entkoppelte Prämienvolumen der Region aus dem Referenzzeitraum auf die förderfähige Fläche der Region verteilt werden, was eine Umverteilung zwischen Betrieben und Betriebstypen implizierte (Lippert 2004). Die Betriebsprämie ist der WTO als „decoupled income support" notifiziert. 56

Die Modulation wurde mit Jahresbeginn 2005 für die Mitgliedstaaten obligatorisch. Allerdings sollten die Kürzungen der Direktzahlungen gegenüber dem Kommissionsvorschlag weniger stark ausfallen. Es wurde beschlossen, die Direktzahlungen über 5.000 EUR je Betrieb und Jahr um 3\% (2006: 4\%, ab 2007: $5 \%$ ) zu reduzieren. Ein Betrag in Höhe von einem Prozentpunkt sollte dem Mitgliedstaat zugewiesen werden, in dem er anfällt. Die übrigen Modulationsmittel sollten unter den Ländern auf Basis der landwirtschaftlichen Fläche, der Anzahl der Beschäftigten in der Landwirtschaft und des Bruttoinlandsprodukts pro Kopf aufgeteilt werden. Insgesamt sollten jedoch nicht weniger als $80 \%$ der Modulationsmittel an den jeweiligen Mitgliedstaat zurückfließen und dort für die Maßnahmen der 2. Säule verwendet werden (mit entsprechender nationaler Ko-Finanzierung). Die Möglichkeiten der einjährigen Rotationsbrache sowie des Anbaus nachwachsender Rohstoffe auf den Stilllegungsflächen blieben bestehen. Auf diesen Flächen wurde

\footnotetext{
${ }^{56}$ Es bleibt anzumerken, dass Swinbank und Tranter (2005) die Vereinbarkeit der Betriebsprämie mit den Green-Box-Kriterien infrage stellen. Dafür führen sie zwei Gründe an. Zum einen ist die Fläche, auf der Obst und Gemüse angebaut werden, nicht beihilfefähig, was eine Kopplung an die Erzeugung suggeriert. Zum anderen muss die beihilfefähige Fläche entweder bewirtschaftet oder in einem guten landwirtschaftlichen und ökologischen Zustand erhalten werden.
} 
der Anbau von Futterleguminosen zugelassen. Von der Verpflichtung, Flächen stillzulegen, wurden Ökobetriebe und Betriebe unter 20 Hektar (Kleinlandwirte) befreit. Den Milchmarkt betreffend sollten ab 2004 die Interventionspreise für Butter und Magermilchpulver schrittweise um durchschnittlich $20 \%$ gesenkt werden. Als Kompensation wurden den Milcherzeugern Ausgleichszahlungen gewährt. Die Milchquotenregelung wurde bis 2014/15 verlängert (ibid.). Im April 2004 beschloss der Ministerrat, die Direktzahlungen auch bei Olivenöl (Entkopplung von mindestens $60 \%$ der Direktzahlungen), Baumwolle (65\%), Hopfen $(75 \%)$ und Tabak (100\% ab 2010) zum großen Teil produktionsunabhängig zu gewähren (Lippert 2004). Im November 2005 wurden die Grundzüge der Reform der Zuckermarktordnung beschlossen. Der Zuckerrübenmindestpreis sollte bis zum Jahr 2009/10 um 39,7\% gesenkt werden. Als Kompensation wurden den Anbauern Direktzahlungen gewährt (Lippert 2006).

Die Luxemburger Beschlüsse enthielten eine Reihe von Überprüfungsklauseln, die je nach Marktentwicklung Anpassungen der bestehenden Politik ermöglichten. Einen Überblick über die notwendigen Änderungen der GAP präsentierte die Kommission in ihrer Mittteilung an das Europäische Parlament und den Rat von 2007 (Kommission 2007). Mit dem somit initiierten „Gesundheitscheck“ der GAP sollte keine grundlegende Reform vorgenommen werden. Vielmehr betrachtete ihn die Kommission (2007: 4) als ,the right opportunity to propose changes that, without altering the fundamental structure of the system, adjust and simplify its implementation“. Ziel sei insbesondere gewesen, die Betriebsprämienregelung einfacher und effizienter zu machen, die Marktstützungsinstrumente dahingehend umzugestalten, dass sie der neuen Realität der globalisierten Märkte und der erweiterten Union entsprechen sowie neue Herausforderungen und bestehende Probleme, wie Klimawandel und Verlust an Biodiversität, in Angriff zu nehmen (ibid.: 3). Konkret schlug die Kommission (2007: 3ff) eine stärkere Entkopplung der Direktzahlungen, Vereinfachung der Betriebsprämienregelung (Anpassungen der Entkopplungsmodelle und Vereinheitlichung der Sätze) und der Cross-Compliance-Regelung, Abschaffung der Flächenstilllegungsregelung sowie Stärkung der 2. Säule über die Anhebung der Modulation um 2\% jährlich im Zeitraum von 2010 bis 2013 vor. Darüber hinaus brachte sie eine Kürzung der Direktzahlungen in Abhängigkeit von den dem Betrieb zufließenden Beträgen (je höher die Tranche desto höher der prozentuale Abschlag) erneut ins Spiel (ibid.: 5).

Im November 2008 einigten sich die 27 Mitgliedstaaten auf folgende Reformen (Lippert 2010):

- Im Jahr 2012 sollten alle Direktzahlungen entkoppelt sein. Ausnahmen wurden für die Mutterkuhprämie und die Prämie für Schafund Ziegenfleisch gemacht.

- Die Modulationssätze sollten erhöht werden (bis auf $10 \%$ in 2012). Für Beträge oberhalb von 300.000 EUR sollten die Abschläge um 4 Prozentpunkte angehoben werden. Die somit gewonnenen Mo- 
dulationsmittel wurden für die Förderung der 2. Säule vorgesehen.

Der Modus für ihre Aufteilung unter den Mitgliedstaaten blieb dabei unverändert.

- Die Flächenstilllegung wurde abgeschafft.

- Ab 2010 sollte die Zahlung für Energiepflanzen ersatzlos wegfallen.

Außerdem wurden die Ergänzungszahlungen aus Art. 69 der Verordnung (EG) Nr. 1782/2003 ausgeweitet, indem die Zahl der beihilfefähigen Ziele erhöht wurde. Die sogenannte besondere Stützung nach Art. 68 der Verordnung (EG) Nr. 73/2009 konnte gewährt werden, u. a. bei Nachteilen, denen sich Betriebsinhaber in ausgewählten Sektoren (Milcherzeugnisse, Rindfleisch, Schaf- und Ziegenfleisch sowie Reis) in wirtschaftlich schwachen oder umweltgefährdeten Gebieten gegenübersahen oder für wirtschaftlich anfällige Formen der landwirtschaftlichen Tätigkeit in diesen Sektoren. Gleichzeitig aber wurde eine engere Limitierung der gekoppelten Zahlungen eingeführt: Die besondere Stützung war auf $10 \%$ der jährlichen nationalen Obergrenze begrenzt; die besondere Stützung in Form von gekoppelten Zahlungen auf 3,5\% dieser Obergrenze.

Die Entkopplung wurde von der Reform von 2008 fortgesetzt, sodass 2013 im letzten Jahr vor Inkrafttreten der jüngsten GAP-Reform - der Anteil der gekoppelten Direktzahlungen an den gesamten EU-Direktzahlungen $7 \%$ betrug. Im Jahr 2008 lag ihr Anteil bei knapp 16\% (vgl. Tabelle 7). Im Jahr 2006 waren noch $96 \%$ der EU-Direktzahlungen gekoppelt (Kommission 2015a: 3).

Tabelle 7: Anteil der gekoppelten Direktzahlungen an den gesamten EU-Direktzahlungen im Zeitablauf

\begin{tabular}{lrrrrrrrrr} 
& $\mathbf{2 0 0 8}$ & $\mathbf{2 0 0 9}$ & $\mathbf{2 0 1 0}$ & $\mathbf{2 0 1 1}$ & $\mathbf{2 0 1 2}$ & $\mathbf{2 0 1 3}$ & $\mathbf{2 0 1 4}$ & $\mathbf{2 0 1 5}$ & $\mathbf{2 0 1 6}$ \\
\hline $\begin{array}{l}\text { EU-Direktzahlungen } \\
\text { gesamt (Mrd. EUR) }\end{array}$ & 36,8 & 37,8 & 39,3 & 39,8 & $\mathbf{4 0 , 5}$ & $\mathbf{4 0 , 9}$ & $\mathbf{4 1 , 4}$ & $\mathbf{4 0 , 9}$ & 39,4 \\
$\begin{array}{l}\text { Gekoppelte Zahlungen } \\
\text { Mrd. EUR) }\end{array}$ & 5,8 & 5,9 & 6,0 & 3,4 & 3,3 & 2,9 & 2,8 & 3,1 & 4,7 \\
Anteil (\%) & 15,8 & 15,6 & 15,3 & 8,5 & 8,1 & 7,0 & 6,8 & 7,6 & 11,9 \\
\hline
\end{tabular}

Quelle: EU-Haushaltspläne für die betreffenden Jahre (Kapitel 0503 - Mittel für Direktzahlungen).

Anmerkung: Die gekoppelten Direktzahlungen bis 2014 umfassten v. a. die Maßnahmen unter Art. 69 der Verordnung (EG) Nr. 1782/2003 und Art. 68 der Verordnung (EG) Nr. 73/2009 (vgl. Kapitel 5). 


\subsection{Beibehaltung der Direktzahlungen}

Die nächste GAP-Reform wurde mit der Mitteilung der Kommission vom November 2010 offiziell angestoßen. Laut der Kommission (2010a: 5) war eine weitere Agrarreform nötig, um die Landwirtschaft der EU auf die künftigen Herausforderungen vorzubereiten. Zu diesen zählte sie u. a. Ernährungssicherung, Nachhaltigkeits- und Umweltfragen, Klimawandel, Erhaltung und Steigerung der Wettbewerbsfähigkeit des Agrarsektors sowie die räumliche Ausgewogenheit im Sinne von vitalen ländlichen Gebieten in der gesamten Union. Die Reform der GAP sollte fortgesetzt werden ,to promote greater competitiveness, efficient use of taxpayer resources and effective public policy returns European citizens expect" (ibid.: 3). Auch für die Zukunft sollte angestrebt werden, die landwirtschaftliche Erzeugung in der gesamten EU aufrechtzuerhalten. Die Verwirklichung dieser Ziele erfordere laut der Kommission eine Aufrechterhaltung der öffentlichen Unterstützung für den Agrarsektor und die ländlichen Gebiete auf europäischer Ebene (ibid.: 7f). Sie wies dabei darauf hin, dass, wie die Eurostat-Daten zeigten, „price and income volatility and natural risks [in der Landwirtschaft, Anm. der Verf.] are more marked than in most other sectors and farmers' incomes and profitability levels are on average below those in the rest of the economy" (ibid.: 7).

An dem Zwei-Säulen-System, bestehend aus den Direktzahlungen und den Maßnahmen zur Entwicklung des ländlichen Raums, wollte die Kommission weiter festhalten. Dabei stützte sie sich auf die Ergebnisse der zum Thema der Ausgestaltung der künftigen GAP durchgeführten öffentlichen Konsultation: „[T]he overwhelming majority of views expressed concurred that the future CAP should remain a strong common policy structured around its two pillars [Herv. im Original]“ (ibid.: 2). Dieses bedeutete, dass auch die Direktzahlungen erhalten bleiben sollten. Ihre Rolle wurde von der Kommission (2010: 3f) positiv beurteilt:

The introduction of direct payments has been a lever for consistent market-oriented reforms, enhancing the competitiveness of the agricultural sector by encouraging farmers to adapt to market conditions. Decoupled direct payments provide today basic income support and support for basic public goods desired by European society.

Trotz dieser positiven Bilanz sprach die Kommission (2010a: 8) über „,necessary adaptations of the direct payment system" im Hinblick auf die Umverteilung, Umgestaltung und bessere Fokussierung der Zahlungen, um Nutzen und Qualität der Ausgaben zu verbessern. Insbesondere sollte die Verteilung der Direktzahlungen dem Steuerzahler verständlicher gemacht werden. Um das zu erreichen, regte die Kommission an, den Direktzahlungen neben wirtschaftlichen Kriterien auch ökologische Kriterien zugrunde zu legen. Bereits am Anfang des Reformprozesses stellte die Kommission klar, dass eine gerechte Verteilung der Direktzahlungen keine einheitliche Direktzahlung in der gesamten EU nach sich ziehen muss. Die- 
se wurde aufgrund der sehr unterschiedlichen wirtschaftlichen und natürlichen Bedingungen, denen die Landwirte in der EU gegenüberstehen, abgelehnt (ibid.).

Ihr Konzept für die Direktzahlungen nach 2013 stellte die Kommission in dem offiziellen Reformvorschlag vom Oktober 2011 vor (Kommission 2011a), u. a. folgende Punkte:

- In Mitgliedstaaten, in denen die Direktzahlungen weniger als $90 \%$ des EU-Durchschnitts betrugen, sollte ein Drittel der Differenz zwischen diesem Betrag und dem aktuellen Betrag ausgeglichen werden (externe Konvergenz). Dieses sollte anteilsmäßig und schrittweise von den Mitgliedstaaten finanziert werden, die über dem EU-Durchschnitt liegende Direktzahlungen (270 EUR/Hektar) erhalten.

- Spätestens bis 2019 sollten einheitliche Direktzahlungen auf nationaler bzw. regionaler Ebene (das heißt in einem Mitgliedstaat bzw. innerhalb der Regionen eines Mitgliedstaates) in Kraft sein (interne Konvergenz).

- Statt einer Einzelzahlung wurden im neuen Direktzahlungssystem mehrere Komponenten vorgesehen. Lediglich die Basisprämie sollte allen aktiven Landwirten automatisch gewährt werden, wobei sie die Cross-Compliance-Vorgaben weiterhin erfüllen müssten. Aktiv war ein Landwirt im Sinne des Kommissionsvorschlags dann, wenn seine Direktzahlungen mindestens 5\% seines Einkommens aus nichtlandwirtschaftlichen Tätigkeiten ausmachten. Ausgenommen von dieser Regelung sollten Betriebe mit weniger als 5.000 EUR Direktzahlungen pro Jahr sein.

- Die Höhe der Basisprämie pro Hektar wurde nicht festgesetzt, sondern sollte nach Abzug aller anderen Zahlungen, die das Direktzahlungssystem künftig bilden sollten, berechnet werden. Dazu gehörte in erster Linie die neue Greening-Prämie, für die 30\% der nationalen Obergrenze zur Verfügung gestellt werden sollten. Auch für junge Landwirte, Kleinbetriebe und Betriebe in benachteiligten Gebieten wurden in der 1. Säule Neuregelungen in Form von $\mathrm{Zu}-$ schüssen oder Pauschalzahlungen entwickelt. Erst der verbleibende Betrag sollte für die Basisprämie bereitgestellt werden. Je mehr optionale Zahlungen ein Mitgliedstaat wählen würde, desto niedrigerer würde dort die Basisprämie ausfallen. Außerdem sollten die Mitgliedstaaten ermächtigt werden, bis zu $10 \%$ der nationalen Obergrenze in die 2. Säule für ländliche Entwicklung umzuschichten, wodurch sich die Basisprämie in den betroffenen Mitgliedstaaten zusätzlich reduzieren würde.

- Darüber hinaus sollte die Basisprämie einer Degression unterliegen (Kürzungen ab 150.000 EUR und eine Deckelung bei 300.000 EUR 
pro Betrieb und Jahr, allerdings sollten vor der Berechnung die Löhne von dem Betrag der Basisprämie abgezogen werden können). Die bisherige Modulationsregelung sollte abgeschafft werden. Außerdem sollten die Mitgliedstaaten die Möglichkeit behalten, gekoppelte Zahlungen für bedeutende, sich in Schwierigkeiten befindende Sektoren zu gewähren, allerdings nur in dem als Anreiz zur Beibehaltung des derzeitigen Produktionsniveaus erforderlichen Umfang.

- Eine der größten Neuerungen des Reformvorschlags war die Greening-Prämie als Teil des künftigen Direktzahlungssystems (Tangermann 2011: 9). Laut Vorschlag der Kommission müssten Landwirte EU-weit drei Greening-Anforderungen erfüllen, um ihre Direktzahlungen in vollem Umfang zu bekommen. Erstens sollten sie jährlich mindestens drei verschiedene Pflanzenkulturen anbauen, dabei maximal $70 \%$ von einer Kulturart. Zweitens dürften sie nicht mehr als 5\% ihres Dauergrünlands in Ackerland umbrechen. Als dritte Bedingung sollten alle Landwirte $7 \%$ ihrer Fläche als sogenannte „ökologische Vorrangfläche“ bzw. „Fläche im Umweltinteresse“ ausweisen. Dazu zählte die Kommission Brachflächen, Terrassen, Landschaftselemente, Pufferstreifen sowie Aufforstungsflächen. Ökobetriebe sollten die Greening-Prämie automatisch erhalten.

- Im Hinblick auf die Zahlungen der 2. Säule schlug die Kommission vor, die Vorruhestandsregelung zu streichen. Außerdem regte sie ein Instrument zur Einkommensstabilisierung an. Die Mitgliedstaaten sollten die Möglichkeit erhalten, zu Fonds auf Gegenseitigkeit finanziell beizutragen, um Landwirte im Falle eines erheblichen Einkommensrückgangs zu unterstützen (Kommission 2011b).

Die politische Einigung über die Reform kam am 26. Juni 2013 zustande. Die wichtigsten Änderungen gegenüber dem Kommissionsvorschlag umfassten folgende Punkte (Verordnung (EU) Nr. 1307/2013):

- Es wurde beschlossen, die einem Betrieb zustehende Basisprämie bei Beträgen von mehr als 150.000 EUR um mindestens $5 \% \mathrm{zu}$ kürzen (Lohnkosten können abgezogen werden). Die Mitgliedstaaten wurden ermächtigt, höhere Kürzungen und eine Deckelung der Direktzahlungen zu beschließen. Die somit gewonnenen Mittel verbleiben in dem jeweiligen Mitgliedstaat, wo sie für die Förderung der Maßnahmen der 2. Säule verwendet werden. Dabei entfällt die nationale Ko-Finanzierung.

- Von der obligatorischen Degression von $5 \%$ sollten die Mitgliedstaaten befreit werden, die mindestens $5 \%$ ihrer Obergrenze zur 
Förderung der ersten Hektarflächen aller Betriebe verwenden (bis maximal 30 Hektar oder aber bis zur Betriebsdurchschnittsgröße im jeweiligen Land). Beide Regelungen können aber auch gleichzeitig angewandt werden. Für die Zahlung für die ersten Hektarflächen (sogenannte Umverteilungsprämie) können bis zu 30\% ihrer nationalen Obergrenze bereitstellt werden.

- Die Flexibilität zwischen den beiden GAP-Säulen wurde erweitert. Die Länder erhielten die Möglichkeit, bis zu 15\% ihrer nationalen Obergrenze in die 2. Säule umzuverteilen. Bei diesen Beträgen sollte keine nationale Ko-Finanzierung erforderlich sein. Zudem wurden die Mitgliedstaaten ermächtigt, bis zu 15\% (Mitgliedstaaten mit unterdurchschnittlichen Direktzahlungen bis zu $25 \%$ ) der Finanzmittel für die 2. Säule in die 1. Säule zu übertragen.

- Auch die für gekoppelte Zahlungen verfügbaren Finanzmittel wurden gegenüber dem ursprünglichen Vorschlag erweitert. Gegenüber der vorherigen Regelung (Art. 69) wurden zwar die Ziele der gekoppelten Zahlungen auf eine Kategorie beschränkt (Sektoren in wirtschaftlichen Schwierigkeiten). Allerdings wurden sowohl die Liste der beihilfefähigen Produkte (auf 26) als auch die verfügbaren Mittel (je nach historischer Nutzung der Kopplung bis zu 15\% der Obergrenze, darunter 2\% für Eiweißpflanzen; ggf. auch darüber hinaus) ausgeweitet. Eine Genehmigung der Kommission ist nur im Falle der Verwendung für die gekoppelte Stützung von mehr als 13\% (bzw. $15 \%$ ) der jährlichen nationalen Obergrenze erforderlich. Unter dem Namen der „fakultativen gekoppelten Stützung“ werden gekoppelte Zahlungen nun in Art. 52 der Verordnung Nr. 1307/2013 geregelt.

- Im Hinblick auf die Definition des „aktiven Landwirts“ wurde eine für die Mitgliedstaaten verbindliche Negativliste von Geschäftstätigkeiten erstellt (z. B. Flughäfen, Eisenbahndienste, Wasserwerke), für die keine Direktzahlungen gewährt werden sollten. Die Liste kann von den Mitgliedstaaten ergänzt werden.

- Es wurden Ausnahmen in Bezug auf die interne Konvergenz beschlossen.

- Abgeändert wurde auch die Regelung zum Greening der Direktzahlungen. Beispielsweise sollte die Fläche im Umweltinteresse von $5 \%$ nur für Betriebe mit einer Fläche von mehr als 15 Hektar (ohne Dauergrünland) gelten. Die ursprünglich vorgeschlagenen $7 \%$ sind erst ab 2018 vorgesehen, vorausgesetzt, dass der Kommissionsbericht zu den Folgen des Greenings auf Umwelt, Einkommen und Agrarproduktion positiv ausfällt und die Gesetzgeber der entsprechenden Empfehlung der Kommission folgen werden. Flächen mit 
Zwischenfruchtanbau und stickstoffbindenden Pflanzen sollten als ökologische Vorrangflächen gelten. Gleichzeitig können eine Reihe gleichwertiger Methoden auf das Greening angerechnet werden (z. B. Umweltzertifizierungssysteme).

Die GAP-Reform von 2013 weitete die gekoppelten Direktzahlungen sowohl in Bezug auf beihilfefähige Sektoren als auch verfügbares Budget aus. Entsprechend stieg ihr Anteil an den gesamten EU-Direktzahlungen von 7\% im Jahr 2013 auf $12 \%$ im Jahr 2016. Diese Zahlungen qualifizieren sich nicht mehr für die Green Box, sondern können unter bestimmten Bedingungen als Blue-Box-Maßnahme notifiziert werden. Vor diesem Hintergrund unterliegt die gekoppelte Stützung nach Art. 52 der Verordnung (EU) Nr. 1307/2013 Mengenbegrenzungen mit festgesetzten Flächen und Erträgen oder Tierzahlen (Delegierte Verordnung (EU) Nr. 639/2014). Außerdem müssen die Mitgliedstaaten die festgesetzten Höchstprozentsätze der nationalen Obergrenzen einhalten.

Tabelle 8: Gekoppelte Direktzahlungen und ihre Budgets laut dem EU-Haushaltsplan 2016

\section{Gekoppelte Direktzahlung}

Prämien für die Mutterkuhhaltung

Zusätzliche Prämien für die Mutterkuhhaltung

Schaf- und Ziegenprämien

Zusätzliche Schaf- und Ziegenprämien

Beihilfen für Seidenraupen

Kulturspezifische Zahlung für Baumwolle

Besondere Stützung (Art. 68 der Verordnung

Nr. 73/2009) - Gekoppelte Direktzahlungen

POSEI ${ }^{57}$ - Förderprogramme der EU

POSEI - Inseln des Ägäischen Meeres

Fakultative gekoppelte Stützung (Art. 52 der

Verordnung Nr. 1307/2013)

Sonstiges

Summe

\section{Finanzmittel (EUR)}

1.500 .000

100.000

500.000

100.000

500.000

247.000 .000

3.000 .000

416.000 .000

17.000 .000

4.046 .000 .000

3.108 .157

4.734.808.157

Quelle: EU-Haushaltsplan 2016 (Kapitel 050302 - Andere Direktzahlungen).

${ }^{57}$ Sondermaßnahmen im Bereich der Landwirtschaft zugunsten der Regionen in äußerster Randlage der Union gemäß der Verordnung (EU) Nr. 228/2013 (Programme d'Options Spécifiques à l'Éloignement et l'Insularité). 
Wie Tabelle 8 zeigt, stellt die 2013 beschlossene fakultative gekoppelte Stützung nach Art. 52 budgetmäßig die wichtigste Kategorie unter den gekoppelten Zahlungen dar. Ihr Anteil an den gesamten EU-Direktzahlungen belief sich 2016 auf 10,3\%. Von der Möglichkeit, die gekoppelte Stützung gemäß Art. 52 ab 2015 zu gewähren, machen die Mitgliedstaaten umfangreichen Gebrauch. Nur Deutschland wendet dieses Instrument nicht an. Dabei wurden 94\% der von 2015 bis 2020 dafür eingeplanten Finanzmittel in nur sechs Sektoren eingesetzt: Rind- und Kalbfleisch (24 Mitgliedstaaten; $42 \%$ der Finanzmittel), Milch und Milchprodukte (19; $20 \%$ ), Schaf- und Ziegenfleisch (22; $12 \%$ ), Eiweißpflanzen (16;10,6\%), Obst und Gemüse (19;5\%) sowie Zuckerrüben (10;4,3\%). Elf Mitgliedstaaten verwenden für die fakultative gekoppelte Stützung ihren maximalen Prozentsatz der Obergrenze (13\% bzw. 15\%); drei Mitgliedstaaten - Belgien, Finnland und Portugal - nutzen für diesen Zweck sogar mehr als 13\% bzw. 15\% der Obergrenze (Kommission 2016b: 8f).

Eine Abkehr vom Entkopplungskurs an dieser Entwicklung abzuleiten wäre falsch: Immerhin sind aktuell $88 \%$ der EU-Direktzahlungen entkoppelt. Trotzdem muss der Anstieg des Anteils der gekoppelten Direktzahlungen an den gesamten EU-Direktzahlungen agrarpolitisch als Rückschritt erachtet werden: Er steht mit den Grundgedanken der vorausgegangenen GAP-Reformen nicht im Einklang. Darüber hinaus wird vereinzelt die Vereinbarkeit von EU-Direktzahlungen mit den Green-Box-Kriterien aufgrund der Greening-Anforderungen und der daraus resultierenden Verknüpfung mit der Produktion infrage gestellt (z. B. Swinbank 2012: 52). ${ }^{58}$ Nichtsdestotrotz ist aus heutiger Sicht das Ergebnis der letzten GAP-Reform - trotz einiger Abweichungen vom Entkopplungskurs - ein Festhalten an den entkoppelten Direktzahlungen.

\footnotetext{
${ }^{58}$ Rein rechnerisch und die politischen Aspekte eines solchen Schrittes ausblendend würde es die aktuelle Höchstgrenze der EU für die AMS-Stützung von 72 Mrd. EUR sogar erlauben, alle Direktzahlungen von der Green- in die Amber-Box umzuschichten. Darauf weist u. a. Swinbank (2015: 209) hin.
} 


\section{Vergleich der Bedeutung der Direktzahlungen für die Landwirtschaft der USA und der EU}

Bevor im nächsten Kapitel die Milestones in der Entwicklung der Direktzahlungen in den USA und der EU bestimmt werden, soll im Folgenden noch die Bedeutung des Instruments der Direktzahlungen auf beiden Seiten des Atlantiks näher beleuchtet werden. Dieses stellt den Kontext für den anschließenden Vergleich der Einflussfaktoren, die den ausgewählten Milestones zugrunde liegen, dar. Wo es sinnvoll erscheint oder die relevanten Daten zum Einsatz von Direktzahlungen fehlen, wird die Bedeutung der einkommensstützenden Zahlungen (EUDirektzahlungen und „commodity payments“ der USA) bzw. der staatlichen Zahlungen an Landwirte im Allgemeinen erläutert.

Zum einen ist das Gewicht der einkommensstützenden Zahlungen und insbesondere der Direktzahlungen unter den Instrumenten der Agrarpolitik in den USA und in der EU höchst unterschiedlich. In den USA werden die Agrarausgaben nach Titeln aufgeteilt. Die einkommensstützenden Zahlungen in Form von „commodity payments“ unter Titel I der Farm Bill „Commodities“ (Agrarrohstoffprogramme) stellen mit 23,6 Mrd. USD nicht den budgetstärksten Posten der Farm Bill von 2014 dar. Den größten Teil der für 2014 bis 2018 projizierten 
Nettoagrarausgaben ${ }^{59}$ macht der Titel IV „Nutrition“ aus, der bedürftigen Verbrauchern zugutekommt (80\% oder knapp $391 \mathrm{Mrd}$. USD für fünf Jahre). Den zweitgrößten Ausgabenposten bilden mit 41,4 Mrd. USD (8,5\%) die Ennteversicherungsprogramme, gefolgt von den Umweltprogrammen (28 Mrd. USD bzw. knapp $6 \%$ ). Erst auf Platz vier findet sich Titel I, der „,commodity payments“ (aktuell sind das „price loss coverage payments“, „agriculture risk coverage payments“, „loan deficiency payments“, „milk margin protection payments“, „cotton transition payments" und "marketing loans") sowie das Zuckerprogramm und einige „disaster payments“ umfasst. Auf diesen Farm-Bill-Titel entfallen bis 2018 mit knapp 23,6 Mrd. USD etwa $5 \%$ der Agrarausgaben (Monke 2014: 2).

Gegenüber der sogenannten „baseline“, die die Verlängerung der Farm Bill von 2008 über das Jahr 2013 hinaus darstellt, wurde für 2014 bis 2018 insbesondere die Ernteversicherung mit zusätzlichen Finanzmitteln (+1,8 Mrd. USD) ausgestattet. Die größten Kürzungen betrafen mit 6,3 Mrd. USD die „commodity payments" des Titels I (vgl. Tabelle 9). Dieses ist vor allem auf die Abschaffung von „fixed direct payments“ zurückzuführen, die bis 2018 Einsparungen von insgesamt 18,2 Mrd. USD mit sich bringt. Diese werden allerdings durch die neuen antizyklischen Zahlungen „price loss coverage payments“ und „agriculture risk coverage payments" zum Teil wieder wettgemacht (geschätzte Aufwendungen von insgesamt 5,1 bzw. 6,5 Mrd. USD im Zeitraum von 2014 bis 2018) (CBO 2014: Tabelle 2 und 4). Unter den für die Farm Bill von 2008 zum Zeitpunkt ihrer Verabschiedung projizierten Ausgaben machten die Agrarrohstoffprogramme samt „commodity payments" noch 15\% der Agrarausgaben aus, gefolgt von den Umweltprogrammen $(8,5 \%)$ und der Ernteversicherung $(7,7 \%)$. Der budgetmäßig stärkste Posten waren auch damals die Ernährungsprogramme (Titel „Nutrition“) mit einem Anteil von 67\% (Johnson und Monke 2014: 6).

\footnotetext{
59 Die Schätzungen des „Congressional Budget Office“ (CBO) für die Ausgaben einer Farm Bill werden dreimal im Jahr erstellt. Als Benchmark dient die sogenannte „baseline“. Das sind die projizierten Ausgaben, die in den nächsten fünf bzw. zehn Jahren bei einer Verlängerung der aktuellen Farm Bill entstehen würden. Diese sind keine festen Größen, sondern werden vom CBO je nach Wirtschafts- und Gesetzeslage aktualisiert. Die Veränderung gegenüber der „baseline“ wird „score“ genannt. Sie misst die Auswirkungen, die die geplante Gesetzgebung auf den Haushalt hätte und ist ein wichtiger Faktor in jedem Reformprozess. Falls bei unveränderter Programmausgestaltung die tatsächlichen Ausgaben die CBO-Schätzungen zum Zeitpunkt der Verabschiedung der Farm Bill überschreiten (weil z. B. die Zahl der Empfangsberechtigten aufgrund einer sich verschlechterten Marktsituation gestiegen ist), müssen keine zusätzlichen Finanzmittel gefunden werden. Umgekehrt erfolgen für den Haushalt aber auch keine Einsparungen, wenn die Ausgaben niedriger ausfallen als ursprünglich angenommen. Dieses Verfahren betrifft die sogenannten Pflichtausgaben, die im Agrarbereich den Großteil der Ernährungsprogramme einschließlich des „Supplemental Nutrition Assistance Program“ (,food stamps“), die Ernteversicherungsprogramme, die meisten Umweltprogramme sowie die Agrarrohstoffprogramme umfassen (Monke 2014).
} 
Tabelle 9: Budget der Farm Bill von 2014 zum Zeitpunkt der Verabschiedung

\begin{tabular}{|c|c|c|}
\hline Farm-Bill-Titel & $\begin{array}{r}\text { Ausgaben } 2014 \text { bis } 2018 \\
\text { Mrd. USD }\end{array}$ & $\begin{array}{l}\text { Veränderung ggü. } \\
\text { „baseline“ Mrd. USD }\end{array}$ \\
\hline „Commodities“ & 23,556 & $-6,332$ \\
\hline „Conservation“ & 28,165 & $-0,208$ \\
\hline „Trade“ & 1,782 & $+0,064$ \\
\hline „Nutrition“" & 390,650 & $-3,280$ \\
\hline „Credit““ & $-1,011$ & 0,000 \\
\hline „Rural Development“ & 0,218 & $+0,205$ \\
\hline „Research/Extension“ & 0,800 & $+0,689$ \\
\hline „Forestry“ & 0,008 & $+0,005$ \\
\hline „Energy“ & 0,625 & $+0,541$ \\
\hline „Horticulture“ & 0,874 & $+0,338$ \\
\hline „Crop Insurance“ & 41,420 & $+1,828$ \\
\hline „Miscellaneous“ & 1,544 & $+0,839$ \\
\hline Gesamt & 488,631 & $-5,310$ \\
\hline
\end{tabular}

Quelle: CBO (2014 Tabelle 2 und 3).

Zum Zeitpunkt der Verabschiedung der neuesten Farm Bill im Januar 2014 übertrafen also nicht nur die voraussichtlichen Ausgaben für die Ernteversicherung, sondern auch die für die Umweltprogramme die Aufwendungen für die „commodity payments“ des Titels I. Dieses geschah zum ersten Mal (Orden und Zulauf 2015: 9). Es bleibt allerdings abzuwarten, wie sich die tatsächlichen Ausgaben entwickeln werden. Bereits die CBO-Prognose vom März 2015 brachte wegen sinkender Marktpreise eine Steigerung der aktuellen (2014) und projizierten Ausgaben für den Titel I mit sich, während die Ausgaben für die Umweltprogramme zurückgingen (CBO 2015). Insbesondere die projizierten Ausgaben für „PLC payments“ wurden aufgestockt. Die Gegenüberstellung der tatsächlichen Ausgaben für die Ernteversicherung (größter Zuwachs 2014 bis 2018) und „commodity payments“ (größte Kürzung) zeigt aber, dass zumindest die Ernteversicherung die „commodity payments“ in den letzten Jahren budgetmäßig wiederholt übertraf (vgl. Tabelle 10). Dieses lag auch daran, dass die Marktpreise ungewöhnlich hoch waren. Denn hohe Marktpreise resultieren in hohen Subventionen für die Versicherungsprämien, während die antizyklisch angelegten Zahlungen sinken. 
Tabelle 10: Tatsächliche Ausgaben für „commodity payments“ und die Ernteversicherung in den USA 2000-2015

\begin{tabular}{|c|c|c|}
\hline & $\begin{array}{r}\text { „Commodity payments“ } \\
\text { Mrd. USD }\end{array}$ & $\begin{array}{r}\text { Ernteversicherungsprogramme } \\
\text { Mrd. USD }\end{array}$ \\
\hline 2000 & 12,6 & 2,2 \\
\hline 2001 & 11,9 & 3,2 \\
\hline 2002 & 8,7 & 3,5 \\
\hline 2003 & 11,2 & 3,6 \\
\hline 2004 & 10,1 & 3,1 \\
\hline 2005 & 18,5 & 2,7 \\
\hline 2006 & 12,5 & 3,6 \\
\hline 2007 & 8,3 & 3,9 \\
\hline 2008 & 7,0 & 5,7 \\
\hline 2009 & 8,7 & 7,3 \\
\hline 2010 & 6,5 & 3,7 \\
\hline 2011 & 5,4 & 11,3 \\
\hline 2012 & 5,8 & 14,0 \\
\hline 2013 & 5,4 & 6,0 \\
\hline 2014 & 1,5 & 8,7 \\
\hline 2015 & 5,4 & 8,2 \\
\hline
\end{tabular}

Quelle: „Commodity payments“ (je Kalenderjahr) nach Tabelle A2. Ernteversicherung (je Haushaltsjahr) 2000-2005 Shields (2013: 17), 2006-2015 RMA-USDA (2015).

Im Rahmen von „commodity payments“ beanspruchten die Direktzahlungen seit der Einführung im Jahr 1996 zwar den Großteil der Ausgaben. Für sie wurden insgesamt $91 \mathrm{Mrd}$. USD ausgegeben. Das ist mehr als für die antizyklischen Zahlungen und die Zahlungen aus der „marketing loan“-Regelung zusammen (vgl. Tabelle 11). Dieses ist aber weniger auf die besonders wichtige Stellung der Direktzahlungen zurückzuführen, sondern ist der Natur der antizyklischen Zahlungen geschuldet. In Zeiten höherer Marktpreise fallen sie kaum ins Gewicht, während die Direktzahlungen jedes Jahr gezahlt wurden. 
Tabelle 11: Ausgaben für die einzelnen Arten von „commodity payments“ in den USA 1996-2016

\begin{tabular}{|c|c|c|c|}
\hline & $\begin{array}{r}\text { Direktzahlungen } \\
\text { Mrd. USD }\end{array}$ & $\begin{array}{r}\text { Antizyklische Zahlungen } \\
\text { Mrd. USD }\end{array}$ & $\begin{array}{l}\text { „Marketing loan“- } \\
\text { Zahlungen Mrd. USD }\end{array}$ \\
\hline 1996 & 6,00 & - & 0,00 \\
\hline 1997 & 6,10 & - & 0,00 \\
\hline 1998 & 6,00 & - & 2,00 \\
\hline 1999 & 5,00 & - & 6,80 \\
\hline 2000 & 5,00 & - & 7,60 \\
\hline 2001 & 4,00 & - & 7,90 \\
\hline 2002 & 3,90 & 0,20 & 2,80 \\
\hline 2003 & 6,40 & 2,30 & 1,30 \\
\hline 2004 & 5,20 & 1,10 & 3,50 \\
\hline 2005 & 5,20 & 4,00 & 7,00 \\
\hline 2006 & 5,00 & 4,00 & 1,80 \\
\hline 2007 & 5,00 & 1,10 & 1,10 \\
\hline 2008 & 5,10 & 0,70 & 0,30 \\
\hline 2009 & 4,70 & 1,20 & 1,10 \\
\hline 2010 & 4,80 & 0,60 & 0,10 \\
\hline 2011 & 4,70 & 0,03 & 0,00 \\
\hline 2012 & 4,70 & 0,04 & 0,00 \\
\hline 2013 & 4,30 & 0,20 & 0,00 \\
\hline 2014 & 0,02 & 0,30 & 0,10 \\
\hline 2015 & - & 5,10 & 0,20 \\
\hline 2016 & - & 8,70 & 0,50 \\
\hline Gesamt & 91,10 & 29,60 & 44,10 \\
\hline
\end{tabular}

Quelle: ERS-USDA (2016c).

Anmerkung: Die Direktzahlungen umfassen „PFC payments“ und „fixed direct payments“. Die antizyklischen Zahlungen umfassen „,counter-cyclical payments“ und „ACRE payments“ bis 2014 bzw. ,price loss coverage payments“ und ,agriculture risk coverage payments“ ab 2014. Die Zahlen für 2016 sind vorläufig. 
In Fällen, in denen der Betrag der „PFC payments“ oder „fixed direct payments“ den Gesamtbetrag der „,commodity payments“ aus Tabelle A2 übertrifft, liegt das daran, dass einige restliche Zahlungen einen negativen Betrag aufweisen.

In der EU hingegen sind die einkommensstützenden Zahlungen in Form von EUDirektzahlungen das Hauptinstrument der Agrarpolitik. Dieses spiegelt sich in der Zusammenstellung der Agrarausgaben wider. Der Anteil der EU-Direktzahlungen an den Gesamtausgaben für die GAP belief sich im Haushaltsjahr 2014 auf $75 \%$; es wurden für sie fast $42 \mathrm{Mrd}$. EUR bereitgestellt (Kommission 2015a: 3). Seit der MacSharry-Reform von 1992 gewann dieses Instrument kontinuierlich an Bedeutung. Noch im Haushaltsjahr 1990 lagen die EU-Direktzahlungen bei knapp 0,6 Mrd. ECU (vgl. Tabelle 6). Bereits im Haushaltsjahr 2000 machten sie mit 25,5 Mrd. EUR 61\% der GAP-Ausgaben aus; im Haushaltsjahr 2006 waren es schon 33,7 Mrd. EUR und 65\% (Kommission 2015a: 3). Da aktuell der Großteil der EU-Direktzahlungen entkoppelt ist (vgl. Tabelle 7) machen auch die (entkoppelten) Direktzahlungen den Hauptanteil an den GAP-Ausgaben aus.

Zum anderen ist der Anwendungsbereich der einkommensstützenden Zahlungen bzw. der Direktzahlungen in den USA und in der EU unterschiedlich. Der Großteil der US-Landwirtschaft profitiert nicht von den „commodity payments“. Denn sie werden in den USA nicht flächendeckend gewährt, sondern stehen nur für ausgewählte Erzeugnisse zur Verfügung (sogenannte „,covered commodities“"). Dieses war schon zu Beginn im Jahr 1933 ihr besonderes Merkmal. Aktuell profitieren von den „commodity payments“ zwei Dutzend Ackerkulturen, die zusammen ein Drittel der landwirtschaftlichen Umsätze („farm sales") ausmachen, sowie Milch (Shields 2014a: 2). Dabei flossen in den Haushaltsjahren 2005 bis $201490 \%$ dieser Zahlungen an nur fünf Erzeugnisse: Weizen, Mais, Soja, Baumwolle und Reis (ibid.). Fleisch, Obst, Gemüse, Nüsse, Heu und Baumschulerzeugnisse waren - mit kleineren Ausnahmen - schon immer von dieser Form der Stützung ausgeschlossen. Zum Vergleich: Die subventionierten Ernteversicherungsprogramme stehen etwa 130 Erzeugnissen offen, inklusive Obst und Gemüse (Shields 2014b: 1). $\mathrm{Zu}$ „conservation payments“ im Rahmen von mindestens einem Programm sind zwar grundsätzlich alle Landwirte berechtigt (Claassen 2012); sie fließen aber an nur etwa 14\% der Betriebe (vgl. Tabelle 13). Auch die Direktzahlungen (,fixed direct payments“ und ihre Vorgänger die „PFC payments“) wurden nur gewährt, wenn der Erzeuger die ausgewählten Kulturen (Weizen, Mais, Sorghum, Gerste, Hafer, Baumwolle und Reis, ab 2002 auch Ölsaaten und Erdnüsse) im Referenzzeitraum anbaute. Dabei konzentrierte sich die Mehrheit dieser Zahlungen auf nur fünf Erzeugnisse: Weizen, Mais, Soja, Baumwolle und Reis (GAO 2012: 2). Ein Viertel der „fixed direct payments“ floss von 2003 bis 2011 an Erzeuger, die keine der Kulturen, für die sie Basisanbauflächen besaßen, mehr anbauten (ibid.: 8). Die durchschnittliche Direktzahlung pro Acre Basisanbaufläche variierte je nach Kultur und lag im Jahr 2012 zwischen 1 USD für Hafer und 97 USD für Reis (Zulauf und Orden 2014: 30). 
Vor diesem Hintergrund machten in den letzten 40 Jahren die Begünstigten der staatlichen Zahlungen (,government payments“) nur etwa 33\% aller Betriebe aus (vgl. Tabelle 12). Die Durchschnittszahlung pro Betrieb veränderte sich seit über 20 Jahren nominal kaum (ibid.). Die Zahl der Begünstigten bei den „commodity payments“ sowie die durchschnittliche „,commodity payment“ werden vom USDA nicht ausgewiesen. Wird der Gesamtbetrag für „commodity payments“ aus dem Jahr 2012 in Höhe von 5,8 Mrd. USD (vgl. Tabelle A2) durch die Anzahl der Begünstigten für ,government payments“ geteilt, ergibt sich eine Durchschnittszahlung von knapp 7.200 USD.

Tabelle 12: Durch „government payments“ begünstigte Betriebe in den USA im Zeitablauf

\begin{tabular}{|c|c|c|c|c|c|}
\hline & $\begin{array}{r}\text { „Government } \\
\text { payments“ }\end{array}$ & $\begin{array}{r}\text { Anzahl } \\
\text { Betriebe } \\
\text { insgesamt }\end{array}$ & $\begin{array}{l}\text { Anzahl } \\
\text { begünstigte } \\
\text { Betriebe (1) }\end{array}$ & $\begin{array}{r}\text { Anteil (1) } \\
\text { an allen } \\
\text { Betrieben }\end{array}$ & $\begin{array}{l}\text { Durchschnittliche } \\
\text { Zahlung je Betrieb }\end{array}$ \\
\hline 2012 & 8,0 Mrd. USD & 2.109 .303 & 811.387 & $38 \%$ & 9.925 USD \\
\hline 2007 & 8,0 Mrd. USD & 2.204 .792 & 838.391 & $38 \%$ & 9.523 USD \\
\hline 2002 & 6,5 Mrd. USD & 2.128 .982 & 707.596 & $33 \%$ & 9.251 USD \\
\hline 1997 & 5,0 Mrd. USD & 1.911 .859 & 685.029 & $36 \%$ & 7.378 USD \\
\hline 1992 & 5,2 Mrd. USD & 1.925 .300 & 569.216 & $30 \%$ & 9.152 USD \\
\hline 1987 & 9,6 Mrd. USD & 2.087 .759 & 699.010 & $33 \%$ & 13.800 USD \\
\hline 1974 & 0,3 Mrd. USD & 2.314 .013 & 215.769 & $9 \%$ & 1.305 USD \\
\hline 1969 & 2,5 Mrd. USD & 2.730 .250 & 1.130 .541 & $46 \%$ & 2.242 USD \\
\hline
\end{tabular}

Quellen: NASS-USDA (1974: Tabelle 4 und 3); NASS-USDA (1987: Tabelle 5 und 1), NASS-USDA (1992: Tabelle 5 und 1), NASS-USDA (1997: Tabelle 5 und 1), NASSUSDA (2002: Tabelle 6 und 3), NASS-USDA (2007: Tabelle 6 und 3), NASS-USDA (2012: Tabelle 6 und 3). Spalte 6 für 1969 und 1974 sowie Spalte 5: eigene Berechnung. Anmerkungen: Der Zensus vom Jahr 1969 erhob diese Daten zum ersten Mal. Für 1969 enthalten „commodity payments“ auch Einkommen aus den landwirtschaftlichen Dienstleistungen für Dritte und aus Freizeitdienstleistungen. Die Höhe der „government payments" stimmt mit Tabelle A1 nicht immer überein, weil dem Zensus eine andere Methodologie zugrunde liegt. 
Tabelle 13: Durch „,conservation payments“ begünstigte Betriebe in den USA im Zeitablauf

\begin{tabular}{lrrrrr}
$\begin{array}{r}\text { „Conservation } \\
\text { payments“ }\end{array}$ & $\begin{array}{r}\text { Anzahl } \\
\text { Betriebe } \\
\text { insgesamt }\end{array}$ & $\begin{array}{r}\text { Anzahl } \\
\text { begünstigte } \\
\text { Betriebe (1) }\end{array}$ & $\begin{array}{r}\text { Anteil (1) } \\
\text { an allen } \\
\text { Betrieben }\end{array}$ & $\begin{array}{r}\text { Durchschnittliche } \\
\text { Zahlung je Betrieb }\end{array}$ \\
\hline 2012 & 1,4 Mrd. USD & 2.109 .303 & 291.706 & $13,8 \%$ & 4.943 USD \\
2007 & $1,8 \mathrm{Mrd}$. USD & 2.204 .792 & 346.227 & $15,7 \%$ & $5.072 \mathrm{USD}$ \\
\hline 2002 & $1,4 \mathrm{Mrd}$. USD & 2.128 .982 & 268.830 & $12,6 \%$ & $5.163 \mathrm{USD}$ \\
1997 & $1,4 \mathrm{Mrd}$. USD & 1.911 .859 & 225.328 & $11,8 \%$ & $6.048 \mathrm{USD}$ \\
\hline 1992 & $1,0 \mathrm{Mrd}$. USD & 1.925 .300 & 150.704 & $7,8 \%$ & $6.412 \mathrm{USD}$ \\
\hline
\end{tabular}

Quellen: Wie für Tabelle 12. Spalte 5: eigene Berechnung.

Von „commodity payments“ profitieren in den USA vor allem größere Betriebe. Beispielsweise machten 2012 die Betriebe mit einem Umsatz (,farm sales“) von 250.000 USD und mehr nur 9\% aller Betriebe aus, sie erhielten aber ,commodity payments“ in der Gesamthöhe von 4,8 Mrd. USD, was $60 \%$ des Gesamtbetrags ausmachte (vgl. Tabelle 14). Ohne konkrete Zahlen zu nennen, stellt das GAO (2012: 16) fest, dass sich auch die „fixed direct payments“ auf Großbetriebe konzentrieren, weil sie ,tied to land and paid on a per-acre basis“ sind. Über $50 \%$ der „fixed direct payments“ flossen an Erzeuger mit Einkommen (,income“) von über 100.000 USD (OMB 2012: 29). Die Begünstigten der zwei Hauptarten der US-Agrarzahlungen - „commodity payments“ und „conservation payments“ sind zum großen Teil unterschiedliche Betriebe. Im Jahr 2011 erhielten nur $6 \%$ aller Betriebe „commodity payments “ und „conservation payments“ zugleich; $10 \%$ aller Betriebe erhielten nur „,conservation payments“ und $19 \%$ der Betriebe nur „,commodity payments“ (Hoppe 2014: 28). Während die meisten „commodity payments“ an größere Betriebe gehen, fließen die meisten „conservation payments" an kleinere Betriebe im Nebenerwerb mit einem Jahresumsatz von weniger als 250.000 USD $^{60}$ - die sogenannten „,rural residence farms“ (Claassen und Morehart 2006: 2f).

\footnotetext{
${ }^{60}$ Die US-Statistik unterscheidet bei den Betrieben mit einem Umsatz unter 250.000 USD zwischen den Nebenerwerbsbetrieben (,rural residence farms") und Haupterwerbsbetrieben (,intermediate farms"). Betriebe mit einem höheren Umsatz werden „commercial farms“ genannt (Claassen und Morehart 2006).
} 
Tabelle 14: Verteilung von „commodity payments“ in den USA je nach Betriebsgröße 2012

\begin{tabular}{|c|c|c|}
\hline Umsatz & Anzahl der Betriebe & „Commodity payments“ \\
\hline$>1.000$ USD & 35.800 & 0,02 Mrd. USD \\
\hline 1.000-2.499 USD & 72.175 & 0,10 Mrd. USD \\
\hline 2.500-4.999 USD & 69.170 & 0,20 Mrd. USD \\
\hline 5.000-9.999 USD & 77.879 & 0,30 Mrd. USD \\
\hline 10.000-24.999 USD & 101.634 & 0,60 Mrd. USD \\
\hline 25.000-49.999 USD & 79.565 & 0,50 Mrd. USD \\
\hline 50.000-99.999 USD & 82.505 & 0,60 Mrd. USD \\
\hline 100.000-249.999 USD & 101.543 & 1,00 Mrd. USD \\
\hline 250.000-499.999 USD & 74.683 & 1,10 Mrd. USD \\
\hline 500.000-999.999 USD & 60.032 & 1,40 Mrd. USD \\
\hline$<1.000 .000 \mathrm{USD}$ & 56.401 & 2,30 Mrd. USD \\
\hline Gesamt & 2.109 .303 & 8,00 Mrd. USD \\
\hline
\end{tabular}

Quelle: NASS-USDA (2012: Tabelle 3).

Von den EU-Direktzahlungen profitieren aktuell rund 7,6 Mio. von insgesamt 10,8 Mio. der landwirtschaftlichen Betriebe (Kommission 2015b; Eurostat 2015). Somit werden auf diese Weise $70 \%$ aller EU-Betriebe unterstützt (vgl. Tabelle 15). Von der Förderung ausgeschlossen sind insbesondere die kleinsten Betriebe, da grundsätzlich keine Direktzahlungen gewährt werden sollten, wenn die beihilfefähige Fläche des Betriebes weniger als 1 Hektar beträgt (Verordnung Nr. 1307/ 2013). Gerade in den beiden 2007 beigetretenen Ländern Bulgarien und Rumänien sowie in Ungarn kommen nur etwa ein Drittel der Betriebe in den Genuss von EU-Direktzahlungen (vgl. Tabelle 15). Dieses dürfte der Betriebsstruktur geschuldet sein: In Rumänien sind $74 \%$, in Ungarn $79 \%$ und in Bulgarien sogar $84 \%$ der Betriebe kleiner als 2 Hektar $^{61}$ (Eurostat 2013a, 2013b und 2013c). Die Zahl der Begünstigten von (entkoppelten) Direktzahlungen ist nur um etwa 100.000 Betriebe geringer als die Gesamtzahl der Begünstigten von EU-Direktzahlungen. Vor dem Hintergrund dieser Daten lässt sich feststellen, dass die EU-Direktzahlungen im Allgemeinen als auch die (entkoppelten) Direktzahlungen flächendeckend gewährt werden. Wie geschildert, ist dieses in den USA bei „commodity payments" nicht der Fall.

\footnotetext{
${ }^{61}$ Eurostat erfasst Betriebe mit weniger als 1 Hektar landwirtschaftlicher Nutzfläche nicht separat.
} 
Der Gesamtbetrag der EU-Direktzahlungen im Jahr ist zwar das Vielfache des jährlichen Gesamtbetrags der „commodity payments“ in den USA. Allerdings ist in der EU auch die Zahl der begünstigten Betriebe bedeutend höher. Deshalb fällt die durchschnittliche EU-Direktzahlung pro Betrieb mit 5.542 EUR kleiner aus als die durchschnittliche „commodity payment" in den USA (7.200 USD, etwa 6.500 EUR nach dem Wechselkurs vom 04.08.2016). Es bestehen allerdings große regionale Unterschiede: In den alten EU-Mitgliedstaaten beträgt die Durchschnittszahlung 7.849 EUR, in den neuen, 2004 beigetretenen Mitgliedstaaten 3.344 EUR. In Rumänien und Bulgarien erhalten die Betriebe durchschnittlich 1.440 EUR und in Kroatien 1.006 EUR (Kommission 2015a: 6).

Tabelle 15: Von EU-Direktzahlungen begünstigte Betriebe 2014

\begin{tabular}{lrrrr}
$\begin{array}{l}\text { Mitgliedstaat } \\
\text { (MS) }\end{array}$ & $\begin{array}{r}\text { Anzahl Betriebe } \\
\text { mit Direktzah- } \\
\text { lungen (DZ) }\end{array}$ & $\begin{array}{r}\text { Anzahl Betriebe } \\
\text { mit entkoppelten } \\
\text { DZ }\end{array}$ & $\begin{array}{r}\text { Anzahl Betriebe } \\
\text { mit gekoppelten } \\
\text { DZ }\end{array}$ & $\begin{array}{r}\text { Anzahl Be- } \\
\text { jeweiligen MS }\end{array}$ \\
\hline Belgien & 35.220 & 34.920 & 12.940 & 37.800 \\
Bulgarien & 90.490 & 87.760 & 10.180 & 254.400 \\
Dänemark & 44.270 & 44.150 & 6.080 & 38.800 \\
\hline Deutschland & 320.290 & 320.270 & 170 & 285.000 \\
\hline Estland & 17.090 & 17.080 & 650 & 19.200 \\
Finnland & 57.070 & 56.980 & 13.330 & 54.400 \\
\hline Frankreich & 358.230 & 347.830 & 161.350 & 472.200 \\
Griechenland & 709.270 & 704.120 & 181.280 & 709.500 \\
\hline Großbritannien & 175.700 & 175.250 & 7.630 & 185.200 \\
\hline Irland & 124.570 & 122.540 & 49.070 & 139.600 \\
\hline Italien & 1.163 .690 & 1.162 .500 & 52.030 & 1.010 .300 \\
Kroatien & 92.640 & 90.600 & 23.200 & 157.500 \\
\hline Lettland & 61.530 & 61.540 & 3.670 & 81.800 \\
\hline Litauen & 148.500 & 148.500 & 21.400 & 171.800 \\
\hline Luxemburg & 1.920 & 1.920 & & 2.100 \\
\hline Malta & 6.070 & 6.070 & - & 9.400 \\
\hline Niederlande & 49.880 & 49.490 & 3.310 & 67.500 \\
Österreich & 110.250 & 109.310 & 58.130 & 140.400 \\
\hline Polen & 1.351 .270 & 1.351 .250 & 229.340 & 1.429 .000
\end{tabular}




\begin{tabular}{lrrrr}
$\begin{array}{l}\text { Mitgliedstaat } \\
(\mathbf{M S})\end{array}$ & $\begin{array}{r}\text { Anzahl Betriebe } \\
\text { mit Direktzah- } \\
\text { lungen (DZ) }\end{array}$ & $\begin{array}{r}\text { Anzahl Betriebe } \\
\text { mit entkoppelten } \\
\text { DZ }\end{array}$ & $\begin{array}{r}\text { Anzahl Betriebe } \\
\text { mit gekoppelten } \\
\text { DZ }\end{array}$ & $\begin{array}{r}\text { Anzahl Be- } \\
\text { jeweiligen MS }\end{array}$ \\
\hline Portugal & 173.310 & 148.800 & 61.620 & 264.400 \\
Rumänien & 1.186 .290 & 1.154 .910 & 104.260 & 3.629 .700 \\
Schweden & 63.140 & 63.130 & 6.900 & 67.200 \\
Slowakei & 17.010 & 17.020 & 1.620 & 23.600 \\
\hline Slowenien & 56.720 & 56.620 & 17.850 & 72.400 \\
Spanien & 869.980 & 854.110 & 118.290 & 965.000 \\
\hline Tschechien & 28.460 & 28.430 & 7.740 & 26.300 \\
Ungarn & 174.870 & 174.850 & 2.620 & 491.300 \\
\hline Zypern & 33.220 & 33.220 & & 35.400 \\
EU gesamt & 7.520 .950 & 7.423 .170 & 1.154 .660 & 10.841 .000 \\
\hline
\end{tabular}

Quelle: Eurostat (2015) für Anzahl der Betriebe in der EU. Ansonsten Kommission (2015b) (Daten „Number of beneficiaries per range of expenditure“).

Die Zahl der Betriebe in der Union, die von Umweltzahlungen (EU: Agrarumweltmaßnahmen) profitieren, ist viel niedriger als die Zahl der Betriebe, denen die EUDirektzahlungen gewährt werden, wobei die Situation je nach Mitgliedstaat unterschiedlich ist (vgl. Tabelle 16).

Tabelle 16: Zahlungen für Agrarumweltmaßnahmen in ausgewählten EU-Mitgliedstaaten 2007-2012

EAFRD-Ausgaben Anzahl begünstigter Betriebe Anzahl Betriebe (neue Verpflichtungen) gesamt 2010

\begin{tabular}{lrrr}
\hline Deutschland & 1.299 .560 .000 EUR & 171.146 & 299.100 \\
Frankreich & 1.101 .480 .000 EUR & 2626 & 516.100 \\
Italien & 681.203 .000 EUR & 112.923 & 1.620 .900 \\
Niederlande & 60.193 .000 EUR & 12.439 & 72.300 \\
Österreich & 1.523 .022 .000 EUR & 114.202 & 150.300 \\
Polen & 494.385 .000 EUR & 106.914 & 1.506 .600 \\
Portugal & 235.613 .000 EUR & 27.107 & 305.300 \\
Rumänien & 624.782 .000 EUR & 276.399 & 3.859 .000 \\
EU gesamt & 10.440 .236 .000 EUR & 1.272 .347 & 12.014 .700 \\
\hline
\end{tabular}

Quelle: Kommission (2013: Tabelle 3.6.2.3 und 3.5.4.1). 
Wie in den USA profitieren auch in der EU insbesondere die größeren Betriebe von den einkommensstützenden Zahlungen. Im Jahr 2009 erhielten 0,5\% der Begünstigten $16 \%$ des Gesamtbetrags der Direktzahlungen (100.000 EUR und mehr), während $80 \%$ der Begünstigten etwa $20 \%$ dieses Betrags bekamen (5.000 EUR und weniger) (Kommission 2011c: 14f). Diese Verteilung der Stützung ist nicht neu: Schon vor der Einführung der Ausgleichszahlungen der MacSharry-Reform beklagte die Kommission, dass 80 \% der EAGFL-Ausgaben an nur $20 \%$ der Betriebe flossen (Kommission 1991a: 2).

Die Bedeutung der einkommensstützenden Zahlungen bzw. der Direktzahlungen für Landwirte ist in der EU und den USA unterschiedlich. Die Frage nach der Bedeutung der staatlichen Förderung für landwirtschaftliche Einkommen ist zwar schwer zu beantworten (OECD 2003: 26) und auch nicht Ziel dieser Arbeit, aber der Anteil der hier interessierenden Agrarzahlungen am landwirtschaftlichen Einkommen auf beiden Seiten des Atlantiks kann berechnet und unter Umständen auch verglichen werden. In den USA wird weder der Anteil der „government payments“ noch der „commodity payments“ am landwirtschaftlichen Einkommen in der Agrarstatistik separat ausgewiesen. In Tabelle A1 wird auf Basis der USDADaten der Anteil der „government payments“ am landwirtschaftlichen Nettoeinkommen $^{62}$ - dem Hauptindikator für landwirtschaftliches Einkommen in den USA (Schnepf 2016: 1) - berechnet. Seinen Höchststand erreichte er im Jahr 1983 mit $65 \%$, schwankte aber stark im Verlauf der Jahre. In den letzten zehn Jahren sank er zunächst nahezu kontinuierlich und stabilisierte sich von 2011 bis 2014 auf einem relativ niedrigen Niveau von etwa $10 \% .2015$ kehrte sich jedoch der Trend um (Tabelle A1). Der Anteil der „commodity payments“ am Einkommen schwankte in den letzten zehn Jahren ebenfalls stark. Er betrug zwischen $2 \%$ im Jahr 2014 und $22 \%$ im Jahr 2006 (eigene Berechnung auf Basis der USDA-Daten in Tabelle A1 und Tabelle A2). Diese Schwankungen hängen mit der Natur der Zahlungen, die zum Großteil antizyklisch angelegt sind, zusammen: Sinken die Marktpreise, sinkt das landwirtschaftliche Einkommen, gleichzeitig aber steigen die Zahlungen und somit auch ihr Anteil am (niedrigeren) Einkommen. Dieses trifft umso mehr nach der Abschaffung der „fixed direct payments“, die unabhängig von der Marktsituation gewährt wurden, zu. Zudem variiert der Anteil der ,government payments" am Einkommen in den USA je nach Sektor und Region (Schnepf 2016: 8f).

Einer der Indikatoren für das Einkommen in der Landwirtschaft in den USA ist das Einkommen des landwirtschaftlichen Betriebsleiterhaushalts (,farm opera-

\footnotetext{
62 Das landwirtschaftliche Nettoeinkommen wird definiert als „, a value of production measure, indicating the farm operator's share of the net value added to the national economy within a calendar year, independent of whether it is received in cash or noncash form. As a result, net farm income includes the value of home consumption, changes in inventories, capital replacement, and implicit rent and expenses related to the farm operator's dwelling that are not reflected in cash transactions" (Schnepf 2016: 1).
} 
tor household income“), das auch Einkommen aus nichtlandwirtschaftlichen Tätigkeiten umfasst. Er ermöglicht einen direkten Vergleich des Durchschnittseinkommens der landwirtschaftlichen Haushalte mit dem Durchschnittseinkommen aller US-Haushalte. Wie Tabelle 17 zeigt, übertraf das durchschnittliche Einkommen landwirtschaftlicher Haushalte bereits im Jahr 1990 das durchschnittliche Einkommen aller Haushalte. In diesem Zusammenhang wies Gardner (1992) darauf hin, dass sich das sogenannte „farm problem“, das sich in der Einkommensdisparität zwischen nichtlandwirtschaftlichen und landwirtschaftlichen Haushalten widerspiegelte, in den USA verringert hat. Dieser Trend festigte sich nach dem Jahr 1995 noch. Seit einigen Jahren übersteigt auch das Median-Einkommen landwirtschaftlicher Haushalte das Median-Einkommen aller Haushalte.

Tabelle 17: Durchschnitts- und Medianeinkommen landwirtschaftlicher Haushalte im Vergleich zum Einkommen aller Haushalte in den USA im Zeitablauf (zu laufenden Preisen)

\begin{tabular}{|c|c|c|c|c|}
\hline & \multicolumn{2}{|c|}{ Durchschnittseinkommen (USD) } & \multicolumn{2}{|c|}{ Medianeinkommen (USD) } \\
\hline & Landw. Haushalte & Alle Haushalte & Landw. Haushalte & Alle Haushalte \\
\hline 1960 & 4.054 (1.913) & 6.227 & k.A. & k.A. \\
\hline 1965 & $6.344(2.552)$ & 7.704 & k.A. & k.A. \\
\hline 1970 & 9.472 (3.498) & 10.001 & k.A. & k.A. \\
\hline 1975 & $15.692 \quad(6.212)$ & 13.779 & k.A. & k.A. \\
\hline 1980 & $18.504 \quad(4.285)$ & 21.063 & k.A. & k.A. \\
\hline 1985 & $35.473(11.417)$ & 29.066 & k.A. & k.A. \\
\hline 1990 & $38.237 \quad(4.626)$ & 37.403 & k.A. & k.A. \\
\hline 1995 & $44.392 \quad(4.720)$ & 44.938 & 30.702 & 34.076 \\
\hline 2000 & $62.223(2.872)$ & 57.135 & 43.520 & 41.990 \\
\hline 2005 & $81.317(14.227)$ & 63.344 & 54.550 & 46.326 \\
\hline 2010 & $84.459(11.788)$ & 67.530 & 54.162 & 49.276 \\
\hline 2014 & $134.164(31.025)$ & 75.738 & 81.637 & 53.657 \\
\hline
\end{tabular}

Quelle: ERS-USDA (2016a).

Anmerkung: Bei landwirtschaftlichen Haushalten ist der Teil des Einkommens kursiv in Klammern ausgewiesen, der aus landwirtschaftlichen Tätigkeiten kommt.

Der Anteil des Gesamteinkommens, den landwirtschaftliche Haushalte aus landwirtschaftlichen Tätigkeiten (,farm income“) beziehen, weist beträchtliche Schwankungen auf. Er erreichte im Jahr 1961 mit knapp 50 \% seinen Höchststand, ging aber auf $10 \%$ im Jahr 1988 zurück und blieb bis einschließlich 2014 auf dem Ni- 
veau von unter $25 \%$ (ERS-USDA 2016a). Im Allgemeinen sind die US-Betriebe also auf Einkommen aus nichtlandwirtschaftlichen Tätigkeiten („,off-farm income") angewiesen. Allerdings ist dieses Bild zum Teil der großzügigen Definition des landwirtschaftlichen Betriebes geschuldet. ${ }^{63}$

Ein ähnlicher Indikator für das Gesamteinkommen eines Betriebes wird in der EU nicht geführt (für die möglichen Gründe, u. a. mangelndes Interesse an Transparenz, s. Hill und Bradley 2015: 27). Die Aussagen der Kommission, dass landwirtschaftliche Einkommen je Arbeitseinheit erheblich niedriger sind als die Einkommen in der übrigen Wirtschaft (z. B. Kommission 2010a: 5, Kommission 2011c: 8) werden in der Wissenschaft als unzureichend erklärt (Swinbank 2012: 26f). In diesem Zusammenhang rügt auch der Europäische Rechnungshof (2016: 8) die Kommission mit klaren Worten:

The Commission has not clearly established the statistical data needed to effectively assess the performance of CAP measures in support of farmers' incomes. No representative data are available on the disposable income of farm households, which would facilitate assessing the achievement of the treaty objective of ensuring a fair standard of living for farmers. Furthermore, there is no reliable system to allow comparisons to be made between agricultural incomes and those in other sectors of the economy, which could justify EU income support for farmers.

Was den Beitrag der EU-Direktzahlungen zum landwirtschaftlichen Einkommen betrifft, bemerkt die Kommission, dass „many farms still depend heavily on direct payments due to the low profitability of agricultural activities" (Kommission 2011c: 11) und „EU producers are highly dependent on public support“ (Kommission 2016b: o.S.). Der Anteil der EU-Direktzahlungen am landwirtschaftlichen Faktoreinkommen ${ }^{64}$ blieb in den letzten zehn Jahren konstant auf einem relativ hohen Niveau von etwa $30 \%$. Konkret machten sie im Zeitraum von 2004 bis 2006 im Durchschnitt $26 \%$ bis $30 \%$ des landwirtschaftlichen Faktoreinkommens aus (Kommission 2010b: 26); in den Jahren 2007 bis $200929 \%$ (Kommission 2011c: 11). Im Zeitraum von 2010 bis 2014 lag ihr Anteil am landwirtschaftlichen Faktoreinkommen bei $28 \%$; der Anteil der Gesamt-Subventionen, einschließlich der EU-Direktzahlungen, betrug 33 \% (Kommission 2016c: o.S.). Die Daten der Kommission zeigen aber auch, dass auf diesem Gebiet große Unterschiede zwischen den Mitgliedstaaten bestehen. Der Anteil der EU-Direktzahlungen am landwirtschaftlichen Faktoreinkommen reicht von $15 \%$ und weniger in Zypern,

\footnotetext{
${ }^{63}$ In den USA gilt als landwirtschaftlicher Betrieb die Stelle, die landwirtschaftliche Produkte im Wert von 1.000 USD in einem Jahr verkauft hat oder normalerweise verkauft hätte (Hoppe 2014: 1).

${ }^{64}$ Das Faktoreinkommen in der Landwirtschaft definiert die Kommission (2016b: o.S.) als „income generated by farming which is used to remunerate (1) borrowed/rented factors of production (capital, wages and land rents), and (2) own production factors (own labour, capital and land)“.
} 
Malta, den Niederlanden und Rumänien bis über $40 \%$ in Irland, Luxemburg, der Slowakei und Schweden (Kommission 2016b: o.S.). Der Anteil der EU-Direktzahlungen am Einkommen variiert zudem je nach Betriebsausrichtung und erreicht die höchsten Werte bei Ackerbau- und Weideviehbetrieben (Kommission 2010b: 27).

Die USA und die EU bedienen sich unterschiedlicher Einkommensindikatoren, die nicht miteinander vergleichbar sind. Nichtsdestotrotz lässt sich zusammenfassend feststellen, dass in der EU der Anteil der EU-Direktzahlungen am landwirtschaftlichen Einkommen in der letzten Dekade konstant blieb, während in den USA der Anteil der „commodity payments“ (und der gesamten staatlichen Zahlungen) erheblich schwankte. In der EU scheinen die Zahlungen eine größere Rolle für die Betriebe zu spielen, weil ihr Anteil am landwirtschaftlichen Einkommen auf einem höheren Niveau als in den USA liegt. Dieses bestätigt sich, wenn das landwirtschaftliche Stützungsniveau in der EU und den USA, ermittelt mit dem PSE-Indikator der OECD, verglichen wird. Seit Beginn seiner Berechnung im Jahr 1986 lag das PSE in Prozent der Bruttoeinnahmen der Landwirtschaft für die EU durchgehend höher als für die USA. Im Jahr 2015 verdankten die Landwirte in der EU knapp $19 \%$ ihrer Bruttoeinnahmen staatlicher Unterstützung; in den USA lag dieser Wert bei 9,4\% (vgl. Tabelle 18).

Tabelle 18: Entwicklung des PSE in der EU und den USA in Prozent der Bruttoeinnahmen der Landwirtschaft seit dem Jahr 1986

\begin{tabular}{lrrrrrrr} 
& $\mathbf{1 9 8 6}$ & $\mathbf{1 9 9 0}$ & $\mathbf{1 9 9 5}$ & $\mathbf{2 0 0 0}$ & $\mathbf{2 0 0 5}$ & $\mathbf{2 0 1 0}$ & $\mathbf{2 0 1 5}$ \\
\hline EU & 38,6 & 32,9 & 35,0 & 32,9 & 30,8 & 20,0 & 18,9 \\
USA & 23,5 & 16,1 & 9,7 & 22,7 & 15,0 & 8,6 & 9,4 \\
\hline
\end{tabular}

Quelle: OECD (2016a).

Auch der Anteil der staatlichen Zahlungen an Bruttoeinnahmen der Landwirtschaft liegt laut OECD-Berechnungen in der EU höher als in den USA. Konkret betrug im Zeitraum von 2013 bis 2015 der Anteil der „payments based on area, animal numbers, receipts and income“, bei denen die Produktion nicht erforderlich ist, an Bruttoeinnahmen in der EU im Durchschnitt fast $10 \%$ und in den USA nur $1 \%$ (vgl. Tabelle 19). Diese Kategorie der Zahlungen umfasst die (entkoppelten) Direktzahlungen in der EU sowie die „fixed direct payments“ bzw. ihre Vorläufer in den USA (Zahlungen mit festen Sätzen) sowie die antizyklischen Zahlungen in den USA (Zahlungen mit variablen Sätzen). Der Anteil der entkoppelten US-Zahlungen (Zahlungen mit festen Sätzen) an Bruttoeinnahmen der Landwirtschaft lag im Zeitraum von 2013 bis 2015 jedes Jahr unter 1,6 \%. 
Tabelle 19: Entwicklung der Zahlungen auf der Grundlage historischer Werte (Produktion nicht erforderlich) in der EU und den USA seit dem Jahr 1986

\begin{tabular}{|c|c|c|c|c|c|c|}
\hline & 1986-88 & 1995-97 & 2013-15 & 2013 & 2014 & 2015 \\
\hline \multicolumn{7}{|l|}{$\mathrm{EU}$} \\
\hline $\begin{array}{l}\text { Bruttoeinnahmen } \\
\text { (Mrd. EUR) }\end{array}$ & 211,380 & 239,230 & 379,526 & 385,031 & 385,031 & 368,516 \\
\hline $\begin{array}{l}\text { Zahlungen mit festen } \\
\text { Sätzen (1) (Mrd. EUR) }\end{array}$ & 0,000 & 0,020 & 36,800 & 38,800 & 37,100 & 34,500 \\
\hline $\begin{array}{l}\text { Anteil (1) an Brutto- } \\
\text { einnahmen }(\%)\end{array}$ & 0,000 & 0,010 & 9,700 & 10,100 & 9,600 & 9,400 \\
\hline $\begin{array}{l}\text { Zahlungen mit } \\
\text { variablen Sätzen (2) } \\
\text { (Mrd. EUR) }\end{array}$ & 0,000 & 0,000 & 0,000 & 0,000 & 0,000 & 0,000 \\
\hline $\begin{array}{l}\text { Anteil (2) an } \\
\text { Bruttoeinnahmen (\%) }\end{array}$ & 0,000 & 0,000 & 0,000 & 0,000 & 0,000 & 0,000 \\
\hline \multicolumn{7}{|l|}{ USA } \\
\hline $\begin{array}{l}\text { Bruttoeinnahmen } \\
\text { (Mrd. USD) }\end{array}$ & 143,469 & 200,325 & 393,982 & 394,251 & 405,217 & 382,477 \\
\hline $\begin{array}{l}\text { Zahlungen mit festen } \\
\text { Sätzen (3) (Mrd. USD) }\end{array}$ & 0,300 & 3,800 & 3,700 & 5,000 & 6,100 & 0,040 \\
\hline $\begin{array}{l}\text { Anteil (3) an Brutto- } \\
\text { einnahmen (\%) }\end{array}$ & 0,230 & 1,900 & 1,000 & 1,300 & 1,500 & 0,010 \\
\hline $\begin{array}{l}\text { Zahlungen mit } \\
\text { variablen Sätzen (4) } \\
\text { (Mrd. USD) }\end{array}$ & 0,000 & 0,000 & 4,900 & 0,000 & 5,200 & 9,600 \\
\hline $\begin{array}{l}\text { Anteil (4) an } \\
\text { Bruttoeinnahmen (\%) }\end{array}$ & 0,000 & 0,000 & 1,300 & 0,000 & 1,300 & 2,500 \\
\hline
\end{tabular}

Quelle: OECD (2016b: 86, 131). Anteil an Bruttoeinnahmen: eigene Berechnung. Jahr 2015: vorläufig.

Die Erhebungen zeigen, dass die einkommensstützenden Zahlungen für die Landwirtschaft in den USA und in der EU von unterschiedlicher Bedeutung sind. Grundsätzlich ist dieses Instrument für die Landwirte in der EU wichtiger. Dieses spiegelt sich im Anteil der Zahlungen am landwirtschaftlichen Einkommen, im Anteil der beihilfefähigen Betriebe an der Gesamtzahl der Betriebe und schließlich auch im Anteil der relevanten Programme am Gesamtbudget wider. Das trifft auch auf das Instrument der (entkoppelten) Direktzahlungen zu. Während sie in der EU das wichtigste Instrument der Agrarpolitik darstellen, ist es in den USA 
die subventionierte Ernteversicherung, die diese Rolle innehat. Die Ernteversicherung gehört zwar nicht zu „commodity payments“ im Sinne der Budgetberichterstattung, aber auch hier handelt es sich um eine Stützung der Landwirtschaft. Würden alle „commodity payments“ in den USA abgeschafft, bliebe die US-Landwirtschaft stark subventioniert. Würden allerdings in der EU die Direktzahlungen ersatzlos wegfallen, würde die Landwirtschaft den Großteil der Stützung verlieren.

Dieses heißt allerdings nicht, dass die Direktzahlungen in den USA vor der Abschaffung im Jahr 2014 unbedeutend waren. Woolverton und Young (2009: 25) identifizieren die Tatsache, dass Landwirte, die am ACRE-Programm teilnehmen wollten, auf $20 \%$ der „fixed direct payments“ verzichten mussten, als einen Grund für den Misserfolg des ACRE-Programms, der sich in niedrigen Teilnahmeraten im ersten Anwendungsjahr manifestierte. Das galt insbesondere für Erzeuger von Hochland-Baumwolle, Erdnüssen und Reis, denn hier war der Wert der „fixed direct payments“ je Acre Basisanbaufläche am höchsten. Die Mehrheit der „fixed direct payments" floss für nur fünf Erzeugnisse: Weizen, Mais, Soja, Baumwolle und Reis (GAO 2012: 2). Zudem konzentrierten sich die Zahlungen auf größere Betriebe: Im Jahr 2011 erhielten 10 \% der Empfänger von „fixed direct payments" $51 \%$ dieser Zahlungen; $25 \%$ der Empfänger bezogen $73 \%$ der Zahlungen (GAO 2012: 16). Auch eine geografische Konzentration der „fixed direct payments" war zu erkennen: Von ihnen profitierten insbesondere die Betriebe im Mittleren Westen sowie dem Einzugsgebiet des Mississippi. Im Jahr 2011 erhielten $9 \%$ der Landkreise („,county“) jeweils weniger als 250.000 USD an „fixed direct payments", während an $8 \%$ der Landkreise jeweils mindestens 5 Mio. USD flossen (ibid.: 9). Daraus lässt sich folgern, dass das Instrument der Direktzahlungen in den USA zwar keinen vergleichbar hohen Stellenwert für die gesamte Landwirtschaft hatte wie in der EU, es für einzelne Sektoren, Betriebe und Regionen aber eine große Rolle spielte. 



\section{Auswahl der Milestones in der Entwicklung der Direktzahlungen beidseits des Atlantiks}

Die Schilderung der Entwicklung der staatlichen Zahlungen an Landwirte in den USA und der EU dient der Bestimmung der Milestones in der Entwicklung der Direktzahlungen auf beiden Seiten des Atlantiks, die im folgenden Kapitel vorgenommen und erklärt wird. Die anschließende Gegenüberstellung der ausgewählten Milestones macht es möglich, den Verlauf des Prozesses der Konvergenz bzw. der Divergenz zwischen den Agrarpolitiken der USA und der EU abzubilden. Es ist insbesondere von Interesse herauszufinden, welcher der beiden Akteure die Vorreiterrolle in Bezug auf die Einführung bestimmter Zahlungstypen innehatte.

An der Stelle bleibt anzumerken, dass in den USA Agrarreformen in regelmäBigen Abständen auf der politischen Agenda stehen, weil die Farm Bills eine Geltungsdauer von fünf Jahren haben. Dieses bietet, wie Johnson und Monke (2014: o.S.) bemerken, ,a predictable opportunity for policy makers to comprehensively and periodically address agricultural and food issues". Falls es nicht gelingt, rechtzeitig eine neue Farm Bill zu verabschieden oder zumindest die geltende zu verlängern, greift die sogenannte permanente Gesetzgebung - der „Agricultural Adjustment Act“ von 1938 sowie der „Agricultural Act“ von 1949 - mit ihren überholten, teuren Preisstützungsmaßnahmen. Dieses stellt eine Garantie dar, dass sich die Gesetzgeber auf eine neue Farm Bill einigen werden. In der EU hingegen fehlt ein solcher Automatismus. In der Vergangenheit waren beispielsweise institutio- 
nelle Faktoren relevant für den Reformstart, wie das der Kommission vom Europäischen Rat erteilte Mandat für die Reform von 2003 (Cunha und Swinbank 2011: 147). Für die Reform von 2013 waren die Notwendigkeit der Festlegung eines neuen Mehrjährigen Finanzrahmens (MFR) für den Zeitraum von 2014 bis 2020 sowie die auf Druck von Großbritannien vereinbarte umfassende Überprüfung sämtlicher Aspekte der Haushaltsausgaben, einschließlich der Agrarpolitik, relevant. Beides „meant that a recalibration of the CAP had to be undertaken“ (Swinbank 2015: 210). Auch die nächste Reform der GAP wird mit der Revision des MFR zusammenfallen.

\subsection{Ausgewählte Milestones in den USA}

Mit Blick auf das Ziel der vorliegenden Untersuchung ist es naheliegend, dass einer der Milestones die Ersteinführung von Direktzahlungen, wie sie in dieser Arbeit definiert sind, darstellen sollte. Die vorstehende Analyse ergab, dass die staatlichen Zahlungen an Landwirte in den USA lange Zeit als gekoppelte Zahlungen geführt wurden. Die Abhängigkeit von bzw. der Bezug zur aktuellen Erzeugung, zu Produktionsfaktoren oder Preisen war auf verschiedene Weise hergestellt. Insbesondere musste der Landwirt das jeweilige Erzeugnis weiter produzieren, um den Anspruch auf die Zahlungen zu behalten. Da für die Berechnung der beihilfeberechtigten Erzeugung keine festen Erträge, sondern die individuellen Erträge aus den jüngsten Jahren bzw. der Vorjahresertrag herangezogen wurden, konnte der Landwirt Einfluss auf die Höhe seiner künftigen Zahlungen nehmen, indem er die Anbauintensität erhöhte. Deshalb können auch die „deficiency payments“ der Jahre 1974 bis 1977 nicht als entkoppelt angesehen werden, obwohl sie unabhängig von der aktuellen Anbaufläche für das beihilfefähige Erzeugnis gewährt wurden. Gleichzeitig waren Auflagen zur Produktionskontrolle in Kraft. Nichtsdestotrotz waren Entkopplungselemente schon von Anbeginn der systematischen Agrarstützung an sichtbar, wenn auch nur punktuell. Beispielsweise wurden die Zahlungen unter dem „Agricultural Adjustment Act“" von 1933 grundsätzlich auf Basis der individuellen Erzeugungsmenge aus jüngster Vergangenheit gewährt.

Die von der aktuellen und zukünftigen Erzeugung sowie von den Marktpreisen entkoppelten Zahlungen wurden im Jahr 1996 unter dem Namen ,production flexibility contract payments“ eingeführt. Ihre Entkopplung wird durch die Notifikation als „decoupled income support" unter der Green Box der WTO bestätigt. „Production flexibility contract payments" und später „fixed direct payments“ waren die einzigen „commodity payments“, die die USA als „,decoupled income support" notifizierten. ${ }^{65}$

65 Auch „transition payments“ für Tabak 2005 bis 2014 in Höhe von insgesamt 9,6 Mrd. USD wurden als „decoupled income support“ gemeldet. Allerdings wurden sie durch eine bei Herstellern und Importeuren von Tabakprodukten erhobene Abgabe finanziert und entsprechen somit nicht den 
Einen Milestone-Charakter verdeutlicht die Tatsache, dass die Einführung von „production flexibility contract payments" für Rucker und Pasour (2007) den Beginn einer neuen Phase in der US-Agrarpolitik, nämlich der Phase der „decoupled payments“, markiert. Die Umstellung auf entkoppelte Zahlungen wird von einigen Experten als ein Paradigmenwechsel in der US-Agrarpolitik bewertet (Coleman et al. 1996, Tweeten und Zulauf 1997, Skogstad 1998, Moyer und Josling 2002). Die New York Times (1996) hatte in diesem Zusammenhang sogar das Ende der Subventionen ausgerufen.66

Vor diesem Hintergrund stellt die Abschaffung der Direktzahlungen im Jahr 2014 einen weiteren Milestone dar. Auch dieser Schritt wurde in der Presse als eine Zäsur bewertet. ${ }^{67}$ Das Verlassen des Entkopplungskurses lief den Empfehlungen der OECD über eine marktorientierte, möglichst wenig handelsverzerrende Agrarpolitik zuwider. Besondere Bedeutung erlangt die Abschaffung der Direktzahlungen in den USA, vergleicht man sie mit den parallelen Entwicklungen in der EU, die in der letzten Agrarreform von 2013 die Direktzahlungen beibehielt. Josling und Tangermann (2015) weisen darauf hin, dass die jüngsten Reformen „have broken [the] trend towards convergence“. Auch Bureau (2012: 76) sieht ,a fundamental divergence“ zwischen den USA und der EU darin, dass „the US is moving towards further isolating its farmers from adverse conditions and in particular from downward market fluctuations“, während ,the EU maintains a system of fixed payments that do not depend on market conditions and yields".

Genau diese Auseinanderentwicklung macht den beabsichtigten Vergleich zwischen den USA und der EU hinsichtlich der Direktzahlungen besonders interessant. Schließlich ist die Auswahl der Abschaffung der Direktzahlungen als Milestone auch der Auseinandersetzung mit der von Josling und Tangermann (2015) aufgestellten Hypothese geschuldet, die divergierende Entwicklung des Instruments der Direktzahlungen in den USA und in der EU in jüngerer Zeit sei das Ergebnis politischer Entscheidungen, die in unterschiedlichen innenpolitischen Umfeldern als Reaktion auf die Legitimationskrise der Direktzahlungen fielen (vgl. Kapitel 1). Die vorliegende Arbeit setzt sich - unter anderem - zum Ziel, diese These zu verifizieren.

Als ein weiterer Milestone wird die Einführung der Ausgleichszahlungen als Kompensation für den Abbau der Preisstützung gewählt. In den USA initiierten Anfang der 1960er-Jahre die „price support payments“ - zunächst bei Futtergetreide und Weizen - den Übergang von der Preis- zur Einkommensstützung. Die

Kriterien der Definition der staatlichen Zahlungen. Darüber hinaus wurden auch die Ausgleichszahlungen für Erdnüsse im Zuge von „peanuts buyout“ 2002 bis 2006 als „,decoupled income support“ notifiziert. Mit 1,3 Mrd. USD über fünf Jahre waren sie aber vergleichsweise gering.

${ }^{66}$ Der Artikel wurde am 5. April 1996 veröffentlicht und mit dem Titel „Clinton Signs Farm Bill Ending Subsidies" versehen.

${ }^{67}$ Wall Street Journal (2012): „,[O]ne of the biggest changes to farm policy in years.“ Politico (2012): „[G]enuinely a landmark shift.“ 
Zahlungen waren aus dem öffentlichen Haushalt gewährt und sollten die negativen Folgen der Absenkung der Stützpreise („loan rates") für landwirtschaftliche Einkommen abfangen, indem sie die Differenz zwischen dem alten, höheren und dem neuen Stützniveau ausgleichen. Bereits 1963 - dem ersten Jahr der Anwendung der „price support payments“ - wurde die „loan rate“ für Mais auf etwa Weltmarktpreisniveau abgesenkt; bei Weizen im zweiten Anwendungsjahr (1964), bei Baumwolle ebenfalls im ersten Anwendungsjahr (1966). Im Jahr 1970 wurden die Zahlungen dahingehend modifiziert, dass sie den Unterschied zwischen der abgesenkten „loan rate“ und dem Marktpreis ausglichen.

Somit waren „price support payments“ Vorboten der „deficiency payments“, die im Jahr 1973 beschlossen und mit der Zeit so modifiziert wurden, dass sie sich als Blue-Box-Zahlungen (teilentkoppelte Zahlungen) qualifizieren konnten. Für das Jahr 1995 - das letzte Jahr ihrer Geltung - wurden sie bei der WTO auch so notifiziert (Schnepf 2014c: 13). Nichtsdestotrotz wird für die Zwecke der vorliegenden Untersuchung nicht die Beschließung der „deficiency payments“, sondern der weniger bekannten „price support payments“ als Milestone ausgewählt. Als erste Ausgleichszahlungen für einen Abbau der Preisstützung waren sie ein Novum. Denn frühere variable Zahlungen, wie beispielsweise „parity payments“, wurden zusätzlich zur auf hohem Niveau festgelegten Preisstützung angewendet. Wie Ingersent und Rayner (1999: 183) konstatieren: „The feed grains provision of the 1962 Food and Agriculture Act broke new ground by giving producers deficiency payments [Herv. durch die Verf.] support for the first time ever in the US.“ Sie weisen auch darauf hin, dass der wahre Charakter dieser Zahlungen unklar bleiben sollte: Der Begriff „deficiency payments“ wurde erst 1973 verwendet. Eine mögliche Erklärung dafür ist, so Ingersent und Rayner (1999: 183), dass ansonsten ein Widerstand von Landwirten, die sich nicht als Empfänger von staatlichen Leistungen sehen wollten, zu befürchten war.

Auch für Rucker und Pasour (2007) ist die Einführung der „price support payments“ - nicht der „deficiency payments“ - eine Wegmarke: Sie markiert den Beginn der neuen Phase in der US-Agrarpolitik, der Phase der „direct payments“. Dass es so kommt, war allerdings nicht selbstverständlich: Alternativkonzepte zu den Ausgleichszahlungen wurden immer wieder vorgeschlagen und debattiert. Im Vorfeld der Farm Bill von 1973 beabsichtigte die Nixon-Regierung, das Zeitfenster der hohen Marktpreise zu nutzen und „price support payments“ innerhalb von drei Jahren auslaufen zu lassen. Staatliche Zahlungen an landwirtschaftliche Erzeuger sollten nur als Entschädigung für die Flächenstilllegung in Zeiten hoher Bestände möglich sein. Der von Demokraten dominierte Kongress entschied mit dem „Agriculture and Consumer Protection Act“ von 1973 aber anders. Ein Versuch, „deficiency payments“ stark zu reduzieren, wurde Mitte der 1980er-Jahre durch die Reagan-Regierung vor dem Hintergrund der hohen Budgetausgaben angestrebt, fand im Kongress aber ebenfalls keine Beachtung. Auch der Plan der Senatoren Boschwitz (Republikaner) und Boren (Demokrat) von 1985, der von der 
Erzeugung und Preisen entkoppelte „transition payments“ über fünf Jahre vorsah (danach würden sie abgeschafft), wurde zu jener Zeit abgelehnt (Orden et al. 1999).

„Price support payments“ für die ersten Erzeugnisse - Weizen sowie Mais, Sorghum und Gerste - wurden mit dem „Food and Agriculture Act“ im Jahr 1962 beschlossen. Eine Konsolidierung der neuen Vorschriften erfolgte durch den „Food and Agricultural Act“" von 1965, der außerdem Baumwolle in das neue System einbezog. Deshalb wird für den nachfolgenden Vergleich der Zeitraum 1962 bis 1965 als Milestone herangezogen.

Zu Beginn waren die „price support payments“ an die aktuelle Erzeugung gekoppelt. Während in der EU im Jahr 1992 gleich teilentkoppelte Ausgleichszahlungen eingeführt wurden, erfolgte die Entwicklung in den USA schrittweise: Ausgleichszahlungen der 1960er-Jahre wurden erst in den Jahren 1985 und 1990 teilweise entkoppelt. Diese beiden Entkopplungsschritte gelten als Milestones im Sinne der vorliegenden Arbeit.

\subsection{Ausgewählte Milestones in der EU}

Analog zum Vorgehen im Falle der USA und aus demselben Grund wird die Ersteinführung der Direktzahlungen in der EU, wie sie in der vorliegenden Arbeit definiert werden, als einer der Milestones bestimmt. Die weitgehende Entkopplung der Agrarzahlungen fand in der EU im Zuge der Fischler-Reform von 2003 - „the most radical reform of the CAP“ (Swinnen 2008b: 135) - statt. Die ab 2005 schrittweise eingeführte einheitliche Betriebsprämie ersetzte die nur teilweise entkoppelten Zahlungen der MacSharry-Reform. Die neue Prämie entsprach den Kriterien für „decoupled income support“ der Green Box der W'TO und wurde als solche notifiziert. Ihr Pendant in den 2004 und später der EU beigetretenen Ländern (mit Ausnahme von Slowenien, Malta und Kroatien) stellt die einheitliche Flächenzahlung dar.

Zwar ist die Betriebsprämie von 2003 nicht die erste EU-Agrarzahlung, die bei der WTO als „decoupled income support“ notifiziert wurde bzw. die den für „decoupled income support" aufgestellten Kriterien entsprach. Bereits die vorübergehenden Einkommensbeihilfen aus dem Jahr 1989 erfüllten diese Kriterien und wurden entsprechend eingeordnet. Da von ihnen aber wenig Gebrauch gemacht wurde und sie bald durch die Reform von 1992 überholt wurden, eignen sie sich nicht als Milestone im Sinne der Fragestellung. Noch weniger trifft dieses auf die entkoppelten Ausgleichszahlungen im Falle der Währungsaufwertung von 1995 $\mathrm{zu}$, die für einige wenige Mitgliedstaaten, zeitlich sehr begrenzt und aus einem spezifischen Grund vorgesehen wurden. Die Zahlungen der MacSharry-Reform von 1992 qualifizierten sich nicht für die Green Box. Eine Einführung von entkoppelten Zahlungen war zu der Zeit politisch problematisch, urteilen Cunha und Swinbank (2011: 91): 
The farming community believed it would be very vulnerable to criticisms of the European Union paying farmers for doing nothing [Herv. im Original] [...]; and the Council could not easily agree to such a huge change in CAP's basic principles given the fierce opposition of the farming community.

Den weiteren Milestone in der Entwicklung der Direktzahlungen in der EU stellt, ähnlich wie in den USA, die letzte Reform dar. Zwar waren die Veränderungen, die die Reform von 2013 brachte, relativ klein, sodass Zweifel bestehen, ob die Bezeichnung „Reform“ überhaupt zutrifft (Swinnen 2015b). In Bezug auf die Forschungsfrage war sie aber nichtsdestotrotz wichtig, weil die (entkoppelten) Direktzahlungen als Kern der Stützung beibehalten wurden. Schon die Mitteilung der Kommission vom November 2010, in der die Optionen für die künftige GAP dargestellt wurden, interpretiert Tangermann (2011: 11) als Versuch, das System der Direktzahlungen in der EU politisch abzusichern. Somit ging die EU in Bezug auf die Direktzahlungen einen anderen Weg als die USA. Vor dem Hintergrund dieser Auseinanderentwicklung wird die Entscheidung von 2013 als Milestone betrachtet.

Als ein weiterer Milestone wird - analog zu den USA - die Ersteinführung der teilentkoppelten Ausgleichszahlungen als Kompensation für den Abbau der Preisstützung gewählt. Diese fand in der EU auf breiter Basis im Zuge der MacSharryReform von 1992 statt: Die Stützpreise wurden schrittweise abgesenkt und die dadurch entstandenen Einkommenseinbußen den Landwirten durch Ausgleichszahlungen kompensiert. Davor dominierte in der EU-Agrarpolitik die Preisstützung; die Zahlungen an Landwirte waren von viel geringerem Gewicht. Die Reform von 1992 verschob das Verhältnis der agrarpolitischen Instrumente zugunsten der Zahlungen. Moyer und Josling (2002: 116) urteilen folgendermaßen: „,The MacSharry reforms established a new path for the Common Agricultural Policy in that they shifted the primary instrument for supporting farmer income from price supports to compensatory payments." Auch für Hill (2012) ist die Reform von 1992 eine Wegmarke: Sie markiert den Beginn einer neuen Phase in der Entwicklung der europäischen Agrarpolitik.

\subsection{Verlauf des Konvergenzprozesses zwischen den USA und der EU in Bezug auf die Direktzahlungen}

Insgesamt wurden für die Zwecke der vorliegenden Untersuchung jeweils drei Milestones als wichtige Einschnitte in der Entwicklung der Direktzahlungen in den USA und in der EU identifiziert (vgl. Tabelle 20). Mit Ausnahme der Beibehaltung der Direktzahlungen in der EU im Jahr 2013 haben sie gemeinsam, dass sie die tief greifenden Veränderungen der Agrarpolitik beidseits des Atlantiks in Bezug auf dieses Instrument markieren: die Entscheidungen über die Einführung 
von Ausgleichszahlungen als Vorstufe zu Direktzahlungen (1962 bis 1965 bzw. 1992), von Direktzahlungen selbst (1996 bzw. 2003) sowie die Abschaffung der Direktzahlungen in den USA (2014). Anders als in der EU wurden in den USA die teilentkoppelten Ausgleichszahlungen schrittweise eingeführt; dementsprechend wurden die beiden Teilentkopplungsschritte 1985 und 1990 dem ersten Milestone zugeteilt. Das Festhalten der EU an den Direktzahlungen in der GAP-Reform von 2013 wurde insbesondere vor dem Hintergrund der darauf zurückgehenden Auseinanderentwicklung zwischen den USA und der EU als Milestone ausgewählt.

Tabelle 20: Die ausgewählten Milestones in der Entwicklung der Direktzahlungen in den USA und in der EU

\begin{tabular}{lrrr} 
& Erster Milestone & Zweiter Milestone & Dritter Milestone \\
\hline USA & $1962-1965$ & 1996 & 2014 \\
EU & 1995 und 1990$)$ & & \\
\hline
\end{tabular}

Es ist festzuhalten, dass die Entwicklungen in den USA und der EU zunächst ähnlich verliefen. Ab Beginn der systematischen Agrarstützung (1933 in den USA, 1962 in der EU) waren staatliche Zahlungen an Landwirte Teil des jeweiligen agrarpolitischen Instrumentariums. Allerdings wurden sie auf beiden Seiten lange Zeit nur punktuell eingesetzt und spielten im Vergleich zur Preisstützung nur eine sekundäre Rolle. Zwar war beidseits des Atlantiks bereits sehr früh eine Phase zu verzeichnen, in der die staatlichen Zahlungen das primäre Instrument der Agrarpolitik darstellten (Zahlungen unter dem „Agricultural Adjustment Act“ von 1933 in den USA, „deficiency payments“ in Großbritannien in der Zwischenkriegszeit). Sie konnten sich aber zu der Zeit nicht dauerhaft etablieren. In den USA beendete das Oberste Gericht das Experiment. In der EU wurden zu Beginn der GAP staatliche Zahlungen aus finanziellen und verwaltungstechnischen Gründen abgelehnt. Die ersten Zahlungen waren auf beiden Seiten an die aktuelle Erzeugung und/oder Marktpreise gekoppelt.

In beiden Ländern bzw. Wirtschaftsräumen erfolgte zunächst die Einführung der Ausgleichszahlungen und später die Einführung von (entkoppelten) Direktzahlungen (,decoupled income support“). Diese Entwicklung wird jeweils durch den ersten und zweiten Milestone abgebildet. Im ersten Schritt fand die Verlagerung der Stützung weg von der Preis- und hin zur direkten Einkommensstützung statt. In der EU entsprachen die Ausgleichszahlungen gleich den Blue-Box-Kriterien; in den USA wurden die bereits in den 1960er-Jahren eingeführten Ausgleichszahlungen im Laufe der Zeit dahingehend modifiziert. Im zweiten Schritt wurden die Ausgleichszahlungen zu Direktzahlungen. An den zwei identisch verlaufenen Milestones wird die Angleichung der Agrarpolitiken der USA und der EU in Bezug auf die Direktzahlungen deutlich erkennbar. Zum einen wurden die- 
selben Instrumente eingeführt (Ausgleichszahlungen als Vorstufe der Direktzahlungen und Direktzahlungen). Zum anderen verlor das Instrument der Preisstützung an Bedeutung. Dieses steht in Einklang mit der Bemerkung von Josling und Tangermann (2015), die eine Annäherung der Agrarpolitiken der USA und der EU u. a. am konvergierenden Instrumentarium festmachen. Allerdings war im Zuge der letzten Agrarreformen 2013 bzw. 2014 mit der gleichartigen Entwicklung in Bezug auf die Direktzahlungen Schluss. Während die USA sie abschafften, hielt die EU an ihnen fest. Durch den Verzicht einer Seite auf das Instrument der Direktzahlungen kam es zu einer divergierenden Entwicklung. Dieses Phänomen, abgebildet jeweils durch den dritten und letzten Milestone, überrascht vor dem Hintergrund des langen, bis dahin stattgefundenen Konvergenzprozesses.

Die Bestimmung und Gegenüberstellung der Milestones ermöglichen, den Verlauf des Prozesses der Konvergenz bzw. der Divergenz zwischen den Agrarpolitiken der USA und der EU abzubilden. Wie im Forschungskonzept dargestellt, stehen dabei die Beta- und Delta-Konvergenz im Fokus (vgl. Kapitel 2.4). Eine der Hauptfragen ist, welcher der beiden Akteure hinsichtlich der Direktzahlungen die Vorreiterrolle innehatte und wer der „Nachzügler“ war. Es ist somit zugleich die Frage nach dem Verlauf der Beta-Konvergenz. Der Blick auf die ersten zwei Milestones verdeutlicht, dass die USA der EU in beiden Fällen voraus waren. Sie beschlossen die Ausgleichszahlungen bereits 1962 in Form von „price support payments" für Futtergetreide. Zu der Zeit wurde die europäische Agrarpolitik als eine Preisstützungspolitik etabliert; die Ausgleichszahlungen wurden in der EU erst 30 Jahre nach den USA im Zuge der MacSharry-Reform von 1992 eingeführt. Mit diesem Schritt glich die EU ihre Agrarpolitik hinsichtlich der Direktzahlungen an die USA an. Die USA setzten auch die Direktzahlungen eher als die EU ein. Als dieses im Jahr 1996 erfolgte, war in der EU gerade die Einführung der Ausgleichszahlungen vollzogen worden. Die Direktzahlungen wurden dort sieben Jahre später als in den USA im Jahr 2003 beschlossen. Wieder war es die EU, die ihre Politik anglich. Dieses Muster - die USA als Vorreiter, die EU als „Nachzügler“ wurde mit den letzten, fast zeitgleich erfolgten Reformen im Dezember 2013 bzw. Januar 2014, die ein unterschiedliches Ergebnis hatten, unterbrochen, aber nicht unbedingt eingestellt. Sollte allerdings die EU in der nächsten Reform der GAP im Jahr 2020 (und später) die Direktzahlungen abschaffen, wäre dieses Konvergenzmuster wieder hergestellt. Diese Entwicklung deutet auf Differenzen zwischen den USA und der EU bezüglich der Bedeutung der externen Faktoren in der Entwicklung der Direktzahlungen hin. Andernfalls hätten insbesondere die jeweiligen ersten zwei Milestones zeitlich nicht so weit auseinandergelegen bzw. wären die bisher letzten Reformen nicht so deutlich auseinander gegangen. Diese Frage wird im nächsten Kapitel näher überprüft.

Allerdings lassen sich die Entwicklungen in den USA und der EU auch aus einer anderen Perspektive betrachten. Rückt die Delta-Konvergenz in den Fokus, wird deutlich, dass sich die EU schneller dem Modell „decoupled income sup- 
port" annäherte als die USA, wenn als Ausgangspunkt der jeweilige Beginn der gezielten Agrarstützung gewählt wird. Beide Akteure führten die Ausgleichszahlungen etwa 30 Jahre nach dem Beginn der Agrarstützung im Jahr 1933 bzw. 1962 ein: im Jahr 1962 bzw. 1992. Die USA brauchten aber weitere 34 Jahre, um die Direktzahlungen einzuführen (1996); die EU nur 11 Jahre (2003). Für den nächsten Schritt - die Abschaffung von Direktzahlungen, die gleichzeitig die Abkehr vom „decoupled income support"-Modell bedeutete - benötigten die USA 18 Jahre. Die EU entschied, die Direktzahlungen beizubehalten und zu „begrünen“, zehn Jahre nach der Entscheidung über die Entkopplung. Vor diesem Hintergrund erscheint die EU als der beweglichere Akteur: Die Intervalle zwischen den Milestones sind in der EU generell kürzer als in den USA. Insbesondere führte sie die Direktzahlungen relativ zügig ein (bereits elf Jahre nach den Ausgleichszahlungen). Werden aber für die Untersuchung der Delta-Konvergenz dieselben Zeitpunkte in der Vergangenheit gewählt, wird klar, dass die EU gegenüber den USA in der Entwicklung der Direktzahlungen die meiste Zeit zurückblieb. Als gemeinsamer Ausgangspunkt kann nachvollziehbar erst das Jahr der Einführung der GAP im Jahr 1962 dienen. Das „decoupled income support“-Modell implementierten die USA 34 Jahre später mit „production flexibility contract payments“; die EU 41 Jahre später mit der Betriebsprämie.

Die Vorreiterrolle der USA wird durch die frühe Einführung von anderen Zahlungstypen unterstrichen. Beispielsweise wurden Umweltzahlungen („,conservation payments") in den USA bereits im Jahr 1936 vor dem Hintergrund der Staubstürme „Dust Bowl“ eingeführt - aber auch, um eine Weiterführung der Agrarstützung nach dem Beenden von AAA 1933 durch das Oberste Gericht zu ermöglichen (Benedict 1953). Es wurden neben den Zahlungen für die Kürzung der Anbaufläche für Überschusserzeugnisse (Flächenstilllegung) auch Zahlungen beschlossen, die Landwirte für ausgewählte umweltfreundliche Leistungen bzw. Produktionsverfahren auf Basis von Verträgen vergüteten (Anderson und Magleby 1997). Das Cross-Compliance (Bindung der Agrarstützung an bestimmte Auflagen) hat seinen Ursprung in der Farm Bill von 1985 (Cain und Lovejoy 2004). Aber bereits die Zahlungen im Zuckersektor von 1934 waren an die Einhaltung von Standards im Bereich landwirtschaftlicher Arbeit gebunden; 1938 kamen noch Umweltstandards hinzu (Davis 1935, Ballinger 1946). Die ersten regulären „disaster payments" wurden in den USA bereits im Jahr 1937 im Zuckersektor eingeführt (vgl. Box 12). Auch ein „bond scheme“ im Sinne des Vorschlags von Tangermann von 1990 (s. Kapitel 3) implementierten die USA - im Gegensatz zur EU - bereits. Anfang der 2000er-Jahre wurden den Quoteninhabern im Erdnussund Tabaksektor zeitlich begrenzte und im Voraus festgelegte Ausgleichszahlungen als Kompensation für die Abschaffung der Quote gewährt (s. Kapitel 4). Allerdings wurden Erdnussanbauer gleichzeitig in das System der zur Zeit des „buyout" gültigen Agrarzahlungen einbezogen, was dem Bond-Konzept nicht entspricht. 
Box 12: „Disaster payments“ in der US-Agrarpolitik

Bei den „disaster payments“ in den USA handelt sich um Zahlungen an landwirtschaftliche Erzeuger, die im Falle natürlicher Katastrophen gewährt werden (,,crop disaster payments"). Im weiteren Sinne umfassen sie auch Notzahlungen bei Marktkrisen (,market loss payments“). Sie machen den größten Teil der „miscellaneous payments“ in Tabelle A2 und Tabelle A3 aus. Die ersten regulären „disaster payments“ im Falle natürlicher Katastrophen wurden in den USA bereits in den 1930er-Jahren für Zuckerrüben und -rohr eingeführt (Ballinger 1946). Für Weizen, Futtergetreide und Baumwolle wurden sie 1973 gleichzeitig mit den „deficiency payments“ beschlossen (Bowers et al. 1984). Im Jahr 1989 standen die „disaster payments" laut „Government Accountability Office“ (GAO) bereits für 472 Erzeugnisse offen (GAO 1989: 30). Lange Zeit waren sie das Hauptinstrument der Katastrophenhilfe im Bereich der Landwirtschaft. Im Zeitraum von 1974 bis 1980 beliefen sie sich im Durchschnitt auf 436 Mio. USD jährlich (ibid.: 3). Aufgrund der hohen Haushaltsausgaben und der Tatsache, dass sie die Kostenplanung erschwerten, standen die „disaster payments“ in der Kritik. Bemängelt wurde außerdem, dass sie einen Anreiz schafften, die landwirtschaftliche Erzeugung in Hochrisikoregionen fortzuführen. Damit erhöhten sie die Wahrscheinlichkeit von Verlusten sowie den Bedarf an Unterstützung. Aus diesen Gründen wurde an Alternativen zu den "disaster payments“ gearbeitet (GAO 1989).

Das erste Versicherungsprogramm für die Landwirtschaft wurde bereits 1938 aufgestellt (Benedict 1953). Im Jahr 1980 wurde ein neues Ernteversicherungsprogramm verabschiedet, dessen Umfang und Verfügbarkeit im Vergleich zum Vorgänger erweitert wurde. Sein Ziel war es, die Landwirte gegen unvermeidbare Ertragsausfälle durch Naturkatastrophen zu schützen. Der Staat bezuschusste in der Regel $30 \%$ der Versicherungsprämie für etwa 50 Erzeugnisse (GAO 1989: 3). Dieses Programm sollte die „disaster payments“ ersetzen: Diese wurden ab dem Jahr 1982 grundsätzlich nicht mehr gezahlt, wenn dem Landwirt eine Ernteversicherung zur Verfügung stand (Johnson et al. 1982). Die versicherte Fläche erhöhte sich zwar von 9,6\% der beihilfefähigen Fläche im Jahr 1980 auf 24,5\% im Jahr 1988, blieb aber immer noch unter dem festgelegten Ziel von $50 \%$ (GAO 1989: 4). Da die Teilnahmerate am Versicherungsprogramm relativ niedrig war, wurden Ad-hoc-,,disaster payments“ fortgeführt. Folglich waren in den Jahren 1980 bis 1988 „disaster payments" mit insgesamt 6,9 Mrd. USD immer noch das Hauptinstrument der Katastrophenhilfe, gefolgt von Notfallkrediten (6,4 Mrd. USD). Dazu trugen vornehmlich die Notfallzah- 
lungen bei, die in den Jahren 1981 und 1988 als Reaktion auf die schwere Dürre aufgestellt und mit insgesamt 5,4 Mrd. USD ausgestattet wurden. Für die Ernteversicherungsprogramme wurden in dem Zeitraum mit 4,3 Mrd. USD deutlich weniger als für „disaster payments“ ausgegeben (ibid.). Die niedrige Teilnahmerate am Versicherungsprogramm war u. a. auf die kostenfreie Verfügbarkeit von „disaster payments“ zurückzuführen, wie das GAO (1989: 4) feststellte. In dieser Konstellation wurde eine Versicherung der Ernte von Landwirten als unnötig angesehen.

Mit der Zeit wurde das Versicherungsprogramm in Bezug auf die Teilnahmequote doch noch zum Erfolg. Im Jahr 2013 wurden 295 Mio. Acres, die 83 \% der Anbaufläche ausmachten, durch eine landwirtschaftliche Versicherung gedeckt (Shields 2013: 3). Laut Shields (2013: 22) liegt der Erfolg der Ernteversicherung in den USA u. a. in der Tatsache, dass in den letzten Jahren die „commodity payments“" weniger Schutz vor einem Preisrückgang boten, als dieses früher der Fall war. Außerdem seien hier eine hohe Zahl der beihilfefähigen Erzeugnisse sowie höhere Zahlungen als im Rahmen der Agrarrohstoffprogramme zu nennen. Die Anzahl der versicherbaren Risiken und Kulturen sowie die Produktvielfalt wurden kontinuierlich erweitert. Aktuell schützt die Versicherung vor Ertragsausfällen sowie vor Erlösverlusten bezogen auf das Erzeugnis oder den Betrieb. Etwa 130 Erzeugnisse werden abgedeckt (Shields 2014b: 1). Der Zuschuss zur Versicherungsprämie betrug 2013 im Durchschnitt 62 \% (ibid.: 3). Im Grundprogramm - „catastrophic yield policies“ bei Ertragsausfällen von über $50 \%$ - übernimmt der Staat die gesamte Versicherungsprämie. Er trägt außerdem die Kosten für den Verkauf und Betreuung von Versicherungen. Es sind keine Obergrenzen für Beträge pro Betrieb bzw. Landwirt in Kraft. Mit der Farm Bill von 2014 wurde die Teilnahme an den staatlich subventionierten Versicherungsprogrammen an die Einhaltung bestimmter Umweltauflagen (CrossCompliance) gebunden (Shields 2014b).

Der Erfolg der Ernteversicherung führte allerdings nicht - wie ursprünglich geplant - zur Abschaffung der ,disaster payments“. Seit 1989 verabschiedete der Kongress fast jedes Jahr zusätzliche Mittelzuweisungen für die Notfallhilfe im Agrarsektor. Zwischen den Haushaltsjahren 1989 und 2012 betrugen die Finanzmittel für diese Zwecke insgesamt $70 \mathrm{Mrd}$. USD, davon $44 \mathrm{Mrd}$. USD für die Zahlungen an landwirtschaftliche Erzeuger (21,7 Mrd. USD für „,market loss payments“ von 1999 bis 2001 und 22,3 Mrd. USD für „crop disaster payments“) (Chite 2011). 



\section{Zusammenstellung der für die Milestones relevanten Einflussfaktoren}

\subsection{Vorgehensweise}

Die im vorherigen Kapitel bestimmten Milestones bilden die substanziellen Entscheidungen der USA und der EU in Bezug auf die jeweilige Entwicklung der Direktzahlungen $\mathrm{ab}$. Sie werden nachstehend näher untersucht. Dabei werden für jeden Milestone die ihm zugrunde liegenden Faktoren ${ }^{68}$ zusammengestellt. Die Fragen lauten hier: Warum wurden jeweils die Ausgleichszahlungen und die Direktzahlungen eingeführt? Warum wurden die Direktzahlungen abgeschafft bzw. nicht abgeschafft?

Einem Politikwandel liegt in der Regel ein komplexes Geflecht aus Faktoren zugrunde, die nicht selten in gewissem Maße miteinander zusammenhängen (z. B. kann ein Rückgang der Marktpreise den Haushaltsdruck erhöhen). Zudem kann vom Reformdruck nicht unbedingt auf die Kausalitäten für die Ausgestaltung der Reformen geschlossen werden. Gerade weil die Reformen mehrere Ursachen haben können, gehört zu ihrer Auswahl und Anordnung ehrlicherweise auch eine Portion an Spekulation. Das trifft umso mehr zu, je tiefer in die Vergangenheit ge-

${ }^{68}$ Ausdrücke wie Auslösefaktoren, Antriebskräfte, treibende Kräfte, Treiber, Ursachen, Reformfaktoren, zugrunde liegende Faktoren und Einflussfaktoren werden hier abwechselnd als Synonyme genutzt. 
schaut wird. Wie Swinbank (2016: 59) im Hinblick auf die EU-Agrarpolitik bemerkt: „Over that long sweep of history it is almost impossible to determine what were the decisive influences shaping the evolution of the CAP." Umso wichtiger ist es, im Vorfeld zu bestimmen, welche Ursachen vor dem Hintergrund der Forschungsfrage von Bedeutung sind. Dazu ist eine Bündelung der Faktoren ratsam, um sich auf diejenigen, die später der Erklärung der Konvergenzprozesse dienlich sind, fokussieren zu können.

Das Konzept des „Politikfensters“ (,policy window“) von Kingdon (1995) kann hier hilfreich sein. Kingdon stellt die Frage, was für Faktoren bestimmen, welche Themen es zu einem Zeitpunkt auf die politische Agenda schaffen. Obwohl das Konzept ursprünglich explizit nur für die Analyse der ersten Phase eines Politikprozesses - das Agenda-Setting - entwickelt wurde, wird es vor dem Hintergrund der ähnlichen Entscheidungslogik auch für die Analyse von Entscheidungsfindungsprozessen verwendet (u. a. Zahariadis 1995).

Anhand einer empirischen Untersuchung des Gesetzgebungsverfahrens in den USA identifiziert Kingdon (1995) drei Ströme, die das politische Geschehen bestimmen. Der erste Strom ist der sogenannte „problem stream“: Probleme und Fehlentwicklungen, die die Aufmerksamkeit von handelnden Akteuren auf sich ziehen und wo offenbar Handlungsbedarf besteht. Dieses kann aufgrund von verschiedenen Ereignissen erfolgen, u. a. durch Katastrophen, Krisen, vertiefte Aufmerksamkeit der Medien, Akkumulierung von kleinen Problemen, die eine kritische Masse erreichen, gescheiterte politische Entscheidungen sowie Bekanntwerden neuer Daten. Im „policy stream“ wiederum befinden sich verfügbare Ideen, Konzepte und mögliche Lösungsvorschläge für das bestimmte Problem. Der „politics stream" schließlich beinhaltet die nationale Stimmungslage, die Machtverteilung, die Prozesse innerhalb der Regierungsorganisation (z. B. Regierungswechsel) sowie organisierte politische Kräfte außerhalb der Regierungsorganisation. „Policy windows“ für politische Veränderungen entstehen aus der Kopplung der drei Ströme, wobei Kingdon (1995: 204) die Rolle, die dabei die sogenannten „policy entrepreneurs“ - ,people willing to invest their resources in return for future policies they favour" - spielen, betont.

Laut Kingdon (1995) kommt der primäre Anstoß zur Entstehung eines „policy window" durch Veränderungen entweder im „problem stream“ oder „politics stream“. Es ist der „problem stream“, der Erklärungen liefert, warum eine Entscheidung getroffen wurde (bzw. - im Sinne von Kingdon - warum ein Problem auf die Agenda platziert wurde). Vor dem Hintergrund der Forschungsfrage steht er im Fokus der vorliegenden Untersuchung: Die darunter fallenden Faktoren sind dazu prädestiniert, Politikkonvergenz zu erklären, weil sie länderübergreifend wirksam sein können. Davon können Kausalmechanismen der Konvergenz abgeleitet werden. Auf die Frage, wie sich die betreffende Entscheidung herbeiführen ließ, liefert der „politics stream“ Antworten. Er bezieht sich auf die politischen bzw. institutionellen Faktoren auf der nationalen Ebene und kann somit bei der 
Erklärung von Politikkonvergenz zweier Länder zwangsläufig nur von sekundärer Bedeutung sein. Die darunter fallenden Faktoren übernehmen die Rolle sogenannter intervenierender Faktoren, die laut Konvergenztheorie die Wirksamkeit von Kausalmechanismen und somit die Geschwindigkeit und den Umfang der Konvergenz beeinflussen.

In der vorliegenden Untersuchung interessieren Entwicklungen außerhalb des Systems der Agrarpolitik im Gegensatz zu Faktoren innerhalb dieses Systems. Als Beispiel für letztere kann das Streben nach Vereinfachung der Agrarpolitik als Ursache für die Reformen Anfang der 1960er-Jahre in den USA (Hadwiger und Talbot 1965) und für die Reform der GAP von 2003 (Daugbjerg 2012) gelten. In der vorliegenden Arbeit wird vielmehr nach Änderungen im externen Umfeld der Agrarpolitik gesucht, die die politischen Präferenzen in eine reformorientierte Richtung verschoben haben, wie von Pokrivcak et al. (2006) definiert. Sie müssen zudem ausreichend groß gewesen sein, um die starke Präferenz zur Fortführung des Status quo zu überwinden (ibid.: 587). Denn wie de Gorter (2008: 15) bemerkt: „A distinguishing feature of agricultural policies is that once a policy is put in place, there is inertia in the political system with respect to changing the instrument, with changes made often only incrementally over long periods of time. This reflects both the path dependency of policy instrument choice and a bias for the status quo."

Der Fokus der Untersuchung liegt auf dem Kontext zu der Zeit des Reformvorschlags. Es wird aber auch geprüft, ob sich die jeweiligen Faktoren tatsächlich in den Reformergebnissen widerspiegeln. Dieses dient der Unterscheidung zwischen „political rhetoric“ und „political reality“ im Sinne von Olper (2008) und letztendlich der Identifizierung von „the true [Herv. im Original] key drivers and constraints" (ibid.: 88) für die ausgewählten Milestones. ${ }^{69}$ Aus dem Grund, dass sie viel „political rhetoric“ enthalten dürften, können die Dokumente der im Gesetzgebungsverfahren involvierten Institutionen, insbesondere derjenigen, die das Recht zur Gesetzesinitiative besitzen, nur unterstützend herangezogen werden. Das gilt auch für die Ziele, die der Gesetzgeber als Begründung für die jeweilige Reform im Gesetz benennt.

Die Vorgehensweise ist wie folgt: Zunächst werden die Ursachen für die agrarpolitischen Reformen, deren Bestandteil die ausgewählten Milestones bilden, anhand der Auswertung der relevanten Literatur zusammengetragen. Sobald klar wird, woher der größte Reformdruck rührte, wird versucht, die Ursachen für die

\footnotetext{
${ }^{69}$ Auch Cunha und Swinbank (2011: 176) weisen auf eine gewisse Inkohärenz zwischen „the factors that were officially invoked to justify the reforms" und „those that really seem to have motivated them, explained their shape and, ultimately, their final outcome“. Die Erklärung dafür sehen sie in der Natur der Politik. Diese „obliges decision-makers to find arguments to justify their decisions that can both be more readily understood by the general public and the farmers themselves, thus preparing the political ground for reform, and which can also serve to protect their political interests by avoiding or minimizing blame“.
} 
Milestones herauszufiltern. Denn nicht immer werden diese in der Literatur ausdrücklich genannt. In solchen Fällen interessieren beim „problem stream“ die Ursachen der jeweiligen Reform, die Einfluss auf Reforminhalte hatten - im Gegensatz zu den Ursachen, die sich eher auf das Timing der Reformen oder den Umfang des Agrarbudgets auswirkten. Es wird untersucht, inwiefern ein direkter Zusammenhang zwischen den Reformfaktoren und der hier interessierenden Entscheidung über die Direktzahlungen besteht bzw. plausibel ausgemacht werden kann. Dieses ist allerdings nicht immer leicht zu ermitteln und somit kann auch die Isolierung der Faktoren für die Milestones erschwert sein. Hinsichtlich des „politics stream“" wird angenommen, dass alle hierzu gehörenden Faktoren sowohl für die Reform als Ganzes als auch für den Milestone relevant waren.

Im darauffolgenden Kapitel werden die zusammengetragenen Faktoren für die jeweiligen Milestones auf beiden Seiten des Atlantiks auf Gemeinsamkeiten und Unterschiede untersucht und verglichen. Die zwei Teilentkopplungsschritte 1985 und 1990 in den USA werden aufgrund von Ähnlichkeiten in den Ursachen zusammen dargestellt. Auf dieser Basis werden anschließend Erklärungen für Konvergenzen bzw. Divergenzen zwischen den Agrarpolitiken der USA und der EU bei der Anwendung des Instruments der Direktzahlungen herausgearbeitet.

\subsection{US-Milestone 1962-1965}

Lange Zeit lehnten Landwirte in den USA eine breitere Anwendung von staatlichen Zahlungen in der Agrarpolitik entschieden ab (z. B. nach dem 2. Weltkrieg im Rahmen des Brannan-Plans, s. Kapitel 4). Hadwiger und Talbot (1965: 22) bemerken dazu, dass „the words compensatory payments or direct payments [Herv. im Original] came to have a high emotional content in the 1950's" und dass die Landwirte gegen sie opponierten, weil sie nicht „on the government dole“ sein wollten. Es stellt sich deshalb die Frage, warum solche Zahlungen für die wichtigsten landwirtschaftlichen Erzeugnisse - Futtergetreide, Weizen und Baumwolle - im Zeitraum von 1962 bis 1965 letztendlich eingeführt wurden.

In der Literatur werden in erster Linie die Auslöser für die Reformen der ersten Hälfte der 1960er-Jahre als Ganzes genannt. Als für sie ausschlaggebend wurden insbesondere die großen Überschüsse landwirtschaftlicher Erzeugnisse und die hohen Haushaltskosten der Stützungsprogramme identifiziert (z. B. Moyer und Josling 1990: 107, Orden et al. 1999: 61). Der US-Agrarsektor verzeichnete spätestens seit Mitte der 1950er-Jahre hohe Produktionsüberschüsse bei Weizen, Mais und Baumwolle (USDA 1963: Tabelle 10, 43 und 105). Die hohen Staatsausgaben für die Verwaltung dieser Überschüsse trugen dazu bei, dass die Landwirtschaft Anfang der 1960er-Jahre den drittgrößten Haushaltsposten darstellte, der lediglich von Zins- und Verteidigungsausgaben übertroffen wurde (Hadwiger und Talbot 1965: 1). Darüber hinaus wiesen die USA zu der Zeit fast jedes Jahr ein Haushaltsdefizit auf (OMB 2016: Tabelle 1.1 und 1.2). Dieser Problemdruck kann 
auch an den Zielen der relevanten Agrargesetze abgelesen werden. Der „Food and Agriculture Act“ von 1962, der „price support payments“ für Futtergetreide und Weizen einführte, hatte - neben Einkommensschutz für Landwirte - auch die Reduzierung der Ausgaben für Agrarprogramme und die Verhinderung der Überschuss-Akkumulation zum Ziel. Der „Agricultural Act“ von 1964 mit der entsprechenden Regelung für Baumwolle ging indirekt auf die Problematik der hohen Überschüsse ein, indem er den Baumwollverbrauch fördern wollte. Schließlich zielte der die neuen Vorschriften konsolidierende „Food and Agricultural Act“ von 1965 neben der Einkommenssicherung auch auf die Reduzierung von Produktionsüberschüssen sowie Agrarausgaben ab. Cochrane und Ryan (1976: 35) bezeichnen den zu der Zeit herrschenden und weiter wachsenden Druck, die Agrarausgaben zu reduzieren, als ,a new element in the boiling farm policy cauldron"; das alte Element stellte das sich verschärfende Überschuss- sowie das Einkommensproblem dar. Es stellt sich die Frage, warum hohe Agrarausgaben in jener Zeit zum dringenden Problem und somit auch zum Auslöser für die Reformen von 1962 bis 1965 wurden.

Moyer und Josling (1990) machen auf die verminderte Stärke des die Landwirtschaft unterstützenden Lagers im US-Kongress aufmerksam: Die Zahl der Kongressmitglieder, die landwirtschaftliche Wahlkreise repräsentierten, sank bedingt durch den Rückgang der landwirtschaftlichen Bevölkerung. Moyer und Josling (1990: 108) stellen deshalb fest: „The decline in strength of the Congressional farm lobby contributed to the demand for limiting farm programme costs". Cochrane und Ryan (1976: 35) weisen in diesem Zusammenhang nicht nur auf den „urbanized“ Kongress, sondern auch auf die „urbanized nation“ hin. Sie betonen die schwindende gesellschaftliche Akzeptanz für hohe Agrarausgaben Anfang der 1960er-Jahre. Die wachsende städtische Bevölkerung war inzwischen immer weniger bereit, die Landwirtschaft im bisherigen Umfang zu unterstützen, und forderte zudem mehr Investitionen in Bereichen wie Bildung und Armutsbekämpfung. Mit 9 Mrd. USD an Steuergeldern, gebunden in Überschusserzeugnissen, wurde das „farm problem“ zu einem „national problem“, zitieren Cochrane und Ryan (1976: 36) den damaligen republikanischen Landwirtschaftsminister E. T. Benson.

Ihr nachlassender Einfluss veranlasste die Vertreter der ländlichen Regionen, die Unterstützung der restlichen Kongressmitglieder zu suchen. Der „Food and Agriculture Act" von 1962, der die ersten „price support payments" einführte, „purposely emphasized concerns beyond the farm gate - in particular those of consumers and rural communities" (Cochrane und Ryan 1976: 42). Die Politik beabsichtigte damit, ,a broader base of political support for farm legislation“ zu finden (ibid.). Hadwiger und Talbot (1965: 96) weisen darauf hin, dass aus diesem Grund die Farm Bill von 1962 „Food and Agriculture Act“ genannt wurde, statt des für Farm Bills lange verwendeten, üblichen Namens „Agricultural Act“. Eine Verlagerung weg von einer rein sektoralen Politik setzte zu der Zeit ein. Wie Paarlberg (1978: 138f) es formuliert, etablierte sich Anfang der 1960er-Jahre „a 
new agenda for agriculture“, in der die Anliegen von „the poor, the rural nonfarm people, hired farm workers, minority groups, consumers, and environmentalists“ einen höheren Stellenwert erlangten.

Vor diesem Hintergrund verwundert es nicht, dass das Food-Stamps-Programm für Bedürftige Anfang der 1960er-Jahre zum ersten Mal Gegenstand des sogenannten Logrolling (Stimmentausch) wurde. Dieses wurde vom Agrarausschuss des Repräsentantenhauses genutzt, um Stimmen der Demokraten aus den urbanen Regionen für den umstrittenen „Agricultural Act" von 1964 mit dem Weizen- und Baumwollprogramm zu sichern (Ferejohn 1986: 233). Sowohl Präsident Kennedy als auch sein Nachfolger Johnson waren bestrebt, das FoodStamps-Programm aus den Jahren 1939-1943, das 1961 auf Pilotbasis wiedereingeführt wurde, dauerhaft zu etablieren. Die Republikaner im Kongress waren gegen beide Gesetzesvorschläge. Was folgte, war laut Ripley (1969: 300) keine formale, sondern eine "gefühlte" Abmachung zwischen den Demokraten aus den ländlichen und den urbanen Wahlkreisen im Repräsentantenhaus:

Gradually during March [1964, Anm. der Verf.] it became clear that the trade would involve the food stamp bill and the wheat-cotton bill [Farm Bill 1964]. No formal announcement was made of such trade. Indeed, no formal meeting was held at which leaders of urban and rural blocks agreed on it. Instead, [...] it was a matter of a favourable psychological climate. The more the individual members and the press talked about a specific trade of rural votes on food stamp for urban votes on wheat-cotton the more firmly the exchange became implanted in the minds of the members.

Wie Ferejohn (1986: 233) feststellt, erhielten die landwirtschaftsnahen Kongressmitglieder „a new weapon that would assist them in finding urban support for farm legislation". Zudem half die Verbindung von Maßnahmen für Weizen und Baumwolle in einem Gesetz, die Demokraten aus dem Süden und dem Mittleren Westen, die die Interessen unterschiedlicher Erzeugergruppen repräsentierten, zusammenzubringen (Cochrane und Ryan 1976: 45). Eine Koalition aus den meisten Republikanern und den südlichen Demokraten hingegen, die früher z. B. den Brannan-Plan erfolgreich torpedierte, war zu der Zeit nicht möglich (Hadwiger und Talbot 1965: 116). Die damaligen Probleme der Landwirtschaft waren „a good election issue for the party out of power“, sodass „Democratic legislators rarely consulted and almost never voted with Republicans, and vice versa“ (ibid.: 51). Beispielsweise sprach sich 1962 der Führer der Republikaner im Agrarausschuss des Repräsentantenhauses für ein auf der freiwilligen Flächenkürzung basierendes Programm als Alternative zur verpflichtenden Angebotskontrolle aus, obwohl er 1961 gegen solche Programme für Futtergetreide und Weizen stimmte (ibid.: 110f). 
Orden et al. (1999: 61) weisen auf den starken Wunsch der Demokratischen Partei nach einem Kurswechsel in der Agrarpolitik hin. Zusammen mit den hohen Agrarausgaben veranlasste dieses die neue Regierung nach einer Alternative zur bisherigen Politik der Preisstützung zu suchen:

Congressional agricultural policies in the Eisenhower era had proven expensive as well as intrusive. Net government expenditures exceeded $\$ 3$ billion in fiscal year 1955 and were nearly as high in 1956 [...]. Continued high program costs through 1960, plus a partisan urge to reverse course, prompted the new Kennedy administration to abandon the faltering squeeze-out strategy. (ibid.)

Der Weg für neue Maßnahmen war somit frei. Allerdings verlief er sehr turbulent (s. Box 13). Die Mehrheit der Republikaner und die konservativen Demokraten stellten sich gegen die Einführung von ,price support payments“: Im Repräsentantenhaus wurde die Farm Bill von 1962, die solche Zahlungen für Futtergetreide und Weizen einführte, mit einer nur sehr knappen Mehrheit von $202 \mathrm{zu}$ 197, die Farm Bill von 1964 mit entsprechenden Zahlungen für Baumwolle mit 211 zu 203 Stimmen angenommen (Hadwiger und Talbot 1965: 233 und 324). Die Periode von 1960 bis 1965 beschreiben Orden et al. (1999: 61 und 63) als ,an important interlude of policy dispute and improvisation, which eventually moved Congress toward a new experiment with cash-out policy reform options - at least for export crops“, in dem ,intrusive market-distorting price supports for these [export] crops would gradually be replaced with income support to farmers in the form of direct cash payments".

Aber auch die neuen Agrarzahlungen waren mit hohen öffentlichen Ausgaben verbunden, dessen sich der Gesetzgeber zum Zeitpunkt ihrer Einführung durchaus bewusst war (Orden et al. 1999: 65)..$^{70}$ Vor diesem Hintergrund entfallen Haushaltszwänge als Antriebskraft für den Milestone 1962-65, auch wenn sie einen wichtigen Auslöser für die Reformbestrebungen Anfang der 1960er-Jahre darstellten.

Box 13: Weg zur Einführung von ,price support payments“ in den USA im Jahr 1962

Die Einführung von „price support payments“ stand nicht auf der politischen Agenda der Kennedy-Regierung. Ihr ursprüngliches Ziel war es vielmehr, eine verpflichtende Angebotssteuerung für alle Erzeugnisse einzuführen, um die Agrarausgaben $\mathrm{zu}$ reduzieren und gleichzeitig landwirtschaftliche Einkommen zu verbessern. Staatliche Zahlungen an landwirtschaftliche Erzeuger waren nicht

\footnotetext{
${ }^{70}$ Cochrane und Ryan (1976: 45) weisen darauf hin, dass - auch wenn sich die Höhe der Ausgaben nach den Reformen der 1960er-Jahre kaum verringerte - staatliche Gelder nun mehr den Landwirten zugutekamen.
} 
vorgesehen; höhere Agrareinkommen sollten durch höhere Agrarpreise, bedingt durch ein kleineres Angebot, erzielt werden. Diesem Plan, der später auf Weizen und Futtergetreide begrenzt wurde, wollte die Kennedy-Regierung nachgehen, nachdem das dringende Problem der Überschüsse bei Futtergetreide im Jahr 1961 mit einem auf freiwilligen Flächenkürzungen basierenden Notprogramm („Feed Grain Program“) angegangen wurde (Cochrane und Ryan 1976: 92). Als Alternative stellte die Regierung bewusst das für Landwirte zu der Zeit nicht akzeptable Anpassungsprogramm des „Committee for Economic Development“ dar. Das im Jahr 1962 unter der Mitarbeit von Professor T. Schultz entworfene Programm empfahl, die Preisstützung abzubauen (s. Kapitel 3.2). Dieser Prozess sollte fünf Jahre lang durch „compensatory payments“ als Ausgleich für den Rückgang der landwirtschaftlichen Einkommen sowie durch Maßnahmen zur Flächenstilllegung und Umschulung begleitet werden. Das Ziel war, in diesem Zeitraum etwa ein Drittel der Landwirte zur Aufgabe der landwirtschaftlichen Produktion zu bewegen (Hadwiger und Talbot 1965: 220). Josling und Tangermann (2015: 48) sprechen von einer langjährigen Debatte in den politischen Kreisen der USA unter dem Motto „,reduce incentives or restrict supplies“.

Den Konflikt, der über den Regierungsplan zur verpflichtenden Angebotskontrolle entbrannte und der in der Entstehung der hier relevanten Agrargesetze mündete, beschreiben die Zeitzeugen Hadwiger und Talbot (1965) ausführlich. Die Regierung scheiterte zweimal, die entsprechenden Gesetzesentwürfe im Kongress durchzusetzen. ${ }^{71}$ Danach griff sie jeweils nach dem aus dem Notprogramm für Futtergetreide bekannten Instrument der freiwilligen Flächenkürzung, die durch Zahlungen begleitet wurde. So entstanden nicht nur der „Agricultural Act“ von 1961, sondern auch der „Food and Agriculture Act“ von 1962. Dabei war das Notprogramm doch lediglich als „a temporary provision for an emergency situation while permanent legislation was worked out" gedacht (Cochrane und Ryan 1976: 40). Wie Hadwiger und Talbot (1965: 48) bemerken, wäre das Notprogramm hinsichtlich der Reduzierung von Futtergetreideüberschüssen nicht erfolgreich gewesen, hätte der Plan der Kennedy-Regierung zur Angebotskontrolle bessere Chancen gehabt, angenommen zu werden.

\footnotetext{
${ }^{71}$ Dem demokratischen Präsidenten Kennedy und seinem Landwirtschaftsminister Freeman gelang es nicht, eine Mehrheit im von den Demokraten kontrollierten Kongress für ihren Plan zu gewinnen. Für die verpflichtende Angebotskontrolle setzten sich nur die meisten Demokraten aus dem Süden, die die Interessen der damit bereits vertrauten Erzeuger von Tabak und Baumwolle repräsentierten, sowie die Demokraten aus den urbanen Wahlbezirken, die sich von dem Plan Ausgabeneinsparungen versprachen, ein. Die Republikaner waren strikt dagegen (Hadwiger und Talbot 1965).
} 
Der „Food and Agriculture Act“ von 1962 sah ein Referendum unter Weizenanbauern vor. In dem im Mai 1963 durchgeführten Referendum sprachen sie sich gegen die verpflichtende Angebotskontrolle aus. Dass die Alternative - die Weizenerzeugung den Kräften des freien Marktes zu überlassen - aus politischen Gründen nicht zum Tragen kommen würde, sahen viele Gegner der Angebotskontrolle voraus (Cochrane und Ryan 1976). Stattdessen wurde ein durch Agrarzahlungen begleitetes freiwilliges Flächenstilllegungsprogramm aufgestellt und die Preisstützung abgebaut: „With the defeat of the mandatory control alternative in the wheat referendum in May 1963 and the acceptance of production payments in the feed grain program in 1963, the stage was set for a voluntary program involving a low level of price support in combination with production payments for cotton and wheat" (ibid.: 81). Der "Agricultural Act" von 1964 wurde verabschiedet.

Vor dem Hintergrund der intensiven Auseinandersetzung über die Ausgestaltung der Agrarpolitik Anfang der 1960er-Jahre waren für Petit (1985: 29) die Reformen 1962-1965 eine Kompromisslösung:

Following these intense struggles, legislation in the mid-1960s can be viewed as a compromise, the two extreme ideological solutions (a completely free market and mandatory production controls) having been defeated. This compromise was embodied in the Food and Agricultural Act of 1965 and, secondarily, in the Food for Peace Act of 1966. The main elements were: price support levels lowered to world equilibrium levels, and farm incomes - if they were to be supported - supplemented by direct income payments to farmers. To be eligible, farmers had to participate in production control programs authorized if supplies became excessive. Storage and surplus disposal programs continued as additional instruments. Thus government was still heavily involved, U.S. budget costs remained relatively high, and farm incomes were supported but at levels that many farmers felt were unsatisfactory. Such is the essence of a compromise: nobody is satisfied, but it is acceptable to most.

Auch Ingersent und Rayner (1999: 182) weisen auf den Kompromisscharakter der Reformen hin: „Following the result of the 1963 [wheat] referendum, legislation on agricultural policy represented a compromise between the extremes of market liberalisation and mandatory supply control.“ Orden et al. (1999: 64) machen zudem darauf aufmerksam, dass die neue Stützungsregelung mit der Zeit die Akzeptanz beider Parteien gewann: „This move toward direct payments [...] was not a move away from income support for farmers and came to enjoy considerable bipartisan support." Hadwiger und Talbot (1965: 113) notieren bereits für 1962, 
dass diejenigen Republikaner, die noch nicht lange im Kongress waren, einer parteiübergreifenden Koalition für ein längerfristiges freiwilliges Flächenkürzungsprogramm zugeneigt waren.

Moyer und Josling (1990: 107) unterstreichen die wichtige Rolle, die die Landwirte bei den Reformen Anfang der 1960er-Jahre spielten: „[F]armers strongly influenced the nature of change when wheat producers voted down the referendum [in 1963] to impose mandatory acreage restrictions." Dieses deutet auf immer noch relativ starke Landwirtschaftsverbände hin: Hadwiger und Talbot (1965) beispielsweise beschreiben das Engagement des den Republikanern nahestehenden Verbandes „Farm Bureau“ gegen das besagte Weizenreferendum. Für Petit (1985: 33) ist dieses Referendum - neben der erfolgreichen Bekämpfung des BrannanPlans - ein Beleg dafür, dass „farm organizations continued to be major actors in the agricultural policy process". Dabei verbündeten sie sich mit der Regierung ,and [did] operate as lobbyists on behalf of their administration ally in moving a legislative proposal through the Congress" (Cochrane und Ryan 1976: 117). Diese Konstellation bildete das berüchtigte ,iron triangle“ zwischen US-Agrarpolitik (insbesondere die Agrarausschüsse des Kongresses), Exekutive (USDA) und Landwirtschaft bzw. Agrarlobby (vgl. z. B. Orden et al. 1999: 53).

Es stellt sich die Frage, warum konkret die „price support payments“ vorgeschlagen und eingeführt wurden. Sie wurden im Laufe des Gesetzgebungsverfahrens für die Farm Bill von 1962 zunächst für Weizen von USDA ins Spiel gebracht. Ein junger republikanischer Abgeordneter im Repräsentantenhaus, Al Quie, der Mitglied des zur Erarbeitung einer Kompromisslösung einberufenen „Conference Committee“ beider Kongresskammern war, schlug ihre Ausweitung auf Futtergetreide vor und setzte sich, unterstützt von der Regierung, mit Erfolg dafür ein, dass die Ausgleichszahlungen am Ende tatsächlich für beide Kulturen beschlossen wurden (Hadwiger und Talbot 1965). Als Alternative wurde die Erhöhung der „diversion payments“ diskutiert. Widerstand gegen Ausgleichszahlungen leisteten insbesondere das „Budget Bureau“ (Vorgänger des „Office of Management and Budget") und der Landwirtschaftsverband „Farm Bureau“, aber "Quie was insistent, and compensatory payments were kept for both temporary programs“ (ibid.: 231). Interessanterweise waren die Initiatoren der „price support payments" nach der Verabschiedung der Farm Bill von 1962 nicht mehr von der Idee überzeugt: „Later in the year, Quie doubted the wisdom of compensatory payments, and the administration also had second thoughts" (ibid.). Dazu bemerken Hadwiger und Talbot (1965: 231): „[]t is accepted practice in Congress to write laws in the helter-skelter atmosphere of the conference committee, and often later to regret it."

Hadwiger und Talbot (1965: 231) berichten über ein Treffen von Al Quie mit einem Vertreter des Handelsunternehmens Cargill und weisen auf den Druck seitens des Getreidehandels hin, Ausgleichszahlungen einzuführen. Der Handel hatte starkes Interesse daran, dass als Anreiz zur Teilnahme an den Flächenstilllegungs- 
programmen zur Begrenzung von Überschüssen nicht eine hohe Preisstützung etabliert wird, um den Staatseinfluss auf das Geschehen am Getreidemarkt zu minimieren. Als Alternativinstrument propagierten sie den Einsatz von Zahlungen (ibid.: 230). Diese sollten nicht nur für einen Einkommensausgleich nach dem Abbau der Preisstützung sorgen, sondern zudem den Anreiz stärken, Flächen stillzulegen (ibid.: 229). Somit erhoffte sich der Handel, den weiteren Anstieg von Getreideüberschüssen zu verhindern, mit deren Veräußerungen der Staat den Getreidemarkt und -preis massiv beeinflusste. Der Zusammenhang zwischen den neuen Zahlungen und den Produktionsüberschüssen ist somit eher indirekter $\mathrm{Na}$ tur. Auch Levins (2000) weist auf das starke Engagement von Getreidehändlern für ein landwirtschaftliches Stützungsprogramm, in dem die Regierung keine aktive Rolle mehr am Getreidemarkt spielen würde, hin. Die Unternehmen gründeten und finanzierten eine „Public Policy Study Group“, deren Aufgabe es war, ein solches Programm zu erstellen und im Kongress voranzubringen. Dabei bediente man sich des Instruments der „deficiency payments“. Nach Levins (2000) verdoppelte Cargill in den fünf Jahren nach der Einführung der Ausgleichszahlungen seine Verkäufe.

Auch andere Forscher weisen darauf hin, dass bei den Reformen Anfang der 1960er-Jahre die Exportinteressen der US-Landwirtschaft eine große Rolle spielten. Noch für die Zeit um das Jahr 1960 herum attestieren Ingersent und Rayner (1999: 179) der Agrarpolitik der USA eine Entkopplung von den Entwicklungen in anderen Teilen der Welt: „By and large, agricultural trade policy, including competition between the US and other countries for export market shares, and the interaction between trade policy and domestic agricultural policy, remained at the margin of the debate." Die USA waren zwar schon immer Netto-Exporteur von Agrarprodukten (Gardner 2009: 185) aber noch bis in die 1960er-Jahre setzten sie sich für Ausnahmeregelungen für die Landwirtschaft im Allgemeinen Zollund Handelsabkommen (GATT) ein (Josling und Tangermann 2015: 24). Der Wandel setzte zum Amtsantritt der Kennedy-Regierung ein. Wie Josling und Tangermann (2015: 24) feststellen: „The US began to consider that its interests lay in reducing barriers to its exports“. Die Exportinteressen der US-Landwirtschaft seien deshalb auch laut Orden et al. (1999: 64) bei den Reformen hin zu „price support payments“ entscheidend: „The largest U.S. grain export companies and lowest-cost farm producers had begun to see damage to commercial sales abroad from high domestic support prices by the early 1960s. These interests were instrumental as early as 1962 in prompting Congress to authorize the secretary of agriculture to reduce loan rates (initially for corn) and to compensate farmers instead with direct payments.“ Weiter bemerken sie: „This move toward direct payments [...] allowed domestic consumption to expand (aiding producers of livestock as well as food processors and consumers) and was less damaging to export sales". 
Nicht nur der Getreidehandel, sondern auch der Staat selbst war daran interessiert, die Preisstützung für landwirtschaftliche Erzeugnisse zurückzufahren. Sein Ziel war, eine Verteuerung von Lebensmitteln zu verhindern. Cochrane und Ryan (1976: 42) nennen „the need to assure little or no increase in food prices as a consequence of farm income support programs" als einen wichtigen Treiber für die Reformen Anfang der 1960er-Jahre. Hadwiger und Talbot (1965: 88) bemerken in diesem Zusammenhang: „The USDA was under orders not to raise the price of food." Vor diesem Hintergrund war das Instrument der direkt aus dem Staatshaushalt finanzierten „price support payments" hilfreich. Cochrane und Ryan (1976: 81) sehen ein wichtiges Merkmal des „Food and Agriculture Act“ aus dem Jahr 1962 in der Bereitstellung eines Mechanismus „for holding down prices to the domestic consumer". Gemeint sind hier die „price support payments“.

Während die wichtigsten Treiber der Reformen der 1960er-Jahre als Ganzes die hohen Überschüsse landwirtschaftlicher Erzeugnisse und die hohen Haushaltskosten der Stützungsprogramme waren, lagen der Entscheidung zur Einführung der Ausgleichszahlungen die Exportinteressen der Landwirtschaft (festgehalten in der Tabelle 21 als internationale Wettbewerbsfähigkeit) und die Interessen der Verbraucher an günstigen Lebensmittelpreisen zugrunde. Diese Einflussfaktoren sind dem „problem stream“ zuzuordnen. Unter den „politics stream“ fallen die starke Unterstützung des Agribusiness und - angesichts dessen, dass die in der Vergangenheit geübte überparteiliche Einigung nicht möglich war - die Bildung einer neuen Koalition mit den (demokratischen) Repräsentanten urbaner Interessen, die für Lebensmittelbeihilfen eintraten (vgl. Tabelle 21).

Tabelle 21: Der US-Reformen 1962-1965 als Ganzes und dem Milestone zugrunde liegende Faktoren

\begin{tabular}{c|c} 
Reformen 1962-1965 & Milestone 1962-1965 \\
\hline Hohe Produktionsüberschüsse & Internationale Wettbewerbsfähigkeit \\
Hohe Haushaltskosten & Anliegen der Verbraucher \\
Koalition mit urbanen Interessen \\
Unterstützung der Agrarwirtschaft
\end{tabular}

\subsection{US-Milestones 1985 und 1990}

Bei der Verabschiedung der beiden dem „FAIR Act“ von 1996 vorausgegangenen Farm Bills von 1985 und 1990 spielte der Haushaltsdruck eine große Rolle (Moyer und Josling 1990: 209, Josling und Tangermann 2015: 46 und 91). Das Kostenargument galt auch für die darin vorgenommene partielle Entkopplung der „deficiency payments“ von der aktuellen Erzeugung. Zu den im Jahr 1985 beschlossenen Entkopplungsschritten schreiben Moyer und Josling (1990: 161): „The budget 
pressures $[\ldots]$ were still not influential enough to cause significant overhaul of any commodity programmes. However, they were strong enough to induce a first step toward decoupling and reform of the deficiency payments system with the freezing of eligible bases and yields." Damit sollte eine größere Erzeugung verhindert werden, als die, die der Markt aufnehmen kann (Thompson 2005: 2).

Auch bei den Entkopplungsschritten der Farm Bill von 1990 standen die Haushaltsauswirkungen im Vordergrund. Webb et al. (1989: 1231) bemerken zur Zeit ihrer Vorbereitung, dass ,,the pressure to reduce the budget deficit is [even] more compelling than it was five years ago". Laut Erdman und Runge (1990: 113) war die Einführung der sogenannten „triple base“-Regel unter der Farm Bill von 1990 keiner „deeply felt attraction for a clear step in the direction of decoupling“, sondern vornehmlich dem Haushaltsdruck geschuldet. Auch Moyer und Josling (2002: 84) zeigen, dass dort der Ursprung dieser Regel liegt. Die Bedeutung des Haushaltsdrucks im Reformprozess 1990 bestätigen Orden et al. (1999: 100f) mit der Anmerkung, die ,triple base“-Regel sei auf die Verpflichtung, im Agrarbereich 13,6 Mrd. USD über fünf Jahre einzusparen („,budget reconciliation“72), zurückzuführen. Vor diesem Hintergrund kann festgestellt werden, dass der Teilentkopplung aus den Farm Bills von 1985 und 1990 eindeutig Haushaltszwänge zugrunde lagen.

Moyer und Josling (2002) führen neben dem Haushaltsdruck einen weiteren Grund an, der die Bush-Regierung dazu bewegte, im Februar 1990 eine Anbauflexibilität auf der gesamten Basisanbaufläche vorzuschlagen: Umweltbelange. Die Regierung argumentierte, dass ,allowing farmers to respond to market signals would make them more efficient and frugal in their use of synthetic pesticides and fertilizers, and would encourage them to rotate their crops" (ibid.: 79). Somit war die vorgeschlagene Flexibilität ,a key element in the Administration proposals for dealing with environmental problems resulting from agriculture“" (ibid.). Auch Erdman und Runge (1990) erwähnen die wichtige Rolle, die Umweltbelange bei der Vorbereitung der Farm Bill von 1990 spielten. In der politischen Debatte wurde insbesondere hervorgehoben, dass gekoppelte „deficiency payments“ Fruchtfolgen einengen und die Verwendung von Pflanzenschutzmitteln fördern würden. Der ursprüngliche Entkopplungsvorschlag der Bush-Regierung konnte sich aber nicht durchsetzen, denn ,total flexibility [...] was neither necessary to achieve budget targets nor desirable to many commodity groups and their supporters in Congress“ (ibid.: 112f). Er mündete schließlich im „triple base“-Kompromiss. Dieses verdeutlicht die Bedeutung der Haushaltszwänge für die Entkopplungsschritte von 1990.

\footnotetext{
${ }^{72}$ Im Falle der bestehenden Gesetze im Bereich der Pflichtausgaben kann über die Haushaltsentschließung zum Instrument der „budget reconciliation“ gegriffen werden. Mit Ausgabenkürzungen sollen die Pflichtausgabengesetze mit dem in der Haushaltsentschließung festgesetzten Haushaltsvolumen in Einklang gebracht werden (Heniff et al. 2012).
} 
Zwar liefen zu der Zeit die Handelsverhandlungen im Rahmen der Uruguay-Runde des GATT. Wie Ingersent und Rayner (1999: 391f) aber bemerken, ,the bill [of 1990] was not drafted and passed in anticipation of any particular outcome from the Uruguay Round agricultural negotiations“. Weiter weisen sie auf die Notwendigkeit der Erarbeitung einer neuen Farm Bill im Falle eines ehrgeizigen Abschlusses der Uruguay-Runde hin (ibid.: 392). Im Umkehrschluss bedeutet das, dass die Farm Bill von 1990 nicht mit der Absicht, einem solchen Abschluss zu entsprechen, entworfen wurde. Laut Moyer und Josling (2002: 77) ging aus den Handelsverhandlungen kaum Druck hervor, weil diese zu der Zeit durch den Unwillen der EU, die Subventionen zu reduzieren, blockiert waren. Erdman und Runge (1990: 114) schreiben, dass ihre Auswirkungen auf die Farm Bill von 1990 „more subtle“ waren als die des Haushaltsdrucks oder der höheren Relevanz des Umweltschutzes: Die USA, die in den Verhandlungen eine liberale Position vertraten, „could not be perceived to be completely out of step in its domestic legislation". Allerdings konnte die Uruguay-Runde die Einführung bzw. Fortsetzung illiberaler Maßnahmen in der Farm Bill von 1990 schlussendlich nicht verhindern. Zu den Entkopplungsschritten von 1985 schreiben Webb et al. (1989: 1232): „The GATT-compatibility elements of the 1985 farm bill were primarily motivated by administration budget concerns“. Auch Paarlberg (1997: 429) sieht keinen Einfluss der Uruguay-Runde auf die beiden Reformen von 1985 und 1990, die „in response to internal budget pressures" verabschiedet wurden.

In beiden Reformen stellten die Exportinteressen der US-Landwirtschaft einen wichtigen Faktor dar (Webb et al. 1989). Bei den hier interessierenden Milestones scheinen sie allerdings keine Rolle gespielt zu haben. Erdman und Runge (1990) nennen außerdem die anstehenden Kongresswahlen im November 1990 als wichtigen Treiber der Reform von 1990. Vor diesem Hintergrund war die Verringerung der für „deficiency payments“ beihilfefähigen Fläche „the least politically damaging way to find budget savings", während eine Kürzung der Richtpreise sichtbarer gewesen wäre und deshalb in der Sorge um Stimmenverluste im ländlichen Raum abgelehnt wurde (ibid.: 112). Somit war dieser Faktor auch für den Milestone von 1990 entscheidend. Zusammenfassend lässt sich feststellen, dass die Treiber der Farm Bills von 1985 und 1990 der starke Haushaltsdruck aufgrund des hohen Defizits sowie Umweltbelange und die Frage der internationalen Wettbewerbsfähigkeit bildeten. Für die Milestones wiederum waren lediglich die ersten zwei Faktoren entscheidend, wobei Umweltbelange nur für die Entkopplungsschritte von 1990 relevant waren. Diese Faktoren gehören zum ,problem stream“. Den „politics stream“ repräsentieren die Kongresswahlen (vgl. Tabelle 22). 
Tabelle 22: Der US-Reformen 1985 und 1990 als Ganzes und den Milestones zugrunde liegende Faktoren

\begin{tabular}{c|c} 
Reformen $\mathbf{1 9 8 5}$ und $\mathbf{1 9 9 0}$ & Milestones $\mathbf{1 9 8 5}$ und $\mathbf{1 9 9 0}$ \\
\hline Haushaltsdruck & Haushaltsdruck \\
\hline Internationale Wettbewerbsfähigkeit & Umweltbelange (nur 1990) \\
Umweltbelange (nur 1990) & \multicolumn{2}{c}{ Bevorstehende Kongresswablen (nur 1990) } \\
\hline
\end{tabular}

\subsection{EU-Milestone 1992}

Die vor dem Jahr 1990 untergenommenen Reformen der EU-Agrarpolitik werden in der Literatur als vom Haushalt bestimmt interpretiert. Beispielsweise schreiben Moyer und Josling (1990: 100) folgendes über die Dynamik dieser Reformen: „The budget created the only constraint to domestic agricultural interests - the growth of farm spending had to be contained. It was largely left to agriculture ministers to decide among themselves how it was to be done. EC decisionmaking, as in all developed countries, was concerned almost exclusively with domestic priorities and interests." Der internationale Druck seitens der USA und anderer Länder sei zu jener Zeit irrelevant gewesen, so Moyer und Josling (1990: 100). ${ }^{73}$ Wie Daugbjerg (2012: 94) bemerkt, traten „exogenous factors“ bei der Entwicklung der GAP erst während der multilateralen Handelsverhandlungen der Uruguay-Runde von 1986 bis 1994 in den Vordergrund. Nicht unumstritten bleibt in der Literatur allerdings die Frage, wie wichtig die parallel zum Reformprozess laufenden Handelsverhandlungen für die Reform von 1992 waren, insbesondere im Vergleich zu den internen Problemlagen in Form von hohen Haushaltsbelastungen und Produktionsüberschüssen.

Während einige Forscher die Reform von 1992 als Ergebnis der GATT-Verhandlungen sehen, finden sich am anderen Ende des Meinungsspektrums Aussagen, dass sie auch ohne die Uruguay-Runde erfolgt wäre (Josling et al. 1996: 173). Coleman und Tangermann (1999: 386) bringen den Dissens mit einem Hinweis auf zwei Denkschulen auf den Punkt:

The first argues that the 1992 CAP reform occurred largely independently of the simultaneous international negotiations. Internal

\footnotetext{
${ }^{73}$ Gleichzeitig aber weisen Moyer und Josling (1990) auf die Bedeutung des makroökonomischen Umfelds für die Entwicklung der EU-Agrarpolitik in den 1980er-Jahren hin. Darunter waren insbesondere zwei externe Ereignisse relevant, infolge deren die Exportpreise für US-Agrarerzeugnisse sanken: die Verringerung der „loan rates“ im Rahmen der US-Farm Bill von 1985 und die USDollar-Abwertung gegenüber dem ECU. Der Reformdruck auf die EU erhöhte sich damit, weil ihre Ausgaben im Bereich der Agrarpolitik in die Höhe schossen (ibid.: 82).
} 
domestic problems related to budgetary constraints and oversupply of some commodities forced political leaders to consider fundamental reforms of the CAP. The second hypothesis does not deny the importance of these domestic problems. It postulates, however, that the timing of CAP reform and the very logic of the reforms introduced represent direct responses to international pressures emanating from the GAT'T negotiations.

$\mathrm{Zu}$ den Verfechtern der These, wonach die Handelsverhandlungen im Rahmen der Uruguay-Runde die wichtigste treibende Kraft der Reform von 1992 waren, gehören Coleman und Tangermann (1999: 386) selbst. Bis zur Uruguay-Runde erfuhr die Landwirtschaft im GAT'T eine Sonderbehandlung gemäß der Ansicht, dass der Agrarsektor sich von allen anderen Wirtschaftssektoren unterscheidet und deshalb besonderer Unterstützung seitens des Staates bedarf (Skogstad 1998). Dieser ,agricultural exceptionalism“ spiegelte sich in den ausdrücklichen Ausnahmen hinsichtlich der für andere Güter geltenden Regeln zur Anwendung von mengenmäßigen Beschränkungen und Ausfuhrsubventionen sowie 1955 in einer Sondergenehmigung für die USA, mengenmäßige Einfuhrbeschränkungen auch ohne Beschränkung der heimischen Produktion zu nutzen, wider (Josling et al. 1996).

Warum die EU-Agrarpolitik im Kontext der internationalen Handelsverhandlungen der 1980er-Jahre zum Problem wurde, erklärt Josling (2008: 65):

By the mid-1980s, the CAP had become a central topic of concern in the multilateral trade system. The twin reasons were the high levels of border protection that had been retained to give a broad umbrella of protection against the overseas competition under which the market orders could continue, and the increasing surpluses of cereals, meat, dairy products and sugar, all of which other OECD countries produced for export. As a result, the CAP came under criticism abroad as a major cause of low world prices and at home for high support costs and (at least in the UK) for high consumer prices.

Josling (2008) untersucht die Auswirkungen der externen Faktoren auf den GAPReformprozess und identifiziert für den Zeitraum rund um die Reform von 1992 die Handelsverhandlungen der Uruguay-Runde als Hauptfaktor. Er resümiert aus seiner Untersuchung folgendes: „In effect, the MacSharry reforms rescued both the CAP and the Uruguay round. External pressures had forced change and set up a framework for monitoring and locking in that change. The winds of reform had come from outside, although internal acquiescence was essential to their implementation at home“ (ibid.: 67). Da - wie Josling erwähnt - den Handelskonflikten dieser Zeit die großen Produktionsüberschüsse der EU zugrunde lagen, besteht ein indirekter Zusammenhang zwischen ihnen und der Reform. 
Tangermann (1998: 19) schreibt über die GATT-Verhandlungen als „major force behind MacSharry's attempts at moving the CAP in a new direction ". Seiner These zufolge stellt das Scheitern der Verhandlungen in Heysel im Dezember 1990 einen Teil der Strategie des Agrarkommissars dar: „He had to let the negotiations come as close as possible to a compromise [...]. But he then had to break the negotiations sharply, before the point where success was in reach" (ibid.: 22). Auf diese Weise beabsichtigte MacSharry, Zeit zu gewinnen, um zum einen die EUAgrarpolitik vor dem Abschluss der Uruguay-Runde zu reformieren und zum anderen die politische Unterstützung vom Rat für die Reform zu erlangen. Tangermann (1998: 33) resümiert: „In my view, the real motivation behind the MacSharry reform was the need to make the CAP consistent with international obligations on agricultural policy which the EU could not have avoided unless it was prepared to let the Uruguay Round of overall GATT negotiations go down the drain." Er erkennt an, dass EU-interne Gründe für die Reform von 1992 bestanden, meint jedoch, dass ,[c]ontrary to earlier CAP reforms, the MacSharry reform was not triggered by budgetary concerns“ und dass ,it also was the first CAP reform which was driven mainly by external forces" (ibid.: 25). Zur Untermauerung seiner These führt er an, dass schon der erste Reformentwurf der Kommission - wenn umgesetzt - einen Ausgabenanstieg zur Folge gehabt hätte. Am Ende war die Reform von 1992 die erste Reform der GAP, die den Haushalt nicht weniger, sondern mehr belastete (ibid.: 18).

Moyer und Josling (2002) stimmen Tangermann weitgehend zu. Die UruguayRunde sei „,a very strong impetus for the MacSharry reforms" gewesen (ibid.: 106), die EU war in den Verhandlungen isoliert, der Druck seitens der Handelspartner war beträchtlich. Die schwierige Haushaltslage hingegen sehen die beiden Autoren lediglich als Faktor an, der die Notwendigkeit einer tiefgreifenden Reform verstärkte: „The rising costs exacerbated the need for fundamental reform of the CAP“ (ibid.: 104). Zur Unterstützung der Ansicht von Tangermann (1998) führen sie die mit ihr im Einklang stehende Hypothese an, dass das Durchsickern des Reformentwurfs der Kommission, der auf den Tag datierte, an dem die Verhandlungen abgebrochen wurden (6. Dezember 1990), vom Agrarkommissar selbst organisiert worden war. Wobei Moyer und Josling (2002: 109) auch Gründe aufzählen, warum der Reformentwurf weder vor noch nach der Konferenz von Heysel veröffentlicht werden konnte. Einige Jahre später hoben Josling und Tangermann (2015: 97) in einer gemeinsamen Publikation einmal mehr die Bedeutung der Uruguay-Runde für die Reform von 1992 hervor: „[]t is difficult to argue that the need to find some way of concluding the Uruguay Round negotiations on agriculture was not in reality one of the major motivations behind the MacSharry reform." Auch weitere Forschungsarbeiten, wie die von Daugbjerg und Swinbank (2010) und Swinbank und Tanner (1996), unterstreichen die bedeutende Rolle der Uruguay-Runde in der Reform von 1992. Dabei wird auch auf starken Druck sei- 
tens der am Abschluss der Uruguay-Runde interessierten deutschen Industrie hingewiesen (Hendriks 1994 nach Daugbjerg und Swinbank 2007: 13).

Kay (1998) hingegen sieht die treibenden Kräfte der Reform von 1992 etwas anders. Er ist der Meinung, dass der internationale Druck Einfluss auf den Start des Reformprozesses hatte. Allerdings war es die Erkenntnis, dass die Stabilisierungsmaßnahmen vom Februar 1988 für die Begrenzung der Agrarausgaben nicht ausreichen, die den Rat zwang, den Reformprozess abzuschließen. In seiner Analyse betont Kay (1998) die Bedeutung der EU-internen Ursachen für die Reform von 1992 und die Rolle des Agrarkommissars MacSharry. Auch für Ingersent und Rayner (1999) stellen nicht die GATT-Verhandlungen, sondern die internen Probleme der EU in Form von Produktionsüberschüssen und den damit verbundenen Haushaltskosten den Hauptgrund für die Reform von 1992 dar; die UruguayRunde diente eher als Beschleuniger. Es heißt u. a.: ,[A]cknowledging that the UR [Uruguay Round] was one of several reasons for CAP reform advanced by the Commission, is not tantamount to contending that international pressure was the sole, or even the dominant reason why the reform occurred when it did" (ibid.: 355). Weiter wird angeführt: „It is difficult to avoid the conclusion that CAP reform was inevitable for principally domestic reasons. The most that can be said of the effect of external pressure as represented by the Uruguay Round negotiations is that this may have somewhat hastened the reform" (ibid.: 356). Mit dem Verweis auf die Dokumente der Kommission aus den 1980er-Jahren bemerken Ingersent und Rayner (1999: 353): ,There is plenty of evidence that, even before the inauguration of the UR [Uruguay Round], internal pressures for CAP reform would eventually have to be addressed by action." In ihrer Schlussfolgerung werden sie konkreter: „Thus, quite apart from the external pressures represented by the UR [Uruguay Round] negotiations the early adoption by the EC [European Community] of measures to reform the CAP was virtually inevitable by the beginning of the 1990s" (ibid.).

Cunha und Swinbank (2011) scheinen die interne Problemlage und die Uruguay-Runde in ihrer Bedeutung für die Reform als insgesamt gleichwertig, aber in unterschiedlichen Phasen für den Reformprozess relevant, anzusehen. Die schwierige Haushaltslage sei ein wichtiger Faktor beim Kommissionsvorschlag gewesen (,motivating [Herv. im Original] the Commission's reform proposal“), bei der Entscheidung des Rates spielte er allerdings keine große Rolle mehr (,determining [Herv. im Original] the Council's final package") (ibid.: 88). Es sei die UruguayRunde gewesen, die „the final reform package“ beeinflusste (ibid.: 90). Die Haushaltssorgen mussten der Notwendigkeit weichen, eine Einigung auf die Reform zunächst im Kollegium der Kommissionsmitglieder und hernach auch im Rat zu finden. In diesem Sinne argumentieren auch Moyer und Josling (2002: 106): „But the budget constraint, while important for placing reform on the political agenda, disappeared in the consideration of the MacSharry reforms, where policy changes were instituted that increased the cost of the CAP." Beispielsweise wurde die von 
der Kommission anfangs vorgeschlagene Modulation der Zahlungen abhängig von der Betriebsgröße als Mittel für die Ausgabensenkung letztlich nicht umgesetzt (ibid.: 239).

Die Kommission selbst bestritt zwar einen Zusammenhang zwischen der Reform von 1992 und den GATT-Verhandlungen. Die Verordnung (EWG) Nr. 17 65/92, mit der die neue Stützungsregelung eingeführt wurde und die in der Präambel die „Gewährleistung eines besseren Marktgleichgewichts“ als Reformziel bezeichnete, deutete auf eine EU-interne Reformursache. Wie Daugbjerg und Swinbank (2010: 50) in diesem Zusammenhang bemerken: „The official motivation for reform was said to be the increasing, and costly, surplus production which put pressure on the agricultural budget of the EU. The international trade implications of surplus production were hardly mentioned in the reform paper of February 1991 [...], and Community spokespersons officially denied any link between the Uruguay Round and the reform debate." Allerdings war die interne Reformbegründung nur vorgeschoben (Tangermann 1998, Moyer und Josling 2002). Das Verhalten der Kommission erklären Daugbjerg und Swinbank (2010: 50) damit, dass der Hinweis auf den Druck seitens der USA als eigentliche Reformursache kontraproduktiv gewesen wäre in Bezug auf Versuche, Landwirte und Mitgliedstaaten von der Reform zu überzeugen.

Eine etwas andere Erklärung für die Reform von 1992 liefert Paarlberg (1997: 437f). Er vertritt die Meinung, dass nicht die Uruguay-Runde an sich, sondern der Druck seitens der USA ${ }^{74}$ hier ausschlaggebend war. Dieser hatte seinen Ursprung in der Dillon-Runde der GATT von 1960 bis 1961, in der die EU das Zugeständnis machte, für Getreidesubstitute keine Zölle zu erheben. Die direkten und indirekten Subventionen, die die EU vor dem Hintergrund der steigenden ÖlsaatenImporte und der Getreideüberschüsse seitdem im Ölsaatenbereich einführte, beanstandeten die USA - in Sorge um ihre Absatzmöglichkeiten - 1988 vor dem GATT-Panel. Weil diese in dem Schiedsspruch von 1990 als Unterlaufen des Zugeständnisses der Dillon-Runde interpretiert wurden, beschloss die EU eine Reform, indem die Stützpreise für Ölsaaten gesenkt und Ausgleichszahlungen eingeführt werden sollten. Doch die USA beanstandeten auch das neue Stützungsregime und das zweite Panel entschied 1992, dass auch dieses das Zugeständnis der EU aus der Dillon-Runde entwertet. Im sogenannten Blair-House-Abkommen vom November 1992 erreichten die USA und die EU eine Einigung sowohl im

\footnotetext{
${ }^{74} \mathrm{Zu}$ der Rolle der Cairns-Gruppe in der Uruguay-Runde bemerkt Paarlberg (1997: 432), dass sie vom bilateralen Blair-House-Abkommen zwischen den USA und der EU praktisch ausgeschlossen war und dass ihre primäre Forderung, Exportsubventionen abzuschaffen, lediglich in einem sehr bescheidenen Umfang erfüllt wurde. Josling und Tangermann (2015: 109) bemerken dazu, dass die Rolle der Cairns-Gruppe ,indeed important in terms of making sure that both the US and the EU remained committed to achieving an outcome“ war. Sie führen jedoch weiter aus: „[T] he talks were to a large extent dominated by the US and the EU, and tensions between them set the tone" (ibid.). In diesem Zusammenhang weisen sie darauf hin, dass der Abschluss der Uruguay-Runde erst erfolgte, nachdem sich die USA und die EU im Blair-House-Abkommen geeinigt hatten.
} 
Ölsaaten-Konflikt als auch zur Uruguay-Runde. Insbesondere konnte die EU die drohenden US-Sanktionen dadurch abwenden, dass Erzeuger einen Teil ihrer mit Ölsaaten bebauten Fläche aus der Produktion nahmen. Laut Paarlberg (1997) wurde der EU zu jener Zeit klar, dass die Aufrechterhaltung der Beihilferegelung für Getreide durch die Verhinderung der Ölsaaten-Importe aussichtslos sein würde, was wiederum auch die Reform dieses Sektors nach sich zog. Wie er bemerkt: „It was this credible threat of U.S. retaliation in a highly charged trade dispute, more than any positive synergistic linkage within the Uruguay Round, that generated external pressures for CAP reform“" (ibid.: 439).

Hohe Haushaltsbelastung und die Handelsverhandlungen sind nicht die einzigen Faktoren, die der Reform von 1992 zugrunde liegen. Josling und Tangermann (2015: 94) schreiben über ,interplay between domestic and international pressures“. Tangermann (1998: 18) bemerkt: „Probably many different factors were involved." Die restlichen Faktoren hält er aber für weniger bedeutsam, auch wenn sie möglicherweise zur Schaffung eines Klimas, in dem eine fundamentale GAPReform nicht mehr verhindert werden konnte, beitrugen (ibid.: 19). Dazu zählt er das wachsende Bewusstsein über die negativen Auswirkungen der bisherigen Agrarpolitik auf die Umwelt. Lynggaard und Nedergaard (2009) weisen in diesem Zusammenhang darauf hin, dass die MacSharry-Reform die erste Reform der EUAgrarpolitik war, die von Umweltsorgen mitgeprägt wurde. Diese stellen eine der „significant ideational changes, which fed into the 1992 CAP reform“ dar (ibid.: 303). Josling und Tangermann (2015: 97) erwähnen darüber hinaus einen weiteren Reformgrund: Das Streben nach Verbesserung der Wettbewerbsfähigkeit der EUGetreideerzeugung - ,a need that was underlined by the EU's position as a major participant in international agricultural trade“. Dieser Grund wurde im Reformprozess zwar von MacSharry selbst angeführt, zählt aber nicht zur „political rhetoric“, denn Josling und Tangermann halten es für ein ,important argument" und „quite rightly" vorgebracht (ibid.).

Tangermann (1998: 18f) unterstreicht zudem die Rolle der OECD, die die Kritik der Agrarökonomen an der auf der Preisstützung basierten Agrarpolitik kanalisierte:

Economists had long argued that agricultural price support was an inefficient, and in the longer run, ineffective method of improving the standard of living of people in rural communities. Though it is doubtful whether such academic arguments have had much direct effect on the thinking of agricultural policy-makers, they may have found their way into actual policy considerations through the activities of the OECD, which had engaged in work on the Ministerial Trade Mandate in the mid-1980s.

Unter den externen Treibern der Reform von 1992 finden sich noch der Zusammenbruch des Kommunismus und die Wiedervereinigung Deutschlands (Moyer 
und Josling 2002: 106), die einerseits einen Ausgabenanstieg (insbesondere im Agrarbereich durch die Integration von Ostdeutschland) und andererseits erhöhten Wettbewerbsdruck nach sich zu ziehen drohten. Ingersent und Rayner (1999: 356) ergänzen, dass auch die antizipierte Erweiterung der Union als Treiber angeführt wird. Ebenso wird die starke Rolle von Agrarkommissar MacSharry im Reformprozess unterstrichen (Tangermann 1998). Er ,recognized the inevitability of change and took the initiative to have DGVI [Directorate-General for Agriculture] develop proposals which would both maintain the CAP and allow for successful completion of the GATT negotiations“, bringen es Moyer und Josling (2002: 116) auf den Punkt.

Forscher stellen zudem einen Einfluss neuer Ideen auf die Reform der GAP von 1992 fest. Laut Moyer und Josling (2002: 38) brachten die Agrarreformen der 1990er-Jahre in den Industrieländern, so auch die MacSharry-Reform in der EU, „a new paradigm for domestic policy“ mit sich, das „much more trade-friendly as well as more efficient" sei und "the manipulation of the commodity price level as the main way to assist the agricultural sector" mit „direct payments to farm families on the basis of present or past activities" ersetzte. Die EU-Agrarpolitik wird seit der MacSharry-Reform zunehmend durch das „Multi-Functional Agriculture paradigm" geprägt (ibid.: 240). Andere Forscher sind in Bezug auf die EU-Reform von 1992 vorsichtiger und sprechen von einer schrittweisen Entwicklung bzw. einem Schritt in Richtung eines neuen Paradigmas (Skogstad 1998, Feindt et al. 2008, Daugbjerg und Swinbank 2010). Daugbjerg (2009: 400) zweifelt gänzlich daran, dass mit der Reform von 1992 das Paradigma einer schutzbedürftigen Landwirtschaft bewältigt werden könne: „Originally, the CAP was underpinned by the state-assisted paradigm [...]. The reform changed nothing in that respect. There was still a consensus that the EU should protect farmers from the market and ensure their economic well-being.“ Er führt weiter aus: „The underlying purpose of the reform was to preserve the core of the policy by undertaking a partial shift in the policy instruments with which farmers were supported. The level of support remained high and the CAP's contribution to the preservation of the incomes of farmers remained unchanged." Einigkeit scheint darüber zu bestehen, dass die neuen Ideen allein nicht ausreichten, den Politikwechsel auszulösen. Die Tatsache, dass das alte Paradigma nicht mehr tragbar war, trug aber zur Entstehung von ,a policy environment conducive to policy reform" bei (Moyer und Josling 2002: 240).

Mangels einer systematischen Zusammenstellung der Ursachen für die Einführung von Ausgleichszahlungen im Rahmen der MacSharry-Reform in der Literatur soll nachfolgend versucht werden, dieser Fragestellung nachzugehen. Für diesen Zweck werden die vorstehend genannten Ursachen für die Reform als Ganzes darauf untersucht, inwiefern sie sich auf die Inhalte der Reform von 1992 auswirkten. 
Ungeachtet der Auseinandersetzung über die Bedeutung der Haushaltszwänge für die MacSharry-Reform als Ganzes scheinen sie bei der Einführung von hier interessierenden Ausgleichszahlungen keine Rolle gespielt zu haben. Denn gerade die Ausgleichszahlungen waren mit hohen Ausgaben verbunden, was sich bereits zu Beginn des Reformprozesses abzeichnete (Tangermann 1998: 33). Die ursprünglich projizierten Ausgaben waren zwar viel niedriger als die tatsächlichen Aufwendungen, allerdings - wie Tangermann (1998: 25) es mit Blick auf die neuen Zahlungen formuliert - „the way in which this reform was designed necessarily meant that it would result in higher budget expenditure".

Forschungsergebnisse zeigen, dass die im Jahr 1986 gestarteten Verhandlungen der Uruguay-Runde nicht nur auf den zeitlichen Ablauf der Reform, sondern gerade auf ihre Inhalte großen Einfluss hatten. Beispielsweise stellt Josling (2008: 67) fest, dass , [t] the pace and the content of the 1992 MacSharry CAP reforms were clearly influenced by the negotiations in the Uruguay round". Cunha und Swinbank (2011: 92) bemerken, dass „the Uruguay Round negotiations explain why the proposed reform had the shape it had, as well as its final configuration". Moyer und Josling (2002: 116) schreiben in diesem Zusammenhang, dass die Reformvorschläge der Kommission, ,would both maintain the CAP and allow for successful completion of the GATT negotiations“. Tangermann (1998: 25) geht indirekt auf den Einfluss der Uruguay-Runde auf Reforminhalte mit der Feststellung ein, dass ,ability to accept a GATT agreement on agriculture was the major reason behind the MacSharry reform“. Die Frage, die er stellt, lautet: „[W] hy was it [Commission] willing, at that particular point of time, to engage in a CAP reform which deviated so fundamentally from the earlier types of budget-driven reforms?"“ (ibid.: 18) und fokussiert somit ausdrücklich auf die Reforminhalte. Eine Erklärung für die oben geschilderte, unterschiedliche Betonung einzelner Reformursachen könnte deshalb darin liegen, dass Tangermann (1998) die Ursachen der Reform von 1992 in ihrer finalen, ehrgeizigen Ausgestaltung untersuchte, während die anderen Forscher eher die Auslöser „einer Reform“ erkundeten.

Hinsichtlich der von der Uruguay-Runde tangierten Reforminhalte werden insbesondere die Preisstützung sowie Importbarrieren und Exportsubventionen genannt (z. B. Moyer und Josling 2002: 117). Cunha und Swinbank (2011: 92) führen aber explizit auch die 1992 eingeführten Ausgleichszahlungen als durch die Verhandlungen beeinflusst an: „The configuration of the proposed aid scheme was not arbitrary with regard to the GATT.“ Josling et al. (1996: 202) bemerken im Hinblick auf die Inlandsstützung als Ganzes: „The domestic support provisions are probably the most innovative feature of the Agreement. They go far beyond the traditional GATT approach of dealing mainly with trade policies and having no more than rather general rules regarding domestic subsidies."

Vor dem Hintergrund der Bedeutung der Uruguay-Runde für die Ausgleichszahlungen lohnt ein genauerer Blick auf ihren Ursprung. Für die vorliegende Arbeit ist von Interesse, dass es die USA waren, die sich Anfang der 1980er-Jahre für 
neue GATT-Verhandlungen stark machten (Josling et al. 1996: 134f). Getrieben waren sie u. a. von Wirtschaftsinteressen im Bereich des Dienstleistungshandels und des geistigen Eigentums, es war aber auch die Agrarpolitik der EU, die für die USA ,a major target" darstellte (ibid.: 173). Darüber hinaus ist anzumerken, dass das volle Einbinden der Landwirtschaft in die GATT-Verhandlungen entgegen der bisherigen Praxis möglich war, weil „the economic conditions were such that it became both possible and urgent to tackle the crisis of agricultural markets, policies, and rules" (ibid.: 102). Josling et al. (1996: 101) führen dazu aus:

In the 1980s, economic conditions [...] changed fundamentally. World markets for agricultural products weakened, and international farm prices collapsed. As a result of these international market trends, and as a consequence of inflexible national policies, the financial and economic costs of farm policies rose sharply. This led to a severe farm policy crisis in most of the major industrialized countries. It was this crisis more than anything else that revived interest in the GATT [...].

Zwischen den hohen Kosten der Agrarprogramme und der Einführung der Ausgleichszahlungen besteht somit zumindest ein indirekter Zusammenhang.

Es bleibt noch festzuhalten, dass der Einfluss des von den USA initiierten Streitbeilegungsverfahrens im Ölsaatenbereich auf die Instrumentenwahl und somit auch auf den Milestone 1992 nicht gegeben zu sein scheint. Zwar bildeten die nach dem ersten von der EU verlorenen GATTT-Panel beschlossenen Ausgleichszahlungen im Ölsaatenbereich „a prototype for the subsequent MacSharry reforms for other crops" (Paarlberg 1997: 438). Allerdings war diese Instrumentenwahl vom Panel nicht vorgeschrieben (GATT 1990). Auch sie wurde von den USA beanstandet und daraufhin ein neues Panelverfahren eingeleitet.

Welche Faktoren außer der Uruguay-Runde hatten Einfluss auf die Reforminhalte? Josling et al. (1996: 102) heben die Tatsache hervor, dass ,when the final preparations were made for the Uruguay Round of GATT negotiations, the conceptual framework was in place“. Sie verweisen in diesem Zusammenhang auf die OECD-Arbeiten zur Inlandsstützung im Agrarbereich in den Mitgliedsländern. Diese begannen mit dem „Ministerial Trade Mandate“ von 1982, im Rahmen dessen die OECD Handelsauswirkungen der nationalen Agrarpolitikmaßnahmen untersuchen sollte. Der erste Synthesebericht und die Länderberichte erschienen kurz nach dem Start der Uruguay-Runde im Jahr 1987 (ibid.: 107). Gleichzeitig bearbeitete der „GATT Committee on Trade in Agriculture“ die Handelsthemen. Josling (1992: 91) unterstreicht die intensive zwischenstaatliche Kommunikation zu der Zeit: „The flurry of activity in the multilateral arena in the 1980s was unusual; governments were engaged in an almost continuous dialogue, leading up to the current discussions of agricultural policy in the GATT Uruguay Round."“ 
Wie Josling (1992: 91) es formuliert, leistete die OECD „[b]ackground work“ für die Verhandlungen der Uruguay-Runde. Diese sei aus zwei Gründen wichtig gewesen. Zum einen konnten die Regierungen überzeugt werden, dass die Wurzeln der Handelsprobleme in ihren heimischen Agrarpolitiken liegen. Zum anderen wurde das Ausmaß der Inlandsstützung sichtbar, wodurch eine neue Dimension an Transparenz in Bezug auf Agrarpolitiken entstand (Josling et al. 1996: 108). Vor diesem Hintergrund stellte die Arbeit der OECD im Rahmen des „Ministerial Trade Mandate“ „a remarkable departure from earlier work on agriculture in the OECD“ dar (ibid.: 105). Zusammenfassend war sie „an important element in the preparation of the political and intellectual ground for the Uruguay Round negotiations on agriculture" (ibid.). Wie beim GATT gaben auch in der OECD die USA, neben Australien und Neuseeland, den Ton bei der Bearbeitung der neuen Themen an (ibid.: 106).

Josling et al. (1996) folgend besteht das Verdienst der OECD darin, auf die Instrumente der Inlandsstützung im Agrarbereich aufmerksam zu machen und sie hinsichtlich ihrer Wirksamkeit und Auswirkungen zu bewerten. Insbesondere spielte die Kritik der OECD an der Preisstützung eine wichtige Rolle, darunter die Botschaften, dass die Preisstützung weniger effektiv in Stützung der landwirtschaftlichen Einkommen ist als von den Entscheidungsträgern bisher angenommen und dass ihre gegenseitige und ausgewogene Senkung in allen Ländern zu verkraften sei (Tangermann 1998: 19). Aus der Unzufriedenheit mit der traditionellen Agrarpolitik resultierte die Bereitschaft, Alternativen zu erwägen (ibid.). Dabei beschränkte sich die OECD in ihren Arbeiten nicht auf die Kritik an der bisherigen Agrarpolitik, sondern zeigte auch Alternativen auf. Statt der Preisstützung und anderen an die aktuelle Produktion gekoppelten Maßnahmen regte sie an, die landwirtschaftlichen Einkommen direkt zu unterstützen. Beispielhaft dafür ist die Empfehlung aus der Erklärung der Minister der OECD-Länder aus dem Jahr 1987: „Rather than being provided through price guarantees or other measures linked to production or to factors of production, farm income support should, as appropriate, be sought through direct income support" (OECD 1987). Mit ihrer Studie aus dem Jahre 1990 führte die OECD zum ersten Mal detailliert aus, wie die Anwendung von „direct income payments“ als Alternative zu den an die Produktion gekoppelten Maßnahmen aussehen könnte (s. Box 1). Vor diesem Hintergrund erscheint es plausibel, den Druck seitens der OECD den Faktoren, die auf die Einführung der Ausgleichszahlungen in der EU im Jahr 1992 einwirkten, zuzuordnen.

Des Weiteren ist auch die antizipierte Osterweiterung der EU zu nennen, denn sie machte einen Wechsel der Instrumente erforderlich. Die Anpassung der niedrigen Preise in den osteuropäischen Kandidatenländern an die EU-Stützpreise wäre nur mit sehr hohen Ausgaben zu bewältigen gewesen (Josling 1993). Auch gesellschaftliche Ansprüche, wie Umweltbelange, scheinen der Einführung der Ausgleichszahlungen von 1992 zugrunde zu liegen. Auf diese antwortete die Politik 
zwar vorwiegend mit den sogenannten flankierenden Maßnahmen (Tangermann 1998: 25). Lynggaard und Nedergaard (2009: 304) lassen aber keine Zweifel daran aufkommen, dass für die 1992 beschlossene Teilentkopplung auch Umweltanliegen relevant waren: „Of course, this [partial decoupling] is meant as an incentive to less intensive exploitation of the farming land.“

Der MacSharry-Reform lag eine Reihe der unter den „,problem stream“ fallenden Faktoren zugrunde. Die meisten von ihnen waren auch für den hier interessierenden Milestone relevant. Darüber hinaus war die Haltung des Agrarkommissars MacSharry für die Reform und für den Milestone ausschlaggebend (vgl. Tabelle 23).

Tabelle 23: Der EU-Reform von 1992 als Ganzes und dem Milestone zugrunde liegende Faktoren

\begin{tabular}{c|c} 
Reform $\mathbf{1 9 9 2}$ & Milestone 1992 \\
\hline Hohe Produktionsüberschüsse & WTO/Uruguay-Runde \\
Hohe Haushaltskosten & Umweltsorgen der Gesellschaft \\
WTO/Uruguay-Runde u. Ölsaaten-Panel & Antizipierte EU-Erweiterung \\
Umweltsorgen der Gesellschaft & OECD \\
Antizipierte EU-Erweiterung & Internationale Wettbewerbsfähigkeit \\
OECD & \\
Internationale Wettbewerbsfähigkeit & \\
\hline
\end{tabular}

\subsection{US-Milestone 1996}

Im Jahr 1994 gewannen die Republikaner die Mehrheit im Senat und - nach 40 Jahren - auch im Repräsentantenhaus zurück. Ihr Wahlprogramm unter dem Namen „Contract with America“ versprach u. a. Haushaltsausgleich und setzte die Clinton-Regierung in der Finanzpolitik unter Druck. Das US-Haushaltsdefizit belief sich im Jahr 1982 auf rund 130 Mrd. USD, zehn Jahre später bereits auf fast 300 Mrd. USD (OMB 2016: Tabelle 1.1). Wie Heniff et al. (2012: 4) bemerken, wurden die Haushaltsdebatten in den USA in den 1980er- und 1990er-Jahren von den Sorgen um das steigende Haushaltsdefizit dominiert. Erst im Haushaltsjahr 1998 wurde wieder ein Überschuss verzeichnet (OMB 2016: Tabelle 1.1).

Zu der für 1995 geplanten Farm Bill berichten zwar Ingersent und Rayner (1999: 395), dass ,[i]t seemed clear from an early stage that, like the 1985 und 1990 bills, the 1995 Farm Bill would be subject to tight budgetary constraints [...] particularly in the area of commodity support payments". Zudem bezeichnen Mo- 
yer und Josling (2002: 159) das chronische Haushaltsdefizit als „[p]erhaps the most important economic trend at the time of the 1995-6 Farm Bill debate". Einer der ersten Reformvorstöße, der vom damaligen Vorsitzenden des Agrarausschusses im Senat kam, sah noch eine Senkung der Agrarausgaben um $15 \mathrm{Mrd}$. USD innerhalb der nächsten fünf Jahre vor (Orden et al. 1999: 128). Und nicht zuletzt wurden Agrarprogramme Teil des - letztendlich im Dezember 1995 von Präsident Clinton blockierten - „budget reconciliation“-Akts, der für die Landwirtschaft Einsparungen von 12,5 Mrd. USD vorsah (Ingersent und Rayner 1999: 395).

Allerdings besteht allgemeine Übereinstimmung darin, dass der Haushaltsdruck letztlich kaum Auswirkungen auf die Farm Bill von 1996 hatte. Stellvertretend können hier Stuart und Runge (1997: 129) zitiert werden, die ein Nachlassen seiner Wirkung im Laufe des Gesetzgebungsprozesses konstatieren: „The final Farm Bill calls for costly additional programmes such as rural development and environmental initiatives. This, combined with the continuation of relatively costly policies such as export subsidies, suggests that budget concerns faded as consideration went on." Moyer und Josling (2002: 172) sehen im Budgetdruck lediglich den Reformkatalysator. Die starke Fokussierung der Republikanischen Partei im Wahlkampf auf den Haushaltsausgleich führte dazu, dass nach ihrem Sieg sich auch die Agrarprogramme einer Überprüfung nicht entziehen konnten. Allerdings verlor dieser Einflussfaktor im Laufe der Zeit an Bedeutung, denn die Farm Bill „developed such a momentum that it could not be stopped, even when it became clear that there would be no budget savings, the original impetus for reform" (ibid.: 173). Auch Harvey (1998: 7) stellt „the supposed dominance of budgetary considerations in setting the farm legislation" infrage. Für Orden et al. (1999: 186) liefert der Budgetdruck ebenfalls keine überzeugende Erklärung für die Reform von 1996. Zum einen stießen die Republikaner schnell an ihre Grenzen, was die angekündigte Bekämpfung des Haushaltsdefizits betrifft. Dazu kam, dass im Wahljahr 1996, ,[t]he allure of spending more through the farm bill eventually became too great, in an election year, for even the House Republican leadership to resist" (ibid.: 187). Vor diesem Hintergrund gelang es dem neuen Vorsitzenden des Agrarausschusses im Repräsentantenhaus, Pat Roberts, seine Parteikollegen zu überzeugen, dass von der Unterstützung der Landwirtschaft in der kommenden Farm Bill der Erfolg der Republikaner bei den Kongresswahlen abhängt (ibid.: 130). Letztendlich wurden die Einsparungen durch den „FAIR Act" vom CBO auf lediglich $2 \mathrm{Mrd}$. USD im Vergleich zur „baseline“ beziffert (Moyer und Josling 2002: 158).

Während im Laufe des Gesetzgebungsprozesses die Bedeutung des Haushaltszwangs als Reformtreiber sank, stieg die Relevanz des Marktumfelds (Stuart und Runge 1997: 129). Die Marktpreise für landwirtschaftliche Erzeugnisse waren nach 1993 deutlich angestiegen und erreichten im Jahr 1995 Rekordhöhen (Moyer und Josling 2002: 161). Es wurde deutlich, dass unter diesen Umständen die von 
Roberts vorgeschlagene Umstellung auf von der Produktion entkoppelte ,production flexibility contract payments "75 den Landwirten Vorteile gegenüber der Beibehaltung von preisabhängigen „deficiency payments“ bringen würde. Die Fortsetzung letzterer hätte bedeutet, dass die Ausgaben für die Landwirtschaft im Rahmen der neuen Farm Bill unter der CBO-,,baseline“" vom Februar 1995 (Verlängerung der Farm Bill von 1990 unter den prognostizierten Marktpreisen) liegen. Mit der Entkopplung hingegen würde das Konzept von „baseline capture“ realisiert werden können: Die vom CBO projizierten Agrarausgaben könnten auch bei einem hohen Marktpreisniveau tatsächlich verwendet werden. Roberts argumentierte, dass durch seinen Vorschlag nicht nur die Agrarausgaben in Zukunft vor Kürzungen besser geschützt, sondern auch kurzfristig die Zahlungen an Landwirte im Vergleich zur bisherigen Regelung erhöht würden (Orden et al. 1999: 185). Diese Erkenntnis machte eine Einigung zwischen Republikanern und Demokraten im Kongress möglich, die in der Farm Bill von 1996 mündete: „As these realities became clear, the idea of decoupling became sufficiently attractive - first to southern Republican agriculturalists and eventually even to a sufficient number of Democrats - to ensure its eventual passage" (ibid.: 148). So wandelten steigende Marktpreise eine „unacceptable option“ in eine ,irresistible choice“ um (ibid.: 185) und der „FAIR Act“ wurde mit einer überwältigenden Mehrheit von $318 \mathrm{zu}$ 89 Stimmen im Repräsentantenhaus und $74 \mathrm{zu} 26$ im Senat angenommen (Moyer und Josling 2002: 158). Ähnlich argumentiert Hofreither (1996). Für ihn ist der Entkopplungsvorschlag ,ganz einfach dem Faktum, daß in der Zwischenzeit die (Welt-)Marktpreise deutlich angestiegen waren“"zuzuschreiben (ibid.: 6). Die mit der Farm Bill von 1996 erzielte stärkere Marktorientierung der US-Agrarpolitik sei „ein Ergebnis optimaler Anpassung an real existierende Agrarmärkte im richtigen Zeitpunkt" (ibid.: 12).

Dabei waren die Haushaltsregeln des Kongresses dem Ergebnis sehr förderlich (Orden et al. 1999: 186). Ohne sie hätten die Republikaner keinen zwingenden Grund gehabt, für die Entkopplung zu stimmen (ibid.: 187). Denn die sogenannte Pay-As-You-Go-Regel ${ }^{76}$ erschwerte die Abschaffung des unter Landwirten unbeliebten Flächenstilllegungsprogramms ARP im Falle einer Verlängerung von „deficiency payments“. Die laufend überholten $\mathrm{CBO}$-Budgetprognosen wiederum

\footnotetext{
75 Der offizielle Vorschlag, entkoppelte Zahlungen anstelle von „deficiency payments“ einzuführen, wurde von Roberts Anfang August 1995 unterbreitet, wobei bereits im Juni 1995 ein anonymer „Freedom to Farm“-Plan zirkulierte, der solche Zahlungen vorsah, ,perhaps just a trial balloon at first" (Orden et al. 1999: 135). Anfangs sah es nicht danach aus, dass diese Idee Erfolg haben könnte (ibid.: 138 und 184). Wie Josling (1998: 38) feststellt, waren Landwirtschaftsthemen direkt im Vorfeld der Farm Bill von 1996 ein Konfliktgegenstand zwischen den beiden Parteien.

${ }^{76}$ Für die Finanzierung von zusätzlichen Ausgaben müssen äquivalente Reduzierungen der Ausgaben in anderen Bereichen oder äquivalente Erhöhungen der Staatseinnahmen erfolgen (Heniff et al. 2012). Solche zusätzlichen Ausgaben würden für „,deficiency payments“ und „,marketing loans“ entstehen, wenn man das unter ARP stillgelegte Land wieder in die Produktion bringen würde.
} 
ermöglichten durch den Wechsel zu entkoppelten Zahlungen höhere Leistungen für die Landwirte, als die Marktpreise zu steigen begannen (ibid.).

Orden et al. (1999: 175ff) benennen gar die Kontrolle des US-Kongresses und folglich der Legislativ-Agenda durch die Republikaner in der Zeit der Vorbereitung der Farm Bill von 1996 als entscheidenden Faktor für die Reform. Grundsätzlich befürworteten die Demokraten hohe Preisstützung und Angebotskontrolle, während die Republikaner eine Vollproduktion und freie Märkte favorisierten. Letztere setzten sich jedoch nicht zwangsweise für die Verringerung der staatlichen Unterstützung der Landwirtschaft ein. Dabei hätte die republikanische Kontrolle des Kongresses allein nicht zum „FAIR Act“ geführt. Erst der plötzliche und starke Anstieg der Marktpreise gab laut Orden et al. (1999: 183) den entscheidenden Impuls als ,a second enabling condition“.

Die meisten Forscher stimmen darin überein, dass die Verhandlungen der Uruguay-Runde bzw. das Übereinkommen über die Landwirtschaft von 1994 keine Rolle bei der Ausgestaltung der Farm Bill von 1996 spielten. In der Reformdebatte blieben sie beinahe unerwähnt, außer in Bezug auf Exportprogramme (Orden et al. 1999: 186). Wie Moyer und Josling (2002: 144) bemerken: „Since US agricultural policy, with the changes instituted in the 1990, seemed well within the parameters set by the Uruguay Round Agricultural Agreement, there really were no strong international pressures for further reform." Orden et al. (1999: 186) stellen vor dem Hintergrund, dass teilentkoppelte Zahlungen wie „deficiency payments“ als Blue-Box-Maßnahme explizit erlaubt waren, fest, dass ,in the United States the international negotiation functioned more as a source of farm policy continuity than as a force for policy change“. Dieses bestätigen Orden et al. (2010: 162) mit der Bemerkung, dass „the broad thrust of agricultural policies in the United States since the URAA [Uruguay Round Agreement on Agriculture] has exhibited a remarkable consistency with earlier decades". Insofern attestieren sie der US-Agrarpolitik im untersuchten Zeitraum ab dem Jahr 1994 „resilience [...] in the context of a potentially increasingly assertive set of multilateral trade rules" (ibid.).

Allenfalls wurde es nach dem erfolgreichen Abschluss der Uruguay-Runde leichter, „to argue for domestic legislation consistent with the liberalization of international trade than to argue for a policy that distorted markets or interfered with US international competitiveness", wie Moyer und Josling anmerken (2002: 162). Im Besonderen stellen sie zu den "production flexibility contract payments“ fest, dass das Übereinkommen über die Landwirtschaft Befürworter der Entkopplung stärkte und Verteidiger der bisherigen Programme in die Defensive drängte (ibid.: 237). Nach deren Meinung hatte dieses Abkommen gewisse Auswirkungen auf die Farm-Bill-Debatte, wobei es sowohl liberale als auch illiberale Elemente in die Diskussion hineintrug (ibid.). Die Notwendigkeit, Stimmen im Kongress für seine Ratifizierung zu gewinnen, führte zur Beibehaltung von illiberalen Elementen in der inländischen Agrarpolitik, wie z. B. Exportprogramme; das liberale 
Element stellte die Entkopplung dar (ibid.: 170). Paarlberg (1997: 416) sieht keinen Einfluss der Uruguay-Runde auf die Inhalte und das Tempo der Reform von 1996. ${ }^{77}$ Harvey (1998) äußert sich zum Einfluss der Uruguay-Runde auf die Farm Bill von 1996 zwar nicht, unterstreicht allerdings die Bedeutung der Farm Bill von 1996 für die auf die Uruguay-Runde folgende WTO-Verhandlungsrunde: „However, whatever else FAIR has achieved, it has set a considerable part of the relevant agenda for the review of the UR AA, for the next round of WTO agricultural trade negotiations and the associated requirements for further European farm policy reform" (ibid.: 14).

Wichtig für die Reform von 1996 waren hingegen die Exportinteressen des Agrarsektors bzw. die Frage seiner internationalen Wettbewerbsfähigkeit. Die Bedeutung der Exporte für die US-Landwirtschaft nahm stetig zu: Lag ihr Wert im Haushaltsjahr 1965 bei knapp 6,1 Mrd. USD, stieg er bis 1980 auf über 40 Mrd. USD (USDA 1982: Tabelle 722). Allerdings ging der Anteil der USA am Weltgetreidehandel in den 1980er-Jahren zurück, nicht zuletzt aufgrund von Flächenstilllegungsprogrammen (Orden et al. 1999: 73). Gleichzeitig wurde eine Zunahme der internationalen Nachfrage nach Agrarprodukten prognostiziert. Die stärkere Marktorientierung der Agrarpolitik sollte es der US-Landwirtschaft ermöglichen, davon zu profitieren (Moyer und Josling 2002: 150). Vor diesem Hintergrund hatte die Idee der entkoppelten Zahlungen ohne verpflichtende Flächenstilllegung auch die Unterstützung der Getreidewirtschaft (ibid.: 149). Wie es Harvey (1998: 9) formuliert, setzte der „FAIR Act“ die volle Produktionskapazität der US-Landwirtschaft frei. Nicht irrelevant für die Reform war das Engagement der „Coalition for a Competitive Food and Agricultural System“ von 125 Unternehmen der Agrarwirtschaft, die sich für eine marktorientierte Landwirtschaft einsetzte und den „Freedom to Farm“-Plan von Anfang an unterstützte (Moyer und Josling 2002: 163).

Die vorstehende Zusammenstellung von Ursachen bezieht sich grundsätzlich auf die Reform von 1996 als Ganzes, ohne näher auf die spezifischen Ursachen für die Einführung von entkoppelten Zahlungen einzugehen. Vor dem Hintergrund aber, dass der größte, innovativste Reformgewinn und mit Abstand die Entkopplung der Zahlungen von der aktuellen und künftigen Produktion sowie von den Marktpreisen darstellte (Orden et al. 1999: 166f), kann davon ausgegangen werden, dass zwischen den Ursachen für die Farm Bill und für die Entkopplung groBe Parallelen bestehen. An der Stelle werden die genannten Reformursachen auf ihre Bedeutung für die Einführung der entkoppelten Zahlungen in Form von „production flexibility contract payments" bzw. für die Reforminhalte untersucht.

\footnotetext{
77 Paarlberg (1997) sieht auch wenig Einfluss der Uruguay-Runde auf die MacSharry-Reform von 1992 in der EU, sondern unterstreicht die Bedeutung der hohen Belastung des EU-Haushalts sowie das Pochen der USA auf die Einhaltung der die Ölsaaten-Importe betreffenden Zugeständnisse durch die EU.
} 
Es sind Orden et al. (1999: 134 und 184), die die Faktoren, die dem Entkopplungsvorschlag von Roberts zugrunde lagen, explizit benennen. Die Entkopplung sollte Roberts helfen, zwei Probleme gleichzeitig zu lösen. Das unbeliebte Flächenstilllegungsprogramm ARP könnte abgeschafft werden, ohne dass dafür die vom $\mathrm{CBO}$ geschätzten 6,5 Mrd. USD fällig sind (wie im Fall der Fortsetzung von „deficiency payments"). Darüber hinaus würde somit auch Landwirten geholfen, die Ernteverluste erlitten, wie z. B. die Weizenerzeuger aus Roberts Bundesstaat Kansas im Sommer 1995 (sie erhielten in dem Jahr nur geringe „deficiency payments" bzw. mussten die vorausgezahlten „deficiency payments" sogar zurückbezahlen, konnten aber aufgrund des Mengenrückgangs von den höheren Marktpreisen nicht profitieren). Diese Ursachen lassen vermuten, dass Roberts nicht aus Überzeugung, sondern aus Pragmatismus handelte. Dafür spricht die Tatsache, dass entkoppelte Zahlungen in seinen früheren Vorschlägen zur Farm Bill von 1996 nicht zu finden sind (ibid.: 129). Roberts war vielmehr als engagierter Beschützer des Status quo in der Agrarpolitik bekannt und ,[t] here was no hint before mid-1995 that he was about to advocate a radical decoupling option " (ibid.: 184). Roberts wurde erst zum Befürworter des Entkopplungskonzeptes, „,when he was convinced that decoupled income payments better served the interests of farmers than existing commodity policy" (Moyer und Josling 2002: 167). Für ihn war die Einführung von Entkopplung ,a question of damage control - how to limit the harm to agriculture" vor dem Hintergrund der schwierigen Defizitdebatte (ibid.: 147).

Für die Einführung der „production flexibility contract payments“ spielte der Anstieg der Marktpreise eine zentrale Rolle. Schließlich bezog sich das oben geschilderte Konzept der „baseline capture“ auf diese Zahlungen. Orden et al. (1999: 183) fassen die Bedeutung der günstigen Marktbedingungen wie folgt zusammen: „Without steadily rising commodity prices in late 1995, Congress would never have been persuaded to embrace Freedom to Farm decoupled payments." Sie gehen sogar noch weiter: „Without above-trend prices, in fact, Roberts never would have proposed this approach in the first place." Dafür, dass die steigenden Marktpreise ausschlaggebend für die Einführung der Entkopplung waren, sprechen außerdem zwei Punkte. Zum einen sei 1985 laut Orden et al. (1999: 183) das Scheitern der Initiative der Senatoren Boschwitz und Boren, entkoppelte „transition payments" anstelle von „deficiency payments" einzuführen, unter anderem den zu der Zeit niedrigen - und fallenden - Marktpreisen für landwirtschaftliche Erzeugnisse geschuldet gewesen. Aus demselben Grund scheiterte auch die Reagan-Regierung Mitte der 1980er-Jahre mit ihrem Vorschlag, die „deficiency payments" stark zu kürzen (ibid.: 74). Zum anderen war die (teilweise) Abkehr von der Entkopplung durch die Einführung von „market loss assistance payments“ Ende der 1990er-Jahre auf den Preisrückgang zurückzuführen. Dieses verdeutlicht die Bedeutung der Marktbedingungen für die Einführung von entkoppelten Zahlungen im Jahr 1996. 
Die Haushaltszwänge, die in der Literatur zumindest als Reformkatalysator anerkannt werden, können bei der Einführung der Entkopplung aufgrund der Tatsache, dass sich die Politik letztendlich für ein kostenintensiveres Zahlungssystem entschied (Orden et al. 1999: 187), als Einflussfaktor ausgeschlossen werden. Das Abkommen der Uruguay-Runde scheint keinen Einfluss auf die Inhalte der Reform zu haben. Die Exportinteressen der US-Landwirtschaft spielten wohl für die Abschaffung des ARP-Programms, die mit der Einführung der ,production flexibility contract payments" einherging, eine größere Rolle als für die Zahlungen selbst.

Zuweilen wird der „FAIR Act“ im Kontext der Entstehung von neuen dominierenden Ideen im Sinne eines Wechsels weg vom Paradigma einer schutzbedürftigen Landwirtschaft hin zum marktliberalen Paradigma interpretiert (Coleman et al. 1996, Tweeten und Zulauf 1997, Skogstad 1998, Moyer und Josling 2002). Coleman et al. (1996: 280) beispielsweise schreiben, dass der spürbare Rückzug des Staates aus der Landwirtschaft, der durch die Verabschiedung der Farm Bill von 1996 verkörpert sei, „followed Hall's proposed trajectory“. Orden et al. (1999: 193) vertreten jedoch die Meinung, dass die Macht der neuen Ideen keine Erklärung für die Reform von 1996 sein kann, denn „old ideas were alive and well“. Unter anderem weisen sie darauf hin, dass die neuen Ideen, wie die der Marktorientierung, lediglich für einige Erzeugnisse realisiert wurden und dass keine gesellschaftliche Auseinandersetzung um die Agrarpolitik stattfand (was laut Hall 1993 zu den Begleiterscheinungen eines Paradigmenwechsels gehört). Diese Skepsis wird von Josling und Tangermann (2015) geteilt. Sie stellen zur Motivation hinter dem Entkopplungsvorschlag Folgendes fest:

The policy instrumentation introduced [in 1996] was well in line with the paradigm of decoupling support from production as developed in academia and international organizations. However, the strongest motivation for moving in that direction was not necessarily interest in improving the economic rationality of farm policy or paying tribute to the AoA [Agreement on Agriculture of 1994], but an attempt at preserving farm benefits that market developments appeared to undermine. Decoupling was advanced not so much because of its overall economic advantages, but in the interest of maintaining a high level of farm support. (ibid.: 101)

Für diese These erbrachte ihnen zufolge die nächste Farm Bill den endgültigen Beweis: „In a way it [Farm Bill 2002] demonstrated that the emphasis on producer flexibility and decoupling that had characterized the FAIR Act of 1996 and its forerunners was not really based on a firm 'philosophical' position but a matter of convenience when market developments and budgetary considerations suggested this was more favorable for the farming lobby" (ibid.: 136). Paarlberg (1997: 435) schreibt in diesem Zusammenhang vom „dirty decoupling“. Auch für Hofreither 
(1996: 6) ist die Umstellung von „deficiency payments“ auf „,contract payments“ weniger „einer ideologischen Orientierung zu einem marktor[i]entierten Farmsektor bzw. dem anfangs verfolgten Haushaltsdruck“ zuzuschreiben, sondern „ganz einfach dem Faktum, daß in der Zwischenzeit die (Welt-)Marktpreise deutlich angestiegen waren“. Er betont dabei die Tatsache, dass „die in den USA verfügbaren Simulationsmodelle zur Abschätzung der Konsequenzen dieser Reform lediglich in Richtung der budgetären Auswirkungen herangezogen wurden, ihre umfangreichen Informationen über zumindest gleich wichtige Variable - wie z. B. Produktionsmengen, Preise, Struktureffekte, usw. - im politischen Prozeß kaum beachtet wurden“ (ibid.: 13). Darauf weist auch Conley (1996: 43) mit der Bemerkung hin, dass ,it would have been useful to have had estimates of efficiency, welfare, and planted acreage impacts of the ARA more fully articulated during the debate".$^{78}$ Darüber hinaus wurde schon sehr früh auf die Anfälligkeit der Reform hingewiesen. Harvey (1998: 9) bemerkt, dass die Farm Bill von 1996 ,has been heralded in Europe as a clear and unambiguous signal that the US is committed to full liberalisation of farm policy, and hence of farm trade“. Allerdings "there remain strong reasons to question whether these reforms will ,stick“" und ,whether FAIR is fair or foul" (ibid.: 2 und 14). Diese Thesen vertrat Harvey bereits im November 1996 auf der Konferenz der „Agricultural Economics Society“ über die Farm Bill von 1995 in London (ibid.: 1). Er war offensichtlich nicht allein. Wie es Stuart and Runge (1997: 118) kurz darauf formulieren: „,P]olitical realists tend to doubt that the end of the seven-year contract will actually terminate transfers to these producers, as advertised.“

Unter den dem „problem stream“ zuzurechnenden Faktoren waren für die Farm Bill von 1996 der Anstieg der Marktpreise bzw. die Erwartung hoher Marktpreise sowie der Haushaltsdruck und die internationale Wettbewerbsfähigkeit der Landwirtschaft entscheidend. Als relevant für den Milestone konnte nur Ersteres identifiziert werden. Erst die einem Instrumentenwechsel förderlichen Haushaltsregeln aber ermöglichten die sogenannte „baseline capture“. „Politics stream" wird darüber hinaus durch die Kontrolle des US-Kongresses durch die Republikanische Partei in der Zeit, als die Farm Bill von 1996 vorbereitet wurde, sowie ein starkes Lobbying seitens der Agrarwirtschaft, insbesondere der Getreidewirtschaft, repräsentiert (vgl. Tabelle 24).

78 ARA = „Agricultural Reconciliation Act“ - die Version der Farm Bill von 1995. 
Tabelle 24: Der US-Reform von 1996 als Ganzes und dem Milestone zugrunde liegende Faktoren

\begin{tabular}{c|c} 
Reform $\mathbf{1 9 9 6}$ & Milestone $\mathbf{1 9 9 6}$ \\
\hline $\begin{array}{c}\text { Anstieg der Marktpreise/Erwartung hoher } \\
\text { Marktpreise }\end{array}$ & $\begin{array}{c}\text { Anstieg der Marktpreise/Erwartung } \\
\text { hoher Marktpreise }\end{array}$ \\
Haushaltsdruck & \multicolumn{2}{c}{ Haushaltsregeln } \\
Republikanische Mebrheit im Kongress \\
Unterstützung der Agrarwirtschaft
\end{tabular}

\subsection{EU-Milestone 2003}

Swinnen (2008b: 162) identifiziert drei Gruppen von Faktoren, die einen ,perfect storm" entstehen ließen, sodass die GAP-Reform von 2003 erfolgreich verabschiedet werden konnte. Neben den Auswirkungen der institutionellen Reformen sowie Änderungen in Zahl und Qualität der politischen Akteure (s. unten), führt er ebenfalls „the optimal conditions for reform“ an. Während die ersten zwei Gruppen eher dem „politics stream“ nach Kingdon (1995) zuzuschreiben sind, liefert letztere Hinweise auf die Frage nach Auslösefaktoren für die Reform von 2003 im Sinne des ,problem stream“. Darunter identifiziert Swinnen (2008b: 162) vier treibende Reformkräfte: die WTO- und andere Handelsverhandlungen, Haushaltszwänge, Lebensmittelsicherheits- und Umweltbelange sowie die Osterweiterung der EU. Sie alle „combined to increase pressure for change in the EU“ (ibid.: 143).

Bereits die vorausgegangene Reform - die „Agenda 2000“ - sollte die GAP auf die neue W'TO-Verhandlungsrunde in Doha, deren Start für 2001 geplant war, vorbereiten, erreichte dieses Ziel aber nicht (Daugbjerg 2012: 97). Vor diesem Hintergrund stellte der in der WTO institutionalisierte internationale Druck einen wichtigen Treiber der Reform von 2003 dar (Swinnen 2008b). Wie Cunha und Swinbank (2011: 147) bemerken: ,While there was a complex array of factors that both motivated and influenced the Fischler reform, most authors conclude that the forthcoming international trade negotiation was its main driving force." Dazu gehören u. a. Daugbjerg und Swinbank (2010: 57), die feststellen, dass die Verhandlungen ,the most important motivation for the CAP reform in 2003" waren. Sie schreiben: „WTO concerns have gradually been accepted as a constraint within which the CAP evolves and which could no longer be ignored, but which instead increasingly had to be taken into consideration. From 1995 on, policy makers designing reforms would strive to ensure conformity with WTO agreements 
and, perhaps, even attempt to anticipate future developments in the WTO" (ibid.: 52). Alle anderen in der Literatur aufgezeigten Faktoren waren ihrer Meinung nach lediglich genutzt, ,to justify continuation of farm support to the European electorate" (ibid.: 57). Laut Nedergaard (2006) war der externe, auf die WTO-Verhandlungen zurückzuführende Druck die notwendige Voraussetzung für die Reform von 2003. Ohne ihn wäre aufgrund von institutionellen Voraussetzungen nicht nur keine Reform verabschiedet worden, sondern die Kommission hätte es nicht gewagt, einen Reformvorschlag vorzulegen: „Without external pressure on the CAP, the super asymmetrical political system of the CAP would probably have resisted a reform proposal and the Commission would not even have dared to present a plan for CAP reform" (ibid.: 216). Und Josling (2008: 68) stellt fest, dass - während bereits die MacSharry-Reform von den Handelsverhandlungen geprägt wurde - ihre Bedeutung für die nachfolgende Entwicklung der GAP sogar noch zunahm.

Wenige Forscher bewerten die Bedeutung der WTO für die Reform von 2003 anders. Aufgrund der Tatsache, dass die Doha-Runde „was drifting to unknown territory after the 2002 U.S. farm Bill“", sieht Haniotis (2006: 53) die Handelsverhandlungen nicht als ausschlaggebend für die Fischler-Reform an (er nennt aber die Green-Box-Konformität als einen der Beweggründe hinter der Entkopplung, s. unten). ${ }^{79}$ Allerdings ist aufgrund seiner Funktion als Mitglied im Kabinett des Agrarkommissars „political rhetoric“ bei seiner Darstellung der Reformgründe nicht unwahrscheinlich. Anania (2009) wiederum hält die Reform von 2003 nicht für WTO-getrieben, weil sie ohne Berücksichtigung der Dynamik der parallel laufenden Verhandlungen verabschiedet wurde. Er bemerkt: „If these policy changes [2003 reform] were to have been part of an effective negotiation strategy, they should have been introduced toward the end, or after the conclusion, of the round, in order to use these significant policy reforms as part of the final offers by the EU in the round“ (ibid.: 13). Aus diesem Grund vermutet er EU-interne Ursachen für die Reform von 2003. Haniotis (2006: 59) weist in diesem Zusammenhang darauf hin, dass viele die WTO-Verhandlungen „more as a card than as a strategy game“ ansahen und behaupteten, „the E.U. needed to keep its cards hidden till the end". Agrarkommissar Fischler teilte diese Sichtweise nicht. In einer Rede vor dem Landwirtschaftsausschuss des Europäischen Parlaments im Januar 2003 sagte er, dass ,in the presence of clear trade rules, the E.U. cards were not as close to its chest as some tended to believe and, worse, they are not as good, thus the risk of changing them is worth taking if this were a card game!" (ibid.: 59).

\footnotetext{
${ }^{79}$ Garzon (2006), die wie Haniotis zur Zeit der Reformvorbereitung in der Europäischen Kommission arbeitete (sie im Kabinett des Handelskommissars, er im Kabinett des Agrarkommissars), teilt die in der Literatur überwiegende Meinung, dass die WTO die Hauptantriebskraft der Reform war. Auf diese interessante Meinungsdifferenz innerhalb der Kommission weisen Daugbjerg und Swinbank (2010: 56) hin.
} 
Auch für die Reform von 1992 waren die multilateralen Handelsverhandlungen ausschlaggebend. Allerdings bestehen hinsichtlich dieses Reformfaktors zwischen beiden Reformen signifikante Differenzen. Der erste Unterschied bezieht sich auf die Positionen der USA und der EU zueinander in der Uruguay- und in der DohaRunde. War die erste Runde durch die Konfrontation zwischen den USA und der EU geprägt, glichen sich in letzterer die Interessen und handelspolitischen Positionen der beiden Akteure weitgehend an (Josling 2008). „While the Uruguay Round negotiations on agriculture were to a large extent characterized by tensions between the US and the EU, these two protagonists found themselves essentially at the same side of the negotiating table in the Doha Round", konstatieren auch Josling und Tangermann (2015: 125). Einen größeren Konfliktpunkt zwischen ihnen bildeten lediglich die Pläne der USA zur Abschaffung der Blue Box (s. unten). Ansonsten waren es nun die Entwicklungs- und Schwellenländer, die von den Industrieländern weitere Agrarreformen forderten: Die EU sollte insbesondere den Außenschutz, die USA die Inlandsstützung reformieren (ibid.).

Zum anderen war die EU-Agrarpolitik im Jahr 2003 in Vorbereitung auf den für 2005 geplanten Abschluss der Doha-Runde reformiert. Zu der Zeit war es noch nicht klar, welche Regelungen für die Landwirtschaft getroffen werden, aber „Fischler wanted to avoid a repeat of the experience in the Uruguay Round, when the EU was on the defensive and under pressure to change its policies" (Josling und Tangermann 2015: 149). Denn es bestanden kaum Zweifel daran, dass die Doha-Runde neue Reduktionsverpflichtungen in Bezug auf Inlandsstützung, AuBenschutz sowie Exportsubventionen vorsehen wird und „[i] $\mathrm{t}$ was certainly preferable to prepare the CAP for these requirements than to be forced to engage in further reform while the negotiations were going on" (ibid.). Swinbank und Daugbjerg (2006) liefern eine Reihe von Argumenten für die Begründung ihrer These, dass die GAP-Reform von 2003 ,accommodat[ed] WTO pressures“ (ibid.: 47). Laut Fouilleux (2004: 253) war „much more proactive and ,aggressive“ stance on agriculture" der EU in der Doha-Runde den ,improved intellectual resources“ der Kommission im Agrarbereich zuzuschreiben. Diese halfen ihr, „to formulate, diffuse and advocate new ideas in the context of the CAP" (ibid.). Während der Uruguay-Runde hingegen „[a] lack of internal expertise and intellectual resources in agricultural economics, policy evaluation and forward-looking analysis kept the EU from taking a proactive stance" (ibid.: 241).

Daugbjerg und Swinbank (2007: 10f) zeigen, dass trotz der Ungewissheit hinsichtlich der Ergebnisse der Doha-Runde in den Agrarfragen der Druck auf die GAP präsent war. Es gelang zwar nicht, bis Ende März 2003 eine Einigung über die Modalitäten im Agrarbereich zu erzielen. Allerdings sah der Entwurf des Leiters der Agrarverhandlungen, S. Harbinson, entweder eine Kürzung der damaligen Blue-Box-Zahlungen um $50 \%$ über einen Zeitraum von fünf Jahren oder eine Integration dieser Zahlungen in die Amber Box vor. Beide Ansätze erhöhten den Druck auf den Rat, dem Reformvorschlag der Kommission im Juni 2003 zuzu- 
stimmen (für September 2003 war die WTO-Ministerkonferenz in Cancún anberaumt). Denn es schien unwahrscheinlich, dass die WTO-Mitglieder eine weniger strengere Regelung zur Blue Box als diese vom Harbinson-Entwurf akzeptieren würden und ,it may be that this led to concern in some EU capitals that the inability of the EU to reform the CAP could disrupt the trade round and have negative implications for the image of the EU“ (ibid.: 11). Schließlich bestehen große Unterschiede im Umgang mit dem Einflussfaktor WTO in der jeweiligen Reformdebatte. Im Reformprozess von 1992 wurde ein Zusammenhang zwischen der Reform und der Uruguay-Runde von der Kommission noch bestritten. Für den Zeitraum danach stellen Daugbjerg und Swinbank (2010: 51) fest, dass „EU agricultural policy makers explicitly and increasingly referred to the WTO when proposing, and deliberating upon, CAP reforms". Anhand der Dokumente der Kommission und des Rates liefern sie dafür mehrere Beispiele aus den Jahren 2002 und 2003 (ibid.). Wie Swinnen (2008b: 142) zudem feststellt, spielten nicht nur die Doha-Runde der WTO, sondern auch andere Handelsinitiativen (z. B. „Everything but Arms"-Initiative und bilaterale Freihandelsabkommen) eine Rolle bei der Reform von 2003, denn sie "further raised awareness of the international effects of the CAP and contributed to the sense of a need to reform the CAP“".

Die WTO-Verhandlungen stellen den wichtigsten Auslösefaktor der Reform von 2003 dar, der alleine zur Erklärung dieser Reform allerdings nicht ausreichen würde (z. B. Nedergaard 2006, Daugbjerg und Swinbank 2007). Laut Swinnen (2008b) hatte auch noch der Haushaltsdruck Einfluss auf den Reformprozess im Jahre 2003. Es herrschten zwar keine ,immediate budgetary pressures challenging the CAP" (Cunha und Swinbank 2011: 148), aber es bestand die Gefahr, dass der Agrarhaushalt der EU aufgrund der Osterweiterung, der bevorstehenden Verhandlungen über den Mehrjährigen Finanzrahmen von 2003 bis 2007 sowie der um die Finanzmittel konkurrierenden Politikbereiche gekürzt wird (ibid.). Eine umfassende Reform der GAP war laut Pirzio-Biroli (2008) notwendig, um die Agrarausgaben vor Kürzungen in den kommenden Verhandlungen über den MFR zu bewahren. Zwar legten im Jahr 2002 in Brüssel der französische Präsident Chirac und der deutsche Bundeskanzler Schröder diese bis 2013 fest. Aber wie PirzioBiroli (2008: 108) bemerkt: „Without reforms [...] the Brussels European Council agreement of 2002 would not have held up against the pressures of the ,one percenters' in connection with the 2007-13 financial perspectives." Auch Swinnen (2015c: 450) geht mit dem Hinweis darauf ein, dass die mutigen Reformschritte von 2003 ,counter the demand for large budget cuts, in effect limiting the cuts planned for the next financial period"“.

Eine Unzufriedenheit mit der Verwendung von GAP-Finanzmitteln war zu jener Zeit zu beobachten. Wie Olper (2008: 91) es formuliert: „In fact, before and during the reform process we were aware of a growing dissatisfaction with respect to CAP money, especially from actors normally outside the CAP decision-making process, such as finance ministers, trade commissioners and industrial sectors, but 
also from traditional supporters of the CAP such as Germany, the biggest contributor to the EU budget." Auch die Kommission selbst hinterfragte die Höhe der Agrarausgaben: Der damalige EU-Kommissionspräsident Prodi, unterstützt von einigen Kommissaren, sprach sich für ihre Kürzung um 30\% aus (PirzioBiroli 2008). Olper (2008: 91) weist darauf hin, dass der Haushaltsdruck auch Auswirkungen auf das Timing der Reform hatte: Agrarkommissar Fischler bemühte sich, die GAP-Reform vor der Debatte über den MFR für den Zeitraum 2003 bis 2007 abzuschließen.

Die dritte Gruppe von Faktoren aus der Zusammenstellung von Swinnen (2008b) bilden Sorgen der Verbraucher bezüglich der Lebensmittelsicherheit und Umwelt, die durch Lebensmittelkrisen verstärkt und von neuen Akteuren in der agrarpolitischen Debatte, wie ökologische Nichtregierungsorganisationen, aufgegriffen wurden. Wie Lynggaard und Nedergaard (2009: 304) bemerken: „Compared with the 1992 CAP reform, the 2003 Fischler reform highlighted even more environmental concerns in combination with other concerns with regard to animal welfare and food security." Eine Kluft zwischen den von der Gesellschaft erwarteten Zielen der Agrarpolitik und der aktuellen Politik wurde registriert (Cunha und Swinbank 2011: 147). Öffentliche Kritik an Inhalten und Auswirkungen der GAP hatte laut Swinbank und Daugbjerg (2006: 60f) ihre Wurzeln bereits in den 1980er-Jahren und war somit nicht neu. Aber „,rises put food safety, animal welfare and environmental concerns on top of the agricultural policy agenda", stellt Swinnen (2008b: 142) für den Anfang der 2000er-Jahre fest. Dabei standen diese starken Reaktionen der Verbraucher bzw. der Öffentlichkeit im großen Kontrast zu ihrer eher passiven Einstellung gegenüber den traditionellen agrarpolitischen Themen in den letzten Dekaden (ibid.: 142).

Swinnen (2008b: 148 und 142) stellt mit dem Hinweis auf die Lebensmittelkrisen fest, dass ,the pressure to reform the CAP had plainly increased by 2001“ und "there were strident calls for the overhaul of the EU's agricultural policy“. Eine besondere Rolle ,as a de-legitimizing factor as far as industrialized agriculture is concerned" spielte die Krise aufgrund der Rinderseuche BSE um das Jahr 2000 (Lynggaard und Nedergaard 2009: 304). Auch wenn keine direkte Verbindung zwischen den Lebensmittelkrisen und der Agrarpolitik bestand, sieht Swinnen (2008b) durchaus ihren Einfluss auf die GAP-Reform. Denn sie trugen zur Stimmung bei, dass „,something needs to be done“ (ibid.: 148). „Consumer groups, environmental organisations and government officials emphasised that large government subsidies for farming practices that did not adhere to appropriate food safety, environmental and animal welfare standards were unacceptable“ (ibid.: 142). Die Antwort der Kommission ,was to take advantage of this reform-minded context to present a substantial reform package" (Cunha und Swinbank 2011: 127).

Die gesellschaftlichen Anliegen für mehr Umweltschutz, Lebensmittelsicherheit und Förderung des ländlichen Raums nutzte Agrarkommissar Fischler ,in selling his MTR [Mid-Term Review] proposals“ (Swinnen 2008b: 145). Wie Cunha 
und Swinbank (2011: 148) dazu bemerken: „Because they were popular with the media, these concerns were constantly re-articulated by Fischler and his team, as they legitimized the reform in the eyes of the wider public, and created the political environment for it to be more acceptable to farmers and the agricultural policy network." Allerdings erklären die neuen gesellschaftlichen Anliegen das Reformergebnis nicht, weil es diesbezüglich eher mager ausfiel (ibid.). Deshalb ordnet Olper (2008) diesen Faktor der „political rhetoric“ zu. Er bemerkt, dass „quite a divergence emerges between political rhetoric and political reality, suggesting that the pressures and concerns brought to the fore by these ,new" players have not been decisive in the reform process, even though they represented an important ally in pursuing the Commission's reform objectives" (ibid.: 90). Swinbank und Daugbjerg (2006) schließen die gesellschaftlichen Anliegen als ausschlaggebenden Reformfaktor aus (u. a. aus dem Grund geringer Finanzierung der Programme der 2. Säule). Daugbjerg und Swinbank (2010: 56) schreiben dazu: „Undoubtedly, the wish to move towards more sustainable agricultural production played a role, but it was not a major driving force behind the reform."

Unstrittig ist aber, dass die neuen Interessengruppen aus den Bereichen Umwelt und Verbraucherschutz im Jahr 2003 eine stärkere Rolle spielten als in den vergangenen GAP-Reformen. Wie Swinnen (2008b: 145) bemerkt: „[T]he MTR [Mid-Term Review] negotiations brought consumer groups and environmental groups to the political table in a much stronger way than had happened before." Gleichzeitig hatte die Agrarlobby weniger Einfluss auf die Reformdebatte und -ergebnisse als in der Vergangenheit (Swinnen 2008b: 159). Die Gründe wurden darin vermutet, dass die landwirtschaftlichen Verbände auf den Entkopplungsvorschlag sowie auf die neue stärkere Rolle anderer Interessenverbände nicht vorbereitet waren. Auch die Tatsache, dass die Landwirte realisierten, dass die entkoppelten Zahlungen ein wirksameres Einkommensstützungsinstrument darstellen, wird in diesem Zusammenhang erwähnt (ibid.).

Schließlich zählt nach Swinnen (2008b) die für 2004 geplante EU-Erweiterung auf 25 Mitgliedstaaten, die eine Ausweitung der GAP auf diese Länder zur Folge hatte, zu den Ursachen für die Reform von 2003. Verglichen mit den restlichen Faktoren sei ihr Einfluss allerdings am schwächsten gewesen (ibid.: 162). Die Osterweiterung beeinflusste insbesondere das Timing der Reform. Denn der Kommission war bewusst, dass eine Einigung auf eine Agrarreform nach der Aufnahme der neuen Mitgliedstaaten schwieriger geworden wäre. Wie Swinnen (2008b: 144) es formuliert, ,the expected eastern enlargement gave a sense of urgency to the reform proposals: they had to be decided before enlargement took place“. In Bezug auf die Einhaltung der WTO-Verpflichtungen sollte die Osterweiterung weniger problematisch sein als ursprünglich befürchtet, wobei das letztendlich vom Ergebnis der Doha-Runde abhängen würde (Swinnen 2008b: 140).

Die Reform von 2003 wird auch im Kontext neuer Ideen bzw. eines Paradigmenwechsels analysiert. Beispielsweise argumentiert Garzon (2006), dass im Laufe 
der GAP-Reformen eine Verlagerung weg vom Paradigma einer schutzbedürftigen Landwirtschaft in Richtung des Multifunktionalitätsparadigmas erfolgte. Für Daugbjerg und Swinbank (2010: 55) hingegen ist es fraglich, ob das Multifunktionalitätsparadigma die Reform von 2003 tatsächlich untermauerte, denn ,[t]he CAP is still based on the view that agriculture is an exceptional industry requiring special treatment". Laut ihnen lässt die Tatsache, dass die GAP weiter auf dem Paradigma einer schutzbedürftigen Landwirtschaft basiert, vermuten, dass ,it exists uneasily within a WTO framework that is far more orientated to the marketliberal paradigm“ (ibid.: 57). Für Fouilleux (2004: 236) sind die Paradigmen Teil des politischen Diskurses und „,[w]hether a discourse is true or false is always a matter of interpretation “. Während, the promotion of CAP through multifunctionality is denounced as a false discourse by many actors within and outside the EU $[\ldots]$, from an international perspective, CAP is more ,multifunctionality-friendly ${ }^{\circ}$ than Australian or US farming policies" (ibid.: 236f).

Wichtig für die Fischler-Reform war auch die Einführung und Nutzung der qualifizierten Mehrheit für die Entscheidungen des Rates im Agrarbereich. Wie Swinnen (2008b: 138) bemerkt, ,the voting rules played a very important role“ und „Fischler and his team spent a lot of effort trying to put together a winning coalition and breaking a blocking minority coalition". Darüber hinaus weist er auf die Relevanz der Erweiterung von 1995, die die Änderungen in Zahl und Qualität der politischen Akteure mit sich brachte, hin (ibid.: 143f). Er hob u. a. hervor, dass keiner der drei neuen Mitgliedstaaten einen natürlichen Verbündeten des in Fragen der Agrarpolitik konservativ ausgerichteten Frankreichs darstellte. Insbesondere der Beitritt Schwedens stärkte das reformorientierte Lager im Rat. Ebenfalls relevant für die Reform war die Haltung des erfahrenen Agrarkommissars Fischler. Swinnen (2008b: 150f) schreibt, dass die Kombination von „Fischler's strategic vision and political tactics“ sowie "the Commission officials" effort and preparation" als entscheidend für den Reformerfolg angesehen wird.

Nachfolgend wird geprüft, inwieweit für die 2003 beschlossene Entkopplung der EU-Direktzahlungen von der Produktion die Faktoren, die die Reform als Ganzes beeinflussten, bedeutsam waren. Außerdem wird untersucht, ob und welche Ursachen für die Entkopplung in der Literatur explizit genannt werden.

Für die Einführung der entkoppelten Direktzahlungen, wie auch für die gesamte Reform von 2003, stellte die Doha-Runde der WTO ${ }^{80}$ eine wichtige treibende Kraft dar. Gleichzeitig zur internen Reformdebatte befand sich die EU unter Druck, ihr Angebot an die WTO-Partner zu unterbreiten inklusive möglicher Änderungen der Regelung zu der Blue Box und der Green Box (Daugbjerg und Swinbank 2007: 10). Der Einfluss der WTO-Verhandlungen auf die Entkopplung

\footnotetext{
${ }^{80}$ Schon bei der Einführung des Wegbereiters der Betriebsprämie - des entkoppelten „Small Farmers Scheme“ von 2001 - spielte die WTO eine wichtige Rolle (Daugbjerg 2012). „By proposing the Small Farmers Scheme, Fischler tested the willingness of the farm ministers to accept the decoupled payments" (ibid.: 99).
} 
resultierte aus den Forderungen der EU-Handelspartner, insbesondere der USA und der Cairns-Gruppe, die Blue Box, in der bisher die im Jahr 1992 eingeführten Ausgleichszahlungen untergebracht wurden, abzuschaffen. Daugbjerg (2009: 405) beschreibt die damalige Situation wie folgt: „In November 2001 a new trade round in the WTO began in Doha [...]. It was soon realized that the direct area and headage payments of the CAP were under pressure. Many of the EU's trading partners, at that time in particular the US and the Cairns Group, wanted to abolish the so-called blue box of the Uruguay Round Agreement on Agriculture." Da die Blue-Box-Ausnahmeregelung infrage gestellt wurde, sollten die Ausgleichszahlungen vereinbar mit den Green-Box-Kriterien gestaltet werden.

Der Ausweg wurde in ihrer Entkopplung von der Produktion gesehen: „The Commission held the view that, with the further decoupling of direct payments, the Fischler reforms would shift direct payments from the blue to the green box, thereby appeasing in part its international critics" (Daugbjerg und Swinbank 2007: 10). Cunha und Swinbank (2011: 148) schreiben, dass die Entkopplung „was believed to be the only way to shelter direct payments from likely future cuts in domestic support under new WTO agreements“. Wie Daugbjerg (2009: 406) bemerkt, konnte die Tatsache, dass die Entkopplung den Druck auf die GAP in der WTO mindern würde, den Widerstand gegen diesen Schritt verringern. Auch Haniotis (2006: 57), der EU-interne Faktoren für die Reform betont, sieht den Transfer der EU-Direktzahlungen in die Green Box als einen der Gründe hinter der Entkopplung an (den anderen Grund bildete die Vereinfachung). Er stellt fest: „Few seemed to dispute the fact that the Commission proposal would strengthen the E.U. negotiating position in the Doha Round, especially with respect to domestic support“ (ibid.: 59). Der Europäische Rat (2003: 3) sieht das Reformergebnis von 2003 als Beitrag der EU zur Doha-Runde an: „The CAP reform is Europe's important contribution to the Doha Development Agenda (DDA), and constitutes the limits for the Commission's negotiating brief in the WTO Round.“ Laut der Kommission (2002: 19) sicherte die Entkopplung der EU einen deutlichen Vorteil in der WTO ,since the Green Box compatibility of the scheme will help secure these payments in an international context".

Zum Einfluss der gesellschaftlichen Anliegen auf die Entscheidung über die Entkopplung zeigt sich ein gemischtes Bild. Vor dem Hintergrund der Lebensmittelkrisen erwartete die breite Öffentlichkeit nichts weniger als eine fundamentale Reform der GAP (Haniotis 2006: 64). Der Kommissionsvorschlag basierte auf den Ergebnissen einer langwierigen öffentlichen Debatte, die auf die BSE-Krise vom November 2000 folgte, und es war klar, dass sich die GAP verändern musste, um den Ansprüchen der modernen Gesellschaft besser zu genügen (ibid.: 56). Swinnen (2008b: 160) merkt an, dass „Fischler looked at the reforms much more from the standpoint of how he could save the support for European agriculture in the $21^{\text {st }}$ century with new demands and new constraints being imposed on European farmers and with new opportunities“ und dass ,[d]ecoupling in this way was 
an attractive choice for several reasons“. Neben den Verbesserungen im Hinblick auf das Budget (effizientere Mittelverwendung) und WTO (Abbau von Handelsverzerrungen), it was an improvement from an environmental perspective as it reduced incentives to use land intensively and the introduction of cross-compliance further enhanced the environmental benefits" (ibid.). Allerdings, wie Daugbjerg und Swinbank (2010: 56) bemerken, war für die Einführung des obligatorischen Cross-Compliance keine Entkopplung der Zahlungen erforderlich. Bezüglich anderer gesellschaftlichen Anliegen macht die Kommission (2002: 11f) deutlich, dass „society's expectations of a policy that promotes food quality, sustainability, and value for money“ eher durch eine Stärkung der Programme der 2. Säule zu erfüllen sind. Somit bleibt die Reduzierung des Drucks auf die Ressourcen durch die Beseitigung der Produktionsanreize als positiver Beitrag der Entkopplung zum Umweltschutz übrig.

Beide für die Entkopplung als relevant identifizierten Faktoren - die WTO und die gesellschaftlichen Ansprüche an die Landwirtschaft - scheinen auch hinter der viel beklagten Legitimationskrise der GAP zu stehen. Wie Swinnen (2008b: 143) bemerkt: „[T] he fact that the CAP was increasingly seen as at the same time hurting EU trade interests, having negative effects on the environment and unable to address the food safety concerns of EU consumers was turning into a major call for reform.“ Ausgedrückt mit den Worten von Fischler: „, [T [he CAP had lost its legitimacy among the EU public" (ibid.).

Es besteht hingegen kein direkter Zusammenhang zwischen der Entscheidung hinsichtlich der Direktzahlungen und den Haushaltszwängen, wie z. B. in der „Agenda 2000“, als der Europäische Rat die vom Agrarrat festgesetzten Preiskürzungen nach unten korrigierte, um den EU-Haushalt nicht mit übermäßigen Ausgaben für die Ausgleichszahlungen zu belasten (Olper 2008: 90f). Auf die Entscheidung von 2003 über die Entkopplung hatte der Haushaltsdruck laut Daugbjerg und Swinbank (2010: 56) keinen Einfluss. Dieses stellen sie mit Hinweis darauf fest, dass ,[a]s originally tabled in 2002, the proposed Farm Income Payment would have maintained [Herv. im Original] CAP expenditure on direct payments at prevailing levels in nominal terms“. Sie weisen weiter darauf hin, dass die Betriebsprämie „was not designed to reduce the budget allocation; although progressively, through modulation, some expenditure would have been switched to Pillar 2“. Auch im Laufe des Gesetzgebungsverfahrens wurden die Ausgaben für die Direktzahlungen nicht beeinträchtigt. Wie Olper (2008: 89) bemerkt: „[T] he cut in the agricultural budget introduced by the agreement for the 2007-13 financial perspective will only affect the pillar II expenditures, not those of pillar I, also because of the 2002 European Council agreement in Brussels that fixed market-related expenditure and direct payments." Vor dem Hintergrund der Festlegung der Agrarleitlinie für den Zeitraum von 2007 bis 2013 und der Entscheidung, die EU-Direktzahlungen auf die neuen Mitgliedstaaten auszuweiten, wurden die Finanzmittel, die von der 1. in die 2. Säule umgeschichtet werden sollten, letztend- 
lich um $70 \%$ gegenüber dem ursprünglichen Vorschlag der Kommission gekürzt (Cunha und Swinbank 2011: 148). Es kann auch nicht mit Sicherheit festgestellt werden, ob zwischen der Entkopplung und dem Schutz der Agrarausgaben vor Kürzungen in den bevorstehenden MFR-Verhandlungen ein direkter Zusammenhang besteht, auf den Pirzio-Biroli (2008) hinwies. Denn es stellt sich die Frage, ob die Entkopplung dafür die einzige mögliche Lösung darstellte. Am Ende stellt Swinnen (2008b: 136) fest: „,T] otal farm support has only marginally been affected by the reforms and remains essentially the same as before. Moreover, there is very little change in the distribution of CAP benefits across countries and farms."

Im Hinblick auf den Einfluss der EU-Osterweiterung auf die Entkopplung vermutet Swinbank (2016: 32), dass ,the recognition in January 2002 [...] that direct payments could not be denied the new Member States“ zusammen mit ,the need to apply a simplified scheme" die Überlegungen der Europäischen Kommission über die Möglichkeit einer weiteren Entkopplung der Zahlungen in den alten Mitgliedstaaten im Jahr 2003 beeinflusste. Wie Daugbjerg (2012: 100) bemerkt: „By applying decoupled payments in the Simplified Scheme [in the new Member States], Fischler had, intentionally or not, laid the ground for the transformation of the direct payments in the old Member States to decoupled payments. Introducing decoupled payments in the new member states could be utilized as a precedent for introducing decoupled payments on a grand scale, extending the principle to the old member states." Er sieht somit die für die östlichen Mitgliedstaaten vorgesehene entkoppelte einheitliche Flächenzahlung als Wegbereiter der Betriebsprämie von 2003 an. ${ }^{81}$ Die Kommission selbst betonte, dass die Entkopplung ,will facilitate the integration of the new Member States into the common agricultural policy" (Kommission 2002: 19).

Im Hinblick auf das Multifunktionalitätsparadigma weisen Daugbjerg und Swinbank (2010: 56) darauf hin, dass die Ausgestaltung der Entkopplung keine auf dieses Paradigma deutenden Elemente zeigt: „Neither the historical nor the regionalized mode of transforming the area and headage payments into single farm payments linked them to the actual production of public goods produced on each farm. A true shift to the multifunctional paradigm would, to a considerable extent, imply that payments would be much more directly related to the provision of public goods on individual farms." Vor diesem Hintergrund sehen Daugbjerg und Swinbank (2009: 12) Grund zu der Annahme, dass ,the rhetoric of multifunctionality in 2003 was just the state-assisted paradigm dressed up in different clothes". Für Feindt et al. (2008: 80) korrespondieren die verschiedenen Elemente der Reform von 2003 mit Ideen aus verschiedenen Paradigmen. Die Entkopplung der Zahlungen von der Produktion entspreche eindeutig dem marktliberalen Paradigma, denn mit diesem Schritt sind Produktionsentscheidungen stärker auf die

\footnotetext{
81 Daugbjerg (2012) sieht die jeweils folgenden Entscheidungen bzw. Reformen als bedingt durch die vorhergegangene Entscheidung/Reform an (,sequencing").
} 
Preissignale und damit die Nachfrage am Markt ausgerichtet. Die Fortsetzung der im Jahr 1992 als Ausgleich für die Preissenkungen eingeführten Ausgleichszahlungen über einen langen Zeitraum hingegen ist für ihn ein Zeichen des Fortwirkens des Paradigmas einer schutzbedürftigen Landwirtschaft (ibid.: 82).

Zum Schluss soll noch der Beitrag der OECD zur Fischler-Reform beleuchtet werden. Es ist ein Einfluss der OECD auf die Entscheidung zur Entkopplung im Jahr 2003 feststellbar. Ahner, der zur Zeit der Reform von 2003 in der Generaldirektion Landwirtschaft tätig war, erinnert sich: „Als wir begannen, an der Ausgestaltung der Entkopplung zu arbeiten, fanden wir nur wenig Material und Ideen in der europäischen Forschung. Allerdings hatte die OECD eine gute analytische und empirische Basis geschaffen, auf die wir uns stützen konnten" (Ahner 2010: 35). Swinnen (2008b: 159) bemerkt in diesem Zusammenhang: „Although both insiders and outsiders are sometimes sceptical about the impact of sound ideas and careful analysis on political decisions, it appeared that a series of studies and analyses had a considerable impact on the CAP reform process." Er hebt insbesondere eine Studie der OECD über die Entkopplung hervor, auf deren Bedeutung Agrarkommissar Fischler selbst verwiesen hätte. Diese Studie belegte, dass die entkoppelte Stützung eine viel größere Transfereffizienz aufweist als das bisherige System. Vor dem Hintergrund, dass die GAP einen halben EU-Haushalt ausmachte, ,[t] $[$ he analysis by the OECD reinforced the argument in favour of decoupling" (ibid.: 160).

Im Vergleich zur Reform als Ganzes fehlt unter den zum „problem stream“ gehörenden Ursachen für den Milestone der Haushaltsdruck. Zusätzlich konnte für die Einführung der Entkopplung noch der Einfluss der OECD und neuer Ideen festgestellt werden. Darüber hinaus spielten die Doha-Verhandlungsrunde der WTO und die bevorstehende Erweiterung der EU um zehn Länder eine wichtige Rolle. Zum „,politics stream“ gehören insbesondere die Anwendung der qualifizierten Mehrheit für die Entscheidungen des Rates sowie die mit der Osterweiterung einhergehenden Änderungen in Zahl und Qualität der politischen Akteure. Auch die Erfahrung und das Engagement des Agrarkommissars waren für die Entkopplung der EU-Direktzahlungen ausschlaggebend. Relevant waren zudem neue Interessengruppen, deren Beteiligung am politischen Prozess im Vergleich zu früher stärker ausgeprägt war (vgl. Tabelle 25). 
Tabelle 25: Der EU-Reform von 2003 als Ganzes und dem Milestone zugrunde liegende Faktoren

\begin{tabular}{c|c} 
Reform 2003 & Milestone 2003 \\
\hline WTO/Doha-Runde & WTO/Doha-Runde \\
Haushaltsdruck & Umweltsorgen der Gesellschaft \\
$\begin{array}{c}\text { Gesellschaftliche Anliegen, inkl. } \\
\text { Umweltsorgen }\end{array}$ & Bevorstehende Osterweiterung \\
Bevorstehende Osterweiterung & OECD \\
Starker Agrarkommissar Ideen \\
Nutzung der qualifizierten Mehrheit \\
Zabl und Qualität der politischen Akteure \\
Neue Interessengruppen \\
\hline
\end{tabular}

\subsection{US-Milestone 2014}

Da die Abschaffung der ,fixed direct payments“ die größte Neuerung der Farm Bill von 2014 bildet, liegt in der Literatur der Fokus auf den dieser Entscheidung zugrunde liegenden Ursachen und weniger auf den Treibern der Reform als Ganzes. Deshalb wird hier unmittelbar mit ersteren begonnen.

Im Vorfeld der Farm Bill von 2008 wurde weder vom Senat noch vom Repräsentantenhaus eine Abschaffung der „fixed direct payments“ angeregt (Monke 2008). Präsident Obama schlug jedoch bereits in seinem ersten Haushaltsvorschlag - für das Haushaltsjahr 2010 - vor, die zu der Zeit geltende Farm Bill von 2008 dahingehend $\mathrm{zu}$ ändern, dass an Betriebe mit einem jährlichen Umsatz (,sales") von über 500.000 USD keine „fixed direct payments“ gezahlt werden. ${ }^{82}$ Dieses würde Einsparungen von etwa $10 \mathrm{Mrd}$. USD in zehn Jahren mit sich bringen (Monke 2009: 2). In seiner Ansprache an den Kongress formulierte Obama sein Anliegen wie folgt: „In this budget, we will ... end direct payments to large agribusinesses that don't need them“ (ibid.: 1). Bereits als designierter Präsident kritisierte er die Tatsache, dass „from 2003 to 2006, millionaire farmers received $\$ 49$ million in crop subsidies [...] it is a prime example of the kind of waste that I intend to end as president" (ibid.). Obama schlug gleichzeitig vor, auch die Ausgaben für die Ernteversicherung zu reduzieren - um 5,2 Mrd. USD in zehn Jahren (ibid.: 3). Diese beiden Vorschläge wurden vom Kongress aber nicht weiterver-

82 Von der Kappung wären $11 \%$ der Betriebe, die „government payments“ erhalten, betroffen (Monke 2009: 2). 
folgt. ${ }^{83} \mathrm{Im}$ Haushaltsvorschlag für das Haushaltsjahr 2013 ging die ObamaRegierung einen Schritt weiter und regte eine vollständige Abschaffung der „fixed direct payments" mit der Begründung an, die landwirtschaftlichen Einkommen würden hoch sein und weiter steigen (OMB 2012: 29). Es wurde unterstrichen, dass die fünf Jahre mit den höchsten Einkommen der letzten dreißig Jahre allesamt nach 2003 lagen (ibid.). Weil die Höhe der „fixed direct payments“ nicht mit den Preisen, Erträgen oder Einkommen variiert, „taxpayers continue to foot the bill for these payments to farmers even when farmers are earning record incomes" (ibid.). „Providing income support payments to farmers that are experiencing near record incomes is not prudent", so begründete die Regierung ihren Vorschlag. Sie stellte abschließend fest, dass „,[i]n a period of severe fiscal restraint, these payments are no longer defensible" (ibid.). Auch die Ausgaben für die Ernteversicherung wollte sie kürzen - um 7,6 Mrd. USD in zehn Jahren (ibid.: 33). Ein solcher Vorschlag zur Abschaffung bzw. Begrenzung von „fixed direct payments“ war zu der Zeit keine Ausnahme. Nur einer von den zehn von Zulauf und Orden (2014: 16) untersuchten Farm-Bill-Vorschlägen von 2012 plädierte für ihre Beibehaltung nach 2013 (er kam vom Republikaner R. Neugebauer). Weder die Verbände, in denen sich Erzeuger einzelner Erzeugnisse zusammenschließen („Corn Growers Association“, „American Soybean Association“ und „National Cotton Council“), noch die allgemeinen landwirtschaftlichen Verbände „Farm Bureau“ und „National Farmers Union“ setzten sich für deren Fortführung ein (ibid.: 49f). Hingegen sprachen sich alle zehn Vorschläge für die Fortsetzung der Ernteversicherung aus (ibid.: 16). Es stellt sich die Frage, was diesen breiten Konsens herbeiführte.

Bereits im Jahr 2010 wiesen Orden et al. (2010: 186) auf eine ,acrimony over the direct payments in the United States" hin. Das würde sie unattraktiv für die auf die Minimierung der Kontroverse über die Agrarstützung bedachten landwirtschaftlichen Verbände und ihre Vertreter machen. Blandford et al. (2011: 2) betont „political vulnerability“ der „fixed direct payments“, die unumstritten sei. In diesem Sinne wird auch auf ,sensitivity within the agricultural industry and among the public to potentially unnecessary budget outlays" hingewiesen, die zu Fragen bezüglich der Notwendigkeit und der Tragfähigkeit von „,fixed direct payments“ führte (GAO 2012: 1). Keeney (2013: 3) bemerkt, dass ,fixed direct payments“ in den politischen Statements sogar verhöhnt wurden. All diese Aussagen deuten deutlich auf schwindende Zustimmung für das Instrument der „fixed direct pay-

\footnotetext{
${ }^{83}$ Die US-Verfassung verleiht dem Kongress „,the power of purse“. Der Haushaltsentwurf, der dem Kongress spätestens zum ersten Montag im Februar für das kommende Haushaltsjahr ab 1. Oktober vorliegen muss, kommt zwar vom Präsidenten. Da aber der jährliche Haushaltsplan kein Gesetz, sondern eine gemeinsame Entschließung beider Kammern des Kongresses ist, bedarf er keiner Unterschrift des Präsidenten. Dieses bedeutet auch, dass der Präsident gegen den Haushaltsplan kein Veto einlegen kann. Die Haushaltsentschließung soll bis zum 15. April fertiggestellt werden, diese Frist wird aber regelmäßig überschritten (Haas 2007).
} 
ments" in der Politik und der breiten Öffentlichkeit hin, dessen sich die Agrarlobby bewusst war.

Die Faktoren, die zu diesem Akzeptanzverlust beitrugen, dürften sich mit den Ursachen für die Abschaffung der Direktzahlungen decken. Dabei ragen der Aspekt der hohen landwirtschaftlichen Einkommen und die ihnen zugrunde liegenden Marktpreise heraus. Als die im Januar 2014 beschlossene Farm Bill diskutiert wurde, befand sich die US-Wirtschaft infolge der Finanzkrise, die als Immobilienkrise im Jahr 2007 begann, in einer schwierigen Lage. Die Rezession 2008/09 führte zu einer starken Senkung der Staatseinnahmen; gleichzeitig stiegen die Staatsausgaben, z. B. für die Ernährungsprogramme, und damit das Haushaltsdefizit (Novoa et al. 2014: o.S.). Im Gegensatz dazu erlebte die Landwirtschaft in den sechs Jahren vor der Verabschiedung der Farm Bill von 2014 aufgrund hoher Marktpreise für landwirtschaftliche Erzeugnisse und damit korrespondierenden hohen landwirtschaftlichen Einkommen einen Boom. Das Nettoeinkommen in der Landwirtschaft betrug im Zeitraum von 2011 bis 2013 im Durchschnitt 121 Mrd. USD im Vergleich zu 74 Mrd. USD in den Jahren von 2008 bis 2010 (ibid.).

Die hohen Einkommen in der Landwirtschaft, die mit der allgemeinen Wirtschaftsentwicklung kontrastierten, stellten eine wichtige Ursache für die Abschaffung der „fixed direct payments“ dar. Wie Keeney (2013: 2) es formuliert:

When agricultural incomes were considerably lower in the 20022008 period, the idea of providing $\$ 5$ billion worth of agricultural income support via fixed annual payments had enough political support to be maintained. Since 2008, while most of the U.S. economy has been strongly affected by recession and a slow recovery, agricultural incomes have soared, setting historical highs in recent years. The prospect of continuing to make direct payments during this period of prosperity no longer has any political champions.

Orden et al. (2010: 177) bemerken, dass „,[w]ith increasing farm commodity prices in 2008, direct payments came under intense scrutiny in the domestic policy debate“. Zulauf und Orden (2014: 16) weisen auf „the difficulty of defending the fairness of large government payments when farm incomes were at or near record high levels, especially when farmers were also receiving high crop insurance net indemnity payments" hin. Vor dem Hintergrund der hohen landwirtschaftlichen Einkommen sei kaum zu rechtfertigen, Zahlungen aus dem öffentlichen Haushalt für einen sehr kleinen Teil der Bevölkerung, der zudem Rekordeinkommen einfährt, zu gewähren (Blandford et al. 2011: 2). Auch GAO (2012: 1) betont neben dem hohen Haushaltsdefizit die Bedeutung hoher Einkommen für die Farm Bill von 2014. Gleichzeitig bemängelte die Behörde die Verteilung der „fixed direct payments" unter den Betrieben: Weil diese flächenbezogen gewährt wurden, profitierten von ihnen vornehmlich größere Betriebe (ibid.: 16f). Indirekt geht Shields (2014a: 5) auf die Relevanz der hohen Einkommen mit der Bemerkung ein, dass 
„direct payments lost political support in recent years because recipients did not need to suffer an income loss in order to receive a payment". Auch Babcock (2011: 10) bemerkt: „[]t is not reasonable to transfer $\$ 5$ billion each year from taxpayers to farmers when farm income is at a record high, farm balance sheets have never been better, and Congress is actively cutting programs that provide public goods such as transportation, parks, disease prevention, and education." "There has never been a better time to reform farm programs", schlussfolgert er (ibid.: 11).

Die Abschaffung der angeschlagenen „direct payments“ erfolgte jedoch nicht ersatzlos. Zum einen, wie Bureau (2012: 10) bemerkt, wurde nur ein Teil der Agrarzahlungen abgeschafft: ,While Congress claims that its proposals will end ,direct payments', it is only the fixed, decoupled, direct payments that will be eliminated, not the many layers of direct payments that depend on market conditions. " Zum anderen fand ein Eintausch gegen die Stärkung der Ernteversicherung statt (Zulauf und Orden 2014: 16). Wie Orden und Zulauf (2015: 3) bemerken: „With high incomes, but facing yield, price and revenue variability, most farm groups endorsed wider calls to eliminate direct payments as unjustified when farm incomes were high. They argued that most of the savings should go into strengthening insurance and other downside risk safety net programs - the eventual outcome." Eine Reihe von Faktoren erleichterte den Landwirten diesen Schritt: die ähnliche Verteilung von Zahlungen aus der Ernteversicherung und von ,fixed direct payments" nach Bundesstaaten, die Tatsache, dass die Netto-Entschädigungszahlungen die „fixed direct payments“ in den Jahren unmittelbar vor der Verabschiedung der Farm Bill von 2014 übertrafen und schließlich die Prognosen, wonach erstere auch in Zukunft höher ausfallen würden als die „,fixed direct payments“ (Zulauf und Orden 2014: 31).

Im Gegensatz zu den „fixed direct payments“ scheint das Instrument der Ernteversicherung die Akzeptanz der Gesellschaft zu genießen. Aus einer Umfrage vom April 2016 geht hervor, dass $79 \%$ der Befragten die Subventionierung der Ernteversicherung, die ,pays claims only in the event of bad weather or low prices“" unterstützen; nur $17 \%$ der Befragten sprechen sich dagegen aus (Crop Insurance 2016: o.S.). Dieses weist darauf hin, dass die Gesellschaft kein Verständnis für die Unterstützung von Landwirten aus dem Staatshaushalt in Zeiten höherer Marktpreise aufbringt. Das agrarpolitische Instrumentarium wird vielmehr als ein Sicherheitsnetz für schwierige Zeiten gesehen: wenn die Preise abstürzen oder die Erträge niedrig ausfallen. Die festen Direktzahlungen passten nicht in dieses Konzept. Wie Keeney (2013: 3) es formuliert: „A payment that is identical when a farm profits or loses can hardly be classified as safety net policy." Die Tatsache, dass dieser Aspekt bei der Einführung der entkoppelten Zahlungen im Jahr 1996 keine Rolle spielte, liefert allerdings einen Hinweis darauf, dass nicht die Sympathie der Gesellschaft für die Ernteversicherung beim Milestone 2014 entscheidend war. 
Laut Zulauf und Orden (2014: 9) spiegeln die Änderungen im agrarpolitischen Instrumentarium von 2014 weg von „fixed direct payments“ hin zur Ernteversicherung ,changes in the economic condition of US farms and farm families" wider. Nur das Risikomanagement „still resonates as a potential basis for US farms receiving support from the public" (ibid.: 10). Die restlichen ursprünglichen Begründungen für die Agrarstützung - hohe volkswirtschaftliche Bedeutung der Landwirtschaft, Einkommensprobleme sowie Ernährungssicherung - erodierten. Denn die Bedeutung der Landwirtschaft als Wirtschaftszweig sinkt kontinuierlich, während das Durchschnittseinkommen landwirtschaftlicher Haushalte nun das Durchschnittseinkommen aller Haushalte übersteigt und die Ernährungssicherung nicht mehr im Vordergrund steht (ibid.).

In der Literatur wird auch der starke Haushaltsdruck als Treiber für die Reform von 2014 genannt. Das im Jahr 2010 gestartete Gesetzgebungsverfahren war durch eine Debatte über das Haushaltsdefizit zwischen dem republikanischen Repräsentantenhaus und dem von den Demokraten dominierten Senat und der Verwaltung stark belastet (Zulauf und Orden 2014: 16). Im Jahr 2009 erreichte das Haushaltsdefizit der USA rekordverdächtige 1,4 Billionen USD, was $10 \%$ des Bruttoinlandsproduktes entsprach (nach knapp 0,5 Billionen USD und 3\% im Vorjahr). Auch im Zeitraum von 2010 bis 2012 überstieg das Defizit die EineBillion-Dollar-Marke; im Jahr 2013 sank es auf knapp 0,7 Billionen USD (OMB 2016: Tabelle 1.1 und 1.2). In dieser Situation stellte das bevorstehende Agrargesetz eine attraktive Möglichkeit für Ausgabensenkungen zwecks Defizitreduktion dar (Smith 2014: 2). Laut Monke (2014: 1) wurde die Ausgestaltung der Farm Bill von 2014 insbesondere von drei Fragen beeinflusst: der Verfügbarkeit von Finanzmitteln für die Pflichtausgaben (inklusive der Agrarrohstoffprogramme), der künftigen Trendentwicklung bei den Preisen sowie der Sequestration. ${ }^{84}$ Novoa et al. (2014: o.S.) bemerken: „The concern over the deficit was one of the contentious issues surrounding the deliberation of the recent farm bill.“

Die im Vorfeld kursierenden Sparpläne bezifferten den Beitrag der neuen Farm Bill zur Defizitverringerung in den nächsten zehn Jahren auf 10 bis sogar 179 Mrd. USD (Monke 2014: 1). Allerdings herrschte Uneinigkeit, in welchen Bereichen vornehmlich gespart werden sollte. Die Republikaner, hier insbesondere die Abgeordneten aus der Tea Party, drängten auf Einsparungen bei den Ernährungsprogrammen, was auf Widerstand der Demokraten stieß. Viele Kongressmitglieder aus nichtruralen Wahlbezirken hingegen wollten eher die Ausgaben für die Agrarrohstoffprogramme kürzen, die sie für ,wasteful, poorly targeted and unnecessary" hielten (Smith 2014: 2). Letztendlich waren im Entwurf des Repräsentantenhauses von 2013 die Einsparungen bei den Ernährungsprogrammen (Titel IV „Nutrition“) auf 39 Mrd. USD und bei den Rohstoffprogrammen (Titel I

\footnotetext{
${ }^{84}$ Eine automatische, pauschale Kürzung der Ausgaben, um die festgelegten Haushaltsziele zu erreichen (Monke 2014).
} 
"Commodities“) auf 18,7 Mrd. USD, im Entwurf des Senats entsprechend auf knapp 4 Mrd. USD und 17,4 Mrd. USD für zehn Jahre festgelegt. Beide Entwürfe sahen die Abschaffung der „fixed direct payments" und somit auch damit verbundene Einsparungen von $40 \mathrm{Mrd}$. USD vor; die vorgeschlagene Abschaffung von „counter-cyclical payments“ und „ACRE payments“ sollte zusätzlich 6,2 Mrd. USD an Einsparungen bringen (Monke 2014: 16 und 31). Zum Zeitpunkt der Verabschiedung der Farm Bill im Januar 2014 wurden die Einsparungen in den nächsten zehn Jahren (Haushaltsjahre von 2014 bis 2023) auf 16,6 Mrd. USD beziffert. Da in der „baseline“ die Sequestration in Höhe von 6,4 Mrd. USD ${ }^{85}$ bereits berücksichtigt wurde, beliefen sich die gesamten Einsparungen auf $23 \mathrm{Mrd}$. USD (ibid.: 15). Die größten Kürzungen betrafen zum einen die Rohstoffprogramme (14,3 Mrd. USD) und zum anderen die Ernährungsprogramme (8 Mrd. USD) (ibid.: 16). Diese fielen aber niedriger aus als in den Vorschlägen der beiden Kongresskammern von 2013.

Die Einsparungen bei den Rohstoffprogrammen sind insbesondere auf die Abschaffung von „fixed direct payments“ zurückzuführen. Diesen Schritt erklärt Keeney (2013: 2) mit ihrer relativ hohen „baseline“ von etwa 5 Mrd. USD jährlich gegenüber der vor dem Hintergrund der höheren Marktpreise seit 2008 vergleichsweise geringen „baseline“ für „,counter-cyclical payments“ und „ACRE payments“. Dieses machte die „fixed direct payments" zum vorrangigen Ziel unter den Agrarrohstoffprogrammen im Hinblick auf den Defizitabbau. Auch Bureau (2012: 60) bemerkt: „Because in the CBO baseline the only farm programs that appear to generate high budget costs are the (fixed) direct payments and the conservation payments [...] attention is drawn to these programs." Das bestätigt Monke (2014: 7): „Fixed direct payments“ waren „the primary program with a mandatory funding baseline“, während die antizyklischen Zahlungen „did not have much baseline in May 2013 because high market prices for farm commodities had reduced payments“. Höher als die „baseline“ für die gesamten Agrarrohstoffprogramme unter Titel I lag die „baseline“ für die Ernteversicherungen (ibid.). Nichtsdestotrotz wurden diese nicht nur nicht gekürzt, sondern sogar aufgestockt.

Wie Bureau (2012: 60) bemerkt, war die relativ hohe „baseline“ für „fixed direct payments“",somewhat artificial since it relie[d] on the bet that current high prices will be maintained over ten years". Zwei Jahre nach der Verabschiedung der Farm Bill untersuchte Smith (2016) die im Gesetzgebungsverfahren gemachten Aussagen der Agrarpolitiker über die möglichen Einsparungen auf Plausibilität. Dazu nimmt er die Prognosen der CBO vom Januar 2014 über die Entwicklung der Marktpreise für Weizen, Mais und Erdnüsse im Zeitraum von 2014 bis 2018 unter die Lupe, die die Grundlage für die Ausgabenschätzung der neuen Farm Bill

\footnotetext{
${ }^{85}$ Es wurden 4,1 Mrd. USD bei den Rohstoffprogrammen (Titel I) und 2,1 Mrd. USD bei den Umweltprogrammen (Titel II) bis 2023 gekürzt (Monke 2014: 13).
} 
bildeten. Diese vergleicht er zum einen mit den entsprechenden Preisprognosen des USDA und des „Food and Agricultural Policy Research Institute“ (FAPRI), die ebenfalls Anfang 2014 veröffentlicht wurden und zum anderen mit den CBOPrognosen vom Januar 2016. Nur in einem einzigen Fall (Weizenpreise im Jahr 2014 in der Prognose von FAPRI) prognostizierte die CBO einen niedrigeren Marktpreis. Zudem waren alle Preise bis auf einen im CBO-Ausblick von 2016 niedriger angesetzt als im Ausblick von 2014 (nur der tatsächliche, im Ausblick 2016 angeführte durchschnittliche nationale Weizenpreis von 2014 lag 3\% höher als im Ausblick vom Januar 2014).

Darauf basierend bemerkt Smith (2016: 2), dass die Prognosen von CBO über die Entwicklung der Marktpreise bereits zur Zeit der Vorbereitung der Farm Bill von 2014 nicht glaubhaft waren. Er führt weiter aus, dass bis zu ihrer Verabschiedung ,there was never any real likelihood that farm subsidy spending would be lower under the 2014 farm bill provisions than under the previous farm policy mix" (ibid.). Die Behauptungen zu Einsparungen waren trügerisch, weil sie auf der CBO-,,baseline“ basierten, die „presumed that the historically high crop prices that many farmers had enjoyed since 2007 would be sustained for major program crops such as corn and wheat over the entire four-year period covered by the 2014 farm bill and well beyond“ (ibid.). Smith (2016: 7) sieht Hinweise darauf, dass „the $\mathrm{CBO}$ was required to use a price baseline in developing its estimates of PLC and ARC budget outlays that included significantly higher price forecasts for some major crops than other groups were predicting". Die treibenden Kräfte hierfür waren die Agrarausschüsse des Kongresses: „A widespread practice by congressional committees interested in obtaining approval for their legislative initiatives has been to utilize the rules of the game that cause the $\mathrm{CBO}$ to use assumptions that minimize scoring estimates" (ibid.: 2). Vor diesem Hintergrund ist für ihn nicht überraschend, dass sich die tatsächlichen Staatsausgaben für die neuen Zahlungsprogramme nicht nur als viel höher als Anfang 2014 vom CBO projiziert, sondern auch höher als die Aufwendungen für die ersetzten „fixed direct payments“ erweisen (ibid.: 6). Es stellte sich heraus, dass „trading out of direct payments into PLC and ARC has caused farm-subsidy spending to go up by about $\$ 1.5$ billion a year (a 30 percent increase), not down by about $\$ 1$ billion, as the House agricultural committee leadership continues to claim“" (ibid.: 10). ${ }^{86}$ Bereits während des Gesetzgebungsverfahrens wurde davor gewarnt, dass die unter der Farm Bill von 2014 angestrebten Einsparungen nicht nur nicht erreicht werden, sondern dass die Agrarausgaben noch höher als zuvor ausfallen könnten. Beispielsweise bemerkt Keeney (2013: 2), dass „,new farm subsidy spending could actually increase dramatically over the 10-year period rather than decline“", weil die neuen Programme von den Preisen und Erträgen abhängen. Diese Sicht teilt Smith (2013: 13), nach

\footnotetext{
86 Die CBO-,,baseline“ vom Januar 2017 beziffert die Ausgaben für den Titel I „Commodities“ im Zeitraum von 2014 bis 2018 auf 37,4 Mrd. USD, was einer Steigerung von 59\% gegenüber der „baseline“ vom Januar 2014 entspricht (Johnson und Monke 2017: 5).
} 
dessen Schätzungen die Ausgaben unter Titel I „Commodities“ 10 bis 20 Mrd. USD jährlich betragen könnten, wenn die Marktpreise von den rekordnahen Levels von 2013 in die Richtung ihres langfristigen Trends sinken sollten. Dieses wäre zwei- bis viermal so viel, wie für die ,fixed direct payments“ floss.

Die obigen Ausführungen legen die Schlussfolgerung nahe, dass die meisten Akteure des Reformprozesses, Agrarlobby und Politik inklusive, davon ausgehen konnten, dass die Marktpreise künftig sinken werden und die neu installierten Programme dafür sorgen würden, dass die Zahlungen deutlich höher ausfallen als dieses bei der Beibehaltung der „,fixed direct payments“ der Fall gewesen wäre. Diese Erwartung dürfte die Abschaffung letzterer begünstigt haben. Nach dem Motto „history repeats itself“ sieht O. Doering von der Purdue-Universität darin Parallelen mit dem Reformprozess von 1996. Er bemerkte bereits 2011, dass „[w]e are seeing a replay of the politics and events of the 1996 Farm Bill“, indem „, farm and commodity groups will work to capture the budget baseline, which is now becoming a standard practice“ (Farm Futures 2011: o.S.). Dabei spielten die Haushaltsregeln die entscheidende Rolle. Es ist in diesem Zusammenhang darauf hinzuweisen, dass ein Teil des durch die Abschaffung von „fixed direct payments“ eingesparten Betrages für insgesamt sieben andere Farm-Bill-Titel bereitgestellt wurde, insbesondere für die Ernteversicherungen, die mit zusätzlichen 5,7 Mrd. USD gegenüber der „baseline“ ausgestattet wurden, gefolgt von der Forschung mit 1,1 Mrd. USD (Monke 2014: 16). Außerdem wurden für die neu eingeführten „price loss coverage payments“ und ,agriculture risk coverage payments“ bis 2023 27,2 Mrd. USD veranschlagt (ibid.: 17). Insgesamt wurden $80 \%$ des durch die Abschaffung von „fixed direct payments“ eingesparten Betrages wieder eingesetzt (Zulauf und Orden 2014: 19). Vor diesem Hintergrund darf daran gezweifelt werden, dass der Haushaltsdruck bei der Abschaffung der „fixed direct payments“ im Vordergrund stand.

Zur Rolle der W'TO bei der Vorbereitung der Farm Bill von 2014 bemerkt Carter (2014: 1), dass diese Farm Bill nicht nur die Agrarstützung ausweitete, sondern sie auch stärker an die aktuelle Erzeugung und die Marktbedingungen koppelte. „On both counts (larger and more distortive subsidies), the 2014 Farm Bill fails the test of being consistent with WTO objectives", lautet deshalb sein Fazit (ibid.). Denn alle neu eingeführten Subventionsprogramme sind „unambiguously amber box programs“ (Smith 2014: 1). Dank der „de-minimis“-Regel halten es Orden und Zulauf (2015) jedoch für eher unwahrscheinlich, dass die USA infolge der Farm Bill von 2014 ihr aktuelles aggregiertes Stützungsmaß überschreiten werden. Gleichzeitig vermuten sie, dass „the U.S. would not have enacted the 2014 farm bill as it is, had a Doha agreement been reached in 2008 and phased in over six years“ (ibid.: 11). Smith (2014: 1) stimmt mit ihnen überein: „While these new programs [PLC and ARC] are unlikely to cause problems for the US in meeting its current WTO Aggregate Measure of Support (AMS) commitments, they may make it more difficult for the US to agree to future reductions in allowable 
caps on AMS expenditures and related de minimis AMS exclusion provisions in a new W'TO agreement." Die Analyse von Glauber und Westhoff (2015) hingegen deutet darauf hin, dass unter gewissen Marktbedingungen die Ausgaben unter der Farm Bill von 2014 die geltenden WTO-Höchstgrenzen für die handelsverzerrende Stützung überschreiten könnten. Sie stellen fest, dass trotz dieser ,high exposure to WTO limits under the 2014 farm bill“ diesem Thema in der Farm-BillDebatte kaum Aufmerksamkeit geschenkt wurde, was sie überraschend finden (ibid.: 8). Dieses bestätigt Carter (2014: 4) mit der Bemerkung, dass ,the new farm bill indicates that international trade commitments have little or no influence over U.S. farm policy choices“. Wie Zulauf und Orden (2014: 42) bemerken, ,, the elimination of direct payments in the 2014 farm bill runs counter to the implicit WTO design of increased reliance on green box measures“, was im Kontrast zum Festhalten der EU an diesem Instrument stehe.

Es besteht allerdings in der Literatur Übereinstimmung darin, dass das neue, von der Farm Bill von 2014 installierte Stützungsprogramm für HochlandBaumwolle hier eine Ausnahme bildet. Für dieses Erzeugnis wurden keine „,price loss coverage payments“ und keine ,agricultural risk coverage payments“, sondern ein separates Programm in Form einer Erlösversicherung - der sogenannte „Stacked Income Protection Plan“ - eingeführt. „The redesign of cotton support compared to other program crops was driven in large part by the WTO BrazilU.S. cotton case“, stellen Orden und Zulauf (2015: 7) fest. Zulauf und Orden (2016: 4) ergänzen, dass das Urteil des WTO-Panels zwar „proximate cause“ der Neuerungen im Baumwollbereich war. „[B] the underlying cause was the program's largesse", denn auf Hochland-Baumwolle entfielen in den Haushaltsjahren 2005 bis 2012 25\% aller Ausgaben für die Agrarrohstoffprogramme. Vor diesem Hintergrund resümieren sie, dass ,upland cotton policy likely would have been revised in some manner even without the WTO case“ (ibid.). Auch lässt es sich darüber streiten, ob die WTO als Ursache für die Abschaffung der „fixed direct payments“ für Baumwolle herangezogen werden kann. Zum einen zielte das Urteil des WTO-Panels nicht auf dieses Instrument, sondern auf die antizyklisch angelegten Maßnahmen ab (s. Box 8). Brasilien lehnte jegliche Verwendung eines festen Referenzpreises für Baumwolle ab (Orden und Zulauf 2015: 7). Des Weiteren wurden die „fixed direct payments“ als sogenannte „transition payments“ gerade im Baumwollbereich für 2014 und ggf. auch 2015 weiter vorgesehen, um die aus den Änderungen der Stützungsregelung entstehenden Verluste zu kompensieren (Schnepf 2014a). Die geringe Relevanz der WTO für die ausgewählten US-Milestones bedeutet nicht, dass dieser Faktor im Agrarreformprozess in den USA durchweg unbedeutend war. Beispielsweise schildert Box 14 den großen Einfluss der WTO auf den Vorschlag des USDA für ,green payments“ von 2007, der an die späteren Ideen der EU-Kommission zum Greening erinnert. 
Box 14: Bedeutung der WTO bei der Vorbereitung der Farm Bill von 2008

Im Januar 2007 stellte das USDA seinen Vorschlag für die neue Farm Bill vor, mit dem die Agrarrohstoffprogramme so reformiert werden sollten, dass sie „more market-oriented, more predictable, less market distorting and better able to withstand challenge in the World Trade Organization" sind (CRS 2007: 1). Gleichzeitig sollten die angeregten Änderungen Einsparungen in Höhe von insgesamt 4,5 Mrd. USD in zehn Jahren bringen. Mit Blick auf die WTO-Verhandlungen sah der USDA-Vorschlag u. a. eine Stärkung des Instruments der „fixed direct payments“ vor: Diese sollten fortgesetzt und erhöht werden. Die von der WTO als nicht mit den Green-Box-Vorschriften vereinbar angesehenen Beschränkungen für den Anbau von Obst und Gemüse auf der Basisanbaufläche sollten abgeschafft und die „loan rates“ (Amber Box) gekürzt werden. Eine Neuerung stellte die sogenannte ,conservation enhanced payment“ im Titel I („Commodities“) dar. Sie sollte den Betrieben mit der Basisanbaufläche gewährt werden, wenn diese bestimmte Anforderungen in Bezug auf den Umweltschutz erfüllen. Dafür müssten sie allerdings auf die Leistungen unter den antizyklischen Programmen („,counter-cyclical payments“ und „marketing loan benefits“, beide Amber Box) verzichten. Die „conservation enhanced payment" wurde als eine Zusatzzahlung zu den regulären „fixed direct payments“ konzipiert und sollte sich auf $10 \%$ des individuellen ,fixed direct payments“-Betrags eines Landwirts belaufen (CRS 2007). Laut CRS (2007: 10) kann dieses als ein Pilotprogramm zur Umwandlung der Amber-Box-Maßnahmen in die Green-BoxMaßnahmen ansehen werden.

Für den Vorschlag solcher WTO-konformen ,green payments“ als Vergütung für die von Landwirten erbrachten Umweltleistungen seien laut Batie und Schweikhardt (2007: 159f) drei Faktoren verantwortlich gewesen. Zum einen nennen sie die Unzufriedenheit mit den Verteilungsfolgen der bisherigen Programme, von denen insbesondere große Betriebe profitieren würden. Dieses Thema wurde von Medien aufgegriffen und diskutiert (z. B. Artikelserie der Washington Post unter dem Titel „Harvesting Cash“). Zum zweiten stieg mit wachsendem Wohlstand die Nachfrage nach Umweltgütern auch in der Lebensmittelproduktion. Schließlich waren die USA einem internationalen Druck ausgesetzt, ihre Agrarpolitik zur reformieren. Dieser war vornehmlich auf das im Jahr 2004 verlorene WTO-Panel über Baumwolle und auf die Doha-Runde, von der erwartet wurde, dass sie einen höheren Grad der Entkopplung erforderlich macht, zurückzuführen. 
Von 2004 bis 2006 sah es tatsächlich danach aus, dass die Agrarrohstoffprogramme infolge des Drucks seitens der WTO reformiert werden könnten. Die WTO-Konformität der vorgeschlagenen ,green payments“ wurde vor dem Hintergrund der laufenden Doha-Runde zum wichtigen Argument in der Reformdebatte. Nach der Suspendierung der Verhandlungen im Sommer 2006 gewannen allerdings andere Faktoren die Oberhand. Insbesondere machten die steigenden Marktpreise die laufenden antizyklischen Programme günstiger; darüber hinaus konnten dank ihnen die zu jener Zeit geltenden W'TO-Limits eingehalten werden. Beides bremste die Bereitschaft zu Reformen (Lehrer und Becker 2010). Die Regelung zu „conservation enhanced payments“ wurde letztlich nicht Teil der Farm Bill von 2008 (Johnson 2008).

Auch im Falle der Farm Bill von 2014 gelang es - trotz anfänglicher Schwierigkeiten - eine breite politische Koalition zu bilden, um eine Mehrheit im Kongress für die Fortsetzung einer hohen Stützung zu erreichen. Diese bestand aus Repräsentanten urbaner, ökologischer und landwirtschaftlicher Interessen. Den Forderungen der ersten beiden Lager wurde u. a. durch die Kürzung der Ausgaben für die Ernährungsprogramme um lediglich $1 \%$ in zehn Jahren und die Ausweitung von Cross-Compliance auf die Ernteversicherung Genüge getan. Darüber hinaus wurden die Ausgaben der Farm Bill für Forstwirtschaft, Handel, ländliche Entwicklung, Gartenbau, Energie und Forschung um 39\% erhöht, um eine noch breitere Unterstützung für die klassischen Agrarprogramme zu sichern (Orden und Zulauf 2015: 10).

Von den Reformursachen, die unter den „problem stream“ fallen, waren die hohen Marktpreise und landwirtschaftlichen Einkommen zum Zeitpunkt der Reform für den Milestone relevant. Die Erwartung sinkender Marktpreise kommt als Ursache für die Abschaffung der Direktzahlungen zusätzlich hinzu. Wie im Jahr 1996 ermöglichten erst die Haushaltsregeln, die dem „politics stream“ zuzuordnen sind, die „baseline capture“. Auch das Eingehen einer strategischen Koalition mit nichtlandwirtschaftlichen Interessen spielte dabei eine Rolle (vgl. Tabelle 26). 
Tabelle 26: Der US-Reform von 2014 als Ganzes und dem Milestone zugrunde liegende Faktoren

\begin{tabular}{c|c} 
Reform 2014 & Milestone 2014 \\
\hline $\begin{array}{c}\text { Hohe Marktpreise und landwirtschaftliche } \\
\text { Einkommen }\end{array}$ & $\begin{array}{c}\text { Hohe Marktpreise und } \\
\text { landwirtschaftliche Einkommen }\end{array}$ \\
Haushaltsdruck & Erwartung sinkender Marktpreise \\
BTO/Streitbeilegungsverfahren (nur bei \\
Baumwolle) \\
Koalition mit anderen Interessen \\
\hline
\end{tabular}

\subsection{EU-Milestone 2013}

Die im vorliegenden Abschnitt interessierende Frage lautet: Warum wurden die (entkoppelten) Direktzahlungen in der neuesten Reform der GAP nicht abgeschafft? Gesucht werden somit Faktoren, die zur Beibehaltung des Status quo beitrugen. Die Frage, der hier nachgegangen wird, unterscheidet sich somit von den Fragen der vorausgegangenen Abschnitte des vorliegenden Kapitels, verlangt aber keine Veränderung der gewählten Vorgehensweise. Zunächst werden die in der Literatur genannten Reformfaktoren zusammengefasst. Anschließend werden sie auf ihren Einfluss auf die Beibehaltung der Direktzahlungen geprüft.

Swinnen (2015c: 450) untersucht ,[p]ressures, institutions and agents“, die zum bekannten Ergebnis der Reformdebatte über die Ausgestaltung der EU-Agrarpolitik nach 2013 führten. Als einen der Haupttreiber der neuesten GAP-Reform sieht er Haushaltszwänge an (ibid.). Sie seien aus zwei Gründen von großer Bedeutung gewesen. Zum einen wurde auf Druck der britischen Regierung eine umfassende Überprüfung aller Aspekte der Haushaltsausgaben, einschließlich der Agrarpolitik, angeordnet. Zum anderen bestand aufgrund der schweren Finanz- und Wirtschaftskrise, mit der die EU-Mitgliedstaaten zu kämpfen hatten, beträchtlicher Druck, die Agrarausgaben zu kürzen und sie ggf. für andere Bereiche zur Verfügung zu stellen. Weil die GAP-Verhandlungen parallel zu den Verhandlungen über den Finanzrahmen für den Zeitraum von 2014 bis 2020 liefen, bestand „an urgency to adapt the CAP“ (Matthews 2015: 169). Die MFR-Debatte wurde von „wide-ranging fiscal austerity in Europe“ in Kombination mit „the clear articulation of an alternative set of spending priorities" geprägt (ibid.: 170). Vor diesem Hintergrund argumentierte die Kommission bereits zu Beginn des Reformprozesses, dass die Beibehaltung des GAP-Budgets im bisherigen Umfang nur möglich sei, wenn eine ehrgeizige Greening-Strategie umgesetzt würde (ibid.: 178). 
Wie Swinnen (2015c: 452) bemerkt, gehörten sowohl hohe Agrarpreise als auch ihre Volatilität zu den wichtigen Treibern der Reform von 2013. Die Jahre 2007 und 2008 sowie 2010 und 2011 (also zur Zeit der Reformdebatte über die GAP nach 2013) verzeichneten jeweils einen Anstieg der Marktpreise für landwirtschaftliche Erzeugnisse und Lebensmittel. In diesem Zusammenhang unterstreicht Swinnen (2015c: 452) die Tatsache, dass unter diesen Umständen „,it was less obvious for farmers to argue that they needed government support". Auf der anderen Seite allerdings stützte der Preisanstieg die Argumentation, die landwirtschaftliche Produktion nicht mit zusätzlichen Auflagen und Vorschriften einzuschränken, um die Nahrungsmittelversorgung nicht zu verschlechtern. Dieses Argument „had a decisive impact on the final decision“, wie Swinnen (2015b: 25) anmerkt.

Die Auswirkungen der Preisschwankungen im Zeitraum von 2007 bis 2011 auf die EU-Politiken, darunter auch auf die GAP, werden von Swinnen et al. (2013) untersucht. Sie konkludieren, dass die Preissprünge die Debatte über die Ausgestaltung der GAP nach 2013 beeinflussten, allerdings waren ihre Auswirkungen auf das Reformergebnis begrenzt, „mostly affecting arguments, much less the main policies“ (ibid.: 18). Dass keine größeren staatlichen Eingriffe in die Märkte als Antwort auf die Preisvolatilität unternommen wurden, führen Swinnen et al. (2013: 19) u. a. auf die Tatsache zurück, dass die realen Einkommen der Landwirte im Zeitraum von 2005 bis $2011 \mathrm{im}$ Durchschnitt anstiegen.

Auch die Erweiterungen der EU von 2004 und 2007 waren laut Swinnen (2015c: 458) für die Reform von 2013 von Bedeutung. In erster Linie wirkten sie sich auf den Prozess der Entscheidungsfindung aus, indem sie die Zahl der Entscheidungsträger im Rat und die Heterogenität der EU erhöhten, neue Präferenzen mit sich brachten sowie die politische Gewichtung der Mitgliedstaaten untereinander insgesamt veränderten. Der Entscheidungsprozess wurde dadurch schwerfälliger.

Darüber hinaus beeinflussten wachsende gesellschaftliche Ansprüche an die Landwirtschaft, die über die Produktqualität hinausgingen und den Produktionsprozess betrafen, die Reform entscheidend (Swinnen 2015c). Knops und Swinnen (2014: 13) nennen „new concerns from the general public“ als „crucial [...] in determining the future design of the CAP“. Das 2013 beschlossene Greening der Direktzahlungen sei auf diese gesellschaftlichen Erwartungen zurückzuführen. Darin sehen Daugbjerg und Swinbank (2015: 12) ,a message to the public that the CAP was there to support the production of public goods, such as environmental protection and the maintenance of the rural economy and landscapes" und somit auch eine Maßnahme „to legitimise the continuation of farm support“.

In der Literatur besteht Übereinstimmung darin, dass bei der GAP-Reform von 2013 - im Gegensatz zu den vergangenen Reformen - die WTO kaum eine Rolle spielte. Wie Swinbank (2015: 210) es formuliert: „In 2013 that force for change was muted." Den Grund dafür sieht er darin, dass sich die Verhandlungen in der Doha-Runde schwierig gestalteten und eine Einigung nicht in Sicht war. 
Zudem boten die bestehenden WTO-Verpflichtungen der EU dank früherer Agrarreformen genügend Spielraum für die Ausgestaltung von inländischen Stützungsmaßnahmen. Aber auch ein neues multilaterales Abkommen würde der EU höchstwahrscheinlich keine Probleme bereiten, vorausgesetzt jedoch, dass die Notifizierung der Direktzahlungen als Green-Box-Maßnahme nicht infrage gestellt würde. Allerdings ,[[w] hy worry about something that might happen in the distant future, following a Doha outcome, particularly when the US had demonstrated that a major power can put off abiding by a DSB [Dispute Settlement Body] ruling for a decade or so? ${ }^{\text {‘87 }}$ (ibid.: 207).

Vor dem Hintergrund, dass ,international trade negotiations played a marginal role in the 2013 CAP reform, compared to previous reform rounds where WTO agreements could be seen as the main driver of reform" erwähnen Knops und Swinnen (2014: 20) in ihrer Liste der Faktoren, die für die Reform von 2013 von Relevanz waren, die WTO nicht. Daugbjerg und Swinbank (2015: 3) bemerken angesichts der zum Stillstand gekommenen Doha-Runde, dass die WTO-Beschränkungen „were no longer of pressing concern“. Hofreither (2012: 2) attestiert der EU-Politik Desinteresse an der WTO-Frage. Insbesondere seien mögliche Probleme hinsichtlich der Konformität der Reformelemente mit den geltenden und möglichen künftigen WTO-Verpflichtungen für sie kein Thema gewesen. Er findet dieses überraschend, zum einen weil die EU das geltende WTO-Regelwerk maßgeblich mitgestaltete und zum anderen weil daraus in der Vergangenheit deutliche Reformimpulse entstanden. Laut Daugbjerg und Swinbank (2015: 12) spiegelt bereits die erste Mitteilung der Kommission zur Reform von 2013 einen verringerten Einfluss der WTO auf die Ausgestaltung der GAP wider, denn die Organisation wurde lediglich zwei Mal erwähnt. Für Swinnen (2015c: 453) stellen die internationalen Handelsabkommen einen eher im Hintergrund agierenden Reformfaktor dar, indem sie eine insbesondere vom Europäischen Parlament befürwortete Rückkehr zur Marktregulierung in der EU-Agrarpolitik verhinderten.

Die neueste GAP-Reform wird auch im Kontext neuer Ideen im Sinne eines Paradigmenwechsels interpretiert. Für Erjavec et al. (2015) war dieses - neben Haushaltszwängen - der wichtigste Treiber der Reform von 2013. Mit dem Umweltparadigma versuchte die Kommission, die Akzeptanz der Gesellschaft für die kostenintensive Agrarpolitik zu verbessern (ibid.: 237). Letztendlich aber stellte der Paradigmenwechsel „,an (half) empty shell“ dar (ibid.: 236). Als Faktor, der die $\mathrm{EU}$ an der Umstellung hin zu mehr zielgerichteten Umweltzahlungen hinderte, identifizieren Erjavec et al. (2015) die institutionellen Veränderungen, insbesondere die mit dem Vertrag von Lissabon erworbenen Befugnisse der Mitentscheidung des Europäischen Parlaments.

${ }^{87}$ Gemeint ist hier das WTO-Panel zu den Agrarsubventionen der USA im Baumwollbereich von 2004 (vgl. Box 8). 
Die Faktoren, die die Reform der EU-Agrarpolitik von 2013 beeinflussten, sind vielfältig. Es stellt sich die Frage, warum sie im Ergebnis lediglich relativ geringe Anpassungen der bisherigen Politik produzierten, sodass mancher Forscher das Wort Reform meidet und stattdessen über „repackaging“ bzw. „recalibration“ spricht (Swinnen 2015b: 5).

Wie Hofreither (2012: 1) bemerkt, war die Debatte um die GAP für den Zeitraum nach 2013 „vom Bemühen um die Erhaltung des Status Quo geprägt“. Bereits der Reformvorschlag der Kommission war „, not very ambitious to begin with“, wie Swinnen (2015b: 7) bemerkt. Tangermann (2011: 32) bewertet die Mitteilung der Kommission vom November 2010 als „focused on maintaining direct payments as the backbone of the CAP and [failing] to make the next step forward in the process of strategic CAP reform ". Tatsächlich stellen Daugbjerg und Swinbank (2015: 14) fest, dass trotz neuer Maßnahmen wie dem Greening die GAP nach 2013 ,strongly resembles the pre-2013 CAP, with its dominant focus on farm interests“. Oder, wie Swinnen (2015b: 25) es formuliert, „the overall assessment is closer to a status-quo evaluation than a significant reform". Allerdings wird dieses nicht von allen kritisch bewertet. Insbesondere das weitere Festhalten an der Entkopplung der Direktzahlungen wird als Erfolg angesehen (ibid.). Swinbank (2015: 210) zeigt sich überrascht, dass die EU ihre agrarpolitischen Entscheidungen der letzten zwei Dekaden nicht rückgängig machte in Anbetracht dessen, dass „a Doha agreement has not yet locked in past CAP reforms“. Bureau und Mahé (2015: 129) stellen fest, dass die Kommission den kursierenden „bad ideas" insbesondere hinsichtlich der Marktverwaltung und der Preisstützung standhalten konnte.

Auf der anderen Seite zeigen sich zahlreiche enttäuschte Stimmen, dass die EU einen entschiedeneren Schritt Richtung „targeting“ versäumte (s. insbesondere Tangermann 2014). Für Ökonomen würde eine stärkere Ausrichtung der Zahlungen auf spezifische, messbare Ziele den nächsten logischen Schritt auf dem Agrarreformweg der EU bilden „from distortive interventions in the 1970s and 1980s to less distortive payments in the 1990s (after MacSharry) to decoupled payments in the 2000s (after Fischler) to (more) targeted payments in the 2010s" (Swinnen 2015c: 452). Im Vorfeld der Reform von 2013 forderten sie ein besseres „targeting" der Agrarsubventionen, indem diese enger an die Bereitstellung der öffentlichen Güter geknüpft und stärker auf ökologische Ziele ausgerichtet werden (Declaration by Agricultural Economists 2010). Wie Tangermann (2011: 32) es zusammenfasst: „The next step now called for is to move from decoupling to targeting." Letztendlich aber war das Ergebnis der Reform diesbezüglich enttäuschend; entsprechend wurde es von Erjavec et al. (2015: 238) nicht als ,greening“, sondern als ,greenwash" bezeichnet. Vor diesem Hintergrund betrachten Bureau und Mahé (2015: 128) die neueste Reform als ,a lost opportunity for making the CAP more targeted on public goods procurement, more equitable, and less permissive to excessive rents inducing leakages, capital deepening and addiction to subsidies". 
Josling und Tangermann (2015: 158) sehen im Reformergebnis sogar „a break in the trend towards CAP reform that began in 1992".

In der Literatur wurden die Ursachen für die weitreichende Beibehaltung des Status quo in der letzten GAP-Reform bereits beleuchtet. Es werden die institutionellen Veränderungen, insbesondere die durch den Vertrag von Lissabon vorgesehene Ausweitung des Mitentscheidungsverfahrens auf die Agrarpolitik mit dem Europäischen Parlament als vollberechtigtem Akteur sowie die Beteiligung von nun 27 Mitgliedstaaten, genannt (Knops und Swinnen 2014). Es wird auf „the rather status quo influence" des Parlaments verwiesen (Knops und Garrone 2015: 436). Auch sein Beharren darauf, über die Inhalte der GAP-Reform erst nach der Festlegung des Finanzrahmens zu verhandeln, „did not bode well for an ambitious reform“ (Bureau und Mahé 2015: 128). Wie Matthews (2015: 178) bemerkt, dieses „worked to strongly favour those holding to a status quo position on the reform proposals (for example, farm groups) while disadvantaging those who sought a more radical change in the orientation of the CAP (for example, environmental groups seeking a greater focus on environmental public goods)“. Diese Faktoren sind dem ,politics stream“ zuzuordnen.

Von Cramon-Taubadel (2017) nennt den Widerstand gegen die Änderungen am bestehenden Lastenausgleich zwischen den EU-Mitgliedstaaten - also an der Verteilung der Beiträge zum und Rückflüsse aus dem EU-Haushalt - als Faktor für die Konservierung des agrarpolitischen Status quo. Dieser Widerstand schützte die EU-Agrarpolitik in der Vergangenheit, „,restricting the politically feasible set of CAP reforms to those that largely preserve the balance of net contributions" (ibid.: 10). Das 2013 eingeführte Greening stelle „an example of this lock-in“ dar: „[P]olicy makers simply wanted to construct an environmental justification for direct payments while avoiding major changes in the member states' net contributions to the budget" (ibid.). Mit dem Hinweis auf die zu erwartenden signifikanten negativen Auswirkungen des Austritts des Vereinigten Königreichs aus der EU (,Brexit“) auf den künftigen EU-Haushalt hofft von Cramon-Taubadel (2017) auf ein Ende dieser gestalterischen Zurückhaltung. Vor diesem Hintergrund kann argumentiert werden, dass dieser Faktor auch für die Beibehaltung der Direktzahlungen an sich eine Rolle spielte. Auch Blandford et al. (2011: 3) sehen die EUHaushaltsregeln - allem voran das System der fixen Mittelzuweisungen - als hinderlich für einen Instrumentenwechsel an. Auch dieser Faktor gehört zum „politics stream".

Des Weiteren vertritt Swinbank (2015) die Meinung, dass das gegenüber den früheren GAP-Reformen wenig ehrgeizige Ergebnis der Reform von 2013 dem Fehlen des Drucks seitens der WTO geschuldet sei. Speziell für den Erhalt der Direktzahlungen dürfte dieses jedoch keine Rolle spielen, denn auch eine Einigung in der Doha-Runde, falls diese an die Modalitäten von 2008 angelehnt wäre, 
würde sie dank früherer Reformen nicht unter Druck setzen (ibid.: 199). ${ }^{88}$ Eine Abschaffung der Direktzahlungen ist vor diesem Hintergrund nicht nötig, solange sie den Kriterien der unbeschränkten Green Box entsprechen (Josling und Swinbank 2008). Vermutlich aber wäre die EU hinsichtlich ihrer Ausgestaltung für die Zeit nach 2013 vorsichtiger gewesen, um ihre Konformität mit der Green Box nicht zu gefährden.

Swinnen (2015c) unternimmt einen Vergleich der neuesten Reform mit der Reform aus dem Jahr 2003, um Erklärungen für eine Reihe von Elementen sowie das gesamte magere Ergebnis von 2013 zu finden (der Erhalt von Direktzahlungen ist nicht dabei). Auch wenn ,the timing and the approach at first sight were not so different" (Swinnen 2015b: 10), identifiziert er zwischen beiden Reformen signifikante Unterschiede. Basierend auf der Vergleichsanalyse sieht er die Ursachen für die Beibehaltung des Status quo nach 2013 darin, dass sich verschiedene Faktoren, die bei der letzten Reform eine Rolle spielten, neutralisierten (Swinnen 2015c: 479). Wie er formuliert: „Different pressures and institutional changes partially offset rather than reinforced each other." Swinnen bezieht seine Aussagen grundsätzlich auf die gesamte Reform. Nachfolgend wird versucht, darzustellen, inwiefern die oben zusammengefassten Reformfaktoren in Bezug auf die Direktzahlungen einander neutralisierten. Dabei sind insbesondere solche Faktoren von Interesse, die der Beibehaltung dieses Instruments dienten.

Der Druck, die EU-Direktzahlungen stark zu reduzieren, war sowohl auf knappe öffentliche Finanzen als auch auf hohe Marktpreise für landwirtschaftliche Erzeugnisse zum Zeitpunkt der Reform zurückzuführen (Swinnen 2015c: 453). In der Wirtschaft und auf den internationalen Agrarmärkten herrschte somit ein „perfect storm“. Allerdings wurden diese Faktoren durch andere Faktoren neutralisiert und auch institutionelle Zwänge waren zu jener Zeit einer tiefergreifenden Reform nicht förderlich, so dass das Resultat nur ein ,imperfect storm“ in der politischen Ökonomie der GAP war (ibid.: 479). Am Ende „there was no dramatic cut to CAP spending“" (Matthews 2015: 187). Die Ausgaben der 1. Säule wurden real um lediglich 1,8\% gekürzt, während die Ausgaben der 2. Säule mit 7,6 \% von Kürzungen stärker betroffen waren als die Direktzahlungen (Josling und Tangermann 2015: 157). Laut Swinnen (2015c: 469) wirkten die Preisvolatilität und die damit verbundene Erwartung, dass die Marktpreise wieder zurückgehen werden, 89 dem Ansinnen entgegen, das Agrarbudget signifikant zu reduzieren. Auch die Verknüpfung mit dem Greening half, es abzusichern (ibid.: 478). Da die (entkop-

\footnotetext{
${ }_{88}$ Bei den gekoppelten Direktzahlungen (Artikel 52 der Verordnung Nr. 1307/2013) hingegen könnte eine Einigung der Doha-Runde Veränderungen bringen, weil die Modalitäten 2008 eine Begrenzung der Blue Box vorsahen (Josling und Swinbank 2008). In der Reform von 2013 bemühte sich die Kommission ausdrücklich um den Einklang der gekoppelten Stützung mit den Blue-BoxKriterien (Kommission 2015c: 2).

${ }^{89}$ Ein Preisrückgang auf den Agrarmärkten wurde zu der Zeit nicht konkret prognostiziert. Es handelte sich hier um eine allgemeine Erwartung, basierend auf der Natur der Preisvolatilität.
} 
pelten) Direktzahlungen den ganz wesentlichen Teil der GAP-Ausgaben ausmachen, gelten diese beiden Argumente auch für sie. Die Preisvolatilität hatte zudem Einfluss auf die Entscheidungsfindung im Hinblick auf „nature of the farm support“, das heißt auf die Wahl zwischen „market regulation“ und „decoupled payments" (Swinnen 2015b: 20). Denn stark schwankende Preise zogen Forderungen nach staatlichen Markteingriffen nach sich (Swinnen 2015c: 478). Vor diesem Hintergrund nutzte die Kommission die Preisvolatilität als Argument für die Beibehaltung der Direktzahlungen als Sicherheitsnetz bzw. als „an insurance against volatility, and one in line with the decades-long strategy towards liberalisation - consistent with [the] commitment to WTO and possible future accords“ (ibid.: 479). Es kann der Eindruck gewonnen werden, dass die Forderungen nach staatlichen Eingriffen in die Märkte Direktzahlungen als das kleinere Übel erscheinen ließen.

Die Osterweiterung war insbesondere bei der Frage der Angleichung der EUDirektzahlungen zwischen den Mitgliedstaaten ein entscheidender Faktor, betraf den Erhalt der (entkoppelten) Direktzahlungen nach 2013 an sich jedoch nicht. Wachsende Ansprüche der Gesellschaft in Bezug auf die Landwirtschaft sowie ihre Umweltsorgen lagen der Einführung des Greening in der 1. Säule zugrunde. Aber auch die schwierige Finanzlage dürfte hier von entscheidender Bedeutung sein. Das Greening zum Schwerpunkt der Reform zu machen sollte der Kommission helfen, das Agrarbudget abzusichern, indem ein Teil nun für die Vergütung von Umweltleistungen verwendet wird (Matthews 2015: 187f). Die parallel laufenden Verhandlungen über den EU-Finanzrahmen bis 2020 beschleunigten die Entwicklung in diese Richtung (ibid.: 188). Allerdings wurde sie durch hohe Agrarpreise und deshalb aufkommende Sorgen um die Ernährungssicherheit erfolgreich gebremst. Diese Beeinträchtigung des Greenings stellen u. a. Erjavec et al. (2015) fest. Zudem entfiel mit der Einigung auf den MFR (und somit auch auf das Agrarbudget bis 2020) der Haushaltsdruck als Reformfaktor, was die Bereitschaft zu signifikanten Änderungen der GAP stark senkte (Swinnen 2015c: 479).

Unter den genannten Reformfaktoren fällt die Preisvolatilität besonders auf. Sie wird des Öfteren als Faktor genannt, der anderen Einflüssen entgegen wirkte, v. a. den Haushaltzwängen und hohen Marktpreisen. Ihretwegen wurden die Ausgaben für die GAP - und somit auch für die Direktzahlungen - nicht signifikant reduziert. Für die vorliegende Untersuchung ist ein Aspekt aber noch wichtiger: Die Preisvolatilität hatte Einfluss auf die Entscheidungsfindung über die Art der Stützung unter der GAP nach 2013 und trug somit zum Erhalt der Direktzahlungen als Sicherheitsnetz bei.

Zu den großen Unterschieden zwischen den Reformen 2003 und 2013 gehören laut Swinnen (2015b: 10) auch die bereits erwähnte neue Rolle des Europäischen Parlaments als Co-Gesetzgeber in der letzten Reform und das Handeln des jeweiligen Agrarkommissars. In Bezug auf Letzteres bemerkt er: „Commissioner Cioloş appears to have been less committed, less experienced or less politically skilled at the European level to keep the coalition and the reform compromise to- 
gether until the end“ (ibid.: 10f). Er schreibt auch: „For most [authors in this book], Cioloş [...] was not the driver of the reforms, but more a reluctant participant" (ibid.: 11).

Knops und Swinnen (2014: 19) listen die Legitimationskrise der GAP, insbesondere in Bezug auf die EU-Direktzahlungen, als entscheidenden Faktor hinter der Reform von 2013 auf. Die Einführung der Ausgleichszahlungen im Jahr 1992 erhöhte die Sichtbarkeit der finanziellen Unterstützung der Landwirtschaft durch „shifting the CAP burden from consumers to taxpayers" und offenbarte somit die hohe politische Brisanz dieses Themas (Olper 2008: 91). Die Notwendigkeit, die Direktzahlungen neu zu legitimieren, ergab sich laut Daugbjerg und Swinbank (2015: 11) insbesondere aus ihrer Entkopplung von der Erzeugung. Bereits im Jahr 2004 sagten Henning und Latacz-Lohmann (2004: 42) voraus, dass die Entkopplung das Potenzial hat, „the farm support game“ grundlegend zu ändern. Den Grund dafür sahen sie in der Schwächung der Koalition der Landwirte, weil die Entkopplung Ungleichheiten der Transferleistungen sichtbar mache: „Increased transparency, in turn, casts increasing doubts on the legitimacy of payments to large farms and wealthy farmers" (ibid.). Im Reformprozess von 2013 hatte die Idee einer Begrenzung der Direktzahlungen für Großbetriebe eine breite Unterstützung der Öffentlichkeit (Sahrbacher et al. 2015: 280f). Zudem ist die Kompensation für den Abbau der Preisstützung als Begründung für die EUDirektzahlungen über 20 Jahre nach der einschlägigen Reform von 1992 kaum vermittelbar, auch deshalb nicht, weil die Marktpreise für landwirtschaftliche Erzeugnisse sogar höher liegen, als zu erwarten war (Tangermann 2014: 24). Zudem weist Tangermann (2011: 21) darauf hin, dass sich die EU-Direktzahlungen mit der neuesten Umverteilung unter den Mitgliedstaaten noch weiter vom ursprünglichen Kompensationsgedanken entfernen.

Die Legitimationskrise 2013 hängt eng mit anderen Faktoren zusammen. Erst die besondere Konstellation von Faktoren, die sich zu jener Zeit etablierte, „force[d] us to raise some fundamental questions regarding all EU policies, including the CAP, and [...] the direct payments in particular", wie Swinnen (2009: 16) bemerkt. Sowohl der wachsende Druck seitens der Steuerzahler als auch die Forderungen von Umweltverbänden stellten ,the need to continue the type of payments as they currently exist" infrage (Swinnen et al. 2013: 20). Knops und Swinnen (2014: 19) betonen den Zusammenhang zwischen der Legitimationskrise der GAP und dem hohen Anteil der Agrarausgaben am EU-Haushalt in Zeiten, in denen öffentliche Haushalte aufgrund der Wirtschaftskrise stark belastet sind. Auch Matthews (2015: 169) weist im Zusammenhang mit der Legitimationskrise auf die Bedeutung der Haushaltszwänge hin. Er bemerkt, dass „direction taken by the 2013 CAP reform was shaped, in part, by the need to create a narrative to legitimise and defend the share of the CAP budget in the 2014-20 MFF [Multiannual Financial Framework]". Einen indirekten Hinweis darauf, dass die Legitimationskrise auch mit Umweltbelangen zusammenhing, liefern Erjavec et al. (2015: 
237), wenn sie schreiben, dass das Umweltparadigma der Erhöhung der gesellschaftlichen Akzeptanz für die weiterhin kostspielige GAP dienen sollte.

Auch wenn für die Reform als Ganzes eine Vielzahl von Einflussfaktoren relevant waren, spielte für die Beibehaltung der Direktzahlungen nach 2013 lediglich die Preisvolatilität bzw. die Erwartung, dass die hohen Marktpreise auch wieder sinken werden, eine wichtige Rolle. Zum „politics stream“ gehören die Haushaltsregeln, die Anwendung des komplexen Mitentscheidungsverfahrens für die Reform sowie ein eher schwacher Agrarkommissar. Auch das Eingehen einer Koalition mit den Vertretern von Umweltinteressen zählt dazu (vgl. Tabelle 27).

Tabelle 27: Der EU-Reform von 2013 als Ganzes und dem Milestone zugrunde liegende Faktoren

\begin{tabular}{c|c} 
Reform 2013 & Milestone 2013 \\
\hline Haushaltsdruck & $\begin{array}{c}\text { Preisvolatilität/Erwartung sinkender } \\
\text { Marktpreise }\end{array}$ \\
Hohe Marktpreise & \\
Preisvolatilität/ & \\
Erwartung sinkender Marktpreise & \\
Vergangene Osterweiterung & Haushaltsregeln \\
Gesellschaftliche Anliegen & Koalition mit Umweltinteressen \\
Neue Ideen $\quad$ Schwacher Agrarkommissar \\
\hline
\end{tabular}





\section{Konvergenzanalyse}

Nachfolgend werden die Ursachen für die ausgewählten Milestones in der Entwicklung der Direktzahlungen in den USA und der EU zusammengestellt und näher untersucht. Auf Basis der Literaturauswertung des vorausgegangenen Kapitels wird zunächst auf die einzelnen Faktoren - ihre Rolle und ihre jeweils unterschiedliche Bedeutung für die hier interessierenden politischen Entscheidungen fokussiert. Danach wird auf die jeweiligen Milestone-Paare eingegangen, um die für sie relevanten Antriebskräfte zu vergleichen. Dieser Analyse geht eine Reihe von Anmerkungen voraus, die bei der Gegenüberstellung der identifizierten Milestone-Ursachen als Erstes auffallen. Der Vergleich der ersten Milestones wird dadurch erschwert, dass der US-Milestone dreigeteilt ist, da in den USA teilentkoppelte Ausgleichszahlungen nicht auf einmal eingeführt wurden, sondern die Entwicklung schrittweise erfolgte. Für die Zwecke der vorliegenden Analyse werden die Entscheidungen von 1962 bis 1965 sowie 1985 und 1990 zu einem Milestone gebündelt, um ein Pendant zum EU-Milestone von 1992 zu bilden. 
Tabelle 28: Zusammenstellung der für die ausgewählten Milestones relevanten Einflussfaktoren

\begin{tabular}{|c|c|c|}
\hline & USA & $\mathbf{E U}$ \\
\hline \multirow[t]{3}{*}{ 1. Milestone } & 1962-1965, 1985, 1990 & 1992 \\
\hline & $\begin{array}{c}\text { Internationale } \\
\text { Wettbewerbsfähigkeit } \\
\text { Anliegen der Verbraucher } \\
\text { Koalition mit urbanen Interessen } \\
\text { Unterstützung der Agrarwirtschaft }\end{array}$ & \multirow{2}{*}{$\begin{array}{c}\text { WTO/Uruguay-Runde } \\
\text { Umweltsorgen der Gesellschaft } \\
\text { Antizipierte EU-Erweiterung } \\
\text { OECD } \\
\text { Internationale } \\
\text { Wettbewerbsfähigkeit } \\
\text { Starker Agrarkommissar }\end{array}$} \\
\hline & $\begin{array}{c}\text { Haushaltsdruck } \\
\text { Umweltbelange (nur 1990) } \\
\text { Kongresswablen (nur 1990) }\end{array}$ & \\
\hline \multirow[t]{2}{*}{ 2. Milestone } & 1996 & 2003 \\
\hline & $\begin{array}{c}\text { Anstieg der Marktpreise/ } \\
\text { Erwartung hoher Marktpreise } \\
\text { Haushaltsregeln } \\
\text { Republikanische Mehrbeit im Kongress } \\
\text { Unterstützung der Agrarwirtschaft }\end{array}$ & $\begin{array}{c}\text { WTO/Doha-Runde } \\
\text { Umweltsorgen der Gesellschaft } \\
\text { Bevorstehende Osterweiterung } \\
\text { OECD } \\
\text { Neue Ideen } \\
\text { Starker Agrarkommissar } \\
\text { Nutzung der qualifizierten Mebrheit } \\
\text { Zabl und Qualität der politischen } \\
\text { Akteure } \\
\text { Neue Interessengruppen }\end{array}$ \\
\hline \multirow[t]{2}{*}{ 3. Milestone } & 2014 & 2013 \\
\hline & $\begin{array}{l}\text { Hohe Marktpreise und landw. } \\
\text { Einkommen } \\
\text { Erwartung sinkender Marktpreise } \\
\text { Haushaltsregeln } \\
\text { Koalition mit anderen Interessen }\end{array}$ & $\begin{array}{c}\text { Preisvolatilität/Erwartung } \\
\text { sinkender Marktpreise } \\
\text { Haushaltsregeln } \\
\text { Anwendung des } \\
\text { Mitentscheidungsverfahrens } \\
\text { Koalition mit Umweltinteressen } \\
\text { Schwacher Agrarkommissar }\end{array}$ \\
\hline
\end{tabular}

Anmerkung: Kursiv sind die Faktoren vermerkt, die unter den „politics stream“ fallen.

Tabelle 28 bietet eine Übersicht über die Einflussfaktoren, auf die die ausgewählten Milestones zurückzuführen sind. Es wird dabei zwischen ,pproblem stream“und ,politics stream“-Faktoren unterschieden. 


\subsection{Analyse der einzelnen Einflussfaktoren}

Dieses vorausgeschickt, ist festzustellen, dass bei den hier beleuchteten Entwicklungen der Direktzahlungen eine große Vielzahl von sowohl „problem stream“als auch „politics stream“-Faktoren von Relevanz waren. Erstere reichen von externen Faktoren, wie WTO, OECD und EU-Erweiterung über die Entwicklungen auf den Agrarmärkten bis zu rein innenpolitischen bzw. internen Faktoren, wie Wettbewerbsfähigkeit der heimischen Landwirtschaft und gesellschaftliche Anliegen (angemessene Verbraucherpreise und Umweltbelange). Alle diese Faktoren mit Ausnahme der EU-Erweiterung sind grundsätzlich auf beide Akteure anwendbar. Die Wirkung der „politics stream“-Faktoren hingegen beschränkt sich naturgemäß auf die nationale Ebene. Ferner ist auffallend, dass in den USA insgesamt weniger Faktoren als in der EU für die Entwicklung der Direktzahlungen bedeutsam waren. Auf den ersten Blick ist zudem erkennbar, dass in den USA externe Faktoren keine Rolle spielten. Die im Kapitel 7.3 aufgestellte Hypothese, dass solche Faktoren für die Entwicklung der Direktzahlungen beidseits des Atlantiks von unterschiedlicher Bedeutung waren, worauf das zeitliche Auseinanderliegen der Milestones hindeutet, wird somit auf Anhieb bestätigt. Dass diesen auch die Auseinanderentwicklung der US- und der EU-Agrarpolitik in ihren jeweils letzten Reformen geschuldet ist, konnte jedoch nicht bestätigt werden. Denn beim Vergleich des dritten Milestone-Paars sind hier und dort keine externen Faktoren als Ursache für die jeweilige Entwicklung feststellbar.

Vergleicht man die unter den „problem stream“ fallenden Ursachen für die einzelnen US- sowie EU-Milestones separat untereinander, wird deutlich, dass sich ihre Bedeutung im Laufe der Zeit änderte. In den USA unterscheiden sich die Ursachen für den ersten Milestone von den Ursachen für die zwei späteren Milestones gänzlich. Zwischen ihnen bestehen keine Überschneidungen. Somit waren gesellschaftliche Anliegen, aber auch die Frage der Wettbewerbsfähigkeit und der Haushaltsdruck lediglich zu Beginn der hier analysierten Entwicklung relevant: bei der Einführung von Ausgleichszahlungen und den ersten Entkopplungsschritten. Hingegen weisen die zwei späteren Milestones - 1996 und 2014 - in Bezug auf die Ursachen große Ähnlichkeiten auf. Sowohl bei der Einführung von Direktzahlungen als auch ihrer Abschaffung waren es die Marktentwicklungen, die die Entwicklung bestimmten.

Anders gestaltet sich die Situation in der EU: Hier sind die Ursachen für die ersten zwei Milestones nahezu identisch, während der dritte Milestone - der einzige, der keinen Instrumentenwechsel mit sich brachte - diesbezüglich hervorsticht. Der einzige Unterschied zwischen den Milestones 1992 und 2003 besteht darin, dass beim erstgenannten der Einfluss neuer Ideen, beim letzteren das Streben nach Wettbewerbsfähigkeit als Ursache fehlen; die restlichen vier Faktoren sind gleich. Beim Milestone 2013 sind die Ursachen neu: Die Preisvolatilität bzw. die Erwartung sinkender Marktpreise sind bei den früheren Milestones 1992 und 2003 keine ursächlichen Faktoren. Die Vermutung liegt nahe, dass, um einen Instru- 
mentenwechsel in der EU zu bewirken, andere - womöglich externe - Faktoren nötig sind. Diese fehlten 2013. Die Preisvolatilität als neuerdings aufgetretene Ursache ist eine mögliche Folgeerscheinung der Reformen der letzten Jahre, die stärker marktwirtschaftlich orientiert waren und die die langjährige Abschottung der EU-Agrarmärkte gegenüber dem Weltmarkt beendeten.

Im Hinblick auf die „politics stream“-Faktoren fällt auf, dass in den USA die Milestones 1996 und 2014 Ähnlichkeiten aufweisen, weil für beide die Haushaltsregeln eine Rolle spielten. Für die zwei letzten EU-Milestones hingegen stellte das Gesetzgebungsverfahren, im Rahmen dessen die Entscheidung zustande kam, einen bedeutenden Faktor dar. Außerdem war dort bei allen drei Reformen die Haltung des jeweiligen Agrarkommissars von Bedeutung.

Den Anmerkungen allgemeiner Natur folgt nun eine tiefergehende Analyse der Einflussfaktoren, in deren Fokus zunächst einzelne Ursachen an sich und ihre Bedeutung für die Entwicklung der Direktzahlungen in den USA und der EU stehen, danach erfolgt der Vergleich der jeweiligen Milestone-Paare.

Die Auswertung der Literatur des vorausgegangenen Kapitels ergibt, dass der Haushaltsdruck bei der Entwicklung der Direktzahlungen keine (EU) bzw. nur eine geringfügige (USA) Rolle spielte. Lediglich bei den ersten Entkopplungsschritten in den USA von 1985 und 1990 war er tatsächlich mitentscheidend. Dieses ist vor allem vor dem Hintergrund der Tatsache bemerkenswert, dass der Haushaltsdruck - wie die Auswertung der Literatur ergab - für jede der relevanten Reformen als Ganzes eine Rolle spielte. Selbst im Laufe der schweren Finanz- und Wirtschaftskrise, die 2007 ausbrach, blieben die Direktzahlungen beidseits des Atlantiks vom Haushaltsdruck im Großen und Ganzen unberührt. Er scheint vielmehr die Rolle eines Reformkatalysators wahrzunehmen. Erklärungen, warum am Ende keine kostengünstigeren Maßnahmen beschlossen wurden, liefern Cunha und Swinbank (2011). Die von ihnen Befragten verweisen zum einen auf die Notwendigkeit, zukünftige Steigerungen der Agrarausgaben zu verhindern, die bei der Fortführung des Status quo anfallen würden. Zum anderen ist die Absage an Kürzungen als politischer Preis für den Kompromiss und die Reduzierung des Widerstands seitens der Landwirte anzusehen (ibid.: 173). Zwar beziehen sich Cunha und Swinbank (2011) nur auf die Reformen der EU-Agrarpolitik von 1992, 1999 und 2003, aber auch für die übrigen hier relevanten Reformen dürften beide Erklärungen zutreffend sein.

Im Gegensatz zum Finanzierungsumfang scheinen Haushaltsregeln für die Entwicklung der Direktzahlungen auf beiden Seiten des Atlantiks überdurchschnittlich wichtig gewesen zu sein. Agrarkommissar Fischler bemerkte während der Vorbereitungen auf die Reform von 2003: „In the E.U. we focus on improving our policy tools under budgetary constraints, notably on restoring the link between farmers and markets. In the U.S. they focus on maximising budgetary outlays with fixed policy tools (or even contemplating returning to old, more trade distorting policy tools)“ (nach Haniotis 2006: 62). In seiner Aussage brachte er 
den Unterschied zwischen den USA und der EU in Bezug auf die im politischen Prozess zu beachtenden Haushaltsregeln auf den Punkt. In den USA bestehen für die sogenannten Pflichtausgaben (dazu gehören die meisten Agrarausgaben) keine festen, im Voraus festgesetzten Mittelzuweisungen. Thre Finanzierung ist für die Laufzeit einer Farm Bill automatisch gesichert, ohne dass ein zusätzliches Gesetz nötig ist. Wenn die tatsächlichen Ausgaben - bei unveränderter Programmausgestaltung - die CBO-Schätzungen zum Zeitpunkt der Verabschiedung der Farm Bill überschreiten (weil z. B. die Zahl der Empfangsberechtigten aufgrund einer sich verschlechterten Marktsituation gestiegen ist), müssen keine zusätzlichen Finanzmittel gefunden werden (Monke 2014). Dadurch ist eine „baseline capture“ bzw. „maximising budgetary outlays“ überhaupt möglich und ein Instrumentenwechsel unter Umständen attraktiv. Wie es Bureau (2012: 59) sehr treffend formuliert:,,The CBO baseline is central to the Farm Bill design.“" Vor diesem Hintergrund beschreiben Orden et al. (1999: 187) die US-Haushaltsregeln hinsichtlich der Einführung der entkoppelten Zahlungen 1996 als ,a source of opportunity and advantage, not as a constraint". Auch für ihre Abschaffung im Jahr 2014 trifft dieses zu. In beiden Fällen führte die „baseline capture“ in Kombination mit der Erwartung hoher bzw. niedriger Marktpreise die jeweiligen Entscheidungen herbei. Beim Milestone 1996 ist dieses vor dem Hintergrund des Fehlens anderer „problem stream“-Faktoren eindeutig. Aber auch für den Milestone 2014 darf gezweifelt werden, dass - angesichts der starken Agrarlobby - allein die sich aus hohen landwirtschaftlichen Einkommen speisende geringe gesellschaftliche Akzeptanz von „fixed direct payments“ zu ihrer Abschaffung geführt hätte.

In der EU hingegen scheinen die Haushaltsregeln grundsätzlich eher als „,constraint" zu fungieren, um bei dem von Orden et al. (1999) genutzten Ausdruck zu bleiben. Im Rahmen des Mehrjährigen Finanzrahmens werden die jährlichen Höchstbeträge für die Haushaltsrubrik „Nachhaltiges Wachstum: natürliche Ressourcen“, die die Ausgaben für die GAP umfasst, festgelegt, die während der MFR-Laufzeit ausgegeben werden dürfen. Darunter wird explizit auch der Höchstbetrag für die Ausgaben der 1. Säule bestimmt. Die Direktzahlungen werden gekürzt, wenn die Prognosen erkennen lassen, dass er überschritten wird (sogenannte finanzielle Disziplin). Dieses System ,prevent large variations in expenditure“ (Blandford et al. 2011: 3). Deshalb sei eine Umgestaltung der Direktzahlungen in von Jahr zu Jahr unterschiedlich hoch ausfallende Zahlungen nach dem Vorbild der USA ohne eine grundlegende Haushaltsreform nicht möglich (ibid.). Nichtsdestotrotz kann auch im Falle der EU von einer Art „baseline capture“ gesprochen werden. Eine Erhöhung der Agrarausgaben scheint zwar ausgeschlossen zu sein, aber es gelang der Agrarlobby, über Jahre ein hohes Ausgabenniveau zu halten: Seit 1993 liegen die Ausgaben für die GAP, ausgedrückt in Preisen von 2011, durchgehend über der Schwelle von 50 Mrd. EUR (Kommission 2017). In diesem Rahmen sind es aktuell die Direktzahlungen, die für die Landwirte den höchsten Leistungsumfang ausmachen. Stabilisierend auf die GAP wirkt die be- 
stehende Balance der nationalen Nettobeiträge zum EU-Haushalt: Nach von Cramon-Taubadel (2017) verhindert die Furcht, diese anzutasten, tiefergreifende Agrarreformen. Wie sich dieses auf die Ausgestaltung der GAP-Reformen auswirken kann, beschreiben Bureau et al. (2009: 10) mit Hinweis auf die im Zuge des „Gesundheitscheck“ der GAP diskutierten Vorschläge für breiter angelegte Ernteversicherungsprogramme, die am Ende den Mitgliedstaaten überlassen wurden, und zwar wegen der ,sensitivity of reforms that might modify net contributions to the EU budget".

In der Literatur wird die WTO als ein wichtiger Faktor für die Entwicklung der Agrarpolitik der Mitgliedsländer genannt. Insbesondere wird ihr Einfluss auf die Wahl des agrarpolitischen Instrumentariums hervorgehoben. Beispielsweise stellt Tangermann (2003: 25) fest, dass die Uruguay-Runde „has not only resulted in new legal rules and quantitative reduction commitments in the areas of market access, domestic support and export competition", sondern sie beeinflusste auch „the nature of the policy debate in agriculture“. Er konstatiert: „The WTO has become a relevant factor in agricultural policy making" (ibid.). Swinnen et al. (2012: 1100) weisen auf ihre ökonometrische Analyse hin, die belegt, dass das Übereinkommen über die Landwirtschaft „did not cause a significant reduction in the total amount of support to agriculture“, sondern „,it caused a significant shift from distortionary to less distortionary instruments". Die These, dass die WTO für die Instrumentenwahl wichtiger ist als für das Stützungsniveau, illustriert Swinnen (2010a: 95) mit dem Beispiel der EU, in der „GATT/WTO accession triggered an important change in the instrument choice $[. .$.$] over the past decades,$ but much less on the level of protection".

Tatsächlich zeigt die Gegenüberstellung von Milestones in der Entwicklung der Direktzahlungen in den USA und der EU, dass die WTO für zwei der drei EU-Milestones einen wichtigen Faktor darstellt. Wie die OECD, lag sie beiden Instrumentenwechseln in der EU zugrunde. Diese zwei Faktoren sind nur beim dritten Milestone - der Fortführung des Status quo - nicht vertreten. In den USA hingegen ist weder ein Einfluss der WTO noch der OECD auf die Entwicklung der Direktzahlungen feststellbar. Wie die Auswertung der einschlägigen Literatur ergab, ist keiner der drei Milestones darauf zurückzuführen, einschließlich des während der Uruguay-Runde beschlossenen Entkopplungsschrittes 1990. Auch der Entkopplungsschritt von 1985 lässt sich nicht als Vorbereitung auf die bevorstehenden GATT-Verhandlungen deuten.

Dass die WTO keine Rolle für die Milestones in den USA spielte, steht im Kontrast zu den ursprünglichen Gründen, warum die USA auf die volle Aufnahme der Landwirtschaft in die multilateralen Handelsverhandlungen drängten. Laut Paarlberg (1992: 30) wollte die US-Regierung damit eine Reform der US-Agrarpolitik in Richtung Deregulierung bewirken, nachdem nationale Gründe offensichtlich nicht ausreichten (die Reagan-Regierung scheiterte im Kongress sowohl 1981 als auch 1985 mit ihren Liberalisierungsvorschlägen). Die auf diese Weise 
herbeigeführte Internationalisierung nationaler Reform sollte den Einfluss der starken landwirtschaftlichen Lobby einschränken und die Folgen der Reformen für den US-Agrarsektor abfedern, weil mit entsprechenden Folgen für die Landwirtschaft in anderen Ländern zu rechnen war. Die US-Regierung hoffte dabei auf Interesse seitens der EU, die über die hohen Haushaltskosten eigener landwirtschaftlicher Programme besorgt sein müsste. Diese Strategie wurde am Ende nicht von den USA, sondern von der EU realisiert. In den USA hingegen sind alle drei Milestones ausschließlich auf innenpolitische bzw. interne Faktoren zurückzuführen.

Wie von de Gorter (2008: 18f) festgehalten, kann die WTO die Agrarpolitik und insbesondere die Instrumentenwahl auf dreierlei Weise beeinflussen: Neben den multilateralen Verhandlungsrunden zur Liberalisierung des Agrarhandels sind es das Streitbeilegungsverfahren sowie der WTO-Beitritt. Wie sich herausstellte, war für die hier interessierenden EU-Milestones lediglich der erste Aspekt - die Verhandlungsrunden - von Bedeutung. Er spielte beim 1. und 2. Milestone eine Rolle. Unterschiedlich wurde aber damit umgegangen. Während beim ersten Milestone 1992 ein Zusammenhang mit den Handelsverhandlungen noch bestritten wurde und die EU auf die Geschehnisse lediglich reagierte, nahm sie beim zweiten Milestone 2003 eine proaktive Rolle ein und kommunizierte die WTO-Verhandlungsrunde als Begründung für die Reform auch offensiv.

Es überrascht nicht, dass neben der WTO auch die OECD sowie neue Ideen keine Rolle in der Entwicklung der Direktzahlungen in den USA spielten, denn beide sind bei näherer Betrachtung mit der WTO verwoben. Wo die WTO eine Rolle spielt, kann man somit auch das Mitwirken der OECD und der neuen Ideen vermuten. Dieses ist im Falle der EU insbesondere bei dem Milestone 2003 erkennbar. Neben Josling (1992) merkt beispielsweise auch Cahill (2010: 14) an, dass die Tätigkeit der OECD politische Folgen in den Mitgliedsländern hatte. Sie weist auf einen Einfluss der OECD auf die WTO-Verhandlungen über das agrarpolitische Instrumentarium hin: „Agricultural policy discussions at the OECD influence negotiations of disciplines on those same policy instruments at the WTO." Die OECD scheint in zweierlei Hinsicht eine Rolle zu spielen: Zum einen stellt sie eine zwischenstaatliche Plattform für Dialog- und Erfahrungsaustausch dar. Laut Josling et al. (2010: 439) leistete die OECD im Agrarreformprozess einen besonderen Beitrag zu mehr Marktorientierung und Handelsliberalisierung als „a platform for dialogue among governments on how to put that paradigm into policy practice". Gleichzeitig verbesserte sich durch die Arbeit der OECD die Transparenz hinsichtlich der Agrarpolitik einzelner Länder erheblich (Josling et al. 1996). Einen Zusammenhang zwischen neuen Ideen, der Wissenschaft und der OECD beschreiben Josling et al. (2010: 427) wie folgt: „Policy dialogue in international bodies such as the Organisation for Economic Co-operation and Development contributed significantly to the paradigm shift, and this dialogue was an extension of the academic discussions of the time." Gerade bei der Idee der Direktzahlun- 
gen als Ersatz für an die Produktion gekoppelte Maßnahmen scheint der Einfluss der OECD besonders groß zu sein. Bereits 1990 führte die OECD detailliert aus, wie die Anwendung solcher „direct income payments“ aussehen könnte (Box 1). Ihre Studie über die Vorteile der Entkopplung spielte eine Rolle in der Reform der GAP von 2003.

Im Kapitel 3 konnte gezeigt werden, dass die Idee der Direktzahlungen ihren Ursprung in der Wissenschaft hat, die sich bereits zum Ende des 2. Weltkriegs mit diesem Thema befasste. Höchstwahrscheinlich schlug W. W. Cochrane 1945 als Erster von der Produktion entkoppelte ,income payments“ vor; eine Reihe von Entkopplungskonzepten wurde in den 1960er-Jahren in den USA und in den 1970er-Jahren in Europa vorgelegt. Somit war die Idee nicht neu, als sich die OECD in den 1980er-Jahren des Themas annahm. Auch die Kritik am Instrument der Preisstützung äußerten die Wissenschaftler schon lange Zeit davor. Es war aber die OECD, die sie kanalisierte (Tangermann 1998: 19). Darüber hinaus gelang ihr das, was die Wissenschaft offensichtlich nicht ausreichend schaffte: Die wissenschaftlichen Erkenntnisse erfolgreich bei den Entscheidungsträgern zu platzieren (Josling et al. 1996: 109). Von nun an konnten Interessierte Informationen ,from an official source rather than from obscure academic research" beziehen (ibid.: 110). Darauf, dass die OECD die Idee der (entkoppelten) Direktzahlungen verbreitete, weist Hofreither (2016) hin. Durch „beharrliche Überzeugungsarbeit der OECD“ wurde sie schrittweise aus der Forschung in die praktische Politik eingeführt (ibid.: 9).

Auch neuen Ideen wird eine bedeutende Rolle für die Gestaltung von Politik zugeschrieben (Hall 1993). Feindt et al. (2008: 74) weisen darauf hin, dass „,seit Mitte der 1980er Jahre erhebliche Innovationen auf der Ebene der politischen Programme und der Politikinstrumente" im Agrarbereich zu verzeichnen sind und deshalb von einem Paradigmenwechsel gesprochen wird. Dabei stehen insbesondere zwei miteinander konkurrierende Paradigmen im Vordergrund: das alte Paradigma einer schutzbedürftigen Landwirtschaft und das neue marktliberale Paradigma. Erstes beruht auf zwei Grundsätzen: „first, the agriculture sector contributes to national policy goals and therefore merits special attention; and, second, the price mechanism is a sub-optimal means of achieving an efficient and productive agricultural sector“ (Coleman et al. 1996: 275). Als Ergebnis ,[i]mperfect markets combine with unmanageable natural risks and concern for food security to give governments a large role in subsidizing agricultural production" (ibid.). Unter dem marktliberalen Paradigma hingegen ,individual producers should be responsible $[\ldots]$ for protecting themselves against income losses due to natural conditions“ (ibid.: 276). Diesem Paradigma entspricht die Idee der entkoppelten Direktzahlungen (Feindt et al. 2008: 80).

Neue Ideen, die das marktliberale Paradigma stützen, scheinen in Agrarpolitikreformen stark mit dem WTO-Faktor verwoben zu sein. Coleman et al. (1996: 275) weisen darauf hin, dass der Druck, die Agrarpolitik weg vom Paradigma einer 
schutzbedürftigen Landwirtschaft zu reformieren, „has intensified further as agriculture has become more fully integrated into international trade discussions at the regional (EU, NAFTA) and global (GATT) levels“. Daugbjerg und Swinbank (2010: 47) weisen darauf hin, dass das Agrarabkommen der Uruguay-Runde „ideational change" in der GATT/WTO mit sich brachte. Als ideelle Fundierung des Weltagrarhandelsregimes ersetzte das marktliberale Paradigma das bisherige, im GATT 1947 institutionalisierte alte Paradigma einer schutzbedürftigen Landwirtschaft. Dieses hatte Auswirkungen auf die Agrarpolitik der WTO-Mitglieder, insbesondere auf die der EU (ibid.). Wie die Auswertung der Literatur des vorausgegangenen Kapitels ergab, ist die Bedeutung neuer Ideen nicht immer eindeutig feststellbar und je nach Interpretation variieren die Analyseergebnisse. Am eindeutigsten und nachhaltigsten ist der Einfluss des marktliberalen Paradigmas auf den zweiten EU-Milestone - die Einführung der entkoppelten Betriebsprämie im Jahr 2003 - zu erkennen.

Eine Begründung der Agrarstützung stellen in neueren Zeiten Umweltbelange dar (Zulauf und Orden 2014: 10). Für die Entwicklung der Direktzahlungen in der EU spielte dieser Faktor eine größere Rolle als in den USA. Das gesellschaftliche Anliegen für mehr Umweltschutz war für zwei EU-Milestones (1992 und 2003) mitentscheidend, während es in den USA lediglich für die Entscheidung von 1990 eine Rolle spielte. Die Motivation hinter den jeweiligen Reformschritten war jedoch ähnlich. Durch die (Teil-)Entkopplung, die die Produktionsanreize beseitigte, sollte der Druck auf die natürlichen Ressourcen reduziert werden.

Indirekt sorgten Umweltbelange auch für die Beibehaltung der EU-Direktzahlungen nach 2013. Sie beantworten zwar nicht die hier interessierende Frage, warum die Direktzahlungen in der letzten GAP-Reform nicht abgeschafft wurden. Denn gesellschaftliche Anliegen hätten auch außerhalb des Systems der Direktzahlungen angegangen werden können, z. B. durch Aufstockung oder Neukonzipierung der 2. Säule, was von vielen Wissenschaftlern propagiert wird (z. B. Wissenschaftlicher Beirat 2010, Isermeyer 2014). Sicher aber trug das Greening - also die Bindung der Direktzahlungen an zusätzliche Umweltauflagen über das CrossCompliance hinaus - zur Akzeptanz für ihren Erhalt bei. Für die USA hingegen scheint eine stärkere Bindung der Zahlungen an Umweltziele keine Option zu sein. Als möglichen Grund dafür nennen Blandford et al. (2011:4) die in den USA stark ausgeprägte Sorge hinsichtlich einer Überregulierung der Landwirtschaft.

Gesellschaftliche Anliegen können neben Umweltschutz auch weitere Anforderungen beinhalten. Verbraucherinteressen im Sinne der Verhinderung der Verteuerung von Lebensmitteln spielten für die Milestones ein einziges Mal eine Rolle: Sie waren für die Einführung der Ausgleichszahlungen in den USA mitentscheidend. Für die EU-Milestones bleiben sie in der Literatur jedoch unerwähnt.

Es ist allgemein bekannt, dass Veränderungen der Marktpreise für landwirtschaftliche Erzeugnisse einen Einfluss auf die Agrarpolitik haben können (Anderson et al. 2013). Die Gegenüberstellung von Milestones in der Entwicklung der 
Direktzahlungen auf beiden Seiten des Atlantiks zeigt eindeutig, dass diese in den USA eine größere Rolle spielten. Zum einen ist das die Marktentwicklung zum Zeitpunkt der Vorbereitung der relevanten Farm Bill. Steigende Marktpreise nach 1993 waren für die Einführung der Entkopplung entscheidend. Hohe Marktpreise in den Jahren vor 2014 wiederum führten zu hohen landwirtschaftlichen Einkommen, die die gesellschaftliche Akzeptanz für „fixed direct payments“ in Zeiten der schweren Finanz- und Wirtschaftskrise untergruben und zu ihrer Abschaffung beitrugen. Dieses deckt sich mit der Anmerkung von Thompson (2005: 15), dass „[e]very Farm Bill is influenced disproportionately by the current economic conditions in the farm sector and agricultural commodity markets at the time the Farm Bill is written". Zum anderen sind in den USA die Erwartungshaltungen der Akteure an die zukünftige Marktentwicklung für die Reformausgestaltung sehr wichtig. Die Erwartung von höheren bzw. niedrigeren Marktpreisen als zum Reformzeitpunkt war für die Einführung bzw. Abschaffung der "fixed direct payments“ mitentscheidend. Orden et al. (2010: 176) weisen im Hinblick sowohl auf die Farm Bill von 1996 als auch auf die Farm Bill von 2002 auf ,responsiveness of U.S. farm policy to proximate market circumstances" hin. Haniotis (2006: 68) bemerkt mit Blick auf die Farm Bill von 2002, mit der eine teilweise Abkehr vom Entkopplungskurs beschlossen wurde, dass „,[e]xpectations about market developments played a big part in this reversal of U.S. policies". Vor diesem Hintergrund überrascht nicht, dass dieser Faktor auch für den Milestone 2014 eine entscheidende Rolle spielte. Dabei halfen die Haushaltsregeln, die Finanzmittel - trotz sich verändernder Bedingungen - voll auszuschöpfen („,baseline capture“).

In der EU hingegen ist der Einfluss der Marktentwicklung nur beim Milestone 2013 feststellbar. Die Preisvolatilität trug dazu bei, dass die Direktzahlungen trotz hoher Marktpreise zur Zeit des Reformbeschlusses - beibehalten wurden. Es bleibt noch anzumerken, dass Erwartungen hoher Marktpreise nicht zu den Antriebskräften der MacSharry-Reform von 1992 gezählt werden. Im Unterschied zu den USA war die Reform in der EU zu der Zeit, als die Marktpreise Mitte der 1990er-Jahre anstiegen, bereits in vollem Gang. ${ }^{90}$ Der Preisanstieg erleichterte jedoch diese Reform, weil der Wert der Marktinterventionen für die Erzeuger sank (Feindt et al. 2008: 86). Dieses hatte eher Folgen für die nächste GAP-Reform -

\footnotetext{
${ }^{90}$ Wie der Europäische Rechnungshof (1999) feststellt, lagen aufgrund der durch schlechte Ernteergebnisse in den Jahren 1994 und 1995 bedingten Angebotsknappheit die Weltmarktpreise für Getreide Mitte der 1990er-Jahre außergewöhnlich hoch. Vor diesem Hintergrund folgte der Senkung der EU-Richtpreise in Folge der MacSharry-Reform von 1992 kein Absinken der Erzeugerpreise in gleichem Maße, sodass der Ausgleich im Verhältnis zu den tatsächlichen Einkommensverlusten der EU-Landwirte zu hoch ausfiel. Im Getreidesektor überstiegen zwischen 1993/94 und 1997/98 die gezahlten Ausgleichszahlungen die Einkommensverluste um etwa 13,1 Mrd. ECU (ibid.: 22). Die durchschnittlichen Marktpreise für Weichweizen variierten 1995 zwischen 132,4 ECU/Tonne in Deutschland und 174,9 ECU/Tonne in Griechenland und lagen in einigen Mitgliedstaaten sogar höher als der ursprüngliche Richtpreis von 155 ECU/Tonne (ibid.: 18).
} 
die „Agenda 2000“ - in Form von positivem Feedback über das neue Instrument der Ausgleichszahlungen (Daugbjerg 2003).

Die Frage der internationalen Wettbewerbsfähigkeit des Sektors stellt in der Entwicklung der Direktzahlungen auf beiden Seiten des Atlantiks einen Faktor dar. Auf Druck der exportorientierten Landwirtschaft wurde in den USA in den 1960er-Jahren die bisherige Preisstützungspolitik aufgegeben. Das Ziel, die Wettbewerbsfähigkeit des heimischen Sektors zu verbessern, spielte auch eine - wenn nicht die wichtigste - Rolle bei der Einführung der Ausgleichszahlungen in der EU im Jahr 1992. Die EU-Osterweiterung ist als ein rein EU-spezifischer Faktor naturgemäß für die US-Milestones nicht von Bedeutung. Deshalb wird sie in der anschließenden Analyse nicht berücksichtigt.

\subsection{Paarvergleich der Milestones: Gemeinsamkeiten und Unterschiede}

Nachdem die einzelnen Ursachen und ihre Bedeutung für die Entwicklung der Direktzahlungen in den USA und der EU beleuchtet wurden, sollte nachfolgend ein Vergleich der Ursachen für die jeweiligen Milestone-Paare angestellt werden. Ziel ist es, auf Basis der Literaturauswertung des vorausgegangenen Kapitels zu untersuchen, ob die Einführung der Ausgleichszahlungen sowie die Einführung der Direktzahlungen auf beiden Seiten des Atlantiks auf ähnliche Faktoren zurückzuführen sind. Auch soll ermittelt werden, welche Unterschiede - aber auch eventuell Gemeinsamkeiten - im Hinblick auf die Ursachen der divergierenden politischen Entscheidungen von 2013 und 2014 bestehen. Auf dieser Grundlage wird versucht, die in Bezug auf die Direktzahlungen stattgefundenen Konvergenz und Divergenz der Agrarpolitiken beider Seiten zu erklären.

Bei den „problem stream“-Faktoren des ersten Milestone-Paars fällt auf, dass im Falle der USA interne (im Sinne von innenpolitischen), im Falle der EU externe Faktoren dominieren. Die Faktoren, die in den USA eine Rolle spielten, sind sogar ausschließlich nationaler Natur und beinhalten die Frage der Wettbewerbsfähigkeit der Landwirtschaft, den Haushaltsdruck sowie die gesellschaftlichen Anliegen (angemessene Verbraucherpreise und Umweltbelange). In der EU hingegen fallen für den Milestone 1992 lediglich zwei von fünf „problem stream“-Ursachen in die Kategorie ,interner Faktor“. Dieses sind die Umweltsorgen der Gesellschaft und die Frage der Wettbewerbsfähigkeit des EU-Agrarsektors.

Die Einführung von (teilentkoppelten) Ausgleichszahlungen ist in den USA und der EU nur teilweise denselben Ursachen zuzuschreiben. Obwohl beidseits des Atlantiks eine Vielzahl von Faktoren für den ersten Milestone bedeutsam waren, bestehen zwischen den beiden Akteuren lediglich zwei Übereinstimmungen: Umweltbelange und Wettbewerbsfähigkeit. Allerdings herrschen hinsichtlich des Relevanzgrades dieser Faktoren zwischen den USA und der EU zum Teil Unter- 
schiede. Die Frage der internationalen Wettbewerbsfähigkeit der Landwirtschaft stellte in den USA eindeutig den wichtigsten Faktor für die Einführung der Ausgleichszahlungen dar, während sie in der EU 1992 eher zweitrangig war. Andere Faktoren waren in der EU für die Einführung der Ausgleichszahlungen bedeutender, allem voran die Uruguay-Runde. Die bei der Teilentkopplung mitentscheidenden Umweltbelange zählten auf beiden Seiten nicht zu den wichtigsten Ursachen. In den USA wurden sie bezüglich der Bedeutung vom Haushaltsdruck, in der EU von der Uruguay-Runde übertroffen. Wird die antizipierte EU-Erweiterung als rein EU-spezifischer Faktor ausgespart, haben immerhin vier Faktoren auf der jeweils anderen Seite des Atlantiks kein Pendant: Sie waren nur in einem der Wirtschaftsräume relevant. Dazu zählen der Haushaltsdruck und die Verbraucherinteressen in den USA sowie der Druck seitens der WTO und der OECD in der EU.

Beim zweiten Milestone-Paar - der Einführung von Direktzahlungen - lassen sich zwischen den USA und der EU keine Gemeinsamkeiten bzw. Überschneidungen in Bezug auf die Ursachen feststellen. Dieses bedeutet, dass die USA und die EU die Entkopplung der Zahlungen von der Produktion aus unterschiedlichen Motiven durchführten. In den USA stellten der Preisanstieg und die Erwartung hoher Marktpreise die entscheidenden „problem stream“-Faktoren dar (gestützt durch die förderlichen Haushaltsregeln). Wie die Auswertung der Literatur zeigt, wäre dort ohne steigende Marktpreise Mitte der 1990er-Jahre keine Entkopplung vorgeschlagen und beschlossen worden. In der EU hingegen bestimmten - wie bereits beim ersten Milestone - größtenteils die externen Faktoren die Entwicklung. Den stärksten Einfluss scheinen dabei die Verhandlungen im Rahmen der Doha-Runde der WTO gehabt zu haben. Die Entwicklung der Agrarmärkte wird in der Literatur als Einflussfaktor nicht erwähnt.

Anders gestaltet sich das dritte Milestone-Paar. Obwohl die politischen Entscheidungen an sich divergierten, weisen die Ursachen beidseits des Atlantiks Ähnlichkeiten auf. Dieses überrascht. Gerade bei den Entscheidungen, die zu einer Auseinanderentwicklung der USA und EU in der Frage der Direktzahlungen führten, bestehen hinsichtlich der Ursachen Überschneidungen. Bei den Entscheidungen wiederum, die die Agrarpolitik beider Akteure konvergieren ließen, sind einmal Gemeinsamkeiten in Bezug auf ihre Ursachen feststellbar (das erste Milestone-Paar); einmal sind gar keine Gemeinsamkeiten erkennbar (das zweite Milestone-Paar). Dieses ist zum Teil dadurch erklärbar, dass - im Gegensatz zu den früheren Milestones - die letzten Entscheidungen fast parallel stattfanden. Damit ist es eher wahrscheinlich, dass gemeinsame Ursachen vorliegen, wobei hier kein Automatismus besteht, was man am Beispiel von teils unterschiedlichen Ursachen für den EU-Milestone 1992 und den US-Milestone 1990 beobachten kann. Ein genauerer Blick auf die jüngsten Entscheidungen ist notwendig, um die Frage zu beantworten, wie es trotz Gemeinsamkeiten bei den Ursachen zu unterschiedlichen Reformergebnissen kommen konnte. 
Ein gemeinsamer Einflussfaktor beim dritten Milestone-Paar ist die Preissituation auf dem Agrarmarkt. Wie Bureau (2012: 75) mit Blick auf die letzten Reformen beidseits des Atlantiks bemerkt: „One shared concern seems to be the need to help farmers cope with price or income volatility." In den USA kam der Druck, die Direktzahlungen abzuschaffen, von den zur Zeit der Vorbereitung der Farm Bill von 2014 herrschenden hohen Marktpreisen, die zu Rekordeinkommen in der Landwirtschaft führten, während die allgemeine Wirtschaftsentwicklung schwach war. Die Erwartung künftig niedrigerer Marktpreise wirkte dabei verstärkend, weil die Direktzahlungen im Vergleich zu den antizyklischen Instrumenten für die Landwirte monetär weniger attraktiv wurden. In der EU wiederum wirkte sich die Erwartung, dass die Preise volatil bleiben und deshalb auch wieder sinken werden, völlig anders aus: Sie trug zum Erhalt der Direktzahlungen bei, zumal es hier - im Gegensatz zu den USA - antizyklische Stützungen nicht gibt. Sie verstärkte nicht, sondern neutralisierte den Druck, der von den herrschenden hohen Marktpreisen ausging, mit dem Hinweis, die Preise - und damit die landwirtschaftlichen Einkommen - würden wieder sinken. Daher werden die hohen Marktpreise zum Zeitpunkt des Reformprozesses lediglich als Einflussfaktor für die Reform von 2013 als Ganzes festgehalten.

Somit wurde auf gleiche Marktbedingungen, also hohe, aber volatile Marktpreise, beidseits des Atlantiks unterschiedlich reagiert. Die entscheidende Rolle spielten dabei die jeweiligen Haushaltsregeln. Diese stellen zwischen den USA und der EU eine weitere Überschneidung hinsichtlich der Ursachen für die letzten Milestones dar, weil sie hier wie dort mitentscheidend waren. Für sich genommen sind die Haushaltsregeln jedoch unterschiedlich und folglich führten sie auch zu unterschiedlichen Ergebnissen. In den USA waren die permanent überholten CBO-Budgetprognosen ohne feste Obergrenzen - erneut - einem Instrumentenwechsel förderlich. Die „fixed direct payments“ mussten den Instrumenten weichen, die bei einem niedrigeren Marktpreisniveau für ein höheres Niveau der Stützung sorgen. In der EU hingegen hatten die Haushaltsregeln - das System der fixen Mittelzuweisungen und der Lastenausgleich - eine eher konservierende Wirkung. Sie trieben die Reform in dieselbe Richtung voran wie die Erwartung sinkender Preise: zur Beibehaltung der Direktzahlungen. Im Jahr 2013 fehlte ein starker externer Faktor, der diese Wirkungen hätte aushebeln können. Wie von von Cramon-Taubadel (2017) ausgeführt, könnte der Austritt des Vereinigten Königreichs aus der EU für die GAP nach 2020 solch ein Reformimpuls werden, weil er den bestehenden Lastenausgleich aus der Balance bringen könnte.

Weitere Erklärungen für die eingetretene Divergenz von Agrarpolitiken von USA und EU kann die Prüfung der Hypothese von Josling und Tangermann (2015) hinsichtlich dieser Auseinanderentwicklung liefern, was eines der eingangs formulierten, wesentlichen Ziele dieser Arbeit ist. Die Annahme von Josling und Tangermann (2015: 265) besagt, dass die jüngste Divergenz von Agrarpolitiken beidseits des Atlantiks auf ein unterschiedliches innenpolitisches Umfeld, in dem 
die jeweiligen letzten Reformen beschlossen werden, zurückzuführen ist. Als die wesentliche Ursache für die politischen Entscheidungen sowohl in den USA im Jahr 2014 als auch in der EU im Jahr 2013 identifizieren sie die Erosion der gesellschaftlich-politischen Basis für die Direktzahlungen. Auf diese Legitimationskrise reagierten beide Akteure aus politischem Kalkül heraus unterschiedlich. Deshalb fiel das Reformergebnis hier wie dort unterschiedlich aus. Vor diesem Hintergrund sehen Josling und Tangermann (2015: 163) keine „,new and fundamentally diverging conditions in agriculture " als Ursache für die stattgefundene Auseinanderentwicklung der USA und der EU in der Frage der Direktzahlungen. In ihrem Buch streifen sie diese Frage allerdings nur kurz mit einem Hinweis auf eine interessante Forschungslücke, die einer Bearbeitung bedarf. Die vorliegende Analyse zeigt, dass die Ursachen für die Milestones 2014 und 2013 in der Tat „neuen Bedingungen" nicht zugeordnet werden können. Die Preisvolatilität und Phasen hoher Marktpreise auf den Agrarmärkten waren zu der Zeit keine neuen Phänomene. Zudem kann angesichts der identifizierten Gemeinsamkeiten in Bezug auf die Ursachen für das dritte Milestone-Paar auch nicht von grundlegend verschiedenen Bedingungen gesprochen werden. Sowohl in den USA als auch in der EU fehlten externe Einflussfaktoren, die als (zusätzlicher) Reformimpuls bzw. Korrektiv fungieren würden und die die unterschiedlichen Reformergebnisse erklären könnten. Daher sind die Ursachen der unterschiedlichen Reformergebnisse eindeutig im innenpolitischen Umfeld zu suchen.

Für Josling und Tangermann (2015) ist die Legitimationskrise der Direktzahlungen die wesentliche - und gemeinsame - Ursache für die politischen Entscheidungen 2013 und 2014, die hier als dritte Milestones gekennzeichnet sind. Die vorliegende Arbeit hingegen sieht die Legitimationskrise nicht als einen eigenständigen Reformtreiber an, weil sie sehr eng mit anderen Einflussfaktoren verknüpft ist. Eine Legitimationskrise kann grundsätzlich als Ausdruck der Unzufriedenheit mit der bisherigen Politik gewertet werden und diese kann verschiedene Gründe haben. Durch die Analyse dieser Gründe verspricht sich die Verfasserin, Erkenntnisse in Bezug auf die Forschungsfrage zu gewinnen. Es stellt sich also die Frage, woraus sich die Legitimationskrise der Direktzahlungen in den USA und in der EU speiste und ob diesbezüglich Gemeinsamkeiten oder eher Unterschiede zwischen den beiden Akteuren bestehen. Vor diesem Hintergrund wird bei der Auswertung der relevanten Literatur im vorausgegangenen Kapitel darauf geachtet, separat die in der Wissenschaft identifizierten Faktoren hinter der jeweiligen Legitimationskrise festzuhalten. Ihr Ursprung und ihre Besonderheiten werden im vorausgegangenen Kapitel jeweils näher beleuchtet. Diese Zusammenstellung liefert Hinweise darauf, dass die Legitimationskrise der Direktzahlungen in den USA und in der EU auf unterschiedliche Gründe zurückzuführen ist. Bevor auf diese im Detail eingegangen wird, bleibt noch anzumerken, dass die agrarpolitische Legitimationskrise mitnichten ein neues Phänomen ist. Einen Akzeptanzverlust erfuhr 
z. B. die US-Agrarpolitik Anfang der 1960er-Jahre (Cochrane und Ryan 1976), aber auch die EU-Agrarpolitik Anfang der 1990er-Jahre (Ahner 2010).

In den USA wurden die Direktzahlungen vornehmlich deshalb kritisiert, weil sie auch in Zeiten hoher Marktpreise gewährt wurden. Vor diesem Hintergrund wundert es nicht, dass sie ab 2008 - als die Marktpreise zu steigen begannen - an Zustimmung verloren. Diese führten zu Rekordeinkommen in der Landwirtschaft, selbst in Zeiten, in denen sich die übrige Wirtschaft in einer schweren Krise befand und das Haushaltsdefizit Rekordhöhe erreichte. In dieser Situation rückte die Agrarstützung in den Mittelpunkt der öffentlichen Aufmerksamkeit. In der Kritik standen insbesondere die "fixed direct payments“, deren Gewährung in Zeiten hoher Marktpreise in der Gesellschaft zunehmend auf Unverständnis stieß. Auch wurde bemängelt, dass von ihnen vornehmlich Großbetriebe profitieren. Folglich wurden sie unter Zustimmung der landwirtschaftlichen Verbände abgeschafft; der Großteil des dadurch eingesparten Betrages wurde aber innerhalb des Agrarhaushalts umgeschichtet. Es ist nicht überraschend, dass die Faktoren, die dieser Legitimationskrise zugrunde lagen, mit den im vorausgegangenen Kapitel identifizierten Ursachen für die Abschaffung der Direktzahlungen übereinstimmen.

Die Auswertung der Literatur ergab, dass in der EU der bröckelnden gesellschaftlich-politischen Basis für die Direktzahlungen die gesellschaftlichen Forderungen nach einer umweltfreundlicheren Landwirtschaft sowie der Haushaltsdruck zugrunde lagen. Letzterer äußerte sich in den Forderungen, die Agrarausgaben stark zu kürzen und sie ggf. für andere Bereiche zur Verfügung stellen zu wollen. Die Fragen hoher Marktpreise und Agrareinkommen gewannen in der EU nicht die gleiche Dynamik und Bedeutung wie in den USA (in beiden Wirtschaftsräumen gab es aber die Kritik, dass die Direktzahlungen überwiegend an große, nicht bedürftige Betriebe fließen). Die EU-Politik reagierte mit dem Greening der Direktzahlungen in der Hoffnung, durch die Erfüllung der Forderungen im Umweltbereich die Gründe hinter der Legitimationskrise zu dämpfen. Dieses half, zumindest vorerst, das Instrument der Direktzahlungen zu erhalten. Anders formuliert: Die Direktzahlungen wurden nicht abgeschafft, weil die Politik auf einen Teil der Gründe für den Akzeptanzverlust einging. Sollte sich das eingeführte Greening in Sachen Ökologie jedoch als wenig wirksam erweisen - was sich seit geraumer Zeit abzeichnet - kann wohl davon ausgegangen werden, dass bei der nächsten GAP-Reform der Haushaltsdruck umso stärker präsent sein wird. Vor diesem Hintergrund haben die Faktoren, die der Legitimationskrise im Vorfeld der GAP-Reform von 2013 zugrunde lagen, immer noch das Potenzial, eine Umgestaltung der Direktzahlungen anzutreiben.

Die USA und die EU reagierten deshalb mit „different political calculations“ (Josling und Tangermann 2015: 163) auf die jeweilige Legitimationskrise der Direktzahlungen, weil diese - bis auf die vorgenannte Kritik an Direktzahlungen zugunsten von Großbetrieben - unterschiedliche Gründe hatten. Dieses hilft zu erklären, warum es in den jüngsten Reformen zu unterschiedlichen Reformergebnis- 
sen in Bezug auf die Direktzahlungen und somit auch zur Divergenz von Agrarpolitiken von USA und EU kam. Da der Legitimationskrise in der EU zwei Faktoren zugrunde lagen, hatte sie mehr Spielräume, mit ihr umzugehen, indem sie geschickt einen Faktor gegen den anderen ausspielte. Die USA hingegen konnten auf die Kritik zur Einkommenslage nur mit einer Abschaffung - eventuell einer Reduzierung - der Direktzahlungen reagieren. In beiden Fällen aber lagen den Legitimationskrisen rein innenpolitische Faktoren zugrunde. Der Reformprozess wurde durch die der jeweiligen Entwicklung förderlichen Haushaltsregeln begünstigt. Sie bestimmten den Rahmen für die „political calculations“ beider Akteure.

Die Hypothese von Josling und Tangermann (2015) wird also zum Teil bestätigt und ergänzt. Die für die Reformen beidseits des Atlantiks relevanten Bedingungen hatten keinen neuen oder grundlegend verschiedenen Charakter. Die Ursachen für die Divergenz sind innenpolitischer Natur. Anders aber als bei Josling und Tangermann (2015), die auf die Legitimationskrise der Direktzahlungen als den für beide Akteure gemeinsamen Reformtreiber fokussieren, wird in der vorliegenden Arbeit hinter die jeweilige Legitimationskrise geschaut. Damit konnten wichtige Unterschiede zwischen den USA und der EU identifiziert werden. Die Analyse der jeweiligen Legitimationskrise bestätigt die Ergebnisse der bisherigen Analyse von Ursachen der dritten Milestones und liefert zudem v. a. für die EU neue Erkenntnisse.

Auf Basis der Gegenüberstellung der USA mit der EU aus den vorausgehenden Kapiteln können weitere Aspekte identifiziert werden, die Hinweise dafür liefern können, warum sich die Abschaffung der Direktzahlungen in der EU schwieriger als in den USA gestaltet und es somit zur Divergenz von Agrarpolitiken kam. Als Faktoren, die eher innerhalb des Systems der Agrarpolitik anzusiedeln sind, interessierten sie bei der Suche nach den Ursachen für die einzelnen Milestones nicht. Sie können aber dazu beitragen, das Bild über die Entwicklung der Direktzahlungen auf beiden Seiten des Atlantiks zu vervollständigen. Auf die Bedeutung solcher Analysen auf Mikroebene weisen Coleman und Grant (1998) hin.

In der EU - im Gegensatz zu den USA - fehlt ein Alternativinstrument für die Direktzahlungen. Das Beispiel der USA zeigt, dass es eines attraktiven Instruments bedarf, um die Akzeptanz der Landwirte für eine Reform zu gewinnen (in der jetzigen Form erfüllt die 2. Säule der GAP dieses Kriterium nicht). Dieses muss auch die Akzeptanz der Gesellschaft bzw. der Steuerzahler genießen. In den USA haben die Ernteversicherungsprogramme diese Rolle inne. Beliebt bei den Landwirten (83\% der Anbaufläche wird laut Shields 2013 durch eine solche Versicherung gedeckt), genießen sie auch das Wohlwollen der Gesellschaft. Laut Crop Insurance (2016) unterstützen 79\% der Befragten die Subventionierung der Ernteversicherung.

Zudem lässt sich feststellen, dass eine Abschaffung der Direktzahlungen in der EU eine radikalere Reform als in den USA bedeuten würde. Das hängt mit der un- 
terschiedlichen Stellung, die dieses Instrument beidseits des Atlantiks hat bzw. hatte, zusammen (s. Kapitel 6). Diese differiert hinsichtlich des Gewichts der Direktzahlungen unter den agrarpolitischen Instrumenten, ihres Anwendungsbereichs und ihrer Bedeutung für die Landwirte. In den USA waren die Direktzahlungen bei weitem kein Hauptinstrument der Agrarstützung und die Mehrheit der US-Landwirtschaft profitierte davon nicht. Zudem weisen die Zahlungen im Rahmen der Ernteversicherung eine ähnliche Verteilung nach Bundesstaaten wie die ,fixed direct payments“ auf. Entsprechend begrenzt war die Reform von 2014. In der EU hingegen wäre ein Großteil der Stützung der Landwirtschaft und damit fast jeder Landwirt von einer entsprechenden Reform betroffen. Solche umfassenden Reformen dürften wesentlich schwieriger zu beschließen sein.

Mit der Entscheidung im Jahr 2014, die „fixed direct payments“ abzuschaffen, wandten sich die USA vom Entkopplungskurs ab. Allerdings ist zu berücksichtigen, dass die Entkopplung in beiden Wirtschaftsräumen schon vor den letzten Reformen einen unterschiedlichen Stellenwert hatte. Während in der EU der Großteil der Einkommensstützung entkoppelt ist, war in den USA auch vor 2014 der Großteil dieser Stützung zumeist an die aktuellen Marktpreise gekoppelt. Auch die nach 1996 eingeführten „commodity payments“ (Titel I der Farm Bill) waren mit der Ausnahme von ,transition payments“ für Baumwolle von 2014 alle zumindest teilweise gekoppelt. Die „fixed direct payments“ genossen in der Agrarpolitik der USA somit eine Exotenstellung, weshalb ihre Abschaffung mit Blick auf die agrarpolitische Systematik der USA als ein logischer Schritt angesehen werden kann. Zulauf und Orden (2014: 48) stellen fest, dass „US farm policy in 2014 has the same underlying countercyclical foundation as it had in the last two decades of the $20^{\text {th }}$ century and from its historical roots“. Schon die im Jahr 1933 eingeführten „benefit payments“ hatten einen antizyklischen Charakter. Denn sie waren Teil eines komplexen Produktionsanpassungsprogramms, das sich am Paritätspreis des jeweiligen Erzeugnisses - einer Art von „target price“ - orientierte.

Es ist schließlich nicht von der Hand zu weisen, dass in den USA möglicherweise eine größere Transparenz in Bezug auf die Entwicklung der landwirtschaftlichen Einkommen die Abschaffung der Direktzahlungen begünstigte. Mit dem Indikator „Durchschnitts- und Medianeinkommen landwirtschaftlicher Haushalte" ist ein direkter Vergleich des Durchschnitts- und Medianeinkommens in der Landwirtschaft mit dem entsprechenden Einkommen aller US-Haushalte möglich. Ein ähnlicher Indikator wird in der EU nicht geführt. Deshalb konnte die Begründung der Kommission (2010a) für ihren Vorschlag für die GAP nach 2013, landwirtschaftliche Einkommen seien erheblich niedriger als die Einkommen in der übrigen Wirtschaft, nicht mit konkreten Zahlen widerlegt werden. Die von dieser Behauptung abgeleitete Notwendigkeit einer fortwährenden Einkommensstützung in Form von EU-finanzierten Direktzahlungen stellt die Wissenschaft regelmäßig infrage (z. B. Swinbank 2012: 26f). 
Somit spielten die spezifische Ausgestaltung der agrarpolitischen Maßnahmen insgesamt und die jeweilige Stellung des Instruments der Direktzahlungen für den Konvergenzprozess zwischen den Agrarpolitiken der USA und der EU hinsichtlich der Direktzahlungen eine bedeutende Rolle. Sie liefern insbesondere für die neuerdings stattgefundene Auseinanderentwicklung beider Akteure Erklärungen.

\subsection{Analyse der Konvergenzmuster}

Im Kapitel 7.3 wurde der Verlauf des Konvergenzprozesses zwischen den USA und der EU in Bezug auf die Anwendung der Direktzahlungen in der Agrarpolitik dargestellt. Nachfolgend wird untersucht, wie die Analyse von Ursachen der ausgewählten Milestones zum besseren Verständnis dieses Prozesses beitragen kann.

\subsubsection{Untersuchung der Kausalmechanismen der Politikkonvergenz}

Als erster Schritt sollen die im vorausgegangenen Kapitel identifizierten, unter den „problem stream“ fallenden Einflussfaktoren für die Milestones den Kausalmechanismen der Politikkonvergenz zugeordnet werden. Die Handelsverhandlungen im Rahmen der Uruguay- und Doha-Runden bzw. die WTO zusammen mit der EU-Erweiterung fallen unter die Harmonisierung nationaler Politiken mittels internationalen Rechts, während der internationale Austausch im Rahmen der OECD eindeutig und neue Ideen zum Teil der transnationalen Kommunikation zuzuordnen sind. Einige Faktoren sind dem vergleichbaren Problemdruck zuzuordnen. Dazu zählen Umweltbelange und die Preisvolatiliät bzw. die Erwartung sinkender Marktpreise. Haushaltsdruck, Verbraucheranliegen sowie hohe Marktpreise haben hingegen kein Pendant auf der anderen Seite des Atlantiks und qualifizieren sich somit nicht als vergleichbarer Problemdruck. Die Frage der internationalen Wettbewerbsfähigkeit der Landwirtschaft schließlich fällt unter den Regulierungswettbewerb. Diese Zuordnung der Einflussfaktoren ist zunächst theoretischer Natur und bedeutet nicht zwangsläufig, dass die Kausalmechanismen in dem hier interessierenden Fall der Politikkonvergenz tatsächlich so gegriffen haben. Dieses wird Gegenstand der nachfolgenden Untersuchung.

Entscheidend für die Interpretation der Analyseergebnisse ist die Wahl des Konvergenztyps. Wie im Kapitel 7.3 ausgeführt, kommen hier grundsätzlich zwei Konvergenztypen infrage: die Beta- und die Delta-Konvergenz. Es wurde festgestellt, dass die Beta-Konvergenz - die die Annäherung an einen Vorreiter beschreibt - ein klares Muster mit den USA als „Pionier“ und der EU als „Nachzügler" aufweist. Bei der Untersuchung der Triebkräfte für die Reformen wird zudem ein Einfluss der USA auf die Entwicklungen in der EU erkennbar. ${ }^{91}$ Eine umge-

\footnotetext{
${ }^{91}$ Es interessieren hier nur die gegenseitigen Einflüsse zwischen den USA und der EU. Einflüsse anderer Länder wie z. B. der Cairns-Gruppe werden hier nicht berücksichtigt.
} 
kehrte Tendenz konnte nicht identifiziert werden. Der indirekte Einfluss der USA erfolgte insbesondere über die WTO, aber auch über die OECD. Denn es waren die USA, die sich für das Einbinden der Landwirtschaft in die Uruguay-Runde stark machten. In der Doha-Runde drängten sie darauf, die Blue Box abzuschaffen. Zudem verweist Paarlberg (1997) auf den direkten Druck der USA auf die EU, Anfang der 1990er-Jahre die Politik im Ölsaatenbereich zu verändern. Die damalige Schlüsselrolle der USA auf der internationalen Bühne unterstreicht Bureau (2012: 15): „After the 1985 and 1996 Farm Bills, the US was a key actor [Herv. durch die Verf.] in promoting the reforms agreed upon within the OECD Ministerial Mandate and then underlined the 1994 WTO Agreement. It championed the removal of the largest distortions in world markets, by shifting from supply control, price management and export subsidies toward more neutral direct payments." In der Literatur wird dieser Einfluss registriert und festgehalten. Beispielsweise bemerkt Harvey (1998: 9), dass „the European Union [is] already adopting set-aside and limited deficiency payments for arable crops, in practical imitation of the U.S. [Herv. durch die Verf.]“. Lynggaard und Nedergaard (2009: 304) schreiben zur MacSharry-Reform, dass "the CAP had undergone a transformation from a high price policy to a low price policy combined with direct payment ad modum the US agricultural policy model [Herv. durch die Verf. und zum Teil im Original]". Laut Delorme (1995: 32) reformierte die EU im Jahr 1992 ihre Agrarpolitik nach dem Vorbild der USA, was zu einer Harmonisierung der Agrarpolitiken beider Akteure führte.

Es stellt sich die Frage, warum sich in den vielen Jahren kein umgekehrter Einfluss feststellen lässt. Mahé und Tavéra (1989) liefern dafür im Hinblick auf die Situation in den 1980er-Jahren zwei Erklärungen. Zum einen hätten die USA grundsätzlich mehr von der Liberalisierung der EU-Agrarpolitik profitiert als umgekehrt. Die Autoren heben insbesondere die positiven Auswirkungen der GAPReformen auf den US-Haushalt ${ }^{22}$ hervor, was daran läge, dass die „deficiency payments“ im US-Getreidesektor von Marktpreisen abhingen, für die sie dann einen Anstieg prognostizierten. Zum anderen seien Exportländer wie die USA grundsätzlich „,more active, display more efforts to alleviate trade barriers, and argue for free trade as a desirable target" (ibid.: 328). Zwar wurde mit der Zeit auch die EU Nettoexporteur vieler Agrarprodukte; ihre Agrarpolitik jedoch war weiter auf einen Nettoimporteur zugeschnitten. Weil „this net exporting situation [was] viewed to be lacking in legitimacy since it [was] largely due to protectionist policies", stand die EU in den 1980er-Jahren unter erheblichem Druck seitens ihrer Handelspartner (ibid.).

\footnotetext{
${ }^{92}$ Nach Berechnungen von Koester et al. (1988: 40) betragen die Haushaltseinsparungen bzw. -verluste der EU im Falle der Liberalisierung der US-Agrarpolitik je nach Option -0,1 bis 0,4 Mrd. ECU, während die Haushaltseinsparungen der USA im Falle der Liberalisierung der EU-Agrarpolitik sich je nach Option auf 1,3 bis 1,8 Mrd. ECU belaufen. Mahé und Tavéra wirkten bei dieser Studie mit.
} 
Eine Erklärung, warum es zu dem besagten Konvergenzmuster mit den USA als Vorreiter und der EU als „Nachzügler“" kommen konnte, könnte darin bestehen, dass in der EU die vergleichbaren Probleme bzw. der Wettbewerbsdruck später als in den USA auftraten bzw. sich zuspitzten. Das würde heißen, dass beide Akteure vergleichbaren Druck verspürten, allerdings zu unterschiedlichen, zum Teil sehr weit auseinanderliegenden Zeitpunkten. Die Reaktionen darauf wären jedoch in Bezug auf die gewählten Instrumente ähnlich. Zu den Problemen, die infrage kommen, weil sie auf beiden Seiten des Atlantiks für die Milestones mitentscheidend waren, gehören die internationale Wettbewerbsfähigkeit, die Preisvolatilität sowie Umweltbelange. Nach dieser These wäre beispielsweise die Frage der internationalen Wettbewerbsfähigkeit der Landwirtschaft für die USA schon in den 1960er-Jahren so wichtig und dringend, dass sie mit einem Instrumentenwechsel reagierten, während diese Frage in der EU erst Anfang der 1990er-Jahre an vergleichbarer Bedeutung gewann. Vor diesem Hintergrund wäre die neuerdings eingetretene Divergenz nichts anderes als ein kurzfristiges Zwischenspiel. Sobald die EU mit einem ähnlich dringenden vergleichbaren Problemdruck konfrontiert wird, könnte vermutet werden, dass sie wie die USA reagieren und die Direktzahlungen abschaffen wird, wodurch das Konvergenzmuster wiederhergestellt wäre. Zwar übten bereits in der letzten Reform der GAP - wie in den USA - hohe Marktpreise für landwirtschaftliche Erzeugnisse Druck auf den EU-Agrarhaushalt aus, dieser konnte jedoch durch die Preisvolatilität (noch) neutralisiert werden.

Diese Erklärung des Musters der Beta-Konvergenz wäre denkbar, wenn die besagten Faktoren die wichtigsten für die jeweiligen EU-Milestones wären bzw. es keine Kausalmechanismen für die Konvergenz gäbe, die stärker wirkten. Ersteres trifft für die zwei Milestones, durch die sich die EU-Agrarpolitik der US-Agrarpolitik in Bezug auf die Direktzahlungen anglich, nicht zu. Die Auswertung der Literatur liefert viele Hinweise darauf, dass die WTO in beiden Fällen den bedeutsamsten Einflussfaktor darstellte. Auf der Suche nach Kausalmechanismen, die stärker als der vergleichbare Problemdruck wirkten, lohnt ein genauerer Blick auf die Delta-Konvergenz, die die Annäherung an das „decoupled income support“Modell, also die Direktzahlungen im Sinne der Definition dieser Arbeit, beschreibt. Im Kapitel 7.3 wird geprüft, wie schnell sich die USA und die EU diesem Modell annäherten. Es stellte sich heraus, dass - beim gemeinsamen Ausgangspunkt - die EU gegenüber den USA in der Entwicklung der Direktzahlungen die meiste Zeit zurückblieb. Jedoch ist die EU der beweglichere Akteur, denn die Abstände zwischen den einzelnen Milestones sind hier kürzer als in den USA.

Sowohl in den USA als auch in der EU konnte eine Zeit lang eine Annäherung an das „decoupled income support"-Modell beobachtet werden. Nach der Abschaffung der „fixed direct payments“ in den USA gilt diese Aussage nur für die EU. Der Blick auf die Einflussfaktoren für ihre ersten zwei Milestones macht deutlich, dass dort die Harmonisierung nationaler Politiken über internationales Recht (WTO) und die transnationale Kommunikation (OECD und neue Ideen) 
zu den wichtigen Kausalmechanismen der Delta-Konvergenz gehören. Daraus, dass die WTO - wie die Literaturauswertung ergab - den bedeutsamsten Einflussfaktor darstellt, kann geschlossen werden, dass im Falle der EU die Harmonisierung nationaler Politiken den wichtigsten Kausalmechanismus der Konvergenz bildet. In den USA hingegen spielte keiner dieser Kausalmechanismen eine Rolle, was den Eindruck entstehen lässt, dass es sich hier um eine Delta-Konvergenz handelt, die von den politischen Akteuren nicht unmittelbar beabsichtigt war. Beispielsweise führten die USA die Ausgleichszahlungen ein, bevor dieses Instrument auf die Agenda der WTO und der OECD kam. Auch die ersten Entkopplungsschritte von 1985 erfolgten vor der wegweisenden Erklärung der OECD-Minister von 1987, die ein Bekenntnis zur Reform der Agrarpolitik und zur UruguayRunde des GATT enthielt. Die Einflussfaktoren hinter diesen Reformschritten waren vielmehr innenpolitischer Natur. Umso leichter fiel es den USA, vom „decoupled income support"-Modell abzukehren, wenn sich die politische und wirtschaftliche Lage im Land änderte.

Es spricht einiges dafür, dass sich die EU nicht an den USA orientierte und die Delta-Konvergenz den Reformprozess in der EU besser als die Beta-Konvergenz beschreibt. Zum einen fällt auf, dass die EU den USA nur insoweit folgte, solange sich die Politik der USA in Bezug auf die Direktzahlungen dem „decoupled income support" "Modell annäherte bzw. diesem entsprach. Die neuerliche Divergenz zwischen beiden Akteuren entstand, weil sich die USA von den Direktzahlungen abwandten, die EU dem jedoch nicht folgte. Zum anderen geht die EU, was die übrige Agrarpolitik angeht, eindeutig andere Wege als die USA. Die Risikomanagementinstrumente - platziert in der 2. Säule und mit der Green Box der WTO vereinbar ${ }^{93}$ - stehen in der EU agrarpolitisch nicht im Fokus; antizyklische Zahlungen gewährt sie nicht. Auch im Umweltbereich bestehen beidseits des Atlantiks andere Schwerpunkte, denn die USA bevorzugen das Instrument der Flächenstilllegung (Bureau 2012: 10). Zudem spielen die Ernährungsprogramme in der US-Agrarpolitik eine Haupt-, in der EU-Agrarpolitik eine marginale Rolle (ibid.: 76). Würde sich die EU in Bezug auf die Direktzahlungen an den USA orientieren, würde eine Annäherung auch bei anderen agrarpolitischen Instrumenten zu erwarten sein.

Schließlich lehnte die EU das in den USA geltende Modell bzw. Paradigma der Landwirtschaft explizit ab - auch in Zeiten der Konvergenz ihrer Agrarpolitiken in Bezug auf die Direktzahlungen. Bereits vor der Reform von 1992, die den ersten Milestone im Sinne dieser Arbeit bildet, schrieb die Kommission (1985a: II): „An agriculture on the model of the USA with vast spaces of land and few farmers, is neither possible nor desirable in European conditions, in which the basic concept remains the family farm." Dieses bekräftigte sie beispielsweise in der fol-

${ }^{93}$ Die Ernte- und Erlösversicherungen in den USA sind so konstruiert, dass sie unter die Amber Box der WTO fallen (Schnepf 2015). 
genden Aussage: „[T] proaches such as those adopted in the United States of America, where farms are very large and farmers few in number, is neither feasible nor desirable" (Kommission 1985b: o.S.). Auch später grenzte die Kommission das EU-Modell vom Modell der USA deutlich ab (z. B. Kommission 1998: 7). Bis heute bestehen große Differenzen zwischen den USA und der EU hinsichtlich der Ausrichtung der Agrarpolitik (Bureau 2012: 26). Während die USA insbesondere die Verbesserung der Wettbewerbsfähigkeit des Sektors sowie die Steigerung der Exporte anstreben, "[s] uch an ambition is less obvious in the EU reforms“, wo ,the focus has been more on social and environmental issues than in structural policies that would boost EU competitiveness in world markets“ (ibid.). Dass die von 1992 bis 2014 zu beobachtende Konvergenz zwischen den Agrarpolitiken der USA und der EU nur in Bezug auf das Instrument der Direktzahlungen, nicht aber in Bezug auf die Paradigmen bzw. Modelle erfolgte, bestätigt die in der Einleitung angeführte These von Knill (2005), dass Politikkonvergenz auf Ebene der politischen Instrumente wahrscheinlicher ist als auf Ebene der Paradigmen.

\subsubsection{Untersuchung der intervenierenden Faktoren}

Aus der vorliegenden Analyse geht hervor, dass hinter der Konvergenz zwischen den Agrarpolitiken der USA und der EU hinsichtlich der Direktzahlungen recht verschiedene Konstellationen standen, auch wenn einige Faktoren beidseits des Atlantiks wirkten. Zwar gibt es Hinweise darauf, dass Regulierungswettbewerb und vergleichbarer Problemdruck eine gewisse Rolle spielten, allerdings erklären diese Kausalmechanismen nicht den gesamten Konvergenzprozess. Weiterhin gibt es Hinweise darauf, dass sich die EU in Fragen der Agrarpolitik nicht an den USA orientierte. Nichtsdestotrotz ist für den Zeitraum 1992 bis 2013 eine faktische Konvergenz zwischen den USA und der EU bei den Direktzahlungen nicht zu leugnen, die 2014 in einen Divergenzprozess umgeschlagen ist. Wenn diese Entwicklung nicht als klares Ergebnis eines Kausalmechanismus gedeutet werden kann, stellt sich die Frage, worauf sie tatsächlich zurückzuführen ist.

Liefern Kausalmechanismen keine befriedigende Erklärung, lohnt ein Blick über sie hinaus auf die sogenannten intervenierenden Faktoren, die laut Konvergenztheorie imstande sind, die Wirksamkeit dieser Mechanismen zu beeinflussen. Zunächst bleibt festzustellen, dass im untersuchten Fall der USA und der EU lediglich institutionelle Faktoren von Bedeutung sind. Kulturelle und sozio-ökonomische Faktoren dürften hier weniger eine Rolle spielen, denn beide Akteure gehören zur sogenannten westlichen Welt mit entsprechenden kulturellen und sozioökonomischen Ähnlichkeiten. Es bleiben institutionelle Faktoren, die in der vorliegenden Arbeit im „politics stream“ für die einzelnen Milestones festgehalten sind. Dieses stimmt mit der These von Lenschow et al. (2005) in Bezug auf intervenierende Faktoren überein, wonach im Falle der Konvergenz auf Ebene der Po- 
litikinstrumente nicht kulturelle und sozio-ökonomische, sondern institutionelle Aspekte eine dominierende Rolle spielen.

Im Hinblick auf die bis zum Jahr 2013 festgestellte Annäherung zwischen den USA und der EU bei den Direktzahlungen lässt sich feststellen, dass auf beiden Seiten des Atlantiks eine Reihe von institutionellen Faktoren die Entwicklung Richtung (entkoppelte) Direktzahlungen begünstigte. In den USA sticht insbesondere der Kongress als der für die hier interessierenden Entscheidungen wichtigste Akteur des politischen Prozesses hervor. Für die Entwicklungen in der EU scheint die Europäische Kommission eine ähnlich wichtige Rolle zu spielen. Darauf, dass dort auch der Rat und (2013) das Europäische Parlament eine Rolle spielten, deutet die Bedeutung des Gesetzgebungsverfahrens für die zwei letzten Milestones hin. Auch scheint in der EU der Einfluss der Interessengruppen außerhalb des Agrarsektors auf die Ausgestaltung der Milestones größer als in den USA gewesen zu sein. Dieses könnte damit zusammenhängen, dass die Direktzahlungen das wichtigste Instrument der EU-Agrarpolitik darstellen und als solches große Aufmerksamkeit auf sich ziehen. Vor diesem Hintergrund überrascht die Koalition der Agrarlobby mit den Vertretern ökologischer Interessen in der jüngsten Reform von 2013 nicht. In den USA wurde bereits beim ersten Milestone mit den Repräsentanten urbaner Interessen koaliert. Die Koalition in den USA scheint vergleichsweise stabil zu sein, während die Allianz in der EU bereits nach der Entscheidung des Jahres 2013 über den Finanzrahmen für die Jahre 2014 bis 2020 ins Wanken geriet. Erstere bietet zudem mehr Spielräume für die Ausgestaltung politischer Entscheidungen, während die politische Allianz in der EU eine stärkere Ausrichtung auf Umweltschutz begünstigt. Nicht zuletzt waren auch die Haushaltsregeln für die Entwicklung der Direktzahlungen in den USA und der EU relevant.

In Bezug auf die Divergenz zwischen den USA und der EU nach 2013 sind die Einflussfaktoren relevant, die für die Abschaffung der Direktzahlungen in den USA und ihre Beibehaltung in der EU verantwortlich waren. Dazu haben insbesondere die jeweiligen Haushaltsregeln beigetragen, weil sie in den USA einem Instrumentenwechsel grundsätzlich förderlich und in der EU hingegen eher hinderlich sind. Da die EU-Haushaltsregeln den vorausgegangenen Konvergenzprozess nicht verhinderten, sind für den untersuchten Konvergenzprozess die USHaushaltsregeln offenbar von größerer Bedeutung. In der EU hatten auch die übrigen, für den Milestone 2013 relevanten „politics stream"-Faktoren eine eher konservierende Wirkung. Insbesondere ist es in der auf 27 bzw. 28 Länder erweiterten Union und mit dem Europäischen Parlament als vollberechtigtem Akteur schwieriger als früher, substanzielle Entscheidungen zu treffen. Dieses kann die relativ bescheidenen Ergebnisse der Reform von 2013 und den Stillstand in Bezug auf die Weiterentwicklung der Direktzahlungen mit erklären. Die US-Politik hingegen scheint entscheidungsfreudiger zu sein. Die stabile Koalition mit den Vertretern urbaner Interessen bietet zudem genug Spielraum für die Ausgestaltung von Reformen. Die institutionellen Faktoren können also auch Erklärungen für 
die Tatsache, dass die USA in Bezug auf die Direktzahlungen gegenüber der EU die Vorreiterrolle innehaben, liefern.

Man kann folglich zu dem Schluss kommen, dass die institutionellen Faktoren beidseits des Atlantiks zur Konvergenz zwischen den Agrarpolitiken der USA und der EU beitrugen, indem sie - unabhängig voneinander - die Einführung von Direktzahlungen im jeweiligen Land begünstigten. Auch trugen sie maßgeblich dazu bei, dass 2014 in Bezug auf die Direktzahlungen eine Divergenz entstand. Mehr noch: Im untersuchten Konvergenzfall scheinen die institutionellen Rahmenbedingungen eine Rolle gespielt zu haben, die über die den intervenierenden Faktoren zugeschriebene Funktion, wonach sie lediglich die Wirksamkeit von Kausalmechanismen beeinflussen, hinausragt.

\subsubsection{Divergenz: Ausblick}

Schließlich wirkt sich die Wahl des Konvergenztyps für die Interpretation der Analyseergebnisse auch auf den Ausblick für die weitere Entwicklung der Agrarpolitik beidseits des Atlantiks aus. Insbesondere ist davon die Prognose der Dauer der neuerdings eingetretenen Divergenz zwischen den USA und der EU in Bezug auf die Direktzahlungen betroffen. Wird die Beta-Konvergenz für richtig erachtet, ist zu erwarten, dass die Konvergenz zwischen den beiden Akteuren wiederhergestellt wird. In diesem Fall müsste die EU die Direktzahlungen gänzlich abschaffen. Für einen solchen Schritt scheint aber kein politischer Wille zu bestehen. Die Divergenz wäre auch beendet, wenn die USA zum Instrument der entkoppelten Direktzahlungen zurückkehren würden. Bureau (2012: 78) hält einen solchen Schritt angesichts des politischen Umfelds jedoch für unwahrscheinlich. Aber auch die Historie der Stützung für die Landwirtschaft spricht dafür, dass sich die USA nicht umentscheiden werden. Im Kapitel 4 konnte gezeigt werden, dass schon die ersten breiteren Agrarzahlungen - „benefit payments“ und „parity payments“ der 1930er-Jahre - einen antizyklischen Charakter hatten. Dieses zog sich wie ein roter Faden durch die Entwicklung der US-Agrarpolitik. Mit der Abschaffung der Direktzahlungen erhöhten die USA sowohl die Konsistenz ihrer Agrarpolitik als auch die gesellschaftliche Akzeptanz für sie.

Wie bereits ausgeführt wurde, bestehen Gründe zu der Annahme, dass die Beta-Konvergenz nicht geeignet ist, um die Agrarreformprozesse in den USA und in der EU in Bezug auf die Direktzahlungen zu analysieren. Wird nach der DeltaKonvergenz gefragt, ist zu erwarten, dass die aktuelle Divergenz kein vorübergehendes Phänomen ist, sondern dass sie auch in Zukunft bestehen bleibt. Dieses würde aber nicht unbedingt bedeuten, dass der Status quo in der EU unverändert fortgeführt wird. Das „decoupled income support"-Modell wird fortentwickelt, denn ,[d] ecoupling is certainly not the end of the story“ (van Tongeren 2008: 3). Anreger ist hier wieder die OECD, die für das Konzept des „targeting“ wirbt: 
Decoupled policies in OECD countries have successfully redirected some agricultural policies towards more economically efficient ways to deliver income support to farmers, but decoupling is in itself not sufficient to achieve all the policy objectives [...]. Reconnecting agricultural polices with these objectives is the major task that lies ahead for policy makers. The concept of targeting attempts to address more effectively and more efficiently the market failures and income problems that sometimes prevail in agriculture [...]. (ibid.: 12)

Durch eine Ausrichtung der Maßnahmen auf explizit definierte und messbare Politikziele verspricht sich die OECD eine erhebliche Verbesserung der Ergebnisse der Agrarpolitik. Dieses Konzept ersetzt nicht das „decoupled income support“Modell, sondern baut darauf auf. Es ist eine Reformrichtung, die die EU möglicherweise mit dem Greening-Konzept von 2013 versuchte einzuschlagen. Dabei ist das „targeting“ nicht auf Umweltziele beschränkt. Auch landwirtschaftliche Einkommen können auf diese Art und Weise unterstützt werden, wenn das Gesamteinkommen eines Betriebes als Grundlage fungiert (van Tongeren 2008). Der jetzige EU-Weg, kleine Betriebe stärker zu fördern, erfüllt die OECD-Kriterien für eine zielgerichtete Agrarpolitik allerdings nicht. Auch in der Wissenschaft besteht Übereinstimmung darüber, dass die Direktzahlungen in der EU in der jetzigen Form - pauschal ausgeschüttet - ausgedient haben. Jambor und Harvey (2010: 20) fassen die aktuelle Debatte über die Direktzahlungen wie folgt zusammen: „Direct payments are not effective or justified, and should be reduced or totally phased out." Die Reformkonzepte reichen vom einkommensabhängigen Sicherheitsnetz (Swinnen 2009) zu Zahlungen zur Verbesserung der Umweltleistung der Landwirtschaft (Bureau und Mahé 2008, Isermeyer 2014, Tangermann 2014). Ohne eine erhebliche Umgestaltung der heutigen Direktzahlungen sind solche Reformkonzepte nicht zu realisieren.

Nichtsdestotrotz erntet die EU für ihre Agrarpolitik auch Lob. Insbesondere dann, wenn diese mit der US-Agrarpolitik verglichen wird, die die Landwirte von Schwankungen auf den Agrarmärkten abschirmt. Vor diesem Hintergrund erfuhren die USA wenig Lob für die Agrarreform von 2014, obwohl die Wissenschaft die Direktzahlungen als Übergangsmaßnahme propagiert, was mit der Entscheidung 2014 - 18 Jahre nach ihrer Einführung - tatsächlich verwirklicht wurde. In der EU werden vor allem das Festhalten an dem System der produktionsunabhängigen, die Marktsignale nicht verfälschenden Direktzahlungen und der Wille, das System der WTO zu erhalten, positiv bewertet (Bureau 2012: 78). Die EU „respects not only the multilateral rules but also acts in a way that favour progress in international discipline“ (ibid.: 75). Laut Josling (2015: 488) ,it can now be reasonably said that the CAP is in the lead in the search for a farm policy that provides a stable environment for farming and is minimally distuptive to trade“. Daugbjerg und Swinbank (2015: 3) bemerken, dass im Vergleich zu den USA ,the EU's 
achievement is remarkable“. Vor diesem Hintergrund wäre eine Wiederherstellung der Beta-Konvergenz, bei der sich die EU-Agrarpolitik an die US-Agrarpolitik angleicht, nicht wünschenswert, sondern eine Fortführung der Bemühungen um die Delta-Konvergenz durch Übernahme des ,targeting“-Modells. 


\section{Resümee}

Die vorliegende Arbeit stellt eine Konvergenzanalyse in Bezug auf das Instrument der Direktzahlungen in der Agrarpolitik der USA und der EU dar. Es wird der Frage nachgegangen, inwiefern sich die Agrarpolitiken der USA und der EU bei der Anwendung der Direktzahlungen im Zeitverlauf einander annäherten. Neben der Beschreibung des Verlaufs dieses Konvergenzprozesses besteht der Kern der Arbeit darin, Erklärungen für festgestellte Konvergenzen bzw. Divergenzen herauszuarbeiten. Damit wird versucht, eine Forschungslücke zu schließen, auf die Josling und Tangermann (2015) hinweisen. Da hier die Frage der Angleichung von Politiken zweier ausgewählter Wirtschaftsräume im Fokus steht, bildet die Konvergenztheorie den theoretischen Rahmen der Untersuchung.

Als erster Schritt wurden die Begrifflichkeiten präzisiert. Als Direktzahlungen werden in der vorliegenden Untersuchung Zahlungen an Landwirte verstanden, die im Sinne des „decoupled income support“ des Anhangs 2 des Übereinkommens über die Landwirtschaft von der Erzeugung sowie von den Marktpreisen entkoppelt sind. Solche Zahlungen sind im WTO-Regelwerk als Green-Box-Maßnahme eingestuft. Mit Blick auf die Forschungsfrage sind die so definierten Direktzahlungen deshalb interessant, weil sie eine zentrale Rolle in den jeweiligen Reformprozessen weg von produktionsabhängigen Stützungsmaßnahmen, insbesondere der Preisstützung, spielten. Auch bildeten sie - angesichts der teilweise sehr ausgeprägten Differenzen hinsichtlich des Instrumentariums und der Zielset- 
zung - eine der wenigen Gemeinsamkeiten zwischen den Agrarpolitiken der USA und der EU. Entsprechend setzte eine divergierende Entwicklung ein, als die USA 2014 die Direktzahlungen abschafften. Sämtliche direkte, aus Steuergeldern finanzierte Zahlungen an Landwirte einschließlich der Direktzahlungen werden für die Zwecke der vorliegenden Untersuchung als staatliche Zahlungen bzw. Agrarzahlungen bezeichnet.

Nachdem die grundlegenden Begriffe geklärt wurden, wurde die Geschichte der staatlichen Zahlungen seit ihrer Ersteinführung in den USA und der EU dargestellt. Die bis 2014 stattgefundenen Agrarreformprozesse auf beiden Seiten des Atlantiks wurden auf die Fragen untersucht, wann das Instrument der Direktzahlungen zum ersten Mal eingesetzt wurde und wie es sich im Laufe der Jahre entwickelt hat. In den USA waren staatliche Zahlungen an Landwirte von Anbeginn der vom „New Deal“ 1933 initiierten, gezielten Agrarstützung Teil des agrarpolitischen Instrumentariums, auch wenn sie sich als primäres Instrument der Agrarpolitik zunächst nicht durchsetzten. Vielmehr waren sie als Anreiz für Produktionsbeschränkungen im Einsatz und bereits damals antizyklisch angelegt. Der erste Versuch einer Umstellung weg von der Preisstützung und hin zu einer auf Zahlungen basierten Einkommensstützung - der Brannan-Plan von 1949 - blieb noch erfolglos. Erst Anfang der 1960er-Jahre wurden schrittweise den Abbau der Preisstützung flankierende Ausgleichszahlungen für Weizen, Futtergetreide und Baumwolle - später „deficiency payments“ genannt - eingeführt. Lange Zeit waren diese Zahlungen als gekoppelte Hilfen in Kraft. Erste größere Schritte Richtung Entkopplung von 1985 und 1990 führten dazu, dass „deficiency payments“ als Blue-Box-Maßnahme notifiziert werden konnten. Dieses war allerdings nur ein Jahr - 1995 - relevant, weil 1996 entkoppelte ,production flexibility contract payments“ im Rahmen des „FAIR Act“ eingeführt wurden. Bereits zwei Jahre später kehrte die US-Politik vom Entkopplungskurs teilweise wieder ab: Auf den Rückgang der Marktpreise reagierte sie mit der Einführung der „market loss assistance payments", die 2002 als Bestandteil der Farm Bill institutionalisiert wurden. In den darauffolgenden Jahren wurden die antizyklischen Programme um weitere Agrarzahlungen ergänzt, während die entkoppelten „fixed direct payments“ - der Nachfolger der Zahlungen von 1996 - im Jahr 2014 abgeschafft wurden. Damit wurde der Entkopplungskurs in der US-Agrarpolitik beendet.

Auch zu Beginn der GAP waren staatliche Zahlungen in Kraft, die aber - ähnlich wie in den USA - eine marginale Rolle spielten. Neben dem prominenten Beispiel der dreijährigen Ausgleichszahlungen an Landwirte in Deutschland, Italien und Luxemburg, die von der Getreidepreissenkung infolge der Getreidepreisharmonisierung von 1967 betroffen waren, stellten sie das Instrument der Wahl für einige wenige Erzeugnisse dar, die nicht in großen Mengen oder flächendeckend produziert wurden. Eine breitere Nutzung dieses Instruments lehnte die Europäische Kommission aus finanziellen sowie verwaltungstechnischen Gründen zwar ab, stellte aber bereits Mitte der 1970er-Jahre einen Einsatz von Zahlungen, die 
nicht an die Produktion einzelner Erzeugnisse gebunden sind, zur Diskussion. Die früheren Versuche einer Ergänzung der Preispolitik um staatliche Zahlungen der Mansholt-Plan von 1968 und die Regelung zur landwirtschaftlichen Einkommensbeihilfe von 1989 - scheiterten zunächst. Die agrarpolitische Bedeutung der Zahlungen an Landwirte stieg erst mit der Einführung von teilentkoppelten Ausgleichszahlungen in Folge der MacSharry-Reform von 1992. Die nächste GAPReform - die „Agenda 2000“ - zementierte die EU-Direktzahlungen als Hauptinstrument der Agrarstützung, während die Reform von 2003 den Entkopplungsprozess entscheidend weiter vorantrieb. Auch im Laufe der Reform von 2013 wich die EU-Politik nicht vom Entkopplungskurs ab, auch wenn die Möglichkeiten, die gekoppelte Stützung anzuwenden, ausgeweitet wurden.

Aufbauend auf dieser Darstellung erfolgte die Bestimmung der Milestones in der Entwicklung der Direktzahlungen auf beiden Seiten des Atlantiks. Unter Milestones werden in der Untersuchung substanzielle Reformen der Agrarpolitik in Bezug auf die Direktzahlungen verstanden. In den USA wurden als solche die Einführung der Ausgleichszahlungen 1962 bis 1965, die Ersteinführung der Direktzahlungen 1996 sowie ihre Abschaffung 2014 identifiziert. Da dort - anders als in der EU - teilentkoppelte Ausgleichszahlungen nicht auf einmal, sondern schrittweise eingeführt wurden, wurden die beiden Teilentkopplungsschritte 1985 und 1990 dem ersten Milestone zugeteilt. Analog zu den USA zählen in der EU die Einführung der Ausgleichszahlungen 1992, die Ersteinführung der Direktzahlungen 2003 sowie ihre Beibehaltung 2013 zu den ausgewählten Milestones. Letztere wurde insbesondere vor dem Hintergrund der darauf zurückgehenden Auseinanderentwicklung zwischen den USA und der EU als Milestone festgelegt.

Die anschließende Gegenüberstellung der ausgewählten Milestones machte es möglich, den Verlauf des Prozesses der Konvergenz bzw. der Divergenz zwischen den Agrarpolitiken der USA und der EU abzubilden. Besonders gut eignete sie sich dafür, die Beta-Konvergenz - die Annäherung an einen Vorreiter - zu untersuchen. Es konnte hinsichtlich der Direktzahlungen ein klares Muster mit den USA als „Pionier“ und der EU als „Nachzügler“ festgestellt werden. Mehr noch: Auch ein gewisser Einfluss der USA auf die EU-Reformen ist erkennbar. Eine umgekehrte Tendenz konnte hingegen nicht festgestellt werden. Dieser Prozess der Angleichung der EU-Politik an die US-Politik wurde in der letzten GAPReform zwar unterbrochen, aber nicht unbedingt beendet. Sollte nämlich die EU in Zukunft die Direktzahlungen abschaffen, wäre dieses Konvergenzmuster wieder hergestellt.

Auch die Delta-Konvergenz, die die Annäherung an ein Politikideal beschreibt, konnte durch die ausgewählten Milestones abgebildet werden. Das anstrebenswerte Modell bildeten hier die Direktzahlungen im Sinne der vorliegenden Arbeit („decoupled income support“). Die Annäherung daran begann im Falle beider Akteure mit der Einführung der Ausgleichszahlungen und mündete in ihrer Entkopplung im Jahr 1996 bzw. 2003. Zwar setzten die USA das Modell früher als 
die EU um; jedoch ist die EU der beweglichere Akteur, denn die Abstände zwischen den einzelnen Milestones sind hier kürzer als in den USA (insbesondere wurden die Direktzahlungen relativ zügig nach den Ausgleichszahlungen eingeführt). Die EU ist es auch, die weiterhin am „decoupled income support“-Modell festhält. In den USA hingegen endete der ursprünglich ähnlich verlaufene Konvergenzprozess mit der Abschaffung der Direktzahlungen 2014, was zu einer Divergenz in der Agrarpolitik der USA einer- und der EU andererseits führte.

Auf die Gegenüberstellung der Milestones folgte der Vergleich der Einflussfaktoren, die ihnen zugrunde lagen. Diese wurden anhand der Auswertung der Literatur zu den agrarpolitischen Reformen, deren Bestandteil die ausgewählten Milestones bilden, für jeden Milestone herausgefiltert und zusammengestellt. Der Fokus lag auf den Einflussfaktoren aus dem externen Umfeld der Agrarpolitik, die sich insbesondere zu der Zeit des Reformvorschlags auf Reforminhalte auswirkten und nicht unter „political rhetoric“ fallen. Vor dem Hintergrund der Forschungsfrage interessierten in erster Linie die Faktoren, die dem „problem stream“ nach Kingdon (1995) zuzuordnen sind, weil sie länderübergreifend wirksam sein können und deswegen besonders geeignet sind, Politikkonvergenz zu erklären. Außer diesen wurden zusätzlich die „politics stream“-Faktoren, die auf nationaler Ebene greifen, für die Milestones zusammengestellt.

Die zusammengetragenen Antriebskräfte für die jeweiligen Milestones in den USA und der EU wurden anschließend auf Gemeinsamkeiten und Unterschiede untersucht, um auf dieser Grundlage Erklärungen für Konvergenzen bzw. Divergenzen zwischen den Agrarpolitiken beider Akteure bei der Anwendung der Direktzahlungen herauszuarbeiten. Im Fokus der Analyse standen zunächst einzelne Einflussfaktoren an sich und ihre Bedeutung für die Entwicklung der Direktzahlungen beidseits des Atlantiks; anschließend wurden die jeweiligen MilestonePaare hinsichtlich der Einflussfaktoren untersucht und verglichen. Die Ergebnisse dieser Auswertung haben gezeigt, dass die festgestellte Konvergenz nicht auf ein Wirken von Kausalmechanismen zurückzuführen ist. Es konnten zwischen den USA und der EU nur einige wenige Gemeinsamkeiten bei den Einflussfaktoren festgestellt werden, die als vergleichbarer Problemdruck bzw. regulativer Wettbewerb interpretiert werden können. Dieses trifft zudem nur auf das erste Milestone-Paar - die Einführung von Ausgleichszahlungen - zu. Für die durch das zweite Milestone-Paar abgebildete Einführung von Direktzahlungen waren beidseits des Atlantiks völlig unterschiedliche Ursachen ausschlaggebend, weswegen hier kein Kausalmechanismus abgeleitet werden kann. Folglich standen hinter der nicht zu leugnenden Konvergenz der US- und der EU-Agrarpolitik hinsichtlich der Direktzahlungen recht verschiedene Konstellationen.

Vor diesem Hintergrund scheint im untersuchten Fall den sogenannten intervenierenden Faktoren in Form von institutionellen Faktoren eine wichtige kausale Rolle zuzukommen. Diese begünstigten - unabhängig voneinander - die Einführung von Direktzahlungen im jeweiligen Wirtschaftsraum. Auch bewirkten die in- 
stitutionellen Faktoren, insbesondere die Haushaltsregeln, dass trotz gleicher Marktbedingungen - hohe, aber volatile Marktpreise - die USA und die EU in den letzten Reformen unterschiedlich reagierten. Somit trugen sie maßgeblich zu der durch das dritte Milestone-Paar abgebildeten divergierenden Entwicklung bei. Weitere Erkenntnisse zu dieser Divergenz brachte die Überprüfung der Hypothese von Josling und Tangermann (2015). Es lässt sich feststellen, dass der von ihnen betonten Legitimationskrise der Direktzahlungen als gemeinsamen Grund für die Entscheidungen 2013 und 2014 unterschiedliche und zudem jeweils rein innenpolitische Faktoren zugrunde lagen. Auch das hilft, die unterschiedlichen Reaktionen der USA und der EU auf gleiche Marktbedingungen in diesen Jahren zu erklären. Außerdem liegt die Vermutung nahe, dass die jeweilige Stellung des Instruments der Direktzahlungen unter den agrarpolitischen Maßnahmen sowie die spezifische Ausgestaltung der Agrarpolitik für die neuerdings stattgefundene Auseinanderentwicklung beider Akteure relevant sind. In der EU würde eine Abschaffung der Direktzahlungen einen Großteil der Agrarstützung und somit fast jeden Landwirt betreffen und damit eine radikalere Reform als in den USA darstellen. In den USA kann die Streichung der Direktzahlungen aufgrund des gekoppelten Charakters der meisten übrigen Agrarstützungsmaßnahmen sogar als ein logischer Schritt angesehen werden. Darüber hinaus war dort ein attraktives, von der Gesellschaft akzeptiertes Alternativinstrument in Form der Ernteversicherung zum Zeitpunkt der Reform vorhanden. Möglicherweise begünstigte auch eine im Vergleich zur EU größere Transparenz in Bezug auf die Entwicklung der landwirtschaftlichen Einkommen die Entscheidung der USA zur Abschaffung der Direktzahlungen.

Im Fokus der vorliegenden Arbeit stehen konkrete agrarpolitische Entwicklungen in zwei ausgewählten Wirtschaftsräumen. Es stellte sich dabei die Frage nach dem Ursprung der Idee einer staatlichen Zahlung und insbesondere einer Direktzahlung. Die Schilderung der Behandlung von staatlichen Zahlungen und insbesondere von Direktzahlungen in der wissenschaftlichen Literatur im Kapitel 3 hatte das Ziel, diesbezüglich mehr Klarheit zu schaffen. Es konnte gezeigt werden, dass die Politik staatliche Zahlungen an die Landwirte bereits relativ stark, insbesondere zur Steigerung der heimischen Erzeugung, einsetzte, bevor sie in der Wissenschaft diskutiert wurden. Die USA als ehemalige Kolonie des Britischen Empire scheinen hier eine Pionier-Rolle zu spielen. Die Idee der Direktzahlungen hingegen scheint ihren Ursprung in der Wissenschaft zu haben. Auch wenn die US-amerikanischen Ökonomen - wie die praktische US-Politik - lange Zeit eine Sympathie für antizyklische Zahlungen hegten, wurden die ersten Entkopplungskonzepte in den USA erarbeitet. Den Anfang scheint W. W. Cochrane gemacht zu haben. Auf seinen noch einsamen Vorschlag von 1945 folgte Ende der 1950erund Anfang der 1960er-Jahre eine regelrechte Welle von Entkopplungskonzepten. In Kontinentaleuropa fand eine ähnliche Entwicklung in der ersten Hälfte der 1970er-Jahre statt. In beiden Ländern bzw. Wirtschaftsräumen dauerte es aber 
sehr lange, bis die theoretisch erarbeiteten Konzepte in der praktischen Politik umgesetzt, das heißt bis die Direktzahlungen eingeführt wurden. Der direkte Einfluss wissenschaftlicher Ideen auf die politische Entscheidungsfindung muss letztendlich als begrenzt beurteilt werden, ihre Wirkungsmöglichkeiten beschränken sich jedoch mitnichten darauf. Was die Ideenflüsse zwischen den USA und der EU in der Frage der Direktzahlungen betrifft, konnten keine direkten gegenseitigen Einflüsse festgestellt werden. Die Voraussetzungen dafür scheinen allerdings gegeben zu sein.

Fest steht, dass die Entwicklung der Direktzahlungen beidseits des Atlantiks bis 2013 von Konvergenz und seit 2014 von Divergenz gekennzeichnet ist. Es stellte sich die Frage, ob diese neuerdings stattgefundene Auseinanderentwicklung ein kurzfristiges Phänomen ist oder auf Dauer bestehen bleibt. Damit verbunden ist die Frage, welches der beiden Konvergenzmuster sich besser eignet, die hier interessierenden Entwicklungen zu beschreiben. Es gibt überzeugende Hinweise darauf, dass sich die EU in Fragen der Agrarpolitik und insbesondere der Direktzahlungen nicht an den USA orientierte und damit die Delta-Konvergenz den EUReformprozess besser als die Beta-Konvergenz beschreibt. Folglich würde die Konvergenz zwischen den Agrarpolitiken beider Akteure hinsichtlich der Direktzahlungen nicht wiederhergestellt werden, weil die EU die Direktzahlungen nicht (gänzlich) abschaffen und/oder die USA sie nicht wieder einführen würden. Dieses würde jedoch nicht unbedingt bedeuten, dass der Status quo in der EU unverändert fortgeführt wird. Wahrscheinlicher scheint hier eine Weiterentwicklung der Direktzahlungen in Richtung umweltbezogenen ,targeted payments“ zu sein, während ein Ausbau von Risikomanagement-Instrumenten aufgrund von aktuell geltenden Haushaltsregeln eher problematisch erscheint.

Es empfiehlt sich, die Konvergenzanalyse erneut durchzuführen, sobald die Ergebnisse der nächsten Reform der GAP vorliegen. Sollte die EU - was sich bereits jetzt abzeichnet - die Direktzahlungen für die Förderperiode nach 2020 beibehalten, würde sich an der Divergenz zwischen den USA und der EU in Bezug auf dieses Instrument zwar nichts ändern. Allerdings würden möglicherweise neue Einflussfaktoren ins Spiel kommen, die für den Vergleich der jeweiligen dritten Milestones weitere Erkenntnisse liefern. 


\section{Literaturverzeichnis}

Ahner, D. (2010). Die Gemeinsame Agrarpolitik - von der Vergangenheit in die Zukunft. Rede zur Verabschiedung von Prof. Dr. Stefan Tangermann. German Journal of Agricultural Economics 59 (Supplement Perspectives on International Agricultural Policy - In Honor of the Retirement of Professor Stefan Tangermann): 33-36.

Ambrosius, G. (2005). Regulativer Wettbewerb und koordinative Standardisierung zwischen Staaten. Theoretische Annahmen und historische Beispiele. Stuttgart: Franz Steiner Verlag.

Anania, G. (2009). Multilateral trade negotiations and the CAP. Working Paper 09/10. Paper presented at the Conference „The Faculty of Economics of Siena University remembers Secondo Tarditi: International Trade Liberalization and EU Agricultural and Consumer Policies“, Siena, 21. Oktober 2008.

Anderson, K. (Hrsg.) (2010). The Political Economy of Agricultural Price Distortions. New York: Cambridge University Press.

Anderson, K., Rausser, G. und Swinnen, J. (2013). Political Economy of Public Policies: Insights from Distortions to Agricultural and Food Markets. Policy Research Working Paper 6433. Washington D.C.: World Bank. 
Anderson, M. und Magleby, R. (Hrsg.) (1997). Agricultural Resources and Environmental Indicators, 1996-97. Agricultural Handbook 712. Economic Research Service, U.S. Department of Agriculture.

Babcock, B.A. (2011). Something for Nothing? Direct Payments and Title I Farm Programs. Washington D.C.: American Enterprise Institute.

Bachmann, H. (1958). Die Integration der Agrarmärkte. Aussenwirtschaft: Zeitschrift für Internationale Wirtschaftsbęiehungen 13: 25-48.

Baffes, J. und de Gorter, H. (2005). Disciplining Agricultural Support through Decoupling. Policy Research Working Paper 3533. Washington D.C.: World Bank.

Ballinger, R.A. (1946). Sugar during World War II. War Records Monograph 3. Bureau of Agricultural Economics, U.S. Department of Agriculture.

Ballinger, R.A. (1975). A history of sugar marketing through 1974. Agricultural Economic Report 382. Economics, Statistics, and Cooperatives Service, U.S. Department of Agriculture.

Batie, S.S. und Schweikhardt, D.B. (2007). The Green Payment Debate: Alternative Paradigms and Resulting Tradeoffs. In Knutson, R.D., Knutson, S.D. und Ernstes, D.P. (Hrsg.), Perspectives on 21st Century Agriculture: A Tribute to Walter J. Armbruster. Oak Brook: Farm Foundation, 158-166.

Beckman, J., Arita, S., Mitchell, L. und Burfisher, M. (2015). Agriculture in the Transatlantic Trade and Investment Partnership: Tariffs, Tariff-Rate Quotas, and Non-Tariff Measures. Economic Research Report 198. Economic Research Service, U.S. Department of Agriculture.

Beer, G.L. (1907). British Colonial Policy 1754-1765. New York: The Macmillan Company.

Benedict, M.R. (1953). Farm policies of the United States 1790-1950: A study of their origins and development. New York: The Twentieth Century Fund.

Black, J.D. (1946a). Agricultural Economists' Views on Farm Price Policy. Journal of Farm Economics 28(2): 604-607.

Black, J.D. (1946b). Professor Schultz and C.E.D. on agricultural policy in 1945. Journal of Farm Economics 28(3): 669-686.

Blandford, D., Josling, T. und Bureau, J.-C. (2011). The Future of Direct Payments. Washington D.C.: International Food and Agricultural Trade Policy Council.

BML (Hrsg.) (1975). Der Wissenschaftliche Beirat beim Bundesministerium für Ernährung, Landwirtschaft und Forsten: Sammelband der Gutachten von 1949 bis 1974. Münster-Hiltrup: Landwirtschaftsverlag. 
Bowers, D.E., Rasmussen, W.D. und Baker, G.L. (1984). History of Agricultural Price-Support and Adjustment Programs, 1933-1984. Background for 1985 Farm Legislation. Agriculture Information Bulletin 485. Economic Research Service, U.S. Department of Agriculture.

Bowers, J.K. (1985). British Agricultural Policy Since the Second World War. Agricultural History Review 33: 66-76.

Brandow, G.E. (1955). A Modified Compensatory Price Program for Agriculture. Journal of Farm Economics 37(4): 716-730.

Bureau J.-C. (2012). Latest U.S. Farm Bill developments. A Note for the European Parliament, IP/B/AGRI/CEI/2011-097/E021/SC01.

Bureau, J.-C. und Mahé, L.-P. (2008). CAP reform beyond 2013: An idea for a longer view. Studies and research 64. Notre Europe.

Bureau, J.-C. und Mahé, L.-P. (2015). Was the CAP reform a success? In Swinnen, J. (Hrsg.), The Political Economy of the 2014-2020 Common Agricultural Policy: An Imperfect Storm. Brussels: Centre for European Policy Studies;

London: Rowman and Littlefield International, 87-135.

Bureau, J.-C., Witzke, H.-P., Gohin, A., Heckelei, T., Wieck, C., Chouinard, H., Rudloff, B. und Bernard, B. (2009). The US Farm Bill 2008 - Comparison with the EU CAP after Health Check. A Study for the European Parliament, IP/B/AGRI/FWC/2006-146-Lot4-C01-SC1.

Burfisher, M.E. und Hopkins, J. (Hrsg.) (2003). Decoupled Payments: Household Income Transfers in Contemporary U.S. Agriculture. Agricultural Economic Report 822. Economic Research Service, U.S. Department of Agriculture.

Cahill, C. (2010). OECD's work on agricultural policy: A bridge between research and government. German Journal of Agricultural Economics 59 (Supplement Perspectives on International Agricultural Policy - In Honor of the Retirement of Professor Stefan Tangermann): 10-18.

Cahill, C. und Legg, W. (1989-1990). Estimation of Agricultural Assistance Using Producer and Consumer Subsidy Equivalents - Theory and Practice. OECD Economic Studies 13, 13-43.

Cain, Z. und Lovejoy, S. (2004). History and Outlook for Farm Bill Conservation Programs. Choices 19(4): 37-42.

Carter, C.A. (2014). Some Trade Implications of the 2014 Agricultural Act. Choices 29(3): 1-4.

CBO (2014). Effects on direct spending and revenues of the conference agreement on H.R. 2642, as reported on January 27, 2014. Congressional Budget Office.

CBO (2015). CBO’s March 2015 Baseline for Farm Programs. Congressional Budget Office. 
CED (1962). An Adaptive Program for Agriculture. A Statement on National Policy by the Research and Policy Committee of the Committee for Economic Development. Committee for Economic Development.

CEPR (2013). Reducing Transatlantic Barriers to Trade and Investment: An Economic Assessment. Final Project Report, prepared under implementing Framework Contract TRADE10/A2/A16. London: Centre for Economic Policy Research.

Cherington, P.T. (1912). State Bounties and the Beet-Sugar Industry. Quarterly Journal of Economics 26(2): 381-386.

Chite, R.M. (2011). Emergency Funding for Agriculture: A Brief History of Supplemental Appropriations, FY1989-FY2012. CRS Report for Congress RL31095. Congressional Research Service.

Chite, R.M. (Coordinator) (2014). The 2014 Farm Bill (P.L. 113-79): Summary and Side-by-Side. CRS Report for Congress R43076. Congressional Research Service.

Claassen, R. (2012). The Future of Environmental Compliance Incentives in U.S. Agriculture: The Role of Commodity, Conservation, and Crop Insurance Programs. Economic Information Bulletin 94. Economic Research Service, U.S. Department of Agriculture.

Claassen, R. und Morehart, M. (2006). Conservation Program Design: Greening Income Support and Supporting Green. Economic Brief 1. Economic Research Service, U.S. Department of Agriculture.

Clawson, M. (1958). Agricultural adjustment reconsidered: Changes needed in the Next 25 Years. Journal of Farm Economics 40(2): 265-277.

Cochrane, W.W. (1945). An Honorable Mention Paper: A price policy for agriculture, consistent with economic progress, that will promote adequate and more stable income from farming. Journal of Farm Economics 27(4): 813-820.

Cochrane, W.W. und Ryan, M.E. (1976). American Farm Policy 1948-1973. Minneapolis: University of Minnesota.

Coleman, W.D. (2001). Agricultural policy reform and policy convergence: An actor-centered institutionalist approach. Journal of Comparative Policy Analysis 3(2): 219-241.

Coleman, W.D. und Grant, W.P. (1998). Policy convergence and policy feedback: Agricultural finance policies in a globalizing era. European Journal of Political Research 34(2): 225-247.

Coleman, W.D. und Tangermann, S. (1999). The 1992 CAP Reform, the Uruguay Round and the Commission: Conceptualizing Linked Policy Games. Journal of Common Market Studies 37(3): 385-405.

Coleman, W.D., Skogstad, G.D. und Atkinson, M.M. (1996). Paradigm Shifts and Policy Networks: Cumulative Change in Agriculture. Journal of Public Policy 16(3): 273-301. 
Collins, E.J.T. (2000). Food supplies and food policy. In Collins, E.J.T. (Hrsg.), The Agrarian History of England and Wales, Volume VII, 1850-1914. Cambridge: Cambridge University Press, 33-71.

Committee on Parity Concepts (1946). Outline of a Price Policy for American Agriculture for the Postwar Period. Journal of Farm Economics 28(1): 380-397.

Conley, H.H. (1996). The 1995 Farm Bill: Opportunities and Challenges for Economists. Journal of Agricultural and Applied Economics 28(1): 35-44.

Corbet, H. und van Riemsdijk, J.F. (Hrsg.) (1973). Wageningen memorandum on the reform of the European Community's Common Agricultural Policy. European Review of Agricultural Economics 1(2): 151-60.

Crop Insurance (2016). National Survey of Registered Voters Regarding Crop Insurance. National Crop Insurance Services, April 2016. URL: https:/ /www.ag-risk.org/NCISPUBS/Survey/CropInsPublicOpinionPoll05-2016.pdf (Abrufdatum: 14.12.2016).

CRS (2007). The USDA 2007 Farm Bill Proposal: Possible Questions. CRS Report for Congress RL33916. Congressional Research Service.

Cunha, A. und Swinbank, A. (2011). An Inside View of the CAP Reform Process: Explaining the MacSharry, Agenda 2000, and Fischler Reforms. Oxford: Oxford University Press.

Daugbjerg, C. (2003). Policy feedback and paradigm shift in EU agricultural policy: the effects of the MacSharry reform on future reform. Journal of European Public Policy 10(3): 421-437.

Daugbjerg, C. (2004). Why a Bond Scheme was not Adopted in 1992. In Swinbank, A. und Tranter, R. (Hrsg.), A Bond Scheme for Common Agricultural Policy Reform. Wallingford: CABI Publishing, 93-109.

Daugbjerg, C. (2009). Sequencing in public policy: the evolution of the CAP over a decade. Journal of European Public Policy 16(3): 395-411.

Daugbjerg, C. (2012). Globalization and Internal Policy Dynamics in the Reform of the Common Agricultural Policy. In Richardson, J. (Hrsg.), Constructing a Policy-Making State? Policy Dynamics in the EU. Oxford: Oxford University Press, 88-103.

Daugbjerg, C. und Swinbank, A. (2007). The Politics of CAP Reform: Trade Negotiations, Institutional Settings and Blame Avoidance. Journal of Common Market Studies 45(1): 1-22.

Daugbjerg, C. und Swinbank, A. (2009). Globalising EU Agricultural Policy Making? The Impact of the WTO, Budget and Multifunctional Paradigm in the Health Check Reform of the Common Agricultural Policy. Paper to be presented at the IPSA 21st World Congress of Political Science, July 12 to 16, 2009, Santiago, Chile. 
Daugbjerg, C. und Swinbank, A. (2010). Ideational Change in the WTO and its impacts on EU Agricultural Policy Institutions and the CAP. In Skogstad G. und Verdun, A. (Hrsg.), The Common Agricultural Policy: Policy Dynamics in a Changing Context. London, New York: Routledge, 44-60.

Daugbjerg, C. und Swinbank, A. (2015). Designing Durable Policy Reforms: Gradual Layering in the EU's Common Agricultural Policy over Three Decades. Paper for the ECPR Joint Sessions of Workshops, Warsaw, 29 March-2 April 2015.

Davis, J.S. (1935). Planned Agricultural Adjustment in the United States. In Holland, W.L. (Hrsg.), Commodity Control in the Pacific Area: A Symposium on Recent Experience. Stanford: Stanford University Press, 17-89.

Davis, J.S. (1939). On Agricultural Policy 1926-1938. Stanford: Stanford University Press.

de Gorter, H. (2008). Explaining Inefficient Policy Instruments. Agricultural Distortions Working Paper 75. Washington D.C.: World Bank.

Declaration by Agricultural Economists (2010). For an Ambitious Reform of the Common Agricultural Policy.

Delorme, H. (1995). L'accord de Marrakech: du conflit à la coopération entre l'Union Européenne et les États-Unis. Économie rurale 226(1): 31-34.

Deutsch, K.G. (2013). Transatlantischer Freihandel: Die wirtschaftlichen Chancen von T-TIP. Frankfurt am Main: Deutsche Bank Research.

Dohlman, E., Foreman, L. und Da Pra, M. (2009). The Post-Buyout Experience: Peanut and Tobacco Sectors Adapt to Policy Reform. Economic Information Bulletin 60. Economic Research Service, U.S. Department of Agriculture.

Drezner, D.W. (2001). Globalization and policy convergence. International Studies Review 3(1): 53-78.

Ecorys (2009). Non-Tariff Measures in EU-US Trade and Investment - An Economic Analysis. Reference: OJ 2007/S 180-219493, Final Report.

Effland, A.B. und Young, E. (2001). U.S. Farm Policy for the 21st Century: A Diversity of Visions for the Future. Agricultural Outlook May 2001. Economic Research Service, U.S. Department of Agriculture.

Eggert, R.J. (1945). An Honorable Mention Paper: A price policy for agriculture, consistent with economic progress, that will promote adequate and more stable income from farming. Journal of Farm Economics 27(4): 821-828.

Engels, W., Gutowski, A., Hamm, W., Möschel, W., Stützel, W., von Weizsäcker, C.C. und Willgerodt, H. (1985). Verordnete Verschwendung? Für eine Neue Agrarordnung in Europa. Stuttgart: Horst Poller Verlag.

Erdman, L. und Runge, C.F. (1990). American agricultural policy and the 1990 farm bill. Review of Marketing and Agricultural Economics 58(2-3): 109-126. 
Erjavec, E., Lovec, M. und Erjavec, K. (2015). From 'Greening' to 'Greenwash': The drivers and discourses of CAP 2020 'reform'. In Swinnen, J. (Hrsg.), The Political Economy of the 2014-2020 Common Agricultural Policy: An Imperfect Storm. Brussels: Centre for European Policy Studies; London: Rowman and Littlefield International, 215-244.

ERS-USDA (2016a). Historic data on mean and median farm operator household income and ratio of farm household to U.S. household income, 19602014. Economic Research Service, U.S. Department of Agriculture. Letzte Aktualisierung: 02.05.2016. URL: http://www.ers.usda.gov/data-products/ farm-household-income-and-characteristics.aspx (Abrufdatum: 01.06.2016).

ERS-USDA (2016b). Farm Income and Wealth Statistics: Value added by U.S. agriculture (includes net farm income). Economic Research Service, U.S. Department of Agriculture. Letzte Aktualisierung: 30.08.2016. URL: https://data.ers.usda.gov/reports.aspx?ID=17830 (Abrufdatum: 02.09.2016).

ERS-USDA (2016c). Farm Income and Wealth Statistics: Federal Government direct farm program payments (current dollars). Economic Research Service, U.S. Department of Agriculture. Letzte Aktualisierung: 30.08.2016. URL: https:/ / data.ers.usda.gov/reports.aspx?ID=17833 (Abrufdatum: 05.09.2016).

ERS-USDA (2016d). Farm Income and Wealth Statistics: Federal Government direct farm program payments (2009 dollars). Economic Research Service, U.S. Department of Agriculture. Letzte Aktualisierung: 30.08.2016. URL: https://data.ers.usda.gov/reports.aspx?ID=17833 (Abrufdatum: 05.09.2016).

Europäische Gemeinschaften (1971). Protokoll Nr. 20 über die norwegische Landwirtschaft zum Vertrag über den Beitritt von Dänemark, Großbritannien, Irland und Norwegen zur Europäischen Wirtschaftsgemeinschaft, Amtsblatt der EWG, Nr. L 73, 27. März 1972.

Europäischer Rat (1987). Schlussfolgerungen des Europäischen Rates, Brüssel, 29./30. Juni 1987.

Europäischer Rat (1988). Schlussfolgerungen des Europäischen Rates, Brüssel, 19. Februar 1988.

Europäischer Rat (1999). Schlussfolgerungen des Vorsitzes, Europäischer Rat in Berlin, 24./25. März 1999.

Europäischer Rat (2003). CAP Reform - Presidency Compromise (in agreement with the Commission).

Europäischer Rechnungshof (1999). Sonderbericht Nr. 2/99 über die Auswirkungen der Reform der GAP auf den Getreidesektor, zusammen mit den Antworten der Kommission (vorgelegt gemäß Artikel 248 Absatz 4 Unterabsatz 2 des EG-Vertrags), (1999/C 192/01). 
Europäischer Rechnungshof (2016). Is the Commission's system for performance measurement in relation to farmers' incomes well designed and based on sound data? Special report 01. Luxembourg: Publications Office of the European Union.

Europäisches Parlament (1981). Entschliessung zu möglichen Verbesserungen der gemeinsamen Agrarpolitik vom 17. Juni 1981. ABl. C 172/32.

Eurostat (2013a). Number of holdings and Utilised Agriculture Area (UAA) by UAA size classes in Romania 2010. Data from 16.07.2013. URL: http://ec.europa.eu/eurostat/statistics-explained/index.php/File:Figure_N umber_of_holdings_and_Utilised_Agriculture_Area_(UAA)_by_UAA_siz e_classes_RO_2010.PNG (Abrufdatum: 31.07.2016).

Eurostat (2013b). Number of holdings and Utilised Agriculture Area (UAA) by UAA size classes in Hungary 2010. Data from 11.07.2013. URL: http://ec.europa.eu/eurostat/statistics-explained/index.php/File:Figure_N umber_of_holdings_and_Utilised_Agriculture_Area_(UAA)_by_UAA_si ze_classes_HU_2010.PNG (Abrufdatum: 31.07.2016).

Eurostat (2013c). Number of holdings and Utilised Agriculture Area (UAA) by UAA size classes in Bulgaria 2010. Data from 02.07.2013. URL: http:/ /ec. europa.eu/eurostat/statistics-explained/index.php/File:Figure_Number_ of_holdings_and_Utilised_Agriculture_Area_(UAA)_by_UAA_size_classes _BG_2010.PNG (Abrufdatum: 31.07.2016).

Eurostat (2015). Key farm variables, by country, 2013. URL: http://ec.euro pa.eu/eurostat/statistics-explained/index.php?title=File:Key_farm_variab les,_by_country,_2013.png\&oldid=271649\#file (Abrufdatum: 31.07.2016).

Farm Futures (2011). The Farm Bill in 250 Words or Less. URL: http:/ /www.farmfutures.com/story-the-farm-bill-in-250-words-or-less-1844548 (Abrufdatum: 13.12.2016).

Feindt, P.H. (2007). Harmonisierung, Problemdruck, Kommunikation: Konvergenz in der Agrarpolitik der OECD-Länder, 1986-2004. In Holzinger, K., Jörgens, H. und Knill, C. (Hrsg.), Transfer, Diffusion und Konvergenz von Politiken. PVS-Sonderheft. Wiesbaden: VS Verlag für Sozialwissenschaften, 496-521.

Feindt, P.H., Gottschick, M., Mölders, T., Müller, F., Sodtke, R. und Weiland, S. (2008). Nachbaltige Agrarpolitik als reflexive Politik. Plädoyer für einen neuen Diskurs zwischen Politik und Wissenschaft. Berlin: Edition Sigma.

Felbermayr, G., Heid, B. und Lehwald, S. (2013). Die Transatlantische Handelsund Investitionspartnerschaft (THIP). Wem nutzt ein transatlantisches Freihandelsabkommen? Teil 1: Makroökonomische Effekte. Gütersloh: Bertelsmann Stiftung. 
Ferejohn, J. (1986). Logrolling in an Institutional Context: A Case Study of Food Stamp Legislation. In Wright, G.C., Rieselbach, L.N. und Dodd, L.C. (Hrsg.), Congress and Policy Change. New York: Agathon Press, 223-253.

Fouilleux, E. (2004). CAP Reforms and Multilateral Trade Negotiations: Another View on Discourse Efficiency. West European Politics 27(2): 235-255.

Froker, R.K. (1945). An Honorable Mention Paper: A price policy for agriculture, consistent with economic progress, that will promote adequate and more stable income from farming. Journal of Farm Economics 27(4): 844-851.

FSA-USDA (2010). Wheat farm programs and participation. Farm Service Agency, U.S. Department of Agriculture. Letzte Aktualisierung: 31.12.2010. URL: http://www.ers.usda.gov/data-products/wheat-data.aspx (Abrufdatum: 15.06.2015).

Galbraith, J.K. (1955). Farm Policy: The Current Position. Journal of Farm Economics 37(2): 292-304.

GAO (1989). Disaster Assistance: Crop Insurance Can Provide Assistance More Effectively Than Other Programs. Report to the Chairman, Committee on Agriculture, House of Representatives GAO/RCED-89-211. General Accounting Office.

GAO (2012). Farm Programs. Direct Payments Should Be Reconsidered. Report to Congressional Requesters GAO-12-640. General Accounting Office.

Gardner, B. (1992). Changing economics perspectives on the farm problem. Journal of Economic Literature 30(1): 62-101.

Gardner, B.L. (1996). Why Experts Have Changed Their Policy Tune? In Antle, J.M. und Sumner, D.A. (Hrsg.), Papers in Honor of D. Gale Johnson. The Economics of Agriculture. Chicago: University of Chicago Press, 225-243.

Gardner, B.L. (2009). United States and Canada. In Anderson, K. (Hrsg.), Distortions to agricultural incentives: A Global Perspective, 1955-2007. London: Palgrave Macmillan and Washington D.C.: World Bank, 177-220.

Garzon, I. (2006). Reforming the Common Agricultural Policy: History of a Paradigm Change. Basingstoke et al.: Palgrave Macmillan.

GATT (1990). European Economic Community - payments and subsidies paid to processors and producers of oilseeds and related animal-feed proteins. Report of the Panel adopted on 25 January 1990, L/6627 - 37S/86.

GEWISOLA (1969). Möglichkeiten und Grenzen der Agrarpolitik in der EWG. Schriften der Gesellschaft für Wirtschafts- und Sozialwissenschaften des Landbaues 6.

Glaser, L.K. (1986). Provisions of the Food Security Act of 1985. Agriculture Information Bulletin 498. Economic Research Service, U.S. Department of Agriculture. 
Glauber, J.W. und Westhoff, P. (2015). 50 Shades of Amber: The 2014 Farm Bill and the WTO. Invited paper presented at the session The 2014 Farm Bill: An Economic Post Mortem, Allied Social Science Associations (ASSA) Annual Meetings, Boston, Massachusetts, 4 January.

Haas, C.M. (2007). Budget und Haushaltsverfahren. In Jäger, W., Haas, C.M. und Welz, W. (Hrsg.), Regierungssystem der US A: Lehr- und Handbuch. München, Wien: Oldenbourg Verlag, 205-227.

Hadwiger, D.F. und Talbot, R.B. (1965). Pressures and Protests. The Kennedy Farm Program and the Wheat Referendum of 1963. San Francisco: Chandler Publishing Company.

Haggard, H.R. (1899). A Farmer's Year Being His Commonplace Book for 1898. London et al.: Longmans, Green, and Co.

Hall, P.A. (1993). Policy paradigms, social learning, and the state. The case of economic policymaking in Britain. Comparative Politics 25(3): 275-296.

Hamilton, D.E. (1991). From New Day to New Deal. American Farm Policy from Hoover to Roosevelt, 1928-1933. Chapel Hill: The University of North Carolina Press.

Hanau, A. (1971). Der Mechanismus der agrarpolitischen Willensbildung - dargestellt am Beispiel der Getreidepreisangleichung in der EU. In Schlotter, H.-G. (Hrsg.), Die Willensbildung in der Agrarpolitik. Schriften der Gesellschaft für Wirtschafts- und Sozialwissenschaften des Landbaues 8: 303-336.

Haniotis, T. (2006). The 2003 Reform of the European Union's Common Agricultural Policy and Its Relevance to the U.S. Farm Policy Debate. In Arha, K., Josling, T., Sumner, D.A. und Thompson, B.H. (Hrsg.), U.S. Agricultural Policy and the 2007 Farm Bill. Stanford: Woods Institute for the Environment.

Harvey, D.R. (1998). The US Farm Act: 'Fair' or 'Foul'?: An Evolutionary Perspective from East of the Atlantic. Conference Paper.

Harwood, J.L. und Young, C.E. (1989). Wheat: Background for 1990 Farm Legislation. Staff Report AGES-89-56. Economic Research Service, U.S. Department of Agriculture.

Haushaltsplan der EU für die Haushaltsjahre 1990, 1995, 1998, 2008, 2009, 2010, 2011, 2012, 2013, 2014, 2015 und 2016.

Heady, E.O. (1962). Agricultural Policy under Economic Development. Ames: Iowa State University Press. 
Heichel, S. und Sommerer, T. (2007). Unterschiedliche Pfade, ein Ziel? Spezifikationen im Forschungsdesign und Vergleichbarkeit der Ergebnisse bei der Suche nach der Konvergenz nationalstaatlicher Politiken. In Holzinger, K., Jörgens, H. und Knill, C. (Hrsg.), Transfer, Diffusion und Konvergenz von Politiken. PVS-Sonderheft. Wiesbaden: VS Verlag für Sozialwissenschaften, 107-130.

Heniff, B., Lynch, M.S. und Tollestrup, J. (2012). Introduction to the Federal Budget Process. CRS Report for Congress 7-5700. Congressional Research Service.

Henning, C.H.C.A. (2000). Macht und Tausch in der europäischen Agrarpolitik. Eine positive Theorie kollektiver Entscheidungen. Frankfurt am Main: Campus.

Henning, C.H.C.A. und Latacz-Lohmann, U. (2004). Will Enlargement Gridlock CAP Reforms? A Political Economy Perspective. EuroChoices 3(1): 38-43.

Herrmann, R, und Schäfer, A. (1985). Wie denken europäische Agrarökonomen über Wirtschafts- und Agrarpolitik? Agrarwirtschaft 34: 278-285.

Hill, B. (2012). Understanding the Common Agricultural Policy. London: Earthscan from Routledge.

Hill, B. und Bradley, B.D. (2015). Comparisons of Farmers' Incomes in the EU Member States. A Study for the European Parliament, IP/B/AGRI/IC/ 2014-68.

Hofreither, M.F. (1996). Inhalte und potentielle Konsequenzen des FAIR-Acts 1996. Diskussionspapier 60-W-96. Institut für Wirtschaft, Politik und Recht der Universität für Bodenkultur Wien.

Hofreither, M.F. (2012). WTO-Verpflichtungen der EU und GAP-Reform 2013 - Passen „begrünte“ Direktzahlungen in die „Greenbox“? Ländlicher Raum 4: 1-12.

Hofreither, M.F. (2016). Dimensionen agrarpolitischer Legitimität. Diskussionspapier DP-60-2016. Institut für nachhaltige Wirtschaftsentwicklung der Universität für Bodenkultur Wien.

Holzinger, K. und Knill, C. (2005). Causes and conditions of cross-national policy convergence. Journal of European Public Policy 12(5) (Special Issue): 775-796.

Holzinger, K., Jörgens, H. und Knill, C. (Hrsg.) (2007a). Transfer, Diffusion und Konvergenz von Politiken. PVS-Sonderheft. Wiesbaden: VS Verlag für Sozialwissenschaften.

Holzinger, K., Jörgens, H. und Knill, C. (2007b). Transfer, Diffusion und Konvergenz: Konzepte und Kausalmechanismen. In Holzinger, K., Jörgens, H. und Knill, C. (Hrsg.), Transfer, Diffusion und Konvergenz von Politiken. PVSSonderheft. Wiesbaden: VS Verlag für Sozialwissenschaften, 11-35. 
Hoppe, R. (2014). Structure and Finances of U.S. Farms: Family Farm Report, 2014 Edition. Economic Information Bulletin 132. Economic Research Service, U.S. Department of Agriculture.

Houthakker, H.S. (1961). Toward a solution of the farm problem. Review of Economics and Statistics 43(1): 63-66.

Ingersent, K.A. und Rayner, A.J. (1999). Agricultural Policy in Western Europe and the United States. Cheltenham, Northampton: Edward Elgar Publishing Limited.

Ingersent, K.A., Hine, R.C. und Rayner, A.J. (Hrsg.) (1998). The Reform of the Common Agricultural Policy. Basingstoke et al.: Macmillan.

Isermeyer, F. (2014). Künftige Anforderungen an die Landwirtschaft - Schlussfolgerungen für die Agrarpolitik. Thünen Working Paper 30. Braunschweig.

Jákli, Z. (1990). Vom Marshallplan zum Kohlepfennig: Grundrisse der Subventionspolitik. in der Bundesrepublik Deutschland 1948-1982. Opladen: Westdeutscher Verlag.

Jambor, A. und Harvey, D. (2010). Review of the challenges of CAP reform. Centre for Rural Economy Discussion Paper Series 27. Centre for Rural Economy.

Jesness, O.B. (1946). Postwar Agricultural Policy - Pressure vs. General Welfare. Journal of Farm Economics 28(1): 1-14.

Johnson, D.G. (1945). Second Award Paper: A price policy for agriculture, consistent with economic progress, that will promote adequate and more stable income from farming. Journal of Farm Economics 27(4): 761-772.

Johnson, D.G. (1947). Forward prices for agriculture. Chicago: University of Chicago Press.

Johnson, D.G. (1954). Agricultural Price Policy and International Trade. Essays in International Finance 19. Princeton: Princeton University.

Johnson, J. und Ericksen, M.H. (1977). Commodity Program Provisions Under the Food and Agriculture Act of 1977. Agricultural Economic Report 389. Economic Research Service, U.S. Department of Agriculture.

Johnson, J., Rizzi, R.W., Short, S.D. und Fulton, R.T. (1982). Provisions of the Agriculture and Food Act of 1981. Staff Report 811228. Economic Research Service, U.S. Department of Agriculture.

Johnson, R. (Coordinator) (2008). The 2008 Farm Bill: Major Provisions and Legislative Action. CRS Report for Congress RL34696. Congressional Research Service.

Johnson, R. und Monke, J. (2014). What Is the Farm Bill? CRS Report for Congress RS22131. Congressional Research Service.

Johnson, R. und Monke, J. (2017). What Is the Farm Bill? CRS Report for Congress RS22131. Congressional Research Service. 
Josling, T. (1973). The reform of the Common Agricultural Policy. In Evans, D. (Hrsg.), Britain in the EEC. London: Gollancz.

Josling, T. (1992). Trade in Agricultural Products. In Borner, S. und Grubel, H. (Hrsg.), The European Community after 1992. Perspectives from the Outside. London et al.: Macmillan, 83-102.

Josling, T. (1993). Agricultural Trade Issues in Transatlantic Trade Relations. World Economy 16(5): 553-573.

Josling, T. (1998). Agricultural Policy Reform in the USA and the EU: A Comparison of CAP Reform and the 1992 US Farm Bill. In Ingersent, K.A., Hine, R.C. und Rayner, A.J. (Hrsg.), The Reform of the Common Agricultural Policy. Basingstoke et al.: Macmillan, 36-53.

Josling, T. (2008). External Influences on CAP Reforms: An Historical Perspective. In Swinnen, J.F.M. (Hrsg.), The Perfect Storm: The Political Economy of the Fischler Reforms of the Common Agricultural Policy. Brussels: Centre for European Policy Studies, 57-75.

Josling, T. (2015). CAP Reform, the US Farm Bill and the TTIP. In Swinnen, J. (Hrsg.), The Political Economy of the 2014-2020 Common Agricultural Policy: An Imperfect Storm. Brussels: Centre for European Policy Studies; London: Rowman and Littlefield International, 485-492.

Josling, T. und Swinbank, A. (2008). European Union: Shadow WTO Agricultural Domestic Support Notifications. International Food Policy Research Institute Discussion Paper 00809. IFPRI.

Josling, T., Anderson, K., Schmitz, A. und Tangermann, S. (2010). Understanding International Trade in Agricultural Products: One Hundred Years of Contributions by Agricultural Economists. American Journal of Agricultural Economics 92(2): 424-446.

Josling, T.E. und Tangermann, S. (2015). Transatlantic Food and Agricultural Trade Policy: 50 Years of Conflict and Convergence. Cheltenham UK: Edward Elgar.

Josling, T.E., Tangermann, S. und Warley, T.K. (1996). Agriculture in the GATT. Basingstoke: Macmillan.

Kay, A. (1998). The Reform of the Common Agricultural Policy. The Case of the MacSharry Reforms. Wallingford: CABI Publishing.

Keeney, R.M. (2013). The End of the Direct Payment Era in U.S. Farm Policy. Purdue Extension - APEX (Ag Policy Explained) Series Paper 1, EC-774-W.

Kingdon, J.W. (1995). Agendas, Alternatives and Public Policies. 2. Auflage. New York: Longman.

Knill, C. (2005). Introduction: Cross-national policy convergence: concepts, approaches and explanatory factors. Journal of European Public Policy 12(5) (Special Issue): 764-774. 
Knops, L. und Garrone, M. (2015). Co-decision and the CAP: An unfinished story - Insights on the role of the European Parliament in the 2013 reform. In Swinnen, J. (Hrsg.), The Political Economy of the 2014-2020 Common Agricultural Policy: An Imperfect Storm. Brussels: Centre for European Policy Studies; London: Rowman and Littlefield International, 413-439.

Knops, L. und Swinnen, J. (2014). The First CAP Reform under the Ordinary Legislative Procedure: A Political Economy Perspective. A Study for the European Parliament, IP/B/AGRI/IC/2013-156.

Koester, U. (2001). Europäische Agrarpolitik: Ein Spannungsfeld divergierender Interessen. In Ohr, R. und Theurl, T. (Hrsg.), Kompendium Europäische Wirtschaftspolitik. München: Vahlen, 309-362.

Koester, U. (Project leader) (1988). Disharmonies in EC and US Agricultural Policy Measures: A Summary of Results and Major Conclusions. Report for the Commission of European Communities by EC/US Study Group. Brussels.

Koester, U. und Bale, M.D. (1984). The Common Agricultural Policy of the European Community: A Blessing or a Curse for Developing Countries? World Bank Staff Working Papers 630, Washington D.C.: World Bank.

Koester, U. und Tangermann, S. (1976). Alternativen der Agrarpolitik. Eine KostenNutzen-Analyse im Auftrag des Bundesministeriums für Ernährung, Landwirtschaft und Forsten. Münster Hiltrup: Landwirtschaftsverlag.

Kommission (1968). Memorandum on the Reform of Agriculture in the European Economic Community and Annexes. Submitted by the Commission to the Council on 21 December 1968. $\operatorname{COM}(68)$ 1000, Parts A and B, 18 December 1968. Bulletin of the European Communities, Supplement to No 1/1969.

Kommission (1970). Third general report on the activities of the Communities 1969. Brussels, Luxembourg.

Kommission (1973). Memorandum Agriculture 1973-1978. Newsletter on the Common Agricultural Policy, Special Issue, November 1973. Brussels: Directorate General Press and Information.

Kommission (1975). Stocktaking of the common agricultural policy (Communication from the Commission to the Parliament and the Council transmitted on 27 February 1975). COM(75) 100, 26 February 1975. Bulletin of the European Communities, Supplement 2/75.

Kommission (1980). Reflections on the common agricultural policy (Commission communication to the Council presented on 8 December 1980). Bulletin of the European Communities, Supplement 6/80. Luxembourg: Office for Official Publications of the European Communities.

Kommission (1981a). Commission's report on the mandate of 30 May 1980. $\operatorname{COM}(81) 300$ final. 24 June 1981. Luxemburg. 
Kommission (1981b). Guidelines for European Agriculture: Memorandum to complement the Commission's report on the Mandate of 30 May 1980. $\operatorname{COM}(81) 608$ final, 23 October 1981. Bulletin of the European Communities, Supplement 4/81.

Kommission (1985a). Perspectives for the Common Agricultural Policy (Communication of the Commission to the Council and the Parliament). $\operatorname{COM}(85) 333$ final, 15 July 1985. Brussels.

Kommission (1985b). A future for Community agriculture: Commission guidelines following the consultations in connection with the Green Paper (Communication of the Commission to the Council and to the European Parliament). COM(85) 750 final, 18 December 1985. Brussels.

Kommission (1987a). The Single Act: A new frontier for Europe (Communication from the Commission $(\operatorname{COM}(87) 100)$ to the Council). $\operatorname{COM}(87) 100$, 15 February 1987. Bulletin of the European Communities, Supplement $1 / 87$.

Kommission (1987b). Bulletin der Europäischen Gemeinschaften, Nr. 4 von 1987, 20. Jahrgang. Brüssel.

Kommission (1987c). Bulletin der Europäischen Gemeinschaften, Nr. 10 von 1987, 20. Jahrgang. Brüssel.

Kommission (1988a). Bulletin der Europäischen Gemeinschaften, Nr. 5 von 1988, 21. Jahrgang. Brüssel.

Kommission (1988b). Bulletin of the European Communities, No 10 of 1988, Volume 21. Brussels.

Kommission (1991a). The Development and Future of the CAP. Reflections Paper of the Commission (Communication of the Commission to the Council). C0M(91) 100 final, 1 February 1991. Brussels.

Kommission (1991b). The Development and Future of the CAP. Follow-up to the Reflections Paper of the Commission (COM (91) 100 of 1 February 1991) - Proposals of the Commission (Communication of the Commission to the Council and to the European Parliament). C0M(91) 258 final, 11 July 1991. Brussels.

Kommission (1991c). The Agricultural Situation in the Community, 1990 Report. Brussels, Luxembourg.

Kommission (1993). The Agricultural Situation in the Community, 1992 Report. Brussels, Luxembourg.

Kommission (1994a). The Agricultural Situation in the Community, 1993 Report. Brussels, Luxembourg.

Kommission (1994b). EC Agricultural Policy for the 21st Century. European Economy Reports and Studies No 4. Brussels, Luxembourg. 
Kommission (1995). Study on alternative strategies for the development of relations in the field of agriculture between the EU and the associated countries with a view to future accession of these countries. Agricultural Strategy Paper, prepared for the European Council Madrid, December 15-16, 1992. CSE (95) 607, 12 December 1995.

Kommission (1997). Agenda 2000: For a stronger and wider Union. Document drawn up on the basis of $\operatorname{COM}(97) 2000$ final. 15 July 1997. Bulletin of the European Union, Supplement 5/97.

Kommission (1998). Proposals for Council Regulations (EC) concerning the reform of the common agricultural policy (presented by the Commission). COM(1998) 158 final, 18.03.1998. Brussels.

Kommission (2002). Mid-Term Review of the Common Agricultural Policy (Communication from the Commission to the Council and the European Parliament). COM(2002) 394 final, 10.7.2002. Brussels.

Kommission (2003). Proposals for Council Regulations. COM(2003) 23 final, 21.1.2003. Brussels.

Kommission (2007). Preparing for the "Health Check" of the CAP reform (Communication from the Commission to the European Parliament and the Council). COM(2007) 722 final, 20.11.2007. Brussels.

Kommission (2010a). The CAP towards 2020: Meeting the food, natural resources and territorial challenges of the future (Communication from the Commission to the European Parliament, the Council, the European Economic and Social Committee and the Committee of the Regions). COM(2010) 672 final, 18.11.2010. Brussels.

Kommission (2010b). Developments in the income situation of the EU agricultural sector. Directorate L. Economic analysis, perspectives and evaluations, December 2010. Brussels.

Kommission (2011a). Proposal for a regulation of the European Parliament and of the Council establishing rules for direct payments to farmers under support schemes within the framework of the common agricultural policy. $\operatorname{COM}(2011) 625$ final/2, 19.10.2011. Brussels.

Kommission (2011b). Proposal for a regulation of the European Parliament and of the Council on support for rural development by the European Agricultural Fund for Rural Development (EAFRD). COM(2011) 627 final/2, 19.10.2011. Brussels.

Kommission (2011c). Common Agricultural Policy towards 2020: Impact Assessment, Annex 3: Direct payments. Commission Staff Working Paper, SEC(2011) 1153 final/2, 20.10.2011. Brussels.

Kommission (2013). Agriculture in the European Union: Statistical and economic information. Report 2013, December 2013. 
Kommission (2015a). Report on the distribution of direct aids to agricultural producers (financial year 2014). Ref. Ares(2015)5004686, 11/11/2015.

Kommission (2015b). Indicative figures on the distribution of aid, by size-class of aid, received in the context of direct aid paid to the producers according to Council Regulation (EC) No 73/2009 (financial year 2014). Annex 1 to Report on the distribution of direct aids to agricultural producers (financial year 2014).

Kommission (2015c). Voluntary coupled support - Sectors mostly supported: Notification of decisions taken by Member States by 1 August 2014. Informative note, 30 July 2015.

Kommission (2016a). Agri-food trade in 2015: China boosts EU exports. Monitoring Agri-trade Policy, MAP 2016-1.

Kommission (2016b). CAP post-2013: Key graphs and figures, Share of direct payments and total subsidies in agricultural factor income (2011-15 average). March 2016. URL: http://ec.europa.eu/agriculture/cap-post2013/graphs/graph5_en.pdf (Abrufdatum: 05.08.2016).

Kommission (2016c). Direct payments 2015-2020. Decisions taken by Member States: State of play as at June 2016. Information note.

Kommission (2017). CAP post-2013: Key graphs and figures, CAP expenditure in the total EU expenditure. March 2017. URL: http:/ /ec.europa.eu/agricul ture/cap-post-2013/graphs/graph5_en.pdf (Abrufdatum: 07.04.2017).

Lehrer, N. und Becker, D.R. (2010). Shifting paths to conservation: policy change discourses and the 2008 US farm bill. Journal of Environmental Planning and Management 53(5): 639-655.

Lenschow, A., Liefferink, D. und Veenman, S. (2005). When the birds sing. A framework for analysing domestic factors behind policy convergence. Journal of European Public Policy 12(5) (Special Issue): 797-816.

Levins, R.A. (2000). Willard Cochrane and the American Family Farm. Lincoln: University of Nebraska Press.

Lippert, C. (2004). Agrar- und Fischereipolitik. In Weidenfeld, W. und Wessels, W. (Hrsg.), Jahrbuch der Europäischen Integration 2003/2004. Institut für Europäische Politik, Bonn: Europa Union Verlag, 121-128.

Lippert, C. (2006). Agrar- und Fischereipolitik. In Weidenfeld, W. und Wessels, W. (Hrsg.), Jahrbuch der Europäischen Integration 2006. Institut für Europäische Politik, Bonn: Europa Union Verlag, 129-136.

Lippert, C. (2010). Agrar- und Fischereipolitik. In Weidenfeld, W. und Wessels, W. (Hrsg.), Jahrbuch der Europäischen Integration 2009. Institut für Europäische Politik, Bonn: Europa Union Verlag, 117-124. 
Little, J., Knapp, T., Sottomayor, M. und Swinbank, A. (2004), Compensation Proposals for EU Farm Policy Reform. In Swinbank, A. und Tranter, R. (Hrsg.), A Bond Scheme for Common Agricultural Policy Reform. Wallingford: CABI Publishing, 39-53.

Lopez, J.A. (2001). Decoupling: A conceptual overview. OECD Papers 10. Paris: OECD.

Luttrell, C.B. (1973). The Russian Wheat Deal - Hindsight vs. Foresight. Federal Reserve Bank of St. Louis.

Lynggaard, K. und Nedergaard, P. (2009). The logic of Policy Development: Lessons learned from Reform and Routine within the CAP 1980-2003. Journal of European Integration 31(3): 291-309.

Mahé, L.-P. und Tavéra, C. (1989). Bilateral Harmonization of EC and U.S. Agricultural Policies. European Review of Agricultural Economics 15(4): 327-348.

Marrison, A.J. (1986). The Tariff Commission, Agricultural Protection and Food Taxes, 1903-13. Agricultural History Review 34(2): 171-187.

Matthews, A. (2015). The Multi-Annual Financial Framework and the 2013 CAP reform. In Swinnen, J. (Hrsg.), The Political Economy of the 2014-2020 Common Agricultural Policy: An Imperfect Storm. Brussels: Centre for European Policy Studies; London: Rowman and Littlefield International, 169-192.

McArthur, W.C., Grise, V.N., Doty Jr., H.O. und Hacklander, D. (1982). U.S. Peanut Industry. Agricultural Economic Report 493. Economic Research Service. U.S. Department of Agriculture.

Meloni, G. und Swinnen, J. (2013). The rise and fall of the world's largest wine exporter (and its institutional legacy). Working Paper 134 Economics. American Association of Wine Economists.

Mollett, J.A. (1960). The Wheat Act of 1932: A Forerunner of Modern Farm Price Support Programmes. Agricultural History Review 8(1): 20-35.

Monke, J. (2004). Marketing Loans, Loan Deficiency Payments, and Commodity Certificates. CRS Report for Congress RS21604. Congressional Research Service.

Monke, J. (2008). Farm Commodity Programs and the 2007 Farm Bill. CRS Report for Congress RS21999. Congressional Research Service.

Monke, J. (2009). Farm Commodity Proposals in the President's FY2010 Budget. CRS Report for Congress R40442. Congressional Research Service.

Monke, J. (2014). Budget Issues That Shaped the 2014 Farm Bill. CRS Report for Congress R42484. Congressional Research Service.

Moyer, H.W. und Josling, T.E. (1990). Agricultural Policy Reform: Politics and Process in the EU and in the USA. Hemel Hempstead: Harvester Wheatsheaf.

Moyer, W. und Josling, T. (2002). Agricultural Policy Reform: Politics and Process in the EU and in the US in the 1990s. Aldershot, Burlington: Ashgate. 
Nash, E.F. (1961). Agriculture. In Selton, A. (Hrsg.), Agenda For A Free Society, Essays on Hayek's The Constitution of Liberty. London: The Institute of Economic Affairs, 171-189.

Nash, E.F. und Attwood, E.A. (1961). The Agricultural Policies of Britain and Denmark: A Study in Reciprocal Trade. London: Land Books.

NASS-USDA. Census of Agriculture 1974, 1987, 1992, 1997, 2002, 2007 und 2012. National Agricultural Statistics Service, U.S. Department of Agriculture.

Nedergaard, P. (2006). The 2003 Reform of the Common Agricultural Policy: Against all Odds or Rational Explanations? Journal of European Integration 28(3): 203-223.

New York Times (1996). Clinton Signs Farm Bill Ending Subsidies. Artikel vom 05.04.1996. URL: http:/ / www.nytimes.com/1996/04/05/us/clintonsigns-farm-bill-ending-subsidies.html (Abrufdatum: 16.04.2015).

Nicholls, W.H. und Johnson, D.G. (1946). The Farm Price Policy Awards, 1945: A Topical Digest of the Winning Essays. Journal of Farm Economics 28(1): 267-283.

Novoa, M.A., Cororaton, C.B. und Orden, D. (2014). Some Notes on the Economic Environment Affecting the Debate on the Agricultural Act of 2014. GII Working Paper 2014-2. Global Issues Initiative, Institute for Society, Culture and Environment, Virginia Tech-National Capital Region.

OECD (1987). Communique: The 1987 OECD Ministerial Principles for agricultural policy reform. Paris: OECD.

OECD (1990). Reforming agricultural policies: Quantitative restrictions on production; Direct Income Support. Paris: OECD.

OECD (1994). Agricultural policy reform: New approaches - The role of direct income payments. Paris: OECD.

OECD (1998). Agricultural Policies in OECD Countries 1998. Monitoring and Evaluation. Paris: OECD.

OECD (2003). Farm Household Income: Issues and Policy Responses. Paris: OECD.

OECD (2005). Decoupling - Policy Implications. Paris: OECD.

OECD (2016a). Monitoring and evaluation: Reference Tables for the United States and the EU. OECD. URL: http://stats.oecd.org/viewhtml.aspx?Que ryId=70966\&vh=0000\&vf=0\&l\&il=\&lang=en (Abrufdatum: 05.08.2016).

OECD (2016b). Agricultural Policy Monitoring and Evaluation 2016. Paris: OECD.

OECD (2016c). Economic Surveys: Norway 2016. Paris: OECD. 
Olper, A. (2008). Constraints and Causes of the 2003 EU Agricultural Policy Reforms. In Swinnen, J.F.M. (Hrsg.), The Perfect Storm: The Political Economy of the Fischler Reforms of the Common Agricultural Policy. Brussels: Centre for European Policy Studies, 83-101.

OMB (2012). Fiscal Year 2013: Cuts, Consolidations, and Savings, Budget of the U.S. Government. Office of Management and Budget.

OMB (2016). Fiscal year 2017: Historical Tables, Budget of the U.S. Government. Office of Management and Budget.

Orden, D. und Zulauf, C. (2015). The Political Economy of the 2014 Farm Bill. Invited paper prepared for the AAEA session The 2014 Farm Bill: An Economic Post Mortem, ASSA Annual Meetings, Boston, MA, January 4, 2015.

Orden, D., Blandford, D. und Josling, T. (2010). Determinants of United States Farm Policies. In Anderson, K. (Hrsg.), The Political Economy of Agricultural Price Distortions. New York: Cambridge University Press, 162-190.

Orden, D., Paarlberg, R. und Roe, T. (1999). Policy reform in American Agriculture: Analysis and Prognosis. Chicago, London: The University of Chicago Press.

Paarlberg, D. (1978). A New Agenda for Agriculture. In Hadwiger, D.F. und Browne, W.P. (Hrsg.), The New Politics of Food. Lexington et al.: Lexington Books, 135-140.

Paarlberg, R. (1992). How Agriculture Blocked the Uruguay Round. SAIS Review 12(1): 27-42.

Paarlberg, R. (1997). Agricultural Policy Reform and the Uruguay Round: Synergistic Linkage in a Two-Level Game? International Organization 51(3): 413-444.

Pasour, Jr., E.C. (2013). Theodore W. Schultz. Economic Journal Watch 10(3): 593-601.

Petit, M. (1985). Determinants of Agricultural Policies in the United States and the European Community. Research report 51. Washington D.C.: International Food Policy Research Institute.

Pirzio-Biroli, C. (2008). An Inside Perspective on the Political Economy of the Fischler Reforms. In Swinnen, J.F.M. (Hrsg.), The Perfect Storm: The Political Economy of the Fischler Reforms of the Common Agricultural Policy. Brussels: Centre for European Policy Studies, 102-114.

Plate, R. (1968). Das „Professorengutachten“ von 1962 aus heutiger Sicht. Agrarwirtschaft 17(7): 193-201.

Plate, R. und Woermann, E. unter Mitarbeit von Grupe, D. (1962). Landwirtschaft im Strukturwandel der Volkswirtschaft. Analyse der Nachkriegsentwicklung in der Bundesrepublik Deutschland und Versuch einer Prognose unter Berücksichtigung der Auswirkungen des Gemeinsamen Marktes. Hannover: Alfred Strothe Verlag. 
PMA-USDA (1952). Agricultural Conservation Program, Statistical Summary 1951. Report ACP-13P. Production and Marketing Administration, U.S. Department of Agriculture.

Pokrivcak, J., Crombez, C. und Swinnen, J.F.M. (2006). The status quo bias and reform of the common agricultural policy: Impact of voting rules, the European Commission and external changes. European Review of Agricultural Economics 33(4): 562-590.

Politico (2012). McCain heated about popcorn. Artikel vom 12.06.2012. URL: http://www.politico.com/story/2012/06/mccain-burned-about-popcornprovision-077299 (Abrufdatum: 15.07.2016).

Pollack, S.L. und Lynch, L. (1991). Provisions of the Food, Agriculture, Conservation, and Trade Act of 1990. Agriculture Information Bulletin 624. Economic Research Service, U.S. Department of Agriculture.

Powell, F.W. (1913). Industrial Bounties and Rewards by American States. Quarterly Journal of Economics 28(1): 191-208.

Rabinowicz, E. (2003). Swedish Agricultural Policy Reforms. Workshop on Agricultural Policy Reform and Adjustment Imperial College, Wye October 23-25, 2003.

Rau, A. (1957). Agricultural Policy and Trade Liberalization in the United States (1934-1956). A Study of Conflicting Policies. Geneva: Librairie E. Droz.

Ripley, R.B. (1969). Legislative Bargaining and the Food Stamp Act, 1964. In Cleaveland, F.N. (Hrsg.), A casebook on the legislative process. Congress and Urban Problems. Washington, D.C.: The Brookings Institute, 279-310.

RMA-USDA (2015). Fiscal year government cost of federal crop insurance. Risk Management Agency, U.S. Department of Agriculture. URL: http://www.rma.usda.gov/aboutrma/budget/15costtable1.pdf (Abrufdatum: 02.08.2016).

Rucker, R.R. und Pasour Jr., E.C. (2007). The Growth of U.S. Farm Programs. In Fishback, P. (Hrsg.), Government and the American Economy: A New History. Chicago, London: The University of Chicago Press, 456-489.

Sahrbacher, A., Balmann, A. und Sahrbacher, C. (2015). The Political Economy of Capping Direct Payments: Applications in - and implications for Germany. In Swinnen, J. (Hrsg.), The Political Economy of the 2014-2020 Common Agricultural Policy: An Imperfect Storm. Brussels: Centre for European Policy Studies; London: Rowman and Littlefield International, 277-306.

Schlotter, H.-G. (1963). Produktionsneutrale Einkommenshilfen an die Landwirtschaft. Berichte über Landwirtschaft 41: 451-465. 
Schmitt, G. (1988). Grundsätze für die Gestaltung der Agrarpolitik in der sozialen Marktwirtschaft. In Henrichsmeyer, W. und Langbehn, C. (Hrsg.), Wirtschaftliche und soziale Auswirkungen unterschiedlicher agrarpolitischer Konzepte.

Schriften der Gesellschaft für Wirtschafts- und Sozialwissenschaften des Landbaues 24: 15-32.

Schmitt, G. und Tangermann, S. (1984). Zur Auswirkung der Agrarpreisstützung auf die landwirtschaftlichen Einkommen. In Grosskopf, W. und Köhne, M. (Hrsg.), Einkommen in der Landwirtschaft - Entstehung, Verteilung, Vervendung und Beeinflussung. Schriften der Gesellschaft für Wirtschafts- und Sozialwissenschaften des Landbaues 21: 759-793.

Schnepf, R. (2014a). Status of the W'TO Brazil-U.S. Cotton Case. CRS Report for Congress R43336. Congressional Research Service.

Schnepf, R. (2014b). Dairy Provisions in the 2014 Farm Bill (P.L. 113-79). CRS Report for Congress R43465. Congressional Research Service.

Schnepf, R. (2014c). Agriculture in the WTO: Rules and Limits on Domestic Support. CRS Report for Congress RS20840. Congressional Research Service.

Schnepf, R. (2015). 2014 Farm Bill Provisions and WTO Compliance. CRS Report for Congress R43817. Congressional Research Service.

Schnepf, R. (2016). U.S. Farm Income Outlook for 2016. CRS Report for Congress R40152. Congressional Research Service.

Scholz, H. (1972). Agrarprojektionen in der EWG: Grundlagen der agrarpolitischen Planung. In Schmitt, G. (Hrsg.), Mobilität der landwirtschaftlichen Produktionsfaktoren und regionale Wirtschaftspolitik. Schriften der Gesellschaft für Wirtschafts- und Sozialwissenschaften des Landbaues 9: 247-281.

Schultz, T. (1943). Redirecting Farm Policy. New York: Macmillan.

Schultz, T. (1944). Two conditions necessary for economic progress in agriculture. Canadian Journal of Economics and Political Science 10(3): 298-311.

Shields, D.A. (2013). Federal Crop Insurance: Background. CRS Report for Congress R40532. Congressional Research Service.

Shields, D.A. (2014a). Farm Commodity Provisions in the 2014 Farm Bill (P.L. 113-79). CRS Report for Congress R43448. Congressional Research Service.

Shields, D.A. (2014b). Crop Insurance Provisions in the 2014 Farm Bill (P.L. 113-79). CRS Report for Congress R43494. Congressional Research Service.

Skogstad, G. (1998). Ideas, Paradigms and Institutions: Agricultural Exceptionalism in the European Union and the United States. Governance 11(4): 463-490. 
Smith, V.H. (2013). The 2013 Farm Bill: Limiting Waste by Limiting FarmSubsidy Budgets. Mercatus Center, George Mason University.

Smith, V.H. (2014). The 2014 Agricultural Act: U.S. Farm Policy in the context of the 1994 Marrakesh Agreement and the Doha Round. Issue Paper 52. Geneva: International Centre for Trade and Sustainable Development.

Smith, V.H. (2016). Midterm Review of the 2014 Farm Bill. Washington D.C.: American Enterprise Institute.

Stuart, K. und Runge, C.F. (1997). Agricultural policy reform in the United States: An Unfinished Agenda. Australian Journal of Agricultural and Resource Economics 41(1): 117-136.

Supreme Court (1942). Wickard v. Filburn, 317 U.S. 111 (1942). URL: https://supreme.justia.com/cases/federal/us/317/111/case.html.

Swerling, B.C. (1959). Income Protection for Farmers: A Possible Approach. Journal of Political Economy 67(2): 173-186.

Swinbank, A. (2004). Decoupled Payments and a Triad of Policy Objectives: Compensation, Farm Income Support and Multifunctionality. In Swinbank, A. und Tranter, R. (Hrsg.), A Bond Scheme for Common Agricultural Policy Reform. Wallingford: CABI Publishing, 1-3.

Swinbank, A. (2012). New direct payments scheme: Targeting and redistribution in the future CAP. A Note for the European Parliament, IP/B/AGRI/CEI/2011-097/E003/SC1.

Swinbank, A. (2015). The WTO: No longer relevant for CAP reform? In Swinnen, J. (Hrsg.), The Political Economy of the 2014-2020 Common Agricultural Policy: An Imperfect Storm. Brussels: Centre for European Policy Studies;

London: Rowman and Littlefield International, 193-213.

Swinbank, A. (2016). Research for AGRI Committee: The Interactions Between the EU's External Action and the Common Agricultural Policy. A Study for the European Parliament, IP/B/AGRI/CEI/2015-070/0/7/1.

Swinbank, A. und Daugbjerg, C. (2006). The 2003 CAP Reform: Accommodating WTO Pressures. Comparative European Politics 4(1): 47-64.

Swinbank, A. und Tangermann, S. (2004). A Bond Scheme to Facilitate CAP Reform, In Swinbank, A. und Tranter R. (Hrsg.), A Bond Scheme for Common Agricultural Policy Reform. Wallingford: CABI Publishing, 55-78.

Swinbank, A. und Tanner, C. (1996). Farm Policy and Trade Conflict: The Uruguay Round and Common Agricultural Policy Reform. Ann Arbor: The University of Michigan Press.

Swinbank, A. und Tranter, R. (2005). Decoupling EU Farm Support: Does the New Single Payment Scheme Fit within the Green Box? Estey Centre Journal of International Law and Trade Policy 6(1): 47-61. 
Swinbank, A. und Tranter, R. (Hrsg.) (2004). A Bond Scheme for Common Agricultural Policy Reform. Wallingford: CABI Publishing.

Swinbank, A., Tranter, R., Daniels, J. und Wooldridge, M. (2004). An examination of various theoretical concepts behind decoupling and review of hypothetical and actual de-coupled support schemes in some OECD countries. GENEDEC Project co-funded by the European Commission within the Sixth Framework Programme, Project 502184.

Swinnen, J. (Hrsg.) (2015a). The Political Economy of the 2014-2020 Common Agricultural Policy: An Imperfect Storm. Brussels: Centre for European Policy Studies; London: Rowman and Littlefield International.

Swinnen, J. (2015b). The Political Economy of the 2014-2020 Common Agricultural Policy: Introduction and key conclusions. In Swinnen, J. (Hrsg.), The Political Economy of the 2014-2020 Common Agricultural Policy: An Imperfect Storm. Brussels: Centre for European Policy Studies; London: Rowman and Littlefield International, 1-30.

Swinnen, J. (2015c). An Imperfect Storm in the Political Economy of the Common Agricultural Policy. In Swinnen, J. (Hrsg.), The Political Economy of the 2014-2020 Common Agricultural Policy: An Imperfect Storm. Brussels: Centre for European Policy Studies; London: Rowman and Littlefield International, 443-484.

Swinnen, J., Knops, L. und van Herck, K. (2013). Food price volatility and EU policies. WIDER Working Paper 2013/032. World Institute for Development Economic Research.

Swinnen, J., Olper, A. und Vandemoortele, T. (2012). Impact of the WTO on Agricultural and Food Policies. World Economy 35(9): 1089-1101.

Swinnen, J., Olper, A. und Vandemoortele, T. (2016). The Political Economy of Policy Instrument Choice: Theory and Evidence from Agricultural and Food Policies. Theoretical Economics Letters 6(1): 106-117.

Swinnen, J.F.M. (2009). On The Future of Direct Payments. Paper presented at the Bureau of Economic Policy Advisors (BEPA) Workshop on "Reflections on the Common Agricultural Policy from a long-run perspective", February 26, 2009, European Commission, Brussels.

Swinnen, J.F.M. (2010a). Political Economy of Agricultural Distortions: The Literature to Date. In Anderson, K. (Hrsg.), The Political Economy of Agricultural Price Distortions. New York: Cambridge University Press, 81-104.

Swinnen, J.F.M. (2010b). Agricultural Protection Growth in Europe, 1870-1969. In Anderson, K. (Hrsg.), The Political Economy of Agricultural Price Distortions. New York: Cambridge University Press, 141-161.

Swinnen, J.F.M. (Hrsg.) (2008a). The Perfect Storm: The Political Economy of the Fischler Reforms of the Common Agricultural Policy. Brussels: Centre for European Policy Studies. 
Swinnen, J.F.M. (2008b). The Political Economy of the Fischler Reforms of the EU's Common Agricultural Policy: The Perfect Storm? In Swinnen, J.F.M. (Hrsg.), The Perfect Storm: The Political Economy of the Fischler Reforms of the Common Agricultural Policy. Brussels: Centre for European Policy Studies, 135-166.

Tangermann, S. (1974). Gemeinsame Agrarpolitik und nationale Wirtschaftspolitik in der EWG. Theoretische Analyse der Funktionsbedingungen gemeinsamer Marktordnungen bei Wechselkursänderungen und empirische Untersuchung der Entwicklung in der Bundesrepublik Deutschland und Frankreich. Tübingen: J.C.B. Mohr.

Tangermann, S. (1990). A bond scheme for supporting farm incomes. In Marsh, J.S., Green, B., Kearney, B., Mahé, L., Tangermann, S. und Tarditi, S. (Hrsg), The Changing Role of the Common Agricultural Policy: The Future of Farming in Europe. Land Use and Food Policy Intergroup. Brussels: European Parliament.

Tangermann, S. (1998). An ex-post review of the 1992 MacSharry Reform. In Ingersent, K.A., Hine, R.C. und Rayner, A.J. (Hrsg.), The Reform of the Common Agricultural Policy. Basingstoke et al.: Macmillan, 12-35.

Tangermann, S. (2003). Agricultural Policies in OECD Countries 10 Years After the Uruguay Round: How Much Progress? Invited paper presented at the International Conference Agricultural policy reform and the WTO: where are we heading? Capri (Italy), June 23-26, 2003.

Tangermann, S. (2011). Direct Payments in the CAP post 2013. A Note for the European Parliament, IP/B/AGRI/IC/2011_003.

Tangermann, S. (2014). Direktzahlungen: Ein bleibender Bestandteil der EUAgrarpolitik? In Schmid, E. und Vogel, S. (Hrsg.), Europäische Agrarpolitik im 21. Jahrhundert. Festschrift für Markus F. Hofreither. Wien: Facultas Universitätsverlag, 19-42.

Thiemeyer, G. (1999). Vom „Pool Vert“ zur europäischen Wirtschaftsgemeinschaft. Europäische Integration, Kalter Krieg und die Anfänge der gemeinsamen europäischen Agrarpolitik 1950-1957. Studien zur Internationalen Geschichte. München: Oldenbourg Wissenschaftsverlag.

Thompson, R.L. (2005). The US Farm Bill and the Doha Negotiations: On Parallel Tracks or a Collision Course? IPC Issue Brief 15. International Policy Council.

Tracy, M. (1989). Government and Agriculture in Western Europe 1880-1988.

3. Auflage. New York, et al.: Harvester Wheatsheaf.

Tsou, S.S. und Black, J.D. (1944). International Commodity Arrangements. Quarterly Journal of Economics 58(4): 521-552.

Tweeten, L. und Zulauf, C. (1997). Public Policy for Agriculture After Commodity Programs. Review of Agricultural Economics 19(2): 263-280. 
Uri, P. (Rapporteur), Bergmann, D., Kaldor, N., Krohn, H.-B., Marsh, J.-S., Rossi-Doria, M., Schnittker, J.A., Thompson, C. und Wilbrandt, H. (1971). Die Zukunft der europäischen Landwirtschaft: Ein Vorschlag zur Neugestaltung der gemeinsamen Agrarpolitik. Bericht einer Expertengruppe. Paris: Atlantisches Institut.

USDA (Hrsg.). Agricultural Statistic für die Jahre 1936, 1938, 1939, 1940, 1946, 1949, 1951, 1957 (publiziert 1958), 1958 (publiziert 1959), 1959 (publiziert 1960), 1960 (publiziert 1961), 1961 (publiziert 1962), 1962 (publiziert 1963), 1963, 1964, 1965, 1966, 1972, 1974, 1982, 1988, 1998, 2006 und 2016.

van Riemsdijk, J.F. (1973). A system of direct compensation payments to farmers as a means of reconciling short-run to long-run interests. European $R_{e-}$ view of Agricultural Economics 1(2): 161-189.

van Tongeren, F. (2008). Agricultural Policy Design and Implementation: A Synthesis. OECD Food, Agriculture and Fisheries Working Papers 7. Paris: OECD.

von Cramon-Taubadel, S. (2017). The Common Agricultural Policy and the Next EU Budget. Reflection Paper 2: Re-adjusting the Goals of the CAP. Paper prepared for Expert Workshop "CAP and the next MFF" Berlin, Federal Foreign Office, 30 March 2017. Bertelsmann Stiftung.

von Urff, W. (1982). Agrar- und Fischereipolitik. In Weidenfeld, W. und Wessels, W. (Hrsg.), Jahrbuch der Europäischen Integration 1981. Institut für Europäische Politik, Bonn: Europa Union Verlag, 147-162.

von Urff, W. (1986). Agrar- und Fischereipolitik. In Weidenfeld, W. und Wessels, W. (Hrsg.), Jahrbuch der Europäischen Integration 1985. Institut für Europäische Politik. Bonn: Europa Union Verlag, 103-115.

von Urff, W. (1987). Agrar- und Fischereipolitik. In Weidenfeld, W. und Wessels, W. (Hrsg.), Jahrbuch der Europäischen Integration 1986-87. Institut für Europäische Politik, Bonn: Europa Union Verlag, 105-116.

von Urff, W. (1988). Agrar- und Fischereipolitik. In Weidenfeld, W. und Wessels, W. (Hrsg.), Jahrbuch der Europäischen Integration 1987-88. Institut für Europäische Politik, Bonn: Europa Union Verlag, 109-120.

von Urff, W. (1989). Agrar- und Fischereipolitik. In Weidenfeld, W. und Wessels, W. (Hrsg.), Jahrbuch der Europäischen Integration 1988-89. Institut für Europäische Politik, Bonn: Europa Union Verlag, 87-98.

von Urff, W. (1991). Agrar- und Fischereipolitik. In Weidenfeld, W. und Wessels, W. (Hrsg.), Jahrbuch der Europäischen Integration 1990-91. Institut für Europäische Politik, Bonn: Europa Union Verlag, 97-108.

von Urff, W. (1993). Agrar- und Fischereipolitik. In Weidenfeld, W. und Wessels, W. (Hrsg.), Jahrbuch der Europäischen Integration 1992-93. Institut für Europäische Politik, Bonn: Europa Union Verlag, 97-108. 
von Urff, W. (1999). Agrar- und Fischereipolitik. In Weidenfeld, W. und Wessels, W. (Hrsg.), Jahrbuch der Europäischen Integration 1998-99. Institut für Europäische Politik, Bonn: Europa Union Verlag, 125-134.

Wall Street Journal (2012). Farming Cuts Sow Discord. Artikel vom 07.06.2012. URL: http://www.wsj.com/articles/SB10001424052702303296604577 450781272349376 (Abrufdatum: 15.07.2016).

Waugh, F.V. (1945). Third Award Paper: A price policy for agriculture, consistent with economic progress, that will promote adequate and more stable income from farming. Journal of Farm Economics 27(4): 773-784.

Webb, A.J., Praveen M.D. und Conley, H. (1989). GATT and the 1990 Farm Bill: Compatibility or Confrontation? American Journal of Agricultural Economics 71(5): 1231-1238.

Weinschenck, G. (1964). Probleme direkter Einkommensübertragungen. In Herlemann, H.-H. (Hrsg.), Grenzen und Möglichkeiten einzelstaatlicher Agrarpolitik. Schriften der Gesellschaft für Wirtschafts- und Sozialwissenschaften des Landbaues 1: 128-179.

Westcott, P.C. und Price, J.M. (2001). Analysis of the U.S. Commodity Loan Program with Marketing Loan Provisions. Agricultural Economic Report 801. Economic Research Service, U.S. Department of Agriculture.

Whetham, E.H. (1974). The Agriculture Act, 1920 and Its Repeal - the "Great Betrayal". Agricultural History Review 22(1): 36-49.

Whetham, E.H. (1978). The Agrarian History of England and Wales, Volume VIII, 1914-1939. Cambridge: Cambridge University Press.

Wille, M. (1971). Möglichkeiten und Probleme einer Trennung von Preis- und Einkommenspolitik in der Landwirtschaft: Zum gegenwärtigen Stand der Diskussion in den USA. Agrarwirtschaft 20(8): 257-267.

Wille, M. (1973). Mögliche Formen künftiger Einkommenspolitik in der Landwirtschaft. In Weinschenck, G. (Hrsg.), Die qukünftige Entwicklung der europäischen Landwirtschaft - Prognosen und Denkmodelle. Schriften der Gesellschaft für Wirtschafts- und Sozialwissenschaften des Landbaues 10: 178-194.

Wille, M. (1976). Formen, Möglichkeiten und Wirkungen direkter Einkommensübertragungen an die Landwirtschaft. Serie: Landwirtschaft - angewandte Wissenschaft, 184. Zugl.: Göttingen, Univ., Diss., 1974. Münster-Hiltrup: Landwirtschaftsverlag.

Wissenschaftlicher Beirat (1968). Vorschläge zur Milchpreispolitik: Gutachten des Wissenschaftlichen Beirats beim Bundesministerium für Ernährung, Landwirtschaft und Forsten. Serie: Landwirtschaft - angewandte Wissenschaft, 132. Münster-Hiltrup: Landwirtschaftsverlag. 
Wissenschaftlicher Beirat (1973). Zur Reform der Agrarpolitik der EWG: Gutachten des Wissenschaftlichen Beirats beim Bundesministerium für Ernährung, Landwirtschaft und Forsten. Serie: Landwirtschaft - angewandte Wissenschaft, 166. Münster-Hiltrup: Landwirtschaftsverlag.

Wissenschaftlicher Beirat (1987). Handlungsalternativen der EG-Agrarmarktpolitik: Gutachten des Wissenschaftlichen Beirats beim Bundesministerium für Ernährung, Landwirtschaft und Forsten. Serie: Landwirtschaft - angewandte Wissenschaft, 348. Münster-Hiltrup: Landwirtschaftsverlag.

Wissenschaftlicher Beirat (1989). Flankierende Maßnahmen in Ergänzung einer Agrarpreispolitik des „mittleren Weges“. Gutachten des Wissenschaftlichen Beirats beim Bundesministerium für Ernährung, Landwirtschaft und Forsten. Handlungsalternativen der EG-Agrarmarktpolitik: Gutachten des Wissenschaftlichen Beirats beim Bundesministerium für Ernährung, Landwirtschaft und Forsten, 366. Münster-Hiltrup: Landwirtschaftsverlag.

Wissenschaftlicher Beirat (2003). Stellungnahme des Wissenschaftlichen Beirats Agrarpolitik, nachhaltige Landbewirtschaftung und Entwicklung ländlicher Räume beim Bundesministerium für Verbraucherschutz, Ernährung und Landwirtschaft zu den Beschlüssen des Rates der Europäischen Union zur Reform der Gemeinsamen Agrarpolitik vom 26. Juni 2003.

Wissenschaftlicher Beirat (2010). EU-Agrarpolitik nach 2013: Plädoyer für eine neue Politik für Ernährung, Landwirtschaft und ländliche Räume. Gutachten des Wissenschaftlichen Beirats für Agrarpolitik beim

Bundesministerium für Ernährung, Landwirtschaft und Verbraucherschutz.

Woolverton, A. und Young, E. (2009). Factors Influencing ACRE Program Enrollment. Economic Research Report 84. Economic Research Service, U.S. Department of Agriculture.

WTO (2004). United States - Subsidies on upland cotton. Report of the Panel, W'T/DS267/R. World Trade Organisation.

WTO (2005). United States - Subsidies on upland cotton, AB-2004-5. Report of the Appellate Body, WT/DS267/AB/R. World Trade Organisation.

W'TO-GATT (1994). Agreement on Agriculture.

Wyant, S. (2008). Memories of Agriculture Secretary Earl Butz. Agri-Pulse Communications. URL: https://www.agri-pulse.com/ext/resources/pdfs/ 0/2/1/0/8/021008.pdf (Abrufdatum: 12.08.2015).

Young, E. (Coordinator) (2002). The 2002 Farm Bill: Provisions and Economic Implications. Electronic Report. Administrative Publication 022. Economic Research Service, U.S. Department of Agriculture. URL: https://www.ers. usda.gov/webdocs/publications/42660/13780_ap022_4_.pdf?v=0 (Abrufdatum: 10.01.2015). 
Young, E. und Effland, A.B. (2001). Recommendations of the Commission on 21st Century Production Agriculture. Agricultural Outlook April 2001. Economic Research Service, U.S. Department of Agriculture.

Young, E. und Shields, D.A. (1996). Provisions of the 1996 Farm Bill. Agricultural Outlook Supplement. Economic Research Service, U.S. Department of Agriculture.

Zahariadis, N. (1995). Markets, States and Public Policy. Ann Arbor: University of Michigan Press.

Zulauf, C. und Orden, D. (2009). ACRE in the U.S. Farm Bill and the WTO. IATRC Working Paper 09-2. International Agricultural Trade Research Consortium.

Zulauf, C. und Orden, D. (2014). The U.S. Agricultural Act of 2014: Overview and Analysis. IFPRI Discussion Paper 01393. International Food Policy Research Institute.

Zulauf, C. und Orden, D. (2016). 80 Years of Farm Bills - Evolutionary Reform. Choices 31(4): 1-7. 



\section{Gesetze}

AAA (1933). Agricultural Adjustment Act 1933. Public Law 73-10.

AAA (1938). Agricultural Adjustment Act 1938. Public Law 75-430.

Agricultural Act 1964. Public Law 88-297.

Delegierte Verordnung (EU) Nr. 639/2014 der Kommission vom 11. März 2014 zur Ergänzung der Verordnung (EU) Nr. 1307/2013 des Europäischen Parlaments und des Rates mit Vorschriften über Direktzahlungen an Inhaber landwirtschaftlicher Betriebe im Rahmen von Stützungsregelungen der Gemeinsamen Agrarpolitik und zur Änderung des Anhangs X der genannten Verordnung. ABl. L 181 vom 20.6.2014, S. 1-47.

Fair and Equitable Tobacco Reform Act 2004. Title VI of Public Law 108-357 (H.R. 4520).

Food and Agriculture Act 1962. Public Law 87-703.

Food and Agricultural Act 1965. Public Law 89-321.

FAIR Act 1996. Public Law 104-127.

Richtlinien des BML für die Gewährung von Ausgleichszahlungen im Rahmen der Getreidepreisharmonisierung vom 29. Februar 1967, IV A 1 - 4106. 
Richtlinie des Rates vom 17. April 1972 zur Förderung der Einstellung der landwirtschaftlichen Erwerbstätigkeit und der Verwendung der landwirtschaftlich genutzten Fläche für Zwecke der Strukturverbesserung (72/160/EWG). ABl. L 96 vom 23.4.1972, S. 9-14.

Richtlinie des Rates vom 17. April 1972 über die Modernisierung der landwirtschaftlichen Betriebe (72/159/EWG). ABl. L 96 vom 23.4.1972, S. 1-8.

Richtlinie 75/268/EWG des Rates vom 28. April 1975 über die Landwirtschaft in Berggebieten und in bestimmten benachteiligten Gebieten. ABl. L 128 vom 19.5.1975, S. 1-7.

Sugar Bounty No. 4 of 1903. An Act to provide for a Bounty to Growers of Sugar-Cane and Beet. 30.07.1903.

Sugar Bounty No. 23 of 1905. An Act to provide for a Bounty to, Growers of Sugar-cane and Beet. 21.12.1905.

Verordnung Nr. 742/67/EWG vom 24. Oktober 1967 über die Bildung der Sonderabteilungen I und II des Europäischen Ausrichtungs- und Garantiefonds für die Landwirtschaft für gemeinschaftliche Ausgleichsmaßnahmen zugunsten Deutschlands, Italiens und Luxemburgs sowie für die Zahlung eines Betrages von 4 Mio. Rechnungseinheiten an Luxemburg. ABl. 258 vom 25.10.1967, S. 4-5.

Verordnung 120/67/EWG vom 13. Juni 1967 über die gemeinsame Marktorganisation für Getreide. ABl. 117 vom 19.6.1967, S. 2269-2283.

Verordnung (EWG) Nr. 804/68 des Rates vom 27. Juni 1968 über die gemeinsame Marktorganisation für Milch und Milcherzeugnisse. ABl. L 148 vom 28.6.1968, S. 13-23.

Verordnung Nr. 729/70 des Rates vom 21. April 1970 über die Finanzierung der gemeinsamen Agrarpolitik. ABl. L 94 vom 28.4.1970, S. 13-18.

Verordnung (EWG) Nr. 1308/70 des Rates vom 29. Juni 1970 über die gemeinsame Marktorganisation für Flachs und Hanf. ABl. L 146 vom 4.7.1970, S. 1-4.

Verordnung (EWG) Nr. 619/71 des Rates vom 22. März 1971 zur Festlegung der Grundregeln für die Gewährung einer Beihilfe für Flachs und Hanf. ABl. L 72 vom 26.3.1971, S. 2-3.

Verordnung (EWG) Nr. 1516/71 des Rates vom 12. Juli 1971 zur Einführung einer Beihilferegelung für Baumwollsaat. ABl. L 160 vom 17.7.1971, S. 1-2.

Verordnung (EWG) Nr. 1696/71 des Rates vom 26. Juli 1971 über die gemeinsame Marktorganisation für Hopfen. ABl. L 175 vom 4.8.1971, S. 1-7.

Verordnung (EWG) Nr. 2358/71 des Rates vom 26. Oktober 1971 zur Errichtung einer gemeinsamen Marktorganisation für Saatgut. ABl. L 246 vom 5.11.1971, S. 1-5. 
Verordnung (EWG) Nr. 845/72 des Rates vom 24. April 1972 über Sondermaßnahmen zur Förderung der Seidenraupenzucht. ABl. L 100 vom 27.4.1972, S. 1-2.

Verordnung (EWG) Nr. 722/74 der Kommission vom 29. März 1974 zur Ermächtigung des Vereinigten Königreichs zur Gewährung einer degressiven einzelstaatlichen Beihilfe für Schweineerzeuger. ABl. L 88 vom 1.4.1974, S. 35-35.

Verordnung (EWG) Nr. 920/74 der Kommission vom 18. April 1974 zur Ermächtigung des Vereinigten Königreichs zur vorübergehenden Gewährung von einzelstaatlichen Beihilfen auf dem Rindfleischsektor. ABl. L 106 vom 19.4.1974, S. 36-37.

Verordnung (EWG) Nr. 1067/74 des Rates vom 30. April 1974 über die gemeinsame Marktorganisation für künstlich getrocknetes Futter. ABl. L 120 vom 1.5.1974, S. 2-4.

Verordnung (EWG) Nr. 464/75 des Rates vom 27. Februar 1975 zur Einführung von Prämienregelungen zugunsten der Rindfleischerzeuger. ABl. L 52 vom 28.2.1975, S. 5-7.

Verordnung (EWG) Nr. 1163/76 des Rates vom 17. Mai 1976 über die Gewährung einer Umstellungsprämie im Weinbau. ABl. L 135 vom 24.5.1976, S. 34-37.

Verordnung (EWG) Nr. 1078/77 des Rates vom 17. Mai 1977 zur Einführung einer Prämienregelung für die Nichtvermarktung von Milch und Milcherzeugnissen und die Umstellung der Milchkuhbestände. ABl. L 131 vom 26.5.1977, S. 1-5.

Verordnung (EWG) Nr. 456/80 des Rates vom 18. Februar 1980 über die Gewährung von Prämien für die vorübergehende Aufgabe und für die endgültige Aufgabe bestimmter Rebflächen sowie von Prämien für den Verzicht auf Wiederbepflanzung. ABl. L 57 vom 29.2.1980, S. 16-22.

Verordnung (EWG) Nr. 1357/80 des Rates vom 5. Juni 1980 zur Einführung einer Prämienregelung für die Erhaltung des Mutterkuhbestands. ABl. L 140 vom 5.6.1980, S. 1-3.

Verordnung (EWG) Nr. 1199/82 des Rates vom 18. Mai 1982 über die Gewährung einer Zusatzprämie für die Erhaltung des Mutterkuhbestands in Irland und in Nordirland und zur Aufhebung der Verordnung (EWG) Nr. 1056/81. ABl. L 140 vom 20.5.1982, S. 30-31.

Verordnung (EWG) Nr. 797/85 des Rates vom 12. März 1985 zur Verbesserung der Effizienz der Agrarstruktur. ABl. L 93 vom 30.3.1985, S. 1-18.

Verordnung (EWG) Nr. 1336/86 des Rates vom 6. Mai 1986 zur Festsetzung einer Vergütung bei der endgültigen Aufgabe der Milcherzeugung. ABl. L 119 vom 8.5.1986, S. 21-24. 
Verordnung (EWG) Nr. 1346/86 des Rates vom 6. Mai 1986 über die Gewährung einer Kalbungsprämie in Griechenland, Irland, Italien und Nordirland sowie die Gewährung einer zusätzlichen einzelstaatlichen Prämie in Italien. ABl. L 119 vom 8.5.1986, S. 39-39.

Verordnung (EWG) Nr. 1347/86 des Rates vom 6. Mai 1986 über die Gewährung einer Prämie bei der Schlachtung bestimmter ausgewachsener Schlachtrinder im Vereinigten Königreich. ABl. L 119 vom 8.5.1986, S. $40-41$.

Verordnung (EWG) Nr. 467/87 des Rates vom 10. Februar 1987 zur Änderung der Verordnung (EWG) Nr. 805/68 über die gemeinsame Marktorganisation für Rindfleisch sowie der Prämienregelungen in diesem Sektor. ABl. L 48 vom 17.2.1987, S. 1-3.

Verordnung (EWG) Nr. 1760/87 des Rates vom 15. Juni 1987 zur Änderung der Verordnungen (EWG) Nr. 797/85, (EWG) Nr. 270/79, (EWG) Nr. 1360/78 und (EWG) Nr. 355/77 im Bereich der Agrarstrukturen und zur Anpassung der Landwirtschaft an die neuen Marktgegebenheiten sowie zur Erhaltung des ländlichen Raums. ABl. L 167 vom 26.6.1987, S. 1-8.

Verordnung (EWG) Nr. 1094/88 des Rates vom 25. April 1988 zur Änderung der Verordnungen (EWG) Nr. 797/85 und Nr. 1760/87 hinsichtlich der Stillegung von Ackerflächen und der Extensivierung und Umstellung der Erzeugung. ABl. L 106 vom 27.4.1988, S. 28-32.

Verordnung (EWG) Nr. 1272/88 der Kommission vom 29. April 1988 mit Durchführungsbestimmungen zur Beihilferegelung für die Förderung der Stillegung von Anbauflächen. ABl. L 121 vom 11.5.1988, S. 36-40.

Verordnung (EWG) Nr. 571/89 des Rates vom 2. März 1989 zur Änderung der Verordnung (EWG) Nr. 805/68 über die gemeinsame Marktorganisation für Rindfleisch, zur Aufhebung der Verordnung (EWG) Nr. 1302/73 und zur Verlängerung der Verordnung (EWG) Nr. 4132/88. ABl. L 61 vom 4.3.1989, S. 43-45.

Verordnung (EWG) Nr. 768/89 des Rates vom 21. März 1989 zur Einführung vorübergehender landwirtschaftlicher Einkommensbeihilfen. ABl. L 84 vom 29.3.1989, S. 8-12.

Verordnung (EWG) Nr. 1765/92 des Rates vom 30. Juni 1992 zur Einführung einer Stützungsregelung für Erzeuger bestimmter landwirtschaftlicher Kulturpflanzen. ABl. L 181 vom 1.7.1992, S. 12-20.

Verordnung (EWG) Nr. 3813/92 des Rates vom 28. Dezember 1992 über die Rechnungseinheit und die im Rahmen der gemeinsamen Agrarpolitik anzuwendenden Umrechnungskurse. ABl. L 387 vom 31.12.1992, S. 1-6.

Verordnung (EG) Nr. 1527/95 des Rates vom 29. Juni 1995 über Ausgleichsmaßnahmen infolge der Verringerung der landwirtschaftlichen Umrechnungskurse einiger Währungen. ABl. L 148 vom 30.6.1995, S. 1-2. 
Verordnung (EG) Nr. 1782/2003 des Rates vom 29. September 2003 mit gemeinsamen Regeln für Direktzahlungen im Rahmen der Gemeinsamen Agrarpolitik und mit bestimmten Stützungsregelungen für Inhaber landwirtschaftlicher Betriebe und zur Änderung der Verordnungen (EWG)

Nr. 2019/93, (EG) Nr. 1452/2001, (EG) Nr. 1453/2001, (EG)

Nr. 1454/2001, (EG) Nr. 1868/94, (EG) Nr. 1251/1999, (EG)

Nr. 1254/1999, (EG) Nr. 1673/2000, (EWG) Nr. 2358/71 und (EG)

Nr. 2529/2001. ABl. L 270 vom 21.10.2003, S. 1-69.

Verordnung (EG) Nr. 73/2009 des Rates vom 19. Januar 2009 mit gemeinsamen Regeln für Direktzahlungen im Rahmen der gemeinsamen Agrarpolitik und mit bestimmten Stützungsregelungen für Inhaber landwirtschaftlicher Betriebe und zur Änderung der Verordnungen (EG) Nr. 1290/2005, (EG) Nr. 247/2006, (EG) Nr. 378/2007 sowie zur Aufhebung der Verordnung (EG) Nr. 1782/2003. ABl. L 30 vom 31.1.2009, S. 16-99.

Verordnung (EU) Nr. 228/2013 des Europäischen Parlaments und des Rates vom 13. März 2013 über Sondermaßnahmen im Bereich der Landwirtschaft zugunsten der Regionen in äußerster Randlage der Union und zur Aufhebung der Verordnung (EG) Nr. 247/2006 des Rates. ABl. L 78 vom 20.3.2013, S. 23-40.

Verordnung (EU) Nr. 1307/2013 des Europäischen Parlaments und des Rates vom 17. Dezember 2013 mit Vorschriften über Direktzahlungen an Inhaber landwirtschaftlicher Betriebe im Rahmen von Stützungsregelungen der Gemeinsamen Agrarpolitik und zur Aufhebung der Verordnung (EG) Nr. 637/2008 des Rates und der Verordnung (EG) Nr. 73/2009 des Rates. ABl. L 347 vom 20.12.2013, S. 608-670. 



\section{Anhang: Übersicht über die Höhe der Agrarzahlungen in den USA im Zeitablauf}

Tabelle A1: Gesamtbetrag der staatlichen Zahlungen an Landwirte in den USA seit 1932 (ausgedrückt in jeweiligen Preisen)

\begin{tabular}{|l|r|r|r|r|}
\hline Jahr & $\begin{array}{r}\text { "Government } \\
\text { payments“ } \\
\text { Mrd. USD }\end{array}$ & $\begin{array}{r}\text { Landwirtschaftl. } \\
\text { Nettoeinkommen } \\
\text { Mrd. USD }\end{array}$ & $\begin{array}{r}\text { "Government payments“ } \\
\text { in des landwirtschaftl. } \\
\text { Nettoeinkommens }\end{array}$ & Preisparität \\
\hline 1932 & 0,000 & 2,033 & 0,0 & 58 \\
\hline 1933 & 0,131 & 2,556 & 5,0 & 64 \\
\hline 1934 & 0,446 & 2,923 & 15,3 & 75 \\
\hline 1935 & 0,573 & 5,277 & 10,9 & 88 \\
\hline 1936 & 0,278 & 4,310 & 6,5 & 92 \\
\hline 1937 & 0,336 & 6,003 & 5,6 & 93 \\
\hline 1938 & 0,446 & 4,361 & 10,2 & 78 \\
\hline 1939 & 0,763 & 4,417 & 17,3 & 77 \\
\hline 1940 & 0,723 & 4,481 & 16,1 & 81 \\
\hline
\end{tabular}


Anhang: Übersicht über die Höhe der Agrarzahlungen in den USA im Zeitablauf

\begin{tabular}{|c|c|c|c|c|}
\hline Jahr & $\begin{array}{l}\text { „Government } \\
\text { payments“" } \\
\text { Mrd. USD }\end{array}$ & $\begin{array}{r}\text { Landwirtschaftl. } \\
\text { Nettoeinkommen } \\
\text { Mrd. USD }\end{array}$ & $\begin{array}{r}\text { „Government payments“ } \\
\text { in \% des landwirtschaftl. } \\
\text { Nettoeinkommens }\end{array}$ & Preisparität \\
\hline 1941 & 0,544 & 6,489 & 8,4 & 93 \\
\hline 1942 & 0,650 & 9,854 & 6,6 & 105 \\
\hline 1943 & 0,645 & 11,735 & 5,5 & 113 \\
\hline 1944 & 0,776 & 11,704 & 6,6 & 108 \\
\hline 1945 & 0,742 & 12,313 & 6,0 & 109 \\
\hline 1946 & 0,772 & 15,067 & 5,1 & 113 \\
\hline 1947 & 0,314 & 15,353 & 2,0 & 115 \\
\hline 1948 & 0,257 & 17,664 & 1,5 & 110 \\
\hline 1949 & 0,185 & 12,805 & 1,4 & 100 \\
\hline 1950 & 0,283 & 13,648 & 2,1 & 101 \\
\hline 1951 & 0,286 & 15,933 & 1,8 & 107 \\
\hline 1952 & 0,275 & 14,961 & 1,8 & 100 \\
\hline 1953 & 0,213 & 12,980 & 1,6 & 92 \\
\hline 1954 & 0,257 & 12,373 & 2,1 & 89 \\
\hline 1955 & 0,229 & 11,305 & 2,0 & 84 \\
\hline 1956 & 0,554 & 11,254 & 4,9 & 83 \\
\hline 1957 & 1,016 & 11,085 & 9,2 & 82 \\
\hline 1958 & 1,089 & 13,168 & 8,3 & 85 \\
\hline 1959 & 0,682 & 10,713 & 6,4 & 81 \\
\hline 1960 & 0,702 & 11,212 & 6,3 & 80 \\
\hline 1961 & 1,493 & 11,957 & 12,5 & 79 \\
\hline 1962 & 1,747 & 12,064 & 14,5 & 80 \\
\hline 1963 & 1,696 & 11,770 & 14,4 & 78 \\
\hline 1964 & 2,181 & 10,492 & 20,8 & 76 \\
\hline 1965 & 2,463 & 12,899 & 19,1 & 77 \\
\hline 1966 & 3,277 & 13,960 & 23,5 & 80 \\
\hline 1967 & 3,079 & 12,339 & 24,5 & 74 \\
\hline
\end{tabular}




\begin{tabular}{|c|c|c|c|c|}
\hline Jahr & $\begin{array}{l}\text { „Government } \\
\text { payments“" } \\
\text { Mrd. USD }\end{array}$ & $\begin{array}{r}\text { Landwirtschaftl. } \\
\text { Nettoeinkommen } \\
\text { Mrd. USD }\end{array}$ & $\begin{array}{r}\text { „Government payments“ } \\
\text { in } \% \text { des landwirtschaftl. } \\
\text { Nettoeinkommens }\end{array}$ & Preisparität \\
\hline 1968 & 3,462 & 12,322 & 28,3 & 73 \\
\hline 1969 & 3,794 & 14,293 & 26,5 & 74 \\
\hline 1970 & 3,717 & 14,366 & 25,9 & 72 \\
\hline 1971 & 3,145 & 15,012 & 20,9 & 69 \\
\hline 1972 & 3,961 & 19,455 & 20,4 & 74 \\
\hline 1973 & 2,607 & 34,356 & 7,6 & 91 \\
\hline 1974 & 0,530 & 27,267 & 1,9 & 86 \\
\hline 1975 & 0,807 & 25,510 & 3,2 & 76 \\
\hline 1976 & 0,734 & 20,175 & 3,6 & 71 \\
\hline 1977 & 1,819 & 19,881 & 9,1 & 66 \\
\hline 1978 & 3,030 & 25,198 & 12,0 & 70 \\
\hline 1979 & 1,375 & 27,415 & 5,0 & 71 \\
\hline 1980 & 1,286 & 16,141 & 8,0 & 65 \\
\hline 1981 & 1,932 & 26,879 & 7,2 & 61 \\
\hline 1982 & 3,492 & 23,841 & 14,6 & 57 \\
\hline 1983 & 9,295 & 14,262 & 65,2 & 56 \\
\hline 1984 & 8,430 & 25,975 & 32,5 & 57 \\
\hline 1985 & 7,704 & 28,509 & 27,0 & 52 \\
\hline 1986 & 11,813 & 31,117 & 38,0 & 51 \\
\hline 1987 & 16,747 & 38,005 & 44,1 & 49 \\
\hline 1988 & 14,480 & 39,633 & 36,5 & 53 \\
\hline 1989 & 10,887 & 46,487 & 23,4 & 53 \\
\hline 1990 & 9,298 & 46,261 & 20,1 & 51 \\
\hline 1991 & 8,214 & 40,234 & 20,4 & 48 \\
\hline 1992 & 9,169 & 50,171 & 18,3 & 47 \\
\hline 1993 & 13,402 & 46,725 & 28,7 & 47 \\
\hline 1994 & 7,879 & 52,567 & 15,0 & 45 \\
\hline
\end{tabular}




\begin{tabular}{|c|c|c|c|c|}
\hline Jahr & $\begin{array}{l}\text { "Government } \\
\text { payments“ } \\
\text { Mrd. USD }\end{array}$ & $\begin{array}{r}\text { Landwirtschaftl. } \\
\text { Nettoeinkommen } \\
\text { Mrd. USD }\end{array}$ & $\begin{array}{r}\text { „Government payments“ } \\
\text { in \% des landwirtschaftl. } \\
\text { Nettoeinkommens }\end{array}$ & Preisparität \\
\hline 1995 & 7,279 & 39,771 & 18,3 & 45 \\
\hline 1996 & 7,340 & 58,936 & 12,5 & 47 \\
\hline 1997 & 7,495 & 51,306 & 14,6 & 43 \\
\hline 1998 & 12,380 & 47,119 & 26,3 & 42 \\
\hline 1999 & 21,513 & 47,690 & 45,1 & 40 \\
\hline 2000 & 23,222 & 50,685 & 45,9 & 38 \\
\hline 2001 & 22,431 & 54,886 & 40,9 & 40 \\
\hline 2002 & 12,415 & 39,145 & 31,7 & 38 \\
\hline 2003 & 16,523 & 60,973 & 27,1 & 40 \\
\hline 2004 & 12,970 & 87,418 & 14,8 & 43 \\
\hline 2005 & 24,396 & 78,763 & 31,0 & 38 \\
\hline 2006 & 15,789 & 57,437 & 27,5 & 37 \\
\hline 2007 & 11,903 & 70,013 & 17,0 & 40 \\
\hline 2008 & 12,242 & 78,091 & 15,7 & 39 \\
\hline 2009 & 12,177 & 62,147 & 19,6 & 35 \\
\hline 2010 & 12,392 & 77.103 & 16,1 & 37 \\
\hline 2011 & 10,421 & 113,536 & 9,2 & 38 \\
\hline 2012 & 10,635 & 96,495 & 11,0 & 38 \\
\hline 2013 & 11,004 & 123,758 & 8,9 & 38 \\
\hline 2014 & 9,767 & 92,649 & 10,5 & 37 \\
\hline 2015 & 10,804 & 80,741 & 13,4 & k.A. \\
\hline 2016 & 13,485 & 71,477 & 18,9 & k.A. \\
\hline
\end{tabular}

Quelle: Spalte 2 und 3: ERS-USDA (2016b); Spalte 4: Eigene Berechnungen; Spalte 5: USDA (1963, 1974, 1988, 1998, 2006 und 2016). Zahlen für 2016 sind vorläufig.

Anmerkungen: Auch wenn „benefit payments“ und „rental payments“ 1933-1935 zum größten Teil nicht aus dem Staatshaushalt finanziert wurden, sind sie in der offiziellen Statistik von USDA als ,government payments“ aufgeführt. Die parallel zu den „,soilconserving payments“ im Jahr 1936 eingeführten Zahlungen für Umweltleistungen wie Gründüngerpflanzen oder Aufforstung wurden hier nicht erfasst. 
Preisparität: das Verhältnis der Erzeugerpreise landwirtschaftlicher Produkte zu nichtlandwirtschaftlichen Preisen. Liegt das Verhältnis unter 100, besitzen landwirtschaftliche Erzeugnisse weniger Kaufkraft als im Referenzzeitraum (1910 bis 1914).

Tabelle A2: Gesamtbetrag der primären Zahlungstypen in den USA seit 1933 (ausgedrückt in jeweiligen Preisen)

\begin{tabular}{|l|r|r|r|r|}
\hline Jahr & $\begin{array}{r}\text { "Commodity } \\
\text { payments“ } \\
\text { Mrd. USD }\end{array}$ & $\begin{array}{r}\text { "Conservation } \\
\text { payments“ } \\
\text { Mrd. USD }\end{array}$ & $\begin{array}{r}\text { "Miscellaneous } \\
\text { payments“ } \\
\text { Mrd. USD }\end{array}$ & $\begin{array}{r}\text { Gesamtzahlungen } \\
\text { Mrd. USD }\end{array}$ \\
\hline 1933 & n. z. & n. z. & n. z. & 0,131 \\
\hline 1934 & 0,051 & n. z. & 0,395 & 0,446 \\
\hline 1935 & 0,015 & n. z. & 0,558 & 0,573 \\
\hline 1936 & 0,041 & 0,024 & 0,213 & 0,278 \\
\hline 1937 & n. z. & 0,324 & 0,011 & 0,336 \\
\hline 1938 & 0,114 & 0,309 & 0,022 & 0,446 \\
\hline 1939 & 0,008 & 0,527 & 0,229 & 0,763 \\
\hline 1940 & n. z. & 0,496 & 0,227 & 0,723 \\
\hline 1941 & n. z. & 0,382 & 0,161 & 0,544 \\
\hline 1942 & n. z. & 0,450 & 0,200 & 0,650 \\
\hline 1943 & n. z. & 0,332 & 0,312 & 0,645 \\
\hline 1944 & n. z. & 0,378 & 0,398 & 0,776 \\
\hline 1945 & n. z. & 0,259 & 0,483 & 0,742 \\
\hline 1946 & n. z. & 0,285 & 0,487 & 0,772 \\
\hline 1947 & n. z. & 0,277 & 0,037 & 0,314 \\
\hline 1948 & n. z. & 0,218 & 0,039 & 0,257 \\
\hline 1949 & n. z. & 0,156 & 0,030 & 0,185 \\
\hline 1950 & n. z. & 0,246 & 0,037 & 0,283 \\
\hline 1951 & n. z. & 0,246 & 0,040 & 0,286 \\
\hline 1952 & n. z. & 0,242 & 0,033 & 0,275 \\
\hline 1953 & n. z. & 0,181 & 0,032 & 0,213 \\
\hline & & & & \\
\hline
\end{tabular}


Anhang: Übersicht über die Höhe der Agrarzahlungen in den USA im Zeitablauf

\begin{tabular}{|c|c|c|c|c|}
\hline Jahr & $\begin{array}{c}\text { „Commodity } \\
\text { payments“ } \\
\text { Mrd. USD }\end{array}$ & $\begin{array}{l}\text { "Conservation } \\
\text { payments“ } \\
\text { Mrd. USD }\end{array}$ & $\begin{array}{l}\text { „Miscellaneous } \\
\text { payments“ } \\
\text { Mrd. USD }\end{array}$ & $\begin{array}{r}\text { Gesamtzahlungen } \\
\text { Mrd. USD }\end{array}$ \\
\hline 1954 & n. z. & 0,217 & 0,040 & 0,257 \\
\hline 1955 & n. $z$. & 0,188 & 0,041 & 0,229 \\
\hline 1956 & 0,054 & 0,220 & 0,280 & 0,554 \\
\hline 1957 & 0,053 & 0,230 & 0,732 & 1,016 \\
\hline 1958 & 0,014 & 0,215 & 0,859 & 1,089 \\
\hline 1959 & 0,082 & 0,233 & 0,367 & 0,682 \\
\hline 1960 & 0,051 & 0,217 & 0,429 & 0,702 \\
\hline 1961 & 0,870 & 0,230 & 0,387 & 1,493 \\
\hline 1962 & 1,148 & 0,224 & 0,368 & 1,747 \\
\hline 1963 & 1,095 & 0,222 & 0,370 & 1,696 \\
\hline 1964 & 1,665 & 0,227 & 0,278 & 2,181 \\
\hline 1965 & 2,004 & 0,215 & 0,235 & 2,463 \\
\hline 1966 & 2,779 & 0,220 & 0,267 & 3,277 \\
\hline 1967 & 2,557 & 0,225 & 0,284 & 3,079 \\
\hline 1968 & 2,966 & 0,215 & 0,268 & 3,462 \\
\hline 1969 & 3,390 & 0,204 & 0,199 & 3,794 \\
\hline 1970 & 3,343 & 0,208 & 0,166 & 3,717 \\
\hline 1971 & 2,823 & 0,173 & 0,149 & 3,145 \\
\hline 1972 & 3,624 & 0,198 & 0,140 & 3,961 \\
\hline 1973 & 2,399 & 0,072 & 0,136 & 2,607 \\
\hline 1974 & 0,213 & 0,192 & 0,125 & 0,530 \\
\hline 1975 & 0,507 & 0,193 & 0,107 & 0,807 \\
\hline 1976 & 0,478 & 0,209 & 0,047 & 0,734 \\
\hline 1977 & 1,299 & 0,328 & 0,192 & 1,819 \\
\hline 1978 & 2,291 & 0,239 & 0,499 & 3,030 \\
\hline 1979 & 0,884 & 0,197 & 0,294 & 1,375 \\
\hline 1980 & 0,795 & 0,214 & 0,276 & 1,286 \\
\hline
\end{tabular}




\begin{tabular}{|c|c|c|c|c|}
\hline Jahr & $\begin{array}{c}\text { "Commodity } \\
\text { payments“ } \\
\text { Mrd. USD }\end{array}$ & $\begin{array}{l}\text { „Conservation } \\
\text { payments“ } \\
\text { Mrd. USD }\end{array}$ & $\begin{array}{l}\text { „Miscellaneous } \\
\text { payments“ } \\
\text { Mrd. USD }\end{array}$ & $\begin{array}{r}\text { Gesamtzahlungen } \\
\text { Mrd. USD }\end{array}$ \\
\hline 1981 & 1,126 & 0,201 & 0,605 & 1,932 \\
\hline 1982 & 2,368 & 0,179 & 0,946 & 3,492 \\
\hline 1983 & 3,234 & 0,188 & 5,874 & 9,295 \\
\hline 1984 & 2,746 & 0,191 & 5,493 & 8,430 \\
\hline 1985 & 6,591 & 0,189 & 0,924 & 7,704 \\
\hline 1986 & 10,235 & 0,254 & 1,325 & 11,813 \\
\hline 1987 & 13,244 & 1,531 & 1,972 & 16,747 \\
\hline 1988 & 10,567 & 1,607 & 2,306 & 14,480 \\
\hline 1989 & 5,680 & 1,771 & 3,436 & 10,887 \\
\hline 1990 & 6,014 & 1,898 & 1,386 & 9,298 \\
\hline 1991 & 5,925 & 1,858 & 0,431 & 8,214 \\
\hline 1992 & 5,354 & 1,899 & 1,916 & 9,169 \\
\hline 1993 & 8,802 & 1,967 & 2,633 & 13,402 \\
\hline 1994 & 3,968 & 1,978 & 1,933 & 7,879 \\
\hline 1995 & 4,572 & 1,896 & 0,811 & 7,279 \\
\hline 1996 & 5,241 & 1,793 & 0,306 & 7,340 \\
\hline 1997 & 5,545 & 1,693 & 0,257 & 7,795 \\
\hline 1998 & 7,950 & 1,475 & 2,955 & 12,380 \\
\hline 1999 & 11,859 & 1,494 & 8,161 & 21,513 \\
\hline 2000 & 12,560 & 1,615 & 8,682 & 23,222 \\
\hline 2001 & 11,916 & 1,903 & 8,612 & 22,431 \\
\hline 2002 & 8,748 & 1,966 & 1,701 & 12,415 \\
\hline 2003 & 11,206 & 2,167 & 3,150 & 16,523 \\
\hline 2004 & 10,063 & 2,320 & 0,588 & 12,970 \\
\hline 2005 & 18,450 & 2,767 & 3,179 & 24,396 \\
\hline 2006 & 12,538 & 2,974 & 0,276 & 15,789 \\
\hline 2007 & 8,305 & 3,072 & 0,527 & 11,903 \\
\hline
\end{tabular}




\begin{tabular}{|l|r|r|r|r|}
\hline Jahr & $\begin{array}{r}\text { "Commodity } \\
\text { payments“ } \\
\text { Mrd. USD }\end{array}$ & $\begin{array}{r}\text { "Conservation } \\
\text { payments“ } \\
\text { Mrd. USD }\end{array}$ & $\begin{array}{r}\text { "Miscellaneous } \\
\text { payments“ } \\
\text { Mrd. USD }\end{array}$ & $\begin{array}{r}\text { Gesamtzahlungen } \\
\text { Mrd. USD }\end{array}$ \\
\hline 2008 & 6,959 & 3,155 & 2,127 & 12,242 \\
\hline 2009 & 8,675 & 2,824 & 0,678 & 12,177 \\
\hline 2010 & 6,527 & 3,219 & 2,646 & 12,392 \\
\hline 2011 & 5,440 & 3,674 & 1,306 & 10,421 \\
\hline 2012 & 5,838 & 3,695 & 1,102 & 10,635 \\
\hline 2013 & 5,381 & 3,680 & 1,943 & 11,004 \\
\hline 2014 & 1,480 & 3,561 & 4,726 & 9,767 \\
\hline 2015 & 5,385 & 3,619 & 1,801 & 10,804 \\
\hline
\end{tabular}

Quelle: ERS-USDA (2016c).

Anmerkung: „Miscellaneous payments“ umfassen v.a. „disaster payments“.

Tabelle A3: Gesamtbetrag der primären Zahlungstypen in den USA seit 1933 (ausgedrückt in Preisen von 2009)

\begin{tabular}{|l|r|r|r|r|}
\hline Jahr & $\begin{array}{r}\text { "Commodity } \\
\text { payments } \\
\text { Mrd. USD }\end{array}$ & $\begin{array}{r}\text { "Conservation } \\
\text { payments“ } \\
\text { Mrd. USD }\end{array}$ & $\begin{array}{r}\text { "Miscellaneous } \\
\text { payments“ } \\
\text { Mrd. USD }\end{array}$ & $\begin{array}{r}\text { Gesamtzahlungen } \\
\text { Mrd. USD }\end{array}$ \\
\hline 1933 & n. z. & n. z. & n. z. & 1,771 \\
\hline 1934 & 0,657 & n. z. & 5,092 & 5,750 \\
\hline 1935 & 0,190 & n. z. & 7,052 & 7,241 \\
\hline 1936 & 0,512 & 0,300 & 2,660 & 3,471 \\
\hline 1937 & n. z. & 3,902 & 0,132 & 4,047 \\
\hline 1938 & 1,399 & 3,791 & 0,270 & 5,472 \\
\hline 1939 & 0,099 & 6,547 & 2,845 & 9,479 \\
\hline 1940 & n. z. & 6,106 & 2,795 & 8,901 \\
\hline 1941 & n. z. & 4,413 & 1,860 & 6,285 \\
\hline 1942 & n. z. & 4,800 & 2,133 & 6,933 \\
\hline 1943 & n. z. & 3,381 & 3,177 & 6,568 \\
\hline 1944 & n. z. & 3,759 & 3,958 & 7,718 \\
\hline
\end{tabular}




\begin{tabular}{|c|c|c|c|c|}
\hline Jahr & $\begin{array}{c}\text { „Commodity } \\
\text { payments“ } \\
\text { Mrd. USD }\end{array}$ & $\begin{array}{l}\text { „Conservation } \\
\text { payments“ } \\
\text { Mrd. USD }\end{array}$ & $\begin{array}{l}\text { „Miscellaneous } \\
\text { payments“ } \\
\text { Mrd. USD }\end{array}$ & $\begin{array}{r}\text { Gesamtzahlungen } \\
\text { Mrd. USD }\end{array}$ \\
\hline 1945 & n. z. & 2,513 & 4,686 & 7,198 \\
\hline 1946 & n. $z$. & 2,456 & 4,197 & 6,653 \\
\hline 1947 & n. $z$. & 2,147 & 0,287 & 2,434 \\
\hline 1948 & n. $z$. & 1,600 & 0,286 & 1,886 \\
\hline 1949 & n. z. & 1,145 & 0,220 & 1,361 \\
\hline 1950 & n. z. & 1,790 & 0,269 & 2,062 \\
\hline 1951 & n. $z$. & 1,676 & 0,273 & 1,946 \\
\hline 1952 & n. $z$. & 1,614 & 0,220 & 1,831 \\
\hline 1953 & n. z. & 1,192 & 0,211 & 1,403 \\
\hline 1954 & n. $z$. & 1,414 & 0,261 & 1,677 \\
\hline 1955 & n. $z$. & 1,208 & 0,263 & 1,468 \\
\hline 1956 & 0,335 & 1,366 & 1,739 & 3,438 \\
\hline 1957 & 0,318 & 1,380 & 4,393 & 6,096 \\
\hline 1958 & 0,082 & 1,261 & 5,038 & 6,390 \\
\hline 1959 & 0,475 & 1,349 & 2,124 & 3,945 \\
\hline 1960 & 0,291 & 1,239 & 2,449 & 4,010 \\
\hline 1961 & 4,913 & 1,298 & 2,185 & 8,433 \\
\hline 1962 & 6,404 & 1,250 & 2,053 & 9,742 \\
\hline 1963 & 6,040 & 1,226 & 2,041 & 9,355 \\
\hline 1964 & 9,045 & 1,234 & 1,510 & 11,847 \\
\hline 1965 & 10,691 & 1,445 & 1,254 & 13,141 \\
\hline 1966 & 14,421 & 1,141 & 1,386 & 17,004 \\
\hline 1967 & 12,894 & 1,136 & 1,432 & 15,525 \\
\hline 1968 & 14,347 & 1,039 & 1,296 & 16,746 \\
\hline 1969 & 15,629 & 0,940 & 0,917 & 17,490 \\
\hline 1970 & 14,639 & 0,911 & 0,727 & 16,279 \\
\hline 1971 & 11,764 & 0,721 & 0,621 & 13,105 \\
\hline
\end{tabular}


Anhang: Übersicht über die Höhe der Agrarzahlungen in den USA im Zeitablauf

\begin{tabular}{|c|c|c|c|c|}
\hline Jahr & $\begin{array}{c}\text { „Commodity } \\
\text { payments“ } \\
\text { Mrd. USD }\end{array}$ & $\begin{array}{l}\text { „Conservation } \\
\text { payments“ } \\
\text { Mrd. USD }\end{array}$ & $\begin{array}{l}\text { „Miscellaneous } \\
\text { payments“ } \\
\text { Mrd. USD }\end{array}$ & $\begin{array}{r}\text { Gesamtzahlungen } \\
\text { Mrd. USD }\end{array}$ \\
\hline 1972 & 14,476 & 0,791 & 0,559 & 15,822 \\
\hline 1973 & 9,088 & 0,273 & 0,515 & 9,877 \\
\hline 1974 & 0,741 & 0,668 & 0,435 & 1,844 \\
\hline 1975 & 1,612 & 0,614 & 0,340 & 2,568 \\
\hline 1976 & 1,442 & 0,630 & 0,142 & 2,213 \\
\hline 1977 & 3,689 & 0,932 & 0,545 & 5,166 \\
\hline 1978 & 6,080 & 0,634 & 1,324 & 8,041 \\
\hline 1979 & 2,168 & 0,482 & 0,721 & 3,371 \\
\hline 1980 & 1,788 & 0,482 & 0,621 & 2,890 \\
\hline 1981 & 2,315 & 0,413 & 1,243 & 3,971 \\
\hline 1982 & 4,587 & 0,346 & 1,832 & 6,764 \\
\hline 1983 & 6,026 & 0,349 & 10,947 & 17,323 \\
\hline 1984 & 4,942 & 0,345 & 9,886 & 15,172 \\
\hline 1985 & 11,495 & 0,329 & 1,612 & 13,436 \\
\hline 1986 & 17,494 & 0,434 & 2,264 & 20,192 \\
\hline 1987 & 22,097 & 2,554 & 3,290 & 27,941 \\
\hline 1988 & 17,033 & 2,591 & 3,717 & 23,341 \\
\hline 1989 & 8,813 & 2,747 & 5,331 & 16,892 \\
\hline 1990 & 8,998 & 2,840 & 2,073 & 13,911 \\
\hline 1991 & 8,580 & 2,691 & 0,625 & 11,895 \\
\hline 1992 & 7,579 & 2,689 & 2,713 & 12,981 \\
\hline 1993 & 12,172 & 2,720 & 3,642 & 18,533 \\
\hline 1994 & 5,373 & 2,678 & 2,618 & 10,669 \\
\hline 1995 & 6,065 & 2,515 & 1,075 & 9,655 \\
\hline 1996 & 6,827 & 2,335 & 0,399 & 9,561 \\
\hline 1997 & 7,101 & 2,168 & 0,329 & 9,599 \\
\hline 1998 & 10,071 & 1,868 & 3,744 & 15,684 \\
\hline
\end{tabular}




\begin{tabular}{|c|c|c|c|c|}
\hline Jahr & $\begin{array}{l}\text { „Commodity } \\
\text { payments“ } \\
\text { Mrd. USD }\end{array}$ & $\begin{array}{l}\text { „Conservation } \\
\text { payments“ } \\
\text { Mrd. USD }\end{array}$ & $\begin{array}{l}\text { „Miscellaneous } \\
\text { payments“ } \\
\text { Mrd. USD }\end{array}$ & $\begin{array}{r}\text { Gesamtzahlungen } \\
\text { Mrd. USD }\end{array}$ \\
\hline 1999 & 14,812 & 1,866 & 10,192 & 26,870 \\
\hline 2000 & 15,386 & 1,972 & 10,602 & 28,357 \\
\hline 2001 & 14,227 & 2,273 & 10,282 & 26,782 \\
\hline 2002 & 10,286 & 2,312 & 2,000 & 14,599 \\
\hline 2003 & 12,920 & 2,499 & 3,632 & 19,051 \\
\hline 2004 & 11,291 & 2,603 & 0,660 & 14,554 \\
\hline 2005 & 20,057 & 3,009 & 3,456 & 26,522 \\
\hline 2006 & 13,224 & 3,137 & 0,291 & 16,653 \\
\hline 2007 & 8,531 & 3,156 & 0,541 & 12,229 \\
\hline 2008 & 7,014 & 3,180 & 2,144 & 12,338 \\
\hline 2009 & 8,675 & 2,825 & 0,678 & 12,177 \\
\hline 2010 & 6,448 & 3,180 & 2,613 & 12,242 \\
\hline 2011 & 5,265 & 3,556 & 1,265 & 10,086 \\
\hline 2012 & 5,549 & 3,512 & 1,047 & 10,108 \\
\hline 2013 & 5,032 & 3,441 & 1,817 & 10,290 \\
\hline 2014 & 1,361 & 3,277 & 4,348 & 8,986 \\
\hline 2015 & 4,901 & 3,294 & 1,639 & 9,834 \\
\hline
\end{tabular}

Quelle: ERS-USDA (2016d). 
ange Zeit stellten die entkoppelten Direktzahlungen ein wichtiges Instrument zur Förderung der Landwirtschaft sowohl in den USA als auch in der EU dar. Ihre Abschaffung in den USA durch die Agrarreform von 2014 markierte den Bruch des bisherigen Trends zur Angleichung der Agrarpolitiken beider Akteure. Die vorliegende Arbeit erklärt mithilfe der Konvergenztheorie die Ursachen für die neu auftretenden Divergenzen. Es werden die Gründe für den Reformdruck in Bezug auf die Direktzahlungen identifiziert sowie die Gemeinsamkeiten und Unterschiede zwischen den USA und der EU herausgearbeitet und bewertet. Schließlich werden die Konvergenzmuster untersucht und der Konvergenzprozess erklärt. Der Analyse liegt ein umfassender Vergleich der jeweiligen Entwicklung der Direktzahlungen zugrunde, einschließlich ihrer Behandlung in der wissenschaftlichen Literatur auf beiden Seiten des Atlantiks. 